\title{
Synthesis and Characterization of Strongly Solvatochromic Molybdenum(III) Complexes
}

Sarah D. Helland, Alison S. Chang, ${ }^{\dagger \neq}$ Keren W. Lee, ${ }^{\dagger \neq}$ Phillips S. Hutchison,,$^{\dagger}$ William W. Brennessel", and William T. Eckenhoff'*

${ }^{\dagger}$ Department of Chemistry, Rhodes College, 2000 N. Parkway, Memphis, Tennessee 38112, United States

"Department of Chemistry, University of Rochester, 120 Trustee Road, Rochester, New York 14627, United States 
REFERENCE NUMBER: eiswe28

CRYSTAL STRUCTURE REPORT

$\mathrm{C}_{20} \mathrm{H}_{16} \mathrm{Cl}_{4} \mathrm{Li} \mathrm{Mo} \mathrm{N} 4$

or

$\left\{\mathrm{Li}(\mathrm{bpy}) \mathrm{MoCl}_{4}(\mathrm{bpy})\right\}_{\mathrm{n}}$

Report prepared for:

Dr. W. Eckenhoff, Prof. R. Eisenberg

February 13, 2013

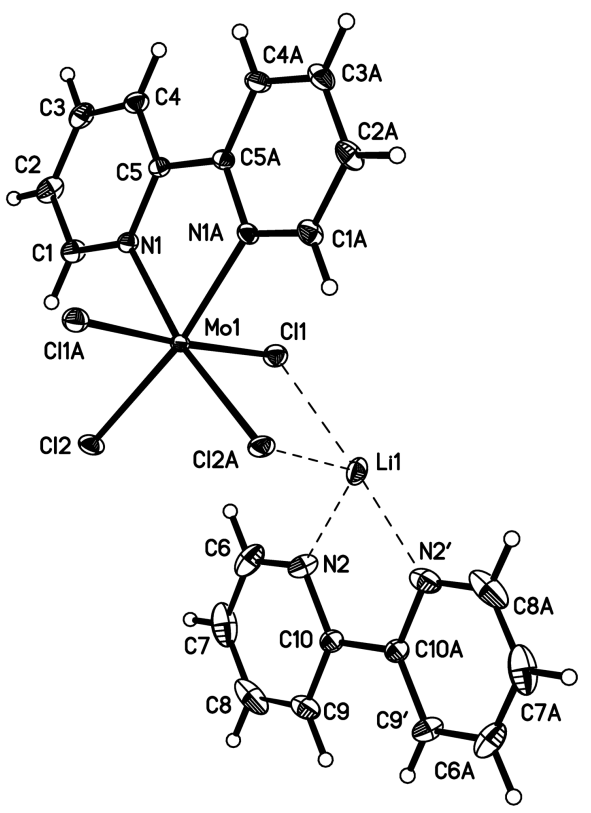

William W. Brennessel

X-ray Crystallographic Facility

Department of Chemistry, University of Rochester

120 Trustee Road

Rochester, NY 14627 


\section{Data collection}

A crystal $\left(0.28 \times 0.14 \times 0.12 \mathrm{~mm}^{3}\right)$ was placed onto the tip of a $0.1 \mathrm{~mm}$ diameter glass capillary tube or fiber and mounted on a Bruker SMART APEX II CCD Platform diffractometer for a data collection at 100.0(5) K. ${ }^{1}$ A preliminary set of cell constants and an orientation matrix were calculated from reflections harvested from three orthogonal wedges of reciprocal space. The full data collection was carried out using MoK $\alpha$ radiation (graphite monochromator) with a frame time of 45 seconds and a detector distance of $4.01 \mathrm{~cm}$. A randomly oriented region of reciprocal space was surveyed: five major sections of frames were collected with $0.50^{\circ}$ steps in $\omega$ at five different $\phi$ settings and a detector position of $-38^{\circ}$ in $2 \theta$. The intensity data were corrected for absorption. ${ }^{2}$ Final cell constants were calculated from the xyz centroids of 4044 strong reflections from the actual data collection after integration. ${ }^{3}$ See Table 1 for additional crystal and refinement information.

\section{Structure solution and refinement}

The structure was solved using SIR97 ${ }^{4}$ and refined using SHELXL-2012..$^{5}$ The space group $C 2 / c$ was determined based on systematic absences and intensity statistics. A direct-methods solution was calculated which provided most non-hydrogen atoms from the E-map. Full-matrix least squares / difference Fourier cycles were performed which located the remaining non-hydrogen atoms. All non-hydrogen atoms were refined with anisotropic displacement parameters. All hydrogen atoms were placed in ideal positions and refined as riding atoms with relative isotropic displacement parameters. The final full matrix least squares refinement converged to $R 1=0.0404$ $\left(F^{2}, I>2 \sigma(I)\right)$ and $w R 2=0.0863\left(F^{2}\right.$, all data).

\section{Structure description}

The structure is related to the one suggested. The asymmetric unit contains one molybdenum complex anion on a crystallographic two-fold axis and one lithium(bipyridine) cation in a crystallographic inversion center. Each lithium cation is coordinated to chloride ligands of one molybdenum complex anion and to the two nitrogen atoms of one cocrystallized bipyridine molecule. Because the cocrystallized bipyridine molecule is in a crystallographic inversion center, the lithium and nitrogen atoms are found either on one side of the bipyridine molecule or the other throughout the crystal, in a 1:1 ratio due to the center. In support of this disorder model, the electron density is modeled best with one lithium cation per molybdenum complex anion.

Due to the disorder (see above), the structure appears to be two-dimensional. However, in a given chain of alternating $\mathrm{Li}$ (bpy) and $\mathrm{MoCl}_{4}$ (bpy) ion pairs, the lithium atoms are only associated with one side of each cocrystallized bipyridine molecule, which leaves aromatic $\mathrm{C}-\mathrm{H}$ donors to (very weakly, if at all) inteact with the next chain's chloride ligands. Thus the structure is only polymeric in one dimension.

The molecules are infinitely pi-stacked in the direction parallel to the $c$-axis (see diagrams). 
Unless noted otherwise all structural diagrams containing thermal displacement ellipsoids are drawn at the $50 \%$ probability level.

Data collection, structure solution, and structure refinement were conducted at the X-ray Crystallographic Facility, B51 Hutchison Hall, Department of Chemistry, University of Rochester. All publications arising from this report MUST either 1) include William W. Brennessel as a coauthor or 2) acknowledge William W. Brennessel and the Xray Crystallographic Facility of the Department of Chemistry at the University of Rochester.

1 APEX2, version 2012.4-3; Bruker AXS: Madison, WI, 2012.

2 Sheldrick, G. M. SADABS, version 2008/1; University of Göttingen: Göttingen, Germany, 2008.

3 SAINT, version 7.68A; Bruker AXS: Madison, WI, 2009.

4 Altomare, A.; Burla, M. C.; Camalli, M.; Cascarano, G. L.; Giacovazzo, C.; Guagliardi, A.; Moliterni, A. G. G.;

Polidori, G.; Spagna, R. SIR97: A new program for solving and refining crystal structures; Istituto di Cristallografia, CNR: Bari, Italy, 1999.

5 Sheldrick, G. M. SHELXL-2012 University of Göttingen: Göttingen, Germany, 2012.

Some equations of interest:

$$
\begin{gathered}
R_{\text {int }}=\Sigma\left|F_{\mathrm{o}}^{2}-<F_{\mathrm{o}}^{2}>\right| / \Sigma\left|F_{\mathrm{o}}{ }^{2}\right| \\
R 1=\Sigma|| F_{\mathrm{o}}|-| F_{\mathrm{c}}|/ \Sigma| F_{\mathrm{o}} \mid \\
w R 2=\left[\Sigma\left[w\left(F_{\mathrm{o}}{ }^{2} F_{\mathrm{c}}{ }^{2}\right)^{2}\right] / \Sigma\left[w\left(F_{\mathrm{o}}{ }^{2}\right)^{2}\right]\right]^{1 / 2} \\
\text { where } w=1 /\left[\sigma^{2}\left(F_{\mathrm{o}}{ }^{2}\right)+(a P)^{2}+b P\right] \text { and } \\
P=1 / 3 \max \left(0, F_{\mathrm{o}}{ }^{2}\right)+2 / 3 F_{\mathrm{c}}{ }^{2} \\
\mathrm{GOF}=S=\left[\Sigma\left[w\left(F_{\mathrm{o}}{ }^{2}-F_{\mathrm{c}}{ }^{2}\right)^{2}\right] /(m-n)\right]^{1 / 2}
\end{gathered}
$$

where $m=$ number of reflections and $n=$ number of parameters 


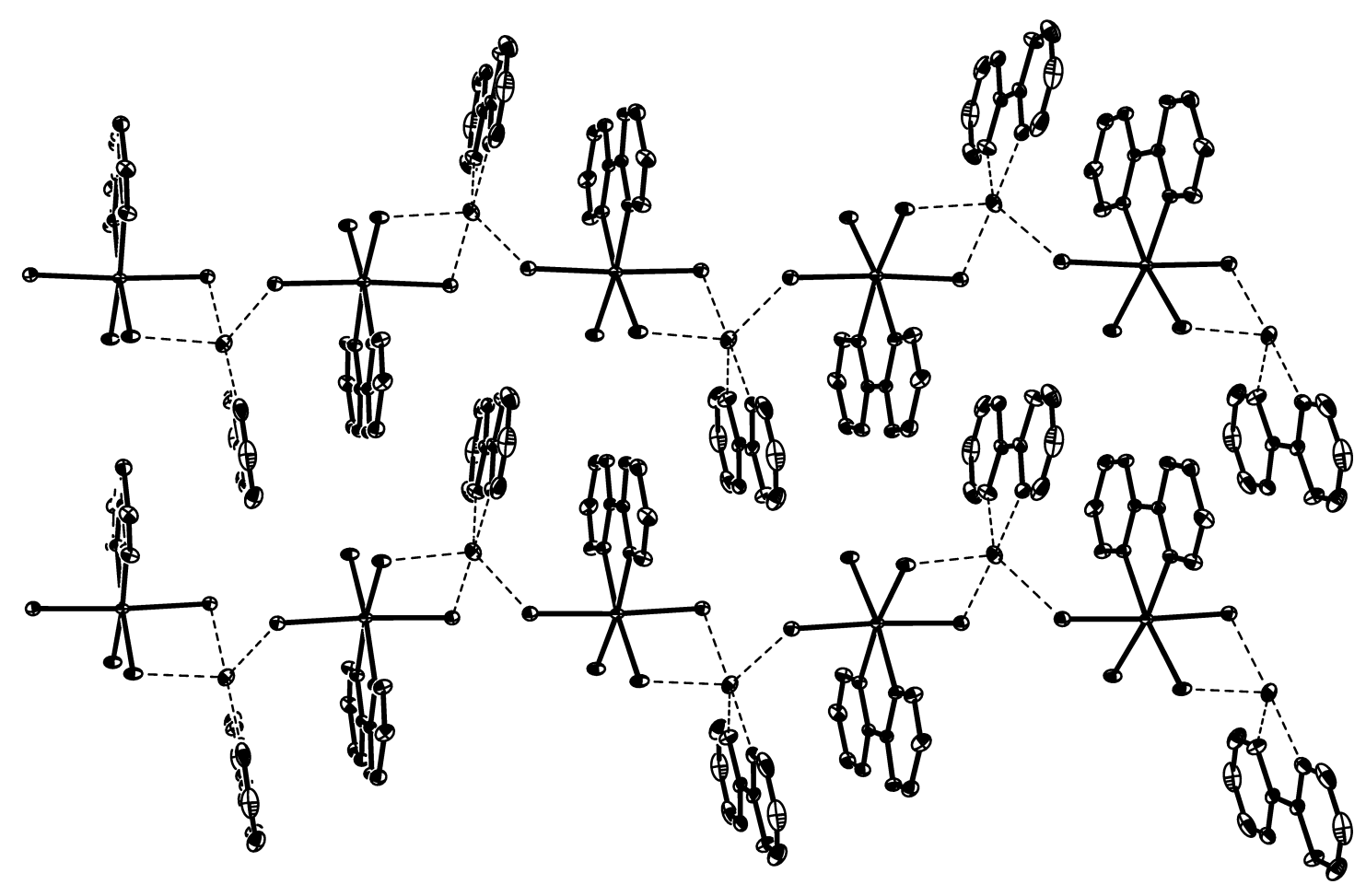

One connectivity scenario.

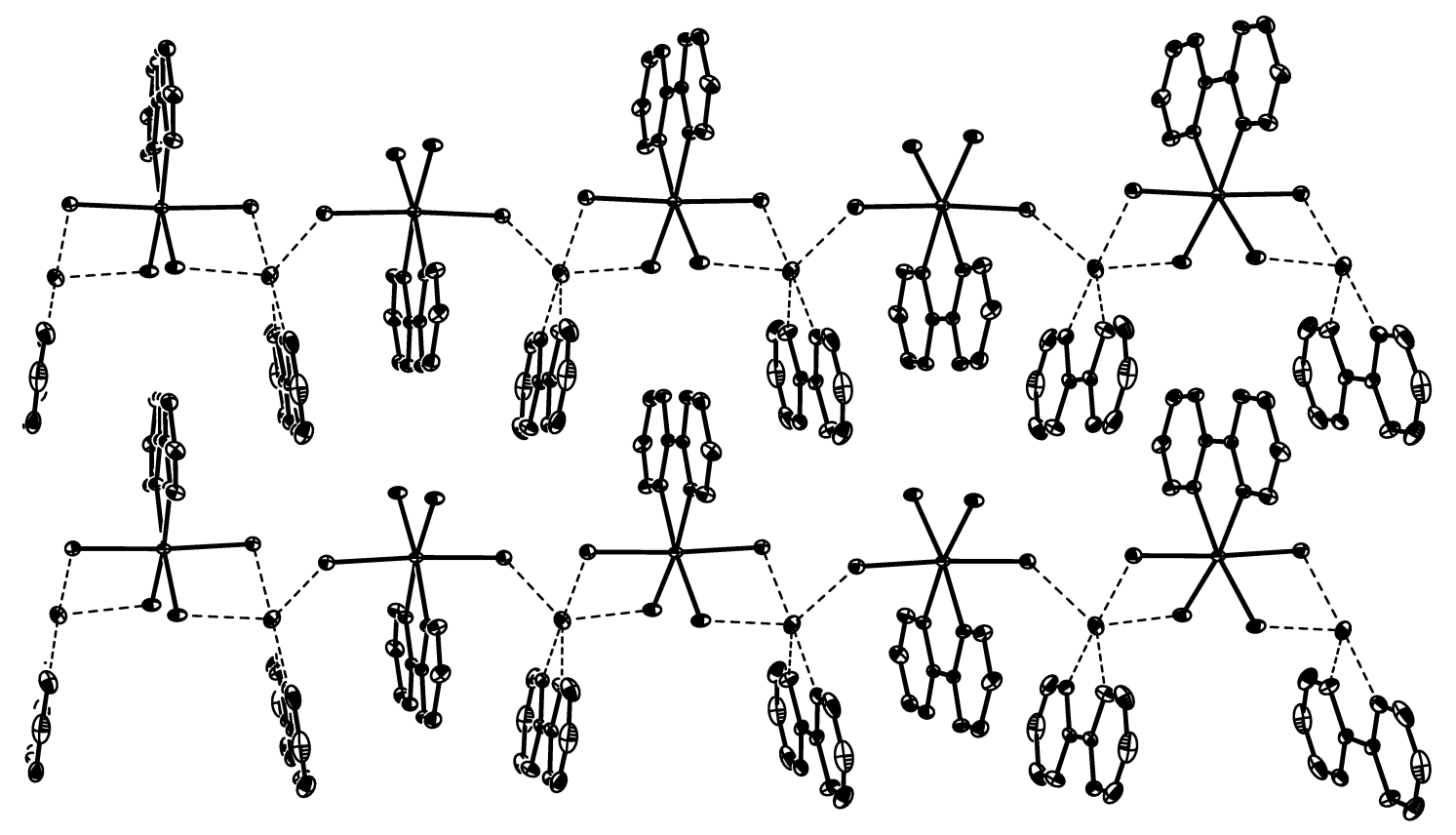

Another connectivity scenario. 
Table 1. Crystal data and structure refinement for eiswe28.

\begin{tabular}{|c|c|c|}
\hline Identification code & \multicolumn{2}{|l|}{ eiswe28 } \\
\hline Empirical formula & \multicolumn{2}{|c|}{ C20 H16 Cl4 Li Mo N4 } \\
\hline Formula weight & \multicolumn{2}{|l|}{557.05} \\
\hline Temperature & \multicolumn{2}{|l|}{$100.0(5) \mathrm{K}$} \\
\hline Wavelength & \multicolumn{2}{|l|}{$0.71073 \AA$} \\
\hline Crystal system & \multicolumn{2}{|l|}{ monoclinic } \\
\hline Space group & \multicolumn{2}{|l|}{$C 2 / c$} \\
\hline \multirow[t]{3}{*}{ Unit cell dimensions } & $a=17.866(2) \AA$ & $\alpha=90^{\circ}$ \\
\hline & $b=9.1865(11) \AA$ & $\beta=115.250(2)^{\circ}$ \\
\hline & $c=13.9860(17) \AA$ & $\gamma=90^{\circ}$ \\
\hline Volume & \multicolumn{2}{|l|}{$2076.2(4) \AA^{3}$} \\
\hline$Z$ & \multicolumn{2}{|l|}{4} \\
\hline Density (calculated) & \multicolumn{2}{|l|}{$1.782 \mathrm{Mg} / \mathrm{m}^{3}$} \\
\hline Absorption coefficient & \multicolumn{2}{|l|}{$1.162 \mathrm{~mm}^{-1}$} \\
\hline$F(000)$ & \multicolumn{2}{|l|}{1108} \\
\hline Crystal color, morphology & \multicolumn{2}{|l|}{ orange, block } \\
\hline Crystal size & \multicolumn{2}{|c|}{$0.28 \times 0.14 \times 0.12 \mathrm{~mm}^{3}$} \\
\hline Theta range for data collection & \multicolumn{2}{|l|}{2.521 to $37.783^{\circ}$} \\
\hline Index ranges & \multicolumn{2}{|c|}{$-30 \leq h \leq 30,-15 \leq k \leq 15,-24 \leq l \leq 24$} \\
\hline Reflections collected & \multicolumn{2}{|l|}{31492} \\
\hline Independent reflections & \multicolumn{2}{|c|}{$5574[R($ int $)=0.0500]$} \\
\hline Observed reflections & \multicolumn{2}{|l|}{4506} \\
\hline Completeness to theta $=37.785^{\circ}$ & \multicolumn{2}{|l|}{$99.9 \%$} \\
\hline Absorption correction & \multicolumn{2}{|l|}{ Multi-scan } \\
\hline Max. and min. transmission & \multicolumn{2}{|l|}{0.7476 and 0.6972} \\
\hline Refinement method & \multicolumn{2}{|c|}{ Full-matrix least-squares on $F^{2}$} \\
\hline Data / restraints / parameters & \multicolumn{2}{|l|}{$5574 / 0 / 141$} \\
\hline Goodness-of-fit on $F^{2}$ & \multicolumn{2}{|l|}{1.144} \\
\hline Final $R$ indices $[I>2 \operatorname{sigma}(I)]$ & \multicolumn{2}{|c|}{$R 1=0.0404, w R 2=0.0833$} \\
\hline$R$ indices (all data) & \multicolumn{2}{|c|}{$R 1=0.0530, w R 2=0.0863$} \\
\hline Extinction coefficient & \multicolumn{2}{|l|}{$\mathrm{n} / \mathrm{a}$} \\
\hline Largest diff. peak and hole & \multicolumn{2}{|c|}{1.331 and -0.795 e. $\AA^{-3}$} \\
\hline
\end{tabular}


Table 2. Atomic coordinates (x $\left.10^{4}\right)$ and equivalent isotropic displacement parameters $\left(\AA^{2} \times 10^{3}\right)$ for eiswe28. $U_{e q}$ is defined as one third of the trace of the orthogonalized $U_{i j}$ tensor.

\begin{tabular}{llrll}
\hline & $\mathrm{x}$ & $\mathrm{y}$ & $\mathrm{z}$ & $\mathrm{U}$ \\
& & & & \\
\hline Mo1 & 5000 & $5099(1)$ & 2500 & $10(1)$ \\
$\mathrm{C} 11$ & $5744(1)$ & $5179(1)$ & $4419(1)$ & $16(1)$ \\
$\mathrm{C} 12$ & $5956(1)$ & $3305(1)$ & $2380(1)$ & $16(1)$ \\
$\mathrm{N} 1$ & $5731(1)$ & $6953(2)$ & $2466(1)$ & $12(1)$ \\
C1 & $6486(1)$ & $6877(2)$ & $2479(2)$ & $16(1)$ \\
C2 & $6958(1)$ & $8099(2)$ & $2550(2)$ & $19(1)$ \\
C3 & $6642(1)$ & $9454(2)$ & $2611(2)$ & $18(1)$ \\
C4 & $5857(1)$ & $9543(2)$ & $2577(2)$ & $16(1)$ \\
C5 & $5417(1)$ & $8278(2)$ & $2508(1)$ & $11(1)$ \\
Li1 & $4841(5)$ & $3092(8)$ & $4623(6)$ & $18(1)$ \\
N2 & $5728(1)$ & $1368(2)$ & $4915(2)$ & $19(1)$ \\
C9 & $5870(1)$ & $-1213(2)$ & $5197(2)$ & $22(1)$ \\
N2' & $5870(1)$ & $-1213(2)$ & $5197(2)$ & $22(1)$ \\
C9' & $5728(1)$ & $1368(2)$ & $4915(2)$ & $19(1)$ \\
C6 & $6514(1)$ & $1427(3)$ & $5001(2)$ & $26(1)$ \\
C7 & $6998(2)$ & $189(3)$ & $5194(2)$ & $34(1)$ \\
C8 & $6653(2)$ & $-1122(3)$ & $5271(2)$ & $34(1)$ \\
C10 & $41(2)$ & $5028(1)$ & $15(1)$ \\
& $5424(1)$ & & & \\
\hline
\end{tabular}


Table 3. Bond lengths $[\AA]$ and angles $\left[{ }^{\circ}\right]$ for eiswe 28 .

\begin{tabular}{|c|c|c|c|}
\hline $\operatorname{Mo}(1)-\mathrm{N}(1)$ & $2.1590(15)$ & $C(10)-C(10) \# 3$ & $1.484(4)$ \\
\hline $\mathrm{Mo}(1)-\mathrm{N}(1) \# 1$ & $2.1590(15)$ & $\mathrm{N}(1)-\mathrm{Mo}(1)-\mathrm{N}(1) \# 1$ & $75.81(8)$ \\
\hline $\operatorname{Mo}(1)-\mathrm{Cl}(2) \# 1$ & $2.4307(5)$ & $\mathrm{N}(1)-\mathrm{Mo}(1)-\mathrm{Cl}(2) \# 1$ & $170.36(4)$ \\
\hline $\mathrm{Mo}(1)-\mathrm{Cl}(2)$ & $2.4307(5)$ & $\mathrm{N}(1) \# 1-\mathrm{Mo}(1)-\mathrm{Cl}(2) \# 1$ & $94.81(4)$ \\
\hline $\operatorname{Mo}(1)-\mathrm{Cl}(1)$ & $2.4359(5)$ & $\mathrm{N}(1)-\mathrm{Mo}(1)-\mathrm{Cl}(2)$ & $94.81(4)$ \\
\hline $\operatorname{Mo}(1)-\mathrm{Cl}(1) \# 1$ & $2.4359(5)$ & $\mathrm{N}(1) \# 1-\mathrm{Mo}(1)-\mathrm{Cl}(2)$ & $170.36(4)$ \\
\hline $\mathrm{Cl}(1)-\operatorname{Li}(1) \# 2$ & $2.569(7)$ & $\mathrm{Cl}(2) \# 1-\mathrm{Mo}(1)-\mathrm{Cl}(2)$ & $94.65(2)$ \\
\hline $\mathrm{Cl}(1)-\operatorname{Li}(1)$ & $2.599(7)$ & $\mathrm{N}(1)-\mathrm{Mo}(1)-\mathrm{Cl}(1)$ & $87.11(4)$ \\
\hline $\mathrm{Cl}(2)-\operatorname{Li}(1) \# 1$ & $2.552(8)$ & $\mathrm{N}(1) \# 1-\mathrm{Mo}(1)-\mathrm{Cl}(1)$ & $90.15(4)$ \\
\hline $\mathrm{N}(1)-\mathrm{C}(1)$ & $1.343(2)$ & $\mathrm{Cl}(2) \# 1-\mathrm{Mo}(1)-\mathrm{Cl}(1)$ & $90.777(17)$ \\
\hline $\mathrm{N}(1)-\mathrm{C}(5)$ & $1.352(2)$ & $\mathrm{Cl}(2)-\mathrm{Mo}(1)-\mathrm{Cl}(1)$ & $91.574(17)$ \\
\hline $\mathrm{C}(1)-\mathrm{C}(2)$ & $1.382(3)$ & $\mathrm{N}(1)-\mathrm{Mo}(1)-\mathrm{Cl}(1) \# 1$ & $90.15(4)$ \\
\hline $\mathrm{C}(1)-\mathrm{H}(1)$ & 0.9500 & $\mathrm{~N}(1) \# 1-\mathrm{Mo}(1)-\mathrm{Cl}(1) \# 1$ & $87.11(4)$ \\
\hline$C(2)-C(3)$ & $1.385(3)$ & $\mathrm{Cl}(2) \# 1-\mathrm{Mo}(1)-\mathrm{Cl}(1) \# 1$ & $91.573(17)$ \\
\hline $\mathrm{C}(2)-\mathrm{H}(2)$ & 0.9500 & $\mathrm{Cl}(2)-\mathrm{Mo}(1)-\mathrm{Cl}(1) \# 1$ & $90.775(17)$ \\
\hline$C(3)-C(4)$ & $1.386(3)$ & $\mathrm{Cl}(1)-\mathrm{Mo}(1)-\mathrm{Cl}(1) \# 1$ & $176.53(2)$ \\
\hline $\mathrm{C}(3)-\mathrm{H}(3)$ & 0.9500 & $\operatorname{Mo}(1)-\mathrm{Cl}(1)-\operatorname{Li}(1) \# 2$ & $116.16(17)$ \\
\hline$C(4)-C(5)$ & $1.382(2)$ & $\operatorname{Mo}(1)-\mathrm{Cl}(1)-\operatorname{Li}(1)$ & $91.50(17)$ \\
\hline $\mathrm{C}(4)-\mathrm{H}(4)$ & 0.9500 & $\mathrm{Li}(1) \# 2-\mathrm{Cl}(1)-\mathrm{Li}(1)$ & $89.4(2)$ \\
\hline $\mathrm{C}(5)-\mathrm{C}(5) \# 1$ & $1.481(4)$ & $\operatorname{Mo}(1)-\mathrm{Cl}(2)-\operatorname{Li}(1) \# 1$ & $92.77(16)$ \\
\hline $\operatorname{Li}(1)-\mathrm{N}(2)$ & $2.153(8)$ & $\mathrm{C}(1)-\mathrm{N}(1)-\mathrm{C}(5)$ & $118.59(15)$ \\
\hline $\mathrm{Li}(1)-\mathrm{N}\left(2^{\prime}\right) \# 3$ & $2.220(7)$ & $\mathrm{C}(1)-\mathrm{N}(1)-\mathrm{Mo}(1)$ & $124.88(12)$ \\
\hline $\mathrm{Li}(1)-\mathrm{Cl}(2) \# 1$ & $2.552(8)$ & $\mathrm{C}(5)-\mathrm{N}(1)-\mathrm{Mo}(1)$ & $116.36(12)$ \\
\hline $\mathrm{Li}(1)-\mathrm{Cl}(1) \# 2$ & $2.569(7)$ & $\mathrm{N}(1)-\mathrm{C}(1)-\mathrm{C}(2)$ & $122.55(18)$ \\
\hline $\mathrm{N}(2)-\mathrm{C}(6)$ & $1.358(3)$ & $\mathrm{N}(1)-\mathrm{C}(1)-\mathrm{H}(1)$ & 118.7 \\
\hline $\mathrm{N}(2)-\mathrm{C}(10)$ & $1.372(3)$ & $\mathrm{C}(2)-\mathrm{C}(1)-\mathrm{H}(1)$ & 118.7 \\
\hline $\mathrm{C}(9)-\mathrm{C}(8)$ & $1.361(4)$ & $\mathrm{C}(1)-\mathrm{C}(2)-\mathrm{C}(3)$ & $118.78(18)$ \\
\hline$C(9)-C(10)$ & $1.363(3)$ & $\mathrm{C}(1)-\mathrm{C}(2)-\mathrm{H}(2)$ & 120.6 \\
\hline $\mathrm{C}(9)-\mathrm{H}(9)$ & 0.9500 & $\mathrm{C}(3)-\mathrm{C}(2)-\mathrm{H}(2)$ & 120.6 \\
\hline$C(6)-C(7)$ & $1.384(4)$ & $\mathrm{C}(2)-\mathrm{C}(3)-\mathrm{C}(4)$ & $118.99(17)$ \\
\hline $\mathrm{C}(6)-\mathrm{H}(6)$ & 0.9500 & $\mathrm{C}(2)-\mathrm{C}(3)-\mathrm{H}(3)$ & 120.5 \\
\hline $\mathrm{C}(7)-\mathrm{C}(8)$ & $1.377(4)$ & $\mathrm{C}(4)-\mathrm{C}(3)-\mathrm{H}(3)$ & 120.5 \\
\hline $\mathrm{C}(7)-\mathrm{H}(7)$ & 0.9500 & $C(5)-C(4)-C(3)$ & $119.31(17)$ \\
\hline $\mathrm{C}(8)-\mathrm{H}(8)$ & 0.9500 & $\mathrm{C}(5)-\mathrm{C}(4)-\mathrm{H}(4)$ & 120.3 \\
\hline
\end{tabular}




$\begin{array}{lcll}\mathrm{C}(3)-\mathrm{C}(4)-\mathrm{H}(4) & 120.3 & \mathrm{C}(8)-\mathrm{C}(9)-\mathrm{C}(10) & 117.9(2) \\ \mathrm{N}(1)-\mathrm{C}(5)-\mathrm{C}(4) & 121.75(16) & \mathrm{C}(8)-\mathrm{C}(9)-\mathrm{H}(9) & 121.1 \\ \mathrm{~N}(1)-\mathrm{C}(5)-\mathrm{C}(5) \# 1 & 115.61(10) & \mathrm{C}(10)-\mathrm{C}(9)-\mathrm{H}(9) & 121.1 \\ \mathrm{C}(4)-\mathrm{C}(5)-\mathrm{C}(5) \# 1 & 122.63(11) & \mathrm{N}(2)-\mathrm{C}(6)-\mathrm{C}(7) & 121.4(2) \\ \mathrm{N}(2)-\mathrm{Li}(1)-\mathrm{Cl}(2) \# 1 & 106.6(3) & \mathrm{N}(2)-\mathrm{C}(6)-\mathrm{H}(6) & 119.3 \\ \mathrm{~N}\left(2^{\prime}\right) \# 3-\mathrm{Li}(1)-\mathrm{Cl}(2) \# 1 & 96.1(3) & \mathrm{C}(7)-\mathrm{C}(6)-\mathrm{H}(6) & 119.3 \\ \mathrm{~N}(2)-\mathrm{Li}(1)-\mathrm{Cl}(1) \# 2 & 141.9(4) & \mathrm{C}(8)-\mathrm{C}(7)-\mathrm{C}(6) & 118.1(2) \\ \mathrm{N}\left(2^{\prime}\right) \# 3-\mathrm{Li}(1)-\mathrm{Cl}(1) \# 2 & 92.4(2) & \mathrm{C}(8)-\mathrm{C}(7)-\mathrm{H}(7) & 120.9 \\ \mathrm{Cl}(2) \# 1-\mathrm{Li}(1)-\mathrm{Cl}(1) \# 2 & 111.4(3) & \mathrm{C}(6)-\mathrm{C}(7)-\mathrm{H}(7) & 120.9 \\ \mathrm{~N}(2)-\mathrm{Li}(1)-\mathrm{Cl}(1) & 97.0(3) & \mathrm{C}(9)-\mathrm{C}(8)-\mathrm{C}(7) & 121.8(2) \\ \mathrm{N}\left(2^{\prime}\right) \# 3-\mathrm{Li}(1)-\mathrm{Cl}(1) & 176.4(3) & \mathrm{C}(9)-\mathrm{C}(8)-\mathrm{H}(8) & 119.1 \\ \mathrm{Cl}(2) \# 1-\mathrm{Li}(1)-\mathrm{Cl}(1) & 84.5(2) & \mathrm{C}(7)-\mathrm{C}(8)-\mathrm{H}(8) & 119.1 \\ \mathrm{Cl}(1) \# 2-\mathrm{Li}(1)-\mathrm{Cl}(1) & 90.6(2) & \mathrm{C}(9)-\mathrm{C}(10)-\mathrm{N}(2) & 122.70(19) \\ \mathrm{C}(6)-\mathrm{N}(2)-\mathrm{C}(10) & 118.08(19) & \mathrm{C}(9)-\mathrm{C}(10)-\mathrm{C}(10) \# 3 & 118.3(2) \\ \mathrm{C}(6)-\mathrm{N}(2)-\mathrm{Li}(1) & 129.6(2) & \mathrm{N}(2)-\mathrm{C}(10)-\mathrm{C}(10) \# 3 & 119.0(2) \\ \mathrm{C}(10)-\mathrm{N}(2)-\mathrm{Li}(1) & 112.3(2) & & \end{array}$

Symmetry transformations used to generate equivalent atoms:

$\# 1-\mathrm{x}+1, \mathrm{y},-\mathrm{z}+1 / 2 \quad \# 2-\mathrm{x}+1,-\mathrm{y}+1,-\mathrm{z}+1 \quad \# 3-\mathrm{x}+1,-\mathrm{y},-\mathrm{z}+1$ 
Table 4. Anisotropic displacement parameters $\left(\AA^{2} \times 10^{3}\right)$ for eiswe28. The anisotropic displacement factor exponent takes the form: $-2 \pi^{2}\left[h^{2} a^{* 2} U_{11}+\ldots+2 h k a^{*} b^{*} U_{12}\right]$

\begin{tabular}{lcccccc}
\hline & $\mathrm{U}_{11}$ & $\mathrm{U}_{22}$ & $\mathrm{U}_{33}$ & $\mathrm{U}_{23}$ & $\mathrm{U}_{13}$ & $\mathrm{U}_{12}$ \\
\hline Mo1 & $10(1)$ & $7(1)$ & $15(1)$ & 0 & $7(1)$ & 0 \\
$\mathrm{C} 11$ & $17(1)$ & $14(1)$ & $16(1)$ & $-1(1)$ & $7(1)$ & $-2(1)$ \\
$\mathrm{C} 2$ & $15(1)$ & $11(1)$ & $26(1)$ & $0(1)$ & $12(1)$ & $3(1)$ \\
$\mathrm{N} 1$ & $10(1)$ & $11(1)$ & $16(1)$ & $1(1)$ & $7(1)$ & $-1(1)$ \\
$\mathrm{C} 1$ & $12(1)$ & $16(1)$ & $23(1)$ & $3(1)$ & $10(1)$ & $0(1)$ \\
$\mathrm{C} 2$ & $11(1)$ & $21(1)$ & $24(1)$ & $4(1)$ & $7(1)$ & $-4(1)$ \\
$\mathrm{C} 3$ & $17(1)$ & $18(1)$ & $18(1)$ & $1(1)$ & $7(1)$ & $-6(1)$ \\
$\mathrm{C} 4$ & $18(1)$ & $11(1)$ & $17(1)$ & $0(1)$ & $7(1)$ & $-3(1)$ \\
$\mathrm{C} 5$ & $11(1)$ & $10(1)$ & $11(1)$ & $0(1)$ & $4(1)$ & $-1(1)$ \\
Li1 & $16(3)$ & $21(3)$ & $20(3)$ & $4(3)$ & $12(3)$ & $0(3)$ \\
$\mathrm{N} 2$ & $16(1)$ & $16(1)$ & $22(1)$ & $5(1)$ & $5(1)$ & $-1(1)$ \\
$\mathrm{C} 9$ & $35(1)$ & $15(1)$ & $12(1)$ & $2(1)$ & $6(1)$ & $8(1)$ \\
$\mathrm{N} 2$ & $35(1)$ & $15(1)$ & $12(1)$ & $2(1)$ & $6(1)$ & $8(1)$ \\
$\mathrm{C} 9$ & $16(1)$ & $16(1)$ & $22(1)$ & $5(1)$ & $5(1)$ & $-1(1)$ \\
$\mathrm{C} 6$ & $20(1)$ & $33(1)$ & $22(1)$ & $6(1)$ & $7(1)$ & $-8(1)$ \\
C7 & $18(1)$ & $61(2)$ & $20(1)$ & $-1(1)$ & $7(1)$ & $10(1)$ \\
C8 & $44(2)$ & $35(1)$ & $14(1)$ & $-1(1)$ & $4(1)$ & $25(1)$ \\
C10 & $19(1)$ & $14(1)$ & $13(1)$ & $4(1)$ & $6(1)$ & $3(1)$ \\
& & & & & & \\
\hline
\end{tabular}


Table 5. Hydrogen coordinates $\left(\times 10^{4}\right)$ and isotropic displacement parameters $\left(\AA^{2} \times 10^{3}\right)$ for eiswe28.

\begin{tabular}{lrrrl}
\hline & \multicolumn{1}{c}{$\mathrm{x}$} & $\mathrm{z}$ & $\mathrm{U}(\mathrm{eq})$ \\
\hline $\mathrm{H} 1$ & & & & \\
$\mathrm{H} 2$ & 6703 & 5948 & 2437 & 19 \\
H3 & 7490 & 8010 & 2556 & 22 \\
H4 & 6959 & 10310 & 2676 & 21 \\
H9 & 5623 & 10463 & 2601 & 19 \\
H9' & 5642 & -2122 & 5260 & 27 \\
H6 & 5398 & 2222 & 4779 & 23 \\
H7 & 6736 & 2338 & 4928 & 31 \\
H8 & 7551 & 242 & 5271 & 40 \\
& 6971 & -1986 & 5379 & 41 \\
\hline
\end{tabular}


Table 6. Torsion angles $\left[{ }^{\circ}\right]$ for eiswe28.

\begin{tabular}{lclc}
\hline C5-N1-C1-C2 & $1.0(3)$ & Li1-N2-C6-C7 & $179.9(3)$ \\
Mo1-N1-C1-C2 & $-174.15(15)$ & N2-C6-C7-C8 & $1.5(4)$ \\
N1-C1-C2-C3 & $0.1(3)$ & C10-C9-C8-C7 & $0.8(3)$ \\
C1-C2-C3-C4 & $-1.4(3)$ & C6-C7-C8-C9 & $-2.1(4)$ \\
C2-C3-C4-C5 & $1.5(3)$ & C8-C9-C10-N2 & $1.2(3)$ \\
C1-N1-C5-C4 & $-0.9(3)$ & C8-C9-C10-C10\#3 & $-178.6(2)$ \\
Mo1-N1-C5-C4 & $174.73(14)$ & C6-N2-C10-C9 & $-1.7(3)$ \\
C1-N1-C5-C5\#1 & $-179.93(19)$ & Li1-N2-C10-C9 & $178.6(3)$ \\
Mo1-N1-C5-C5\#1 & $-4.3(3)$ & C6-N2-C10-C10\#3 & $178.0(2)$ \\
C3-C4-C5-N1 & $-0.4(3)$ & Li1-N2-C10-C10\#3 & $-1.6(4)$ \\
C3-C4-C5-C5\#1 & $178.6(2)$ & & \\
C10-N2-C6-C7 & $0.3(3)$ & & \\
& & & \\
\hline
\end{tabular}

Symmetry transformations used to generate equivalent atoms:

$\# 1-\mathrm{x}+1, \mathrm{y},-\mathrm{z}+1 / 2 \quad \# 2-\mathrm{x}+1,-\mathrm{y}+1,-\mathrm{z}+1 \quad \# 3-\mathrm{x}+1,-\mathrm{y},-\mathrm{z}+1$ 


\section{REFERENCE NUMBER: rhowe05}

\section{CRYSTAL STRUCTURE REPORT}

$\mathrm{C}_{34} \mathrm{H}_{28} \mathrm{Cl}_{4} \mathrm{Mo} \mathrm{N}_{2} \mathrm{P}$

or

$\left[\mathrm{PPh}_{4}\right]\left[\mathrm{MoCl}_{4}(\mathrm{bpy})\right]$

Report prepared for:

Prof. W. Eckenhoff

February 20, 2017
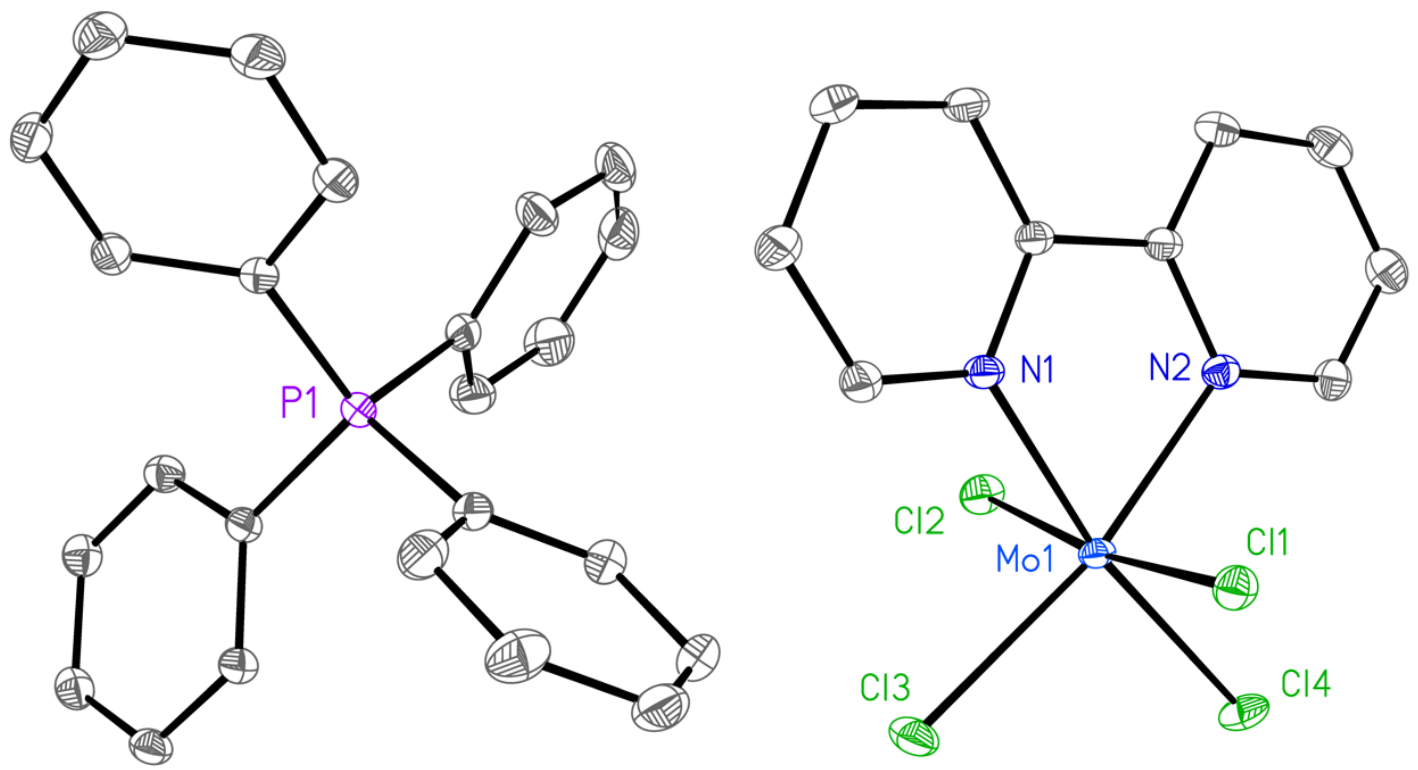

William W. Brennessel

X-ray Crystallographic Facility

Department of Chemistry, University of Rochester

120 Trustee Road

Rochester, NY 14627 


\section{Data collection}

A crystal $\left(0.25 \times 0.20 \times 0.15 \mathrm{~mm}^{3}\right)$ was placed onto the tip of a thin glass optical fiber and mounted on a Bruker SMART APEX II CCD platform diffractometer for a data collection at 100.0(5) K. ${ }^{1}$ A preliminary set of cell constants and an orientation matrix were calculated from reflections harvested from three orthogonal wedges of reciprocal space. The full data collection was carried out using MoK $\alpha$ radiation (graphite monochromator) with a frame time of 10 seconds and a detector distance of $4.05 \mathrm{~cm}$. A randomly oriented region of reciprocal space was surveyed: ten major sections of frames were collected with $0.50^{\circ}$ steps in $\omega$ at ten different $\phi$ settings and a detector position of $-38^{\circ}$ in $2 \theta$. The intensity data were corrected for absorption. ${ }^{2}$ Final cell constants were calculated from the xyz centroids of 4078 strong reflections from the actual data collection after integration. ${ }^{3}$ See Table 1 for additional crystal and refinement information.

\section{Structure solution and refinement}

The structure was solved using SHELXT 2014/54 and refined using SHELXL-2016/6. ${ }^{5}$ The space group $P-1$ was determined based on intensity statistics. A direct-methods solution was calculated which provided most nonhydrogen atoms from the E-map. Full-matrix least squares / difference Fourier cycles were performed which located the remaining non-hydrogen atoms. All non-hydrogen atoms were refined with anisotropic displacement parameters. All hydrogen atoms were placed in ideal positions and refined as riding atoms with relative isotropic displacement parameters. The final full matrix least squares refinement converged to $R 1=0.0342\left(F^{2}, I>2 \sigma(I)\right)$ and $w R 2=0.0760\left(F^{2}\right.$, all data $)$.

\section{Structure description}

The structure is the one suggested. The asymmetric unit contains one $\mathrm{PPh}_{4}$ cation and one anionic Mo complex, both in general positions. The structure has been reported previously (refcode FUCPIC). ${ }^{6}$

Unless noted otherwise all structural diagrams containing thermal displacement ellipsoids are drawn at the $50 \%$ probability level.

Data collection, structure solution, and structure refinement were conducted at the X-ray Crystallographic Facility, B04 Hutchison Hall, Department of Chemistry, University of Rochester. All publications arising from this report MUST either 1) include William W. Brennessel as a coauthor or 2) acknowledge William W. Brennessel and the Xray Crystallographic Facility of the Department of Chemistry at the University of Rochester. 
1 APEX3, version 2016.5-0; Bruker AXS: Madison, WI, 2016.

2 Krause, L.; Herbst-Irmer, R.; Sheldrick, G. M.; Stalke, D. SADABS, version 2016/2; J. Appl. Cryst. 2015, 48, 3-

10.

3 SAINT, version 8.34A; Bruker AXS: Madison, WI, 2013.

4 Sheldrick, G. M. SHELXT, version 2014/5; Acta. Cryst. 2015, A71, 3-8.

5 Sheldrick, G. M. SHELXL, version 2016/6; Acta. Cryst. 2015, C71, 3-8.

6 Richards, R. L.; Shortman, C.; Povey, D. C.; Smith, G. W. Acta Cryst. 1987, C43, 2091-2093.

Some equations of interest:

$$
\begin{gathered}
R_{\text {int }}=\Sigma\left|F_{\mathrm{o}}{ }^{2}-<F_{\mathrm{o}}{ }^{2}>\right| / \Sigma\left|F_{\mathrm{o}}{ }^{2}\right| \\
R 1=\Sigma|| F_{\mathrm{o}}|-| F_{\mathrm{c}} \| / \Sigma\left|F_{\mathrm{o}}\right| \\
w R 2=\left[\Sigma\left[w\left(F_{\mathrm{o}}{ }^{2}-F_{\mathrm{c}}{ }^{2}\right)^{2}\right] / \Sigma\left[w\left(F_{\mathrm{o}}{ }^{2}\right)^{2}\right]\right]^{1 / 2} \\
\text { where } w=1 /\left[\sigma^{2}\left(F_{\mathrm{o}}{ }^{2}\right)+(a P)^{2}+b P\right] \text { and } \\
P=1 / 3 \max \left(0, F_{\mathrm{o}}{ }^{2}\right)+2 / 3 F_{\mathrm{c}}{ }^{2} \\
\text { GOF }=S=\left[\Sigma\left[w\left(F_{\mathrm{o}}{ }^{2}-F_{\mathrm{c}}{ }^{2}\right)^{2}\right] /(m-n)\right]^{1 / 2}
\end{gathered}
$$

where $m=$ number of reflections and $n=$ number of parameters
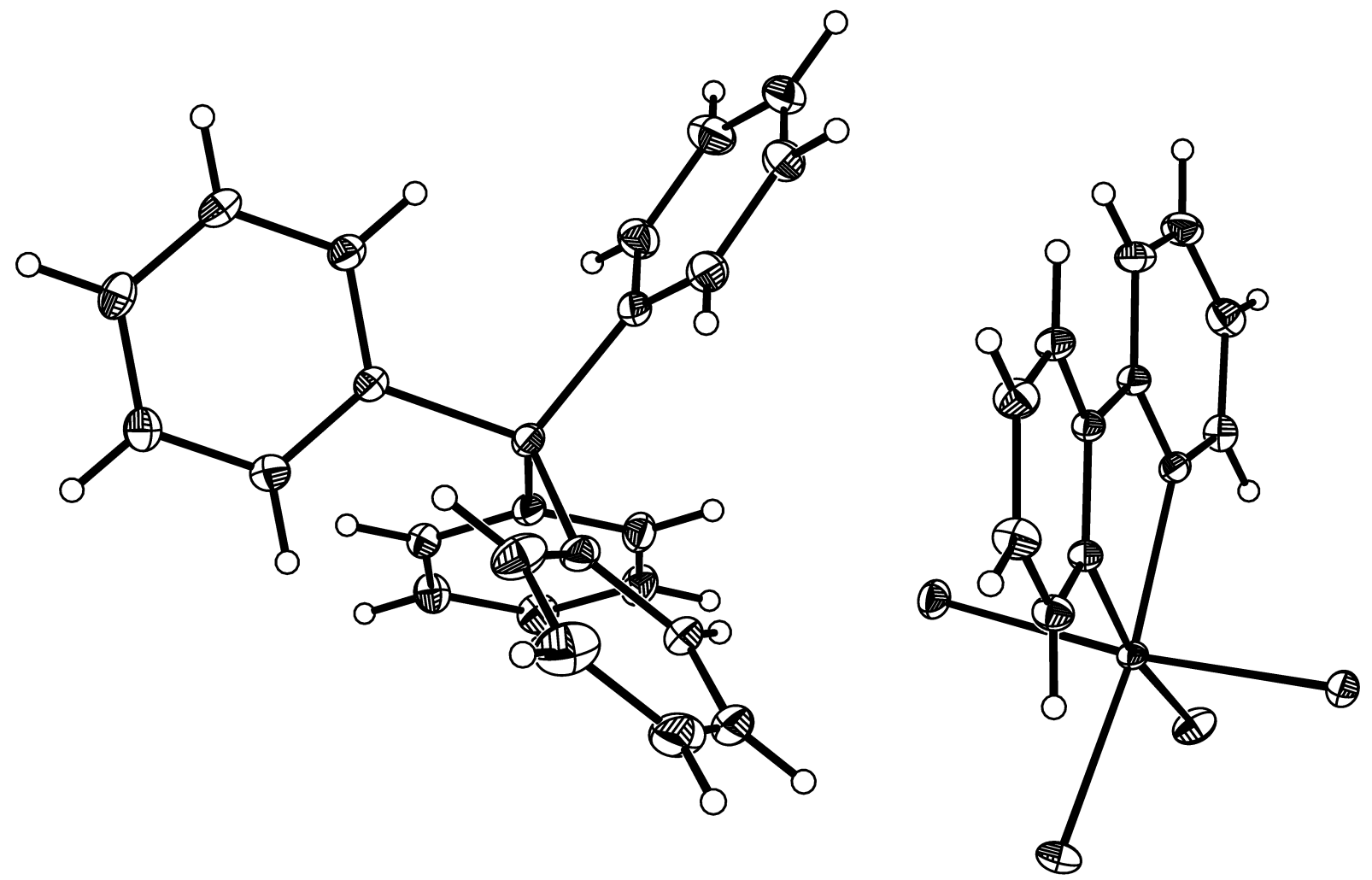


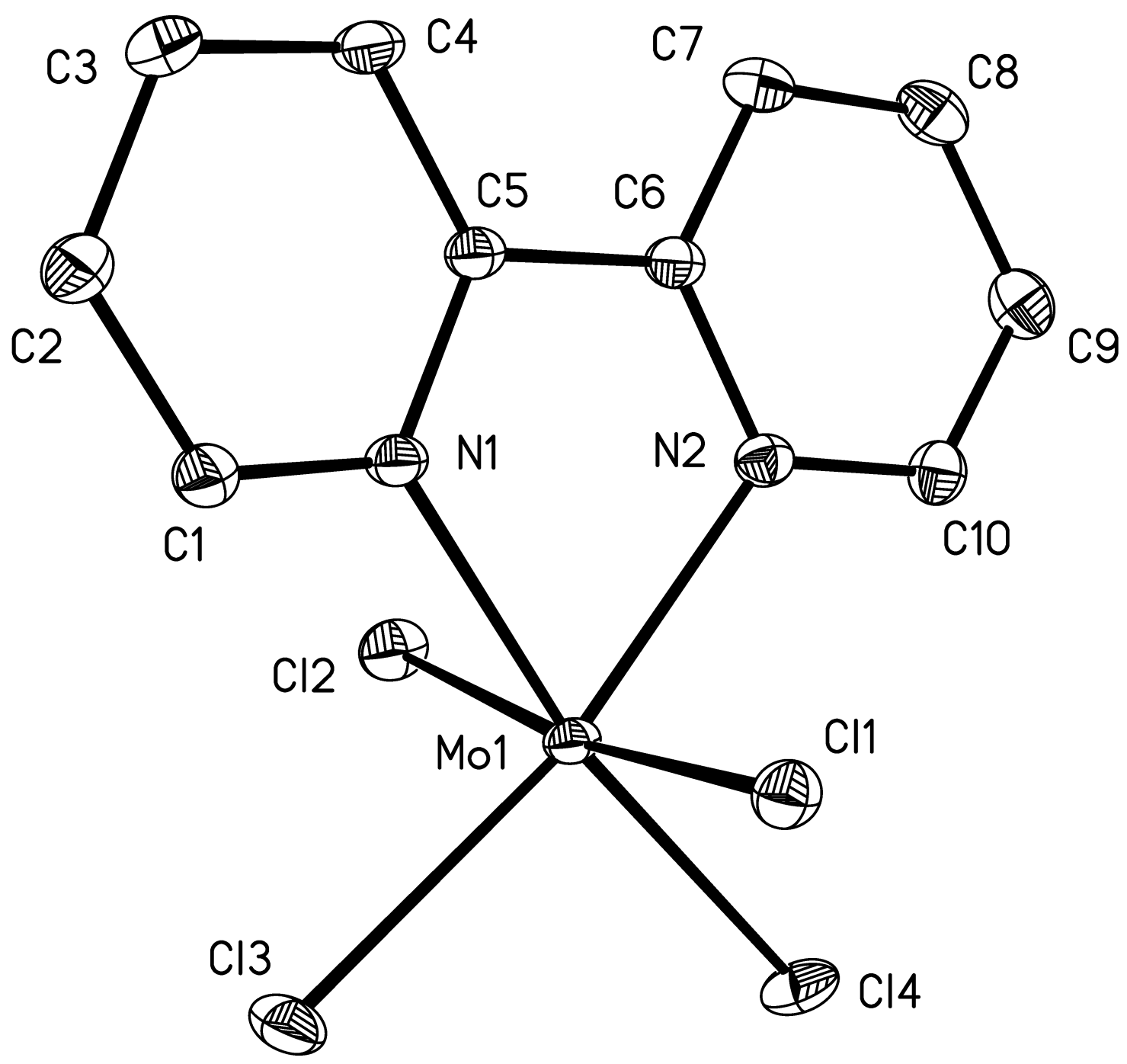




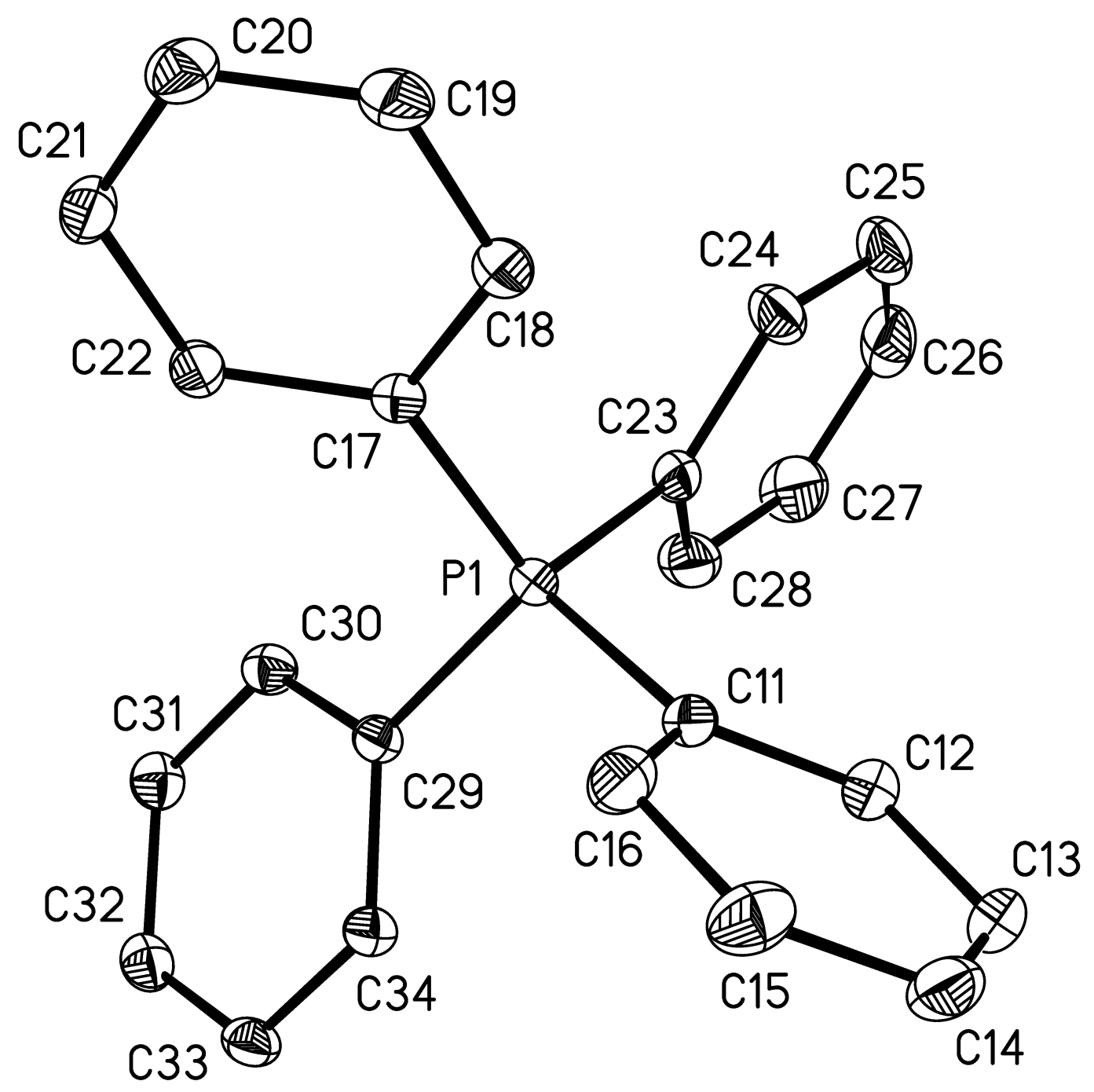


Table 1. Crystal data and structure refinement for rhowe05.

\begin{tabular}{|c|c|c|}
\hline Identification code & \multicolumn{2}{|l|}{ rhowe05 } \\
\hline Empirical formula & \multicolumn{2}{|c|}{ C34 H28 C14 Mo N2 P } \\
\hline Formula weight & \multicolumn{2}{|l|}{733.29} \\
\hline Temperature & \multicolumn{2}{|l|}{$100.0(5) \mathrm{K}$} \\
\hline Wavelength & \multicolumn{2}{|l|}{$0.71073 \AA$} \\
\hline Crystal system & \multicolumn{2}{|l|}{ triclinic } \\
\hline Space group & \multicolumn{2}{|l|}{$P-1$} \\
\hline \multirow[t]{3}{*}{ Unit cell dimensions } & $a=9.1566(5) \AA$ & $\alpha=104.4260(10)^{\circ}$ \\
\hline & $b=12.7273(7) \AA$ & $\beta=101.6820(10)^{\circ}$ \\
\hline & $c=14.2980(8) \AA$ & $\gamma=90.6540(10)^{\circ}$ \\
\hline Volume & \multicolumn{2}{|l|}{$1576.94(15) \AA^{3}$} \\
\hline$Z$ & \multicolumn{2}{|l|}{2} \\
\hline Density (calculated) & \multicolumn{2}{|l|}{$1.544 \mathrm{Mg} / \mathrm{m}^{3}$} \\
\hline Absorption coefficient & \multicolumn{2}{|l|}{$0.833 \mathrm{~mm}^{-1}$} \\
\hline$F(000)$ & \multicolumn{2}{|l|}{742} \\
\hline Crystal color, morphology & \multicolumn{2}{|l|}{ red, block } \\
\hline Crystal size & \multicolumn{2}{|c|}{$0.25 \times 0.20 \times 0.15 \mathrm{~mm}^{3}$} \\
\hline Theta range for data collection & \multicolumn{2}{|l|}{1.930 to $38.651^{\circ}$} \\
\hline Index ranges & \multicolumn{2}{|c|}{$-15 \leq h \leq 15,-22 \leq k \leq 22,-24 \leq l \leq 24$} \\
\hline Reflections collected & \multicolumn{2}{|l|}{118820} \\
\hline Independent reflections & \multicolumn{2}{|c|}{$17356[R($ int $)=0.0562]$} \\
\hline Observed reflections & \multicolumn{2}{|l|}{13707} \\
\hline Completeness to theta $=37.785^{\circ}$ & \multicolumn{2}{|l|}{$99.3 \%$} \\
\hline Absorption correction & \multicolumn{2}{|l|}{ Multi-scan } \\
\hline Max. and min. transmission & \multicolumn{2}{|l|}{0.7476 and 0.7033} \\
\hline Refinement method & \multicolumn{2}{|c|}{ Full-matrix least-squares on $F^{2}$} \\
\hline Data / restraints / parameters & \multicolumn{2}{|l|}{$17356 / 0 / 379$} \\
\hline Goodness-of-fit on $F^{2}$ & \multicolumn{2}{|l|}{1.079} \\
\hline Final $R$ indices $[I>2 \operatorname{sigma}(I)]$ & \multicolumn{2}{|c|}{$R 1=0.0342, w R 2=0.0712$} \\
\hline$R$ indices (all data) & \multicolumn{2}{|c|}{$R 1=0.0492, w R 2=0.0760$} \\
\hline Largest diff. peak and hole & \multicolumn{2}{|c|}{0.794 and -0.786 e. $\AA^{-3}$} \\
\hline
\end{tabular}


Table 2. Atomic coordinates $\left(\times 10^{4}\right)$ and equivalent isotropic displacement parameters $\left(\AA^{2} \times 10^{3}\right)$ for rhowe $05 . \mathrm{U}_{\mathrm{eq}}$ is defined as one third of the trace of the orthogonalized $\mathrm{U}_{\mathrm{ij}}$ tensor.

\begin{tabular}{|c|c|c|c|c|}
\hline & $\mathrm{x}$ & $\mathrm{y}$ & $\mathrm{z}$ & $\mathrm{U}_{\mathrm{eq}}$ \\
\hline Mo1 & 9921(1) & 8881(1) & $2457(1)$ & $11(1)$ \\
\hline $\mathrm{Cl1}$ & 9974(1) & $10815(1)$ & $3232(1)$ & $15(1)$ \\
\hline $\mathrm{Cl} 2$ & $9515(1)$ & 6938(1) & $1677(1)$ & $18(1)$ \\
\hline $\mathrm{Cl} 3$ & $11880(1)$ & $8598(1)$ & $3779(1)$ & 19(1) \\
\hline $\mathrm{Cl} 4$ & $11617(1)$ & $9146(1)$ & $1407(1)$ & $19(1)$ \\
\hline N1 & $8132(1)$ & $8705(1)$ & $3189(1)$ & $13(1)$ \\
\hline N2 & 7904(1) & $8985(1)$ & $1395(1)$ & $13(1)$ \\
\hline $\mathrm{C} 1$ & $8332(1)$ & $8715(1)$ & $4146(1)$ & $15(1)$ \\
\hline $\mathrm{C} 2$ & 7144(1) & $8579(1)$ & $4581(1)$ & $18(1)$ \\
\hline $\mathrm{C} 3$ & $5708(1)$ & $8438(1)$ & $4015(1)$ & $18(1)$ \\
\hline $\mathrm{C} 4$ & $5486(1)$ & $8456(1)$ & $3027(1)$ & $16(1)$ \\
\hline $\mathrm{C} 5$ & $6718(1)$ & $8595(1)$ & 2631(1) & $12(1)$ \\
\hline C6 & 6601(1) & $8699(1)$ & 1611(1) & $13(1)$ \\
\hline $\mathrm{C} 7$ & $5259(1)$ & $8540(1)$ & $917(1)$ & $17(1)$ \\
\hline $\mathrm{C} 8$ & $5254(2)$ & $8708(1)$ & $-7(1)$ & $20(1)$ \\
\hline C9 & $6576(2)$ & $9043(1)$ & $-212(1)$ & $19(1)$ \\
\hline $\mathrm{C} 10$ & $7876(1)$ & $9166(1)$ & $504(1)$ & $16(1)$ \\
\hline P1 & $6603(1)$ & $3998(1)$ & $2790(1)$ & $14(1)$ \\
\hline C11 & $5207(1)$ & $4720(1)$ & $2177(1)$ & $16(1)$ \\
\hline $\mathrm{C} 12$ & $4558(2)$ & $5583(1)$ & $2730(1)$ & $21(1)$ \\
\hline C13 & $3388(2)$ & $6080(1)$ & $2258(1)$ & $24(1)$ \\
\hline C14 & $2889(2)$ & $5730(1)$ & 1243(1) & $25(1)$ \\
\hline $\mathrm{C} 15$ & $3526(2)$ & $4872(1)$ & $690(1)$ & $25(1)$ \\
\hline C16 & $4686(2)$ & $4360(1)$ & $1155(1)$ & $21(1)$ \\
\hline $\mathrm{C} 17$ & $7916(1)$ & $3567(1)$ & $2015(1)$ & $15(1)$ \\
\hline C18 & $8492(2)$ & $4333(1)$ & $1607(1)$ & 19(1) \\
\hline C19 & $9559(2)$ & $4038(1)$ & 1041(1) & $22(1)$ \\
\hline $\mathrm{C} 20$ & 10041(2) & 2989(1) & $866(1)$ & $21(1)$ \\
\hline $\mathrm{C} 21$ & $9471(2)$ & 2231(1) & $1272(1)$ & 19(1) \\
\hline $\mathrm{C} 22$ & $8404(1)$ & 2514(1) & $1845(1)$ & $15(1)$ \\
\hline $\mathrm{C} 23$ & 7511(1) & $4882(1)$ & $3962(1)$ & $18(1)$ \\
\hline
\end{tabular}




$\begin{array}{lllll}\text { C24 } & 8600(2) & 5681(1) & 4001(1) & 22(1) \\ \text { C25 } & 9234(2) & 6398(1) & 4905(1) & 28(1) \\ \text { C26 } & 8798(2) & 6326(1) & 5760(1) & 29(1) \\ \text { C27 } & 7725(2) & 5528(1) & 5719(1) & 30(1) \\ \text { C28 } & 7072(2) & 4805(1) & 4824(1) & 24(1) \\ \text { C29 } & 5714(1) & 2849(1) & 3011(1) & 14(1) \\ \text { C30 } & 6568(1) & 2203(1) & 3552(1) & 16(1) \\ \text { C31 } & 5890(2) & 1296(1) & 3705(1) & 18(1) \\ \text { C32 } & 4361(2) & 1040(1) & 3332(1) & 18(1) \\ \text { C33 } & 3512(1) & 1691(1) & 2814(1) & 18(1) \\ \text { C34 } & 4182(1) & 2595(1) & 2647(1) & 15(1) \\ \end{array}$


Table 3. Bond lengths $[\AA]$ and angles $\left[{ }^{\circ}\right]$ for rhowe 05 .

\begin{tabular}{|c|c|c|c|}
\hline $\operatorname{Mo}(1)-\mathrm{N}(1)$ & $2.1526(9)$ & $\mathrm{C}(12)-\mathrm{H}(12)$ & 0.9500 \\
\hline $\operatorname{Mo}(1)-\mathrm{N}(2)$ & $2.1685(10)$ & $\mathrm{C}(13)-\mathrm{C}(14)$ & $1.385(2)$ \\
\hline $\operatorname{Mo}(1)-\mathrm{Cl}(3)$ & $2.4290(3)$ & $\mathrm{C}(13)-\mathrm{H}(13)$ & 0.9500 \\
\hline $\operatorname{Mo}(1)-\mathrm{Cl}(1)$ & $2.4306(3)$ & $\mathrm{C}(14)-\mathrm{C}(15)$ & $1.389(2)$ \\
\hline $\operatorname{Mo}(1)-\mathrm{Cl}(2)$ & $2.4362(3)$ & $\mathrm{C}(14)-\mathrm{H}(14)$ & 0.9500 \\
\hline $\operatorname{Mo}(1)-\mathrm{Cl}(4)$ & $2.4408(3)$ & $C(15)-C(16)$ & $1.3931(19)$ \\
\hline $\mathrm{N}(1)-\mathrm{C}(1)$ & $1.3406(15)$ & $\mathrm{C}(15)-\mathrm{H}(15)$ & 0.9500 \\
\hline $\mathrm{N}(1)-\mathrm{C}(5)$ & $1.3621(15)$ & $\mathrm{C}(16)-\mathrm{H}(16)$ & 0.9500 \\
\hline $\mathrm{N}(2)-\mathrm{C}(10)$ & $1.3452(15)$ & $\mathrm{C}(17)-\mathrm{C}(22)$ & $1.3963(16)$ \\
\hline $\mathrm{N}(2)-\mathrm{C}(6)$ & $1.3595(15)$ & $\mathrm{C}(17)-\mathrm{C}(18)$ & $1.4019(17)$ \\
\hline $\mathrm{C}(1)-\mathrm{C}(2)$ & $1.3868(17)$ & $\mathrm{C}(18)-\mathrm{C}(19)$ & $1.3882(18)$ \\
\hline $\mathrm{C}(1)-\mathrm{H}(1)$ & 0.9500 & $\mathrm{C}(18)-\mathrm{H}(18)$ & 0.9500 \\
\hline$C(2)-C(3)$ & $1.3811(18)$ & $\mathrm{C}(19)-\mathrm{C}(20)$ & $1.3906(19)$ \\
\hline $\mathrm{C}(2)-\mathrm{H}(2)$ & 0.9500 & $\mathrm{C}(19)-\mathrm{H}(19)$ & 0.9500 \\
\hline $\mathrm{C}(3)-\mathrm{C}(4)$ & $1.3926(18)$ & $\mathrm{C}(20)-\mathrm{C}(21)$ & $1.3895(18)$ \\
\hline $\mathrm{C}(3)-\mathrm{H}(3)$ & 0.9500 & $\mathrm{C}(20)-\mathrm{H}(20)$ & 0.9500 \\
\hline$C(4)-C(5)$ & $1.3882(16)$ & $\mathrm{C}(21)-\mathrm{C}(22)$ & $1.3927(17)$ \\
\hline $\mathrm{C}(4)-\mathrm{H}(4)$ & 0.9500 & $\mathrm{C}(21)-\mathrm{H}(21)$ & 0.9500 \\
\hline$C(5)-C(6)$ & $1.4788(16)$ & $\mathrm{C}(22)-\mathrm{H}(22)$ & 0.9500 \\
\hline $\mathrm{C}(6)-\mathrm{C}(7)$ & $1.3918(17)$ & $\mathrm{C}(23)-\mathrm{C}(28)$ & $1.395(2)$ \\
\hline$C(7)-C(8)$ & $1.3885(18)$ & $\mathrm{C}(23)-\mathrm{C}(24)$ & $1.3991(18)$ \\
\hline $\mathrm{C}(7)-\mathrm{H}(7)$ & 0.9500 & $\mathrm{C}(24)-\mathrm{C}(25)$ & $1.392(2)$ \\
\hline $\mathrm{C}(8)-\mathrm{C}(9)$ & $1.3890(19)$ & $\mathrm{C}(24)-\mathrm{H}(24)$ & 0.9500 \\
\hline $\mathrm{C}(8)-\mathrm{H}(8)$ & 0.9500 & $C(25)-C(26)$ & $1.383(2)$ \\
\hline $\mathrm{C}(9)-\mathrm{C}(10)$ & $1.3835(18)$ & $\mathrm{C}(25)-\mathrm{H}(25)$ & 0.9500 \\
\hline $\mathrm{C}(9)-\mathrm{H}(9)$ & 0.9500 & $C(26)-C(27)$ & $1.387(2)$ \\
\hline $\mathrm{C}(10)-\mathrm{H}(10)$ & 0.9500 & $\mathrm{C}(26)-\mathrm{H}(26)$ & 0.9500 \\
\hline $\mathrm{P}(1)-\mathrm{C}(29)$ & $1.7906(12)$ & $C(27)-C(28)$ & $1.390(2)$ \\
\hline $\mathrm{P}(1)-\mathrm{C}(11)$ & $1.7946(12)$ & $\mathrm{C}(27)-\mathrm{H}(27)$ & 0.9500 \\
\hline $\mathrm{P}(1)-\mathrm{C}(17)$ & $1.7961(12)$ & $\mathrm{C}(28)-\mathrm{H}(28)$ & 0.9500 \\
\hline $\mathrm{P}(1)-\mathrm{C}(23)$ & $1.8005(13)$ & C(29)-C(34) & $1.3977(16)$ \\
\hline$C(11)-C(16)$ & $1.3974(19)$ & $\mathrm{C}(29)-\mathrm{C}(30)$ & $1.4041(17)$ \\
\hline$C(11)-C(12)$ & $1.3984(18)$ & $\mathrm{C}(30)-\mathrm{C}(31)$ & $1.3901(17)$ \\
\hline$C(12)-C(13)$ & $1.3925(19)$ & $\mathrm{C}(30)-\mathrm{H}(30)$ & 0.9500 \\
\hline
\end{tabular}




\begin{tabular}{|c|c|c|c|}
\hline $\mathrm{C}(31)-\mathrm{C}(32)$ & $1.3968(18)$ & $\mathrm{C}(4)-\mathrm{C}(3)-\mathrm{H}(3)$ & 120.4 \\
\hline $\mathrm{C}(31)-\mathrm{H}(31)$ & 0.9500 & $C(5)-C(4)-C(3)$ & $119.08(11)$ \\
\hline C(32)-C(33) & $1.3882(18)$ & $\mathrm{C}(5)-\mathrm{C}(4)-\mathrm{H}(4)$ & 120.5 \\
\hline $\mathrm{C}(32)-\mathrm{H}(32)$ & 0.9500 & $\mathrm{C}(3)-\mathrm{C}(4)-\mathrm{H}(4)$ & 120.5 \\
\hline C(33)-C(34) & $1.3925(17)$ & $\mathrm{N}(1)-\mathrm{C}(5)-\mathrm{C}(4)$ & $121.23(11)$ \\
\hline $\mathrm{C}(33)-\mathrm{H}(33)$ & 0.9500 & $\mathrm{~N}(1)-\mathrm{C}(5)-\mathrm{C}(6)$ & $115.27(9)$ \\
\hline C(34)-H(34) & 0.9500 & $C(4)-C(5)-C(6)$ & $123.41(11)$ \\
\hline $\mathrm{N}(1)-\mathrm{Mo}(1)-\mathrm{N}(2)$ & $75.44(4)$ & $\mathrm{N}(2)-\mathrm{C}(6)-\mathrm{C}(7)$ & $121.43(11)$ \\
\hline $\mathrm{N}(1)-\mathrm{Mo}(1)-\mathrm{Cl}(3)$ & $94.76(3)$ & $\mathrm{N}(2)-\mathrm{C}(6)-\mathrm{C}(5)$ & $115.28(10)$ \\
\hline $\mathrm{N}(2)-\mathrm{Mo}(1)-\mathrm{Cl}(3)$ & $169.51(3)$ & $C(7)-C(6)-C(5)$ & $123.28(10)$ \\
\hline $\mathrm{N}(1)-\mathrm{Mo}(1)-\mathrm{Cl}(1)$ & $86.12(3)$ & $\mathrm{C}(8)-\mathrm{C}(7)-\mathrm{C}(6)$ & $118.95(11)$ \\
\hline $\mathrm{N}(2)-\mathrm{Mo}(1)-\mathrm{Cl}(1)$ & $90.77(3)$ & $\mathrm{C}(8)-\mathrm{C}(7)-\mathrm{H}(7)$ & 120.5 \\
\hline $\mathrm{Cl}(3)-\mathrm{Mo}(1)-\mathrm{Cl}(1)$ & $92.289(11)$ & $\mathrm{C}(6)-\mathrm{C}(7)-\mathrm{H}(7)$ & 120.5 \\
\hline $\mathrm{N}(1)-\mathrm{Mo}(1)-\mathrm{Cl}(2)$ & $87.38(3)$ & $\mathrm{C}(9)-\mathrm{C}(8)-\mathrm{C}(7)$ & $119.48(12)$ \\
\hline $\mathrm{N}(2)-\mathrm{Mo}(1)-\mathrm{Cl}(2)$ & $83.81(3)$ & $\mathrm{C}(9)-\mathrm{C}(8)-\mathrm{H}(8)$ & 120.3 \\
\hline $\mathrm{Cl}(3)-\mathrm{Mo}(1)-\mathrm{Cl}(2)$ & $92.139(12)$ & $\mathrm{C}(7)-\mathrm{C}(8)-\mathrm{H}(8)$ & 120.3 \\
\hline $\mathrm{Cl}(1)-\mathrm{Mo}(1)-\mathrm{Cl}(2)$ & $172.417(11)$ & $C(10)-C(9)-C(8)$ & $118.67(12)$ \\
\hline $\mathrm{N}(1)-\mathrm{Mo}(1)-\mathrm{Cl}(4)$ & $170.23(3)$ & $\mathrm{C}(10)-\mathrm{C}(9)-\mathrm{H}(9)$ & 120.7 \\
\hline $\mathrm{N}(2)-\mathrm{Mo}(1)-\mathrm{Cl}(4)$ & $94.85(3)$ & $\mathrm{C}(8)-\mathrm{C}(9)-\mathrm{H}(9)$ & 120.7 \\
\hline $\mathrm{Cl}(3)-\mathrm{Mo}(1)-\mathrm{Cl}(4)$ & $95.003(13)$ & $\mathrm{N}(2)-\mathrm{C}(10)-\mathrm{C}(9)$ & $122.48(11)$ \\
\hline $\mathrm{Cl}(1)-\mathrm{Mo}(1)-\mathrm{Cl}(4)$ & $92.989(11)$ & $\mathrm{N}(2)-\mathrm{C}(10)-\mathrm{H}(10)$ & 118.8 \\
\hline $\mathrm{Cl}(2)-\mathrm{Mo}(1)-\mathrm{Cl}(4)$ & $92.754(11)$ & $\mathrm{C}(9)-\mathrm{C}(10)-\mathrm{H}(10)$ & 118.8 \\
\hline $\mathrm{C}(1)-\mathrm{N}(1)-\mathrm{C}(5)$ & $119.22(10)$ & $\mathrm{C}(29)-\mathrm{P}(1)-\mathrm{C}(11)$ & $109.03(6)$ \\
\hline $\mathrm{C}(1)-\mathrm{N}(1)-\mathrm{Mo}(1)$ & $124.14(8)$ & $\mathrm{C}(29)-\mathrm{P}(1)-\mathrm{C}(17)$ & $110.60(5)$ \\
\hline $\mathrm{C}(5)-\mathrm{N}(1)-\mathrm{Mo}(1)$ & $116.63(7)$ & $\mathrm{C}(11)-\mathrm{P}(1)-\mathrm{C}(17)$ & $108.63(6)$ \\
\hline $\mathrm{C}(10)-\mathrm{N}(2)-\mathrm{C}(6)$ & $118.91(10)$ & $\mathrm{C}(29)-\mathrm{P}(1)-\mathrm{C}(23)$ & $108.47(6)$ \\
\hline $\mathrm{C}(10)-\mathrm{N}(2)-\mathrm{Mo}(1)$ & $124.70(8)$ & $\mathrm{C}(11)-\mathrm{P}(1)-\mathrm{C}(23)$ & 108.91(6) \\
\hline $\mathrm{C}(6)-\mathrm{N}(2)-\mathrm{Mo}(1)$ & $115.76(8)$ & $\mathrm{C}(17)-\mathrm{P}(1)-\mathrm{C}(23)$ & $111.16(6)$ \\
\hline $\mathrm{N}(1)-\mathrm{C}(1)-\mathrm{C}(2)$ & $122.04(11)$ & $C(16)-C(11)-C(12)$ & $120.16(12)$ \\
\hline $\mathrm{N}(1)-\mathrm{C}(1)-\mathrm{H}(1)$ & 119.0 & $\mathrm{C}(16)-\mathrm{C}(11)-\mathrm{P}(1)$ & $119.59(9)$ \\
\hline $\mathrm{C}(2)-\mathrm{C}(1)-\mathrm{H}(1)$ & 119.0 & $\mathrm{C}(12)-\mathrm{C}(11)-\mathrm{P}(1)$ & $120.04(10)$ \\
\hline$C(3)-C(2)-C(1)$ & $119.16(12)$ & $\mathrm{C}(13)-\mathrm{C}(12)-\mathrm{C}(11)$ & $119.75(13)$ \\
\hline $\mathrm{C}(3)-\mathrm{C}(2)-\mathrm{H}(2)$ & 120.4 & $\mathrm{C}(13)-\mathrm{C}(12)-\mathrm{H}(12)$ & 120.1 \\
\hline $\mathrm{C}(1)-\mathrm{C}(2)-\mathrm{H}(2)$ & 120.4 & $\mathrm{C}(11)-\mathrm{C}(12)-\mathrm{H}(12)$ & 120.1 \\
\hline$C(2)-C(3)-C(4)$ & $119.22(11)$ & $\mathrm{C}(14)-\mathrm{C}(13)-\mathrm{C}(12)$ & $119.89(13)$ \\
\hline $\mathrm{C}(2)-\mathrm{C}(3)-\mathrm{H}(3)$ & 120.4 & $\mathrm{C}(14)-\mathrm{C}(13)-\mathrm{H}(13)$ & 120.1 \\
\hline
\end{tabular}




\begin{tabular}{|c|c|c|c|}
\hline $\mathrm{C}(12)-\mathrm{C}(13)-\mathrm{H}(13)$ & 120.1 & $\mathrm{C}(25)-\mathrm{C}(24)-\mathrm{H}(24)$ & 120.3 \\
\hline$C(13)-C(14)-C(15)$ & $120.66(13)$ & $\mathrm{C}(23)-\mathrm{C}(24)-\mathrm{H}(24)$ & 120.3 \\
\hline $\mathrm{C}(13)-\mathrm{C}(14)-\mathrm{H}(14)$ & 119.7 & $C(26)-C(25)-C(24)$ & $120.62(14)$ \\
\hline $\mathrm{C}(15)-\mathrm{C}(14)-\mathrm{H}(14)$ & 119.7 & $\mathrm{C}(26)-\mathrm{C}(25)-\mathrm{H}(25)$ & 119.7 \\
\hline$C(14)-C(15)-C(16)$ & $119.98(14)$ & $\mathrm{C}(24)-\mathrm{C}(25)-\mathrm{H}(25)$ & 119.7 \\
\hline $\mathrm{C}(14)-\mathrm{C}(15)-\mathrm{H}(15)$ & 120.0 & $C(25)-C(26)-C(27)$ & $119.84(14)$ \\
\hline $\mathrm{C}(16)-\mathrm{C}(15)-\mathrm{H}(15)$ & 120.0 & $\mathrm{C}(25)-\mathrm{C}(26)-\mathrm{H}(26)$ & 120.1 \\
\hline$C(15)-C(16)-C(11)$ & $119.55(13)$ & $\mathrm{C}(27)-\mathrm{C}(26)-\mathrm{H}(26)$ & 120.1 \\
\hline $\mathrm{C}(15)-\mathrm{C}(16)-\mathrm{H}(16)$ & 120.2 & $\mathrm{C}(26)-\mathrm{C}(27)-\mathrm{C}(28)$ & $120.58(15)$ \\
\hline $\mathrm{C}(11)-\mathrm{C}(16)-\mathrm{H}(16)$ & 120.2 & $\mathrm{C}(26)-\mathrm{C}(27)-\mathrm{H}(27)$ & 119.7 \\
\hline$C(22)-C(17)-C(18)$ & $120.12(11)$ & $\mathrm{C}(28)-\mathrm{C}(27)-\mathrm{H}(27)$ & 119.7 \\
\hline $\mathrm{C}(22)-\mathrm{C}(17)-\mathrm{P}(1)$ & $121.74(9)$ & $\mathrm{C}(27)-\mathrm{C}(28)-\mathrm{C}(23)$ & $119.46(14)$ \\
\hline $\mathrm{C}(18)-\mathrm{C}(17)-\mathrm{P}(1)$ & $118.09(9)$ & $\mathrm{C}(27)-\mathrm{C}(28)-\mathrm{H}(28)$ & 120.3 \\
\hline$C(19)-C(18)-C(17)$ & $119.47(12)$ & $\mathrm{C}(23)-\mathrm{C}(28)-\mathrm{H}(28)$ & 120.3 \\
\hline $\mathrm{C}(19)-\mathrm{C}(18)-\mathrm{H}(18)$ & 120.3 & $C(34)-C(29)-C(30)$ & $120.16(11)$ \\
\hline $\mathrm{C}(17)-\mathrm{C}(18)-\mathrm{H}(18)$ & 120.3 & $\mathrm{C}(34)-\mathrm{C}(29)-\mathrm{P}(1)$ & $120.30(9)$ \\
\hline$C(18)-C(19)-C(20)$ & $120.52(12)$ & $C(30)-C(29)-P(1)$ & $119.54(9)$ \\
\hline $\mathrm{C}(18)-\mathrm{C}(19)-\mathrm{H}(19)$ & 119.7 & $C(31)-C(30)-C(29)$ & $119.75(12)$ \\
\hline $\mathrm{C}(20)-\mathrm{C}(19)-\mathrm{H}(19)$ & 119.7 & $\mathrm{C}(31)-\mathrm{C}(30)-\mathrm{H}(30)$ & 120.1 \\
\hline$C(21)-C(20)-C(19)$ & $119.95(12)$ & $\mathrm{C}(29)-\mathrm{C}(30)-\mathrm{H}(30)$ & 120.1 \\
\hline $\mathrm{C}(21)-\mathrm{C}(20)-\mathrm{H}(20)$ & 120.0 & $\mathrm{C}(30)-\mathrm{C}(31)-\mathrm{C}(32)$ & $119.87(12)$ \\
\hline $\mathrm{C}(19)-\mathrm{C}(20)-\mathrm{H}(20)$ & 120.0 & $\mathrm{C}(30)-\mathrm{C}(31)-\mathrm{H}(31)$ & 120.1 \\
\hline$C(20)-C(21)-C(22)$ & $120.26(12)$ & $\mathrm{C}(32)-\mathrm{C}(31)-\mathrm{H}(31)$ & 120.1 \\
\hline $\mathrm{C}(20)-\mathrm{C}(21)-\mathrm{H}(21)$ & 119.9 & $\mathrm{C}(33)-\mathrm{C}(32)-\mathrm{C}(31)$ & $120.34(11)$ \\
\hline $\mathrm{C}(22)-\mathrm{C}(21)-\mathrm{H}(21)$ & 119.9 & $\mathrm{C}(33)-\mathrm{C}(32)-\mathrm{H}(32)$ & 119.8 \\
\hline$C(21)-C(22)-C(17)$ & $119.67(11)$ & $\mathrm{C}(31)-\mathrm{C}(32)-\mathrm{H}(32)$ & 119.8 \\
\hline $\mathrm{C}(21)-\mathrm{C}(22)-\mathrm{H}(22)$ & 120.2 & $\mathrm{C}(32)-\mathrm{C}(33)-\mathrm{C}(34)$ & $120.29(12)$ \\
\hline $\mathrm{C}(17)-\mathrm{C}(22)-\mathrm{H}(22)$ & 120.2 & $\mathrm{C}(32)-\mathrm{C}(33)-\mathrm{H}(33)$ & 119.9 \\
\hline$C(28)-C(23)-C(24)$ & $120.16(12)$ & $\mathrm{C}(34)-\mathrm{C}(33)-\mathrm{H}(33)$ & 119.9 \\
\hline $\mathrm{C}(28)-\mathrm{C}(23)-\mathrm{P}(1)$ & $119.87(10)$ & $\mathrm{C}(33)-\mathrm{C}(34)-\mathrm{C}(29)$ & $119.57(11)$ \\
\hline $\mathrm{C}(24)-\mathrm{C}(23)-\mathrm{P}(1)$ & $119.88(11)$ & $\mathrm{C}(33)-\mathrm{C}(34)-\mathrm{H}(34)$ & 120.2 \\
\hline $\mathrm{C}(25)-\mathrm{C}(24)-\mathrm{C}(23)$ & $119.35(14)$ & $\mathrm{C}(29)-\mathrm{C}(34)-\mathrm{H}(34)$ & 120.2 \\
\hline
\end{tabular}


Table 4. Anisotropic displacement parameters $\left(\AA^{2} \times 10^{3}\right)$ for rhowe 05 . The anisotropic displacement factor exponent takes the form: $-2 \pi^{2}\left[h^{2} a^{* 2} U_{11}+\ldots+2 h k a^{*} b^{*} U_{12}\right]$

\begin{tabular}{|c|c|c|c|c|c|c|}
\hline & $\mathrm{U}_{11}$ & $\mathrm{U}_{22}$ & $\mathrm{U}_{33}$ & $\mathrm{U}_{23}$ & $\mathrm{U}_{13}$ & $\mathrm{U}_{12}$ \\
\hline Mo1 & $8(1)$ & $12(1)$ & $13(1)$ & $3(1)$ & $3(1)$ & $0(1)$ \\
\hline $\mathrm{Cl1}$ & $15(1)$ & $12(1)$ & $17(1)$ & $3(1)$ & $3(1)$ & $0(1)$ \\
\hline $\mathrm{Cl} 2$ & $17(1)$ & $12(1)$ & $25(1)$ & $2(1)$ & $6(1)$ & $1(1)$ \\
\hline $\mathrm{Cl} 3$ & $11(1)$ & $24(1)$ & $20(1)$ & $7(1)$ & $1(1)$ & $4(1)$ \\
\hline $\mathrm{Cl} 4$ & $14(1)$ & $24(1)$ & $19(1)$ & $3(1)$ & $8(1)$ & $-3(1)$ \\
\hline N1 & $9(1)$ & $13(1)$ & $15(1)$ & $4(1)$ & $3(1)$ & $1(1)$ \\
\hline N2 & 11(1) & $12(1)$ & $14(1)$ & $2(1)$ & $3(1)$ & $0(1)$ \\
\hline $\mathrm{C} 1$ & $13(1)$ & $19(1)$ & $14(1)$ & $6(1)$ & $3(1)$ & $1(1)$ \\
\hline $\mathrm{C} 2$ & $15(1)$ & $25(1)$ & $16(1)$ & $8(1)$ & $6(1)$ & $2(1)$ \\
\hline $\mathrm{C} 3$ & $13(1)$ & $23(1)$ & $21(1)$ & $8(1)$ & $8(1)$ & $2(1)$ \\
\hline $\mathrm{C} 4$ & $10(1)$ & $17(1)$ & $20(1)$ & $5(1)$ & $4(1)$ & $0(1)$ \\
\hline C5 & $10(1)$ & $12(1)$ & $14(1)$ & $3(1)$ & $3(1)$ & $1(1)$ \\
\hline C6 & $10(1)$ & $13(1)$ & $15(1)$ & $3(1)$ & $2(1)$ & $1(1)$ \\
\hline C7 & 11(1) & $20(1)$ & $18(1)$ & $4(1)$ & $1(1)$ & $1(1)$ \\
\hline $\mathrm{C} 8$ & $17(1)$ & $22(1)$ & $17(1)$ & $3(1)$ & $-2(1)$ & $2(1)$ \\
\hline C9 & $21(1)$ & $20(1)$ & $14(1)$ & $4(1)$ & $2(1)$ & $3(1)$ \\
\hline $\mathrm{C} 10$ & $18(1)$ & $15(1)$ & $14(1)$ & $4(1)$ & $4(1)$ & $1(1)$ \\
\hline P1 & $12(1)$ & 11(1) & $18(1)$ & $3(1)$ & $3(1)$ & $0(1)$ \\
\hline C11 & $13(1)$ & $14(1)$ & $24(1)$ & $6(1)$ & $4(1)$ & $1(1)$ \\
\hline C12 & $17(1)$ & $16(1)$ & $28(1)$ & $3(1)$ & $4(1)$ & $3(1)$ \\
\hline C13 & $17(1)$ & $17(1)$ & $37(1)$ & $6(1)$ & $7(1)$ & $6(1)$ \\
\hline C14 & $16(1)$ & $26(1)$ & $37(1)$ & $16(1)$ & $5(1)$ & $5(1)$ \\
\hline C15 & $20(1)$ & $32(1)$ & $25(1)$ & $13(1)$ & $3(1)$ & $5(1)$ \\
\hline C16 & $18(1)$ & $21(1)$ & $24(1)$ & $7(1)$ & $6(1)$ & $5(1)$ \\
\hline C17 & $12(1)$ & $14(1)$ & $18(1)$ & $4(1)$ & $3(1)$ & $0(1)$ \\
\hline $\mathrm{C} 18$ & $18(1)$ & $15(1)$ & $27(1)$ & $7(1)$ & $8(1)$ & $1(1)$ \\
\hline C19 & $20(1)$ & $22(1)$ & $27(1)$ & $9(1)$ & $9(1)$ & $-1(1)$ \\
\hline $\mathrm{C} 20$ & $20(1)$ & $24(1)$ & $21(1)$ & $5(1)$ & $9(1)$ & $2(1)$ \\
\hline $\mathrm{C} 21$ & $21(1)$ & $16(1)$ & $20(1)$ & $3(1)$ & $7(1)$ & $4(1)$ \\
\hline $\mathrm{C} 22$ & $16(1)$ & $14(1)$ & $16(1)$ & $3(1)$ & $3(1)$ & $1(1)$ \\
\hline $\mathrm{C} 23$ & $14(1)$ & $13(1)$ & $22(1)$ & $0(1)$ & $1(1)$ & $1(1)$ \\
\hline
\end{tabular}




\begin{tabular}{lllllll}
$\mathrm{C} 24$ & $18(1)$ & $16(1)$ & $30(1)$ & $5(1)$ & $-2(1)$ & $-3(1)$ \\
$\mathrm{C} 25$ & $22(1)$ & $15(1)$ & $38(1)$ & $3(1)$ & $-7(1)$ & $-2(1)$ \\
$\mathrm{C} 26$ & $26(1)$ & $20(1)$ & $30(1)$ & $-6(1)$ & $-7(1)$ & $4(1)$ \\
$\mathrm{C} 27$ & $27(1)$ & $31(1)$ & $24(1)$ & $-7(1)$ & $6(1)$ & $1(1)$ \\
$\mathrm{C} 28$ & $20(1)$ & $24(1)$ & $24(1)$ & $-4(1)$ & $6(1)$ & $-2(1)$ \\
$\mathrm{C} 29$ & $13(1)$ & $13(1)$ & $15(1)$ & $3(1)$ & $3(1)$ & $-1(1)$ \\
$\mathrm{C} 30$ & $15(1)$ & $18(1)$ & $16(1)$ & $4(1)$ & $2(1)$ & $0(1)$ \\
$\mathrm{C} 31$ & $21(1)$ & $17(1)$ & $17(1)$ & $6(1)$ & $5(1)$ & $1(1)$ \\
$\mathrm{C} 32$ & $21(1)$ & $16(1)$ & $18(1)$ & $4(1)$ & $9(1)$ & $-1(1)$ \\
$\mathrm{C} 33$ & $14(1)$ & $18(1)$ & $19(1)$ & $2(1)$ & $5(1)$ & $-3(1)$ \\
$\mathrm{C} 34$ & $13(1)$ & $15(1)$ & $17(1)$ & $3(1)$ & $4(1)$ & $0(1)$ \\
& & & & & & \\
\hline
\end{tabular}


Table 5. Hydrogen coordinates $\left(\times 10^{4}\right)$ and isotropic displacement parameters $\left(\AA^{2} \times 10^{3}\right)$ for rhowe 05 .

\begin{tabular}{|c|c|c|c|c|}
\hline & $\mathrm{x}$ & $\mathrm{y}$ & z & $\mathrm{U}(\mathrm{eq})$ \\
\hline H1 & 9318 & 8817 & 4538 & 18 \\
\hline $\mathrm{H} 2$ & 7316 & 8584 & 5260 & 22 \\
\hline $\mathrm{H} 3$ & 4882 & 8329 & 4297 & 22 \\
\hline $\mathrm{H} 4$ & 4505 & 8375 & 2629 & 19 \\
\hline $\mathrm{H} 7$ & 4362 & 8321 & 1072 & 21 \\
\hline $\mathrm{H} 8$ & 4352 & 8594 & -495 & 24 \\
\hline H9 & 6587 & 9186 & -832 & 23 \\
\hline $\mathrm{H} 10$ & 8784 & 9386 & 361 & 19 \\
\hline $\mathrm{H} 12$ & 4914 & 5828 & 3425 & 25 \\
\hline H13 & 2934 & 6659 & 2632 & 29 \\
\hline H14 & 2103 & 6080 & 922 & 30 \\
\hline H15 & 3171 & 4635 & -5 & 30 \\
\hline H16 & 5119 & 3769 & 780 & 25 \\
\hline H18 & 8155 & 5047 & 1716 & 23 \\
\hline H19 & 9964 & 4558 & 772 & 26 \\
\hline $\mathrm{H} 20$ & 10760 & 2790 & 469 & 25 \\
\hline $\mathrm{H} 21$ & 9810 & 1516 & 1158 & 22 \\
\hline $\mathrm{H} 22$ & 8011 & 1994 & 2119 & 18 \\
\hline $\mathrm{H} 24$ & 8903 & 5733 & 3416 & 27 \\
\hline $\mathrm{H} 25$ & 9973 & 6943 & 4935 & 33 \\
\hline H26 & 9234 & 6820 & 6373 & 35 \\
\hline $\mathrm{H} 27$ & 7433 & 5476 & 6307 & 36 \\
\hline $\mathrm{H} 28$ & 6333 & 4263 & 4799 & 29 \\
\hline $\mathrm{H} 30$ & 7604 & 2386 & 3812 & 20 \\
\hline H31 & 6465 & 850 & 4063 & 22 \\
\hline H32 & 3899 & 417 & 3434 & 22 \\
\hline H33 & 2469 & 1519 & 2573 & 21 \\
\hline H34 & 3602 & 3036 & 2287 & 18 \\
\hline
\end{tabular}


Table 6. Torsion angles $\left[{ }^{\circ}\right]$ for rhowe 05 .

\begin{tabular}{|c|c|c|c|}
\hline $\mathrm{C} 5-\mathrm{N} 1-\mathrm{C} 1-\mathrm{C} 2$ & $2.19(18)$ & C11-C12-C13-C14 & $-1.0(2)$ \\
\hline Mo1-N1-C1-C2 & $-178.84(9)$ & C12-C13-C14-C15 & $1.1(2)$ \\
\hline $\mathrm{N} 1-\mathrm{C} 1-\mathrm{C} 2-\mathrm{C} 3$ & $-0.46(19)$ & C13-C14-C15-C16 & $-0.4(2)$ \\
\hline $\mathrm{C} 1-\mathrm{C} 2-\mathrm{C} 3-\mathrm{C} 4$ & $-1.27(19)$ & C14-C15-C16-C11 & $-0.5(2)$ \\
\hline $\mathrm{C} 2-\mathrm{C} 3-\mathrm{C} 4-\mathrm{C} 5$ & $1.25(19)$ & C12-C11-C16-C15 & $0.6(2)$ \\
\hline C1-N1-C5-C4 & $-2.20(17)$ & P1-C11-C16-C15 & $175.34(10)$ \\
\hline Mo1-N1-C5-C4 & $178.76(9)$ & $\mathrm{C} 29-\mathrm{P} 1-\mathrm{C} 17-\mathrm{C} 22$ & $-14.82(12)$ \\
\hline C1-N1-C5-C6 & $174.44(10)$ & $\mathrm{C} 11-\mathrm{P} 1-\mathrm{C} 17-\mathrm{C} 22$ & $-134.45(10)$ \\
\hline Mo1-N1-C5-C6 & $-4.60(12)$ & C23-P1-C17-C22 & $105.74(11)$ \\
\hline $\mathrm{C} 3-\mathrm{C} 4-\mathrm{C} 5-\mathrm{N} 1$ & $0.49(18)$ & C29-P1-C17-C18 & $167.55(10)$ \\
\hline $\mathrm{C} 3-\mathrm{C} 4-\mathrm{C} 5-\mathrm{C} 6$ & $-175.87(11)$ & C11-P1-C17-C18 & $47.92(12)$ \\
\hline C10-N2-C6-C7 & $3.04(17)$ & C23-P1-C17-C18 & $-71.89(12)$ \\
\hline Mo1-N2-C6-C7 & $-168.28(9)$ & $\mathrm{C} 22-\mathrm{C} 17-\mathrm{C} 18-\mathrm{C} 19$ & $-0.6(2)$ \\
\hline C10-N2-C6-C5 & $-175.67(10)$ & P1-C17-C18-C19 & $177.10(11)$ \\
\hline Mo1-N2-C6-C5 & $13.00(12)$ & C17-C18-C19-C20 & $1.0(2)$ \\
\hline $\mathrm{N} 1-\mathrm{C} 5-\mathrm{C} 6-\mathrm{N} 2$ & $-5.61(15)$ & $\mathrm{C} 18-\mathrm{C} 19-\mathrm{C} 20-\mathrm{C} 21$ & $-1.0(2)$ \\
\hline $\mathrm{C} 4-\mathrm{C} 5-\mathrm{C} 6-\mathrm{N} 2$ & $170.95(11)$ & $\mathrm{C} 19-\mathrm{C} 20-\mathrm{C} 21-\mathrm{C} 22$ & $0.7(2)$ \\
\hline N1-C5-C6-C7 & $175.70(11)$ & $\mathrm{C} 20-\mathrm{C} 21-\mathrm{C} 22-\mathrm{C} 17$ & $-0.4(2)$ \\
\hline $\mathrm{C} 4-\mathrm{C} 5-\mathrm{C} 6-\mathrm{C} 7$ & $-7.74(18)$ & $\mathrm{C} 18-\mathrm{C} 17-\mathrm{C} 22-\mathrm{C} 21$ & $0.27(19)$ \\
\hline N2-C6-C7-C8 & $-1.73(18)$ & P1-C17-C22-C21 & $-177.31(10)$ \\
\hline $\mathrm{C} 5-\mathrm{C} 6-\mathrm{C} 7-\mathrm{C} 8$ & $176.88(11)$ & C29-P1-C23-C28 & $-20.03(12)$ \\
\hline C6-C7-C8-C9 & $-0.89(19)$ & C11-P1-C23-C28 & $98.51(12)$ \\
\hline $\mathrm{C} 7-\mathrm{C} 8-\mathrm{C} 9-\mathrm{C} 10$ & $2.13(19)$ & C17-P1-C23-C28 & $-141.84(11)$ \\
\hline C6-N2-C10-C9 & $-1.75(17)$ & C29-P1-C23-C24 & $163.31(10)$ \\
\hline Mo1-N2-C10-C9 & $168.74(9)$ & C11-P1-C23-C24 & $-78.15(11)$ \\
\hline $\mathrm{C} 8-\mathrm{C} 9-\mathrm{C} 10-\mathrm{N} 2$ & $-0.83(19)$ & C17-P1-C23-C24 & $41.50(12)$ \\
\hline C29-P1-C11-C16 & $-82.13(11)$ & $\mathrm{C} 28-\mathrm{C} 23-\mathrm{C} 24-\mathrm{C} 25$ & $-0.2(2)$ \\
\hline C17-P1-C11-C16 & $38.48(12)$ & $\mathrm{P} 1-\mathrm{C} 23-\mathrm{C} 24-\mathrm{C} 25$ & $176.43(10)$ \\
\hline C23-P1-C11-C16 & $159.68(10)$ & $\mathrm{C} 23-\mathrm{C} 24-\mathrm{C} 25-\mathrm{C} 26$ & $0.1(2)$ \\
\hline C29-P1-C11-C12 & $92.58(11)$ & $\mathrm{C} 24-\mathrm{C} 25-\mathrm{C} 26-\mathrm{C} 27$ & $0.3(2)$ \\
\hline C17-P1-C11-C12 & $-146.82(10)$ & $\mathrm{C} 25-\mathrm{C} 26-\mathrm{C} 27-\mathrm{C} 28$ & $-0.5(2)$ \\
\hline C23-P1-C11-C12 & $-25.61(12)$ & $\mathrm{C} 26-\mathrm{C} 27-\mathrm{C} 28-\mathrm{C} 23$ & $0.4(2)$ \\
\hline C16-C11-C12-C13 & $0.1(2)$ & $\mathrm{C} 24-\mathrm{C} 23-\mathrm{C} 28-\mathrm{C} 27$ & $0.0(2)$ \\
\hline P1-C11-C12-C13 & $-174.60(10)$ & $\mathrm{P} 1-\mathrm{C} 23-\mathrm{C} 28-\mathrm{C} 27$ & $-176.64(12)$ \\
\hline
\end{tabular}




$\begin{array}{lclc}\text { C11-P1-C29-C34 } & 4.98(12) & \text { P1-C29-C30-C31 } & -178.46(9) \\ \text { C17-P1-C29-C34 } & -114.41(10) & \text { C29-C30-C31-C32 } & -0.87(18) \\ \text { C23-P1-C29-C34 } & 123.44(10) & \text { C30-C31-C32-C33 } & -0.40(19) \\ \text { C11-P1-C29-C30 } & -175.15(9) & \text { C31-C32-C33-C34 } & 1.13(19) \\ \text { C17-P1-C29-C30 } & 65.46(11) & \text { C32-C33-C34-C29 } & -0.58(18) \\ \text { C23-P1-C29-C30 } & -56.69(11) & \text { C30-C29-C34-C33 } & -0.69(17) \\ \text { C34-C29-C30-C31 } & 1.41(18) & \text { P1-C29-C34-C33 } & 179.18(9)\end{array}$


REFERENCE NUMBER: rhowe10

CRYSTAL STRUCTURE REPORT

$\mathrm{C}_{18} \mathrm{H}_{24.19} \mathrm{Cl}_{4} \mathrm{Li}$ Mo N2 $\mathrm{O}_{4.10}$

or

$\left[\mathrm{Li}(12\right.$-crown-4) $]\left[\mathrm{MoCl}_{4}(\mathrm{bpy})\right] \cdot 0.1 \mathrm{H}_{2} \mathrm{O}$

Report prepared for:

Prof. W. Eckenhoff

August 29, 2017

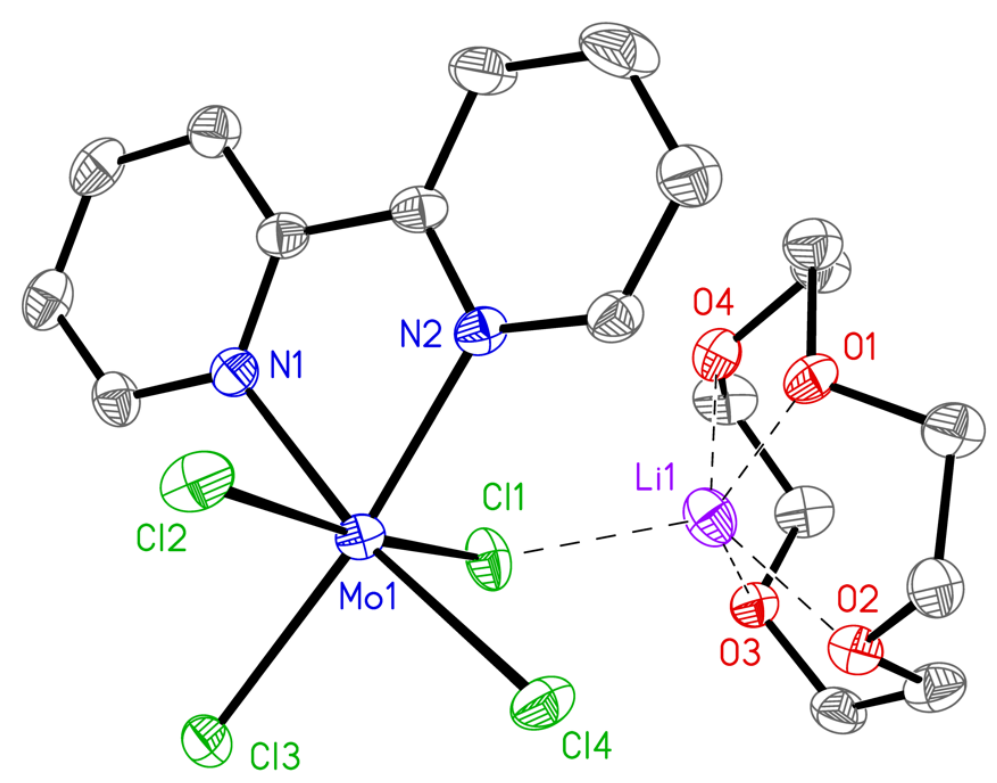

William W. Brennessel

X-ray Crystallographic Facility

Department of Chemistry, University of Rochester

120 Trustee Road

Rochester, NY 14627 


\section{Data collection}

A crystal $\left(0.14 \times 0.12 \times 0.06 \mathrm{~mm}^{3}\right)$ was placed onto the tip of a thin glass optical fiber and mounted on a Bruker SMART APEX II CCD platform diffractometer for a data collection at 100.0(5) K. ${ }^{1}$ A preliminary set of cell constants and an orientation matrix were calculated from reflections harvested from three orthogonal wedges of reciprocal space. The full data collection was carried out using MoK $\alpha$ radiation (graphite monochromator) with a frame time of 50 seconds and a detector distance of $4.03 \mathrm{~cm}$. A randomly oriented region of reciprocal space was surveyed: six major sections of frames were collected with $0.50^{\circ}$ steps in $\omega$ at six different $\phi$ settings and a detector position of $-38^{\circ}$ in $2 \theta$. The intensity data were corrected for absorption. ${ }^{2}$ Final cell constants were calculated from the xyz centroids of 3781 strong reflections from the actual data collection after integration. ${ }^{3}$ See Table 1 for additional crystal and refinement information.

\section{Structure solution and refinement}

The structure was solved using SHELXT-2014/54 and refined using SHELXL-2017/1..$^{5}$ The space group Pbca was determined based on systematic absences. A direct-methods solution was calculated which provided most nonhydrogen atoms from the E-map. Full-matrix least squares / difference Fourier cycles were performed which located the remaining non-hydrogen atoms. All non-hydrogen atoms were refined with anisotropic displacement parameters. All hydrogen atoms were placed in ideal positions and refined as riding atoms with relative isotropic displacement parameters. The final full matrix least squares refinement converged to $R 1=0.0406\left(F^{2}, I>2 \sigma(I)\right)$ and $w R 2=0.0938\left(F^{2}\right.$, all data $)$.

\section{Structure description}

The structure is the one suggested. The asymmetric unit contains two $\mathrm{Li}\left(12\right.$-crown-4) cations, two $\mathrm{MoCl}_{4}(\mathrm{bpy})$ anions, a partially occupied water solvent molecule (0.19), all in general positions. The cations and anions are contact ion pairs via $\mathrm{Li}$...Cl interactions and both $\mathrm{Li}(12$-crown-4) cations are modeled as disordered, Lilover two positions (0.55:0.45) and Li2 over three positions (0.61:0.24:0.15).

Unless noted otherwise all structural diagrams containing thermal displacement ellipsoids are drawn at the $50 \%$ probability level.

Data collection, structure solution, and structure refinement were conducted at the X-ray Crystallographic Facility, B04 Hutchison Hall, Department of Chemistry, University of Rochester. All publications arising from this report MUST either 1) include William W. Brennessel as a coauthor or 2) acknowledge William W. Brennessel and the Xray Crystallographic Facility of the Department of Chemistry at the University of Rochester. 
1 APEX3, version 2017.3-0; Bruker AXS: Madison, WI, 2017.

2 Krause, L.; Herbst-Irmer, R.; Sheldrick, G. M.; Stalke, D. SADABS, version 2016/2; J. Appl. Cryst. 2015, 48, 3-

10.

3 SAINT, version 8.34A; Bruker AXS: Madison, WI, 2013.

4 Sheldrick, G. M. SHELXT, version 2014/5; Acta. Cryst. 2015, A71, 3-8.

5 Sheldrick, G. M. SHELXL, version 2017/1; Acta. Cryst. 2015, C71, 3-8.

Some equations of interest:

$$
\begin{gathered}
R_{\mathrm{int}}=\Sigma\left|F_{\mathrm{o}}{ }^{2}-<F_{\mathrm{o}}{ }^{2}>\right| / \Sigma\left|F_{\mathrm{o}}{ }^{2}\right| \\
R 1=\Sigma|| F_{\mathrm{o}}|-| F_{\mathrm{c}} \| / \Sigma\left|F_{\mathrm{o}}\right| \\
w R 2=\left[\Sigma\left[w\left(F_{\mathrm{o}}{ }^{2}-F_{\mathrm{c}}{ }^{2}\right)^{2}\right] / \Sigma\left[w\left(F_{\mathrm{o}}{ }^{2}\right)^{2}\right]\right]^{1 / 2} \\
\text { where } w=1 /\left[\sigma^{2}\left(F_{\mathrm{o}}{ }^{2}\right)+(a P)^{2}+b P\right] \text { and } \\
P=1 / 3 \max \left(0, F_{\mathrm{o}}{ }^{2}\right)+2 / 3 F_{\mathrm{c}}{ }^{2} \\
\text { GOF }=S=\left[\Sigma\left[w\left(F_{\mathrm{o}}{ }^{2}-F_{\mathrm{c}}{ }^{2}\right)^{2}\right] /(m-n)\right]^{1 / 2}
\end{gathered}
$$

where $m=$ number of reflections and $n=$ number of parameters
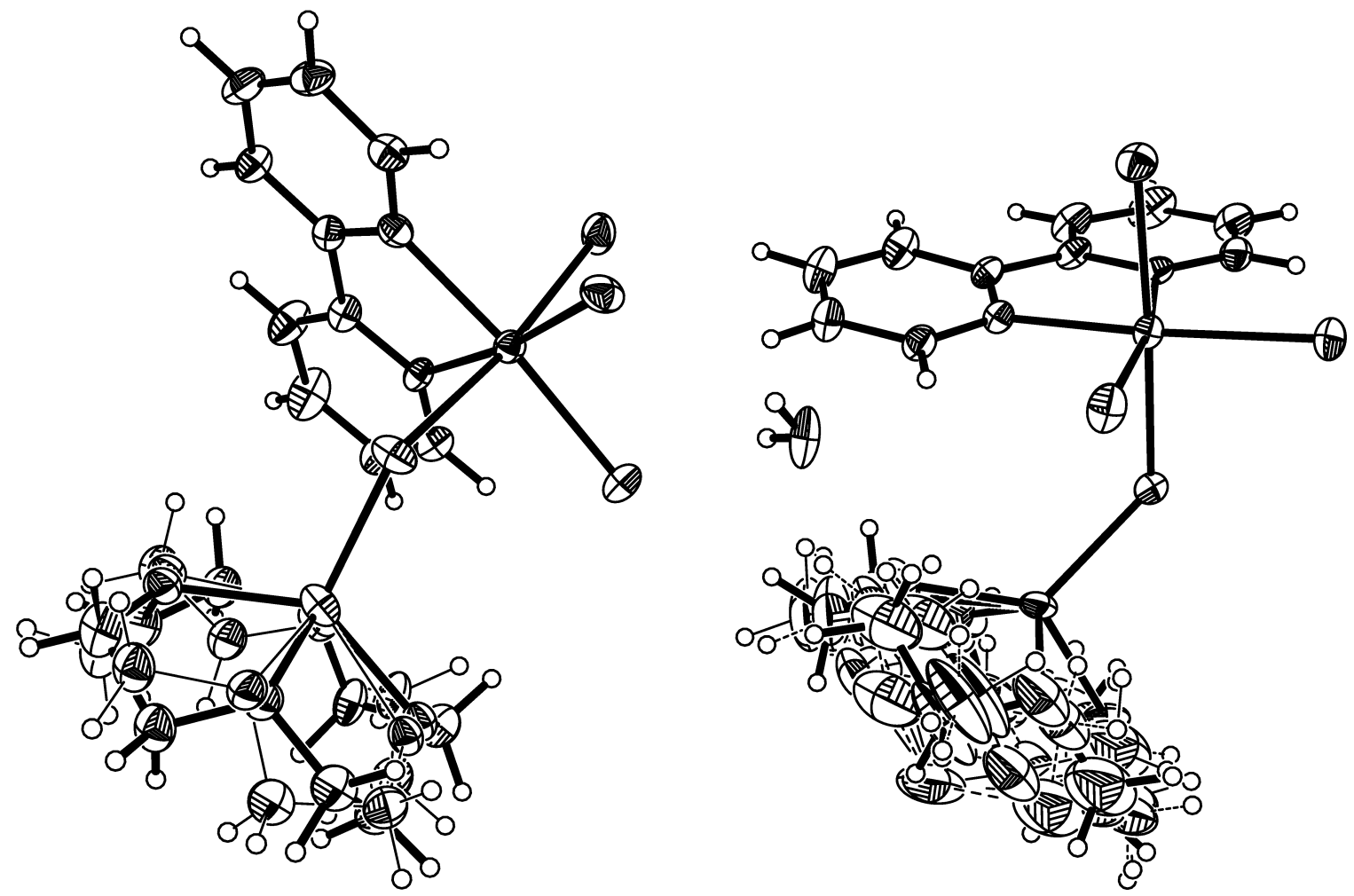

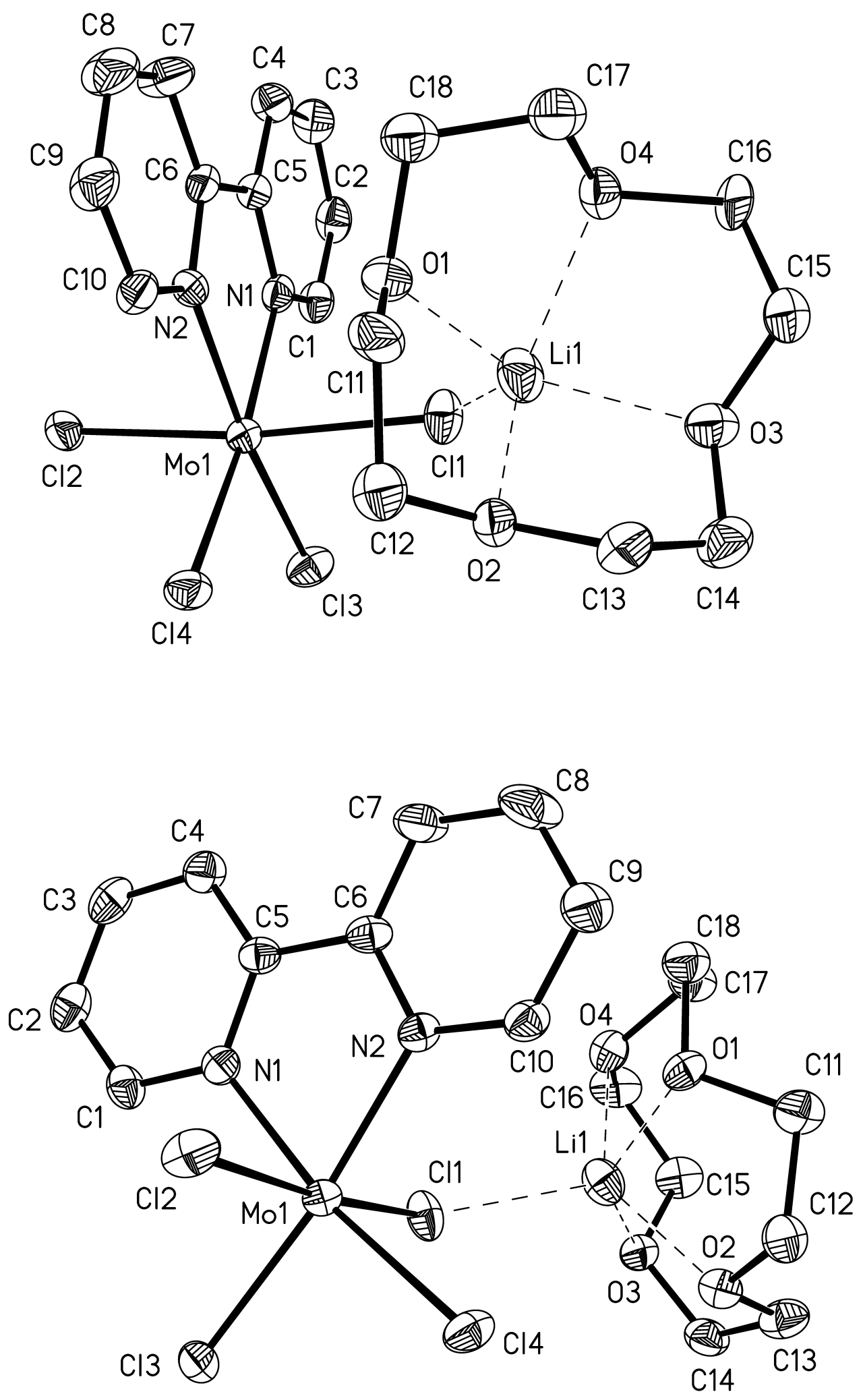


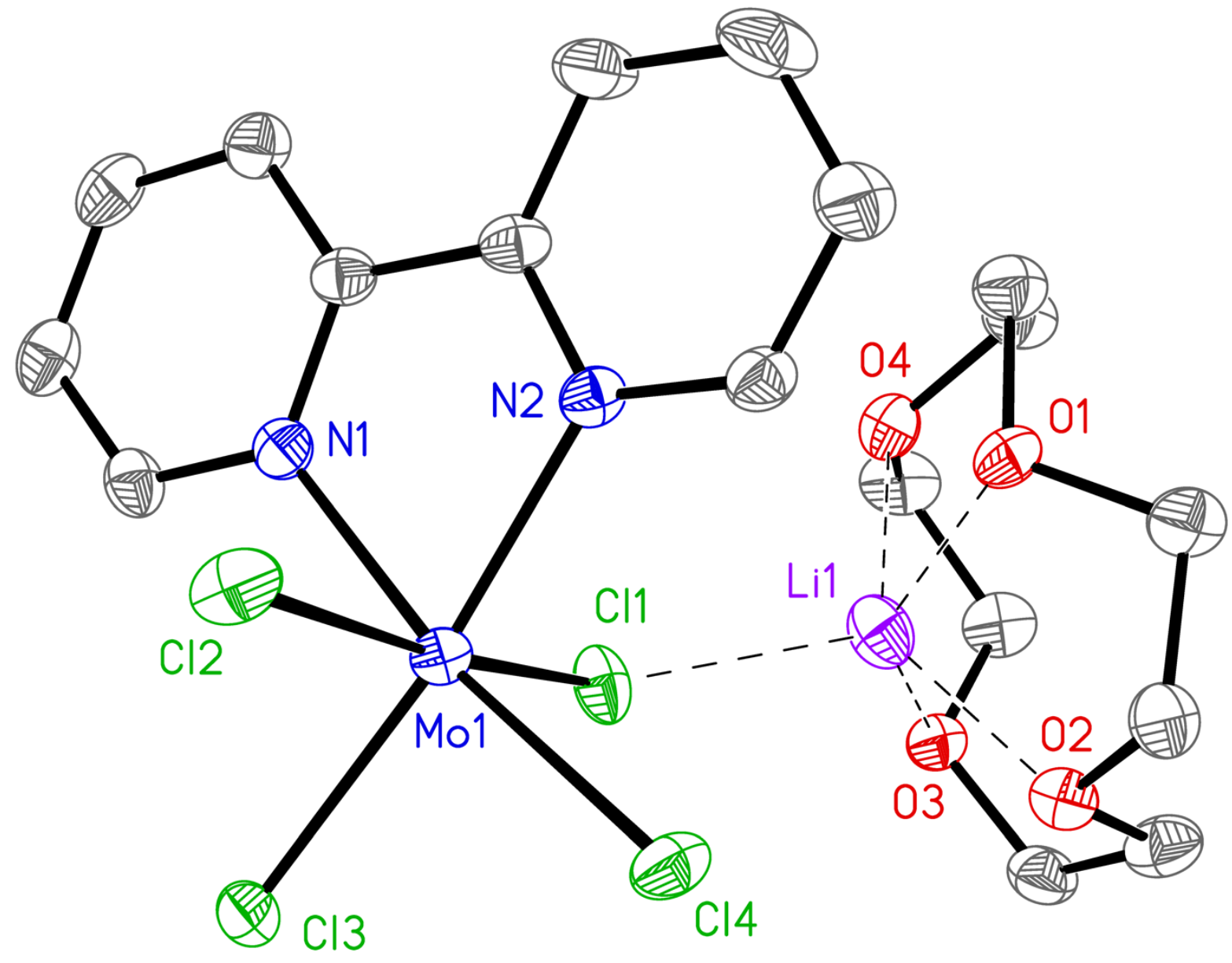



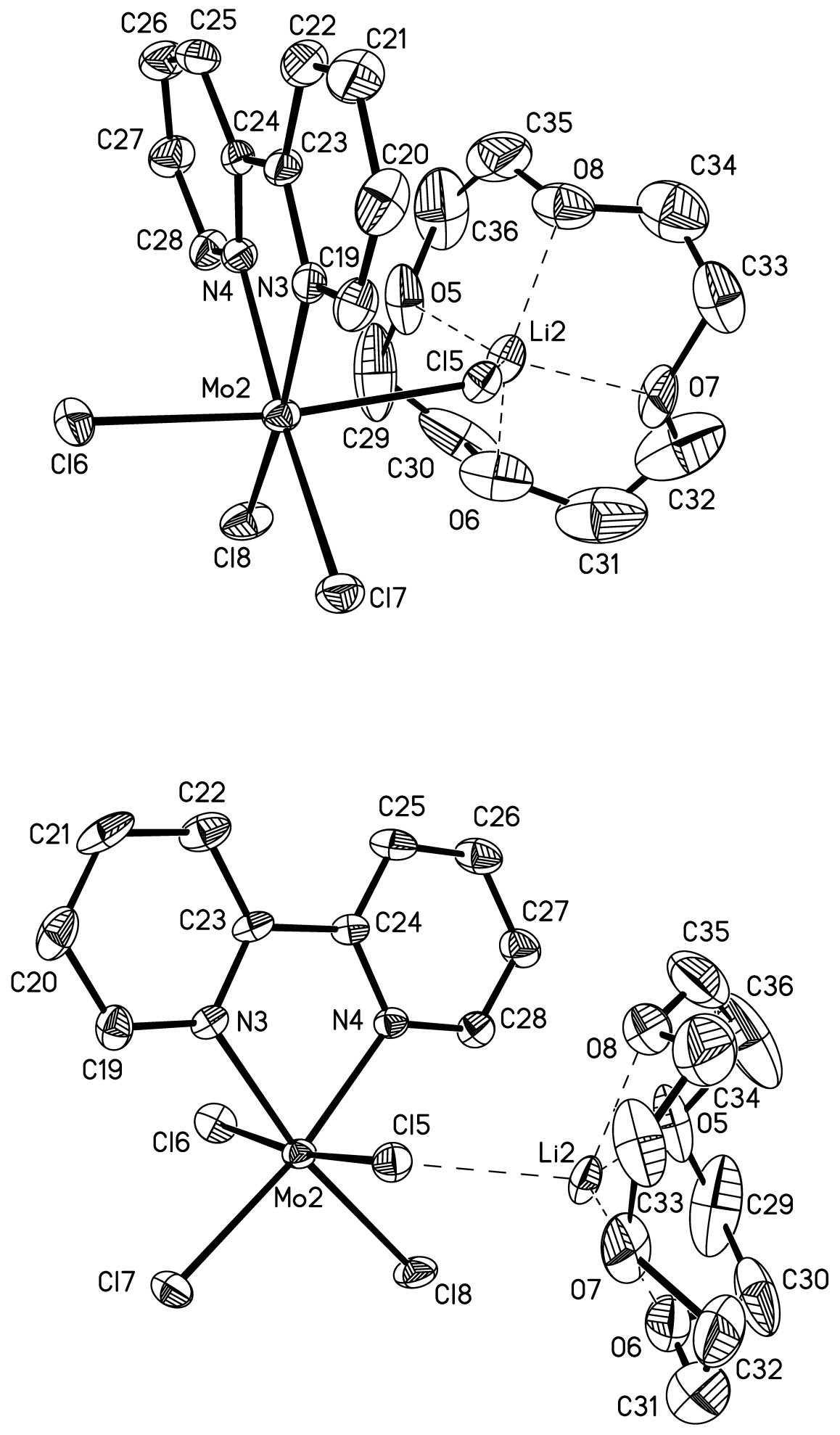
Table 1. Crystal data and structure refinement for rhowe10.

\begin{tabular}{|c|c|c|}
\hline Identification code & \multicolumn{2}{|l|}{ rhowe10 } \\
\hline Empirical formula & \multicolumn{2}{|c|}{ C18 H24.19 C14 Li Mo N2 O4.10 } \\
\hline Formula weight & \multicolumn{2}{|l|}{578.82} \\
\hline Temperature & \multicolumn{2}{|l|}{$100.0(5) \mathrm{K}$} \\
\hline Wavelength & \multicolumn{2}{|l|}{$0.71073 \AA$} \\
\hline Crystal system & \multicolumn{2}{|l|}{ orthorhombic } \\
\hline Space group & \multicolumn{2}{|l|}{ Pbca } \\
\hline \multirow[t]{3}{*}{ Unit cell dimensions } & $a=16.5660(14) \AA$ & $\alpha=90^{\circ}$ \\
\hline & $b=20.9617(18) \AA$ & $\beta=90^{\circ}$ \\
\hline & $c=27.061(2) \AA$ & $\gamma=90^{\circ}$ \\
\hline Volume & \multicolumn{2}{|l|}{$9397.0(14) \AA^{3}$} \\
\hline$Z$ & \multicolumn{2}{|l|}{16} \\
\hline Density (calculated) & \multicolumn{2}{|l|}{$1.637 \mathrm{Mg} / \mathrm{m}^{3}$} \\
\hline Absorption coefficient & \multicolumn{2}{|l|}{$1.040 \mathrm{~mm}^{-1}$} \\
\hline$F(000)$ & \multicolumn{2}{|l|}{4672} \\
\hline Crystal color, morphology & \multicolumn{2}{|l|}{ red, plate } \\
\hline Crystal size & \multicolumn{2}{|c|}{$0.14 \times 0.12 \times 0.06 \mathrm{~mm}^{3}$} \\
\hline Theta range for data collection & \multicolumn{2}{|l|}{1.505 to $25.278^{\circ}$} \\
\hline Index ranges & \multicolumn{2}{|c|}{$-19 \leq h \leq 19,-25 \leq k \leq 25,-32 \leq l \leq 32$} \\
\hline Reflections collected & \multicolumn{2}{|l|}{150032} \\
\hline Independent reflections & \multicolumn{2}{|c|}{$8522[R($ int $)=0.1600]$} \\
\hline Observed reflections & \multicolumn{2}{|l|}{6108} \\
\hline Completeness to theta $=25.242^{\circ}$ & \multicolumn{2}{|l|}{$100.0 \%$} \\
\hline Absorption correction & \multicolumn{2}{|l|}{ Multi-scan } \\
\hline Max. and min. transmission & \multicolumn{2}{|l|}{0.7452 and 0.6647} \\
\hline Refinement method & \multicolumn{2}{|c|}{ Full-matrix least-squares on $F^{2}$} \\
\hline Data / restraints / parameters & \multicolumn{2}{|l|}{$8522 / 336 / 675$} \\
\hline Goodness-of-fit on $F^{2}$ & \multicolumn{2}{|l|}{1.006} \\
\hline Final $R$ indices $[I>2 \operatorname{sigma}(I)]$ & \multicolumn{2}{|c|}{$R 1=0.0406, w R 2=0.0810$} \\
\hline$R$ indices (all data) & \multicolumn{2}{|c|}{$R 1=0.0708, w R 2=0.0938$} \\
\hline Largest diff. peak and hole & \multicolumn{2}{|c|}{0.543 and -0.519 e. $\AA^{-3}$} \\
\hline
\end{tabular}


Table 2. Atomic coordinates $\left(\times 10^{4}\right)$ and equivalent isotropic displacement parameters $\left(\AA^{2} \times 10^{3}\right)$ for rhowe10. $U_{\text {eq }}$ is defined as one third of the trace of the orthogonalized $U_{i j}$ tensor.

\begin{tabular}{|c|c|c|c|c|}
\hline & $\mathrm{x}$ & $\mathrm{y}$ & $\mathrm{z}$ & $\mathrm{U}_{\mathrm{eq}}$ \\
\hline Mo1 & $1543(1)$ & $3273(1)$ & $4940(1)$ & $19(1)$ \\
\hline $\mathrm{Cl1}$ & $2110(1)$ & $2476(1)$ & $5512(1)$ & $28(1)$ \\
\hline $\mathrm{Cl} 2$ & $845(1)$ & $4058(1)$ & $4450(1)$ & $30(1)$ \\
\hline $\mathrm{Cl} 3$ & $2496(1)$ & $4059(1)$ & $5257(1)$ & $29(1)$ \\
\hline $\mathrm{Cl} 4$ & $2437(1)$ & $2950(1)$ & $4272(1)$ & $28(1)$ \\
\hline N1 & $609(2)$ & $3447(2)$ & $5485(1)$ & $21(1)$ \\
\hline N2 & $599(2)$ & $2605(2)$ & $4756(1)$ & $20(1)$ \\
\hline $\mathrm{C} 1$ & $651(3)$ & $3894(2)$ & $5845(2)$ & $24(1)$ \\
\hline $\mathrm{C} 2$ & $44(3)$ & 3991(2) & $6185(2)$ & $28(1)$ \\
\hline $\mathrm{C} 3$ & $-640(3)$ & $3610(2)$ & $6153(2)$ & $31(1)$ \\
\hline $\mathrm{C} 4$ & $-703(3)$ & $3157(2)$ & $5781(2)$ & $26(1)$ \\
\hline $\mathrm{C} 5$ & $-70(3)$ & $3086(2)$ & $5452(2)$ & $21(1)$ \\
\hline C6 & $-77(3)$ & $2620(2)$ & $5037(2)$ & $22(1)$ \\
\hline $\mathrm{C} 7$ & $-729(3)$ & $2231(2)$ & $4940(2)$ & $34(1)$ \\
\hline $\mathrm{C} 8$ & $-687(3)$ & 1812(3) & $4545(2)$ & $40(1)$ \\
\hline C9 & $2(3)$ & $1796(2)$ & $4259(2)$ & $31(1)$ \\
\hline $\mathrm{C} 10$ & $631(3)$ & $2197(2)$ & $4373(2)$ & $23(1)$ \\
\hline Li1 & $2243(5)$ & $1394(4)$ & $5398(3)$ & $30(2)$ \\
\hline $\mathrm{O} 1$ & $1440(6)$ & $1047(7)$ & $4885(3)$ & $26(1)$ \\
\hline $\mathrm{C} 11$ & $1852(6)$ & $637(5)$ & $4538(4)$ & $31(2)$ \\
\hline $\mathrm{C} 12$ & $2629(5)$ & $971(5)$ & $4419(3)$ & $30(2)$ \\
\hline $\mathrm{O} 2$ & $3052(5)$ & $1134(6)$ & $4864(3)$ & $24(2)$ \\
\hline C13 & $3549(6)$ & $639(5)$ & $5066(3)$ & $33(2)$ \\
\hline $\mathrm{C} 14$ & $3772(5)$ & $839(5)$ & $5582(3)$ & $32(2)$ \\
\hline $\mathrm{O} 3$ & $3080(5)$ & $990(7)$ & $5871(4)$ & $28(1)$ \\
\hline $\mathrm{C} 15$ & $2705(5)$ & $455(5)$ & $6111(3)$ & $33(2)$ \\
\hline $\mathrm{C} 16$ & $1877(6)$ & $654(6)$ & $6280(3)$ & $32(2)$ \\
\hline $\mathrm{O} 4$ & $1449(6)$ & $894(7)$ & $5863(4)$ & $26(1)$ \\
\hline $\mathrm{C} 17$ & $1023(6)$ & $420(5)$ & $5574(3)$ & $34(2)$ \\
\hline $\mathrm{C} 18$ & $752(6)$ & $744(5)$ & $5109(4)$ & $32(2)$ \\
\hline O1' & $1549(7)$ & $1020(9)$ & $4847(4)$ & $26(1)$ \\
\hline
\end{tabular}




\begin{tabular}{|c|c|c|c|c|}
\hline C11' & $2057(7)$ & $862(7)$ & $4441(5)$ & $31(2)$ \\
\hline $\mathrm{C} 12^{\prime}$ & $2843(6)$ & $593(5)$ & 4623(4) & $30(2)$ \\
\hline $\mathrm{O} 2^{\prime}$ & $3167(6)$ & $1056(8)$ & 4964(4) & $24(2)$ \\
\hline C13' & $3837(7)$ & $826(6)$ & $5249(4)$ & $33(2)$ \\
\hline C14' & $3542(7)$ & $534(6)$ & 5719(4) & $32(2)$ \\
\hline O3' & $2988(7)$ & $990(8)$ & $5942(4)$ & $28(1)$ \\
\hline $\mathrm{C} 15^{\prime}$ & $2517(7)$ & $734(6)$ & 6341(4) & $33(2)$ \\
\hline C16' & 1751(8) & $464(7)$ & $6126(5)$ & $32(2)$ \\
\hline O4' & 1371(7) & $960(9)$ & $5844(4)$ & $26(1)$ \\
\hline $\mathrm{C} 17^{\prime}$ & $737(7)$ & $730(6)$ & 5532(4) & $34(2)$ \\
\hline $\mathrm{C} 18^{\prime}$ & $1068(8)$ & $514(6)$ & $5048(4)$ & $32(2)$ \\
\hline Mo2 & $3969(1)$ & $5711(1)$ & 2794(1) & $18(1)$ \\
\hline $\mathrm{Cl} 5$ & $4326(1)$ & 4851(1) & $2215(1)$ & $24(1)$ \\
\hline $\mathrm{Cl} 6$ & $3436(1)$ & $6552(1)$ & $3307(1)$ & $29(1)$ \\
\hline $\mathrm{Cl} 7$ & $5022(1)$ & 6391(1) & $2443(1)$ & $26(1)$ \\
\hline $\mathrm{Cl} 8$ & $4830(1)$ & $5280(1)$ & $3443(1)$ & $31(1)$ \\
\hline N3 & $3056(2)$ & $5983(2)$ & $2267(1)$ & $19(1)$ \\
\hline N4 & $2922(2)$ & $5164(2)$ & $3015(1)$ & $19(1)$ \\
\hline C19 & $3173(3)$ & $6373(2)$ & $1886(2)$ & $26(1)$ \\
\hline $\mathrm{C} 20$ & $2569(3)$ & $6504(2)$ & $1545(2)$ & $34(1)$ \\
\hline $\mathrm{C} 21$ & $1829(3)$ & $6220(3)$ & $1605(2)$ & $37(1)$ \\
\hline $\mathrm{C} 22$ & 1698(3) & $5821(2)$ & $2006(2)$ & $31(1)$ \\
\hline $\mathrm{C} 23$ & $2319(3)$ & $5712(2)$ & $2334(2)$ & $21(1)$ \\
\hline $\mathrm{C} 24$ & $2232(3)$ & $5290(2)$ & $2765(2)$ & $19(1)$ \\
\hline $\mathrm{C} 25$ & 1501(3) & $5038(2)$ & $2914(2)$ & $28(1)$ \\
\hline $\mathrm{C} 26$ & $1476(3)$ & $4641(2)$ & $3325(2)$ & $33(1)$ \\
\hline $\mathrm{C} 27$ & 2192(3) & $4488(2)$ & $3563(2)$ & $31(1)$ \\
\hline $\mathrm{C} 28$ & 2904(3) & $4760(2)$ & $3399(2)$ & $24(1)$ \\
\hline Li2 & $4517(8)$ & $3832(6)$ & $2545(5)$ & $27(3)$ \\
\hline O5 & $3967(6)$ & $3458(3)$ & $3152(3)$ & $52(2)$ \\
\hline $\mathrm{C} 29$ & $4534(10)$ & $3335(6)$ & $3532(4)$ & $88(4)$ \\
\hline $\mathrm{C} 30$ & $5325(11)$ & $3080(8)$ & $3316(6)$ & $78(5)$ \\
\hline O6 & $5531(7)$ & $3536(5)$ & 2968(4) & $59(2)$ \\
\hline $\mathrm{C} 31$ & $6116(8)$ & $3290(7)$ & $2593(7)$ & $96(5)$ \\
\hline $\mathrm{C} 32$ & $5779(9)$ & $3032(10)$ & $2162(7)$ & $94(5)$ \\
\hline $\mathrm{O} 7$ & $5187(4)$ & $3459(3)$ & 2004(3) & $46(2)$ \\
\hline
\end{tabular}




\begin{tabular}{|c|c|c|c|c|}
\hline $\mathrm{C} 33$ & $4602(7)$ & $3229(8)$ & $1625(6)$ & $57(2)$ \\
\hline C34 & $3953(9)$ & $2899(10)$ & $1832(6)$ & $72(4)$ \\
\hline $\mathrm{O} 8$ & $3599(5)$ & $3303(3)$ & $2207(3)$ & $48(2)$ \\
\hline $\mathrm{C} 35$ & $3137(7)$ & $3079(6)$ & $2546(6)$ & $68(3)$ \\
\hline $\mathrm{C} 36$ & $3447(12)$ & $2930(11)$ & $3026(6)$ & $94(5)$ \\
\hline Li2' & $4709(13)$ & $2429(18)$ & $2455(8)$ & $73(10)$ \\
\hline O5' & $4032(17)$ & $2767(12)$ & $3042(8)$ & $52(2)$ \\
\hline C29' & $4370(20)$ & $3378(17)$ & $3150(20)$ & $88(4)$ \\
\hline $\mathrm{C} 30^{\prime}$ & $5290(20)$ & $3320(20)$ & $3202(13)$ & $78(5)$ \\
\hline O6' & $5579(15)$ & 2993(13) & $2795(10)$ & $59(2)$ \\
\hline C31' & $5820(20)$ & $3473(19)$ & $2399(13)$ & $96(5)$ \\
\hline C $32^{\prime}$ & $5790(19)$ & $3150(30)$ & $1947(14)$ & $94(5)$ \\
\hline O7' & $5126(14)$ & $2842(12)$ & $1810(8)$ & $46(2)$ \\
\hline C33' & $4410(20)$ & $3210(30)$ & $1620(20)$ & $57(2)$ \\
\hline C34' & $3720(20)$ & $2830(30)$ & $1624(12)$ & $72(4)$ \\
\hline O8' & $3601(12)$ & $2602(12)$ & $2120(9)$ & $48(2)$ \\
\hline $\mathrm{C} 35^{\prime}$ & $3120(20)$ & $2850(20)$ & $2430(13)$ & $68(3)$ \\
\hline C36' & $3180(20)$ & $2780(40)$ & 2954(13) & $94(5)$ \\
\hline Li2" & $4756(11)$ & $2441(15)$ & $2520(6)$ & $73(10)$ \\
\hline O5" & $4633(15)$ & $2878(9)$ & $3205(5)$ & $88(4)$ \\
\hline C29" & $5320(30)$ & $3250(20)$ & $3333(14)$ & $78(5)$ \\
\hline C30" & $5690(30)$ & $3484(18)$ & $2840(16)$ & $59(2)$ \\
\hline O6" & $5837(11)$ & $2933(12)$ & $2568(10)$ & $96(5)$ \\
\hline C31" & $5958(17)$ & $3110(20)$ & $2038(12)$ & $94(5)$ \\
\hline C32" & $5251(15)$ & $3306(11)$ & 1791(10) & $46(2)$ \\
\hline O7" & $4755(12)$ & $2786(8)$ & $1802(5)$ & $57(2)$ \\
\hline C33" & $3886(18)$ & $2880(20)$ & $1672(13)$ & $72(4)$ \\
\hline C34" & $3464(19)$ & $3121(13)$ & 2077(9) & $48(2)$ \\
\hline O8" & $3544(9)$ & $2678(8)$ & $2486(7)$ & $68(3)$ \\
\hline C35" & $3348(19)$ & $2870(20)$ & $2922(12)$ & $94(5)$ \\
\hline C36" & $3898(18)$ & $3243(15)$ & $3207(12)$ & $52(2)$ \\
\hline O9 & $3815(11)$ & $4066(9)$ & $4388(7)$ & $44(7)$ \\
\hline
\end{tabular}


Table 3. Bond lengths $[\AA]$ and angles $\left[{ }^{\circ}\right]$ for rhowe 10 .

\begin{tabular}{|c|c|c|c|}
\hline $\operatorname{Mo}(1)-\mathrm{N}(2)$ & $2.158(4)$ & $\operatorname{Li}(1)-\mathrm{O}\left(3^{\prime}\right)$ & $2.099(13)$ \\
\hline $\operatorname{Mo}(1)-\mathrm{N}(1)$ & $2.169(4)$ & $\mathrm{Li}(1)-\mathrm{O}(4)$ & $2.100(12)$ \\
\hline $\operatorname{Mo}(1)-\mathrm{Cl}(2)$ & $2.4080(12)$ & $\mathrm{O}(1)-\mathrm{C}(18)$ & $1.437(10)$ \\
\hline $\operatorname{Mo}(1)-\mathrm{Cl}(4)$ & $2.4341(12)$ & $\mathrm{O}(1)-\mathrm{C}(11)$ & $1.444(11)$ \\
\hline $\operatorname{Mo}(1)-\mathrm{Cl}(3)$ & $2.4372(12)$ & $\mathrm{C}(11)-\mathrm{C}(12)$ & $1.501(10)$ \\
\hline $\operatorname{Mo}(1)-\mathrm{Cl}(1)$ & $2.4650(12)$ & $\mathrm{C}(11)-\mathrm{H}(11 \mathrm{~A})$ & 0.9900 \\
\hline $\mathrm{Cl}(1)-\operatorname{Li}(1)$ & $2.302(8)$ & $\mathrm{C}(11)-\mathrm{H}(11 \mathrm{~B})$ & 0.9900 \\
\hline $\mathrm{N}(1)-\mathrm{C}(1)$ & $1.353(5)$ & $\mathrm{C}(12)-\mathrm{O}(2)$ & $1.435(10)$ \\
\hline $\mathrm{N}(1)-\mathrm{C}(5)$ & $1.358(6)$ & $\mathrm{C}(12)-\mathrm{H}(12 \mathrm{~A})$ & 0.9900 \\
\hline $\mathrm{N}(2)-\mathrm{C}(10)$ & $1.344(5)$ & $\mathrm{C}(12)-\mathrm{H}(12 \mathrm{~B})$ & 0.9900 \\
\hline $\mathrm{N}(2)-\mathrm{C}(6)$ & $1.353(5)$ & $\mathrm{O}(2)-\mathrm{C}(13)$ & $1.433(11)$ \\
\hline $\mathrm{C}(1)-\mathrm{C}(2)$ & $1.377(6)$ & $\mathrm{C}(13)-\mathrm{C}(14)$ & $1.503(10)$ \\
\hline $\mathrm{C}(1)-\mathrm{H}(1)$ & 0.9500 & $\mathrm{C}(13)-\mathrm{H}(13 \mathrm{~A})$ & 0.9900 \\
\hline $\mathrm{C}(2)-\mathrm{C}(3)$ & $1.388(7)$ & $\mathrm{C}(13)-\mathrm{H}(13 \mathrm{~B})$ & 0.9900 \\
\hline $\mathrm{C}(2)-\mathrm{H}(2)$ & 0.9500 & $\mathrm{C}(14)-\mathrm{O}(3)$ & $1.423(10)$ \\
\hline$C(3)-C(4)$ & $1.390(6)$ & $\mathrm{C}(14)-\mathrm{H}(14 \mathrm{~A})$ & 0.9900 \\
\hline $\mathrm{C}(3)-\mathrm{H}(3)$ & 0.9500 & $\mathrm{C}(14)-\mathrm{H}(14 \mathrm{~B})$ & 0.9900 \\
\hline$C(4)-C(5)$ & $1.383(6)$ & $\mathrm{O}(3)-\mathrm{C}(15)$ & $1.438(11)$ \\
\hline $\mathrm{C}(4)-\mathrm{H}(4)$ & 0.9500 & $C(15)-C(16)$ & $1.504(11)$ \\
\hline$C(5)-C(6)$ & $1.489(6)$ & $\mathrm{C}(15)-\mathrm{H}(15 \mathrm{~A})$ & 0.9900 \\
\hline$C(6)-C(7)$ & $1.379(6)$ & $\mathrm{C}(15)-\mathrm{H}(15 \mathrm{~B})$ & 0.9900 \\
\hline $\mathrm{C}(7)-\mathrm{C}(8)$ & $1.385(7)$ & $\mathrm{C}(16)-\mathrm{O}(4)$ & $1.424(10)$ \\
\hline $\mathrm{C}(7)-\mathrm{H}(7)$ & 0.9500 & $\mathrm{C}(16)-\mathrm{H}(16 \mathrm{~A})$ & 0.9900 \\
\hline $\mathrm{C}(8)-\mathrm{C}(9)$ & $1.379(7)$ & $\mathrm{C}(16)-\mathrm{H}(16 \mathrm{~B})$ & 0.9900 \\
\hline $\mathrm{C}(8)-\mathrm{H}(8)$ & 0.9500 & $\mathrm{O}(4)-\mathrm{C}(17)$ & $1.447(11)$ \\
\hline$C(9)-C(10)$ & $1.374(6)$ & $\mathrm{C}(17)-\mathrm{C}(18)$ & $1.501(10)$ \\
\hline $\mathrm{C}(9)-\mathrm{H}(9)$ & 0.9500 & $\mathrm{C}(17)-\mathrm{H}(17 \mathrm{~A})$ & 0.9900 \\
\hline $\mathrm{C}(10)-\mathrm{H}(10)$ & 0.9500 & $\mathrm{C}(17)-\mathrm{H}(17 \mathrm{~B})$ & 0.9900 \\
\hline $\mathrm{Li}(1)-\mathrm{O}\left(1^{\prime}\right)$ & $2.038(13)$ & $\mathrm{C}(18)-\mathrm{H}(18 \mathrm{~A})$ & 0.9900 \\
\hline $\mathrm{Li}(1)-\mathrm{O}(2)$ & $2.045(12)$ & $\mathrm{C}(18)-\mathrm{H}(18 \mathrm{~B})$ & 0.9900 \\
\hline $\mathrm{Li}(1)-\mathrm{O}\left(2^{\prime}\right)$ & $2.054(13)$ & $\mathrm{O}\left(1^{\prime}\right)-\mathrm{C}\left(11^{\prime}\right)$ & $1.425(11)$ \\
\hline $\mathrm{Li}(1)-\mathrm{O}(1)$ & $2.056(12)$ & $\mathrm{O}\left(1^{\prime}\right)-\mathrm{C}\left(18^{\prime}\right)$ & $1.434(13)$ \\
\hline $\mathrm{Li}(1)-\mathrm{O}(3)$ & $2.068(12)$ & $\mathrm{C}\left(11^{\prime}\right)-\mathrm{C}\left(12^{\prime}\right)$ & $1.502(12)$ \\
\hline $\mathrm{Li}(1)-\mathrm{O}\left(4^{\prime}\right)$ & $2.091(13)$ & $\mathrm{C}\left(11^{\prime}\right)-\mathrm{H}(11 \mathrm{C})$ & 0.9900 \\
\hline
\end{tabular}




\begin{tabular}{|c|c|c|c|}
\hline $\mathrm{C}\left(11^{\prime}\right)-\mathrm{H}(11 \mathrm{D})$ & 0.9900 & $\mathrm{~N}(4)-\mathrm{C}(24)$ & $1.355(5)$ \\
\hline $\mathrm{C}\left(12^{\prime}\right)-\mathrm{O}\left(2^{\prime}\right)$ & $1.442(12)$ & $C(19)-C(20)$ & $1.387(7)$ \\
\hline $\mathrm{C}\left(12^{\prime}\right)-\mathrm{H}(12 \mathrm{C})$ & 0.9900 & $\mathrm{C}(19)-\mathrm{H}(19)$ & 0.9500 \\
\hline $\mathrm{C}\left(12^{\prime}\right)-\mathrm{H}(12 \mathrm{D})$ & 0.9900 & $C(20)-C(21)$ & $1.372(7)$ \\
\hline $\mathrm{O}\left(2^{\prime}\right)-\mathrm{C}\left(13^{\prime}\right)$ & $1.436(11)$ & $\mathrm{C}(20)-\mathrm{H}(20)$ & 0.9500 \\
\hline $\mathrm{C}\left(13^{\prime}\right)-\mathrm{C}\left(14^{\prime}\right)$ & $1.493(11)$ & $C(21)-C(22)$ & $1.388(7)$ \\
\hline $\mathrm{C}\left(13^{\prime}\right)-\mathrm{H}(13 \mathrm{C})$ & 0.9900 & $\mathrm{C}(21)-\mathrm{H}(21)$ & 0.9500 \\
\hline $\mathrm{C}\left(13^{\prime}\right)-\mathrm{H}(13 \mathrm{D})$ & 0.9900 & $C(22)-C(23)$ & $1.378(6)$ \\
\hline $\mathrm{C}\left(14^{\prime}\right)-\mathrm{O}\left(3^{\prime}\right)$ & $1.455(13)$ & $\mathrm{C}(22)-\mathrm{H}(22)$ & 0.9500 \\
\hline $\mathrm{C}\left(14^{\prime}\right)-\mathrm{H}(14 \mathrm{C})$ & 0.9900 & $C(23)-C(24)$ & $1.470(6)$ \\
\hline $\mathrm{C}\left(14^{\prime}\right)-\mathrm{H}(14 \mathrm{D})$ & 0.9900 & $C(24)-C(25)$ & $1.382(6)$ \\
\hline $\mathrm{O}\left(3^{\prime}\right)-\mathrm{C}\left(15^{\prime}\right)$ & $1.437(11)$ & $C(25)-C(26)$ & $1.390(7)$ \\
\hline$C\left(15^{\prime}\right)-C\left(16^{\prime}\right)$ & $1.507(11)$ & $\mathrm{C}(25)-\mathrm{H}(25)$ & 0.9500 \\
\hline $\mathrm{C}\left(15^{\prime}\right)-\mathrm{H}(15 \mathrm{C})$ & 0.9900 & $C(26)-C(27)$ & $1.387(7)$ \\
\hline $\mathrm{C}\left(15^{\prime}\right)-\mathrm{H}(15 \mathrm{D})$ & 0.9900 & $\mathrm{C}(26)-\mathrm{H}(26)$ & 0.9500 \\
\hline$C\left(16^{\prime}\right)-O\left(4^{\prime}\right)$ & $1.435(13)$ & $\mathrm{C}(27)-\mathrm{C}(28)$ & $1.384(6)$ \\
\hline $\mathrm{C}\left(16^{\prime}\right)-\mathrm{H}(16 \mathrm{C})$ & 0.9900 & $\mathrm{C}(27)-\mathrm{H}(27)$ & 0.9500 \\
\hline $\mathrm{C}\left(16^{\prime}\right)-\mathrm{H}(16 \mathrm{D})$ & 0.9900 & $\mathrm{C}(28)-\mathrm{H}(28)$ & 0.9500 \\
\hline $\mathrm{O}\left(4^{\prime}\right)-\mathrm{C}\left(17^{\prime}\right)$ & $1.432(11)$ & $\mathrm{Li}(2)-\mathrm{O}(7)$ & $1.996(14)$ \\
\hline $\mathrm{C}\left(17^{\prime}\right)-\mathrm{C}\left(18^{\prime}\right)$ & $1.490(11)$ & $\mathrm{Li}(2)-\mathrm{O}(5)$ & $2.036(15)$ \\
\hline $\mathrm{C}\left(17^{\prime}\right)-\mathrm{H}(17 \mathrm{C})$ & 0.9900 & $\mathrm{Li}(2)-\mathrm{O}(8)$ & $2.091(15)$ \\
\hline $\mathrm{C}\left(17^{\prime}\right)-\mathrm{H}(17 \mathrm{D})$ & 0.9900 & $\mathrm{Li}(2)-\mathrm{O}(6)$ & $2.126(19)$ \\
\hline $\mathrm{C}\left(18^{\prime}\right)-\mathrm{H}(18 \mathrm{C})$ & 0.9900 & $\operatorname{Li}(2)-C(35)$ & $2.777(17)$ \\
\hline $\mathrm{C}\left(18^{\prime}\right)-\mathrm{H}(18 \mathrm{D})$ & 0.9900 & $\mathrm{O}(5)-\mathrm{C}(29)$ & $1.416(17)$ \\
\hline $\mathrm{Mo}(2)-\mathrm{N}(3)$ & $2.156(3)$ & $\mathrm{O}(5)-\mathrm{C}(36)$ & $1.444(17)$ \\
\hline $\mathrm{Mo}(2)-\mathrm{N}(4)$ & $2.163(4)$ & $C(29)-C(30)$ & $1.53(2)$ \\
\hline $\mathrm{Mo}(2)-\mathrm{Cl}(6)$ & $2.4106(12)$ & $\mathrm{C}(29)-\mathrm{H}(29 \mathrm{~A})$ & 0.9900 \\
\hline $\mathrm{Mo}(2)-\mathrm{Cl}(8)$ & $2.4345(12)$ & $\mathrm{C}(29)-\mathrm{H}(29 \mathrm{~B})$ & 0.9900 \\
\hline $\operatorname{Mo}(2)-\mathrm{Cl}(7)$ & $2.4438(12)$ & $\mathrm{C}(30)-\mathrm{O}(6)$ & $1.385(14)$ \\
\hline $\operatorname{Mo}(2)-\mathrm{Cl}(5)$ & $2.4625(11)$ & $\mathrm{C}(30)-\mathrm{H}(30 \mathrm{~A})$ & 0.9900 \\
\hline $\mathrm{Cl}(5)-\mathrm{Li}(2)$ & $2.337(12)$ & $\mathrm{C}(30)-\mathrm{H}(30 \mathrm{~B})$ & 0.9900 \\
\hline Cl(7)-Li(2')\#1 & $2.24(4)$ & $\mathrm{O}(6)-\mathrm{C}(31)$ & $1.495(17)$ \\
\hline $\mathrm{Cl}(7)-\operatorname{Li}\left(2^{\prime \prime}\right) \# 1$ & $2.23(3)$ & $C(31)-C(32)$ & $1.40(2)$ \\
\hline $\mathrm{N}(3)-\mathrm{C}(19)$ & $1.333(5)$ & $\mathrm{C}(31)-\mathrm{H}(31 \mathrm{~A})$ & 0.9900 \\
\hline $\mathrm{N}(3)-\mathrm{C}(23)$ & $1.358(5)$ & $\mathrm{C}(31)-\mathrm{H}(31 \mathrm{~B})$ & 0.9900 \\
\hline $\mathrm{N}(4)-\mathrm{C}(28)$ & $1.342(5)$ & $\mathrm{C}(32)-\mathrm{O}(7)$ & $1.394(16)$ \\
\hline
\end{tabular}




\begin{tabular}{|c|c|c|c|}
\hline $\mathrm{C}(32)-\mathrm{H}(32 \mathrm{~A})$ & 0.9900 & $\mathrm{O}\left(7^{\prime}\right)-\mathrm{C}\left(33^{\prime}\right)$ & $1.50(3)$ \\
\hline $\mathrm{C}(32)-\mathrm{H}(32 \mathrm{~B})$ & 0.9900 & $C\left(33^{\prime}\right)-C\left(34^{\prime}\right)$ & $1.40(3)$ \\
\hline $\mathrm{O}(7)-\mathrm{C}(33)$ & $1.492(16)$ & $\mathrm{C}\left(33^{\prime}\right)-\mathrm{H}(33 \mathrm{C})$ & 0.9900 \\
\hline$C(33)-C(34)$ & $1.398(19)$ & $\mathrm{C}\left(33^{\prime}\right)-\mathrm{H}(33 \mathrm{D})$ & 0.9900 \\
\hline $\mathrm{C}(33)-\mathrm{H}(33 \mathrm{~A})$ & 0.9900 & $\mathrm{C}\left(34^{\prime}\right)-\mathrm{O}\left(8^{\prime}\right)$ & $1.44(2)$ \\
\hline $\mathrm{C}(33)-\mathrm{H}(33 \mathrm{~B})$ & 0.9900 & $\mathrm{C}\left(34^{\prime}\right)-\mathrm{H}(34 \mathrm{C})$ & 0.9900 \\
\hline $\mathrm{C}(34)-\mathrm{O}(8)$ & $1.445(16)$ & $\mathrm{C}\left(34^{\prime}\right)-\mathrm{H}(34 \mathrm{D})$ & 0.9900 \\
\hline $\mathrm{C}(34)-\mathrm{H}(34 \mathrm{~A})$ & 0.9900 & $\mathrm{O}\left(8^{\prime}\right)-\mathrm{C}\left(35^{\prime}\right)$ & $1.27(2)$ \\
\hline $\mathrm{C}(34)-\mathrm{H}(34 \mathrm{~B})$ & 0.9900 & $C\left(35^{\prime}\right)-C\left(36^{\prime}\right)$ & $1.43(3)$ \\
\hline $\mathrm{O}(8)-\mathrm{C}(35)$ & $1.284(14)$ & $\mathrm{C}\left(35^{\prime}\right)-\mathrm{H}(35 \mathrm{C})$ & 0.9900 \\
\hline$C(35)-C(36)$ & $1.43(2)$ & $\mathrm{C}\left(35^{\prime}\right)-\mathrm{H}(35 \mathrm{D})$ & 0.9900 \\
\hline $\mathrm{C}(35)-\mathrm{H}(35 \mathrm{~A})$ & 0.9900 & $\mathrm{C}\left(36^{\prime}\right)-\mathrm{H}(36 \mathrm{C})$ & 0.9900 \\
\hline $\mathrm{C}(35)-\mathrm{H}(35 \mathrm{~B})$ & 0.9900 & $\mathrm{C}\left(36^{\prime}\right)-\mathrm{H}(36 \mathrm{D})$ & 0.9900 \\
\hline $\mathrm{C}(36)-\mathrm{H}(36 \mathrm{~A})$ & 0.9900 & Li(2")-O(7") & $2.072(17)$ \\
\hline $\mathrm{C}(36)-\mathrm{H}(36 \mathrm{~B})$ & 0.9900 & Li(2")-O(8") & $2.071(17)$ \\
\hline $\operatorname{Li}\left(2^{\prime}\right)-\mathrm{O}\left(7^{\prime}\right)$ & $2.067(19)$ & Li(2")-O(6") & $2.071(18)$ \\
\hline $\operatorname{Li}\left(2^{\prime}\right)-\mathrm{O}\left(5^{\prime}\right)$ & $2.069(18)$ & Li(2")-O(5") & $2.078(17)$ \\
\hline $\mathrm{Li}\left(2^{\prime}\right)-\mathrm{O}\left(8^{\prime}\right)$ & $2.078(18)$ & Li(2")-C(35") & $2.72(3)$ \\
\hline $\operatorname{Li}\left(2^{\prime}\right)-\mathrm{O}\left(6^{\prime}\right)$ & $2.079(19)$ & Li(2")-C(31") & $2.76(4)$ \\
\hline $\operatorname{Li}\left(2^{\prime}\right)-\mathrm{C}\left(32^{\prime}\right)$ & $2.72(4)$ & O(5")-C(36") & $1.44(2)$ \\
\hline $\operatorname{Li}\left(2^{\prime}\right)-\mathrm{C}\left(31^{\prime}\right)$ & $2.86(4)$ & O(5")-C(29") & $1.43(3)$ \\
\hline $\mathrm{O}\left(5^{\prime}\right)-\mathrm{C}\left(29^{\prime}\right)$ & $1.43(2)$ & C(29")-C(30") & $1.54(3)$ \\
\hline $\mathrm{O}\left(5^{\prime}\right)-\mathrm{C}\left(36^{\prime}\right)$ & $1.44(2)$ & C(29")-H(29E) & 0.9900 \\
\hline $\mathrm{C}\left(29^{\prime}\right)-\mathrm{C}\left(30^{\prime}\right)$ & $1.53(3)$ & C(29")-H(29F) & 0.9900 \\
\hline $\mathrm{C}\left(29^{\prime}\right)-\mathrm{H}(29 \mathrm{C})$ & 0.9900 & C(30")-O(6") & $1.39(2)$ \\
\hline $\mathrm{C}\left(29^{\prime}\right)-\mathrm{H}(29 \mathrm{D})$ & 0.9900 & C(30")-H(30E) & 0.9900 \\
\hline $\mathrm{C}\left(30^{\prime}\right)-\mathrm{O}\left(6^{\prime}\right)$ & $1.39(2)$ & $\mathrm{C}\left(30^{\prime \prime}\right)-\mathrm{H}(30 \mathrm{~F})$ & 0.9900 \\
\hline $\mathrm{C}\left(30^{\prime}\right)-\mathrm{H}(30 \mathrm{C})$ & 0.9900 & $\mathrm{O}\left(6^{\prime \prime)}-\mathrm{C}\left(31^{\prime \prime}\right)\right.$ & $1.50(2)$ \\
\hline $\mathrm{C}\left(30^{\prime}\right)-\mathrm{H}(30 \mathrm{D})$ & 0.9900 & C(31")-C(32") & $1.41(3)$ \\
\hline $\mathrm{O}\left(6^{\prime}\right)-\mathrm{C}\left(31^{\prime}\right)$ & $1.52(2)$ & C(31")-H(31E) & 0.9900 \\
\hline $\mathrm{C}\left(31^{\prime}\right)-\mathrm{C}\left(32^{\prime}\right)$ & $1.39(3)$ & $\mathrm{C}\left(31^{\prime \prime}\right)-\mathrm{H}(31 \mathrm{~F})$ & 0.9900 \\
\hline $\mathrm{C}\left(31^{\prime}\right)-\mathrm{H}(31 \mathrm{C})$ & 0.9900 & C(32")-O(7") & $1.37(2)$ \\
\hline $\mathrm{C}\left(31^{\prime}\right)-\mathrm{H}(31 \mathrm{D})$ & 0.9900 & C(32")-H(32E) & 0.9900 \\
\hline $\mathrm{C}\left(32^{\prime}\right)-\mathrm{O}\left(7^{\prime}\right)$ & $1.33(2)$ & $\mathrm{C}\left(32^{\prime \prime}\right)-\mathrm{H}(32 \mathrm{~F})$ & 0.9900 \\
\hline $\mathrm{C}\left(32^{\prime}\right)-\mathrm{H}(32 \mathrm{C})$ & 0.9900 & O(7")-C(33") & $1.49(2)$ \\
\hline $\mathrm{C}\left(32^{\prime}\right)-\mathrm{H}(32 \mathrm{D})$ & 0.9900 & C(33")-C(34") & $1.40(3)$ \\
\hline
\end{tabular}




\begin{tabular}{|c|c|c|c|}
\hline C(33")-H(33E) & 0.9900 & $\mathrm{~N}(1)-\mathrm{C}(1)-\mathrm{H}(1)$ & 118.5 \\
\hline C(33")-H(33F) & 0.9900 & $\mathrm{C}(2)-\mathrm{C}(1)-\mathrm{H}(1)$ & 118.5 \\
\hline $\mathrm{C}(34 ")-\mathrm{O}\left(8^{\prime \prime}\right)$ & $1.45(2)$ & $\mathrm{C}(1)-\mathrm{C}(2)-\mathrm{C}(3)$ & $118.0(4)$ \\
\hline C(34")-H(34E) & 0.9900 & $\mathrm{C}(1)-\mathrm{C}(2)-\mathrm{H}(2)$ & 121.0 \\
\hline C(34")-H(34F) & 0.9900 & $\mathrm{C}(3)-\mathrm{C}(2)-\mathrm{H}(2)$ & 121.0 \\
\hline $\mathrm{O}\left(8^{\prime \prime}\right)-\mathrm{C}\left(35^{\prime \prime}\right)$ & $1.29(2)$ & $\mathrm{C}(2)-\mathrm{C}(3)-\mathrm{C}(4)$ & $119.9(4)$ \\
\hline C(35")-C(36") & $1.43(3)$ & $\mathrm{C}(2)-\mathrm{C}(3)-\mathrm{H}(3)$ & 120.0 \\
\hline C(35")-H(35E) & 0.9900 & $\mathrm{C}(4)-\mathrm{C}(3)-\mathrm{H}(3)$ & 120.0 \\
\hline C(35")-H(35F) & 0.9900 & $C(5)-C(4)-C(3)$ & $118.8(5)$ \\
\hline C(36")-H(36E) & 0.9900 & $\mathrm{C}(5)-\mathrm{C}(4)-\mathrm{H}(4)$ & 120.6 \\
\hline C(36")-H(36F) & 0.9900 & $\mathrm{C}(3)-\mathrm{C}(4)-\mathrm{H}(4)$ & 120.6 \\
\hline $\mathrm{O}(9)-\mathrm{H}(9 \mathrm{~A})$ & 0.8500 & $\mathrm{~N}(1)-\mathrm{C}(5)-\mathrm{C}(4)$ & $121.7(4)$ \\
\hline $\mathrm{O}(9)-\mathrm{H}(9 \mathrm{~B})$ & 0.8500 & $\mathrm{~N}(1)-\mathrm{C}(5)-\mathrm{C}(6)$ & $114.9(4)$ \\
\hline $\mathrm{N}(2)-\mathrm{Mo}(1)-\mathrm{N}(1)$ & $75.44(13)$ & $\mathrm{C}(4)-\mathrm{C}(5)-\mathrm{C}(6)$ & $123.4(4)$ \\
\hline $\mathrm{N}(2)-\mathrm{Mo}(1)-\mathrm{Cl}(2)$ & $88.22(10)$ & $\mathrm{N}(2)-\mathrm{C}(6)-\mathrm{C}(7)$ & $121.8(4)$ \\
\hline N(1)-Mo(1)-Cl(2) & $85.31(10)$ & $\mathrm{N}(2)-\mathrm{C}(6)-\mathrm{C}(5)$ & $115.7(4)$ \\
\hline $\mathrm{N}(2)-\mathrm{Mo}(1)-\mathrm{Cl}(4)$ & $95.10(10)$ & $C(7)-C(6)-C(5)$ & $122.5(4)$ \\
\hline N(1)-Mo(1)-Cl(4) & $170.54(10)$ & $\mathrm{C}(6)-\mathrm{C}(7)-\mathrm{C}(8)$ & $118.8(5)$ \\
\hline $\mathrm{Cl}(2)-\mathrm{Mo}(1)-\mathrm{Cl}(4)$ & $94.20(4)$ & $\mathrm{C}(6)-\mathrm{C}(7)-\mathrm{H}(7)$ & 120.6 \\
\hline N(2)-Mo(1)-Cl(3) & $171.63(10)$ & $\mathrm{C}(8)-\mathrm{C}(7)-\mathrm{H}(7)$ & 120.6 \\
\hline N(1)-Mo(1)-Cl(3) & $96.29(10)$ & $\mathrm{C}(9)-\mathrm{C}(8)-\mathrm{C}(7)$ & $119.3(5)$ \\
\hline $\mathrm{Cl}(2)-\mathrm{Mo}(1)-\mathrm{Cl}(3)$ & $92.46(4)$ & $\mathrm{C}(9)-\mathrm{C}(8)-\mathrm{H}(8)$ & 120.3 \\
\hline $\mathrm{Cl}(4)-\mathrm{Mo}(1)-\mathrm{Cl}(3)$ & $93.17(4)$ & $\mathrm{C}(7)-\mathrm{C}(8)-\mathrm{H}(8)$ & 120.3 \\
\hline N(2)-Mo(1)-Cl(1) & $88.93(10)$ & $\mathrm{C}(10)-\mathrm{C}(9)-\mathrm{C}(8)$ & 119.1(5) \\
\hline $\mathrm{N}(1)-\mathrm{Mo}(1)-\mathrm{Cl}(1)$ & $87.57(10)$ & $\mathrm{C}(10)-\mathrm{C}(9)-\mathrm{H}(9)$ & 120.4 \\
\hline $\mathrm{Cl}(2)-\mathrm{Mo}(1)-\mathrm{Cl}(1)$ & $172.80(4)$ & $\mathrm{C}(8)-\mathrm{C}(9)-\mathrm{H}(9)$ & 120.4 \\
\hline $\mathrm{Cl}(4)-\mathrm{Mo}(1)-\mathrm{Cl}(1)$ & $92.65(4)$ & $\mathrm{N}(2)-\mathrm{C}(10)-\mathrm{C}(9)$ & $122.2(4)$ \\
\hline $\mathrm{Cl}(3)-\mathrm{Mo}(1)-\mathrm{Cl}(1)$ & $89.40(4)$ & $\mathrm{N}(2)-\mathrm{C}(10)-\mathrm{H}(10)$ & 118.9 \\
\hline $\mathrm{Li}(1)-\mathrm{Cl}(1)-\mathrm{Mo}(1)$ & $128.3(2)$ & $\mathrm{C}(9)-\mathrm{C}(10)-\mathrm{H}(10)$ & 118.9 \\
\hline$C(1)-N(1)-C(5)$ & $118.4(4)$ & $\mathrm{O}\left(1^{\prime}\right)-\mathrm{Li}(1)-\mathrm{O}\left(2^{\prime}\right)$ & $82.5(6)$ \\
\hline $\mathrm{C}(1)-\mathrm{N}(1)-\mathrm{Mo}(1)$ & $124.7(3)$ & $\mathrm{O}(2)-\mathrm{Li}(1)-\mathrm{O}(1)$ & $81.5(5)$ \\
\hline $\mathrm{C}(5)-\mathrm{N}(1)-\mathrm{Mo}(1)$ & $116.9(3)$ & $\mathrm{O}(2)-\mathrm{Li}(1)-\mathrm{O}(3)$ & $83.6(5)$ \\
\hline $\mathrm{C}(10)-\mathrm{N}(2)-\mathrm{C}(6)$ & $118.7(4)$ & $\mathrm{O}(1)-\mathrm{Li}(1)-\mathrm{O}(3)$ & $135.0(7)$ \\
\hline $\mathrm{C}(10)-\mathrm{N}(2)-\mathrm{Mo}(1)$ & $124.2(3)$ & $\mathrm{O}\left(1^{\prime}\right)-\mathrm{Li}(1)-\mathrm{O}\left(4^{\prime}\right)$ & $82.3(5)$ \\
\hline $\mathrm{C}(6)-\mathrm{N}(2)-\mathrm{Mo}(1)$ & $117.0(3)$ & $\mathrm{O}\left(2^{\prime}\right)-\mathrm{Li}(1)-\mathrm{O}\left(4^{\prime}\right)$ & $134.0(8)$ \\
\hline $\mathrm{N}(1)-\mathrm{C}(1)-\mathrm{C}(2)$ & $123.0(4)$ & $\mathrm{O}\left(1^{\prime}\right)-\mathrm{Li}(1)-\mathrm{O}\left(3^{\prime}\right)$ & $133.6(8)$ \\
\hline
\end{tabular}




\begin{tabular}{|c|c|c|c|}
\hline $\mathrm{O}\left(2^{\prime}\right)-\mathrm{Li}(1)-\mathrm{O}\left(3^{\prime}\right)$ & $79.8(5)$ & $\mathrm{H}(13 \mathrm{~A})-\mathrm{C}(13)-\mathrm{H}(13 \mathrm{~B})$ & 108.5 \\
\hline $\mathrm{O}\left(4^{\prime}\right)-\mathrm{Li}(1)-\mathrm{O}\left(3^{\prime}\right)$ & $80.0(5)$ & $\mathrm{O}(3)-\mathrm{C}(14)-\mathrm{C}(13)$ & $112.0(8)$ \\
\hline $\mathrm{O}(2)-\mathrm{Li}(1)-\mathrm{O}(4)$ & $134.5(7)$ & $\mathrm{O}(3)-\mathrm{C}(14)-\mathrm{H}(14 \mathrm{~A})$ & 109.2 \\
\hline $\mathrm{O}(1)-\mathrm{Li}(1)-\mathrm{O}(4)$ & $79.8(5)$ & $\mathrm{C}(13)-\mathrm{C}(14)-\mathrm{H}(14 \mathrm{~A})$ & 109.2 \\
\hline $\mathrm{O}(3)-\mathrm{Li}(1)-\mathrm{O}(4)$ & $81.1(5)$ & $\mathrm{O}(3)-\mathrm{C}(14)-\mathrm{H}(14 \mathrm{~B})$ & 109.2 \\
\hline $\mathrm{O}\left(1^{\prime}\right)-\mathrm{Li}(1)-\mathrm{Cl}(1)$ & $115.0(7)$ & $\mathrm{C}(13)-\mathrm{C}(14)-\mathrm{H}(14 \mathrm{~B})$ & 109.2 \\
\hline $\mathrm{O}(2)-\mathrm{Li}(1)-\mathrm{Cl}(1)$ & $114.9(5)$ & $\mathrm{H}(14 \mathrm{~A})-\mathrm{C}(14)-\mathrm{H}(14 \mathrm{~B})$ & 107.9 \\
\hline $\mathrm{O}\left(2^{\prime}\right)-\mathrm{Li}(1)-\mathrm{Cl}(1)$ & $119.2(6)$ & $\mathrm{C}(14)-\mathrm{O}(3)-\mathrm{C}(15)$ & $115.0(10)$ \\
\hline $\mathrm{O}(1)-\mathrm{Li}(1)-\mathrm{Cl}(1)$ & $112.2(6)$ & $\mathrm{C}(14)-\mathrm{O}(3)-\mathrm{Li}(1)$ & $106.9(7)$ \\
\hline $\mathrm{O}(3)-\mathrm{Li}(1)-\mathrm{Cl}(1)$ & $112.6(6)$ & $\mathrm{C}(15)-\mathrm{O}(3)-\mathrm{Li}(1)$ & $108.0(7)$ \\
\hline $\mathrm{O}\left(4^{\prime}\right)-\mathrm{Li}(1)-\mathrm{Cl}(1)$ & $106.5(6)$ & $\mathrm{O}(3)-\mathrm{C}(15)-\mathrm{C}(16)$ & $108.3(8)$ \\
\hline $\mathrm{O}\left(3^{\prime}\right)-\mathrm{Li}(1)-\mathrm{Cl}(1)$ & $111.1(6)$ & $\mathrm{O}(3)-\mathrm{C}(15)-\mathrm{H}(15 \mathrm{~A})$ & 110.0 \\
\hline $\mathrm{O}(4)-\mathrm{Li}(1)-\mathrm{Cl}(1)$ & $110.6(6)$ & $\mathrm{C}(16)-\mathrm{C}(15)-\mathrm{H}(15 \mathrm{~A})$ & 110.0 \\
\hline $\mathrm{C}(18)-\mathrm{O}(1)-\mathrm{C}(11)$ & $112.7(10)$ & $\mathrm{O}(3)-\mathrm{C}(15)-\mathrm{H}(15 \mathrm{~B})$ & 110.0 \\
\hline $\mathrm{C}(18)-\mathrm{O}(1)-\mathrm{Li}(1)$ & $112.6(7)$ & $\mathrm{C}(16)-\mathrm{C}(15)-\mathrm{H}(15 \mathrm{~B})$ & 110.0 \\
\hline $\mathrm{C}(11)-\mathrm{O}(1)-\mathrm{Li}(1)$ & $110.1(8)$ & $\mathrm{H}(15 \mathrm{~A})-\mathrm{C}(15)-\mathrm{H}(15 \mathrm{~B})$ & 108.4 \\
\hline $\mathrm{O}(1)-\mathrm{C}(11)-\mathrm{C}(12)$ & $105.5(8)$ & $\mathrm{O}(4)-\mathrm{C}(16)-\mathrm{C}(15)$ & 108.2(9) \\
\hline $\mathrm{O}(1)-\mathrm{C}(11)-\mathrm{H}(11 \mathrm{~A})$ & 110.6 & $\mathrm{O}(4)-\mathrm{C}(16)-\mathrm{H}(16 \mathrm{~A})$ & 110.1 \\
\hline $\mathrm{C}(12)-\mathrm{C}(11)-\mathrm{H}(11 \mathrm{~A})$ & 110.6 & $\mathrm{C}(15)-\mathrm{C}(16)-\mathrm{H}(16 \mathrm{~A})$ & 110.1 \\
\hline $\mathrm{O}(1)-\mathrm{C}(11)-\mathrm{H}(11 \mathrm{~B})$ & 110.6 & $\mathrm{O}(4)-\mathrm{C}(16)-\mathrm{H}(16 \mathrm{~B})$ & 110.1 \\
\hline $\mathrm{C}(12)-\mathrm{C}(11)-\mathrm{H}(11 \mathrm{~B})$ & 110.6 & $\mathrm{C}(15)-\mathrm{C}(16)-\mathrm{H}(16 \mathrm{~B})$ & 110.1 \\
\hline $\mathrm{H}(11 \mathrm{~A})-\mathrm{C}(11)-\mathrm{H}(11 \mathrm{~B})$ & 108.8 & $\mathrm{H}(16 \mathrm{~A})-\mathrm{C}(16)-\mathrm{H}(16 \mathrm{~B})$ & 108.4 \\
\hline $\mathrm{O}(2)-\mathrm{C}(12)-\mathrm{C}(11)$ & $110.4(8)$ & $\mathrm{C}(16)-\mathrm{O}(4)-\mathrm{C}(17)$ & $115.3(10)$ \\
\hline $\mathrm{O}(2)-\mathrm{C}(12)-\mathrm{H}(12 \mathrm{~A})$ & 109.6 & $\mathrm{C}(16)-\mathrm{O}(4)-\mathrm{Li}(1)$ & $109.8(7)$ \\
\hline $\mathrm{C}(11)-\mathrm{C}(12)-\mathrm{H}(12 \mathrm{~A})$ & 109.6 & $\mathrm{C}(17)-\mathrm{O}(4)-\mathrm{Li}(1)$ & $109.0(7)$ \\
\hline $\mathrm{O}(2)-\mathrm{C}(12)-\mathrm{H}(12 \mathrm{~B})$ & 109.6 & $\mathrm{O}(4)-\mathrm{C}(17)-\mathrm{C}(18)$ & $106.7(8)$ \\
\hline $\mathrm{C}(11)-\mathrm{C}(12)-\mathrm{H}(12 \mathrm{~B})$ & 109.6 & $\mathrm{O}(4)-\mathrm{C}(17)-\mathrm{H}(17 \mathrm{~A})$ & 110.4 \\
\hline $\mathrm{H}(12 \mathrm{~A})-\mathrm{C}(12)-\mathrm{H}(12 \mathrm{~B})$ & 108.1 & $\mathrm{C}(18)-\mathrm{C}(17)-\mathrm{H}(17 \mathrm{~A})$ & 110.4 \\
\hline $\mathrm{C}(13)-\mathrm{O}(2)-\mathrm{C}(12)$ & $115.5(9)$ & $\mathrm{O}(4)-\mathrm{C}(17)-\mathrm{H}(17 \mathrm{~B})$ & 110.4 \\
\hline $\mathrm{C}(13)-\mathrm{O}(2)-\mathrm{Li}(1)$ & $107.4(7)$ & $\mathrm{C}(18)-\mathrm{C}(17)-\mathrm{H}(17 \mathrm{~B})$ & 110.4 \\
\hline$C(12)-O(2)-\operatorname{Li}(1)$ & $109.7(6)$ & $\mathrm{H}(17 \mathrm{~A})-\mathrm{C}(17)-\mathrm{H}(17 \mathrm{~B})$ & 108.6 \\
\hline $\mathrm{O}(2)-\mathrm{C}(13)-\mathrm{C}(14)$ & $107.1(8)$ & $\mathrm{O}(1)-\mathrm{C}(18)-\mathrm{C}(17)$ & $108.5(9)$ \\
\hline $\mathrm{O}(2)-\mathrm{C}(13)-\mathrm{H}(13 \mathrm{~A})$ & 110.3 & $\mathrm{O}(1)-\mathrm{C}(18)-\mathrm{H}(18 \mathrm{~A})$ & 110.0 \\
\hline $\mathrm{C}(14)-\mathrm{C}(13)-\mathrm{H}(13 \mathrm{~A})$ & 110.3 & $\mathrm{C}(17)-\mathrm{C}(18)-\mathrm{H}(18 \mathrm{~A})$ & 110.0 \\
\hline $\mathrm{O}(2)-\mathrm{C}(13)-\mathrm{H}(13 \mathrm{~B})$ & 110.3 & $\mathrm{O}(1)-\mathrm{C}(18)-\mathrm{H}(18 \mathrm{~B})$ & 110.0 \\
\hline $\mathrm{C}(14)-\mathrm{C}(13)-\mathrm{H}(13 \mathrm{~B})$ & 110.3 & $\mathrm{C}(17)-\mathrm{C}(18)-\mathrm{H}(18 \mathrm{~B})$ & 110.0 \\
\hline
\end{tabular}




\begin{tabular}{|c|c|c|c|}
\hline $\mathrm{H}(18 \mathrm{~A})-\mathrm{C}(18)-\mathrm{H}(18 \mathrm{~B})$ & 108.4 & $\mathrm{C}\left(16^{\prime}\right)-\mathrm{C}\left(15^{\prime}\right)-\mathrm{H}(15 \mathrm{C})$ & 110.1 \\
\hline $\mathrm{C}\left(11^{\prime}\right)-\mathrm{O}\left(1^{\prime}\right)-\mathrm{C}\left(18^{\prime}\right)$ & $116.7(12)$ & $\mathrm{O}\left(3^{\prime}\right)-\mathrm{C}\left(15^{\prime}\right)-\mathrm{H}(15 \mathrm{D})$ & 110.1 \\
\hline$C\left(11^{\prime}\right)-\mathrm{O}\left(1^{\prime}\right)-\operatorname{Li}(1)$ & $108.8(9)$ & $\mathrm{C}\left(16^{\prime}\right)-\mathrm{C}\left(15^{\prime}\right)-\mathrm{H}(15 \mathrm{D})$ & 110.1 \\
\hline $\mathrm{C}\left(18^{\prime}\right)-\mathrm{O}\left(1^{\prime}\right)-\mathrm{Li}(1)$ & $108.7(9)$ & $\mathrm{H}(15 \mathrm{C})-\mathrm{C}\left(15^{\prime}\right)-\mathrm{H}(15 \mathrm{D})$ & 108.4 \\
\hline $\mathrm{O}\left(1^{\prime}\right)-\mathrm{C}\left(11^{\prime}\right)-\mathrm{C}\left(12^{\prime}\right)$ & $110.2(11)$ & $\mathrm{O}\left(4^{\prime}\right)-\mathrm{C}\left(16^{\prime}\right)-\mathrm{C}\left(15^{\prime}\right)$ & $107.6(11)$ \\
\hline $\mathrm{O}\left(1^{\prime}\right)-\mathrm{C}\left(11^{\prime}\right)-\mathrm{H}(11 \mathrm{C})$ & 109.6 & $\mathrm{O}\left(4^{\prime}\right)-\mathrm{C}\left(16^{\prime}\right)-\mathrm{H}(16 \mathrm{C})$ & 110.2 \\
\hline $\mathrm{C}\left(12^{\prime}\right)-\mathrm{C}\left(11^{\prime}\right)-\mathrm{H}(11 \mathrm{C})$ & 109.6 & $\mathrm{C}\left(15^{\prime}\right)-\mathrm{C}\left(16^{\prime}\right)-\mathrm{H}(16 \mathrm{C})$ & 110.2 \\
\hline $\mathrm{O}\left(1^{\prime}\right)-\mathrm{C}\left(11^{\prime}\right)-\mathrm{H}(11 \mathrm{D})$ & 109.6 & $\mathrm{O}\left(4^{\prime}\right)-\mathrm{C}\left(16^{\prime}\right)-\mathrm{H}(16 \mathrm{D})$ & 110.2 \\
\hline $\mathrm{C}\left(12^{\prime}\right)-\mathrm{C}\left(11^{\prime}\right)-\mathrm{H}(11 \mathrm{D})$ & 109.6 & $\mathrm{C}\left(15^{\prime}\right)-\mathrm{C}\left(16^{\prime}\right)-\mathrm{H}(16 \mathrm{D})$ & 110.2 \\
\hline $\mathrm{H}(11 \mathrm{C})-\mathrm{C}\left(11^{\prime}\right)-\mathrm{H}(11 \mathrm{D})$ & 108.1 & $\mathrm{H}(16 \mathrm{C})-\mathrm{C}\left(16^{\prime}\right)-\mathrm{H}(16 \mathrm{D})$ & 108.5 \\
\hline $\mathrm{O}\left(2^{\prime}\right)-\mathrm{C}\left(12^{\prime}\right)-\mathrm{C}\left(11^{\prime}\right)$ & $106.4(9)$ & $\mathrm{C}\left(17^{\prime}\right)-\mathrm{O}\left(4^{\prime}\right)-\mathrm{C}\left(16^{\prime}\right)$ & $113.0(12)$ \\
\hline $\mathrm{O}\left(2^{\prime}\right)-\mathrm{C}\left(12^{\prime}\right)-\mathrm{H}(12 \mathrm{C})$ & 110.5 & $\mathrm{C}\left(17^{\prime}\right)-\mathrm{O}\left(4^{\prime}\right)-\mathrm{Li}(1)$ & $108.2(8)$ \\
\hline $\mathrm{C}\left(11^{\prime}\right)-\mathrm{C}\left(12^{\prime}\right)-\mathrm{H}(12 \mathrm{C})$ & 110.5 & $\mathrm{C}\left(16^{\prime}\right)-\mathrm{O}\left(4^{\prime}\right)-\mathrm{Li}(1)$ & $108.6(10)$ \\
\hline $\mathrm{O}\left(2^{\prime}\right)-\mathrm{C}\left(12^{\prime}\right)-\mathrm{H}(12 \mathrm{D})$ & 110.5 & $\mathrm{O}\left(4^{\prime}\right)-\mathrm{C}\left(17^{\prime}\right)-\mathrm{C}\left(18^{\prime}\right)$ & $110.5(10)$ \\
\hline $\mathrm{C}\left(11^{\prime}\right)-\mathrm{C}\left(12^{\prime}\right)-\mathrm{H}(12 \mathrm{D})$ & 110.5 & $\mathrm{O}\left(4^{\prime}\right)-\mathrm{C}\left(17^{\prime}\right)-\mathrm{H}(17 \mathrm{C})$ & 109.5 \\
\hline $\mathrm{H}(12 \mathrm{C})-\mathrm{C}\left(12^{\prime}\right)-\mathrm{H}(12 \mathrm{D})$ & 108.6 & $\mathrm{C}\left(18^{\prime}\right)-\mathrm{C}\left(17^{\prime}\right)-\mathrm{H}(17 \mathrm{C})$ & 109.5 \\
\hline $\mathrm{C}\left(13^{\prime}\right)-\mathrm{O}\left(2^{\prime}\right)-\mathrm{C}\left(12^{\prime}\right)$ & $113.9(11)$ & $\mathrm{O}\left(4^{\prime}\right)-\mathrm{C}\left(17^{\prime}\right)-\mathrm{H}(17 \mathrm{D})$ & 109.5 \\
\hline $\mathrm{C}\left(13^{\prime}\right)-\mathrm{O}\left(2^{\prime}\right)-\mathrm{Li}(1)$ & $112.7(8)$ & $\mathrm{C}\left(18^{\prime}\right)-\mathrm{C}\left(17^{\prime}\right)-\mathrm{H}(17 \mathrm{D})$ & 109.5 \\
\hline $\mathrm{C}\left(12^{\prime}\right)-\mathrm{O}\left(2^{\prime}\right)-\mathrm{Li}(1)$ & $108.7(8)$ & $\mathrm{H}(17 \mathrm{C})-\mathrm{C}\left(17^{\prime}\right)-\mathrm{H}(17 \mathrm{D})$ & 108.1 \\
\hline $\mathrm{O}\left(2^{\prime}\right)-\mathrm{C}\left(13^{\prime}\right)-\mathrm{C}\left(14^{\prime}\right)$ & $110.0(10)$ & $\mathrm{O}\left(1^{\prime}\right)-\mathrm{C}\left(18^{\prime}\right)-\mathrm{C}\left(17^{\prime}\right)$ & $108.2(10)$ \\
\hline $\mathrm{O}\left(2^{\prime}\right)-\mathrm{C}\left(13^{\prime}\right)-\mathrm{H}(13 \mathrm{C})$ & 109.7 & $\mathrm{O}\left(1^{\prime}\right)-\mathrm{C}\left(18^{\prime}\right)-\mathrm{H}(18 \mathrm{C})$ & 110.1 \\
\hline $\mathrm{C}\left(14^{\prime}\right)-\mathrm{C}\left(13^{\prime}\right)-\mathrm{H}(13 \mathrm{C})$ & 109.7 & $\mathrm{C}\left(17^{\prime}\right)-\mathrm{C}\left(18^{\prime}\right)-\mathrm{H}(18 \mathrm{C})$ & 110.1 \\
\hline $\mathrm{O}\left(2^{\prime}\right)-\mathrm{C}\left(13^{\prime}\right)-\mathrm{H}(13 \mathrm{D})$ & 109.7 & $\mathrm{O}\left(1^{\prime}\right)-\mathrm{C}\left(18^{\prime}\right)-\mathrm{H}(18 \mathrm{D})$ & 110.1 \\
\hline $\mathrm{C}\left(14^{\prime}\right)-\mathrm{C}\left(13^{\prime}\right)-\mathrm{H}(13 \mathrm{D})$ & 109.7 & $\mathrm{C}\left(17^{\prime}\right)-\mathrm{C}\left(18^{\prime}\right)-\mathrm{H}(18 \mathrm{D})$ & 110.1 \\
\hline $\mathrm{H}(13 \mathrm{C})-\mathrm{C}\left(13^{\prime}\right)-\mathrm{H}(13 \mathrm{D})$ & 108.2 & $\mathrm{H}(18 \mathrm{C})-\mathrm{C}\left(18^{\prime}\right)-\mathrm{H}(18 \mathrm{D})$ & 108.4 \\
\hline $\mathrm{O}\left(3^{\prime}\right)-\mathrm{C}\left(14^{\prime}\right)-\mathrm{C}\left(13^{\prime}\right)$ & $106.8(9)$ & $\mathrm{N}(3)-\mathrm{Mo}(2)-\mathrm{N}(4)$ & $76.10(13)$ \\
\hline $\mathrm{O}\left(3^{\prime}\right)-\mathrm{C}\left(14^{\prime}\right)-\mathrm{H}(14 \mathrm{C})$ & 110.4 & $\mathrm{~N}(3)-\mathrm{Mo}(2)-\mathrm{Cl}(6)$ & $86.03(10)$ \\
\hline $\mathrm{C}\left(13^{\prime}\right)-\mathrm{C}\left(14^{\prime}\right)-\mathrm{H}(14 \mathrm{C})$ & 110.4 & $\mathrm{~N}(4)-\mathrm{Mo}(2)-\mathrm{Cl}(6)$ & $86.30(10)$ \\
\hline $\mathrm{O}\left(3^{\prime}\right)-\mathrm{C}\left(14^{\prime}\right)-\mathrm{H}(14 \mathrm{D})$ & 110.4 & $\mathrm{~N}(3)-\mathrm{Mo}(2)-\mathrm{Cl}(8)$ & $170.33(10)$ \\
\hline $\mathrm{C}\left(13^{\prime}\right)-\mathrm{C}\left(14^{\prime}\right)-\mathrm{H}(14 \mathrm{D})$ & 110.4 & $\mathrm{~N}(4)-\mathrm{Mo}(2)-\mathrm{Cl}(8)$ & $94.25(10)$ \\
\hline $\mathrm{H}(14 \mathrm{C})-\mathrm{C}\left(14^{\prime}\right)-\mathrm{H}(14 \mathrm{D})$ & 108.6 & $\mathrm{Cl}(6)-\mathrm{Mo}(2)-\mathrm{Cl}(8)$ & $94.05(4)$ \\
\hline $\mathrm{C}\left(15^{\prime}\right)-\mathrm{O}\left(3^{\prime}\right)-\mathrm{C}\left(14^{\prime}\right)$ & $114.1(12)$ & $\mathrm{N}(3)-\mathrm{Mo}(2)-\mathrm{Cl}(7)$ & $95.13(10)$ \\
\hline $\mathrm{C}\left(15^{\prime}\right)-\mathrm{O}\left(3^{\prime}\right)-\mathrm{Li}(1)$ & $111.0(8)$ & $\mathrm{N}(4)-\mathrm{Mo}(2)-\mathrm{Cl}(7)$ & $171.23(10)$ \\
\hline $\mathrm{C}\left(14^{\prime}\right)-\mathrm{O}\left(3^{\prime}\right)-\mathrm{Li}(1)$ & $110.2(8)$ & $\mathrm{Cl}(6)-\mathrm{Mo}(2)-\mathrm{Cl}(7)$ & $93.42(4)$ \\
\hline $\mathrm{O}\left(3^{\prime}\right)-\mathrm{C}\left(15^{\prime}\right)-\mathrm{C}\left(16^{\prime}\right)$ & $107.9(10)$ & $\mathrm{Cl}(8)-\mathrm{Mo}(2)-\mathrm{Cl}(7)$ & $94.51(4)$ \\
\hline $\mathrm{O}\left(3^{\prime}\right)-\mathrm{C}\left(15^{\prime}\right)-\mathrm{H}(15 \mathrm{C})$ & 110.1 & $\mathrm{~N}(3)-\mathrm{Mo}(2)-\mathrm{Cl}(5)$ & $86.59(9)$ \\
\hline
\end{tabular}




\begin{tabular}{|c|c|c|c|}
\hline $\mathrm{N}(4)-\mathrm{Mo}(2)-\mathrm{Cl}(5)$ & $88.82(10)$ & $\mathrm{C}(27)-\mathrm{C}(26)-\mathrm{H}(26)$ & 120.5 \\
\hline $\mathrm{Cl}(6)-\mathrm{Mo}(2)-\mathrm{Cl}(5)$ & $171.95(4)$ & $\mathrm{C}(25)-\mathrm{C}(26)-\mathrm{H}(26)$ & 120.5 \\
\hline $\mathrm{Cl}(8)-\mathrm{Mo}(2)-\mathrm{Cl}(5)$ & $92.68(4)$ & $\mathrm{C}(28)-\mathrm{C}(27)-\mathrm{C}(26)$ & $119.0(5)$ \\
\hline $\mathrm{Cl}(7)-\mathrm{Mo}(2)-\mathrm{Cl}(5)$ & $90.43(4)$ & $\mathrm{C}(28)-\mathrm{C}(27)-\mathrm{H}(27)$ & 120.5 \\
\hline $\mathrm{Li}(2)-\mathrm{Cl}(5)-\mathrm{Mo}(2)$ & $117.3(3)$ & $\mathrm{C}(26)-\mathrm{C}(27)-\mathrm{H}(27)$ & 120.5 \\
\hline $\operatorname{Li}\left(2^{\prime}\right) \# 1-\mathrm{Cl}(7)-\operatorname{Li}\left(2^{\prime \prime}\right) \# 1$ & $5.0(8)$ & $\mathrm{N}(4)-\mathrm{C}(28)-\mathrm{C}(27)$ & $121.8(4)$ \\
\hline $\mathrm{Li}\left(2^{\prime}\right) \# 1-\mathrm{Cl}(7)-\mathrm{Mo}(2)$ & $131.3(6)$ & $\mathrm{N}(4)-\mathrm{C}(28)-\mathrm{H}(28)$ & 119.1 \\
\hline Li(2")\#1-Cl(7)-Mo(2) & $132.4(5)$ & $\mathrm{C}(27)-\mathrm{C}(28)-\mathrm{H}(28)$ & 119.1 \\
\hline $\mathrm{C}(19)-\mathrm{N}(3)-\mathrm{C}(23)$ & $119.4(4)$ & $\mathrm{O}(7)-\mathrm{Li}(2)-\mathrm{O}(5)$ & $133.7(7)$ \\
\hline $\mathrm{C}(19)-\mathrm{N}(3)-\mathrm{Mo}(2)$ & $125.0(3)$ & $\mathrm{O}(7)-\mathrm{Li}(2)-\mathrm{O}(8)$ & $82.9(6)$ \\
\hline $\mathrm{C}(23)-\mathrm{N}(3)-\mathrm{Mo}(2)$ & $115.6(3)$ & $\mathrm{O}(5)-\mathrm{Li}(2)-\mathrm{O}(8)$ & $79.9(6)$ \\
\hline $\mathrm{C}(28)-\mathrm{N}(4)-\mathrm{C}(24)$ & $119.4(4)$ & $\mathrm{O}(7)-\mathrm{Li}(2)-\mathrm{O}(6)$ & $80.8(6)$ \\
\hline $\mathrm{C}(28)-\mathrm{N}(4)-\mathrm{Mo}(2)$ & $124.5(3)$ & $\mathrm{O}(5)-\mathrm{Li}(2)-\mathrm{O}(6)$ & $78.9(6)$ \\
\hline $\mathrm{C}(24)-\mathrm{N}(4)-\mathrm{Mo}(2)$ & $115.8(3)$ & $\mathrm{O}(8)-\mathrm{Li}(2)-\mathrm{O}(6)$ & $131.0(7)$ \\
\hline $\mathrm{N}(3)-\mathrm{C}(19)-\mathrm{C}(20)$ & $122.1(4)$ & $\mathrm{O}(7)-\mathrm{Li}(2)-\mathrm{Cl}(5)$ & $98.8(5)$ \\
\hline $\mathrm{N}(3)-\mathrm{C}(19)-\mathrm{H}(19)$ & 118.9 & $\mathrm{O}(5)-\mathrm{Li}(2)-\mathrm{Cl}(5)$ & $126.8(6)$ \\
\hline $\mathrm{C}(20)-\mathrm{C}(19)-\mathrm{H}(19)$ & 118.9 & $\mathrm{O}(8)-\mathrm{Li}(2)-\mathrm{Cl}(5)$ & $102.7(6)$ \\
\hline$C(21)-C(20)-C(19)$ & $118.7(4)$ & $\mathrm{O}(6)-\mathrm{Li}(2)-\mathrm{Cl}(5)$ & $125.4(7)$ \\
\hline $\mathrm{C}(21)-\mathrm{C}(20)-\mathrm{H}(20)$ & 120.6 & $\mathrm{O}(7)-\mathrm{Li}(2)-\mathrm{C}(35)$ & $103.7(6)$ \\
\hline $\mathrm{C}(19)-\mathrm{C}(20)-\mathrm{H}(20)$ & 120.6 & $\mathrm{O}(5)-\mathrm{Li}(2)-\mathrm{C}(35)$ & $54.0(5)$ \\
\hline$C(20)-C(21)-C(22)$ & $119.5(4)$ & $\mathrm{O}(8)-\mathrm{Li}(2)-\mathrm{C}(35)$ & $26.0(4)$ \\
\hline $\mathrm{C}(20)-\mathrm{C}(21)-\mathrm{H}(21)$ & 120.2 & $\mathrm{O}(6)-\mathrm{Li}(2)-\mathrm{C}(35)$ & $119.0(6)$ \\
\hline $\mathrm{C}(22)-\mathrm{C}(21)-\mathrm{H}(21)$ & 120.2 & $\mathrm{Cl}(5)-\mathrm{Li}(2)-\mathrm{C}(35)$ & $114.1(6)$ \\
\hline$C(23)-C(22)-C(21)$ & $119.2(5)$ & $\mathrm{C}(29)-\mathrm{O}(5)-\mathrm{C}(36)$ & $115.2(13)$ \\
\hline $\mathrm{C}(23)-\mathrm{C}(22)-\mathrm{H}(22)$ & 120.4 & $\mathrm{C}(29)-\mathrm{O}(5)-\mathrm{Li}(2)$ & 111.1(9) \\
\hline $\mathrm{C}(21)-\mathrm{C}(22)-\mathrm{H}(22)$ & 120.4 & $\mathrm{C}(36)-\mathrm{O}(5)-\mathrm{Li}(2)$ & $111.7(9)$ \\
\hline $\mathrm{N}(3)-\mathrm{C}(23)-\mathrm{C}(22)$ & $121.0(4)$ & $\mathrm{O}(5)-\mathrm{C}(29)-\mathrm{C}(30)$ & $110.8(10)$ \\
\hline $\mathrm{N}(3)-\mathrm{C}(23)-\mathrm{C}(24)$ & $116.5(4)$ & $\mathrm{O}(5)-\mathrm{C}(29)-\mathrm{H}(29 \mathrm{~A})$ & 109.5 \\
\hline$C(22)-C(23)-C(24)$ & $122.5(4)$ & $\mathrm{C}(30)-\mathrm{C}(29)-\mathrm{H}(29 \mathrm{~A})$ & 109.5 \\
\hline $\mathrm{N}(4)-\mathrm{C}(24)-\mathrm{C}(25)$ & $121.3(4)$ & $\mathrm{O}(5)-\mathrm{C}(29)-\mathrm{H}(29 \mathrm{~B})$ & 109.5 \\
\hline $\mathrm{N}(4)-\mathrm{C}(24)-\mathrm{C}(23)$ & $115.5(4)$ & $\mathrm{C}(30)-\mathrm{C}(29)-\mathrm{H}(29 \mathrm{~B})$ & 109.5 \\
\hline$C(25)-C(24)-C(23)$ & $123.2(4)$ & $\mathrm{H}(29 \mathrm{~A})-\mathrm{C}(29)-\mathrm{H}(29 \mathrm{~B})$ & 108.1 \\
\hline$C(24)-C(25)-C(26)$ & $119.3(4)$ & $\mathrm{O}(6)-\mathrm{C}(30)-\mathrm{C}(29)$ & $103.3(12)$ \\
\hline $\mathrm{C}(24)-\mathrm{C}(25)-\mathrm{H}(25)$ & 120.4 & $\mathrm{O}(6)-\mathrm{C}(30)-\mathrm{H}(30 \mathrm{~A})$ & 111.1 \\
\hline $\mathrm{C}(26)-\mathrm{C}(25)-\mathrm{H}(25)$ & 120.4 & $\mathrm{C}(29)-\mathrm{C}(30)-\mathrm{H}(30 \mathrm{~A})$ & 111.1 \\
\hline $\mathrm{C}(27)-\mathrm{C}(26)-\mathrm{C}(25)$ & $119.0(5)$ & $\mathrm{O}(6)-\mathrm{C}(30)-\mathrm{H}(30 \mathrm{~B})$ & 111.1 \\
\hline
\end{tabular}




\begin{tabular}{|c|c|c|c|}
\hline $\mathrm{C}(29)-\mathrm{C}(30)-\mathrm{H}(30 \mathrm{~B})$ & 111.1 & $\mathrm{O}(8)-\mathrm{C}(35)-\mathrm{Li}(2)$ & $45.6(5)$ \\
\hline $\mathrm{H}(30 \mathrm{~A})-\mathrm{C}(30)-\mathrm{H}(30 \mathrm{~B})$ & 109.1 & $\mathrm{C}(36)-\mathrm{C}(35)-\mathrm{Li}(2)$ & $80.2(9)$ \\
\hline $\mathrm{C}(30)-\mathrm{O}(6)-\mathrm{C}(31)$ & $112.6(12)$ & $\mathrm{O}(8)-\mathrm{C}(35)-\mathrm{H}(35 \mathrm{~A})$ & 107.1 \\
\hline $\mathrm{C}(30)-\mathrm{O}(6)-\mathrm{Li}(2)$ & $111.9(10)$ & $\mathrm{C}(36)-\mathrm{C}(35)-\mathrm{H}(35 \mathrm{~A})$ & 107.1 \\
\hline $\mathrm{C}(31)-\mathrm{O}(6)-\mathrm{Li}(2)$ & $104.3(9)$ & $\mathrm{Li}(2)-\mathrm{C}(35)-\mathrm{H}(35 \mathrm{~A})$ & 144.5 \\
\hline $\mathrm{C}(32)-\mathrm{C}(31)-\mathrm{O}(6)$ & $116.1(13)$ & $\mathrm{O}(8)-\mathrm{C}(35)-\mathrm{H}(35 \mathrm{~B})$ & 107.1 \\
\hline $\mathrm{C}(32)-\mathrm{C}(31)-\mathrm{H}(31 \mathrm{~A})$ & 108.3 & $\mathrm{C}(36)-\mathrm{C}(35)-\mathrm{H}(35 \mathrm{~B})$ & 107.1 \\
\hline $\mathrm{O}(6)-\mathrm{C}(31)-\mathrm{H}(31 \mathrm{~A})$ & 108.3 & $\mathrm{Li}(2)-\mathrm{C}(35)-\mathrm{H}(35 \mathrm{~B})$ & 103.8 \\
\hline $\mathrm{C}(32)-\mathrm{C}(31)-\mathrm{H}(31 \mathrm{~B})$ & 108.3 & $\mathrm{H}(35 \mathrm{~A})-\mathrm{C}(35)-\mathrm{H}(35 \mathrm{~B})$ & 106.8 \\
\hline $\mathrm{O}(6)-\mathrm{C}(31)-\mathrm{H}(31 \mathrm{~B})$ & 108.3 & $\mathrm{C}(35)-\mathrm{C}(36)-\mathrm{O}(5)$ & $105.1(15)$ \\
\hline $\mathrm{H}(31 \mathrm{~A})-\mathrm{C}(31)-\mathrm{H}(31 \mathrm{~B})$ & 107.4 & $\mathrm{C}(35)-\mathrm{C}(36)-\mathrm{H}(36 \mathrm{~A})$ & 110.7 \\
\hline $\mathrm{C}(31)-\mathrm{C}(32)-\mathrm{O}(7)$ & $106.7(15)$ & $\mathrm{O}(5)-\mathrm{C}(36)-\mathrm{H}(36 \mathrm{~A})$ & 110.7 \\
\hline $\mathrm{C}(31)-\mathrm{C}(32)-\mathrm{H}(32 \mathrm{~A})$ & 110.4 & $\mathrm{C}(35)-\mathrm{C}(36)-\mathrm{H}(36 \mathrm{~B})$ & 110.7 \\
\hline $\mathrm{O}(7)-\mathrm{C}(32)-\mathrm{H}(32 \mathrm{~A})$ & 110.4 & $\mathrm{O}(5)-\mathrm{C}(36)-\mathrm{H}(36 \mathrm{~B})$ & 110.7 \\
\hline $\mathrm{C}(31)-\mathrm{C}(32)-\mathrm{H}(32 \mathrm{~B})$ & 110.4 & $\mathrm{H}(36 \mathrm{~A})-\mathrm{C}(36)-\mathrm{H}(36 \mathrm{~B})$ & 108.8 \\
\hline $\mathrm{O}(7)-\mathrm{C}(32)-\mathrm{H}(32 \mathrm{~B})$ & 110.4 & $\mathrm{O}\left(7^{\prime}\right)-\mathrm{Li}\left(2^{\prime}\right)-\mathrm{O}\left(5^{\prime}\right)$ & $133(2)$ \\
\hline $\mathrm{H}(32 \mathrm{~A})-\mathrm{C}(32)-\mathrm{H}(32 \mathrm{~B})$ & 108.6 & $\mathrm{O}\left(7^{\prime}\right)-\mathrm{Li}\left(2^{\prime}\right)-\mathrm{O}\left(8^{\prime}\right)$ & $81.6(11)$ \\
\hline $\mathrm{C}(32)-\mathrm{O}(7)-\mathrm{C}(33)$ & $117.4(11)$ & $\mathrm{O}\left(5^{\prime}\right)-\mathrm{Li}\left(2^{\prime}\right)-\mathrm{O}\left(8^{\prime}\right)$ & $78.3(12)$ \\
\hline $\mathrm{C}(32)-\mathrm{O}(7)-\mathrm{Li}(2)$ & $114.7(10)$ & $\mathrm{O}\left(7^{\prime}\right)-\mathrm{Li}\left(2^{\prime}\right)-\mathrm{O}\left(6^{\prime}\right)$ & $84.6(12)$ \\
\hline $\mathrm{C}(33)-\mathrm{O}(7)-\mathrm{Li}(2)$ & $105.6(8)$ & $\mathrm{O}\left(5^{\prime}\right)-\mathrm{Li}\left(2^{\prime}\right)-\mathrm{O}\left(6^{\prime}\right)$ & $80.9(12)$ \\
\hline $\mathrm{C}(34)-\mathrm{C}(33)-\mathrm{O}(7)$ & $112.5(13)$ & $\mathrm{O}\left(8^{\prime}\right)-\mathrm{Li}\left(2^{\prime}\right)-\mathrm{O}\left(6^{\prime}\right)$ & $135(2)$ \\
\hline $\mathrm{C}(34)-\mathrm{C}(33)-\mathrm{H}(33 \mathrm{~A})$ & 109.1 & $\mathrm{O}\left(7^{\prime}\right)-\mathrm{Li}\left(2^{\prime}\right)-\mathrm{C}\left(32^{\prime}\right)$ & $28.4(8)$ \\
\hline $\mathrm{O}(7)-\mathrm{C}(33)-\mathrm{H}(33 \mathrm{~A})$ & 109.1 & $\mathrm{O}\left(5^{\prime}\right)-\mathrm{Li}\left(2^{\prime}\right)-\mathrm{C}\left(32^{\prime}\right)$ & $123.6(18)$ \\
\hline $\mathrm{C}(34)-\mathrm{C}(33)-\mathrm{H}(33 \mathrm{~B})$ & 109.1 & $\mathrm{O}\left(8^{\prime}\right)-\mathrm{Li}\left(2^{\prime}\right)-\mathrm{C}\left(32^{\prime}\right)$ & 105.3(16) \\
\hline $\mathrm{O}(7)-\mathrm{C}(33)-\mathrm{H}(33 \mathrm{~B})$ & 109.1 & $\mathrm{O}\left(6^{\prime}\right)-\mathrm{Li}\left(2^{\prime}\right)-\mathrm{C}\left(32^{\prime}\right)$ & $56.6(9)$ \\
\hline $\mathrm{H}(33 \mathrm{~A})-\mathrm{C}(33)-\mathrm{H}(33 \mathrm{~B})$ & 107.8 & $\mathrm{Cl}(7) \# 2-\operatorname{Li}\left(2^{\prime}\right)-\mathrm{C}\left(32^{\prime}\right)$ & $118.3(16)$ \\
\hline $\mathrm{C}(33)-\mathrm{C}(34)-\mathrm{O}(8)$ & $107.6(14)$ & $\mathrm{O}\left(7^{\prime}\right)-\mathrm{Li}\left(2^{\prime}\right)-\mathrm{C}\left(31^{\prime}\right)$ & $54.6(9)$ \\
\hline $\mathrm{C}(33)-\mathrm{C}(34)-\mathrm{H}(34 \mathrm{~A})$ & 110.2 & $\mathrm{O}\left(5^{\prime}\right)-\mathrm{Li}\left(2^{\prime}\right)-\mathrm{C}\left(31^{\prime}\right)$ & $97.3(14)$ \\
\hline $\mathrm{O}(8)-\mathrm{C}(34)-\mathrm{H}(34 \mathrm{~A})$ & 110.2 & $\mathrm{O}\left(8^{\prime}\right)-\mathrm{Li}\left(2^{\prime}\right)-\mathrm{C}\left(31^{\prime}\right)$ & $114.4(16)$ \\
\hline $\mathrm{C}(33)-\mathrm{C}(34)-\mathrm{H}(34 \mathrm{~B})$ & 110.2 & $\mathrm{O}\left(6^{\prime}\right)-\mathrm{Li}\left(2^{\prime}\right)-\mathrm{C}\left(31^{\prime}\right)$ & $31.1(8)$ \\
\hline $\mathrm{O}(8)-\mathrm{C}(34)-\mathrm{H}(34 \mathrm{~B})$ & 110.2 & $\mathrm{Cl}(7) \# 2-\operatorname{Li}\left(2^{\prime}\right)-\mathrm{C}\left(31^{\prime}\right)$ & $128.4(13)$ \\
\hline $\mathrm{H}(34 \mathrm{~A})-\mathrm{C}(34)-\mathrm{H}(34 \mathrm{~B})$ & 108.5 & $C\left(32^{\prime}\right)-\operatorname{Li}\left(2^{\prime}\right)-C\left(31^{\prime}\right)$ & $28.8(6)$ \\
\hline $\mathrm{C}(35)-\mathrm{O}(8)-\mathrm{C}(34)$ & $121.8(11)$ & $\mathrm{C}\left(29^{\prime}\right)-\mathrm{O}\left(5^{\prime}\right)-\mathrm{C}\left(36^{\prime}\right)$ & $114(3)$ \\
\hline $\mathrm{C}(35)-\mathrm{O}(8)-\mathrm{Li}(2)$ & $108.4(8)$ & $\mathrm{C}\left(29^{\prime}\right)-\mathrm{O}\left(5^{\prime}\right)-\mathrm{Li}\left(2^{\prime}\right)$ & $105(2)$ \\
\hline $\mathrm{C}(34)-\mathrm{O}(8)-\mathrm{Li}(2)$ & $108.9(8)$ & $\mathrm{C}\left(36^{\prime}\right)-\mathrm{O}\left(5^{\prime}\right)-\mathrm{Li}\left(2^{\prime}\right)$ & $115(2)$ \\
\hline $\mathrm{O}(8)-\mathrm{C}(35)-\mathrm{C}(36)$ & $120.9(12)$ & $\mathrm{O}\left(5^{\prime}\right)-\mathrm{C}\left(29^{\prime}\right)-\mathrm{C}\left(30^{\prime}\right)$ & $110(2)$ \\
\hline
\end{tabular}




\begin{tabular}{|c|c|c|c|}
\hline $\mathrm{O}\left(5^{\prime}\right)-\mathrm{C}\left(29^{\prime}\right)-\mathrm{H}(29 \mathrm{C})$ & 109.7 & $\mathrm{C}\left(33^{\prime}\right)-\mathrm{O}\left(7^{\prime}\right)-\mathrm{Li}\left(2^{\prime}\right)$ & $104(2)$ \\
\hline $\mathrm{C}\left(30^{\prime}\right)-\mathrm{C}\left(29^{\prime}\right)-\mathrm{H}(29 \mathrm{C})$ & 109.7 & $\mathrm{C}\left(34^{\prime}\right)-\mathrm{C}\left(33^{\prime}\right)-\mathrm{O}\left(7^{\prime}\right)$ & 111(3) \\
\hline $\mathrm{O}\left(5^{\prime}\right)-\mathrm{C}\left(29^{\prime}\right)-\mathrm{H}(29 \mathrm{D})$ & 109.7 & $\mathrm{C}\left(34^{\prime}\right)-\mathrm{C}\left(33^{\prime}\right)-\mathrm{H}(33 \mathrm{C})$ & 109.5 \\
\hline $\mathrm{C}\left(30^{\prime}\right)-\mathrm{C}\left(29^{\prime}\right)-\mathrm{H}(29 \mathrm{D})$ & 109.7 & $\mathrm{O}\left(7^{\prime}\right)-\mathrm{C}\left(33^{\prime}\right)-\mathrm{H}(33 \mathrm{C})$ & 109.5 \\
\hline $\mathrm{H}(29 \mathrm{C})-\mathrm{C}\left(29^{\prime}\right)-\mathrm{H}(29 \mathrm{D})$ & 108.2 & $\mathrm{C}\left(34^{\prime}\right)-\mathrm{C}\left(33^{\prime}\right)-\mathrm{H}(33 \mathrm{D})$ & 109.5 \\
\hline $\mathrm{O}\left(6^{\prime}\right)-\mathrm{C}\left(30^{\prime}\right)-\mathrm{C}\left(29^{\prime}\right)$ & $108(2)$ & $\mathrm{O}\left(7^{\prime}\right)-\mathrm{C}\left(33^{\prime}\right)-\mathrm{H}(33 \mathrm{D})$ & 109.5 \\
\hline $\mathrm{O}\left(6^{\prime}\right)-\mathrm{C}\left(30^{\prime}\right)-\mathrm{H}(30 \mathrm{C})$ & 110.1 & $\mathrm{H}(33 \mathrm{C})-\mathrm{C}\left(33^{\prime}\right)-\mathrm{H}(33 \mathrm{D})$ & 108.1 \\
\hline $\mathrm{C}\left(29^{\prime}\right)-\mathrm{C}\left(30^{\prime}\right)-\mathrm{H}(30 \mathrm{C})$ & 110.1 & $\mathrm{C}\left(33^{\prime}\right)-\mathrm{C}\left(34^{\prime}\right)-\mathrm{O}\left(8^{\prime}\right)$ & $108(3)$ \\
\hline $\mathrm{O}\left(6^{\prime}\right)-\mathrm{C}\left(30^{\prime}\right)-\mathrm{H}(30 \mathrm{D})$ & 110.1 & $\mathrm{C}\left(33^{\prime}\right)-\mathrm{C}\left(34^{\prime}\right)-\mathrm{H}(34 \mathrm{C})$ & 110.0 \\
\hline $\mathrm{C}\left(29^{\prime}\right)-\mathrm{C}\left(30^{\prime}\right)-\mathrm{H}(30 \mathrm{D})$ & 110.1 & $\mathrm{O}\left(8^{\prime}\right)-\mathrm{C}\left(34^{\prime}\right)-\mathrm{H}(34 \mathrm{C})$ & 110.0 \\
\hline $\mathrm{H}(30 \mathrm{C})-\mathrm{C}\left(30^{\prime}\right)-\mathrm{H}(30 \mathrm{D})$ & 108.4 & $\mathrm{C}\left(33^{\prime}\right)-\mathrm{C}\left(34^{\prime}\right)-\mathrm{H}(34 \mathrm{D})$ & 110.0 \\
\hline $\mathrm{C}\left(30^{\prime}\right)-\mathrm{O}\left(6^{\prime}\right)-\mathrm{C}\left(31^{\prime}\right)$ & $109(2)$ & $\mathrm{O}\left(8^{\prime}\right)-\mathrm{C}\left(34^{\prime}\right)-\mathrm{H}(34 \mathrm{D})$ & 110.0 \\
\hline $\mathrm{C}\left(30^{\prime}\right)-\mathrm{O}\left(6^{\prime}\right)-\mathrm{Li}\left(2^{\prime}\right)$ & 113.2(19) & $\mathrm{H}(34 \mathrm{C})-\mathrm{C}\left(34^{\prime}\right)-\mathrm{H}(34 \mathrm{D})$ & 108.4 \\
\hline $\mathrm{C}\left(31^{\prime}\right)-\mathrm{O}\left(6^{\prime}\right)-\mathrm{Li}\left(2^{\prime}\right)$ & $104.2(16)$ & $\mathrm{C}\left(35^{\prime}\right)-\mathrm{O}\left(8^{\prime}\right)-\mathrm{C}\left(34^{\prime}\right)$ & $124(3)$ \\
\hline $\mathrm{C}\left(32^{\prime}\right)-\mathrm{C}\left(31^{\prime}\right)-\mathrm{O}\left(6^{\prime}\right)$ & $107(2)$ & $\mathrm{C}\left(35^{\prime}\right)-\mathrm{O}\left(8^{\prime}\right)-\mathrm{Li}\left(2^{\prime}\right)$ & $110(2)$ \\
\hline $\mathrm{C}\left(32^{\prime}\right)-\mathrm{C}\left(31^{\prime}\right)-\mathrm{Li}\left(2^{\prime}\right)$ & $69.9(17)$ & $\mathrm{C}\left(34^{\prime}\right)-\mathrm{O}\left(8^{\prime}\right)-\mathrm{Li}\left(2^{\prime}\right)$ & 110.1(19) \\
\hline $\mathrm{O}\left(6^{\prime}\right)-\mathrm{C}\left(31^{\prime}\right)-\mathrm{Li}\left(2^{\prime}\right)$ & $44.7(10)$ & $\mathrm{O}\left(8^{\prime}\right)-\mathrm{C}\left(35^{\prime}\right)-\mathrm{C}\left(36^{\prime}\right)$ & $125(3)$ \\
\hline $\mathrm{C}\left(32^{\prime}\right)-\mathrm{C}\left(31^{\prime}\right)-\mathrm{H}(31 \mathrm{C})$ & 110.3 & $\mathrm{O}\left(8^{\prime}\right)-\mathrm{C}\left(35^{\prime}\right)-\mathrm{H}(35 \mathrm{C})$ & 106.1 \\
\hline $\mathrm{O}\left(6^{\prime}\right)-\mathrm{C}\left(31^{\prime}\right)-\mathrm{H}(31 \mathrm{C})$ & 110.3 & $\mathrm{C}\left(36^{\prime}\right)-\mathrm{C}\left(35^{\prime}\right)-\mathrm{H}(35 \mathrm{C})$ & 106.1 \\
\hline $\mathrm{Li}\left(2^{\prime}\right)-\mathrm{C}\left(31^{\prime}\right)-\mathrm{H}(31 \mathrm{C})$ & 100.8 & $\mathrm{O}\left(8^{\prime}\right)-\mathrm{C}\left(35^{\prime}\right)-\mathrm{H}(35 \mathrm{D})$ & 106.1 \\
\hline $\mathrm{C}\left(32^{\prime}\right)-\mathrm{C}\left(31^{\prime}\right)-\mathrm{H}(31 \mathrm{D})$ & 110.3 & $\mathrm{C}\left(36^{\prime}\right)-\mathrm{C}\left(35^{\prime}\right)-\mathrm{H}(35 \mathrm{D})$ & 106.1 \\
\hline $\mathrm{O}\left(6^{\prime}\right)-\mathrm{C}\left(31^{\prime}\right)-\mathrm{H}(31 \mathrm{D})$ & 110.3 & $\mathrm{H}(35 \mathrm{C})-\mathrm{C}\left(35^{\prime}\right)-\mathrm{H}(35 \mathrm{D})$ & 106.3 \\
\hline Li(2')-C(31')-H(31D) & 147.8 & $\mathrm{C}\left(35^{\prime}\right)-\mathrm{C}\left(36^{\prime}\right)-\mathrm{O}\left(5^{\prime}\right)$ & $103(2)$ \\
\hline $\mathrm{H}(31 \mathrm{C})-\mathrm{C}\left(31^{\prime}\right)-\mathrm{H}(31 \mathrm{D})$ & 108.6 & $\mathrm{C}\left(35^{\prime}\right)-\mathrm{C}\left(36^{\prime}\right)-\mathrm{H}(36 \mathrm{C})$ & 111.1 \\
\hline $\mathrm{O}\left(7^{\prime}\right)-\mathrm{C}\left(32^{\prime}\right)-\mathrm{C}\left(31^{\prime}\right)$ & $121(2)$ & $\mathrm{O}\left(5^{\prime}\right)-\mathrm{C}\left(36^{\prime}\right)-\mathrm{H}(36 \mathrm{C})$ & 111.1 \\
\hline $\mathrm{O}\left(7^{\prime}\right)-\mathrm{C}\left(32^{\prime}\right)-\mathrm{Li}\left(2^{\prime}\right)$ & $47.4(15)$ & $\mathrm{C}\left(35^{\prime}\right)-\mathrm{C}\left(36^{\prime}\right)-\mathrm{H}(36 \mathrm{D})$ & 111.1 \\
\hline $\mathrm{C}\left(31^{\prime}\right)-\mathrm{C}\left(32^{\prime}\right)-\mathrm{Li}\left(2^{\prime}\right)$ & $81.3(18)$ & $\mathrm{O}\left(5^{\prime}\right)-\mathrm{C}\left(36^{\prime}\right)-\mathrm{H}(36 \mathrm{D})$ & 111.1 \\
\hline $\mathrm{O}\left(7^{\prime}\right)-\mathrm{C}\left(32^{\prime}\right)-\mathrm{H}(32 \mathrm{C})$ & 107.1 & $\mathrm{H}(36 \mathrm{C})-\mathrm{C}\left(36^{\prime}\right)-\mathrm{H}(36 \mathrm{D})$ & 109.1 \\
\hline $\mathrm{C}\left(31^{\prime}\right)-\mathrm{C}\left(32^{\prime}\right)-\mathrm{H}(32 \mathrm{C})$ & 107.1 & $\mathrm{O}(7 ")-\mathrm{Li}(2 ")-\mathrm{O}\left(8^{\prime \prime}\right)$ & $82.7(10)$ \\
\hline $\mathrm{Li}\left(2^{\prime}\right)-\mathrm{C}\left(32^{\prime}\right)-\mathrm{H}(32 \mathrm{C})$ & 150.0 & $\mathrm{O}\left(7^{\prime \prime}\right)-\mathrm{Li}\left(2^{\prime \prime}\right)-\mathrm{O}\left(6^{\prime \prime}\right)$ & $83.4(11)$ \\
\hline $\mathrm{O}\left(7^{\prime}\right)-\mathrm{C}\left(32^{\prime}\right)-\mathrm{H}(32 \mathrm{D})$ & 107.1 & $\mathrm{O}\left(8^{\prime \prime}\right)-\mathrm{Li}(2 ")-\mathrm{O}\left(6^{\prime \prime}\right)$ & $136.2(18)$ \\
\hline $\mathrm{C}\left(31^{\prime}\right)-\mathrm{C}\left(32^{\prime}\right)-\mathrm{H}(32 \mathrm{D})$ & 107.1 & $\mathrm{O}\left(7^{\prime \prime}\right)-\mathrm{Li}\left(2^{\prime \prime}\right)-\mathrm{O}\left(5^{\prime \prime}\right)$ & $133.0(17)$ \\
\hline $\mathrm{Li}\left(2^{\prime}\right)-\mathrm{C}\left(32^{\prime}\right)-\mathrm{H}(32 \mathrm{D})$ & 97.5 & $\mathrm{O}\left(8^{\prime \prime}\right)-\mathrm{Li}(2 ")-\mathrm{O}\left(5^{\prime \prime}\right)$ & $80.7(10)$ \\
\hline $\mathrm{H}(32 \mathrm{C})-\mathrm{C}\left(32^{\prime}\right)-\mathrm{H}(32 \mathrm{D})$ & 106.8 & $\mathrm{O}\left(6^{\prime \prime}\right)-\mathrm{Li}\left(2^{\prime \prime}\right)-\mathrm{O}\left(5^{\prime \prime}\right)$ & $79.0(10)$ \\
\hline $\mathrm{C}\left(32^{\prime}\right)-\mathrm{O}\left(7^{\prime}\right)-\mathrm{C}\left(33^{\prime}\right)$ & $120(3)$ & O(7")-Li(2")-C(35") & $105.0(13)$ \\
\hline $\mathrm{C}\left(32^{\prime}\right)-\mathrm{O}\left(7^{\prime}\right)-\mathrm{Li}\left(2^{\prime}\right)$ & $104(2)$ & O(8")-Li(2")-C(35") & $27.0(8)$ \\
\hline
\end{tabular}




\begin{tabular}{|c|c|c|c|}
\hline O(6")-Li(2")-C(35") & $123.4(15)$ & H(31E)-C(31")-H(31F) & 107.6 \\
\hline O(5")-Li(2")-C(35") & $54.2(9)$ & O(7")-C(32")-C(31") & $105(2)$ \\
\hline Cl(7)\#2-Li(2")-C(35") & $116.5(14)$ & $\mathrm{O}(7 ")-\mathrm{C}(32 ")-\mathrm{H}(32 \mathrm{E})$ & 110.8 \\
\hline O(7")-Li(2")-C(31") & $51.7(8)$ & C(31")-C(32")-H(32E) & 110.8 \\
\hline O(8")-Li(2")-C(31") & $123.7(15)$ & $\mathrm{O}(7 ")-\mathrm{C}(32 ")-\mathrm{H}(32 \mathrm{~F})$ & 110.8 \\
\hline O(6")-Li(2")-C(31") & $32.2(8)$ & $\mathrm{C}\left(31^{\prime \prime}\right)-\mathrm{C}(32 ")-\mathrm{H}(32 \mathrm{~F})$ & 110.8 \\
\hline O(5")-Li(2")-C(31") & $105.6(14)$ & $\mathrm{H}(32 \mathrm{E})-\mathrm{C}(32 ")-\mathrm{H}(32 \mathrm{~F})$ & 108.8 \\
\hline Cl(7)\#2-Li(2")-C(31") & $113.8(12)$ & C(32")-O(7")-C(33") & $118(2)$ \\
\hline C(35")-Li(2")-C(31") & $129.6(19)$ & C(32")-O(7")-Li(2") & $107.4(16)$ \\
\hline C(36")-O(5")-C(29") & $113(2)$ & C(33")-O(7")-Li(2") & $105.4(18)$ \\
\hline C(36")-O(5")-Li(2") & $108.8(18)$ & C(34")-C(33")-O(7") & $110(2)$ \\
\hline C(29")-O(5")-Li(2") & $112.3(18)$ & C(34")-C(33")-H(33E) & 109.6 \\
\hline O(5")-C(29")-C(30") & $106(3)$ & O(7")-C(33")-H(33E) & 109.6 \\
\hline O(5")-C(29")-H(29E) & 110.5 & $\mathrm{C}(34 ")-\mathrm{C}\left(33^{\prime \prime}\right)-\mathrm{H}(33 \mathrm{~F})$ & 109.6 \\
\hline C(30")-C(29")-H(29E) & 110.5 & $\mathrm{O}(7 ")-\mathrm{C}\left(33^{\prime \prime}\right)-\mathrm{H}(33 \mathrm{~F})$ & 109.6 \\
\hline O(5")-C(29")-H(29F) & 110.5 & H(33E)-C(33")-H(33F) & 108.1 \\
\hline C(30")-C(29")-H(29F) & 110.5 & C(33")-C(34")-O(8") & $108(2)$ \\
\hline H(29E)-C(29")-H(29F) & 108.7 & C(33")-C(34")-H(34E) & 110.0 \\
\hline $\mathrm{O}(6 ")-\mathrm{C}\left(30^{\prime \prime}\right)-\mathrm{C}\left(29^{\prime \prime}\right)$ & $106(2)$ & $\mathrm{O}\left(8^{\prime \prime}\right)-\mathrm{C}(34 ")-\mathrm{H}(34 \mathrm{E})$ & 110.0 \\
\hline $\mathrm{O}\left(6^{\prime \prime}\right)-\mathrm{C}\left(30^{\prime \prime}\right)-\mathrm{H}(30 \mathrm{E})$ & 110.6 & C(33")-C(34")-H(34F) & 110.0 \\
\hline C(29")-C(30")-H(30E) & 110.6 & $\mathrm{O}\left(8^{\prime \prime}\right)-\mathrm{C}(34 ")-\mathrm{H}(34 \mathrm{~F})$ & 110.0 \\
\hline $\mathrm{O}\left(6^{\prime \prime}\right)-\mathrm{C}\left(30^{\prime \prime}\right)-\mathrm{H}(30 \mathrm{~F})$ & 110.6 & H(34E)-C(34")-H(34F) & 108.4 \\
\hline C(29")-C(30")-H(30F) & 110.6 & C(35")-O(8")-C(34") & $118(3)$ \\
\hline $\mathrm{H}(30 \mathrm{E})-\mathrm{C}(30 ")-\mathrm{H}(30 \mathrm{~F})$ & 108.8 & $\mathrm{C}\left(35^{\prime \prime}\right)-\mathrm{O}\left(8^{\prime \prime}\right)-\mathrm{Li}\left(2^{\prime \prime}\right)$ & 106.1(19) \\
\hline C(30")-O(6")-C(31") & $109(2)$ & C(34")-O(8")-Li(2") & 106.1(16) \\
\hline $\mathrm{C}\left(30^{\prime \prime}\right)-\mathrm{O}\left(6^{\prime \prime}\right)-\mathrm{Li}\left(2^{\prime \prime}\right)$ & $107(2)$ & $\mathrm{O}\left(8^{\prime \prime}\right)-\mathrm{C}\left(35^{\prime \prime}\right)-\mathrm{C}\left(36^{\prime \prime}\right)$ & $120(2)$ \\
\hline C(31")-O(6")-Li(2") & $100.3(17)$ & $\mathrm{O}\left(8^{\prime \prime}\right)-\mathrm{C}\left(35^{\prime \prime}\right)-\mathrm{Li}\left(2^{\prime \prime}\right)$ & $46.9(13)$ \\
\hline $\mathrm{C}(32 ")-\mathrm{C}(31 ")-\mathrm{O}(6 ")$ & $115(2)$ & C(36")-C(35")-Li(2") & $81.4(16)$ \\
\hline C(32")-C(31")-Li(2") & $76.9(15)$ & $\mathrm{O}\left(8^{\prime \prime}\right)-\mathrm{C}\left(35^{\prime \prime}\right)-\mathrm{H}(35 \mathrm{E})$ & 107.2 \\
\hline O(6")-C(31")-Li(2") & $47.5(11)$ & $\mathrm{C}\left(36^{\prime \prime}\right)-\mathrm{C}\left(35^{\prime \prime}\right)-\mathrm{H}(35 \mathrm{E})$ & 107.2 \\
\hline C(32")-C(31")-H(31E) & 108.6 & Li(2")-C(35")-H(35E) & 97.8 \\
\hline $\mathrm{O}\left(6^{\prime \prime}\right)-\mathrm{C}(31 ")-\mathrm{H}(31 \mathrm{E})$ & 108.6 & $\mathrm{O}\left(8^{\prime \prime}\right)-\mathrm{C}\left(35^{\prime \prime}\right)-\mathrm{H}(35 \mathrm{~F})$ & 107.2 \\
\hline Li(2")-C(31")-H(31E) & 151.8 & $\mathrm{C}\left(36^{\prime \prime}\right)-\mathrm{C}\left(35^{\prime \prime}\right)-\mathrm{H}(35 \mathrm{~F})$ & 107.2 \\
\hline C(32")-C(31")-H(31F) & 108.6 & Li(2")-C(35")-H(35F) & 149.6 \\
\hline $\mathrm{O}(6 ")-\mathrm{C}(31 ")-\mathrm{H}(31 \mathrm{~F})$ & 108.6 & $\mathrm{H}(35 \mathrm{E})-\mathrm{C}\left(35^{\prime \prime}\right)-\mathrm{H}(35 \mathrm{~F})$ & 106.9 \\
\hline Li(2")-C(31")-H(31F) & 96.1 & $\mathrm{C}\left(35^{\prime \prime}\right)-\mathrm{C}\left(36^{\prime \prime}\right)-\mathrm{O}\left(5^{\prime \prime}\right)$ & $104(2)$ \\
\hline
\end{tabular}




$\begin{array}{llll}\mathrm{C}\left(35^{\prime \prime}\right)-\mathrm{C}\left(36^{\prime \prime}\right)-\mathrm{H}(36 \mathrm{E}) & 110.9 & \mathrm{O}\left(5^{\prime \prime}\right)-\mathrm{C}(36 ")-\mathrm{H}(36 \mathrm{~F}) & 110.9 \\ \mathrm{O}\left(5^{\prime \prime}\right)-\mathrm{C}\left(36^{\prime \prime}\right)-\mathrm{H}(36 \mathrm{E}) & 110.9 & \mathrm{H}(36 \mathrm{E})-\mathrm{C}(36 ")-\mathrm{H}(36 \mathrm{~F}) & 108.9 \\ \mathrm{C}\left(35^{\prime \prime}\right)-\mathrm{C}(36 ")-\mathrm{H}(36 \mathrm{~F}) & 110.9 & \mathrm{H}(9 \mathrm{~A})-\mathrm{O}(9)-\mathrm{H}(9 \mathrm{~B}) & 109.5\end{array}$

Symmetry transformations used to generate equivalent atoms:

$\# 1-\mathrm{x}+1, \mathrm{y}+1 / 2,-\mathrm{z}+1 / 2 \quad \# 2-\mathrm{x}+1, \mathrm{y}-1 / 2,-\mathrm{z}+1 / 2$ 
Table 4. Anisotropic displacement parameters $\left(\AA^{2} \times 10^{3}\right)$ for rhowe10. The anisotropic displacement factor exponent takes the form: $-2 \pi^{2}\left[h^{2} a^{* 2} U_{11}+\ldots+2 h k a^{*} b^{*} U_{12}\right]$

\begin{tabular}{|c|c|c|c|c|c|c|}
\hline & $\mathrm{U}_{11}$ & $\mathrm{U}_{22}$ & $\mathrm{U}_{33}$ & $\mathrm{U}_{23}$ & $\mathrm{U}_{13}$ & $\mathrm{U}_{12}$ \\
\hline Mo1 & $17(1)$ & $21(1)$ & $19(1)$ & $1(1)$ & $0(1)$ & $0(1)$ \\
\hline $\mathrm{Cl1}$ & $37(1)$ & $19(1)$ & $26(1)$ & $-1(1)$ & $-9(1)$ & $3(1)$ \\
\hline $\mathrm{Cl} 2$ & $24(1)$ & $36(1)$ & $29(1)$ & 13(1) & $2(1)$ & $4(1)$ \\
\hline $\mathrm{Cl} 3$ & $23(1)$ & $21(1)$ & $41(1)$ & $-3(1)$ & $-2(1)$ & $-2(1)$ \\
\hline $\mathrm{Cl} 4$ & $20(1)$ & $37(1)$ & $25(1)$ & $-2(1)$ & $4(1)$ & $1(1)$ \\
\hline N1 & $20(2)$ & $20(2)$ & $22(2)$ & $2(2)$ & $-4(2)$ & $2(2)$ \\
\hline N2 & $19(2)$ & $25(2)$ & $14(2)$ & $0(2)$ & $2(2)$ & $0(2)$ \\
\hline $\mathrm{C} 1$ & $28(3)$ & $22(2)$ & $22(2)$ & $-3(2)$ & $-5(2)$ & $-1(2)$ \\
\hline $\mathrm{C} 2$ & $33(3)$ & $32(3)$ & $20(2)$ & $-4(2)$ & $-1(2)$ & $9(2)$ \\
\hline $\mathrm{C} 3$ & $29(3)$ & $39(3)$ & $24(3)$ & $-3(2)$ & $6(2)$ & $9(2)$ \\
\hline $\mathrm{C} 4$ & $26(3)$ & $30(3)$ & $21(2)$ & $-3(2)$ & $3(2)$ & $0(2)$ \\
\hline $\mathrm{C} 5$ & $19(2)$ & $24(2)$ & $21(2)$ & $3(2)$ & $-1(2)$ & $0(2)$ \\
\hline C6 & $18(2)$ & $29(3)$ & $18(2)$ & $1(2)$ & $-3(2)$ & $-1(2)$ \\
\hline C7 & $23(3)$ & $47(3)$ & $31(3)$ & $-12(2)$ & $3(2)$ & $-10(2)$ \\
\hline $\mathrm{C} 8$ & $32(3)$ & $58(4)$ & $29(3)$ & $-11(3)$ & $-3(2)$ & $-18(3)$ \\
\hline C9 & $30(3)$ & $42(3)$ & $22(3)$ & $-8(2)$ & $-3(2)$ & $-3(2)$ \\
\hline $\mathrm{C} 10$ & $19(2)$ & $31(3)$ & $20(2)$ & $-4(2)$ & $-2(2)$ & $2(2)$ \\
\hline Li1 & $37(5)$ & $22(4)$ & $32(4)$ & $5(3)$ & $-4(4)$ & $-1(4)$ \\
\hline $\mathrm{O} 1$ & $22(3)$ & $31(2)$ & $23(2)$ & $0(2)$ & $6(2)$ & $2(2)$ \\
\hline $\mathrm{C} 11$ & $31(6)$ & $43(8)$ & $17(5)$ & $0(4)$ & $4(4)$ & $-7(5)$ \\
\hline $\mathrm{C} 12$ & $32(4)$ & $37(5)$ & $20(4)$ & $-6(3)$ & $-2(3)$ & $-2(4)$ \\
\hline $\mathrm{O} 2$ & $24(3)$ & $24(3)$ & $24(4)$ & $5(3)$ & $-3(2)$ & $-1(2)$ \\
\hline $\mathrm{C} 13$ & $22(6)$ & $38(6)$ & $40(6)$ & $5(4)$ & $0(4)$ & $3(4)$ \\
\hline C14 & $24(5)$ & $32(6)$ & $39(5)$ & $-1(4)$ & $-5(4)$ & $-5(4)$ \\
\hline $\mathrm{O} 3$ & $25(3)$ & $27(2)$ & $33(3)$ & $-1(2)$ & $5(2)$ & $-1(2)$ \\
\hline $\mathrm{C} 15$ & $32(4)$ & $41(6)$ & $26(5)$ & $7(3)$ & $-4(4)$ & $1(4)$ \\
\hline C16 & $31(5)$ & $54(8)$ & $12(6)$ & $5(4)$ & $-13(4)$ & $-3(5)$ \\
\hline $\mathrm{O} 4$ & $29(2)$ & $26(3)$ & $24(2)$ & $3(2)$ & $-1(2)$ & $5(2)$ \\
\hline $\mathrm{C} 17$ & $31(6)$ & $39(7)$ & $33(4)$ & $1(5)$ & $1(4)$ & $-6(4)$ \\
\hline C18 & $27(6)$ & $34(7)$ & $35(4)$ & $1(4)$ & $3(4)$ & $-2(4)$ \\
\hline O1' & $22(3)$ & $31(2)$ & $23(2)$ & $0(2)$ & $6(2)$ & $2(2)$ \\
\hline
\end{tabular}




\begin{tabular}{|c|c|c|c|c|c|c|}
\hline C11' & $31(6)$ & $43(8)$ & $17(5)$ & $0(4)$ & $4(4)$ & $-7(5)$ \\
\hline $\mathrm{C} 12^{\prime}$ & $32(4)$ & $37(5)$ & $20(4)$ & $-6(3)$ & $-2(3)$ & $-2(4)$ \\
\hline $\mathrm{O} 2^{\prime}$ & $24(3)$ & $24(3)$ & $24(4)$ & $5(3)$ & $-3(2)$ & $-1(2)$ \\
\hline C13' & $22(6)$ & $38(6)$ & $40(6)$ & $5(4)$ & $0(4)$ & $3(4)$ \\
\hline C14' & $24(5)$ & $32(6)$ & $39(5)$ & $-1(4)$ & $-5(4)$ & $-5(4)$ \\
\hline O3' & $25(3)$ & $27(2)$ & $33(3)$ & $-1(2)$ & $5(2)$ & $-1(2)$ \\
\hline $\mathrm{C} 15^{\prime}$ & $32(4)$ & $41(6)$ & $26(5)$ & $7(3)$ & $-4(4)$ & 1(4) \\
\hline C16' & $31(5)$ & $54(8)$ & $12(6)$ & $5(4)$ & $-13(4)$ & $-3(5)$ \\
\hline O4' & $29(2)$ & $26(3)$ & $24(2)$ & $3(2)$ & $-1(2)$ & $5(2)$ \\
\hline $\mathrm{C} 17^{\prime}$ & $31(6)$ & $39(7)$ & $33(4)$ & $1(5)$ & $1(4)$ & $-6(4)$ \\
\hline C18' & $27(6)$ & $34(7)$ & $35(4)$ & $1(4)$ & $3(4)$ & $-2(4)$ \\
\hline Mo2 & $16(1)$ & $23(1)$ & $16(1)$ & $2(1)$ & $-2(1)$ & $-1(1)$ \\
\hline $\mathrm{Cl} 5$ & $22(1)$ & $24(1)$ & $26(1)$ & $0(1)$ & $4(1)$ & $2(1)$ \\
\hline $\mathrm{Cl} 6$ & $32(1)$ & $31(1)$ & $22(1)$ & $-7(1)$ & $1(1)$ & $-2(1)$ \\
\hline $\mathrm{Cl} 7$ & $23(1)$ & $30(1)$ & $26(1)$ & $1(1)$ & $1(1)$ & $-7(1)$ \\
\hline $\mathrm{Cl} 8$ & $20(1)$ & $45(1)$ & $27(1)$ & 11(1) & $-6(1)$ & $-1(1)$ \\
\hline N3 & $18(2)$ & $23(2)$ & $15(2)$ & $-2(2)$ & $-1(2)$ & $4(2)$ \\
\hline N4 & $17(2)$ & $23(2)$ & $16(2)$ & $1(2)$ & $0(2)$ & $0(2)$ \\
\hline $\mathrm{C} 19$ & $29(3)$ & $26(3)$ & $22(2)$ & $4(2)$ & $6(2)$ & $3(2)$ \\
\hline $\mathrm{C} 20$ & $37(3)$ & $42(3)$ & 21(3) & $13(2)$ & $5(2)$ & $13(3)$ \\
\hline $\mathrm{C} 21$ & $27(3)$ & $62(4)$ & $23(3)$ & $3(2)$ & $-10(2)$ & $14(3)$ \\
\hline $\mathrm{C} 22$ & $23(3)$ & $48(3)$ & $20(2)$ & $0(2)$ & $-1(2)$ & $5(2)$ \\
\hline $\mathrm{C} 23$ & $15(2)$ & $31(3)$ & $16(2)$ & $-1(2)$ & $-2(2)$ & $4(2)$ \\
\hline $\mathrm{C} 24$ & $17(2)$ & $26(2)$ & $14(2)$ & $-3(2)$ & $0(2)$ & $1(2)$ \\
\hline $\mathrm{C} 25$ & $20(2)$ & $40(3)$ & $25(3)$ & $-4(2)$ & $-3(2)$ & $-7(2)$ \\
\hline $\mathrm{C} 26$ & $24(3)$ & $46(3)$ & $28(3)$ & $2(2)$ & $4(2)$ & $-8(2)$ \\
\hline $\mathrm{C} 27$ & $28(3)$ & $35(3)$ & $30(3)$ & $5(2)$ & $6(2)$ & $-6(2)$ \\
\hline $\mathrm{C} 28$ & $23(2)$ & $29(3)$ & $22(2)$ & $2(2)$ & $3(2)$ & $2(2)$ \\
\hline Li2 & $31(8)$ & $20(7)$ & $30(7)$ & $5(5)$ & $5(6)$ & 11(6) \\
\hline O5 & $97(5)$ & $21(4)$ & $37(4)$ & $-2(3)$ & $28(4)$ & $-10(4)$ \\
\hline $\mathrm{C} 29$ & 171(13) & $57(7)$ & $35(5)$ & $8(5)$ & $28(7)$ & $39(8)$ \\
\hline $\mathrm{C} 30$ & $125(9)$ & $39(12)$ & $71(6)$ & $29(6)$ & $-66(6)$ & $-34(8)$ \\
\hline O6 & $55(6)$ & $37(4)$ & $85(8)$ & $16(4)$ & $-24(5)$ & $-4(4)$ \\
\hline C31 & $57(9)$ & $56(10)$ & $174(14)$ & $7(10)$ & $-18(9)$ & $3(7)$ \\
\hline C32 & $60(8)$ & $41(8)$ & $182(16)$ & $23(10)$ & $21(9)$ & $20(6)$ \\
\hline $\mathrm{O} 7$ & $58(4)$ & $24(3)$ & $57(5)$ & $-14(3)$ & $26(4)$ & 11(3) \\
\hline
\end{tabular}




\begin{tabular}{|c|c|c|c|c|c|c|}
\hline $\mathrm{C} 33$ & $100(8)$ & $32(4)$ & $38(4)$ & 1(3) & $4(5)$ & $-12(6)$ \\
\hline C34 & $75(8)$ & $49(6)$ & 91(13) & $-8(9)$ & $-19(8)$ & $2(6)$ \\
\hline O8 & $43(4)$ & $30(4)$ & $72(5)$ & $-3(3)$ & $-13(4)$ & $-2(3)$ \\
\hline $\mathrm{C} 35$ & $46(6)$ & $43(7)$ & 116(9) & $-23(7)$ & $11(6)$ & $-15(5)$ \\
\hline $\mathrm{C} 36$ & $142(12)$ & $57(9)$ & $82(8)$ & $-13(8)$ & $60(9)$ & $-49(8)$ \\
\hline Li2' & $60(20)$ & $47(18)$ & $110(30)$ & $-6(18)$ & $-8(19)$ & $25(16)$ \\
\hline O5' & $97(5)$ & $21(4)$ & $37(4)$ & $-2(3)$ & $28(4)$ & $-10(4)$ \\
\hline C29' & 171(13) & $57(7)$ & $35(5)$ & $8(5)$ & $28(7)$ & $39(8)$ \\
\hline $\mathrm{C} 30^{\prime}$ & $125(9)$ & $39(12)$ & $71(6)$ & $29(6)$ & $-66(6)$ & $-34(8)$ \\
\hline O6' & $55(6)$ & $37(4)$ & $85(8)$ & $16(4)$ & $-24(5)$ & $-4(4)$ \\
\hline $\mathrm{C} 31^{\prime}$ & $57(9)$ & $56(10)$ & $174(14)$ & $7(10)$ & $-18(9)$ & $3(7)$ \\
\hline $\mathrm{C} 32^{\prime}$ & $60(8)$ & $41(8)$ & $182(16)$ & $23(10)$ & $21(9)$ & $20(6)$ \\
\hline O7' & $58(4)$ & $24(3)$ & $57(5)$ & $-14(3)$ & $26(4)$ & 11(3) \\
\hline C33' & $100(8)$ & $32(4)$ & $38(4)$ & $1(3)$ & $4(5)$ & $-12(6)$ \\
\hline C34' & $75(8)$ & $49(6)$ & 91(13) & $-8(9)$ & $-19(8)$ & $2(6)$ \\
\hline O8' & $43(4)$ & $30(4)$ & $72(5)$ & $-3(3)$ & $-13(4)$ & $-2(3)$ \\
\hline $\mathrm{C} 35^{\prime}$ & $46(6)$ & $43(7)$ & 116(9) & $-23(7)$ & $11(6)$ & $-15(5)$ \\
\hline $\mathrm{C} 36^{\prime}$ & $142(12)$ & $57(9)$ & $82(8)$ & $-13(8)$ & $60(9)$ & $-49(8)$ \\
\hline Li2" & $60(20)$ & $47(18)$ & $110(30)$ & $-6(18)$ & $-8(19)$ & $25(16)$ \\
\hline O5" & 171(13) & $57(7)$ & $35(5)$ & $8(5)$ & $28(7)$ & $39(8)$ \\
\hline C29" & $125(9)$ & $39(12)$ & $71(6)$ & $29(6)$ & $-66(6)$ & $-34(8)$ \\
\hline C30" & $55(6)$ & $37(4)$ & $85(8)$ & $16(4)$ & $-24(5)$ & $-4(4)$ \\
\hline O6" & $57(9)$ & $56(10)$ & $174(14)$ & $7(10)$ & $-18(9)$ & $3(7)$ \\
\hline C31" & $60(8)$ & $41(8)$ & 182(16) & $23(10)$ & $21(9)$ & $20(6)$ \\
\hline C32" & $58(4)$ & $24(3)$ & $57(5)$ & $-14(3)$ & $26(4)$ & 11(3) \\
\hline O7" & $100(8)$ & $32(4)$ & $38(4)$ & $1(3)$ & $4(5)$ & $-12(6)$ \\
\hline C33" & $75(8)$ & $49(6)$ & $91(13)$ & $-8(9)$ & $-19(8)$ & $2(6)$ \\
\hline C34" & $43(4)$ & $30(4)$ & $72(5)$ & $-3(3)$ & $-13(4)$ & $-2(3)$ \\
\hline O8" & $46(6)$ & $43(7)$ & 116(9) & $-23(7)$ & $11(6)$ & $-15(5)$ \\
\hline C35" & $142(12)$ & $57(9)$ & $82(8)$ & $-13(8)$ & $60(9)$ & $-49(8)$ \\
\hline C36" & $97(5)$ & $21(4)$ & $37(4)$ & $-2(3)$ & $28(4)$ & $-10(4)$ \\
\hline O9 & 43(13) & $55(14)$ & 34(11) & $15(9)$ & $12(9)$ & $-16(10)$ \\
\hline
\end{tabular}


Table 5. Hydrogen coordinates $\left(\times 10^{4}\right)$ and isotropic displacement parameters $\left(\AA^{2} \times 10^{3}\right)$ for rhowe10.

\begin{tabular}{|c|c|c|c|c|}
\hline & $\mathrm{x}$ & $\mathrm{y}$ & $\mathrm{z}$ & $\mathrm{U}(\mathrm{eq})$ \\
\hline $\mathrm{H} 1$ & 1120 & 4154 & 5863 & 29 \\
\hline $\mathrm{H} 2$ & 92 & 4309 & 6433 & 34 \\
\hline $\mathrm{H} 3$ & -1064 & 3660 & 6386 & 37 \\
\hline $\mathrm{H} 4$ & -1173 & 2900 & 5752 & 31 \\
\hline $\mathrm{H} 7$ & -1199 & 2250 & 5141 & 41 \\
\hline H8 & -1128 & 1538 & 4472 & 48 \\
\hline H9 & 42 & 1511 & 3987 & 38 \\
\hline $\mathrm{H} 10$ & 1103 & 2187 & 4175 & 28 \\
\hline $\mathrm{H} 11 \mathrm{~A}$ & 1523 & 577 & 4236 & 37 \\
\hline H11B & 1959 & 214 & 4688 & 37 \\
\hline $\mathrm{H} 12 \mathrm{~A}$ & 2971 & 690 & 4212 & 36 \\
\hline H12B & 2513 & 1364 & 4228 & 36 \\
\hline $\mathrm{H} 13 \mathrm{~A}$ & 4041 & 583 & 4863 & 40 \\
\hline H13B & 3251 & 230 & 5073 & 40 \\
\hline $\mathrm{H} 14 \mathrm{~A}$ & 4075 & 489 & 5744 & 38 \\
\hline H14B & 4131 & 1216 & 5566 & 38 \\
\hline $\mathrm{H} 15 \mathrm{~A}$ & 3033 & 319 & 6399 & 39 \\
\hline H15B & 2664 & 92 & 5879 & 39 \\
\hline H16A & 1587 & 284 & 6422 & 39 \\
\hline H16B & 1920 & 988 & 6537 & 39 \\
\hline H17A & 552 & 256 & 5760 & 41 \\
\hline H17B & 1384 & 57 & 5496 & 41 \\
\hline $\mathrm{H} 18 \mathrm{~A}$ & 517 & 428 & 4878 & 39 \\
\hline H18B & 334 & 1067 & 5186 & 39 \\
\hline $\mathrm{H} 11 \mathrm{C}$ & 2161 & 1248 & 4240 & 37 \\
\hline H11D & 1784 & 544 & 4228 & 37 \\
\hline $\mathrm{H} 12 \mathrm{C}$ & 2754 & 180 & 4792 & 36 \\
\hline H12D & 3220 & 526 & 4344 & 36 \\
\hline $\mathrm{H} 13 \mathrm{C}$ & 4208 & 1183 & 5325 & 40 \\
\hline H13D & 4140 & 504 & 5056 & 40 \\
\hline
\end{tabular}




\begin{tabular}{|c|c|c|c|c|}
\hline $\mathrm{H} 14 \mathrm{C}$ & 3261 & 126 & 5651 & 38 \\
\hline H14D & 4000 & 448 & 5945 & 38 \\
\hline $\mathrm{H} 15 \mathrm{C}$ & 2388 & 1074 & 6582 & 39 \\
\hline H15D & 2823 & 395 & 6514 & 39 \\
\hline $\mathrm{H} 16 \mathrm{C}$ & 1876 & 95 & 5910 & 39 \\
\hline H16D & 1387 & 318 & 6393 & 39 \\
\hline $\mathrm{H} 17 \mathrm{C}$ & 337 & 1074 & 5477 & 41 \\
\hline H17D & 458 & 370 & 5696 & 41 \\
\hline $\mathrm{H} 18 \mathrm{C}$ & 1402 & 127 & 5095 & 39 \\
\hline H18D & 621 & 409 & 4819 & 39 \\
\hline H19 & 3686 & 6569 & 1845 & 31 \\
\hline $\mathrm{H} 20$ & 2667 & 6784 & 1275 & 40 \\
\hline $\mathrm{H} 21$ & 1409 & 6297 & 1373 & 45 \\
\hline $\mathrm{H} 22$ & 1187 & 5625 & 2053 & 37 \\
\hline $\mathrm{H} 25$ & 1020 & 5135 & 2738 & 34 \\
\hline $\mathrm{H} 26$ & 976 & 4476 & 3442 & 39 \\
\hline $\mathrm{H} 27$ & 2193 & 4202 & 3835 & 37 \\
\hline $\mathrm{H} 28$ & 3395 & 4657 & 3563 & 29 \\
\hline $\mathrm{H} 29 \mathrm{~A}$ & 4307 & 3018 & 3765 & 106 \\
\hline H29B & 4641 & 3733 & 3718 & 106 \\
\hline $\mathrm{H} 30 \mathrm{~A}$ & 5745 & 3044 & 3575 & 94 \\
\hline H30B & 5245 & 2658 & 3161 & 94 \\
\hline $\mathrm{H} 31 \mathrm{~A}$ & 6479 & 3644 & 2497 & 115 \\
\hline H31B & 6452 & 2957 & 2751 & 115 \\
\hline $\mathrm{H} 32 \mathrm{~A}$ & 5537 & 2609 & 2231 & 113 \\
\hline H32B & 6198 & 2980 & 1904 & 113 \\
\hline $\mathrm{H} 33 \mathrm{~A}$ & 4394 & 3599 & 1437 & 68 \\
\hline H33B & 4886 & 2945 & 1389 & 68 \\
\hline $\mathrm{H} 34 \mathrm{~A}$ & 4142 & 2494 & 1982 & 86 \\
\hline H34B & 3548 & 2797 & 1575 & 86 \\
\hline $\mathrm{H} 35 \mathrm{~A}$ & 2890 & 2685 & 2413 & 82 \\
\hline H35B & 2693 & 3390 & 2593 & 82 \\
\hline H36A & 3756 & 2526 & 3018 & 112 \\
\hline H36B & 3003 & 2888 & 3269 & 112 \\
\hline $\mathrm{H} 29 \mathrm{C}$ & 4138 & 3543 & 3463 & 106 \\
\hline H29D & 4238 & 3681 & 2883 & 106 \\
\hline
\end{tabular}




\begin{tabular}{|c|c|c|c|c|}
\hline $\mathrm{H} 30 \mathrm{C}$ & 5535 & 3752 & 3218 & 94 \\
\hline H30D & 5427 & 3091 & 3510 & 94 \\
\hline $\mathrm{H} 31 \mathrm{C}$ & 5445 & 3840 & 2399 & 115 \\
\hline H31D & 6374 & 3634 & 2462 & 115 \\
\hline $\mathrm{H} 32 \mathrm{C}$ & 5908 & 3472 & 1687 & 113 \\
\hline H32D & 6240 & 2843 & 1945 & 113 \\
\hline $\mathrm{H} 33 \mathrm{C}$ & 4328 & 3591 & 1820 & 68 \\
\hline H33D & 4524 & 3344 & 1272 & 68 \\
\hline $\mathrm{H} 34 \mathrm{C}$ & 3782 & 2460 & 1396 & 86 \\
\hline H34D & 3246 & 3080 & 1517 & 86 \\
\hline $\mathrm{H} 35 \mathrm{C}$ & 2574 & 2708 & 2338 & 82 \\
\hline H35D & 3136 & 3319 & 2365 & 82 \\
\hline $\mathrm{H} 36 \mathrm{C}$ & 2916 & 2382 & 3064 & 112 \\
\hline $\mathrm{H} 36 \mathrm{D}$ & 2921 & 3148 & 3126 & 112 \\
\hline $\mathrm{H} 29 \mathrm{E}$ & 5721 & 2993 & 3519 & 94 \\
\hline $\mathrm{H} 29 \mathrm{~F}$ & 5164 & 3622 & 3540 & 94 \\
\hline H30E & 5304 & 3765 & 2664 & 71 \\
\hline $\mathrm{H} 30 \mathrm{~F}$ & 6196 & 3722 & 2899 & 71 \\
\hline H31E & 6358 & 3461 & 2021 & 113 \\
\hline $\mathrm{H} 31 \mathrm{~F}$ & 6188 & 2740 & 1861 & 113 \\
\hline H32E & 4999 & 3673 & 1962 & 56 \\
\hline $\mathrm{H} 32 \mathrm{~F}$ & 5373 & 3431 & 1446 & 56 \\
\hline H33E & 3840 & 3174 & 1390 & 86 \\
\hline $\mathrm{H} 33 \mathrm{~F}$ & 3648 & 2462 & 1573 & 86 \\
\hline H34E & 2887 & 3176 & 1991 & 58 \\
\hline $\mathrm{H} 34 \mathrm{~F}$ & 3686 & 3542 & 2171 & 58 \\
\hline H35E & 3218 & 2484 & 3119 & 112 \\
\hline $\mathrm{H} 35 \mathrm{~F}$ & 2843 & 3117 & 2890 & 112 \\
\hline $\mathrm{H} 36 \mathrm{E}$ & 3696 & 3302 & 3548 & 62 \\
\hline $\mathrm{H} 36 \mathrm{~F}$ & 3982 & 3667 & 3054 & 62 \\
\hline H9A & 3761 & 4179 & 4688 & 66 \\
\hline H9B & 3398 & 3861 & 4297 & 66 \\
\hline
\end{tabular}


Table 6. Torsion angles $\left[{ }^{\circ}\right]$ for rhowe 10 .

\begin{tabular}{|c|c|c|c|}
\hline C5-N1-C1-C2 & $1.5(6)$ & C13-C14-O3-C15 & $85.8(11)$ \\
\hline Mo1-N1-C1-C2 & $179.9(3)$ & C13-C14-O3-Li1 & $-34.1(12)$ \\
\hline $\mathrm{N} 1-\mathrm{C} 1-\mathrm{C} 2-\mathrm{C} 3$ & $-0.2(7)$ & C14-O3-C15-C16 & $-164.6(8)$ \\
\hline $\mathrm{C} 1-\mathrm{C} 2-\mathrm{C} 3-\mathrm{C} 4$ & $-1.2(7)$ & Li1-O3-C15-C16 & $-45.3(10)$ \\
\hline $\mathrm{C} 2-\mathrm{C} 3-\mathrm{C} 4-\mathrm{C} 5$ & $1.3(7)$ & $\mathrm{O} 3-\mathrm{C} 15-\mathrm{C} 16-\mathrm{O} 4$ & $55.1(12)$ \\
\hline C1-N1-C5-C4 & $-1.4(6)$ & C15-C16-O4-C17 & $87.1(12)$ \\
\hline Mo1-N1-C5-C4 & $-180.0(3)$ & C15-C16-O4-Li1 & $-36.4(11)$ \\
\hline C1-N1-C5-C6 & $178.1(4)$ & C16-O4-C17-C18 & $-169.4(9)$ \\
\hline Mo1-N1-C5-C6 & $-0.5(5)$ & Li1-O4-C17-C18 & $-45.5(11)$ \\
\hline $\mathrm{C} 3-\mathrm{C} 4-\mathrm{C} 5-\mathrm{N} 1$ & $0.1(7)$ & C11-O1-C18-C17 & $90.8(11)$ \\
\hline C3-C4-C5-C6 & $-179.4(4)$ & Li1-O1-C18-C17 & $-34.4(12)$ \\
\hline C10-N2-C6-C7 & $-0.1(7)$ & $\mathrm{O} 4-\mathrm{C} 17-\mathrm{C} 18-\mathrm{O} 1$ & $52.5(12)$ \\
\hline Mo1-N2-C6-C7 & $177.5(4)$ & $\mathrm{C} 18^{\prime}-\mathrm{O} 1^{\prime}-\mathrm{C} 11^{\prime}-\mathrm{C} 12^{\prime}$ & $-85.0(15)$ \\
\hline C10-N2-C6-C5 & $-179.6(4)$ & Li1-O1'-C11'-C12' & $38.4(14)$ \\
\hline Mo1-N2-C6-C5 & $-2.1(5)$ & $\mathrm{O} 1^{\prime}-\mathrm{C} 11^{\prime}-\mathrm{C} 12^{\prime}-\mathrm{O} 2^{\prime}$ & $-54.1(14)$ \\
\hline N1-C5-C6-N2 & $1.7(6)$ & $\mathrm{C} 11^{\prime}-\mathrm{C} 12^{\prime}-\mathrm{O} 2{ }^{\prime}-\mathrm{C} 13^{\prime}$ & $168.4(10)$ \\
\hline C4-C5-C6-N2 & $-178.8(4)$ & $\mathrm{C} 11$ '-C12'-O2'-Li1 & $41.9(12)$ \\
\hline N1-C5-C6-C7 & $-177.9(4)$ & $\mathrm{C} 12^{\prime}-\mathrm{O} 2{ }^{\prime}-\mathrm{C} 13^{\prime}-\mathrm{C} 14^{\prime}$ & $-90.6(13)$ \\
\hline C4-C5-C6-C7 & $1.6(7)$ & Li1-O2'-C13'-C14' & $33.8(15)$ \\
\hline N2-C6-C7-C8 & $0.3(8)$ & $\mathrm{O} 2{ }^{\prime}-\mathrm{C} 13^{\prime}-\mathrm{C} 14{ }^{\prime}-\mathrm{O} 3{ }^{\prime}$ & $-49.4(14)$ \\
\hline C5-C6-C7-C8 & $179.8(5)$ & C13'-C14'-O3'-C15' & $167.5(10)$ \\
\hline C6-C7-C8-C9 & $-0.2(8)$ & C13'-C14'-O3'-Li1 & $41.8(13)$ \\
\hline C7-C8-C9-C10 & $-0.1(8)$ & C14'-O3'-C15'-C16' & $-89.9(14)$ \\
\hline C6-N2-C10-C9 & $-0.2(7)$ & Li1-O3'-C15'-C16' & $35.4(15)$ \\
\hline Mo1-N2-C10-C9 & $-177.6(3)$ & O3'-C15'-C16'-O4' & $-54.7(15)$ \\
\hline C8-C9-C10-N2 & $0.3(7)$ & C15'-C16'-O4'-C17' & $166.9(11)$ \\
\hline C18-O1-C11-C12 & $-169.2(9)$ & C15'-C16'-O4'-Li1 & $46.8(14)$ \\
\hline Li1-O1-C11-C12 & $-42.6(11)$ & C16'-O4'-C17'-C18' & $-86.5(14)$ \\
\hline $\mathrm{O} 1-\mathrm{C} 11-\mathrm{C} 12-\mathrm{O} 2$ & $52.6(12)$ & Li1-O4'-C17'-C18' & $33.7(15)$ \\
\hline C11-C12-O2-C13 & $84.8(11)$ & C11'-O1'-C18'-C17' & $166.9(11)$ \\
\hline C11-C12-O2-Li1 & $-36.7(11)$ & Li1-O1'-C18'-C17' & $43.5(14)$ \\
\hline C12-O2-C13-C14 & $-166.5(8)$ & O4'-C17'-C18'-O1' & $-52.3(16)$ \\
\hline Li1-O2-C13-C14 & $-43.8(10)$ & C23-N3-C19-C20 & $-1.5(7)$ \\
\hline $\mathrm{O} 2-\mathrm{C} 13-\mathrm{C} 14-\mathrm{O} 3$ & $53.7(12)$ & Mo2-N3-C19-C20 & $176.6(3)$ \\
\hline
\end{tabular}




\begin{tabular}{|c|c|c|c|}
\hline N3-C19-C20-C21 & $0.0(7)$ & $\mathrm{O} 7-\mathrm{C} 33-\mathrm{C} 34-\mathrm{O} 8$ & $53.4(15)$ \\
\hline C19-C20-C21-C22 & $1.0(8)$ & C33-C34-O8-C35 & $-161.6(12)$ \\
\hline $\mathrm{C} 20-\mathrm{C} 21-\mathrm{C} 22-\mathrm{C} 23$ & $-0.6(8)$ & C33-C34-O8-Li2 & $-34.5(15)$ \\
\hline C19-N3-C23-C22 & $1.8(6)$ & $\mathrm{C} 34-\mathrm{O} 8-\mathrm{C} 35-\mathrm{C} 36$ & $97.2(17)$ \\
\hline Mo2-N3-C23-C22 & $-176.4(3)$ & Li2-O8-C35-C36 & $-30.2(16)$ \\
\hline C19-N3-C23-C24 & $-179.6(4)$ & C34-O8-C35-Li2 & $127.4(13)$ \\
\hline Mo2-N3-C23-C24 & $2.1(5)$ & $\mathrm{O} 8-\mathrm{C} 35-\mathrm{C} 36-\mathrm{O} 5$ & $43(2)$ \\
\hline $\mathrm{C} 21-\mathrm{C} 22-\mathrm{C} 23-\mathrm{N} 3$ & $-0.8(7)$ & Li2-C35-C36-O5 & $22.1(11)$ \\
\hline $\mathrm{C} 21-\mathrm{C} 22-\mathrm{C} 23-\mathrm{C} 24$ & $-179.3(4)$ & C29-O5-C36-C35 & $-161.0(13)$ \\
\hline C28-N4-C24-C25 & $3.6(6)$ & Li2-O5-C36-C35 & $-32.9(18)$ \\
\hline Mo2-N4-C24-C25 & $-171.0(3)$ & C36'-O5'-C29'-C30' & $-176(3)$ \\
\hline C28-N4-C24-C23 & $-177.0(4)$ & Li2'-O5'-C29'-C30' & $-50(4)$ \\
\hline Mo2-N4-C24-C23 & $8.4(5)$ & O5'-C29'-C30'-O6' & $49(5)$ \\
\hline N3-C23-C24-N4 & $-7.0(6)$ & C29'-C30'-O6'-C31' & $93(4)$ \\
\hline $\mathrm{C} 22-\mathrm{C} 23-\mathrm{C} 24-\mathrm{N} 4$ & $171.5(4)$ & C29'-C30'-O6'-Li2' & $-22(4)$ \\
\hline N3-C23-C24-C25 & $172.4(4)$ & C30'-O6'-C31'-C32' & $-156(3)$ \\
\hline C22-C23-C24-C25 & $-9.1(7)$ & Li2'-O6'-C31'-C32' & $-35(3)$ \\
\hline N4-C24-C25-C26 & $-0.8(7)$ & C30'-O6'-C31'-Li2' & $-121(2)$ \\
\hline $\mathrm{C} 23-\mathrm{C} 24-\mathrm{C} 25-\mathrm{C} 26$ & $179.8(4)$ & O6'-C31'-C32'-O7' & $53(5)$ \\
\hline $\mathrm{C} 24-\mathrm{C} 25-\mathrm{C} 26-\mathrm{C} 27$ & $-2.5(7)$ & Li2'-C31'-C32'-O7' & $28(4)$ \\
\hline $\mathrm{C} 25-\mathrm{C} 26-\mathrm{C} 27-\mathrm{C} 28$ & $3.0(7)$ & O6'-C31'-C32'-Li2' & $26(2)$ \\
\hline C24-N4-C28-C27 & $-3.0(7)$ & C31'-C32'-O7'-C33' & $78(7)$ \\
\hline Mo2-N4-C28-C27 & $171.1(3)$ & $\mathrm{Li2}-\mathrm{C} 32^{\prime}-\mathrm{O} 7^{\prime}-\mathrm{C} 33^{\prime}$ & $116(4)$ \\
\hline $\mathrm{C} 26-\mathrm{C} 27-\mathrm{C} 28-\mathrm{N} 4$ & $-0.3(7)$ & C31'-C32'-O7'-Li2' & $-38(5)$ \\
\hline $\mathrm{C} 36-\mathrm{O} 5-\mathrm{C} 29-\mathrm{C} 30$ & $90.0(14)$ & C32'-O7'-C33'-C34' & $-163(3)$ \\
\hline Li2-O5-C29-C30 & $-38.3(13)$ & Li2'-O7'-C33'-C34' & $-47(5)$ \\
\hline $\mathrm{O} 5-\mathrm{C} 29-\mathrm{C} 30-\mathrm{O} 6$ & $53.7(14)$ & O7'-C33'-C34'-O8' & $56(6)$ \\
\hline C29-C30-O6-C31 & $-160.2(12)$ & C33'-C34'-O 8'-C35' & $98(5)$ \\
\hline C29-C30-O6-Li2 & $-43.1(13)$ & C33'-C34'-O8'-Li2' & $-35(5)$ \\
\hline C30-O6-C31-C32 & $92.3(17)$ & C34'-O8'-C35'-C36' & $-159(4)$ \\
\hline Li2-O6-C31-C32 & $-29.2(15)$ & Li2'-O8'-C35'-C36' & $-25(6)$ \\
\hline O6-C31-C32-O7 & $46.2(18)$ & O8'-C35'-C36'-O5' & $36(7)$ \\
\hline C31-C32-O7-C33 & $-165.7(11)$ & C29'-O5'-C36'-C35' & $93(5)$ \\
\hline C31-C32-O7-Li2 & $-40.7(15)$ & Li2'-O5'-C36'-C35' & $-27(5)$ \\
\hline C32-O7-C33-C34 & $84.7(16)$ & C36"-O5"-C29"-C30" & $-94(3)$ \\
\hline Li2-O7-C33-C34 & $-44.7(13)$ & Li2"-O5"-C29"-C30" & $29(3)$ \\
\hline
\end{tabular}




\begin{tabular}{lclr} 
O5"-C29"-C30"-O6" & $-56(4)$ & Li2"-O7"-C33"-C34" & $40(3)$ \\
C29"-C30"-O6"-C31" & $163(3)$ & O7"-C33"-C34"-O8" & $-59(4)$ \\
C29"-C30"-O6"-Li2" & $56(4)$ & C33"-C34"-O8"-C35" & $166(2)$ \\
C30"-O6"-C31"-C32" & $-72(5)$ & C33"-C34"-O8"-Li2" & $47(3)$ \\
Li2"-O6"-C31"-C32" & $41(4)$ & C34"-O8"-C35"-C36" & $-81(4)$ \\
C30"-O6"-C31"-Li2" & $-112(3)$ & Li2"-O8"-C35"-C36" & $38(5)$ \\
O6"-C31"-C32"-O7" & $-62(4)$ & C34"-O8"-C35"-Li2" & $-119(2)$ \\
Li2"-C31"-C32"-O7" & $-32.7(18)$ & O8"-C35"-C36"-O5" & $-53(5)$ \\
C31"-C32"-O7"-C33" & $166(2)$ & Li2"-C35"-C36"-O5" & $-26(2)$ \\
C31"-C32"-O7"-Li2" & $47(2)$ & C29"-O5"-C36"-C35" & $161(3)$ \\
C32"-O7"-C33"-C34" & $-80(4)$ & & \\
Li2"-O5"-C36"-C35" & $36(3)$ & & \\
\hline
\end{tabular}

Symmetry transformations used to generate equivalent atoms:

$\# 1-\mathrm{x}+1, \mathrm{y}+1 / 2,-\mathrm{z}+1 / 2 \quad \# 2-\mathrm{x}+1, \mathrm{y}-1 / 2,-\mathrm{z}+1 / 2$ 
REFERENCE NUMBER: rhowe31

\title{
[Renumbered 12/19/2018]
}

\author{
CRYSTAL STRUCTURE REPORT
}

$\mathrm{C}_{26} \mathrm{H}_{44} \mathrm{Cl}_{4} \mathrm{Mo} \mathrm{N}_{3}$

or

$\left[\mathrm{NBu}_{4}\right]\left[\mathrm{MoCl}_{4}(\mathrm{bpy})\right]$

Report prepared for:

Prof. W. Eckenhoff

December 13, 2018

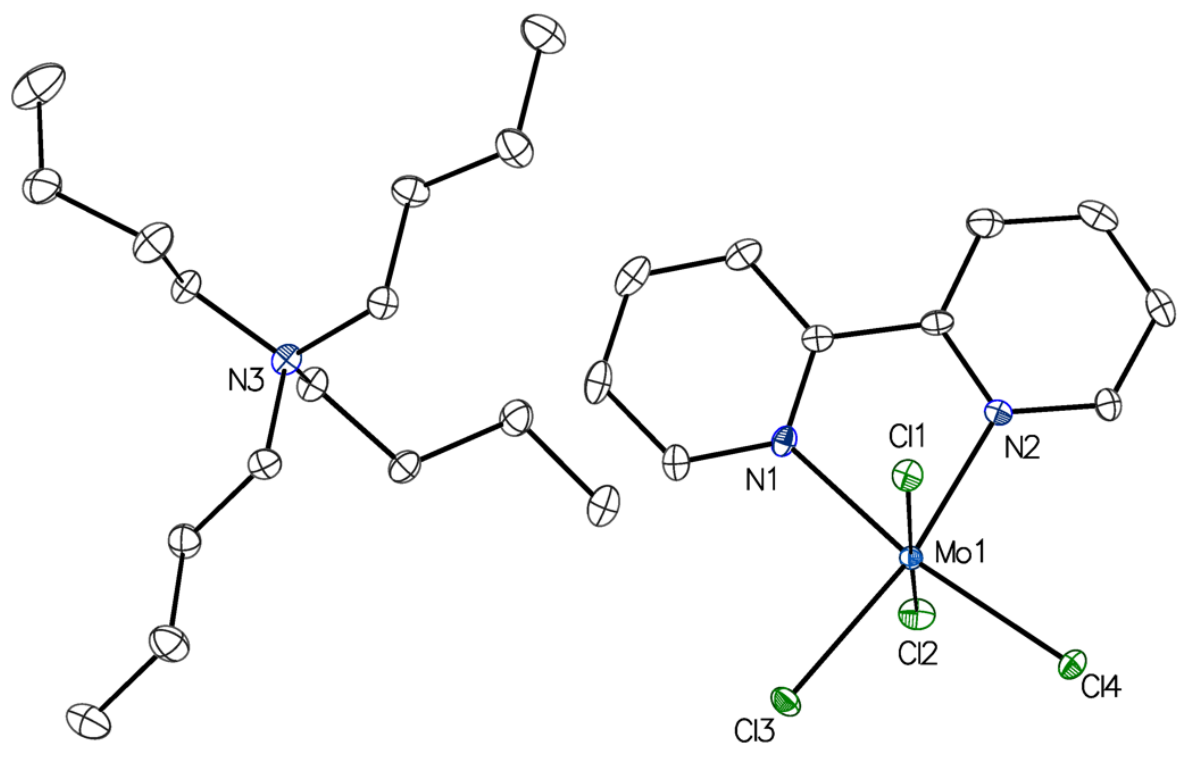

William W. Brennessel

X-ray Crystallographic Facility

Department of Chemistry, University of Rochester

120 Trustee Road

Rochester, NY 14627 


\section{Data collection}

A crystal $\left(0.201 \times 0.165 \times 0.099 \mathrm{~mm}^{3}\right)$ was placed onto a thin glass optical fiber or a nylon loop and mounted on a Rigaku XtaLab Synergy-S Dualflex diffractometer equipped with a HyPix-6000HE HPC area detector for data collection at $100.00(10) \mathrm{K}$. A preliminary set of cell constants and an orientation matrix were calculated from a small sampling of reflections. ${ }^{1}$ A short pre-experiment was run, from which an optimal data collection strategy was determined. The full data collection was carried out using a PhotonJet $(\mathrm{Cu})$ X-ray Source with frame times of 0.05 and 0.16 seconds and a detector distance of $31.2 \mathrm{~mm}$. Series of frames were collected in $0.50^{\circ}$ steps in $\omega$ at different $2 \theta, \kappa$, and $\phi$ settings. After the intensity data were corrected for absorption, the final cell constants were calculated from the xyz centroids of 22477 strong reflections from the actual data collection after integration. ${ }^{1}$ See Table 1 for additional crystal and refinement information.

\section{Structure solution and refinement}

The structure was solved using ShelXT ${ }^{2}$ and refined using ShelXL. ${ }^{3}$ The space group Pbca was determined based on systematic absences. Most or all non-hydrogen atoms were assigned from the solution. Full-matrix least squares / difference Fourier cycles were performed which located any remaining non-hydrogen atoms. All non-hydrogen atoms were refined with anisotropic displacement parameters. All hydrogen atoms were placed in ideal positions and refined as riding atoms with relative isotropic displacement parameters. The final full matrix least squares refinement converged to $R 1=0.0273\left(F^{2}, I>2 \sigma(I)\right)$ and $w R 2=0.0731\left(F^{2}\right.$, all data).

\section{Structure description}

The structure is the one suggested. The asymmetric unit contains one cation and one anion in general positions.

Structure manipulation and figure generation were performed using Olex2. ${ }^{4}$ Unless noted otherwise all structural diagrams containing thermal displacement ellipsoids are drawn at the $50 \%$ probability level.

Data collection, structure solution, and structure refinement were conducted at the X-ray Crystallographic Facility, B04 Hutchison Hall, Department of Chemistry, University of Rochester. The instrument was purchased with funding from NSF MRI program grant CHE-1725028. All publications arising from this report MUST either 1) include William W. Brennessel as a coauthor or 2) acknowledge William W. Brennessel and the X-ray Crystallographic Facility of the Department of Chemistry at the University of Rochester. 
1 CrysAlisPro, version 171.39.46; Rigaku Corporation: Oxford, UK, 2018.

2 Sheldrick, G. M. SHELXT, version 2018/2; Acta. Crystallogr. 2015, A71, 3-8.

3 Sheldrick, G. M. SHELXL, version 2018/3; Acta. Crystallogr. 2015, C71, 3-8.

4 Dolomanov, O. V.; Bourhis, L. J.; Gildea, R. J.; Howard, J. A. K.; Puschmann, H. Olex2, version 1.2-ac3; J. Appl. Cryst. 2009, 42, 339-341.

Some equations of interest:

$$
\begin{gathered}
R_{\mathrm{int}}=\Sigma\left|F_{\mathrm{o}}^{2}-<F_{\mathrm{o}}^{2}>\right| / \Sigma\left|F_{\mathrm{o}}{ }^{2}\right| \\
R 1=\Sigma|| F_{\mathrm{o}}|-| F_{\mathrm{c}}|/ \Sigma| F_{\mathrm{o}} \mid \\
w R 2=\left[\Sigma\left[w\left(F_{\mathrm{o}}{ }^{2} F_{\mathrm{c}}{ }^{2}\right)^{2}\right] / \Sigma\left[w\left(F_{\mathrm{o}}{ }^{2}\right)^{2}\right]\right]^{1 / 2} \\
\text { where } w=1 /\left[\sigma^{2}\left(F_{\mathrm{o}}{ }^{2}\right)+(a P)^{2}+b P\right] \text { and } \\
P=1 / 3 \max \left(0, F_{\mathrm{o}}{ }^{2}\right)+2 / 3 F_{\mathrm{c}}{ }^{2} \\
\mathrm{GOF}=S=\left[\Sigma\left[w\left(F_{\mathrm{o}}{ }^{2}-F_{\mathrm{c}}{ }^{2}\right)^{2}\right] /(m-n)\right]^{1 / 2}
\end{gathered}
$$

where $m=$ number of reflections and $n=$ number of parameters

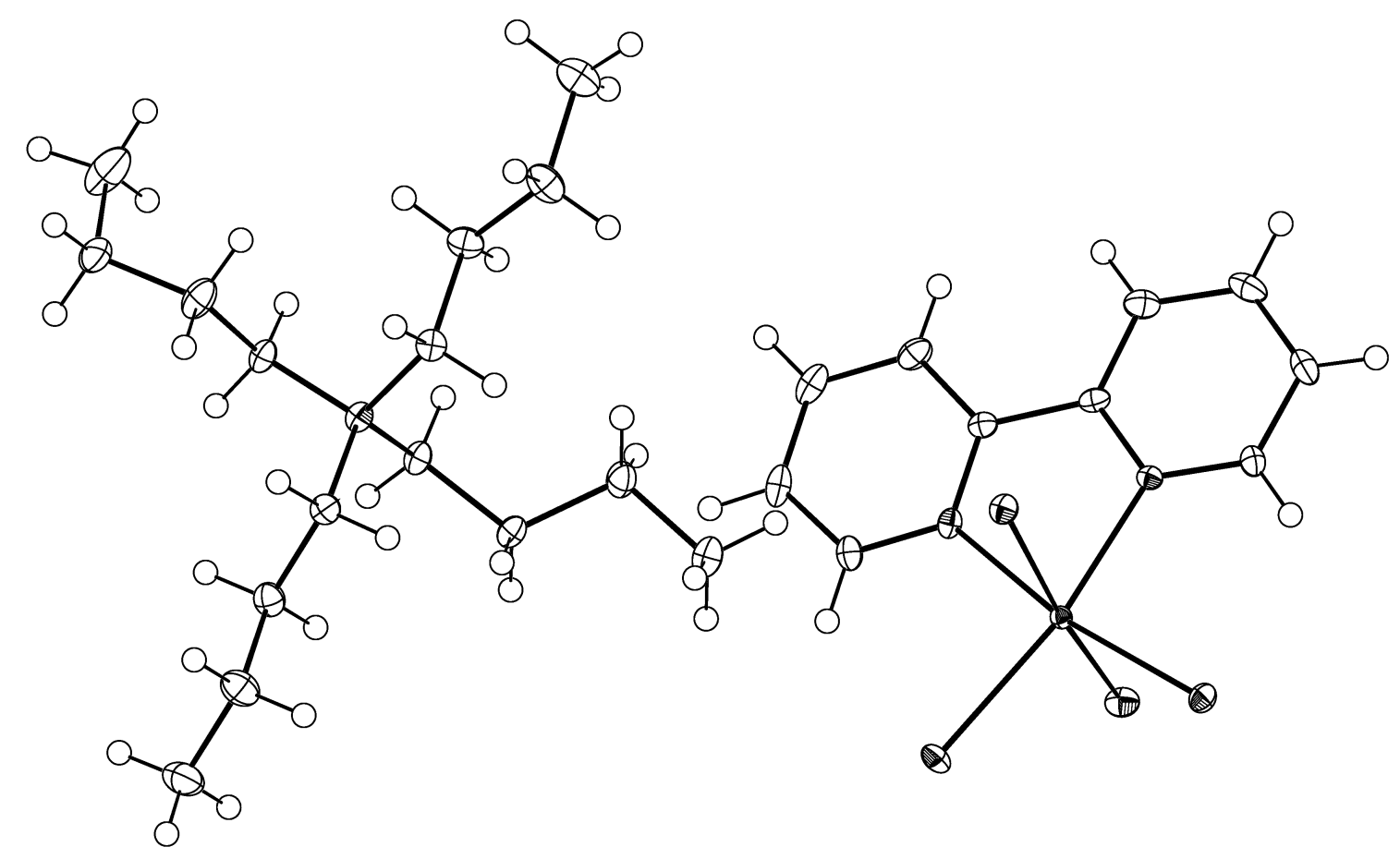



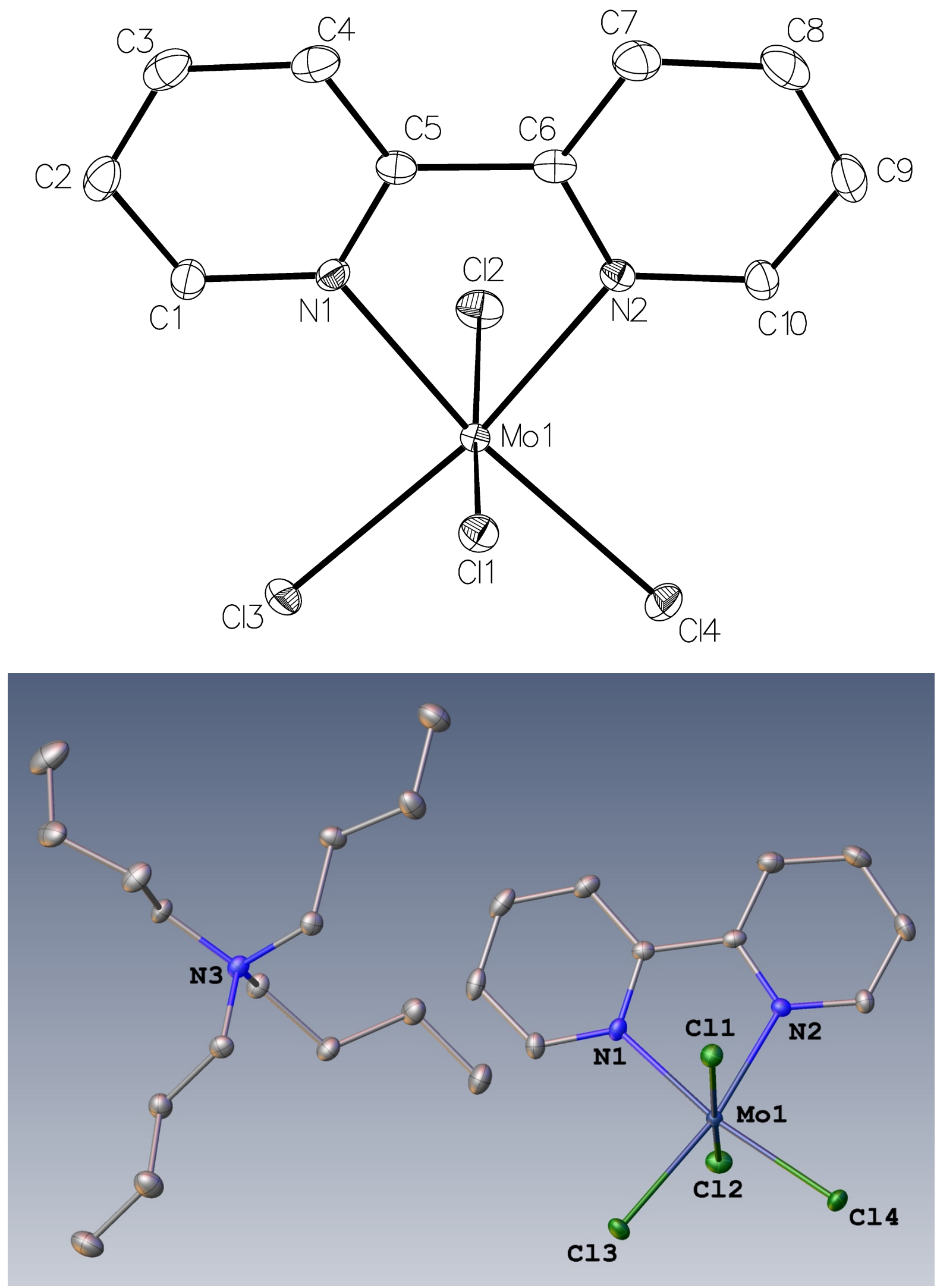
Table 1. Crystal data and structure refinement for rhowe31.

\begin{tabular}{|c|c|c|}
\hline Identification code & \multicolumn{2}{|l|}{ rhowe31 } \\
\hline Empirical formula & \multicolumn{2}{|l|}{ C26 H44 Cl4 Mo N3 } \\
\hline Formula weight & \multicolumn{2}{|l|}{636.38} \\
\hline Temperature & \multicolumn{2}{|l|}{$100.00(10) \mathrm{K}$} \\
\hline Wavelength & \multicolumn{2}{|l|}{$1.54184 \AA$} \\
\hline Crystal system & \multicolumn{2}{|l|}{ orthorhombic } \\
\hline Space group & \multicolumn{2}{|l|}{$P b c a$} \\
\hline \multirow[t]{3}{*}{ Unit cell dimensions } & $a=18.79080(10) \AA$ & $\alpha=90^{\circ}$ \\
\hline & $b=16.02020(10) \AA$ & $\beta=90^{\circ}$ \\
\hline & $c=19.74150(10) \AA$ & $\gamma=90^{\circ}$ \\
\hline Volume & \multicolumn{2}{|l|}{$5942.83(6) \AA^{3}$} \\
\hline$Z$ & \multicolumn{2}{|l|}{8} \\
\hline Density (calculated) & \multicolumn{2}{|l|}{$1.423 \mathrm{Mg} / \mathrm{m}^{3}$} \\
\hline Absorption coefficient & \multicolumn{2}{|l|}{$7.060 \mathrm{~mm}^{-1}$} \\
\hline$F(000)$ & \multicolumn{2}{|l|}{2648} \\
\hline Crystal color, morphology & \multicolumn{2}{|l|}{ red-black, block } \\
\hline Crystal size & \multicolumn{2}{|c|}{$0.201 \times 0.165 \times 0.099 \mathrm{~mm}^{3}$} \\
\hline Theta range for data collection & \multicolumn{2}{|l|}{4.262 to $77.783^{\circ}$} \\
\hline Index ranges & \multicolumn{2}{|c|}{$-20 \leq h \leq 23,-15 \leq k \leq 20,-24 \leq l \leq 24$} \\
\hline Reflections collected & \multicolumn{2}{|l|}{33790} \\
\hline Independent reflections & \multicolumn{2}{|l|}{$6246[R(\mathrm{int})=0.0406]$} \\
\hline Observed reflections & \multicolumn{2}{|l|}{5960} \\
\hline Completeness to theta $=74.504^{\circ}$ & \multicolumn{2}{|l|}{$99.7 \%$} \\
\hline Absorption correction & \multicolumn{2}{|l|}{ Multi-scan } \\
\hline Max. and min. transmission & \multicolumn{2}{|l|}{1.00000 and 0.73785} \\
\hline Refinement method & \multicolumn{2}{|c|}{ Full-matrix least-squares on $F^{2}$} \\
\hline Data / restraints / parameters & \multicolumn{2}{|l|}{$6246 / 0 / 311$} \\
\hline Goodness-of-fit on $F^{2}$ & \multicolumn{2}{|l|}{1.045} \\
\hline Final $R$ indices $[I>2 \operatorname{sigma}(I)]$ & \multicolumn{2}{|c|}{$R 1=0.0273, w R 2=0.0721$} \\
\hline$R$ indices (all data) & \multicolumn{2}{|c|}{$R 1=0.0286, w R 2=0.0731$} \\
\hline Largest diff. peak and hole & \multicolumn{2}{|l|}{0.485 and -0.957 e. $\AA^{-3}$} \\
\hline
\end{tabular}


Table 2. Atomic coordinates (x $\left.10^{4}\right)$ and equivalent isotropic displacement parameters $\left(\AA^{2} \times 10^{3}\right)$ for rhowe $31 . \mathrm{U}_{\mathrm{eq}}$ is defined as one third of the trace of the orthogonalized $\mathrm{U}_{\mathrm{ij}}$ tensor.

\begin{tabular}{|c|c|c|c|c|}
\hline & $\mathrm{x}$ & $\mathrm{y}$ & $\mathrm{z}$ & $\mathrm{U}_{\mathrm{eq}}$ \\
\hline Mo1 & $8594(1)$ & $5456(1)$ & 7192(1) & $8(1)$ \\
\hline $\mathrm{Cl1}$ & $9090(1)$ & $4827(1)$ & $6172(1)$ & $13(1)$ \\
\hline $\mathrm{Cl} 2$ & $8190(1)$ & $5934(1)$ & 8301(1) & $15(1)$ \\
\hline $\mathrm{Cl} 3$ & $7480(1)$ & $5807(1)$ & $6642(1)$ & $14(1)$ \\
\hline $\mathrm{Cl} 4$ & $9136(1)$ & $6798(1)$ & 6931(1) & $14(1)$ \\
\hline N1 & $8277(1)$ & $4195(1)$ & $7456(1)$ & 11(1) \\
\hline N2 & $9515(1)$ & 4933(1) & $7690(1)$ & $10(1)$ \\
\hline $\mathrm{C} 1$ & $7627(1)$ & $3868(1)$ & $7341(1)$ & $14(1)$ \\
\hline $\mathrm{C} 2$ & $7472(1)$ & $3034(1)$ & $7465(1)$ & $17(1)$ \\
\hline $\mathrm{C} 3$ & $8006(1)$ & $2521(1)$ & $7717(1)$ & $19(1)$ \\
\hline $\mathrm{C} 4$ & $8671(1)$ & $2858(1)$ & $7843(1)$ & $17(1)$ \\
\hline $\mathrm{C} 5$ & 8793(1) & $3698(1)$ & $7710(1)$ & $12(1)$ \\
\hline C6 & $9480(1)$ & 4111(1) & 7851(1) & $12(1)$ \\
\hline $\mathrm{C} 7$ & $10056(1)$ & $3698(1)$ & $8135(1)$ & $18(1)$ \\
\hline $\mathrm{C} 8$ & $10680(1)$ & $4136(1)$ & $8255(1)$ & $21(1)$ \\
\hline C9 & $10716(1)$ & $4978(1)$ & $8095(1)$ & $18(1)$ \\
\hline $\mathrm{C} 10$ & 10121(1) & $5354(1)$ & $7814(1)$ & $13(1)$ \\
\hline N3 & $6457(1)$ & $1326(1)$ & $4461(1)$ & $12(1)$ \\
\hline $\mathrm{C} 11$ & $6049(1)$ & $1863(1)$ & $3955(1)$ & $14(1)$ \\
\hline $\mathrm{C} 12$ & $5280(1)$ & $2038(1)$ & $4152(1)$ & $17(1)$ \\
\hline C13 & 4921(1) & $2586(1)$ & $3626(1)$ & $22(1)$ \\
\hline $\mathrm{C} 14$ & 4149(1) & $2762(2)$ & $3815(1)$ & $25(1)$ \\
\hline $\mathrm{C} 15$ & $6358(1)$ & $1654(1)$ & $5183(1)$ & $13(1)$ \\
\hline $\mathrm{C} 16$ & $6539(1)$ & $2579(1)$ & $5280(1)$ & $15(1)$ \\
\hline $\mathrm{C} 17$ & $7273(1)$ & 2761(1) & $5578(1)$ & 19(1) \\
\hline $\mathrm{C} 18$ & $7424(1)$ & $3695(1)$ & $5586(1)$ & $22(1)$ \\
\hline C19 & $7238(1)$ & $1352(1)$ & $4260(1)$ & $14(1)$ \\
\hline $\mathrm{C} 20$ & $7725(1)$ & $786(1)$ & $4669(1)$ & $17(1)$ \\
\hline $\mathrm{C} 21$ & $8497(1)$ & $929(1)$ & $4471(1)$ & $22(1)$ \\
\hline $\mathrm{C} 22$ & $9006(1)$ & $349(2)$ & $4835(1)$ & $29(1)$ \\
\hline $\mathrm{C} 23$ & $6177(1)$ & $435(1)$ & $4454(1)$ & $14(1)$ \\
\hline
\end{tabular}


C24

C25

C26
6294(1)

5895(1)

6138(2)
$-42(1)$

$-870(1)$

$-1484(2)$
3796(1)

3798(1)

4332(1)
22(1)

24(1)

36(1) 
Table 3. Bond lengths $[\AA]$ and angles $\left[{ }^{\circ}\right]$ for rhowe 31 .

\begin{tabular}{|c|c|c|c|}
\hline $\operatorname{Mo}(1)-\mathrm{Cl}(1)$ & $2.4373(4)$ & $\mathrm{C}(12)-\mathrm{H}(12 \mathrm{~A})$ & 0.9700 \\
\hline $\operatorname{Mo}(1)-\mathrm{Cl}(2)$ & $2.4404(4)$ & $\mathrm{C}(12)-\mathrm{H}(12 \mathrm{~B})$ & 0.9700 \\
\hline $\operatorname{Mo}(1)-\mathrm{Cl}(3)$ & $2.4241(4)$ & $\mathrm{C}(12)-\mathrm{C}(13)$ & $1.519(3)$ \\
\hline $\operatorname{Mo}(1)-\mathrm{Cl}(4)$ & $2.4339(4)$ & $\mathrm{C}(13)-\mathrm{H}(13 \mathrm{~A})$ & 0.9700 \\
\hline $\operatorname{Mo}(1)-\mathrm{N}(1)$ & $2.1696(15)$ & $\mathrm{C}(13)-\mathrm{H}(13 \mathrm{~B})$ & 0.9700 \\
\hline $\operatorname{Mo}(1)-\mathrm{N}(2)$ & $2.1596(15)$ & $\mathrm{C}(13)-\mathrm{C}(14)$ & $1.523(3)$ \\
\hline $\mathrm{N}(1)-\mathrm{C}(1)$ & $1.350(2)$ & $\mathrm{C}(14)-\mathrm{H}(14 \mathrm{~A})$ & 0.9600 \\
\hline $\mathrm{N}(1)-\mathrm{C}(5)$ & $1.351(2)$ & $\mathrm{C}(14)-\mathrm{H}(14 \mathrm{~B})$ & 0.9600 \\
\hline $\mathrm{N}(2)-\mathrm{C}(6)$ & $1.356(2)$ & $\mathrm{C}(14)-\mathrm{H}(14 \mathrm{C})$ & 0.9600 \\
\hline $\mathrm{N}(2)-\mathrm{C}(10)$ & $1.345(2)$ & $\mathrm{C}(15)-\mathrm{H}(15 \mathrm{~A})$ & 0.9700 \\
\hline $\mathrm{C}(1)-\mathrm{H}(1)$ & 0.9300 & $\mathrm{C}(15)-\mathrm{H}(15 \mathrm{~B})$ & 0.9700 \\
\hline $\mathrm{C}(1)-\mathrm{C}(2)$ & $1.389(3)$ & $\mathrm{C}(15)-\mathrm{C}(16)$ & $1.532(3)$ \\
\hline $\mathrm{C}(2)-\mathrm{H}(2)$ & 0.9300 & $\mathrm{C}(16)-\mathrm{H}(16 \mathrm{~A})$ & 0.9700 \\
\hline $\mathrm{C}(2)-\mathrm{C}(3)$ & $1.389(3)$ & $\mathrm{C}(16)-\mathrm{H}(16 \mathrm{~B})$ & 0.9700 \\
\hline $\mathrm{C}(3)-\mathrm{H}(3)$ & 0.9300 & $C(16)-C(17)$ & $1.529(3)$ \\
\hline $\mathrm{C}(3)-\mathrm{C}(4)$ & $1.382(3)$ & $\mathrm{C}(17)-\mathrm{H}(17 \mathrm{~A})$ & 0.9700 \\
\hline $\mathrm{C}(4)-\mathrm{H}(4)$ & 0.9300 & $\mathrm{C}(17)-\mathrm{H}(17 \mathrm{~B})$ & 0.9700 \\
\hline$C(4)-C(5)$ & $1.391(3)$ & $\mathrm{C}(17)-\mathrm{C}(18)$ & $1.522(3)$ \\
\hline $\mathrm{C}(5)-\mathrm{C}(6)$ & $1.476(3)$ & $\mathrm{C}(18)-\mathrm{H}(18 \mathrm{~A})$ & 0.9600 \\
\hline $\mathrm{C}(6)-\mathrm{C}(7)$ & $1.387(3)$ & $\mathrm{C}(18)-\mathrm{H}(18 \mathrm{~B})$ & 0.9600 \\
\hline $\mathrm{C}(7)-\mathrm{H}(7)$ & 0.9300 & $\mathrm{C}(18)-\mathrm{H}(18 \mathrm{C})$ & 0.9600 \\
\hline$C(7)-C(8)$ & $1.387(3)$ & $\mathrm{C}(19)-\mathrm{H}(19 \mathrm{~A})$ & 0.9700 \\
\hline $\mathrm{C}(8)-\mathrm{H}(8)$ & 0.9300 & $\mathrm{C}(19)-\mathrm{H}(19 \mathrm{~B})$ & 0.9700 \\
\hline $\mathrm{C}(8)-\mathrm{C}(9)$ & $1.386(3)$ & $\mathrm{C}(19)-\mathrm{C}(20)$ & $1.522(3)$ \\
\hline $\mathrm{C}(9)-\mathrm{H}(9)$ & 0.9300 & $\mathrm{C}(20)-\mathrm{H}(20 \mathrm{~A})$ & 0.9700 \\
\hline $\mathrm{C}(9)-\mathrm{C}(10)$ & $1.386(3)$ & $\mathrm{C}(20)-\mathrm{H}(20 \mathrm{~B})$ & 0.9700 \\
\hline $\mathrm{C}(10)-\mathrm{H}(10)$ & 0.9300 & $C(20)-C(21)$ & $1.521(3)$ \\
\hline $\mathrm{N}(3)-\mathrm{C}(11)$ & $1.525(2)$ & $\mathrm{C}(21)-\mathrm{H}(21 \mathrm{~A})$ & 0.9700 \\
\hline $\mathrm{N}(3)-\mathrm{C}(15)$ & $1.530(2)$ & $\mathrm{C}(21)-\mathrm{H}(21 \mathrm{~B})$ & 0.9700 \\
\hline $\mathrm{N}(3)-\mathrm{C}(19)$ & $1.521(2)$ & $C(21)-C(22)$ & $1.516(3)$ \\
\hline $\mathrm{N}(3)-\mathrm{C}(23)$ & $1.522(2)$ & $\mathrm{C}(22)-\mathrm{H}(22 \mathrm{~A})$ & 0.9600 \\
\hline $\mathrm{C}(11)-\mathrm{H}(11 \mathrm{~A})$ & 0.9700 & $\mathrm{C}(22)-\mathrm{H}(22 \mathrm{~B})$ & 0.9600 \\
\hline $\mathrm{C}(11)-\mathrm{H}(11 \mathrm{~B})$ & 0.9700 & $\mathrm{C}(22)-\mathrm{H}(22 \mathrm{C})$ & 0.9600 \\
\hline $\mathrm{C}(11)-\mathrm{C}(12)$ & $1.521(3)$ & $\mathrm{C}(23)-\mathrm{H}(23 \mathrm{~A})$ & 0.9700 \\
\hline
\end{tabular}




\begin{tabular}{|c|c|c|c|}
\hline $\mathrm{C}(23)-\mathrm{H}(23 \mathrm{~B})$ & 0.9700 & $\mathrm{C}(1)-\mathrm{C}(2)-\mathrm{C}(3)$ & $118.74(17)$ \\
\hline$C(23)-C(24)$ & $1.522(3)$ & $\mathrm{C}(3)-\mathrm{C}(2)-\mathrm{H}(2)$ & 120.6 \\
\hline $\mathrm{C}(24)-\mathrm{H}(24 \mathrm{~A})$ & 0.9700 & $\mathrm{C}(2)-\mathrm{C}(3)-\mathrm{H}(3)$ & 120.5 \\
\hline $\mathrm{C}(24)-\mathrm{H}(24 \mathrm{~B})$ & 0.9700 & $\mathrm{C}(4)-\mathrm{C}(3)-\mathrm{C}(2)$ & $119.06(18)$ \\
\hline$C(24)-C(25)$ & $1.524(3)$ & $\mathrm{C}(4)-\mathrm{C}(3)-\mathrm{H}(3)$ & 120.5 \\
\hline $\mathrm{C}(25)-\mathrm{H}(25 \mathrm{~A})$ & 0.9700 & $\mathrm{C}(3)-\mathrm{C}(4)-\mathrm{H}(4)$ & 120.2 \\
\hline $\mathrm{C}(25)-\mathrm{H}(25 \mathrm{~B})$ & 0.9700 & $C(3)-C(4)-C(5)$ & $119.58(19)$ \\
\hline$C(25)-C(26)$ & $1.512(3)$ & $\mathrm{C}(5)-\mathrm{C}(4)-\mathrm{H}(4)$ & 120.2 \\
\hline $\mathrm{C}(26)-\mathrm{H}(26 \mathrm{~A})$ & 0.9600 & $\mathrm{~N}(1)-\mathrm{C}(5)-\mathrm{C}(4)$ & $121.45(17)$ \\
\hline $\mathrm{C}(26)-\mathrm{H}(26 \mathrm{~B})$ & 0.9600 & $\mathrm{~N}(1)-\mathrm{C}(5)-\mathrm{C}(6)$ & $115.63(16)$ \\
\hline $\mathrm{C}(26)-\mathrm{H}(26 \mathrm{C})$ & 0.9600 & $C(4)-C(5)-C(6)$ & $122.90(17)$ \\
\hline $\mathrm{Cl}(1)-\mathrm{Mo}(1)-\mathrm{Cl}(2)$ & $171.920(15)$ & $\mathrm{N}(2)-\mathrm{C}(6)-\mathrm{C}(5)$ & $115.72(15)$ \\
\hline $\mathrm{Cl}(3)-\mathrm{Mo}(1)-\mathrm{Cl}(1)$ & $93.179(14)$ & $\mathrm{N}(2)-\mathrm{C}(6)-\mathrm{C}(7)$ & $121.27(17)$ \\
\hline $\mathrm{Cl}(3)-\mathrm{Mo}(1)-\mathrm{Cl}(2)$ & $93.442(15)$ & $C(7)-C(6)-C(5)$ & $123.00(17)$ \\
\hline $\mathrm{Cl}(3)-\mathrm{Mo}(1)-\mathrm{Cl}(4)$ & $93.539(15)$ & $\mathrm{C}(6)-\mathrm{C}(7)-\mathrm{H}(7)$ & 120.4 \\
\hline $\mathrm{Cl}(4)-\mathrm{Mo}(1)-\mathrm{Cl}(1)$ & $91.737(15)$ & $\mathrm{C}(8)-\mathrm{C}(7)-\mathrm{C}(6)$ & $119.19(18)$ \\
\hline $\mathrm{Cl}(4)-\mathrm{Mo}(1)-\mathrm{Cl}(2)$ & $92.471(15)$ & $\mathrm{C}(8)-\mathrm{C}(7)-\mathrm{H}(7)$ & 120.4 \\
\hline $\mathrm{N}(1)-\mathrm{Mo}(1)-\mathrm{Cl}(1)$ & $85.32(4)$ & $\mathrm{C}(7)-\mathrm{C}(8)-\mathrm{H}(8)$ & 120.2 \\
\hline $\mathrm{N}(1)-\mathrm{Mo}(1)-\mathrm{Cl}(2)$ & $89.50(4)$ & $\mathrm{C}(9)-\mathrm{C}(8)-\mathrm{C}(7)$ & $119.62(18)$ \\
\hline $\mathrm{N}(1)-\mathrm{Mo}(1)-\mathrm{Cl}(3)$ & $94.95(4)$ & $\mathrm{C}(9)-\mathrm{C}(8)-\mathrm{H}(8)$ & 120.2 \\
\hline $\mathrm{N}(1)-\mathrm{Mo}(1)-\mathrm{Cl}(4)$ & $171.15(4)$ & $\mathrm{C}(8)-\mathrm{C}(9)-\mathrm{H}(9)$ & 120.8 \\
\hline $\mathrm{N}(2)-\mathrm{Mo}(1)-\mathrm{Cl}(1)$ & $84.80(4)$ & $\mathrm{C}(8)-\mathrm{C}(9)-\mathrm{C}(10)$ & $118.36(18)$ \\
\hline $\mathrm{N}(2)-\mathrm{Mo}(1)-\mathrm{Cl}(2)$ & $87.90(4)$ & $\mathrm{C}(10)-\mathrm{C}(9)-\mathrm{H}(9)$ & 120.8 \\
\hline $\mathrm{N}(2)-\mathrm{Mo}(1)-\mathrm{Cl}(3)$ & $170.32(4)$ & $\mathrm{N}(2)-\mathrm{C}(10)-\mathrm{C}(9)$ & $122.49(18)$ \\
\hline $\mathrm{N}(2)-\mathrm{Mo}(1)-\mathrm{Cl}(4)$ & $95.98(4)$ & $\mathrm{N}(2)-\mathrm{C}(10)-\mathrm{H}(10)$ & 118.8 \\
\hline $\mathrm{N}(2)-\mathrm{Mo}(1)-\mathrm{N}(1)$ & $75.47(6)$ & $\mathrm{C}(9)-\mathrm{C}(10)-\mathrm{H}(10)$ & 118.8 \\
\hline $\mathrm{C}(1)-\mathrm{N}(1)-\mathrm{Mo}(1)$ & $124.76(12)$ & $\mathrm{C}(11)-\mathrm{N}(3)-\mathrm{C}(15)$ & $110.86(14)$ \\
\hline$C(1)-N(1)-C(5)$ & $118.88(16)$ & $\mathrm{C}(19)-\mathrm{N}(3)-\mathrm{C}(11)$ & $107.37(13)$ \\
\hline $\mathrm{C}(5)-\mathrm{N}(1)-\mathrm{Mo}(1)$ & $116.19(12)$ & $\mathrm{C}(19)-\mathrm{N}(3)-\mathrm{C}(15)$ & $110.52(13)$ \\
\hline $\mathrm{C}(6)-\mathrm{N}(2)-\mathrm{Mo}(1)$ & $116.36(12)$ & $\mathrm{C}(19)-\mathrm{N}(3)-\mathrm{C}(23)$ & $110.84(14)$ \\
\hline $\mathrm{C}(10)-\mathrm{N}(2)-\mathrm{Mo}(1)$ & $124.49(12)$ & $\mathrm{C}(23)-\mathrm{N}(3)-\mathrm{C}(11)$ & $110.48(14)$ \\
\hline $\mathrm{C}(10)-\mathrm{N}(2)-\mathrm{C}(6)$ & $119.05(15)$ & $\mathrm{C}(23)-\mathrm{N}(3)-\mathrm{C}(15)$ & $106.80(14)$ \\
\hline $\mathrm{N}(1)-\mathrm{C}(1)-\mathrm{H}(1)$ & 118.9 & N(3)-C(11)-H(11A) & 108.7 \\
\hline $\mathrm{N}(1)-\mathrm{C}(1)-\mathrm{C}(2)$ & $122.29(18)$ & $\mathrm{N}(3)-\mathrm{C}(11)-\mathrm{H}(11 \mathrm{~B})$ & 108.7 \\
\hline $\mathrm{C}(2)-\mathrm{C}(1)-\mathrm{H}(1)$ & 118.9 & $\mathrm{H}(11 \mathrm{~A})-\mathrm{C}(11)-\mathrm{H}(11 \mathrm{~B})$ & 107.6 \\
\hline $\mathrm{C}(1)-\mathrm{C}(2)-\mathrm{H}(2)$ & 120.6 & $\mathrm{C}(12)-\mathrm{C}(11)-\mathrm{N}(3)$ & $114.42(15)$ \\
\hline
\end{tabular}




\begin{tabular}{|c|c|c|c|}
\hline $\mathrm{C}(12)-\mathrm{C}(11)-\mathrm{H}(11 \mathrm{~A})$ & 108.7 & $\mathrm{C}(18)-\mathrm{C}(17)-\mathrm{H}(17 \mathrm{~A})$ & 109.4 \\
\hline $\mathrm{C}(12)-\mathrm{C}(11)-\mathrm{H}(11 \mathrm{~B})$ & 108.7 & $\mathrm{C}(18)-\mathrm{C}(17)-\mathrm{H}(17 \mathrm{~B})$ & 109.4 \\
\hline $\mathrm{C}(11)-\mathrm{C}(12)-\mathrm{H}(12 \mathrm{~A})$ & 109.5 & $\mathrm{C}(17)-\mathrm{C}(18)-\mathrm{H}(18 \mathrm{~A})$ & 109.5 \\
\hline $\mathrm{C}(11)-\mathrm{C}(12)-\mathrm{H}(12 \mathrm{~B})$ & 109.5 & $\mathrm{C}(17)-\mathrm{C}(18)-\mathrm{H}(18 \mathrm{~B})$ & 109.5 \\
\hline $\mathrm{H}(12 \mathrm{~A})-\mathrm{C}(12)-\mathrm{H}(12 \mathrm{~B})$ & 108.1 & $\mathrm{C}(17)-\mathrm{C}(18)-\mathrm{H}(18 \mathrm{C})$ & 109.5 \\
\hline $\mathrm{C}(13)-\mathrm{C}(12)-\mathrm{C}(11)$ & $110.72(16)$ & $\mathrm{H}(18 \mathrm{~A})-\mathrm{C}(18)-\mathrm{H}(18 \mathrm{~B})$ & 109.5 \\
\hline $\mathrm{C}(13)-\mathrm{C}(12)-\mathrm{H}(12 \mathrm{~A})$ & 109.5 & $\mathrm{H}(18 \mathrm{~A})-\mathrm{C}(18)-\mathrm{H}(18 \mathrm{C})$ & 109.5 \\
\hline $\mathrm{C}(13)-\mathrm{C}(12)-\mathrm{H}(12 \mathrm{~B})$ & 109.5 & $\mathrm{H}(18 \mathrm{~B})-\mathrm{C}(18)-\mathrm{H}(18 \mathrm{C})$ & 109.5 \\
\hline $\mathrm{C}(12)-\mathrm{C}(13)-\mathrm{H}(13 \mathrm{~A})$ & 109.4 & $\mathrm{~N}(3)-\mathrm{C}(19)-\mathrm{H}(19 \mathrm{~A})$ & 108.5 \\
\hline $\mathrm{C}(12)-\mathrm{C}(13)-\mathrm{H}(13 \mathrm{~B})$ & 109.4 & N(3)-C(19)-H(19B) & 108.5 \\
\hline $\mathrm{C}(12)-\mathrm{C}(13)-\mathrm{C}(14)$ & $111.26(17)$ & $\mathrm{N}(3)-\mathrm{C}(19)-\mathrm{C}(20)$ & $115.19(15)$ \\
\hline $\mathrm{H}(13 \mathrm{~A})-\mathrm{C}(13)-\mathrm{H}(13 \mathrm{~B})$ & 108.0 & H(19A)-C(19)-H(19B) & 107.5 \\
\hline $\mathrm{C}(14)-\mathrm{C}(13)-\mathrm{H}(13 \mathrm{~A})$ & 109.4 & $\mathrm{C}(20)-\mathrm{C}(19)-\mathrm{H}(19 \mathrm{~A})$ & 108.5 \\
\hline $\mathrm{C}(14)-\mathrm{C}(13)-\mathrm{H}(13 \mathrm{~B})$ & 109.4 & $\mathrm{C}(20)-\mathrm{C}(19)-\mathrm{H}(19 \mathrm{~B})$ & 108.5 \\
\hline $\mathrm{C}(13)-\mathrm{C}(14)-\mathrm{H}(14 \mathrm{~A})$ & 109.5 & $\mathrm{C}(19)-\mathrm{C}(20)-\mathrm{H}(20 \mathrm{~A})$ & 109.6 \\
\hline $\mathrm{C}(13)-\mathrm{C}(14)-\mathrm{H}(14 \mathrm{~B})$ & 109.5 & $\mathrm{C}(19)-\mathrm{C}(20)-\mathrm{H}(20 \mathrm{~B})$ & 109.6 \\
\hline $\mathrm{C}(13)-\mathrm{C}(14)-\mathrm{H}(14 \mathrm{C})$ & 109.5 & $\mathrm{H}(20 \mathrm{~A})-\mathrm{C}(20)-\mathrm{H}(20 \mathrm{~B})$ & 108.1 \\
\hline $\mathrm{H}(14 \mathrm{~A})-\mathrm{C}(14)-\mathrm{H}(14 \mathrm{~B})$ & 109.5 & $\mathrm{C}(21)-\mathrm{C}(20)-\mathrm{C}(19)$ & $110.31(16)$ \\
\hline $\mathrm{H}(14 \mathrm{~A})-\mathrm{C}(14)-\mathrm{H}(14 \mathrm{C})$ & 109.5 & $C(21)-C(20)-H(20 A)$ & 109.6 \\
\hline $\mathrm{H}(14 \mathrm{~B})-\mathrm{C}(14)-\mathrm{H}(14 \mathrm{C})$ & 109.5 & $\mathrm{C}(21)-\mathrm{C}(20)-\mathrm{H}(20 \mathrm{~B})$ & 109.6 \\
\hline $\mathrm{N}(3)-\mathrm{C}(15)-\mathrm{H}(15 \mathrm{~A})$ & 108.5 & $\mathrm{C}(20)-\mathrm{C}(21)-\mathrm{H}(21 \mathrm{~A})$ & 109.0 \\
\hline $\mathrm{N}(3)-\mathrm{C}(15)-\mathrm{H}(15 \mathrm{~B})$ & 108.5 & $\mathrm{C}(20)-\mathrm{C}(21)-\mathrm{H}(21 \mathrm{~B})$ & 109.0 \\
\hline $\mathrm{N}(3)-\mathrm{C}(15)-\mathrm{C}(16)$ & $114.89(15)$ & $\mathrm{H}(21 \mathrm{~A})-\mathrm{C}(21)-\mathrm{H}(21 \mathrm{~B})$ & 107.8 \\
\hline $\mathrm{H}(15 \mathrm{~A})-\mathrm{C}(15)-\mathrm{H}(15 \mathrm{~B})$ & 107.5 & $\mathrm{C}(22)-\mathrm{C}(21)-\mathrm{C}(20)$ & $112.76(18)$ \\
\hline $\mathrm{C}(16)-\mathrm{C}(15)-\mathrm{H}(15 \mathrm{~A})$ & 108.5 & $\mathrm{C}(22)-\mathrm{C}(21)-\mathrm{H}(21 \mathrm{~A})$ & 109.0 \\
\hline $\mathrm{C}(16)-\mathrm{C}(15)-\mathrm{H}(15 \mathrm{~B})$ & 108.5 & $\mathrm{C}(22)-\mathrm{C}(21)-\mathrm{H}(21 \mathrm{~B})$ & 109.0 \\
\hline $\mathrm{C}(15)-\mathrm{C}(16)-\mathrm{H}(16 \mathrm{~A})$ & 108.4 & $\mathrm{C}(21)-\mathrm{C}(22)-\mathrm{H}(22 \mathrm{~A})$ & 109.5 \\
\hline $\mathrm{C}(15)-\mathrm{C}(16)-\mathrm{H}(16 \mathrm{~B})$ & 108.4 & $\mathrm{C}(21)-\mathrm{C}(22)-\mathrm{H}(22 \mathrm{~B})$ & 109.5 \\
\hline $\mathrm{H}(16 \mathrm{~A})-\mathrm{C}(16)-\mathrm{H}(16 \mathrm{~B})$ & 107.4 & $\mathrm{C}(21)-\mathrm{C}(22)-\mathrm{H}(22 \mathrm{C})$ & 109.5 \\
\hline$C(17)-C(16)-C(15)$ & $115.61(16)$ & $\mathrm{H}(22 \mathrm{~A})-\mathrm{C}(22)-\mathrm{H}(22 \mathrm{~B})$ & 109.5 \\
\hline $\mathrm{C}(17)-\mathrm{C}(16)-\mathrm{H}(16 \mathrm{~A})$ & 108.4 & $\mathrm{H}(22 \mathrm{~A})-\mathrm{C}(22)-\mathrm{H}(22 \mathrm{C})$ & 109.5 \\
\hline $\mathrm{C}(17)-\mathrm{C}(16)-\mathrm{H}(16 \mathrm{~B})$ & 108.4 & $\mathrm{H}(22 \mathrm{~B})-\mathrm{C}(22)-\mathrm{H}(22 \mathrm{C})$ & 109.5 \\
\hline $\mathrm{C}(16)-\mathrm{C}(17)-\mathrm{H}(17 \mathrm{~A})$ & 109.4 & $\mathrm{~N}(3)-\mathrm{C}(23)-\mathrm{H}(23 \mathrm{~A})$ & 108.4 \\
\hline $\mathrm{C}(16)-\mathrm{C}(17)-\mathrm{H}(17 \mathrm{~B})$ & 109.4 & $\mathrm{~N}(3)-\mathrm{C}(23)-\mathrm{H}(23 \mathrm{~B})$ & 108.4 \\
\hline H(17A)-C(17)-H(17B) & 108.0 & $\mathrm{H}(23 \mathrm{~A})-\mathrm{C}(23)-\mathrm{H}(23 \mathrm{~B})$ & 107.5 \\
\hline$C(18)-C(17)-C(16)$ & $111.07(16)$ & $\mathrm{C}(24)-\mathrm{C}(23)-\mathrm{N}(3)$ & $115.47(15)$ \\
\hline
\end{tabular}




$\begin{array}{llll}\mathrm{C}(24)-\mathrm{C}(23)-\mathrm{H}(23 \mathrm{~A}) & 108.4 & \mathrm{H}(25 \mathrm{~A})-\mathrm{C}(25)-\mathrm{H}(25 \mathrm{~B}) & 107.6 \\ \mathrm{C}(24)-\mathrm{C}(23)-\mathrm{H}(23 \mathrm{~B}) & 108.4 & \mathrm{C}(26)-\mathrm{C}(25)-\mathrm{C}(24) & 114.74(19) \\ \mathrm{C}(23)-\mathrm{C}(24)-\mathrm{H}(24 \mathrm{~A}) & 109.3 & \mathrm{C}(26)-\mathrm{C}(25)-\mathrm{H}(25 \mathrm{~A}) & 108.6 \\ \mathrm{C}(23)-\mathrm{C}(24)-\mathrm{H}(24 \mathrm{~B}) & 109.3 & \mathrm{C}(26)-\mathrm{C}(25)-\mathrm{H}(25 \mathrm{~B}) & 108.6 \\ \mathrm{C}(23)-\mathrm{C}(24)-\mathrm{C}(25) & 111.39(17) & \mathrm{C}(25)-\mathrm{C}(26)-\mathrm{H}(26 \mathrm{~A}) & 109.5 \\ \mathrm{H}(24 \mathrm{~A})-\mathrm{C}(24)-\mathrm{H}(24 \mathrm{~B}) & 108.0 & \mathrm{C}(25)-\mathrm{C}(26)-\mathrm{H}(26 \mathrm{~B}) & 109.5 \\ \mathrm{C}(25)-\mathrm{C}(24)-\mathrm{H}(24 \mathrm{~A}) & 109.3 & \mathrm{C}(25)-\mathrm{C}(26)-\mathrm{H}(26 \mathrm{C}) & 109.5 \\ \mathrm{C}(25)-\mathrm{C}(24)-\mathrm{H}(24 \mathrm{~B}) & 109.3 & \mathrm{H}(26 \mathrm{~A})-\mathrm{C}(26)-\mathrm{H}(26 \mathrm{~B}) & 109.5 \\ \mathrm{C}(24)-\mathrm{C}(25)-\mathrm{H}(25 \mathrm{~A}) & 108.6 & \mathrm{H}(26 \mathrm{~A})-\mathrm{C}(26)-\mathrm{H}(26 \mathrm{C}) & 109.5 \\ \mathrm{C}(24)-\mathrm{C}(25)-\mathrm{H}(25 \mathrm{~B}) & 108.6 & \mathrm{H}(26 \mathrm{~B})-\mathrm{C}(26)-\mathrm{H}(26 \mathrm{C}) & 109.5\end{array}$


Table 4. Anisotropic displacement parameters $\left(\AA^{2} \times 10^{3}\right)$ for rhowe 31 . The anisotropic displacement factor exponent takes the form: $-2 \pi^{2}\left[h^{2} a^{* 2} U_{11}+\ldots+2 h k a^{*} b^{*} U_{12}\right]$

\begin{tabular}{|c|c|c|c|c|c|c|}
\hline & $\mathrm{U}_{11}$ & $\mathrm{U}_{22}$ & $\mathrm{U}_{33}$ & $\mathrm{U}_{23}$ & $\mathrm{U}_{13}$ & $\mathrm{U}_{12}$ \\
\hline Mo1 & $8(1)$ & $8(1)$ & $8(1)$ & $1(1)$ & $-1(1)$ & $0(1)$ \\
\hline $\mathrm{Cl1}$ & $15(1)$ & $14(1)$ & $10(1)$ & $-1(1)$ & $1(1)$ & $0(1)$ \\
\hline $\mathrm{Cl} 2$ & $18(1)$ & $17(1)$ & $11(1)$ & $-1(1)$ & $2(1)$ & $3(1)$ \\
\hline $\mathrm{Cl} 3$ & $10(1)$ & $17(1)$ & $15(1)$ & $3(1)$ & $-2(1)$ & $1(1)$ \\
\hline $\mathrm{Cl} 4$ & $15(1)$ & $10(1)$ & $17(1)$ & $2(1)$ & $0(1)$ & $-2(1)$ \\
\hline N1 & $13(1)$ & $11(1)$ & $9(1)$ & $1(1)$ & $0(1)$ & $-3(1)$ \\
\hline N2 & $10(1)$ & $11(1)$ & $8(1)$ & $0(1)$ & $0(1)$ & $1(1)$ \\
\hline $\mathrm{C} 1$ & $13(1)$ & $17(1)$ & $12(1)$ & $0(1)$ & $0(1)$ & $-4(1)$ \\
\hline $\mathrm{C} 2$ & 21(1) & $19(1)$ & $12(1)$ & $-3(1)$ & $2(1)$ & $-10(1)$ \\
\hline $\mathrm{C} 3$ & $30(1)$ & $13(1)$ & $13(1)$ & $-1(1)$ & $2(1)$ & $-7(1)$ \\
\hline $\mathrm{C} 4$ & $24(1)$ & $12(1)$ & $14(1)$ & $2(1)$ & $1(1)$ & $0(1)$ \\
\hline $\mathrm{C} 5$ & $14(1)$ & $12(1)$ & $9(1)$ & $0(1)$ & $1(1)$ & $2(1)$ \\
\hline C6 & $15(1)$ & $11(1)$ & $10(1)$ & $0(1)$ & $1(1)$ & $3(1)$ \\
\hline C7 & $19(1)$ & $16(1)$ & $19(1)$ & $3(1)$ & $0(1)$ & $4(1)$ \\
\hline $\mathrm{C} 8$ & $16(1)$ & $25(1)$ & $22(1)$ & $2(1)$ & $-4(1)$ & $7(1)$ \\
\hline C9 & $11(1)$ & $24(1)$ & $18(1)$ & $-1(1)$ & $-2(1)$ & $-2(1)$ \\
\hline $\mathrm{C} 10$ & $12(1)$ & $15(1)$ & $12(1)$ & $-1(1)$ & $-1(1)$ & $-2(1)$ \\
\hline N3 & $16(1)$ & $12(1)$ & $7(1)$ & $0(1)$ & $1(1)$ & $-1(1)$ \\
\hline $\mathrm{C} 11$ & $18(1)$ & $15(1)$ & $9(1)$ & $1(1)$ & $-2(1)$ & $1(1)$ \\
\hline $\mathrm{C} 12$ & $17(1)$ & $18(1)$ & $16(1)$ & $2(1)$ & $-1(1)$ & $-1(1)$ \\
\hline $\mathrm{C} 13$ & $21(1)$ & $26(1)$ & $20(1)$ & $5(1)$ & $-2(1)$ & $2(1)$ \\
\hline $\mathrm{C} 14$ & $21(1)$ & $29(1)$ & $26(1)$ & $0(1)$ & $-4(1)$ & $8(1)$ \\
\hline $\mathrm{C} 15$ & $18(1)$ & $14(1)$ & $7(1)$ & $-2(1)$ & $1(1)$ & $-2(1)$ \\
\hline C16 & $19(1)$ & $13(1)$ & $14(1)$ & $-2(1)$ & $0(1)$ & $-1(1)$ \\
\hline $\mathrm{C} 17$ & $22(1)$ & $18(1)$ & $17(1)$ & $-1(1)$ & $-4(1)$ & $-3(1)$ \\
\hline C18 & $24(1)$ & $18(1)$ & $23(1)$ & $-2(1)$ & $0(1)$ & $-6(1)$ \\
\hline C19 & $15(1)$ & $16(1)$ & $11(1)$ & $1(1)$ & $2(1)$ & $-1(1)$ \\
\hline $\mathrm{C} 20$ & $18(1)$ & $19(1)$ & $14(1)$ & $3(1)$ & $0(1)$ & $3(1)$ \\
\hline $\mathrm{C} 21$ & $17(1)$ & $29(1)$ & $18(1)$ & $7(1)$ & $-1(1)$ & $0(1)$ \\
\hline $\mathrm{C} 22$ & $23(1)$ & $33(1)$ & $30(1)$ & $4(1)$ & $-5(1)$ & $5(1)$ \\
\hline $\mathrm{C} 23$ & $18(1)$ & $11(1)$ & $13(1)$ & $0(1)$ & $0(1)$ & $-3(1)$ \\
\hline
\end{tabular}




\begin{tabular}{lllllll}
$\mathrm{C} 24$ & $33(1)$ & $18(1)$ & $14(1)$ & $-3(1)$ & $0(1)$ & $-3(1)$ \\
$\mathrm{C} 25$ & $29(1)$ & $18(1)$ & $27(1)$ & $-8(1)$ & $-9(1)$ & $3(1)$ \\
$\mathrm{C} 26$ & $61(2)$ & $19(1)$ & $26(1)$ & $-2(1)$ & $-6(1)$ & $-2(1)$ \\
\hline
\end{tabular}


Table 5. Hydrogen coordinates $\left(\times 10^{4}\right)$ and isotropic displacement parameters $\left(\AA^{2} \times 10^{3}\right)$ for rhowe31.

\begin{tabular}{|c|c|c|c|c|}
\hline & $\mathrm{x}$ & $\mathrm{y}$ & $\mathrm{z}$ & $\mathrm{U}(\mathrm{eq})$ \\
\hline H1 & 7270 & 4212 & 7172 & 17 \\
\hline $\mathrm{H} 2$ & 7019 & 2823 & 7382 & 21 \\
\hline $\mathrm{H} 3$ & 7918 & 1959 & 7801 & 23 \\
\hline $\mathrm{H} 4$ & 9033 & 2524 & 8014 & 20 \\
\hline $\mathrm{H} 7$ & 10024 & 3135 & 8244 & 22 \\
\hline H8 & 11072 & 3867 & 8441 & 25 \\
\hline H9 & 11129 & 5282 & 8175 & 21 \\
\hline H10 & 10141 & 5919 & 7707 & 15 \\
\hline $\mathrm{H} 11 \mathrm{~A}$ & 6295 & 2392 & 3904 & 17 \\
\hline H11B & 6053 & 1587 & 3518 & 17 \\
\hline $\mathrm{H} 12 \mathrm{~A}$ & 5024 & 1515 & 4192 & 20 \\
\hline H12B & 5268 & 2315 & 4589 & 20 \\
\hline $\mathrm{H} 13 \mathrm{~A}$ & 4936 & 2310 & 3189 & 27 \\
\hline H13B & 5177 & 3109 & 3587 & 27 \\
\hline $\mathrm{H} 14 \mathrm{~A}$ & 3938 & 3115 & 3478 & 38 \\
\hline H14B & 4133 & 3036 & 4247 & 38 \\
\hline $\mathrm{H} 14 \mathrm{C}$ & 3891 & 2246 & 3840 & 38 \\
\hline $\mathrm{H} 15 \mathrm{~A}$ & 6656 & 1327 & 5485 & 16 \\
\hline H15B & 5867 & 1565 & 5317 & 16 \\
\hline H16A & 6504 & 2854 & 4844 & 18 \\
\hline H16B & 6182 & 2825 & 5574 & 18 \\
\hline H17A & 7297 & 2545 & 6037 & 22 \\
\hline H17B & 7634 & 2479 & 5311 & 22 \\
\hline H18A & 7423 & 3904 & 5130 & 32 \\
\hline H18B & 7881 & 3794 & 5788 & 32 \\
\hline $\mathrm{H} 18 \mathrm{C}$ & 7063 & 3975 & 5844 & 32 \\
\hline H19A & 7406 & 1922 & 4304 & 17 \\
\hline H19B & 7276 & 1199 & 3786 & 17 \\
\hline $\mathrm{H} 20 \mathrm{~A}$ & 7664 & 899 & 5148 & 20 \\
\hline H20B & 7599 & 207 & 4588 & 20 \\
\hline
\end{tabular}




$\begin{array}{lrrrl}\text { H21A } & 8547 & 849 & 3986 & 26 \\ \text { H21B } & 8625 & 1502 & 4573 & 26 \\ \text { H22A } & 9486 & 476 & 4704 & 43 \\ \text { H22B } & 8899 & -219 & 4717 & 43 \\ \text { H22C } & 8956 & 421 & 5316 & 43 \\ \text { H23A } & 6404 & 129 & 4818 & 17 \\ \text { H23B } & 5671 & 448 & 4548 & 17 \\ \text { H24A } & 6799 & -147 & 3737 & 26 \\ \text { H24B } & 6132 & 295 & 3418 & 26 \\ \text { H25A } & 5392 & -759 & 3865 & 29 \\ \text { H25B } & 5948 & -1128 & 3356 & 29 \\ \text { H26A } & 6630 & -1619 & 4260 & 53 \\ \text { H26B } & 5856 & -1983 & 4303 & 53 \\ \text { H26C } & 6081 & -1239 & 4773 & 53 \\ \end{array}$


Table 6. Torsion angles $\left[{ }^{\circ}\right]$ for rhowe 31.

\begin{tabular}{|c|c|c|c|}
\hline Mo1-N1-C1-C2 & $174.15(13)$ & $\mathrm{C} 8-\mathrm{C} 9-\mathrm{C} 10-\mathrm{N} 2$ & $-0.2(3)$ \\
\hline Mo1-N1-C5-C4 & $-174.31(13)$ & $\mathrm{C} 10-\mathrm{N} 2-\mathrm{C} 6-\mathrm{C} 5$ & $178.97(15)$ \\
\hline Mo1-N1-C5-C6 & $7.19(19)$ & C10-N2-C6-C7 & $-0.5(3)$ \\
\hline Mo1-N2-C6-C5 & $-4.39(19)$ & N3-C11-C12-C13 & $179.16(16)$ \\
\hline Mo1-N2-C6-C7 & $176.14(13)$ & N3-C15-C16-C17 & $-99.39(19)$ \\
\hline Mo1-N2-C10-C9 & $-175.70(13)$ & N3-C19-C20-C21 & $-174.31(16)$ \\
\hline $\mathrm{N} 1-\mathrm{C} 1-\mathrm{C} 2-\mathrm{C} 3$ & $-0.1(3)$ & N3-C23-C24-C25 & $-170.85(17)$ \\
\hline $\mathrm{N} 1-\mathrm{C} 5-\mathrm{C} 6-\mathrm{N} 2$ & $-1.9(2)$ & C11-N3-C15-C16 & $-53.6(2)$ \\
\hline $\mathrm{N} 1-\mathrm{C} 5-\mathrm{C} 6-\mathrm{C} 7$ & $177.58(16)$ & C11-N3-C19-C20 & $-174.64(15)$ \\
\hline N2-C6-C7-C8 & $-0.1(3)$ & C11-N3-C23-C24 & $65.3(2)$ \\
\hline C1-N1-C5-C4 & $1.1(3)$ & $\mathrm{C} 11-\mathrm{C} 12-\mathrm{C} 13-\mathrm{C} 14$ & $179.73(17)$ \\
\hline C1-N1-C5-C6 & $-177.40(15)$ & C15-N3-C11-C12 & $-48.6(2)$ \\
\hline $\mathrm{C} 1-\mathrm{C} 2-\mathrm{C} 3-\mathrm{C} 4$ & $0.7(3)$ & C15-N3-C19-C20 & $64.3(2)$ \\
\hline $\mathrm{C} 2-\mathrm{C} 3-\mathrm{C} 4-\mathrm{C} 5$ & $-0.4(3)$ & C15-N3-C23-C24 & $-174.00(16)$ \\
\hline $\mathrm{C} 3-\mathrm{C} 4-\mathrm{C} 5-\mathrm{N} 1$ & $-0.5(3)$ & C15-C16-C17-C18 & $174.29(16)$ \\
\hline $\mathrm{C} 3-\mathrm{C} 4-\mathrm{C} 5-\mathrm{C} 6$ & 177.92(17) & C19-N3-C11-C12 & $-169.40(15)$ \\
\hline C4-C5-C6-N2 & $179.65(17)$ & C19-N3-C15-C16 & $65.34(19)$ \\
\hline $\mathrm{C} 4-\mathrm{C} 5-\mathrm{C} 6-\mathrm{C} 7$ & $-0.9(3)$ & C19-N3-C23-C24 & $-53.5(2)$ \\
\hline C5-N1-C1-C2 & $-0.8(3)$ & C19-C20-C21-C22 & $-176.92(18)$ \\
\hline $\mathrm{C} 5-\mathrm{C} 6-\mathrm{C} 7-\mathrm{C} 8$ & $-179.56(17)$ & C23-N3-C11-C12 & $69.62(19)$ \\
\hline C6-N2-C10-C9 & $0.6(3)$ & C23-N3-C15-C16 & $-174.00(15)$ \\
\hline C6-C7-C8-C9 & $0.6(3)$ & C23-N3-C19-C20 & $-53.9(2)$ \\
\hline C7-C8-C9-C10 & $-0.5(3)$ & $\mathrm{C} 23-\mathrm{C} 24-\mathrm{C} 25-\mathrm{C} 26$ & $-63.5(3)$ \\
\hline
\end{tabular}




\section{REFERENCE NUMBER: rhowe22}

\section{CRYSTAL STRUCTURE REPORT}

$\mathrm{C}_{20} \mathrm{H}_{18} \mathrm{Cl}_{4} \mathrm{Co} \mathrm{Mo} \mathrm{N} 2$

or

$\left[\mathrm{CoCp}_{2}\right]\left[\mathrm{MoCl}_{4}(\mathrm{bpy})\right]$

Report prepared for:

Prof. W. Eckenhoff

June 02, 2018

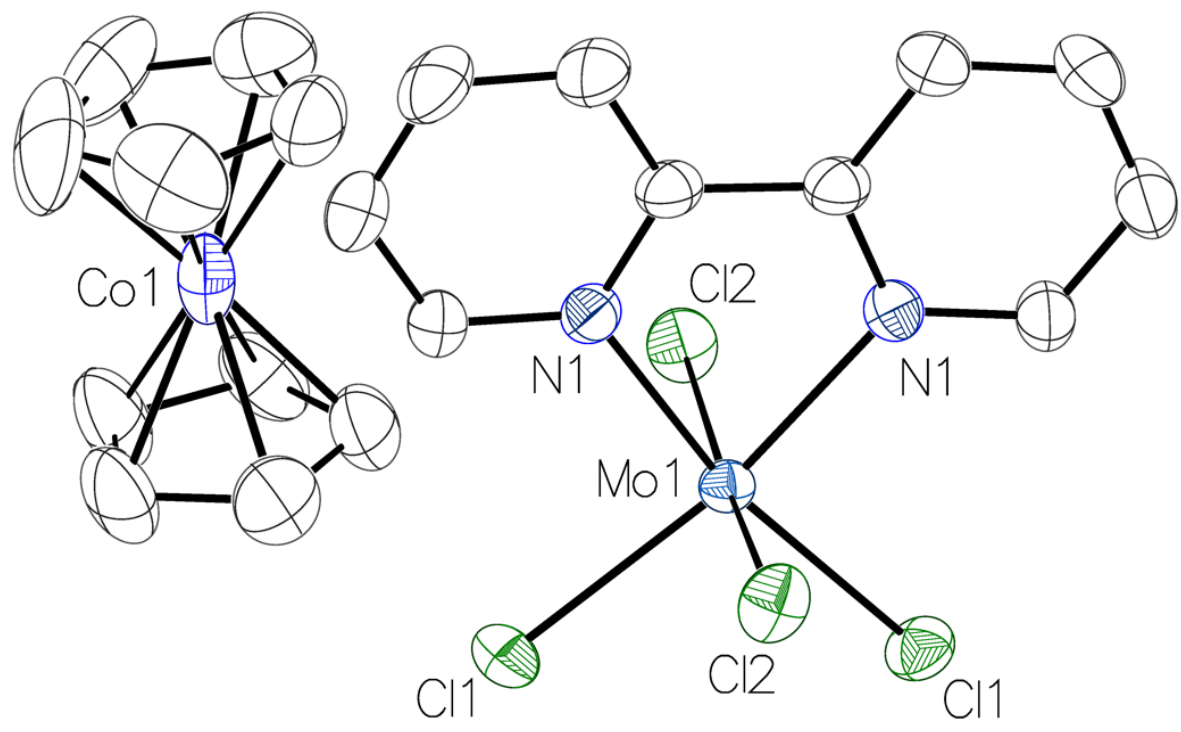

William W. Brennessel

X-ray Crystallographic Facility

Department of Chemistry, University of Rochester

120 Trustee Road

Rochester, NY 14627 


\section{Data collection}

A crystal $\left(0.129 \times 0.068 \times 0.026 \mathrm{~mm}^{3}\right)$ was placed onto a thin glass optical fiber or a nylon loop and mounted on a XtaLab Synergy-S Dualflex diffractometer equipped with a HyPix-6000HE HPC area detector for data collection at 172.99(10) K. A preliminary set of cell constants and an orientation matrix were calculated from a small sampling of reflections. ${ }^{1}$ A short pre-experiment was run, from which an optimal data collection strategy was determined. The full data collection was carried out using a PhotonJet $(\mathrm{Cu})$ X-ray Source with frame times of 0.60 and 2.38 seconds and a detector distance of $31.2 \mathrm{~mm}$. Series of frames were collected in $0.50^{\circ}$ steps in $\omega$ at different $2 \theta, \kappa$, and $\phi$ settings. After the intensity data were corrected for absorption, the final cell constants were calculated from the xyz centroids of 17323 strong reflections from the actual data collection after integration. ${ }^{1}$ See Table 1 for additional crystal and refinement information.

\section{Structure solution and refinement}

The structure was solved using ShelXT ${ }^{2}$ and refined using ShelXL. ${ }^{3}$ The space group $C 2 / c$ was determined based on systematic absences and intensity statistics. Most or all non-hydrogen atoms were assigned from the solution. Full-matrix least squares / difference Fourier cycles were performed which located any remaining non-hydrogen atoms. All non-hydrogen atoms were refined with anisotropic displacement parameters. All hydrogen atoms were placed in ideal positions and refined as riding atoms with relative isotropic displacement parameters. The final full matrix least squares refinement converged to $R 1=0.0276\left(F^{2}, I>2 \sigma(I)\right)$ and $w R 2=0.0764$ ( $F^{2}$, all data).

\section{Structure description}

The structure is the one suggested. The asymmetric unit contains one cobaltocenium cation in a general position and one-half of two independent anionic molybdenum complexes that are located along crystal two-fold axes that include the Mo centers.

Structure manipulation and figure generation were performed using Olex2. ${ }^{4}$ Unless noted otherwise all structural diagrams containing thermal displacement ellipsoids are drawn at the $50 \%$ probability level.

Data collection, structure solution, and structure refinement were conducted at the X-ray Crystallographic Facility, B04 Hutchison Hall, Department of Chemistry, University of Rochester. The instrument was purchased with funding from NSF MRI program grant CHE-1725028. All publications arising from this report MUST either 1) include William W. Brennessel as a coauthor or 2) acknowledge William W. Brennessel and the X-ray Crystallographic Facility of the Department of Chemistry at the University of Rochester. 
1 CrysAlisPro, version 171.39.46; Rigaku Corporation: Oxford, UK, 2018.

2 Sheldrick, G. M. SHELXT, version 2018/2; Acta. Crystallogr. 2015, A71, 3-8.

3 Sheldrick, G. M. SHELXL, version 2018/3; Acta. Crystallogr. 2015, C71, 3-8.

4 Dolomanov, O. V.; Bourhis, L. J.; Gildea, R. J.; Howard, J. A. K.; Puschmann, H. Olex2, version 1.2-ac3; J. Appl. Cryst. 2009, 42, 339-341.

Some equations of interest:

$$
\begin{gathered}
R_{\text {int }}=\Sigma\left|F_{\mathrm{o}}{ }^{2}-<F_{\mathrm{o}}{ }^{2}>\right| / \Sigma\left|F_{\mathrm{o}}{ }^{2}\right| \\
R 1=\Sigma|| F_{\mathrm{o}}|-| F_{\mathrm{c}} \| / \Sigma\left|F_{\mathrm{o}}\right| \\
w R 2=\left[\Sigma\left[w\left(F_{\mathrm{o}}{ }^{2}-F_{\mathrm{c}}{ }^{2}\right)^{2}\right] / \Sigma\left[w\left(F_{\mathrm{o}}{ }^{2}\right)^{2}\right]\right]^{1 / 2} \\
\text { where } w=1 /\left[\sigma^{2}\left(F_{\mathrm{o}}{ }^{2}\right)+(a P)^{2}+b P\right] \text { and } \\
P=1 / 3 \max \left(0, F_{\mathrm{o}}{ }^{2}\right)+2 / 3 F_{\mathrm{c}}{ }^{2} \\
\mathrm{GOF}=S=\left[\Sigma\left[w\left(F_{\mathrm{o}}{ }^{2}-F_{\mathrm{c}}{ }^{2}\right)^{2}\right] /(m-n)\right]^{1 / 2}
\end{gathered}
$$

where $m=$ number of reflections and $n=$ number of parameters
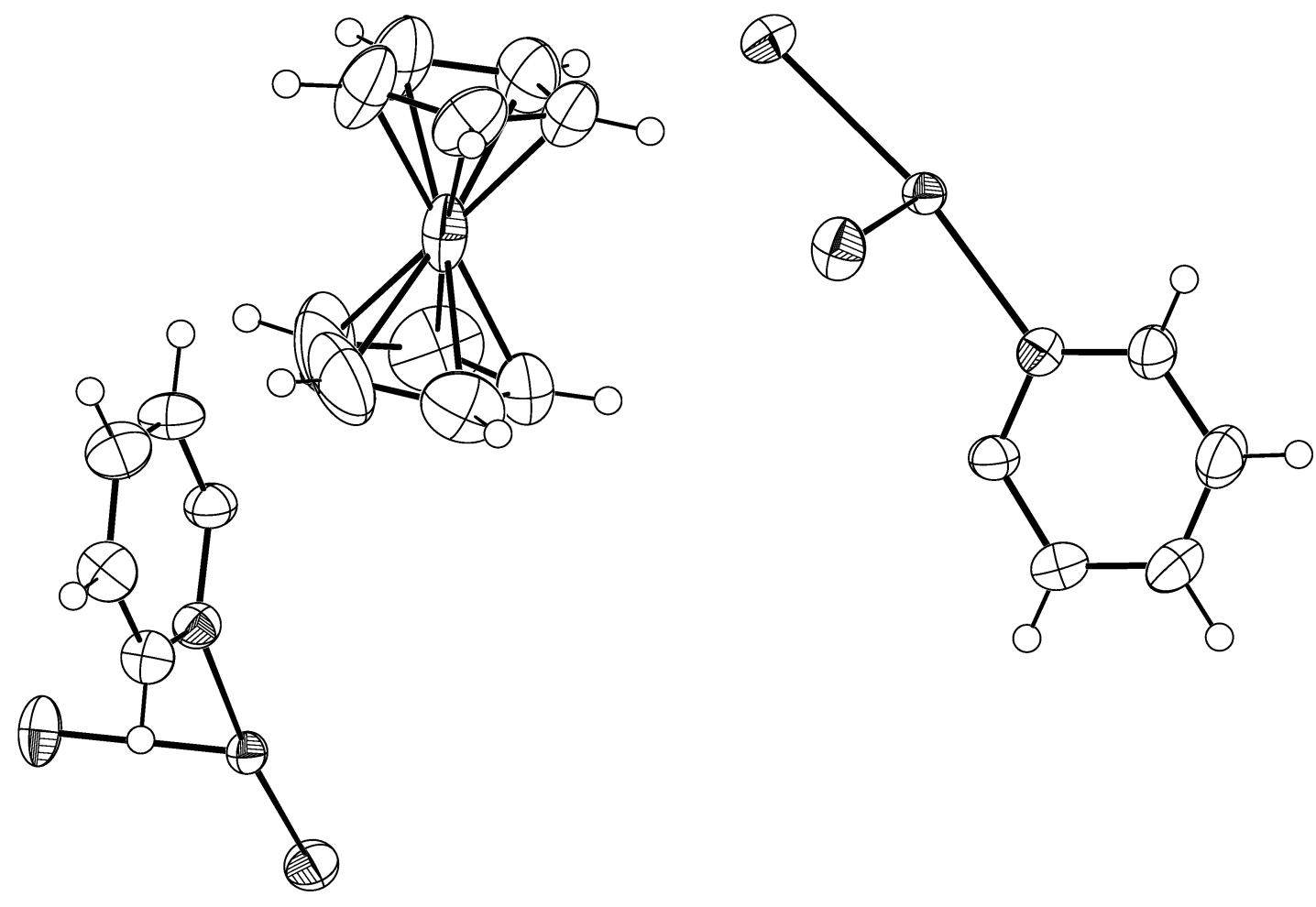

Asymmetric unit. 

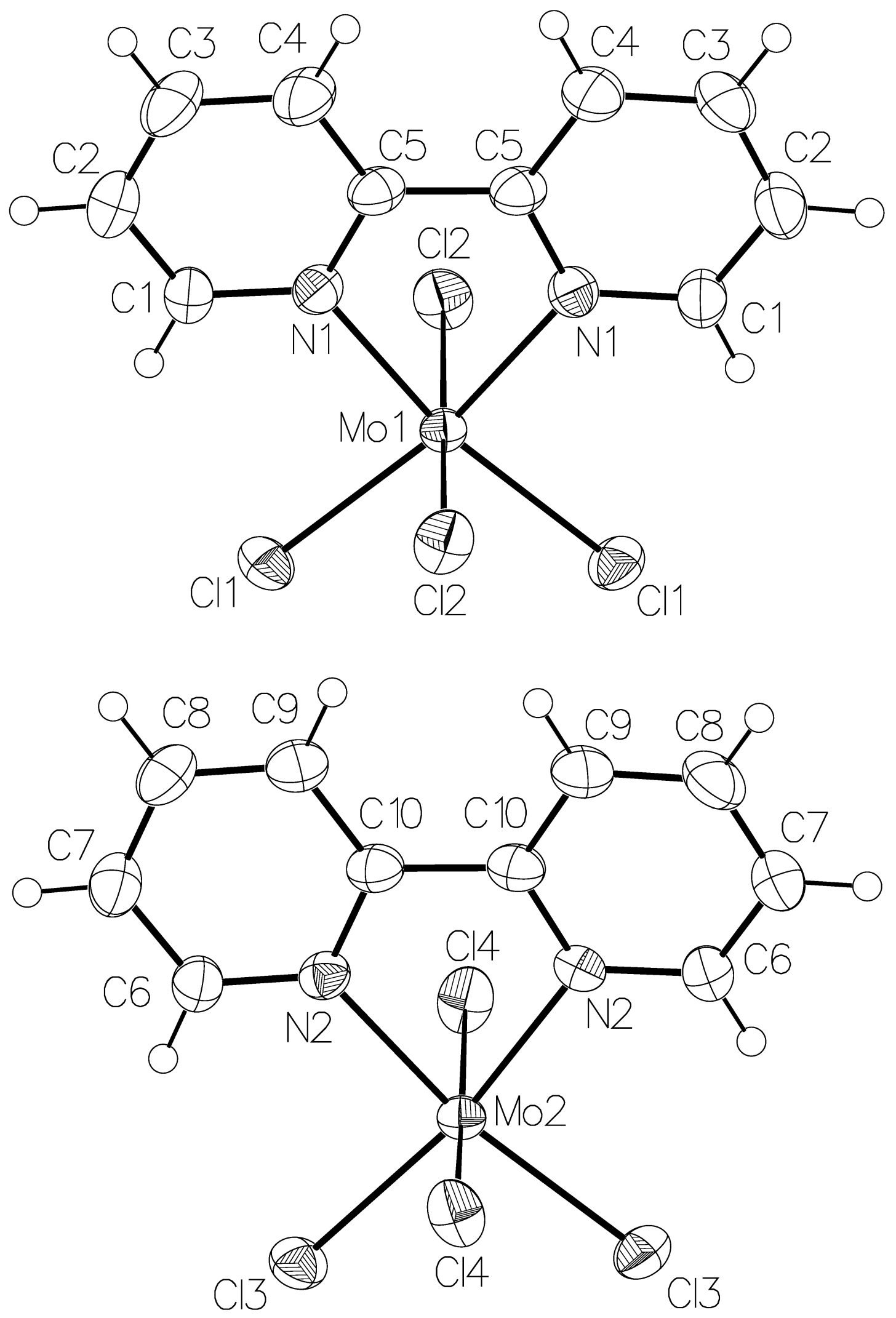

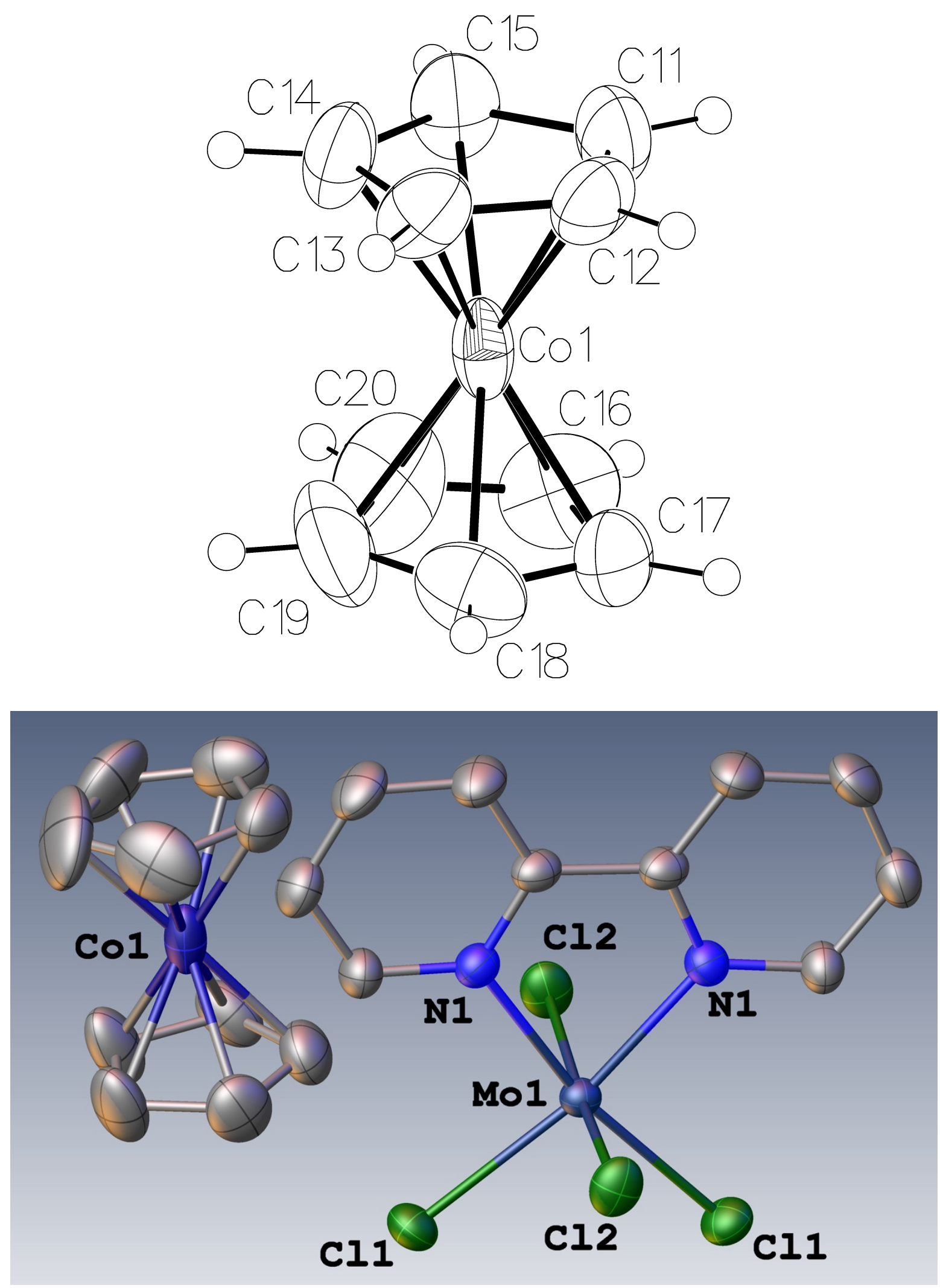
Table 1. Crystal data and structure refinement for rhowe22.

\begin{tabular}{|c|c|c|}
\hline Identification code & \multicolumn{2}{|l|}{ rhowe22 } \\
\hline Empirical formula & \multicolumn{2}{|c|}{ C20 H18 Cl4 Co Mo N2 } \\
\hline Formula weight & \multicolumn{2}{|l|}{583.03} \\
\hline Temperature & \multicolumn{2}{|l|}{$172.99(10) \mathrm{K}$} \\
\hline Wavelength & \multicolumn{2}{|l|}{$1.54184 \AA$} \\
\hline Crystal system & \multicolumn{2}{|l|}{ monoclinic } \\
\hline Space group & \multicolumn{2}{|l|}{$C 2 / c$} \\
\hline \multirow[t]{3}{*}{ Unit cell dimensions } & $a=16.6565(2) \AA$ & $\alpha=90^{\circ}$ \\
\hline & $b=16.8727(2) \AA$ & $\beta=90.4790(10)^{\circ}$ \\
\hline & $c=16.1456(2) \AA$ & $\gamma=90^{\circ}$ \\
\hline Volume & \multicolumn{2}{|l|}{$4537.40(9) \AA^{3}$} \\
\hline$Z$ & \multicolumn{2}{|l|}{8} \\
\hline Density (calculated) & \multicolumn{2}{|l|}{$1.707 \mathrm{Mg} / \mathrm{m}^{3}$} \\
\hline Absorption coefficient & \multicolumn{2}{|l|}{$14.654 \mathrm{~mm}^{-1}$} \\
\hline$F(000)$ & \multicolumn{2}{|l|}{2312} \\
\hline Crystal color, morphology & \multicolumn{2}{|l|}{ red, plate } \\
\hline Crystal size & \multicolumn{2}{|c|}{$0.129 \times 0.068 \times 0.026 \mathrm{~mm}^{3}$} \\
\hline Theta range for data collection & \multicolumn{2}{|l|}{3.729 to $78.117^{\circ}$} \\
\hline Index ranges & \multicolumn{2}{|c|}{$-10 \leq h \leq 20,-21 \leq k \leq 20,-20 \leq l \leq 20$} \\
\hline Reflections collected & \multicolumn{2}{|l|}{28501} \\
\hline Independent reflections & \multicolumn{2}{|c|}{$4783[R($ int $)=0.0356]$} \\
\hline Observed reflections & \multicolumn{2}{|l|}{4384} \\
\hline Completeness to theta $=74.504^{\circ}$ & \multicolumn{2}{|l|}{$99.8 \%$} \\
\hline Absorption correction & \multicolumn{2}{|l|}{ Multi-scan } \\
\hline Max. and min. transmission & \multicolumn{2}{|c|}{1.00000 and 0.53434} \\
\hline Refinement method & \multicolumn{2}{|c|}{ Full-matrix least-squares on $F^{2}$} \\
\hline Data / restraints / parameters & \multicolumn{2}{|l|}{$4783 / 0 / 254$} \\
\hline Goodness-of-fit on $F^{2}$ & \multicolumn{2}{|l|}{1.099} \\
\hline Final $R$ indices $[I>2 \operatorname{sigma}(I)]$ & \multicolumn{2}{|c|}{$R 1=0.0276, w R 2=0.0747$} \\
\hline$R$ indices (all data) & \multicolumn{2}{|c|}{$R 1=0.0304, w R 2=0.0764$} \\
\hline Largest diff. peak and hole & \multicolumn{2}{|c|}{0.506 and -0.773 e. $\AA^{-3}$} \\
\hline
\end{tabular}


Table 2. Atomic coordinates $\left(\times 10^{4}\right)$ and equivalent isotropic displacement parameters $\left(\AA^{2} \times 10^{3}\right)$ for rhowe22. $U_{\text {eq }}$ is defined as one third of the trace of the orthogonalized $U_{i j}$ tensor.

\begin{tabular}{|c|c|c|c|c|}
\hline & $\mathrm{x}$ & $\mathrm{y}$ & $\mathrm{z}$ & $\mathrm{U}_{\mathrm{eq}}$ \\
\hline Mo1 & 5000 & $5530(1)$ & 7500 & 23(1) \\
\hline Mo2 & 5000 & 9446(1) & 2500 & 23(1) \\
\hline $\mathrm{Co} 1$ & $7537(1)$ & 5363(1) & 4982(1) & $45(1)$ \\
\hline $\mathrm{Cl1}$ & 4901(1) & $4568(1)$ & $6373(1)$ & $36(1)$ \\
\hline $\mathrm{Cl} 3$ & 3923(1) & $10416(1)$ & $2538(1)$ & $36(1)$ \\
\hline $\mathrm{Cl} 4$ & $4966(1)$ & 9368(1) & $1002(1)$ & $40(1)$ \\
\hline $\mathrm{Cl} 2$ & $6449(1)$ & 5599(1) & $7409(1)$ & $39(1)$ \\
\hline N2 & 5799(1) & $8435(1)$ & $2465(1)$ & $28(1)$ \\
\hline N1 & $5058(1)$ & 6539(1) & $8322(1)$ & $29(1)$ \\
\hline $\mathrm{C} 10$ & $5441(2)$ & 7714(2) & $2457(2)$ & $32(1)$ \\
\hline C6 & $6599(2)$ & 8482(2) & $2402(2)$ & $34(1)$ \\
\hline $\mathrm{C} 7$ & $7076(2)$ & 7816(2) & $2307(2)$ & $41(1)$ \\
\hline $\mathrm{C} 1$ & $5125(2)$ & 6494(2) & $9152(2)$ & $36(1)$ \\
\hline $\mathrm{C} 5$ & $5041(2)$ & 7261(2) & $7956(2)$ & $32(1)$ \\
\hline $\mathrm{C} 8$ & $6714(2)$ & 7087(2) & $2279(2)$ & $46(1)$ \\
\hline C9 & $5892(2)$ & 7026(2) & $2363(2)$ & $42(1)$ \\
\hline $\mathrm{C} 2$ & $5180(2)$ & 7159(2) & $9646(2)$ & $45(1)$ \\
\hline $\mathrm{C} 4$ & $5095(2)$ & 7952(2) & $8426(2)$ & $46(1)$ \\
\hline $\mathrm{C} 3$ & $5168(2)$ & 7897(2) & $9279(2)$ & $49(1)$ \\
\hline $\mathrm{C} 12$ & $7512(2)$ & 4539(2) & $5900(2)$ & $55(1)$ \\
\hline $\mathrm{C} 11$ & $6849(2)$ & 4443(2) & $5361(2)$ & $60(1)$ \\
\hline $\mathrm{C} 13$ & $8216(2)$ & 4460(2) & $5439(2)$ & $57(1)$ \\
\hline $\mathrm{C} 17$ & $7236(2)$ & 6445(2) & $5415(2)$ & $61(1)$ \\
\hline $\mathrm{C} 18$ & $8068(2)$ & 6398(3) & $5272(3)$ & $67(1)$ \\
\hline $\mathrm{C} 14$ & $7992(2)$ & 4312(3) & $4614(2)$ & $68(1)$ \\
\hline $\mathrm{C} 16$ & $6854(3)$ & 6289(3) & $4641(3)$ & $82(1)$ \\
\hline $\mathrm{C} 15$ & $7150(2)$ & $4297(3)$ & $4560(2)$ & $69(1)$ \\
\hline C19 & $8190(3)$ & $6216(3)$ & $4451(3)$ & $92(2)$ \\
\hline $\mathrm{C} 20$ & $7454(4)$ & $6150(4)$ & $4063(3)$ & $103(2)$ \\
\hline
\end{tabular}


Table 3. Bond lengths $[\AA]$ and angles $\left[{ }^{\circ}\right]$ for rhowe 22 .

\begin{tabular}{|c|c|c|c|}
\hline $\mathrm{Mo}(1)-\mathrm{Cl}(1)$ & $2.4424(6)$ & $C(5)-C(5) \# 1$ & $1.479(5)$ \\
\hline $\operatorname{Mo}(1)-\mathrm{Cl}(1) \# 1$ & $2.4424(6)$ & $C(5)-C(4)$ & $1.393(4)$ \\
\hline $\mathrm{Mo}(1)-\mathrm{Cl}(2)$ & $2.4223(6)$ & $\mathrm{C}(8)-\mathrm{H}(8)$ & 0.9300 \\
\hline $\mathrm{Mo}(1)-\mathrm{Cl}(2) \# 1$ & $2.4223(6)$ & $\mathrm{C}(8)-\mathrm{C}(9)$ & $1.381(4)$ \\
\hline $\mathrm{Mo}(1)-\mathrm{N}(1)$ & $2.160(2)$ & $\mathrm{C}(9)-\mathrm{H}(9)$ & 0.9300 \\
\hline Mo(1)-N(1)\#1 & $2.160(2)$ & $\mathrm{C}(2)-\mathrm{H}(2)$ & 0.9300 \\
\hline $\mathrm{Mo}(2)-\mathrm{Cl}(3)$ & $2.4307(6)$ & $\mathrm{C}(2)-\mathrm{C}(3)$ & $1.379(5)$ \\
\hline $\mathrm{Mo}(2)-\mathrm{Cl}(3) \# 2$ & $2.4306(6)$ & $\mathrm{C}(4)-\mathrm{H}(4)$ & 0.9300 \\
\hline $\mathrm{Mo}(2)-\mathrm{Cl}(4) \# 2$ & $2.4219(6)$ & $\mathrm{C}(4)-\mathrm{C}(3)$ & $1.385(4)$ \\
\hline $\mathrm{Mo}(2)-\mathrm{Cl}(4)$ & $2.4219(6)$ & $\mathrm{C}(3)-\mathrm{H}(3)$ & 0.9300 \\
\hline $\mathrm{Mo}(2)-\mathrm{N}(2)$ & $2.1638(19)$ & $\mathrm{C}(12)-\mathrm{H}(12)$ & 0.9300 \\
\hline $\mathrm{Mo}(2)-\mathrm{N}(2) \# 2$ & $2.1638(19)$ & $C(12)-C(11)$ & $1.410(5)$ \\
\hline $\mathrm{Co}(1)-\mathrm{C}(12)$ & $2.032(4)$ & $C(12)-C(13)$ & $1.400(5)$ \\
\hline $\mathrm{Co}(1)-\mathrm{C}(11)$ & $2.027(4)$ & $\mathrm{C}(11)-\mathrm{H}(11)$ & 0.9300 \\
\hline $\mathrm{Co}(1)-\mathrm{C}(13)$ & $2.032(4)$ & $C(11)-C(15)$ & $1.413(5)$ \\
\hline $\mathrm{Co}(1)-\mathrm{C}(17)$ & $2.019(4)$ & $\mathrm{C}(13)-\mathrm{H}(13)$ & 0.9300 \\
\hline $\mathrm{Co}(1)-\mathrm{C}(18)$ & $2.011(4)$ & $C(13)-C(14)$ & $1.403(5)$ \\
\hline $\mathrm{Co}(1)-\mathrm{C}(14)$ & $2.021(4)$ & $\mathrm{C}(17)-\mathrm{H}(17)$ & 0.9300 \\
\hline $\mathrm{Co}(1)-\mathrm{C}(16)$ & $2.007(4)$ & $C(17)-C(18)$ & $1.408(5)$ \\
\hline Co(1)-C(15) & $2.027(4)$ & $C(17)-C(16)$ & $1.421(6)$ \\
\hline $\mathrm{Co}(1)-\mathrm{C}(19)$ & $2.001(5)$ & $\mathrm{C}(18)-\mathrm{H}(18)$ & 0.9300 \\
\hline $\mathrm{Co}(1)-\mathrm{C}(20)$ & $1.995(5)$ & $C(18)-C(19)$ & $1.378(7)$ \\
\hline $\mathrm{N}(2)-\mathrm{C}(10)$ & $1.356(3)$ & $\mathrm{C}(14)-\mathrm{H}(14)$ & 0.9300 \\
\hline $\mathrm{N}(2)-\mathrm{C}(6)$ & $1.339(3)$ & $C(14)-C(15)$ & $1.406(5)$ \\
\hline $\mathrm{N}(1)-\mathrm{C}(1)$ & $1.346(3)$ & $\mathrm{C}(16)-\mathrm{H}(16)$ & 0.9300 \\
\hline $\mathrm{N}(1)-\mathrm{C}(5)$ & $1.354(3)$ & $C(16)-C(20)$ & $1.393(7)$ \\
\hline$C(10)-C(10) \# 2$ & $1.476(5)$ & $\mathrm{C}(15)-\mathrm{H}(15)$ & 0.9300 \\
\hline$C(10)-C(9)$ & $1.391(4)$ & $\mathrm{C}(19)-\mathrm{H}(19)$ & 0.9300 \\
\hline $\mathrm{C}(6)-\mathrm{H}(6)$ & 0.9300 & $C(19)-C(20)$ & $1.377(8)$ \\
\hline$C(6)-C(7)$ & $1.384(4)$ & $\mathrm{C}(20)-\mathrm{H}(20)$ & 0.9800 \\
\hline $\mathrm{C}(7)-\mathrm{H}(7)$ & 0.9300 & $\mathrm{Cl}(1) \# 1-\mathrm{Mo}(1)-\mathrm{Cl}(1)$ & $96.74(3)$ \\
\hline $\mathrm{C}(7)-\mathrm{C}(8)$ & $1.370(5)$ & $\mathrm{Cl}(2)-\mathrm{Mo}(1)-\mathrm{Cl}(1)$ & $92.74(2)$ \\
\hline $\mathrm{C}(1)-\mathrm{H}(1)$ & 0.9300 & $\mathrm{Cl}(2)-\mathrm{Mo}(1)-\mathrm{Cl}(1) \# 1$ & $90.94(2)$ \\
\hline $\mathrm{C}(1)-\mathrm{C}(2)$ & $1.380(4)$ & $\mathrm{Cl}(2) \# 1-\mathrm{Mo}(1)-\mathrm{Cl}(1) \# 1$ & $92.74(2)$ \\
\hline
\end{tabular}




\begin{tabular}{|c|c|}
\hline $\mathrm{Cl}(2) \# 1-\mathrm{Mo}(1)-\mathrm{Cl}(1)$ & $90.94(2)$ \\
\hline $\mathrm{Cl}(2)-\mathrm{Mo}(1)-\mathrm{Cl}(2) \# 1$ & $174.46(4)$ \\
\hline $\mathrm{N}(1)-\mathrm{Mo}(1)-\mathrm{Cl}(1) \# 1$ & $93.66(6)$ \\
\hline $\mathrm{N}(1)-\mathrm{Mo}(1)-\mathrm{Cl}(1)$ & $169.59(6)$ \\
\hline $\mathrm{N}(1) \# 1-\mathrm{Mo}(1)-\mathrm{Cl}(1) \# 1$ & $169.59(6)$ \\
\hline $\mathrm{N}(1) \# 1-\mathrm{Mo}(1)-\mathrm{Cl}(1)$ & $93.65(6)$ \\
\hline $\mathrm{N}(1)-\mathrm{Mo}(1)-\mathrm{Cl}(2)$ & $87.67(5)$ \\
\hline $\mathrm{N}(1) \# 1-\mathrm{Mo}(1)-\mathrm{Cl}(2)$ & $87.96(6)$ \\
\hline $\mathrm{N}(1)-\mathrm{Mo}(1)-\mathrm{Cl}(2) \# 1$ & $87.96(6)$ \\
\hline $\mathrm{N}(1) \# 1-\mathrm{Mo}(1)-\mathrm{Cl}(2) \# 1$ & $87.67(5)$ \\
\hline $\mathrm{N}(1) \# 1-\mathrm{Mo}(1)-\mathrm{N}(1)$ & $75.96(11)$ \\
\hline $\mathrm{Cl}(3) \# 2-\mathrm{Mo}(2)-\mathrm{Cl}(3)$ & $95.27(3)$ \\
\hline $\mathrm{Cl}(4) \# 2-\mathrm{Mo}(2)-\mathrm{Cl}(3) \# 2$ & $92.87(2)$ \\
\hline $\mathrm{Cl}(4)-\mathrm{Mo}(2)-\mathrm{Cl}(3)$ & $92.87(2)$ \\
\hline $\mathrm{Cl}(4)-\mathrm{Mo}(2)-\mathrm{Cl}(3) \# 2$ & $91.27(2)$ \\
\hline $\mathrm{Cl}(4) \# 2-\mathrm{Mo}(2)-\mathrm{Cl}(3)$ & $91.27(2)$ \\
\hline $\mathrm{Cl}(4)-\mathrm{Mo}(2)-\mathrm{Cl}(4) \# 2$ & $173.85(4)$ \\
\hline $\mathrm{N}(2)-\mathrm{Mo}(2)-\mathrm{Cl}(3) \# 2$ & $94.34(6)$ \\
\hline $\mathrm{N}(2) \# 2-\mathrm{Mo}(2)-\mathrm{Cl}(3) \# 2$ & $170.38(6)$ \\
\hline $\mathrm{N}(2)-\mathrm{Mo}(2)-\mathrm{Cl}(3)$ & $170.38(6)$ \\
\hline $\mathrm{N}(2) \# 2-\mathrm{Mo}(2)-\mathrm{Cl}(3)$ & $94.34(6)$ \\
\hline $\mathrm{N}(2)-\mathrm{Mo}(2)-\mathrm{Cl}(4) \# 2$ & $88.55(5)$ \\
\hline $\mathrm{N}(2)-\mathrm{Mo}(2)-\mathrm{Cl}(4)$ & $86.61(5)$ \\
\hline $\mathrm{N}(2) \# 2-\mathrm{Mo}(2)-\mathrm{Cl}(4) \# 2$ & $86.61(5)$ \\
\hline $\mathrm{N}(2) \# 2-\mathrm{Mo}(2)-\mathrm{Cl}(4)$ & $88.55(5)$ \\
\hline $\mathrm{N}(2) \# 2-\mathrm{Mo}(2)-\mathrm{N}(2)$ & $76.05(11)$ \\
\hline $\mathrm{C}(11)-\mathrm{Co}(1)-\mathrm{C}(12)$ & $40.64(16)$ \\
\hline $\mathrm{C}(11)-\mathrm{Co}(1)-\mathrm{C}(13)$ & $68.31(16)$ \\
\hline $\mathrm{C}(11)-\mathrm{Co}(1)-\mathrm{C}(15)$ & $40.78(16)$ \\
\hline $\mathrm{C}(13)-\mathrm{Co}(1)-\mathrm{C}(12)$ & $40.31(15)$ \\
\hline $\mathrm{C}(17)-\mathrm{Co}(1)-\mathrm{C}(12)$ & $111.06(14)$ \\
\hline $\mathrm{C}(17)-\mathrm{Co}(1)-\mathrm{C}(11)$ & $116.46(15)$ \\
\hline $\mathrm{C}(17)-\mathrm{Co}(1)-\mathrm{C}(13)$ & $133.73(15)$ \\
\hline $\mathrm{C}(17)-\mathrm{Co}(1)-\mathrm{C}(14)$ & $172.02(16)$ \\
\hline $\mathrm{C}(17)-\mathrm{Co}(1)-\mathrm{C}(15)$ & $147.07(17)$ \\
\hline $\mathrm{C}(18)-\mathrm{Co}(1)-\mathrm{C}(12)$ & $115.84(16)$ \\
\hline
\end{tabular}

\begin{tabular}{|c|c|}
\hline $\mathrm{C}(18)-\mathrm{Co}(1)-\mathrm{C}(11)$ & $147.50(17)$ \\
\hline $\mathrm{C}(18)-\mathrm{Co}(1)-\mathrm{C}(13)$ & $108.94(15)$ \\
\hline $\mathrm{C}(18)-\mathrm{Co}(1)-\mathrm{C}(17)$ & $40.90(16)$ \\
\hline $\mathrm{C}(18)-\mathrm{Co}(1)-\mathrm{C}(14)$ & $131.73(16)$ \\
\hline $\mathrm{C}(18)-\mathrm{Co}(1)-\mathrm{C}(15)$ & $170.75(17)$ \\
\hline $\mathrm{C}(14)-\mathrm{Co}(1)-\mathrm{C}(12)$ & $67.94(16)$ \\
\hline $\mathrm{C}(14)-\mathrm{Co}(1)-\mathrm{C}(11)$ & $68.35(16)$ \\
\hline $\mathrm{C}(14)-\mathrm{Co}(1)-\mathrm{C}(13)$ & $40.50(16)$ \\
\hline $\mathrm{C}(14)-\mathrm{Co}(1)-\mathrm{C}(15)$ & $40.64(16)$ \\
\hline $\mathrm{C}(16)-\mathrm{Co}(1)-\mathrm{C}(12)$ & $135.83(19)$ \\
\hline $\mathrm{C}(16)-\mathrm{Co}(1)-\mathrm{C}(11)$ & $111.02(19)$ \\
\hline $\mathrm{C}(16)-\mathrm{Co}(1)-\mathrm{C}(13)$ & $174.61(18)$ \\
\hline $\mathrm{C}(16)-\mathrm{Co}(1)-\mathrm{C}(17)$ & $41.33(18)$ \\
\hline $\mathrm{C}(16)-\mathrm{Co}(1)-\mathrm{C}(18)$ & $68.56(18)$ \\
\hline $\mathrm{C}(16)-\mathrm{Co}(1)-\mathrm{C}(14)$ & $144.69(19)$ \\
\hline $\mathrm{C}(16)-\mathrm{Co}(1)-\mathrm{C}(15)$ & $114.88(18)$ \\
\hline $\mathrm{C}(15)-\mathrm{Co}(1)-\mathrm{C}(12)$ & $68.30(16)$ \\
\hline $\mathrm{C}(15)-\mathrm{Co}(1)-\mathrm{C}(13)$ & $68.37(16)$ \\
\hline $\mathrm{C}(19)-\mathrm{Co}(1)-\mathrm{C}(12)$ & $145.0(2)$ \\
\hline $\mathrm{C}(19)-\mathrm{Co}(1)-\mathrm{C}(11)$ & $172.2(2)$ \\
\hline $\mathrm{C}(19)-\mathrm{Co}(1)-\mathrm{C}(13)$ & 113.1(2) \\
\hline $\mathrm{C}(19)-\mathrm{Co}(1)-\mathrm{C}(17)$ & $68.60(19)$ \\
\hline $\mathrm{C}(19)-\mathrm{Co}(1)-\mathrm{C}(18)$ & $40.2(2)$ \\
\hline $\mathrm{C}(19)-\mathrm{Co}(1)-\mathrm{C}(14)$ & $107.4(2)$ \\
\hline $\mathrm{C}(19)-\mathrm{Co}(1)-\mathrm{C}(16)$ & $68.3(2)$ \\
\hline $\mathrm{C}(19)-\mathrm{Co}(1)-\mathrm{C}(15)$ & $131.8(2)$ \\
\hline $\mathrm{C}(20)-\mathrm{Co}(1)-\mathrm{C}(12)$ & $174.7(2)$ \\
\hline $\mathrm{C}(20)-\mathrm{Co}(1)-\mathrm{C}(11)$ & $134.3(2)$ \\
\hline $\mathrm{C}(20)-\mathrm{Co}(1)-\mathrm{C}(13)$ & $143.5(2)$ \\
\hline $\mathrm{C}(20)-\mathrm{Co}(1)-\mathrm{C}(17)$ & $68.89(19)$ \\
\hline $\mathrm{C}(20)-\mathrm{Co}(1)-\mathrm{C}(18)$ & $67.9(2)$ \\
\hline $\mathrm{C}(20)-\mathrm{Co}(1)-\mathrm{C}(14)$ & $112.9(2)$ \\
\hline $\mathrm{C}(20)-\mathrm{Co}(1)-\mathrm{C}(16)$ & $40.7(2)$ \\
\hline $\mathrm{C}(20)-\mathrm{Co}(1)-\mathrm{C}(15)$ & $108.7(2)$ \\
\hline $\mathrm{C}(20)-\mathrm{Co}(1)-\mathrm{C}(19)$ & $40.3(2)$ \\
\hline $\mathrm{C}(10)-\mathrm{N}(2)-\mathrm{Mo}(2)$ & $115.90(16)$ \\
\hline
\end{tabular}




\begin{tabular}{|c|c|}
\hline $\mathrm{C}(6)-\mathrm{N}(2)-\mathrm{Mo}(2)$ & $124.67(17)$ \\
\hline$C(6)-N(2)-C(10)$ & $119.3(2)$ \\
\hline $\mathrm{C}(1)-\mathrm{N}(1)-\mathrm{Mo}(1)$ & $124.75(17)$ \\
\hline $\mathrm{C}(1)-\mathrm{N}(1)-\mathrm{C}(5)$ & $119.0(2)$ \\
\hline $\mathrm{C}(5)-\mathrm{N}(1)-\mathrm{Mo}(1)$ & $116.19(17)$ \\
\hline $\mathrm{N}(2)-\mathrm{C}(10)-\mathrm{C}(10) \# 2$ & $115.93(14)$ \\
\hline $\mathrm{N}(2)-\mathrm{C}(10)-\mathrm{C}(9)$ & $120.8(2)$ \\
\hline $\mathrm{C}(9)-\mathrm{C}(10)-\mathrm{C}(10) \# 2$ & $123.31(17)$ \\
\hline $\mathrm{N}(2)-\mathrm{C}(6)-\mathrm{H}(6)$ & 118.9 \\
\hline $\mathrm{N}(2)-\mathrm{C}(6)-\mathrm{C}(7)$ & $122.2(3)$ \\
\hline $\mathrm{C}(7)-\mathrm{C}(6)-\mathrm{H}(6)$ & 118.9 \\
\hline $\mathrm{C}(6)-\mathrm{C}(7)-\mathrm{H}(7)$ & 120.7 \\
\hline$C(8)-C(7)-C(6)$ & $118.6(3)$ \\
\hline $\mathrm{C}(8)-\mathrm{C}(7)-\mathrm{H}(7)$ & 120.7 \\
\hline $\mathrm{N}(1)-\mathrm{C}(1)-\mathrm{H}(1)$ & 118.8 \\
\hline $\mathrm{N}(1)-\mathrm{C}(1)-\mathrm{C}(2)$ & $122.3(3)$ \\
\hline $\mathrm{C}(2)-\mathrm{C}(1)-\mathrm{H}(1)$ & 118.8 \\
\hline $\mathrm{N}(1)-\mathrm{C}(5)-\mathrm{C}(5) \# 1$ & $115.81(14)$ \\
\hline$N(1)-C(5)-C(4)$ & $121.0(3)$ \\
\hline$C(4)-C(5)-C(5) \# 1$ & $123.18(17)$ \\
\hline $\mathrm{C}(7)-\mathrm{C}(8)-\mathrm{H}(8)$ & 120.0 \\
\hline $\mathrm{C}(7)-\mathrm{C}(8)-\mathrm{C}(9)$ & $119.9(3)$ \\
\hline $\mathrm{C}(9)-\mathrm{C}(8)-\mathrm{H}(8)$ & 120.0 \\
\hline $\mathrm{C}(10)-\mathrm{C}(9)-\mathrm{H}(9)$ & 120.5 \\
\hline$C(8)-C(9)-C(10)$ & $119.1(3)$ \\
\hline $\mathrm{C}(8)-\mathrm{C}(9)-\mathrm{H}(9)$ & 120.5 \\
\hline $\mathrm{C}(1)-\mathrm{C}(2)-\mathrm{H}(2)$ & 120.5 \\
\hline$C(3)-C(2)-C(1)$ & $119.0(3)$ \\
\hline $\mathrm{C}(3)-\mathrm{C}(2)-\mathrm{H}(2)$ & 120.5 \\
\hline $\mathrm{C}(5)-\mathrm{C}(4)-\mathrm{H}(4)$ & 120.3 \\
\hline$C(3)-C(4)-C(5)$ & $119.3(3)$ \\
\hline $\mathrm{C}(3)-\mathrm{C}(4)-\mathrm{H}(4)$ & 120.3 \\
\hline$C(2)-C(3)-C(4)$ & $119.3(3)$ \\
\hline $\mathrm{C}(2)-\mathrm{C}(3)-\mathrm{H}(3)$ & 120.4 \\
\hline $\mathrm{C}(4)-\mathrm{C}(3)-\mathrm{H}(3)$ & 120.4 \\
\hline $\mathrm{Co}(1)-\mathrm{C}(12)-\mathrm{H}(12)$ & 126.5 \\
\hline
\end{tabular}

\begin{tabular}{|c|c|}
\hline $\mathrm{C}(11)-\mathrm{C}(12)-\mathrm{Co}(1)$ & $69.5(2)$ \\
\hline C(11)-C(12)-H(12) & 125.8 \\
\hline $\mathrm{C}(13)-\mathrm{C}(12)-\mathrm{Co}(1)$ & $69.8(2)$ \\
\hline $\mathrm{C}(13)-\mathrm{C}(12)-\mathrm{H}(12)$ & 125.8 \\
\hline C(13)-C(12)-C(11) & $108.4(3)$ \\
\hline $\mathrm{Co}(1)-\mathrm{C}(11)-\mathrm{H}(11)$ & 125.9 \\
\hline C(12)-C(11)-Co(1) & $69.9(2)$ \\
\hline $\mathrm{C}(12)-\mathrm{C}(11)-\mathrm{H}(11)$ & 126.2 \\
\hline $\mathrm{C}(12)-\mathrm{C}(11)-\mathrm{C}(15)$ & $107.7(3)$ \\
\hline $\mathrm{C}(15)-\mathrm{C}(11)-\mathrm{Co}(1)$ & $69.6(2)$ \\
\hline C(15)-C(11)-H(11) & 126.2 \\
\hline $\mathrm{Co}(1)-\mathrm{C}(13)-\mathrm{H}(13)$ & 126.3 \\
\hline $\mathrm{C}(12)-\mathrm{C}(13)-\mathrm{Co}(1)$ & $69.9(2)$ \\
\hline C(12)-C(13)-H(13) & 126.1 \\
\hline$C(12)-C(13)-C(14)$ & $107.8(3)$ \\
\hline $\mathrm{C}(14)-\mathrm{C}(13)-\mathrm{Co}(1)$ & $69.3(2)$ \\
\hline $\mathrm{C}(14)-\mathrm{C}(13)-\mathrm{H}(13)$ & 126.1 \\
\hline $\mathrm{Co}(1)-\mathrm{C}(17)-\mathrm{H}(17)$ & 126.5 \\
\hline $\mathrm{C}(18)-\mathrm{C}(17)-\mathrm{Co}(1)$ & $69.2(2)$ \\
\hline C(18)-C(17)-H(17) & 126.9 \\
\hline$C(18)-C(17)-C(16)$ & $106.3(4)$ \\
\hline $\mathrm{C}(16)-\mathrm{C}(17)-\mathrm{Co}(1)$ & $68.9(2)$ \\
\hline $\mathrm{C}(16)-\mathrm{C}(17)-\mathrm{H}(17)$ & 126.9 \\
\hline $\mathrm{Co}(1)-\mathrm{C}(18)-\mathrm{H}(18)$ & 126.6 \\
\hline C(17)-C(18)-Co(1) & $69.9(2)$ \\
\hline C(17)-C(18)-H(18) & 125.6 \\
\hline C(19)-C(18)-Co(1) & $69.6(3)$ \\
\hline C(19)-C(18)-C(17) & $108.9(4)$ \\
\hline C(19)-C(18)-H(18) & 125.6 \\
\hline $\mathrm{Co}(1)-\mathrm{C}(14)-\mathrm{H}(14)$ & 125.8 \\
\hline $\mathrm{C}(13)-\mathrm{C}(14)-\mathrm{Co}(1)$ & $70.2(2)$ \\
\hline C(13)-C(14)-H(14) & 125.7 \\
\hline$C(13)-C(14)-C(15)$ & $108.6(3)$ \\
\hline $\mathrm{C}(15)-\mathrm{C}(14)-\mathrm{Co}(1)$ & $69.9(2)$ \\
\hline C(15)-C(14)-H(14) & 125.7 \\
\hline $\mathrm{Co}(1)-\mathrm{C}(16)-\mathrm{H}(16)$ & 126.4 \\
\hline
\end{tabular}




$\begin{array}{lccc}\mathrm{C}(17)-\mathrm{C}(16)-\mathrm{Co}(1) & 69.8(2) & \mathrm{C}(18)-\mathrm{C}(19)-\mathrm{Co}(1) & 70.3(2) \\ \mathrm{C}(17)-\mathrm{C}(16)-\mathrm{H}(16) & 126.2 & \mathrm{C}(18)-\mathrm{C}(19)-\mathrm{H}(19) & 125.7 \\ \mathrm{C}(20)-\mathrm{C}(16)-\mathrm{Co}(1) & 69.2(3) & \mathrm{C}(20)-\mathrm{C}(19)-\mathrm{Co}(1) & 69.6(3) \\ \mathrm{C}(20)-\mathrm{C}(16)-\mathrm{C}(17) & 107.6(4) & \mathrm{C}(20)-\mathrm{C}(19)-\mathrm{C}(18) & 108.5(4) \\ \mathrm{C}(20)-\mathrm{C}(16)-\mathrm{H}(16) & 126.2 & \mathrm{C}(20)-\mathrm{C}(19)-\mathrm{H}(19) & 125.7 \\ \mathrm{Co}(1)-\mathrm{C}(15)-\mathrm{H}(15) & 126.3 & \mathrm{Co}(1)-\mathrm{C}(20)-\mathrm{H}(20) & 125.6 \\ \mathrm{C}(11)-\mathrm{C}(15)-\mathrm{Co}(1) & 69.6(2) & \mathrm{C}(16)-\mathrm{C}(20)-\mathrm{Co}(1) & 70.1(3) \\ \mathrm{C}(11)-\mathrm{C}(15)-\mathrm{H}(15) & 126.2 & \mathrm{C}(16)-\mathrm{C}(20)-\mathrm{H}(20) & 125.6 \\ \mathrm{C}(14)-\mathrm{C}(15)-\mathrm{Co}(1) & 69.4(2) & \mathrm{C}(19)-\mathrm{C}(20)-\mathrm{Co}(1) & 70.1(3) \\ \mathrm{C}(14)-\mathrm{C}(15)-\mathrm{C}(11) & 107.5(3) & \mathrm{C}(19)-\mathrm{C}(20)-\mathrm{C}(16) & 108.8(4) \\ \mathrm{C}(14)-\mathrm{C}(15)-\mathrm{H}(15) & 126.2 & \mathrm{C}(19)-\mathrm{C}(20)-\mathrm{H}(20) & 125.6 \\ \mathrm{Co}(1)-\mathrm{C}(19)-\mathrm{H}(19) & 126.0 & & \end{array}$

Symmetry transformations used to generate equivalent atoms:

$\# 1-\mathrm{x}+1, \mathrm{y},-\mathrm{z}+3 / 2 \quad \# 2-\mathrm{x}+1, \mathrm{y},-\mathrm{z}+1 / 2$ 
Table 4. Anisotropic displacement parameters $\left(\AA^{2} \times 10^{3}\right)$ for rhowe22. The anisotropic displacement factor exponent takes the form: $-2 \pi^{2}\left[h^{2} a^{* 2} U_{11}+\ldots+2 h k a^{*} b^{*} U_{12}\right]$

\begin{tabular}{|c|c|c|c|c|c|c|}
\hline & $\mathrm{U}_{11}$ & $\mathrm{U}_{22}$ & $\mathrm{U}_{33}$ & $\mathrm{U}_{23}$ & $\mathrm{U}_{13}$ & $\mathrm{U}_{12}$ \\
\hline Mo1 & $22(1)$ & $22(1)$ & $25(1)$ & 0 & $1(1)$ & 0 \\
\hline Mo2 & $27(1)$ & $22(1)$ & $20(1)$ & 0 & $-1(1)$ & 0 \\
\hline $\mathrm{Co} 1$ & $28(1)$ & $80(1)$ & $26(1)$ & $-6(1)$ & $1(1)$ & $-2(1)$ \\
\hline $\mathrm{Cl1}$ & $41(1)$ & $30(1)$ & $36(1)$ & $-8(1)$ & $-2(1)$ & $-1(1)$ \\
\hline $\mathrm{Cl} 3$ & $38(1)$ & $30(1)$ & $40(1)$ & $0(1)$ & $4(1)$ & $7(1)$ \\
\hline $\mathrm{Cl} 4$ & $37(1)$ & $61(1)$ & $21(1)$ & $-1(1)$ & $-1(1)$ & $7(1)$ \\
\hline $\mathrm{Cl} 2$ & $24(1)$ & $54(1)$ & $40(1)$ & $-5(1)$ & $3(1)$ & $-3(1)$ \\
\hline N2 & $30(1)$ & $25(1)$ & $27(1)$ & $1(1)$ & $-2(1)$ & $4(1)$ \\
\hline N1 & $31(1)$ & $28(1)$ & $29(1)$ & $-2(1)$ & $2(1)$ & $1(1)$ \\
\hline $\mathrm{C} 10$ & $38(1)$ & $24(1)$ & $34(1)$ & $0(1)$ & $-2(1)$ & $2(1)$ \\
\hline C6 & $30(1)$ & $35(1)$ & $36(1)$ & $0(1)$ & $0(1)$ & $2(1)$ \\
\hline C7 & $32(1)$ & $44(2)$ & $48(2)$ & $3(1)$ & $1(1)$ & $5(1)$ \\
\hline $\mathrm{C} 1$ & $44(1)$ & $36(1)$ & $27(1)$ & $-1(1)$ & $0(1)$ & $2(1)$ \\
\hline $\mathrm{C} 5$ & $36(1)$ & $24(1)$ & $37(1)$ & $-1(1)$ & $6(1)$ & $0(1)$ \\
\hline $\mathrm{C} 8$ & $44(2)$ & $37(2)$ & $56(2)$ & $0(1)$ & $-1(1)$ & $15(1)$ \\
\hline C9 & $45(2)$ & $26(1)$ & $56(2)$ & $0(1)$ & $-2(1)$ & $2(1)$ \\
\hline $\mathrm{C} 2$ & $53(2)$ & $50(2)$ & $31(1)$ & $-8(1)$ & $0(1)$ & $-1(1)$ \\
\hline $\mathrm{C} 4$ & $64(2)$ & $29(1)$ & $44(2)$ & $-5(1)$ & $7(1)$ & $-3(1)$ \\
\hline $\mathrm{C} 3$ & $63(2)$ & $40(2)$ & $43(2)$ & $-17(1)$ & $6(1)$ & $-6(1)$ \\
\hline $\mathrm{C} 12$ & $71(2)$ & $53(2)$ & $41(2)$ & $-11(1)$ & $4(2)$ & $2(2)$ \\
\hline $\mathrm{C} 11$ & $40(2)$ & $77(3)$ & $64(2)$ & $-8(2)$ & $12(2)$ & $-6(2)$ \\
\hline $\mathrm{C} 13$ & $44(2)$ & $61(2)$ & $65(2)$ & $-20(2)$ & $-13(2)$ & $4(1)$ \\
\hline $\mathrm{C} 17$ & $72(2)$ & $64(2)$ & $49(2)$ & $1(2)$ & $17(2)$ & $0(2)$ \\
\hline $\mathrm{C} 18$ & $53(2)$ & $69(3)$ & $79(3)$ & $9(2)$ & $-18(2)$ & $-11(2)$ \\
\hline $\mathrm{C} 14$ & $48(2)$ & $97(3)$ & $59(2)$ & $-39(2)$ & $12(2)$ & $-7(2)$ \\
\hline $\mathrm{C} 16$ & $55(2)$ & $90(3)$ & $99(3)$ & $-3(3)$ & $-32(2)$ & $13(2)$ \\
\hline $\mathrm{C} 15$ & $51(2)$ & $105(3)$ & $50(2)$ & $-28(2)$ & $-5(2)$ & $-21(2)$ \\
\hline C19 & $85(3)$ & $110(4)$ & $81(3)$ & $25(3)$ & $42(3)$ & $-7(3)$ \\
\hline $\mathrm{C} 20$ & $152(5)$ & $120(5)$ & $36(2)$ & 19(2) & $-3(3)$ & $33(4)$ \\
\hline
\end{tabular}


Table 5. Hydrogen coordinates $\left(\times 10^{4}\right)$ and isotropic displacement parameters $\left(\AA^{2} \times 10^{3}\right)$ for rhowe22.

\begin{tabular}{|c|c|c|c|c|}
\hline & $\mathrm{x}$ & $\mathrm{y}$ & z & $\mathrm{U}(\mathrm{eq})$ \\
\hline H6 & 6842 & 8977 & 2423 & 41 \\
\hline $\mathrm{H} 7$ & 7630 & 7863 & 2263 & 49 \\
\hline H1 & 5134 & 5997 & 9401 & 43 \\
\hline H8 & 7022 & 6634 & 2204 & 55 \\
\hline H9 & 5644 & 6533 & 2356 & 51 \\
\hline $\mathrm{H} 2$ & 5224 & 7111 & 10219 & 54 \\
\hline $\mathrm{H} 4$ & 5083 & 8445 & 8169 & 55 \\
\hline H3 & 5208 & 8353 & 9601 & 58 \\
\hline H12 & 7487 & 4639 & 6466 & 66 \\
\hline H11 & 6311 & 4470 & 5507 & 72 \\
\hline H13 & 8738 & 4499 & 5643 & 68 \\
\hline H17 & 6987 & 6555 & 5915 & 74 \\
\hline H18 & 8469 & 6477 & 5668 & 81 \\
\hline H14 & 8343 & 4236 & 4176 & 82 \\
\hline H16 & 6304 & 6282 & 4540 & 98 \\
\hline H15 & 6845 & 4207 & 4083 & 82 \\
\hline H19 & 8686 & 6149 & 4201 & 110 \\
\hline $\mathrm{H} 20$ & 7366 & 6023 & 3477 & 123 \\
\hline
\end{tabular}


Table 6. Torsion angles $\left[{ }^{\circ}\right]$ for rhowe22.

\begin{tabular}{|c|c|c|c|}
\hline Mo1-N1-C1-C2 & $178.3(2)$ & C1-N1-C5-C5\#1 & $-179.5(3)$ \\
\hline Mo1-N1-C5-C5\#1 & $1.8(4)$ & C1-N1-C5-C4 & $0.4(4)$ \\
\hline Mo1-N1-C5-C4 & $-178.4(2)$ & $\mathrm{C} 1-\mathrm{C} 2-\mathrm{C} 3-\mathrm{C} 4$ & $0.5(5)$ \\
\hline Mo2-N2-C10-C10\#2 & $-4.7(4)$ & $\mathrm{C} 5-\mathrm{N} 1-\mathrm{C} 1-\mathrm{C} 2$ & $-0.3(4)$ \\
\hline Mo2-N2-C10-C9 & $174.4(2)$ & C5\#1-C5-C4-C3 & $179.8(3)$ \\
\hline Mo2-N2-C6-C7 & $-174.0(2)$ & $\mathrm{C} 5-\mathrm{C} 4-\mathrm{C} 3-\mathrm{C} 2$ & $-0.4(5)$ \\
\hline Co1-C12-C11-C15 & $59.6(3)$ & $\mathrm{C} 12-\mathrm{C} 11-\mathrm{C} 15-\mathrm{Co} 1$ & $-59.8(3)$ \\
\hline Co1-C12-C13-C14 & $-59.2(3)$ & $\mathrm{C} 12-\mathrm{C} 11-\mathrm{C} 15-\mathrm{C} 14$ & $-0.5(5)$ \\
\hline Co1-C11-C15-C14 & $59.3(3)$ & C12-C13-C14-Co1 & $59.5(3)$ \\
\hline Co1-C13-C14-C15 & $-59.6(3)$ & $\mathrm{C} 12-\mathrm{C} 13-\mathrm{C} 14-\mathrm{C} 15$ & $-0.1(5)$ \\
\hline Co1-C17-C18-C19 & $58.8(3)$ & $\mathrm{C} 11-\mathrm{C} 12-\mathrm{C} 13-\mathrm{Co} 1$ & $59.0(3)$ \\
\hline Co1-C17-C16-C20 & $-59.0(4)$ & C11-C12-C13-C14 & $-0.2(4)$ \\
\hline Co1-C18-C19-C20 & $59.3(4)$ & $\mathrm{C} 13-\mathrm{C} 12-\mathrm{C} 11-\mathrm{Co} 1$ & $-59.2(3)$ \\
\hline Co1-C14-C15-C11 & $-59.4(3)$ & $\mathrm{C} 13-\mathrm{C} 12-\mathrm{C} 11-\mathrm{C} 15$ & $0.4(5)$ \\
\hline Co1-C16-C20-C19 & $-59.6(4)$ & $\mathrm{C} 13-\mathrm{C} 14-\mathrm{C} 15-\mathrm{Co} 1$ & $59.7(3)$ \\
\hline Co1-C19-C20-C16 & $59.7(4)$ & C13-C14-C15-C11 & $0.4(5)$ \\
\hline N2-C10-C9-C8 & $0.2(4)$ & C17-C18-C19-Co1 & $-59.0(3)$ \\
\hline N2-C6-C7-C8 & $-0.2(4)$ & C17-C18-C19-C20 & $0.3(6)$ \\
\hline $\mathrm{N} 1-\mathrm{C} 1-\mathrm{C} 2-\mathrm{C} 3$ & $-0.1(5)$ & C17-C16-C20-Co1 & $59.4(3)$ \\
\hline $\mathrm{N} 1-\mathrm{C} 5-\mathrm{C} 4-\mathrm{C} 3$ & $0.0(5)$ & C17-C16-C20-C19 & $-0.2(7)$ \\
\hline C10-N2-C6-C7 & $1.8(4)$ & $\mathrm{C} 18-\mathrm{C} 17-\mathrm{C} 16-\mathrm{Co} 1$ & $59.4(3)$ \\
\hline $\mathrm{C} 10 \# 2-\mathrm{C} 10-\mathrm{C} 9-\mathrm{C} 8$ & 179.3(3) & $\mathrm{C} 18-\mathrm{C} 17-\mathrm{C} 16-\mathrm{C} 20$ & $0.4(5)$ \\
\hline C6-N2-C10-C10\#2 & 179.1(3) & C18-C19-C20-Co1 & $-59.7(4)$ \\
\hline C6-N2-C10-C9 & $-1.8(4)$ & $\mathrm{C} 18-\mathrm{C} 19-\mathrm{C} 20-\mathrm{C} 16$ & $-0.1(7)$ \\
\hline C6-C7-C8-C9 & $-1.3(5)$ & $\mathrm{C} 16-\mathrm{C} 17-\mathrm{C} 18-\mathrm{Co} 1$ & $-59.2(3)$ \\
\hline C7-C8-C9-C10 & $1.3(5)$ & C16-C17-C18-C19 & $-0.5(5)$ \\
\hline
\end{tabular}

Symmetry transformations used to generate equivalent atoms:

$\# 1-\mathrm{x}+1, \mathrm{y},-\mathrm{z}+3 / 2 \quad \# 2-\mathrm{x}+1, \mathrm{y},-\mathrm{z}+1 / 2$ 
REFERENCE NUMBER: rhowe29

CRYSTAL STRUCTURE REPORT

$\mathrm{C}_{22} \mathrm{H}_{18} \mathrm{Cl}_{4} \mathrm{Co} \mathrm{Mo} \mathrm{N} 2$

or

$\left[\mathrm{CoCp}_{2}\right]\left[\mathrm{MoCl}_{4}(\right.$ phen $\left.)\right] \cdot \mathrm{xSolvent}$

Report prepared for:

Prof. W. Eckenhoff

October 22, 2018

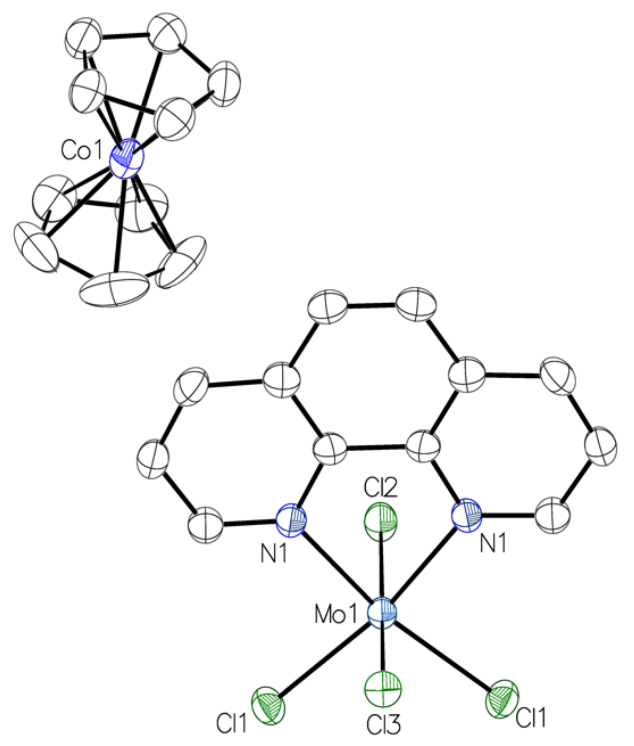

William W. Brennessel

X-ray Crystallographic Facility

Department of Chemistry, University of Rochester

120 Trustee Road

Rochester, NY 14627 


\section{Data collection}

A crystal $\left(0.201 \times 0.066 \times 0.047 \mathrm{~mm}^{3}\right)$ was placed onto a thin glass optical fiber or a nylon loop and mounted on a XtaLab Synergy-S Dualflex diffractometer equipped with a HyPix-6000HE HPC area detector for data collection at 100.00(10) K. A preliminary set of cell constants and an orientation matrix were calculated from a small sampling of reflections. ${ }^{1}$ A short pre-experiment was run, from which an optimal data collection strategy was determined. The full data collection was carried out using a PhotonJet $(\mathrm{Cu})$ X-ray Source with frame times of 0.65 and 2.59 seconds and a detector distance of $31.2 \mathrm{~mm}$. Series of frames were collected in $0.50^{\circ}$ steps in $\omega$ at different $2 \theta, \kappa$, and $\phi$ settings. After the intensity data were corrected for absorption, the final cell constants were calculated from the xyz centroids of 14467 strong reflections from the actual data collection after integration. ${ }^{1}$ See Table 1 for additional crystal and refinement information.

\section{Structure solution and refinement}

The structure was solved using ShelXT ${ }^{2}$ and refined using ShelXL. ${ }^{3}$ The space group Pnnm was determined based on systematic absences and intensity statistics. Most or all non-hydrogen atoms were assigned from the solution. Full-matrix least squares / difference Fourier cycles were performed which located any remaining non-hydrogen atoms. All non-hydrogen atoms were refined with anisotropic displacement parameters. All hydrogen atoms were placed in ideal positions and refined as riding atoms with relative isotropic displacement parameters.

Reflection contributions from highly disordered solvent in intersecting channels along both [010] and [001] were fixed and added to the calculated structure factors using the SQUEEZE routine of program Platon, ${ }^{4}$ which determined there to be 434 electrons in $1540 \AA^{3}$ treated this way per unit cell. Because the identity and exact amount of solvent were unknown, no solvent was included in the atom list or molecular formula. Thus all calculated quantities that derive from the molecular formula (e.g., $F(000)$, density, molecular weight, etc.) are known to be incorrect.

The final full matrix least squares refinement converged to $R 1=0.0444\left(F^{2}, I>2 \sigma(I)\right)$ and $w R 2=0.1219\left(F^{2}\right.$, all data).

\section{Structure description}

The structure is the one suggested. The asymmetric unit contains one cobaltocenium cation in a general position, two half-molecules of the anionic molybdenum complex located on crystallographic mirror planes that each coincide with a metal center and two chlorido ligands, and solvent whose atoms were not assigned (see above). One cyclopentadienyl ring ligand is modeled as disordered over two positions $(0.67: 0.33)$.

Structure manipulation and figure generation were performed using Olex2. ${ }^{5}$ Unless noted otherwise all structural diagrams containing thermal displacement ellipsoids are drawn at the $50 \%$ probability level. 
Data collection, structure solution, and structure refinement were conducted at the X-ray Crystallographic Facility, B04 Hutchison Hall, Department of Chemistry, University of Rochester. The instrument was purchased with funding from NSF MRI program grant CHE-1725028. All publications arising from this report MUST either 1) include William W. Brennessel as a coauthor or 2) acknowledge William W. Brennessel and the X-ray Crystallographic Facility of the Department of Chemistry at the University of Rochester.

1 CrysAlisPro, version 171.39.46; Rigaku Corporation: Oxford, UK, 2018.

2 Sheldrick, G. M. SHELXT, version 2018/2; Acta. Crystallogr. 2015, A71, 3-8.

3 Sheldrick, G. M. SHELXL, version 2018/3; Acta. Crystallogr. 2015, C71, 3-8.

4 Spek, A. L. PLATON, version 150216; Acta. Crystallogr. 2015, C71, 9-18.

5 Dolomanov, O. V.; Bourhis, L. J.; Gildea, R. J.; Howard, J. A. K.; Puschmann, H. Olex2, version 1.2-ac3; J. Appl. Cryst. 2009, 42, 339-341.

Some equations of interest:

$$
\begin{gathered}
R_{\text {int }}=\Sigma\left|F_{\mathrm{o}}{ }^{2}-<F_{\mathrm{o}}{ }^{2}>\right| / \Sigma\left|F_{\mathrm{o}}{ }^{2}\right| \\
R 1=\Sigma|| F_{\mathrm{o}}|-| F_{\mathrm{c}} \| / \Sigma\left|F_{\mathrm{o}}\right| \\
w R 2=\left[\Sigma\left[w\left(F_{\mathrm{o}}{ }^{2} F_{\mathrm{c}}{ }^{2}\right)^{2}\right] / \Sigma\left[w\left(F_{\mathrm{o}}{ }^{2}\right)^{2}\right]\right]^{1 / 2} \\
\text { where } w=1 /\left[\sigma^{2}\left(F_{\mathrm{o}}{ }^{2}\right)+(a P)^{2}+b P\right] \text { and } \\
P=1 / 3 \max \left(0, F_{\mathrm{o}}{ }^{2}\right)+2 / 3 F_{\mathrm{c}}{ }^{2} \\
\text { GOF }=S=\left[\Sigma\left[w\left(F_{\mathrm{o}}{ }^{2}-F_{\mathrm{c}}{ }^{2}\right)^{2}\right] /(m-n)\right]^{1 / 2}
\end{gathered}
$$

where $m=$ number of reflections and $n=$ number of parameters 

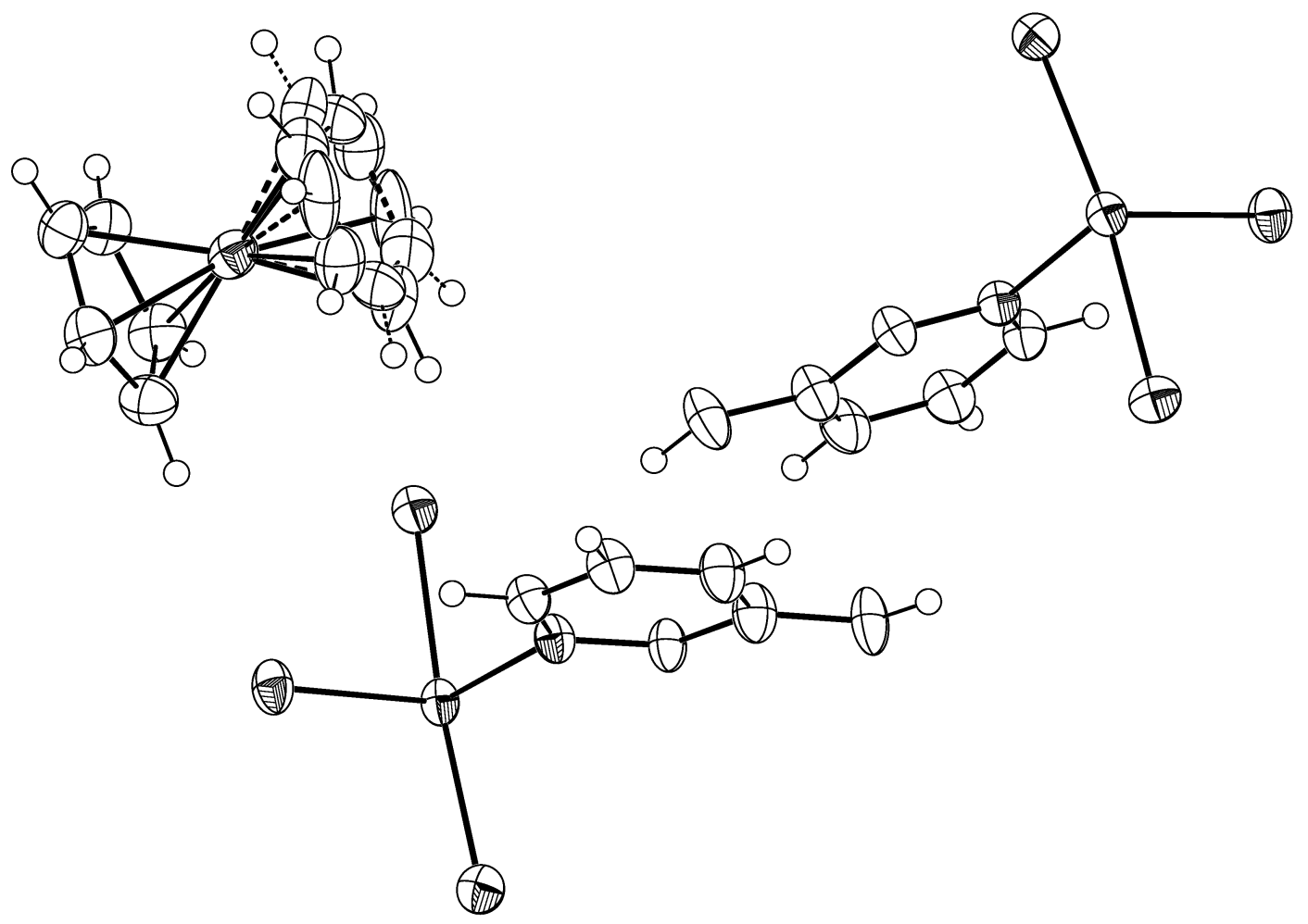

Asymmetric unit (minus solvent). 

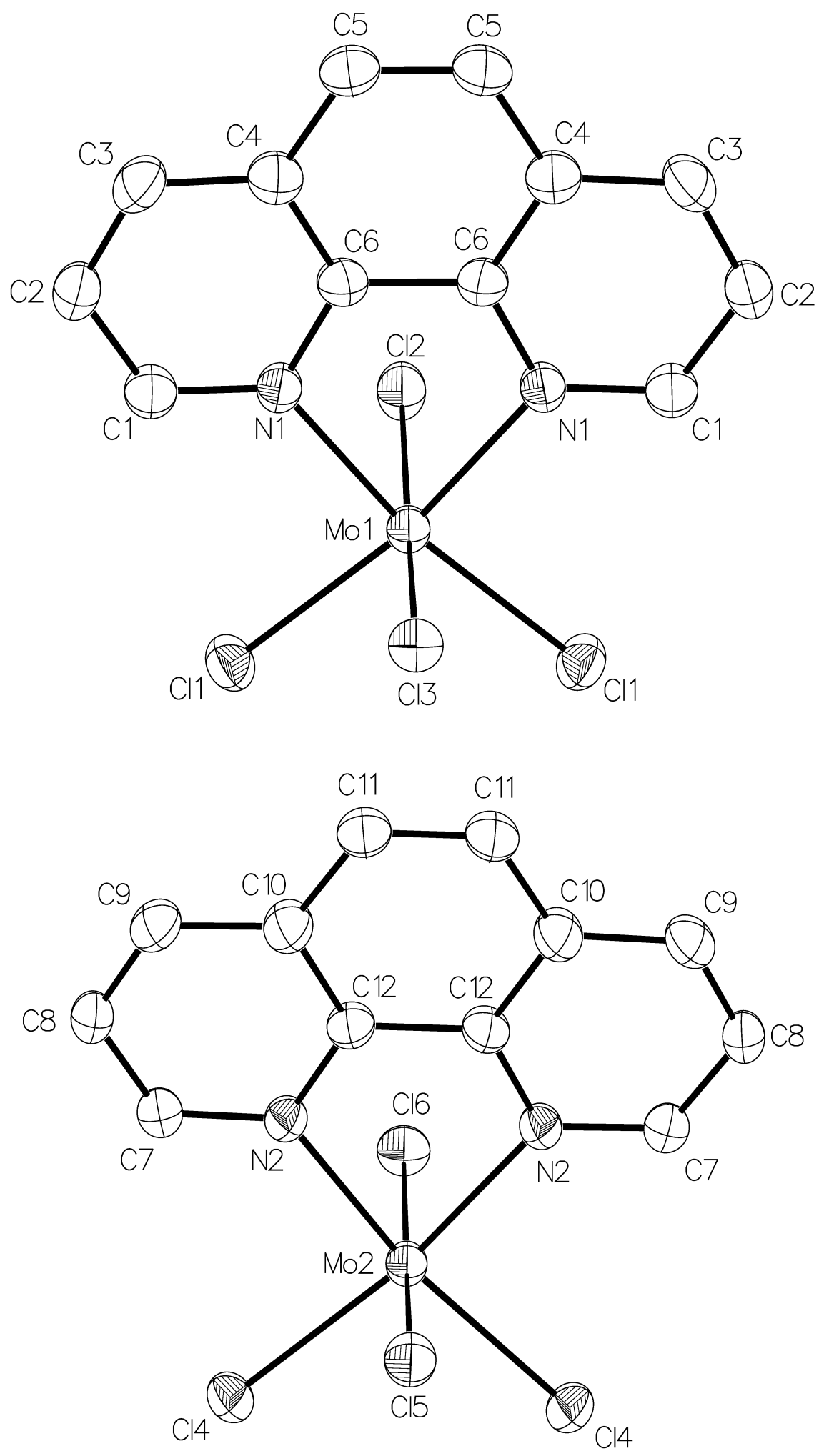


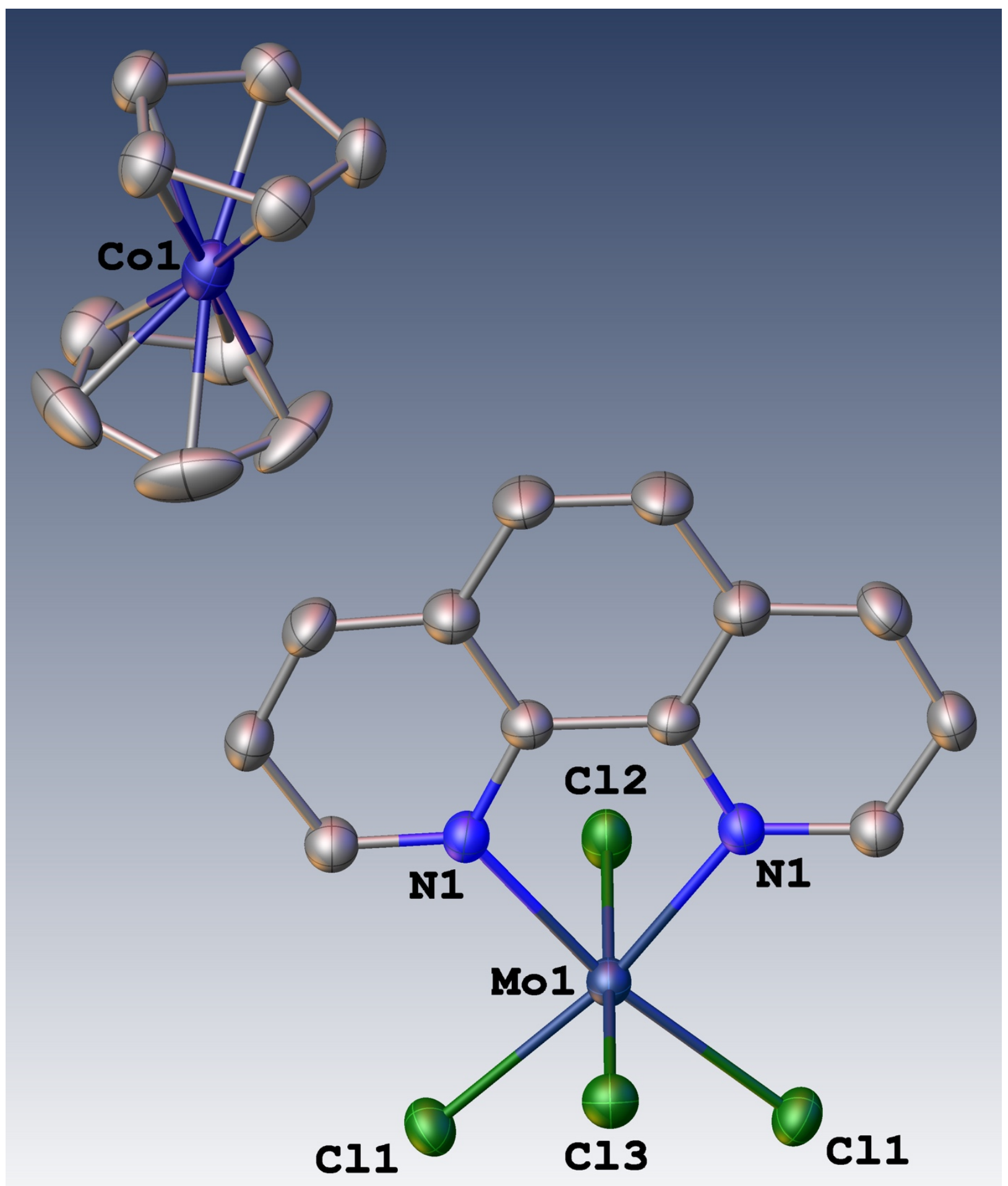


Table 1. Crystal data and structure refinement for rhowe29.

\begin{tabular}{|c|c|c|}
\hline Identification code & \multicolumn{2}{|l|}{ rhowe 29} \\
\hline Empirical formula & \multicolumn{2}{|c|}{ C22 H18 C14 Co Mo N2 } \\
\hline Formula weight & \multicolumn{2}{|l|}{607.05} \\
\hline Temperature & \multicolumn{2}{|l|}{$100.00(10) \mathrm{K}$} \\
\hline Wavelength & \multicolumn{2}{|l|}{$1.54184 \AA$} \\
\hline Crystal system & \multicolumn{2}{|l|}{ orthorhombic } \\
\hline Space group & \multicolumn{2}{|l|}{ Pnnm } \\
\hline \multirow[t]{3}{*}{ Unit cell dimensions } & $a=20.9110(2) \AA$ & $\alpha=90^{\circ}$ \\
\hline & $b=17.7892(3) \AA$ & $\beta=90^{\circ}$ \\
\hline & $c=15.2598(2) \AA$ & $\gamma=90^{\circ}$ \\
\hline Volume & \multicolumn{2}{|l|}{$5676.49(13) \AA^{3}$} \\
\hline$Z$ & \multicolumn{2}{|l|}{8} \\
\hline Density (calculated) & \multicolumn{2}{|l|}{$1.421 \mathrm{Mg} / \mathrm{m}^{3}$} \\
\hline Absorption coefficient & \multicolumn{2}{|l|}{$11.739 \mathrm{~mm}^{-1}$} \\
\hline$F(000)$ & \multicolumn{2}{|l|}{2408} \\
\hline Crystal color, morphology & \multicolumn{2}{|l|}{ red-black, needle } \\
\hline Crystal size & \multicolumn{2}{|c|}{$0.201 \times 0.066 \times 0.047 \mathrm{~mm}^{3}$} \\
\hline Theta range for data collection & \multicolumn{2}{|l|}{3.262 to $77.737^{\circ}$} \\
\hline Index ranges & \multicolumn{2}{|c|}{$-26 \leq h \leq 25,-22 \leq k \leq 22,-19 \leq l \leq 6$} \\
\hline Reflections collected & \multicolumn{2}{|l|}{34105} \\
\hline Independent reflections & \multicolumn{2}{|c|}{$6192[R($ int $)=0.0423]$} \\
\hline Observed reflections & \multicolumn{2}{|l|}{5519} \\
\hline Completeness to theta $=74.504^{\circ}$ & \multicolumn{2}{|l|}{$99.7 \%$} \\
\hline Absorption correction & \multicolumn{2}{|l|}{ Multi-scan } \\
\hline Max. and min. transmission & \multicolumn{2}{|c|}{1.00000 and 0.47251} \\
\hline Refinement method & \multicolumn{2}{|c|}{ Full-matrix least-squares on $F^{2}$} \\
\hline Data / restraints / parameters & \multicolumn{2}{|l|}{$6192 / 120 / 296$} \\
\hline Goodness-of-fit on $F^{2}$ & \multicolumn{2}{|l|}{1.054} \\
\hline Final $R$ indices $[I>2 \operatorname{sigma}(I)]$ & \multicolumn{2}{|c|}{$R 1=0.0444, w R 2=0.1192$} \\
\hline$R$ indices (all data) & \multicolumn{2}{|c|}{$R 1=0.0488, w R 2=0.1219$} \\
\hline Largest diff. peak and hole & \multicolumn{2}{|c|}{0.843 and -1.470 e..$\AA^{-3}$} \\
\hline
\end{tabular}


Table 2. Atomic coordinates $\left(\times 10^{4}\right)$ and equivalent isotropic displacement parameters $\left(\AA^{2} \times 10^{3}\right)$ for rhowe29. $U_{\text {eq }}$ is defined as one third of the trace of the orthogonalized $U_{i j}$ tensor.

\begin{tabular}{|c|c|c|c|c|}
\hline & $\mathrm{x}$ & $\mathrm{y}$ & $\mathrm{z}$ & $\mathrm{U}_{\mathrm{eq}}$ \\
\hline Mo1 & $7288(1)$ & $1670(1)$ & 5000 & $25(1)$ \\
\hline $\mathrm{Cl1}$ & $8067(1)$ & $1620(1)$ & $6179(1)$ & $33(1)$ \\
\hline $\mathrm{Cl} 2$ & 7301(1) & $3032(1)$ & 5000 & $33(1)$ \\
\hline $\mathrm{Cl} 3$ & $7142(1)$ & $314(1)$ & 5000 & $33(1)$ \\
\hline N1 & $6479(1)$ & $1758(2)$ & $5885(2)$ & $26(1)$ \\
\hline $\mathrm{C} 1$ & $6488(2)$ & $1737(2)$ & $6751(2)$ & $32(1)$ \\
\hline $\mathrm{C} 2$ & $5934(2)$ & $1832(2)$ & $7258(2)$ & $37(1)$ \\
\hline $\mathrm{C} 3$ & $5360(2)$ & 1953(3) & $6848(2)$ & $40(1)$ \\
\hline $\mathrm{C} 4$ & $5335(2)$ & 1973(2) & $5934(3)$ & $38(1)$ \\
\hline $\mathrm{C} 5$ & $4755(2)$ & 2087(3) & $5446(3)$ & $44(1)$ \\
\hline C6 & $5907(2)$ & $1868(2)$ & $5471(2)$ & $31(1)$ \\
\hline Mo2 & $2936(1)$ & $3618(1)$ & 5000 & $25(1)$ \\
\hline $\mathrm{Cl} 4$ & $2155(1)$ & $3562(1)$ & $3825(1)$ & $33(1)$ \\
\hline $\mathrm{Cl} 5$ & 2904(1) & $4975(1)$ & 5000 & $33(1)$ \\
\hline $\mathrm{Cl} 6$ & $3060(1)$ & $2254(1)$ & 5000 & $32(1)$ \\
\hline N2 & $3747(1)$ & $3717(2)$ & $4118(2)$ & $27(1)$ \\
\hline $\mathrm{C} 7$ & $3748(2)$ & $3685(2)$ & $3247(2)$ & $30(1)$ \\
\hline $\mathrm{C} 8$ & $4299(2)$ & $3795(2)$ & $2746(2)$ & $34(1)$ \\
\hline C9 & $4865(2)$ & $3939(2)$ & $3151(2)$ & $37(1)$ \\
\hline $\mathrm{C} 10$ & $4893(2)$ & $3971(2)$ & $4066(3)$ & $37(1)$ \\
\hline C11 & $5468(2)$ & 4101(3) & $4557(3)$ & $42(1)$ \\
\hline $\mathrm{C} 12$ & $4319(2)$ & $3855(2)$ & $4533(2)$ & $30(1)$ \\
\hline Co1 & $2657(1)$ & $945(1)$ & 2594(1) & $34(1)$ \\
\hline $\mathrm{C} 13$ & $1929(2)$ & $324(2)$ & $3098(3)$ & $42(1)$ \\
\hline C14 & $1790(2)$ & $547(2)$ & $2230(3)$ & $40(1)$ \\
\hline $\mathrm{C} 15$ & $1799(2)$ & $1340(3)$ & $2195(3)$ & $41(1)$ \\
\hline C16 & $1946(2)$ & $1616(2)$ & $3034(3)$ & $43(1)$ \\
\hline $\mathrm{C} 17$ & $2029(2)$ & $984(2)$ & $3601(3)$ & $43(1)$ \\
\hline $\mathrm{C} 18$ & $3438(4)$ & 295(4) & $2433(13)$ & $67(3)$ \\
\hline C19 & $3279(4)$ & 690(9) & $1660(7)$ & $65(4)$ \\
\hline $\mathrm{C} 20$ & $3318(4)$ & $1458(7)$ & $1845(6)$ & $48(2)$ \\
\hline
\end{tabular}




\begin{tabular}{llrll}
$\mathrm{C} 21$ & $3475(4)$ & $1538(6)$ & $2720(8)$ & $57(2)$ \\
$\mathrm{C} 22$ & $3533(4)$ & $824(9)$ & $3105(7)$ & $65(3)$ \\
$\mathrm{C} 18^{\prime}$ & $3444(11)$ & $1558(9)$ & $2290(30)$ & $67(3)$ \\
$\mathrm{C} 19^{\prime}$ & $3576(10)$ & $1136(19)$ & $3040(15)$ & $65(4)$ \\
$\mathrm{C}^{\prime} 0^{\prime}$ & $3476(11)$ & $376(14)$ & $2864(13)$ & $48(2)$ \\
$\mathrm{C} 21^{\prime}$ & $3310(10)$ & $316(12)$ & $1978(17)$ & $57(2)$ \\
$\mathrm{C} 22^{\prime}$ & $3313(11)$ & $1040(20)$ & $1626(15)$ & $65(3)$ \\
\hline
\end{tabular}


Table 3. Bond lengths $[\AA]$ and angles $\left[{ }^{\circ}\right]$ for rhowe 29 .

\begin{tabular}{|c|c|c|c|}
\hline $\mathrm{Mo}(1)-\mathrm{Cl}(1)$ & $2.4292(8)$ & $C(10)-C(12)$ & $1.411(5)$ \\
\hline $\operatorname{Mo}(1)-\mathrm{Cl}(1) \# 1$ & $2.4292(8)$ & $\mathrm{C}(11)-\mathrm{C}(11) \# 1$ & $1.352(8)$ \\
\hline $\mathrm{Mo}(1)-\mathrm{Cl}(2)$ & $2.4220(13)$ & $\mathrm{C}(11)-\mathrm{H}(11)$ & 0.9300 \\
\hline $\mathrm{Mo}(1)-\mathrm{Cl}(3)$ & $2.4325(13)$ & $\mathrm{C}(12)-\mathrm{C}(12) \# 1$ & $1.426(7)$ \\
\hline $\mathrm{Mo}(1)-\mathrm{N}(1)$ & $2.171(3)$ & Co(1)-C(14) & $2.024(4)$ \\
\hline $\mathrm{Mo}(1)-\mathrm{N}(1) \# 1$ & $2.171(3)$ & $\mathrm{Co}(1)-\mathrm{C}(15)$ & $2.022(4)$ \\
\hline $\mathrm{N}(1)-\mathrm{C}(1)$ & $1.321(5)$ & $\mathrm{Co}(1)-\mathrm{C}(16)$ & $2.021(4)$ \\
\hline $\mathrm{N}(1)-\mathrm{C}(6)$ & $1.366(4)$ & $\mathrm{Co}(1)-\mathrm{C}(17)$ & $2.023(4)$ \\
\hline $\mathrm{C}(1)-\mathrm{H}(1)$ & 0.9300 & $\mathrm{Co}(1)-\mathrm{C}(18)$ & $2.016(8)$ \\
\hline $\mathrm{C}(1)-\mathrm{C}(2)$ & $1.404(5)$ & $\mathrm{Co}(1)-\mathrm{C}(19)$ & $1.982(8)$ \\
\hline $\mathrm{C}(2)-\mathrm{H}(2)$ & 0.9300 & $\mathrm{Co}(1)-\mathrm{C}(20)$ & $2.011(8)$ \\
\hline $\mathrm{C}(2)-\mathrm{C}(3)$ & $1.369(5)$ & $\mathrm{Co}(1)-\mathrm{C}(21)$ & $2.018(8)$ \\
\hline $\mathrm{C}(3)-\mathrm{H}(3)$ & 0.9300 & $\mathrm{Co}(1)-\mathrm{C}(22)$ & $2.002(8)$ \\
\hline $\mathrm{C}(3)-\mathrm{C}(4)$ & $1.396(5)$ & $\mathrm{Co}(1)-\mathrm{C}\left(18^{\prime}\right)$ & $2.03(2)$ \\
\hline $\mathrm{C}(4)-\mathrm{C}(5)$ & $1.439(5)$ & $\mathrm{Co}(1)-\mathrm{C}\left(21^{\prime}\right)$ & $2.00(2)$ \\
\hline$C(4)-C(6)$ & $1.402(5)$ & $\mathrm{Co}(1)-\mathrm{C}\left(22^{\prime}\right)$ & $2.02(2)$ \\
\hline$C(5)-C(5) \# 1$ & $1.360(8)$ & $\mathrm{C}(13)-\mathrm{H}(13)$ & 0.9300 \\
\hline $\mathrm{C}(5)-\mathrm{H}(5)$ & 0.9300 & $C(13)-C(14)$ & $1.413(6)$ \\
\hline $\mathrm{C}(6)-\mathrm{C}(6) \# 1$ & $1.436(7)$ & $\mathrm{C}(13)-\mathrm{C}(17)$ & $1.419(6)$ \\
\hline $\mathrm{Mo}(2)-\mathrm{Cl}(4)$ & $2.4275(8)$ & $\mathrm{C}(14)-\mathrm{H}(14)$ & 0.9300 \\
\hline $\mathrm{Mo}(2)-\mathrm{Cl}(4) \# 1$ & $2.4275(8)$ & $C(14)-C(15)$ & $1.412(6)$ \\
\hline $\mathrm{Mo}(2)-\mathrm{Cl}(5)$ & $2.4160(13)$ & $\mathrm{C}(15)-\mathrm{H}(15)$ & 0.9300 \\
\hline $\mathrm{Mo}(2)-\mathrm{Cl}(6)$ & $2.4390(13)$ & $C(15)-C(16)$ & $1.406(6)$ \\
\hline $\mathrm{Mo}(2)-\mathrm{N}(2)$ & $2.172(3)$ & $\mathrm{C}(16)-\mathrm{H}(16)$ & 0.9300 \\
\hline $\mathrm{Mo}(2)-\mathrm{N}(2) \# 1$ & $2.172(3)$ & $C(16)-C(17)$ & $1.428(6)$ \\
\hline $\mathrm{N}(2)-\mathrm{C}(7)$ & $1.331(4)$ & $\mathrm{C}(17)-\mathrm{H}(17)$ & 0.9300 \\
\hline $\mathrm{N}(2)-\mathrm{C}(12)$ & $1.374(4)$ & $\mathrm{C}(18)-\mathrm{H}(18)$ & 0.9300 \\
\hline $\mathrm{C}(7)-\mathrm{H}(7)$ & 0.9300 & $\mathrm{C}(18)-\mathrm{C}(19)$ & $1.412(10)$ \\
\hline$C(7)-C(8)$ & $1.398(5)$ & $C(18)-C(22)$ & $1.406(10)$ \\
\hline $\mathrm{C}(8)-\mathrm{H}(8)$ & 0.9300 & $\mathrm{C}(19)-\mathrm{H}(19)$ & 0.9300 \\
\hline $\mathrm{C}(8)-\mathrm{C}(9)$ & $1.359(5)$ & $C(19)-C(20)$ & $1.399(10)$ \\
\hline $\mathrm{C}(9)-\mathrm{H}(9)$ & 0.9300 & $\mathrm{C}(20)-\mathrm{H}(20)$ & 0.9300 \\
\hline$C(9)-C(10)$ & $1.399(5)$ & $C(20)-C(21)$ & $1.382(9)$ \\
\hline $\mathrm{C}(10)-\mathrm{C}(11)$ & $1.436(5)$ & $\mathrm{C}(21)-\mathrm{H}(21)$ & 0.9300 \\
\hline
\end{tabular}




\begin{tabular}{|c|c|}
\hline $\mathrm{C}(21)-\mathrm{C}(22)$ & $1.405(10)$ \\
\hline $\mathrm{C}(22)-\mathrm{H}(22)$ & 0.9300 \\
\hline $\mathrm{C}\left(18^{\prime}\right)-\mathrm{H}\left(18^{\prime}\right)$ & 0.9300 \\
\hline $\mathrm{C}\left(18^{\prime}\right)-\mathrm{C}\left(19^{\prime}\right)$ & $1.395(13)$ \\
\hline $\mathrm{C}\left(18^{\prime}\right)-\mathrm{C}\left(22^{\prime}\right)$ & $1.399(13)$ \\
\hline $\mathrm{C}\left(19^{\prime}\right)-\mathrm{H}\left(19^{\prime}\right)$ & 0.9300 \\
\hline $\mathrm{C}\left(19^{\prime}\right)-\mathrm{C}\left(20^{\prime}\right)$ & $1.394(13)$ \\
\hline $\mathrm{C}\left(20^{\prime}\right)-\mathrm{H}\left(20^{\prime}\right)$ & 0.9300 \\
\hline$C\left(20^{\prime}\right)-C\left(21^{\prime}\right)$ & $1.400(13)$ \\
\hline $\mathrm{C}\left(21^{\prime}\right)-\mathrm{H}\left(21^{\prime}\right)$ & 0.9300 \\
\hline $\mathrm{C}\left(21^{\prime}\right)-\mathrm{C}\left(22^{\prime}\right)$ & $1.395(13)$ \\
\hline $\mathrm{C}\left(22^{\prime}\right)-\mathrm{H}\left(22^{\prime}\right)$ & 0.9300 \\
\hline $\mathrm{Cl}(1) \# 1-\mathrm{Mo}(1)-\mathrm{Cl}(1)$ & $95.61(4)$ \\
\hline $\mathrm{Cl}(1) \# 1-\mathrm{Mo}(1)-\mathrm{Cl}(3)$ & $92.71(3)$ \\
\hline $\mathrm{Cl}(1)-\mathrm{Mo}(1)-\mathrm{Cl}(3)$ & $92.71(3)$ \\
\hline $\mathrm{Cl}(2)-\mathrm{Mo}(1)-\mathrm{Cl}(1) \# 1$ & $91.68(3)$ \\
\hline $\mathrm{Cl}(2)-\mathrm{Mo}(1)-\mathrm{Cl}(1)$ & $91.68(3)$ \\
\hline $\mathrm{Cl}(2)-\mathrm{Mo}(1)-\mathrm{Cl}(3)$ & $173.46(4)$ \\
\hline $\mathrm{N}(1)-\mathrm{Mo}(1)-\mathrm{Cl}(1) \# 1$ & $170.54(8)$ \\
\hline $\mathrm{N}(1) \# 1-\mathrm{Mo}(1)-\mathrm{Cl}(1) \# 1$ & $93.69(8)$ \\
\hline $\mathrm{N}(1) \# 1-\mathrm{Mo}(1)-\mathrm{Cl}(1)$ & $170.55(8)$ \\
\hline $\mathrm{N}(1)-\mathrm{Mo}(1)-\mathrm{Cl}(1)$ & $93.70(8)$ \\
\hline $\mathrm{N}(1)-\mathrm{Mo}(1)-\mathrm{Cl}(2)$ & $86.38(8)$ \\
\hline $\mathrm{N}(1) \# 1-\mathrm{Mo}(1)-\mathrm{Cl}(2)$ & $86.38(8)$ \\
\hline $\mathrm{N}(1) \# 1-\mathrm{Mo}(1)-\mathrm{Cl}(3)$ & $88.50(8)$ \\
\hline $\mathrm{N}(1)-\mathrm{Mo}(1)-\mathrm{Cl}(3)$ & $88.50(8)$ \\
\hline $\mathrm{N}(1) \# 1-\mathrm{Mo}(1)-\mathrm{N}(1)$ & $76.96(15)$ \\
\hline $\mathrm{C}(1)-\mathrm{N}(1)-\mathrm{Mo}(1)$ & $127.5(2)$ \\
\hline $\mathrm{C}(1)-\mathrm{N}(1)-\mathrm{C}(6)$ & $118.6(3)$ \\
\hline $\mathrm{C}(6)-\mathrm{N}(1)-\mathrm{Mo}(1)$ & $113.8(2)$ \\
\hline $\mathrm{N}(1)-\mathrm{C}(1)-\mathrm{H}(1)$ & 118.8 \\
\hline $\mathrm{N}(1)-\mathrm{C}(1)-\mathrm{C}(2)$ & $122.4(3)$ \\
\hline $\mathrm{C}(2)-\mathrm{C}(1)-\mathrm{H}(1)$ & 118.8 \\
\hline $\mathrm{C}(1)-\mathrm{C}(2)-\mathrm{H}(2)$ & 120.3 \\
\hline $\mathrm{C}(3)-\mathrm{C}(2)-\mathrm{C}(1)$ & $119.3(3)$ \\
\hline $\mathrm{C}(3)-\mathrm{C}(2)-\mathrm{H}(2)$ & 120.3 \\
\hline
\end{tabular}

\begin{tabular}{|c|c|}
\hline $\mathrm{C}(2)-\mathrm{C}(3)-\mathrm{H}(3)$ & 120.2 \\
\hline$C(2)-C(3)-C(4)$ & $119.6(4)$ \\
\hline $\mathrm{C}(4)-\mathrm{C}(3)-\mathrm{H}(3)$ & 120.2 \\
\hline$C(3)-C(4)-C(5)$ & $123.6(4)$ \\
\hline$C(3)-C(4)-C(6)$ & $117.9(3)$ \\
\hline$C(6)-C(4)-C(5)$ & $118.5(3)$ \\
\hline $\mathrm{C}(4)-\mathrm{C}(5)-\mathrm{H}(5)$ & 119.4 \\
\hline$C(5) \# 1-C(5)-C(4)$ & $121.2(2)$ \\
\hline $\mathrm{C}(5) \# 1-\mathrm{C}(5)-\mathrm{H}(5)$ & 119.4 \\
\hline $\mathrm{N}(1)-\mathrm{C}(6)-\mathrm{C}(4)$ & $122.1(3)$ \\
\hline $\mathrm{N}(1)-\mathrm{C}(6)-\mathrm{C}(6) \# 1$ & $117.57(19)$ \\
\hline$C(4)-C(6)-C(6) \# 1$ & $120.3(2)$ \\
\hline $\mathrm{Cl}(4)-\mathrm{Mo}(2)-\mathrm{Cl}(4) \# 1$ & $95.22(4)$ \\
\hline $\mathrm{Cl}(4)-\mathrm{Mo}(2)-\mathrm{Cl}(6)$ & $91.79(3)$ \\
\hline $\mathrm{Cl}(4) \# 1-\mathrm{Mo}(2)-\mathrm{Cl}(6)$ & $91.78(3)$ \\
\hline $\mathrm{Cl}(5)-\mathrm{Mo}(2)-\mathrm{Cl}(4) \# 1$ & $91.27(3)$ \\
\hline $\mathrm{Cl}(5)-\mathrm{Mo}(2)-\mathrm{Cl}(4)$ & $91.27(3)$ \\
\hline $\mathrm{Cl}(5)-\mathrm{Mo}(2)-\mathrm{Cl}(6)$ & $175.47(4)$ \\
\hline $\mathrm{N}(2)-\mathrm{Mo}(2)-\mathrm{Cl}(4) \# 1$ & $170.48(8)$ \\
\hline $\mathrm{N}(2) \# 1-\mathrm{Mo}(2)-\mathrm{Cl}(4)$ & $170.48(8)$ \\
\hline $\mathrm{N}(2)-\mathrm{Mo}(2)-\mathrm{Cl}(4)$ & $94.10(8)$ \\
\hline $\mathrm{N}(2) \# 1-\mathrm{Mo}(2)-\mathrm{Cl}(4) \# 1$ & $94.10(8)$ \\
\hline $\mathrm{N}(2) \# 1-\mathrm{Mo}(2)-\mathrm{Cl}(5)$ & $86.58(8)$ \\
\hline $\mathrm{N}(2)-\mathrm{Mo}(2)-\mathrm{Cl}(5)$ & $86.58(8)$ \\
\hline $\mathrm{N}(2)-\mathrm{Mo}(2)-\mathrm{Cl}(6)$ & $89.86(8)$ \\
\hline $\mathrm{N}(2) \# 1-\mathrm{Mo}(2)-\mathrm{Cl}(6)$ & $89.86(8)$ \\
\hline $\mathrm{N}(2) \# 1-\mathrm{Mo}(2)-\mathrm{N}(2)$ & $76.53(15)$ \\
\hline $\mathrm{C}(7)-\mathrm{N}(2)-\mathrm{Mo}(2)$ & $128.0(2)$ \\
\hline$C(7)-N(2)-C(12)$ & $117.8(3)$ \\
\hline $\mathrm{C}(12)-\mathrm{N}(2)-\mathrm{Mo}(2)$ & $114.1(2)$ \\
\hline $\mathrm{N}(2)-\mathrm{C}(7)-\mathrm{H}(7)$ & 118.6 \\
\hline $\mathrm{N}(2)-\mathrm{C}(7)-\mathrm{C}(8)$ & $122.7(3)$ \\
\hline $\mathrm{C}(8)-\mathrm{C}(7)-\mathrm{H}(7)$ & 118.6 \\
\hline $\mathrm{C}(7)-\mathrm{C}(8)-\mathrm{H}(8)$ & 120.1 \\
\hline$C(9)-C(8)-C(7)$ & $119.7(3)$ \\
\hline $\mathrm{C}(9)-\mathrm{C}(8)-\mathrm{H}(8)$ & 120.1 \\
\hline
\end{tabular}




\begin{tabular}{|c|c|}
\hline $\mathrm{C}(8)-\mathrm{C}(9)-\mathrm{H}(9)$ & 120.0 \\
\hline $\mathrm{C}(8)-\mathrm{C}(9)-\mathrm{C}(10)$ & 119.9(3) \\
\hline $\mathrm{C}(10)-\mathrm{C}(9)-\mathrm{H}(9)$ & 120.0 \\
\hline $\mathrm{C}(9)-\mathrm{C}(10)-\mathrm{C}(11)$ & $124.3(3)$ \\
\hline$C(9)-C(10)-C(12)$ & $117.5(3)$ \\
\hline $\mathrm{C}(12)-\mathrm{C}(10)-\mathrm{C}(11)$ & $118.2(3)$ \\
\hline $\mathrm{C}(10)-\mathrm{C}(11)-\mathrm{H}(11)$ & 119.3 \\
\hline $\mathrm{C}(11) \# 1-\mathrm{C}(11)-\mathrm{C}(10)$ & $121.4(2)$ \\
\hline $\mathrm{C}(11) \# 1-\mathrm{C}(11)-\mathrm{H}(11)$ & 119.3 \\
\hline $\mathrm{N}(2)-\mathrm{C}(12)-\mathrm{C}(10)$ & $122.3(3)$ \\
\hline $\mathrm{N}(2)-\mathrm{C}(12)-\mathrm{C}(12) \# 1$ & $117.40(18)$ \\
\hline $\mathrm{C}(10)-\mathrm{C}(12)-\mathrm{C}(12) \# 1$ & $120.3(2)$ \\
\hline $\mathrm{C}(14)-\mathrm{Co}(1)-\mathrm{C}\left(18^{\prime}\right)$ & $148.5(11)$ \\
\hline $\mathrm{C}(15)-\mathrm{Co}(1)-\mathrm{C}(14)$ & $40.86(18)$ \\
\hline $\mathrm{C}(15)-\mathrm{Co}(1)-\mathrm{C}(17)$ & $68.90(17)$ \\
\hline $\mathrm{C}(15)-\mathrm{Co}(1)-\mathrm{C}\left(18^{\prime}\right)$ & $117.7(7)$ \\
\hline $\mathrm{C}(15)-\mathrm{Co}(1)-\mathrm{C}\left(22^{\prime}\right)$ & $110.7(5)$ \\
\hline $\mathrm{C}(16)-\mathrm{Co}(1)-\mathrm{C}(14)$ & $68.84(18)$ \\
\hline $\mathrm{C}(16)-\mathrm{Co}(1)-\mathrm{C}(15)$ & $40.70(17)$ \\
\hline $\mathrm{C}(16)-\mathrm{Co}(1)-\mathrm{C}(17)$ & $41.35(17)$ \\
\hline $\mathrm{C}(16)-\mathrm{Co}(1)-\mathrm{C}\left(18^{\prime}\right)$ & $110.8(6)$ \\
\hline$C(16)-\operatorname{Co}(1)-C\left(22^{\prime}\right)$ & $133.9(9)$ \\
\hline $\mathrm{C}(17)-\mathrm{Co}(1)-\mathrm{C}(14)$ & $68.84(17)$ \\
\hline $\mathrm{C}(17)-\mathrm{Co}(1)-\mathrm{C}\left(18^{\prime}\right)$ & $133.0(10)$ \\
\hline $\mathrm{C}(18)-\mathrm{Co}(1)-\mathrm{C}(14)$ & $119.5(3)$ \\
\hline $\mathrm{C}(18)-\mathrm{Co}(1)-\mathrm{C}(15)$ & $151.9(5)$ \\
\hline $\mathrm{C}(18)-\mathrm{Co}(1)-\mathrm{C}(16)$ & 167.1(6) \\
\hline $\mathrm{C}(18)-\mathrm{Co}(1)-\mathrm{C}(17)$ & $129.7(5)$ \\
\hline $\mathrm{C}(18)-\mathrm{Co}(1)-\mathrm{C}(21)$ & $68.0(3)$ \\
\hline $\mathrm{C}(19)-\mathrm{Co}(1)-\mathrm{C}(14)$ & $108.1(3)$ \\
\hline $\mathrm{C}(19)-\mathrm{Co}(1)-\mathrm{C}(15)$ & $116.5(4)$ \\
\hline $\mathrm{C}(19)-\mathrm{Co}(1)-\mathrm{C}(16)$ & $149.0(5)$ \\
\hline $\mathrm{C}(19)-\mathrm{Co}(1)-\mathrm{C}(17)$ & $168.6(5)$ \\
\hline $\mathrm{C}(19)-\mathrm{Co}(1)-\mathrm{C}(18)$ & $41.4(3)$ \\
\hline $\mathrm{C}(19)-\mathrm{Co}(1)-\mathrm{C}(20)$ & $41.0(3)$ \\
\hline$C(19)-C o(1)-C(21)$ & $68.4(4)$ \\
\hline
\end{tabular}

\begin{tabular}{|c|c|}
\hline $\mathrm{C}(19)-\mathrm{Co}(1)-\mathrm{C}(22)$ & $69.9(4)$ \\
\hline $\mathrm{C}(20)-\mathrm{Co}(1)-\mathrm{C}(14)$ & $128.2(3)$ \\
\hline $\mathrm{C}(20)-\mathrm{Co}(1)-\mathrm{C}(15)$ & $106.3(2)$ \\
\hline $\mathrm{C}(20)-\mathrm{Co}(1)-\mathrm{C}(16)$ & $115.2(3)$ \\
\hline $\mathrm{C}(20)-\mathrm{Co}(1)-\mathrm{C}(17)$ & $149.5(4)$ \\
\hline $\mathrm{C}(20)-\mathrm{Co}(1)-\mathrm{C}(18)$ & $68.6(4)$ \\
\hline $\mathrm{C}(20)-\mathrm{Co}(1)-\mathrm{C}(21)$ & $40.1(3)$ \\
\hline $\mathrm{C}(21)-\mathrm{Co}(1)-\mathrm{C}(14)$ & $165.5(4)$ \\
\hline $\mathrm{C}(21)-\mathrm{Co}(1)-\mathrm{C}(15)$ & $126.8(4)$ \\
\hline $\mathrm{C}(21)-\mathrm{Co}(1)-\mathrm{C}(16)$ & $106.4(3)$ \\
\hline $\mathrm{C}(21)-\mathrm{Co}(1)-\mathrm{C}(17)$ & $117.3(3)$ \\
\hline $\mathrm{C}(22)-\mathrm{Co}(1)-\mathrm{C}(14)$ & $152.7(5)$ \\
\hline $\mathrm{C}(22)-\mathrm{Co}(1)-\mathrm{C}(15)$ & $165.2(5)$ \\
\hline $\mathrm{C}(22)-\mathrm{Co}(1)-\mathrm{C}(16)$ & $127.3(5)$ \\
\hline $\mathrm{C}(22)-\mathrm{Co}(1)-\mathrm{C}(17)$ & $107.5(3)$ \\
\hline $\mathrm{C}(22)-\mathrm{Co}(1)-\mathrm{C}(18)$ & $41.0(3)$ \\
\hline $\mathrm{C}(22)-\mathrm{Co}(1)-\mathrm{C}(20)$ & $69.0(3)$ \\
\hline $\mathrm{C}(22)-\mathrm{Co}(1)-\mathrm{C}(21)$ & $40.9(3)$ \\
\hline $\mathrm{C}\left(21^{\prime}\right)-\mathrm{Co}(1)-\mathrm{C}(14)$ & $106.7(5)$ \\
\hline $\mathrm{C}\left(21^{\prime}\right)-\mathrm{Co}(1)-\mathrm{C}(15)$ & $131.3(7)$ \\
\hline $\mathrm{C}\left(21^{\prime}\right)-\mathrm{Co}(1)-\mathrm{C}(16)$ & $171.4(8)$ \\
\hline $\mathrm{C}\left(21^{\prime}\right)-\mathrm{Co}(1)-\mathrm{C}(17)$ & $145.0(8)$ \\
\hline $\mathrm{C}\left(21^{\prime}\right)-\mathrm{Co}(1)-\mathrm{C}\left(18^{\prime}\right)$ & $68.9(6)$ \\
\hline $\mathrm{C}\left(21^{\prime}\right)-\mathrm{Co}(1)-\mathrm{C}\left(22^{\prime}\right)$ & $40.6(5)$ \\
\hline $\mathrm{C}\left(22^{\prime}\right)-\mathrm{Co}(1)-\mathrm{C}(14)$ & $115.8(7)$ \\
\hline $\mathrm{C}\left(22^{\prime}\right)-\mathrm{Co}(1)-\mathrm{C}(17)$ & $172.9(11)$ \\
\hline $\mathrm{C}\left(22^{\prime}\right)-\mathrm{Co}(1)-\mathrm{C}\left(18^{\prime}\right)$ & $40.4(5)$ \\
\hline $\mathrm{Co}(1)-\mathrm{C}(13)-\mathrm{H}(13)$ & 127.0 \\
\hline $\mathrm{C}(14)-\mathrm{C}(13)-\mathrm{Co}(1)$ & $69.3(2)$ \\
\hline $\mathrm{C}(14)-\mathrm{C}(13)-\mathrm{H}(13)$ & 126.1 \\
\hline $\mathrm{C}(14)-\mathrm{C}(13)-\mathrm{C}(17)$ & $107.8(4)$ \\
\hline $\mathrm{C}(17)-\mathrm{C}(13)-\mathrm{Co}(1)$ & $69.2(2)$ \\
\hline $\mathrm{C}(17)-\mathrm{C}(13)-\mathrm{H}(13)$ & 126.1 \\
\hline $\mathrm{Co}(1)-\mathrm{C}(14)-\mathrm{H}(14)$ & 126.3 \\
\hline $\mathrm{C}(13)-\mathrm{C}(14)-\mathrm{Co}(1)$ & $70.0(2)$ \\
\hline $\mathrm{C}(13)-\mathrm{C}(14)-\mathrm{H}(14)$ & 125.9 \\
\hline
\end{tabular}




\begin{tabular}{|c|c|c|c|}
\hline $\mathrm{C}(15)-\mathrm{C}(14)-\mathrm{Co}(1)$ & $69.5(2)$ & $\mathrm{C}(21)-\mathrm{C}(20)-\mathrm{Co}(1)$ & $70.2(5)$ \\
\hline$C(15)-C(14)-C(13)$ & $108.3(4)$ & $C(21)-C(20)-C(19)$ & $108.0(6)$ \\
\hline $\mathrm{C}(15)-\mathrm{C}(14)-\mathrm{H}(14)$ & 125.9 & $\mathrm{C}(21)-\mathrm{C}(20)-\mathrm{H}(20)$ & 126.0 \\
\hline $\mathrm{Co}(1)-\mathrm{C}(15)-\mathrm{H}(15)$ & 126.5 & $\mathrm{Co}(1)-\mathrm{C}(21)-\mathrm{H}(21)$ & 127.7 \\
\hline$C(14)-C(15)-\operatorname{Co}(1)$ & $69.7(2)$ & $\mathrm{C}(20)-\mathrm{C}(21)-\mathrm{Co}(1)$ & $69.7(5)$ \\
\hline $\mathrm{C}(14)-\mathrm{C}(15)-\mathrm{H}(15)$ & 125.8 & $\mathrm{C}(20)-\mathrm{C}(21)-\mathrm{H}(21)$ & 125.3 \\
\hline$C(16)-C(15)-C o(1)$ & $69.6(2)$ & $C(20)-C(21)-C(22)$ & $109.4(7)$ \\
\hline$C(16)-C(15)-C(14)$ & $108.5(4)$ & $\mathrm{C}(22)-\mathrm{C}(21)-\mathrm{Co}(1)$ & $68.9(5)$ \\
\hline $\mathrm{C}(16)-\mathrm{C}(15)-\mathrm{H}(15)$ & 125.8 & $\mathrm{C}(22)-\mathrm{C}(21)-\mathrm{H}(21)$ & 125.3 \\
\hline $\mathrm{Co}(1)-\mathrm{C}(16)-\mathrm{H}(16)$ & 126.3 & $\mathrm{Co}(1)-\mathrm{C}(22)-\mathrm{H}(22)$ & 124.7 \\
\hline $\mathrm{C}(15)-\mathrm{C}(16)-\mathrm{Co}(1)$ & $69.7(2)$ & $\mathrm{C}(18)-\mathrm{C}(22)-\mathrm{Co}(1)$ & $70.1(5)$ \\
\hline $\mathrm{C}(15)-\mathrm{C}(16)-\mathrm{H}(16)$ & 126.1 & $\mathrm{C}(18)-\mathrm{C}(22)-\mathrm{H}(22)$ & 126.7 \\
\hline$C(15)-C(16)-C(17)$ & $107.7(4)$ & $\mathrm{C}(21)-\mathrm{C}(22)-\mathrm{Co}(1)$ & $70.2(5)$ \\
\hline$C(17)-C(16)-C o(1)$ & $69.4(2)$ & $\mathrm{C}(21)-\mathrm{C}(22)-\mathrm{C}(18)$ & $106.7(7)$ \\
\hline $\mathrm{C}(17)-\mathrm{C}(16)-\mathrm{H}(16)$ & 126.1 & $\mathrm{C}(21)-\mathrm{C}(22)-\mathrm{H}(22)$ & 126.7 \\
\hline $\mathrm{Co}(1)-\mathrm{C}(17)-\mathrm{H}(17)$ & 126.4 & $\mathrm{Co}(1)-\mathrm{C}\left(18^{\prime}\right)-\mathrm{H}\left(18^{\prime}\right)$ & 123.5 \\
\hline$C(13)-C(17)-C o(1)$ & $69.9(2)$ & $\mathrm{C}\left(19^{\prime}\right)-\mathrm{C}\left(18^{\prime}\right)-\mathrm{Co}(1)$ & $71.6(12)$ \\
\hline$C(13)-C(17)-C(16)$ & $107.8(4)$ & $\mathrm{C}\left(19^{\prime}\right)-\mathrm{C}\left(18^{\prime}\right)-\mathrm{H}\left(18^{\prime}\right)$ & 126.9 \\
\hline $\mathrm{C}(13)-\mathrm{C}(17)-\mathrm{H}(17)$ & 126.1 & $\mathrm{C}\left(19^{\prime}\right)-\mathrm{C}\left(18^{\prime}\right)-\mathrm{C}\left(22^{\prime}\right)$ & $106.1(10)$ \\
\hline$C(16)-C(17)-C o(1)$ & $69.2(2)$ & $\mathrm{C}\left(22^{\prime}\right)-\mathrm{C}\left(18^{\prime}\right)-\mathrm{Co}(1)$ & $69.6(13)$ \\
\hline $\mathrm{C}(16)-\mathrm{C}(17)-\mathrm{H}(17)$ & 126.1 & $\mathrm{C}\left(22^{\prime}\right)-\mathrm{C}\left(18^{\prime}\right)-\mathrm{H}\left(18^{\prime}\right)$ & 126.9 \\
\hline $\mathrm{Co}(1)-\mathrm{C}(18)-\mathrm{H}(18)$ & 128.6 & $\mathrm{Co}(1)-\mathrm{C}\left(19^{\prime}\right)-\mathrm{H}\left(19^{\prime}\right)$ & 129.1 \\
\hline C(19)-C(18)-Co(1) & $68.0(4)$ & $\mathrm{C}\left(18^{\prime}\right)-\mathrm{C}\left(19^{\prime}\right)-\mathrm{Co}(1)$ & $68.5(12)$ \\
\hline $\mathrm{C}(19)-\mathrm{C}(18)-\mathrm{H}(18)$ & 126.0 & $\mathrm{C}\left(18^{\prime}\right)-\mathrm{C}\left(19^{\prime}\right)-\mathrm{H}\left(19^{\prime}\right)$ & 125.2 \\
\hline $\mathrm{C}(22)-\mathrm{C}(18)-\mathrm{Co}(1)$ & $69.0(5)$ & $\mathrm{C}\left(20^{\prime}\right)-\mathrm{C}\left(19^{\prime}\right)-\mathrm{Co}(1)$ & $68.8(12)$ \\
\hline $\mathrm{C}(22)-\mathrm{C}(18)-\mathrm{H}(18)$ & 126.0 & $\mathrm{C}\left(20^{\prime}\right)-\mathrm{C}\left(19^{\prime}\right)-\mathrm{C}\left(18^{\prime}\right)$ & $109.6(11)$ \\
\hline$C(22)-C(18)-C(19)$ & $108.1(6)$ & $\mathrm{C}\left(20^{\prime}\right)-\mathrm{C}\left(19^{\prime}\right)-\mathrm{H}\left(19^{\prime}\right)$ & 125.2 \\
\hline $\mathrm{Co}(1)-\mathrm{C}(19)-\mathrm{H}(19)$ & 124.2 & $\mathrm{Co}(1)-\mathrm{C}\left(20^{\prime}\right)-\mathrm{H}\left(20^{\prime}\right)$ & 125.2 \\
\hline C(18)-C(19)-Co(1) & $70.6(5)$ & $\mathrm{C}\left(19^{\prime}\right)-\mathrm{C}\left(20^{\prime}\right)-\mathrm{Co}(1)$ & $71.5(12)$ \\
\hline $\mathrm{C}(18)-\mathrm{C}(19)-\mathrm{H}(19)$ & 126.2 & $\mathrm{C}\left(19^{\prime}\right)-\mathrm{C}\left(20^{\prime}\right)-\mathrm{H}\left(20^{\prime}\right)$ & 126.4 \\
\hline $\mathrm{C}(20)-\mathrm{C}(19)-\mathrm{Co}(1)$ & $70.6(4)$ & $\mathrm{C}\left(19^{\prime}\right)-\mathrm{C}\left(20^{\prime}\right)-\mathrm{C}\left(21^{\prime}\right)$ & $107.3(11)$ \\
\hline$C(20)-C(19)-C(18)$ & $107.6(6)$ & $\mathrm{C}\left(21^{\prime}\right)-\mathrm{C}\left(20^{\prime}\right)-\mathrm{Co}(1)$ & $68.5(11)$ \\
\hline $\mathrm{C}(20)-\mathrm{C}(19)-\mathrm{H}(19)$ & 126.2 & $\mathrm{C}\left(21^{\prime}\right)-\mathrm{C}\left(20^{\prime}\right)-\mathrm{H}\left(20^{\prime}\right)$ & 126.4 \\
\hline $\mathrm{Co}(1)-\mathrm{C}(20)-\mathrm{H}(20)$ & 127.0 & $\mathrm{Co}(1)-\mathrm{C}\left(21^{\prime}\right)-\mathrm{H}\left(21^{\prime}\right)$ & 123.9 \\
\hline C(19)-C(20)-Co(1) & $68.4(4)$ & $\mathrm{C}\left(20^{\prime}\right)-\mathrm{C}\left(21^{\prime}\right)-\mathrm{Co}(1)$ & $70.9(12)$ \\
\hline $\mathrm{C}(19)-\mathrm{C}(20)-\mathrm{H}(20)$ & 126.0 & $\mathrm{C}\left(20^{\prime}\right)-\mathrm{C}\left(21^{\prime}\right)-\mathrm{H}\left(21^{\prime}\right)$ & 126.3 \\
\hline
\end{tabular}




$\begin{array}{llll}\mathrm{C}\left(22^{\prime}\right)-\mathrm{C}\left(21^{\prime}\right)-\mathrm{Co}(1) & 70.6(13) & \mathrm{C}\left(18^{\prime}\right)-\mathrm{C}\left(22^{\prime}\right)-\mathrm{H}\left(22^{\prime}\right) & 125.4 \\ \mathrm{C}\left(22^{\prime}\right)-\mathrm{C}\left(21^{\prime}\right)-\mathrm{C}\left(20^{\prime}\right) & 107.5(11) & \mathrm{C}\left(21^{\prime}\right)-\mathrm{C}\left(22^{\prime}\right)-\mathrm{Co}(1) & 68.9(12) \\ \mathrm{C}\left(22^{\prime}\right)-\mathrm{C}\left(21^{\prime}\right)-\mathrm{H}\left(21^{\prime}\right) & 126.3 & \mathrm{C}\left(21^{\prime}\right)-\mathrm{C}\left(22^{\prime}\right)-\mathrm{C}\left(18^{\prime}\right) & 109.3(11) \\ \mathrm{Co}(1)-\mathrm{C}\left(22^{\prime}\right)-\mathrm{H}\left(22^{\prime}\right) & 127.4 & \mathrm{C}\left(21^{\prime}\right)-\mathrm{C}\left(22^{\prime}\right)-\mathrm{H}\left(22^{\prime}\right) & 125.4 \\ \mathrm{C}\left(18^{\prime}\right)-\mathrm{C}\left(22^{\prime}\right)-\mathrm{Co}(1) & 70.0(13) & & \end{array}$

Symmetry transformations used to generate equivalent atoms:

$\# 1 \mathrm{x}, \mathrm{y},-\mathrm{z}+1$ 
Table 4. Anisotropic displacement parameters $\left(\AA^{2} \times 10^{3}\right)$ for rhowe29. The anisotropic displacement factor exponent takes the form: $-2 \pi^{2}\left[h^{2} a^{* 2} U_{11}+\ldots+2 h k a^{*} b^{*} U_{12}\right]$

\begin{tabular}{|c|c|c|c|c|c|c|}
\hline & $\mathrm{U}_{11}$ & $\mathrm{U}_{22}$ & $\mathrm{U}_{33}$ & $\mathrm{U}_{23}$ & $\mathrm{U}_{13}$ & $\mathrm{U}_{12}$ \\
\hline Mo1 & $24(1)$ & $31(1)$ & $21(1)$ & 0 & 0 & $1(1)$ \\
\hline $\mathrm{Cl1}$ & $30(1)$ & $44(1)$ & $27(1)$ & $2(1)$ & $-5(1)$ & $-1(1)$ \\
\hline $\mathrm{Cl} 2$ & $41(1)$ & $32(1)$ & $26(1)$ & 0 & 0 & $0(1)$ \\
\hline $\mathrm{Cl} 3$ & $32(1)$ & $33(1)$ & $33(1)$ & 0 & 0 & $1(1)$ \\
\hline N1 & $26(1)$ & $31(1)$ & $22(1)$ & $-1(1)$ & $1(1)$ & $0(1)$ \\
\hline $\mathrm{C} 1$ & $27(2)$ & $39(2)$ & $30(2)$ & $0(2)$ & $0(1)$ & $-2(1)$ \\
\hline $\mathrm{C} 2$ & $37(2)$ & $50(2)$ & $25(2)$ & $-3(2)$ & $2(2)$ & $3(2)$ \\
\hline $\mathrm{C} 3$ & $32(2)$ & $57(3)$ & $31(2)$ & $-5(2)$ & $8(2)$ & $4(2)$ \\
\hline $\mathrm{C} 4$ & $29(2)$ & $51(2)$ & $34(2)$ & $1(2)$ & $0(2)$ & $5(2)$ \\
\hline $\mathrm{C} 5$ & $24(2)$ & $68(3)$ & $40(2)$ & $-1(2)$ & $3(2)$ & $9(2)$ \\
\hline C6 & $25(2)$ & $38(2)$ & $29(2)$ & $2(2)$ & $1(1)$ & $4(1)$ \\
\hline Mo2 & $21(1)$ & $34(1)$ & $20(1)$ & 0 & 0 & $-2(1)$ \\
\hline $\mathrm{Cl} 4$ & $26(1)$ & $48(1)$ & $26(1)$ & $-1(1)$ & $-5(1)$ & $-1(1)$ \\
\hline $\mathrm{Cl} 5$ & $34(1)$ & $35(1)$ & $31(1)$ & 0 & 0 & $-1(1)$ \\
\hline $\mathrm{Cl} 6$ & $29(1)$ & $34(1)$ & $33(1)$ & 0 & 0 & $0(1)$ \\
\hline N2 & $23(1)$ & $37(2)$ & $21(1)$ & $2(1)$ & $2(1)$ & $-1(1)$ \\
\hline C7 & $28(2)$ & $37(2)$ & $24(2)$ & $0(1)$ & $-1(1)$ & $1(1)$ \\
\hline $\mathrm{C} 8$ & $31(2)$ & $49(2)$ & $21(2)$ & $2(2)$ & $1(1)$ & $1(2)$ \\
\hline C9 & $27(2)$ & $55(2)$ & $30(2)$ & $1(2)$ & $6(1)$ & $-4(2)$ \\
\hline $\mathrm{C} 10$ & $28(2)$ & $54(2)$ & $28(2)$ & $2(2)$ & $3(2)$ & $-5(2)$ \\
\hline C11 & $19(2)$ & $72(3)$ & $34(2)$ & $1(2)$ & $2(1)$ & $-8(2)$ \\
\hline $\mathrm{C} 12$ & $20(2)$ & $43(2)$ & $27(2)$ & $1(2)$ & $2(1)$ & $-5(1)$ \\
\hline Co1 & $40(1)$ & $33(1)$ & $29(1)$ & $1(1)$ & $-7(1)$ & $-1(1)$ \\
\hline $\mathrm{C} 13$ & $45(2)$ & $39(2)$ & $42(2)$ & $7(2)$ & $-1(2)$ & $-3(2)$ \\
\hline $\mathrm{C} 14$ & $41(2)$ & $45(2)$ & $34(2)$ & $1(2)$ & $-8(2)$ & $-4(2)$ \\
\hline C15 & $39(2)$ & $50(2)$ & $33(2)$ & $6(2)$ & $-3(2)$ & $1(2)$ \\
\hline $\mathrm{C} 16$ & $49(2)$ & $41(2)$ & $38(2)$ & $-3(2)$ & $0(2)$ & $6(2)$ \\
\hline C17 & $52(2)$ & $51(3)$ & $27(2)$ & $0(2)$ & $-4(2)$ & $1(2)$ \\
\hline $\mathrm{C} 18$ & $45(4)$ & $32(3)$ & $123(11)$ & $-2(5)$ & $-17(7)$ & $10(3)$ \\
\hline C19 & $39(4)$ & $103(9)$ & $51(5)$ & $-41(6)$ & $13(4)$ & $-8(6)$ \\
\hline \multirow[t]{2}{*}{$\mathrm{C} 20$} & $39(4)$ & $57(5)$ & $49(4)$ & $12(4)$ & $-1(3)$ & $-11(3)$ \\
\hline & & & & S103 & & \\
\hline
\end{tabular}




\begin{tabular}{llccccc}
$\mathrm{C} 21$ & $40(4)$ & $59(5)$ & $72(6)$ & $-8(4)$ & $-13(4)$ & $-12(3)$ \\
$\mathrm{C} 22$ & $36(4)$ & $94(9)$ & $66(5)$ & $33(6)$ & $-29(3)$ & $-13(5)$ \\
$\mathrm{C} 18^{\prime}$ & $45(4)$ & $32(3)$ & $123(11)$ & $-2(5)$ & $-17(7)$ & $10(3)$ \\
$\mathrm{C} 19^{\prime}$ & $39(4)$ & $103(9)$ & $51(5)$ & $-41(6)$ & $13(4)$ & $-8(6)$ \\
$\mathrm{C} 20^{\prime}$ & $39(4)$ & $57(5)$ & $49(4)$ & $12(4)$ & $-1(3)$ & $-11(3)$ \\
$\mathrm{C} 21^{\prime}$ & $40(4)$ & $59(5)$ & $72(6)$ & $-8(4)$ & $-13(4)$ & $-12(3)$ \\
$\mathrm{C} 22^{\prime}$ & $36(4)$ & $94(9)$ & $66(5)$ & $33(6)$ & $-29(3)$ & $-13(5)$ \\
\hline
\end{tabular}


Table 5. Hydrogen coordinates $\left(\times 10^{4}\right)$ and isotropic displacement parameters $\left(\AA^{2} \times 10^{3}\right)$ for rhowe29.

\begin{tabular}{|c|c|c|c|c|}
\hline & $\mathrm{x}$ & $\mathrm{y}$ & z & $\mathrm{U}(\mathrm{eq})$ \\
\hline H1 & 6876 & 1657 & 7034 & 38 \\
\hline $\mathrm{H} 2$ & 5956 & 1812 & 7866 & 45 \\
\hline $\mathrm{H} 3$ & 4990 & 2022 & 7176 & 48 \\
\hline H5 & 4372 & 2162 & 5745 & 53 \\
\hline $\mathrm{H} 7$ & 3365 & 3584 & 2960 & 36 \\
\hline $\mathrm{H} 8$ & 4279 & 3771 & 2138 & 41 \\
\hline H9 & 5232 & 4016 & 2820 & 45 \\
\hline H11 & 5849 & 4187 & 4259 & 50 \\
\hline H13 & 1951 & -168 & 3303 & 50 \\
\hline H14 & 1708 & 226 & 1762 & 48 \\
\hline H15 & 1720 & 1631 & 1700 & 49 \\
\hline H16 & 1983 & 2119 & 3192 & 51 \\
\hline H17 & 2130 & 1003 & 4194 & 52 \\
\hline H18 & 3475 & -224 & 2488 & 80 \\
\hline H19 & 3169 & 476 & 1124 & 78 \\
\hline $\mathrm{H} 20$ & 3249 & 1847 & 1449 & 58 \\
\hline $\mathrm{H} 21$ & 3533 & 1994 & 3009 & 68 \\
\hline $\mathrm{H} 22$ & 3617 & 721 & 3692 & 78 \\
\hline H18' & 3443 & 2079 & 2243 & 80 \\
\hline H19' & 3711 & 1332 & 3574 & 78 \\
\hline $\mathrm{H} 20^{\prime}$ & 3513 & -18 & 3262 & 58 \\
\hline $\mathrm{H} 21^{\prime}$ & 3216 & -126 & 1680 & 68 \\
\hline H22' & 3239 & 1158 & 1041 & 78 \\
\hline
\end{tabular}


Table 6. Torsion angles $\left[{ }^{\circ}\right]$ for rhowe29.

\begin{tabular}{|c|c|c|c|}
\hline Mo1-N1-C1-C2 & 177.3(3) & Co1-C14-C15-C16 & $-59.0(3)$ \\
\hline Mo1-N1-C6-C4 & $-177.0(3)$ & Co1-C15-C16-C17 & $-59.2(3)$ \\
\hline Mo1-N1-C6-C6\#1 & $3.5(3)$ & Co1-C16-C17-C13 & $-59.5(3)$ \\
\hline $\mathrm{N} 1-\mathrm{C} 1-\mathrm{C} 2-\mathrm{C} 3$ & $-0.2(6)$ & Co1-C18-C19-C20 & $61.2(6)$ \\
\hline C1-N1-C6-C4 & $1.2(6)$ & Co1-C18-C22-C21 & $-60.9(7)$ \\
\hline C1-N1-C6-C6\#1 & $-178.4(2)$ & Co1-C19-C20-C21 & $59.2(6)$ \\
\hline $\mathrm{C} 1-\mathrm{C} 2-\mathrm{C} 3-\mathrm{C} 4$ & $0.6(7)$ & Co1-C20-C21-C22 & $57.5(7)$ \\
\hline $\mathrm{C} 2-\mathrm{C} 3-\mathrm{C} 4-\mathrm{C} 5$ & $179.2(4)$ & Co1-C21-C22-C18 & $60.9(7)$ \\
\hline $\mathrm{C} 2-\mathrm{C} 3-\mathrm{C} 4-\mathrm{C} 6$ & $-0.2(7)$ & Co1-C18'-C19'-C20' & $56.4(17)$ \\
\hline C3-C4-C5-C5\#1 & $-178.7(3)$ & Co1-C18'-C22'-C21' & $-57.7(17)$ \\
\hline C3-C4-C6-N1 & $-0.8(6)$ & Co1-C19'-C20'-C21' & $59.4(15)$ \\
\hline C3-C4-C6-C6\#1 & $178.7(3)$ & Co1-C20'-C21'-C22' & $61.4(17)$ \\
\hline C5-C4-C6-N1 & $179.9(4)$ & Co1-C21'-C22'-C18' & $58.4(18)$ \\
\hline C5-C4-C6-C6\#1 & $-0.6(5)$ & C13-C14-C15-Co1 & $59.4(3)$ \\
\hline C6-N1-C1-C2 & $-0.7(6)$ & $\mathrm{C} 13-\mathrm{C} 14-\mathrm{C} 15-\mathrm{C} 16$ & $0.4(5)$ \\
\hline C6-C4-C5-C5\#1 & $0.6(5)$ & C14-C13-C17-Co1 & $-58.7(3)$ \\
\hline Mo2-N2-C7-C8 & $176.6(3)$ & C14-C13-C17-C16 & $0.3(5)$ \\
\hline Mo2-N2-C12-C10 & $-177.0(3)$ & C14-C15-C16-Co1 & $59.0(3)$ \\
\hline Mo2-N2-C12-C12\#1 & $4.9(3)$ & $\mathrm{C} 14-\mathrm{C} 15-\mathrm{C} 16-\mathrm{C} 17$ & $-0.2(5)$ \\
\hline N2-C7-C8-C9 & $0.4(6)$ & C15-C16-C17-Co1 & $59.4(3)$ \\
\hline C7-N2-C12-C10 & $0.9(6)$ & C15-C16-C17-C13 & $-0.1(5)$ \\
\hline C7-N2-C12-C12\#1 & $-177.3(2)$ & C17-C13-C14-Co1 & $58.6(3)$ \\
\hline C7-C8-C9-C10 & $0.4(6)$ & C17-C13-C14-C15 & $-0.5(5)$ \\
\hline C8-C9-C10-C11 & $178.5(4)$ & C18-C19-C20-Co1 & $-61.2(6)$ \\
\hline C8-C9-C10-C12 & $-0.4(6)$ & C18-C19-C20-C21 & $-2.0(10)$ \\
\hline C9-C10-C11-C11\#1 & $-177.8(3)$ & C19-C18-C22-Co1 & $56.8(6)$ \\
\hline C9-C10-C12-N2 & $-0.2(6)$ & C19-C18-C22-C21 & $-4.1(11)$ \\
\hline C9-C10-C12-C12\#1 & $177.9(3)$ & C19-C20-C21-Co1 & $-58.1(6)$ \\
\hline C11-C10-C12-N2 & $-179.2(4)$ & $\mathrm{C} 19-\mathrm{C} 20-\mathrm{C} 21-\mathrm{C} 22$ & $-0.6(10)$ \\
\hline $\mathrm{C} 11-\mathrm{C} 10-\mathrm{C} 12-\mathrm{C} 12 \# 1$ & $-1.1(5)$ & C20-C21-C22-Co1 & $-58.0(7)$ \\
\hline C12-N2-C7-C8 & $-1.0(6)$ & $\mathrm{C} 20-\mathrm{C} 21-\mathrm{C} 22-\mathrm{C} 18$ & $2.9(11)$ \\
\hline C12-C10-C11-C11\#1 & $1.1(5)$ & C22-C18-C19-Co1 & $-57.4(7)$ \\
\hline Co1-C13-C14-C15 & $-59.1(3)$ & C22-C18-C19-C20 & $3.8(11)$ \\
\hline Co1-C13-C17-C16 & $59.1(3)$ & C18'-C19'-C20'-Co1 & $-56.3(17)$ \\
\hline
\end{tabular}




$\begin{array}{lclc}\mathrm{C} 18^{\prime}-\mathrm{C} 19^{\prime}-\mathrm{C} 20^{\prime}-\mathrm{C} 21^{\prime} & 3(3) & \mathrm{C} 20^{\prime}-\mathrm{C} 21^{\prime}-\mathrm{C} 22^{\prime}-\mathrm{Co} 1 & -61.6(16) \\ \mathrm{C} 19^{\prime}-\mathrm{C} 18^{\prime}-\mathrm{C} 22^{\prime}-\mathrm{Co} 1 & 62.8(17) & \mathrm{C} 20^{\prime}-\mathrm{C} 21^{\prime}-\mathrm{C} 22^{\prime}-\mathrm{C} 18^{\prime} & -3(3) \\ \mathrm{C} 19^{\prime}-\mathrm{C} 18^{\prime}-\mathrm{C} 22^{\prime}-\mathrm{C} 21^{\prime} & 5(3) & \mathrm{C} 22^{\prime}-\mathrm{C} 18^{\prime}-\mathrm{C} 19^{\prime}-\mathrm{Co} 1 & -61.4(17) \\ \mathrm{C} 19^{\prime}-\mathrm{C} 20^{\prime}-\mathrm{C} 21^{\prime}-\mathrm{Co} 1 & -61.3(16) & \mathrm{C} 22^{\prime}-\mathrm{C} 18^{\prime}-\mathrm{C} 19^{\prime}-\mathrm{C} 20^{\prime} & -5(3) \\ \mathrm{C} 19^{\prime}-\mathrm{C} 20^{\prime}-\mathrm{C} 21^{\prime}-\mathrm{C} 22^{\prime} & 0(3) & & \end{array}$

Symmetry transformations used to generate equivalent atoms:

$\# 1 \mathrm{x}, \mathrm{y},-\mathrm{z}+1$ 
REFERENCE NUMBER: rhowe28

CRYSTAL STRUCTURE REPORT

$\mathrm{C}_{22} \mathrm{H}_{18.51} \mathrm{Cl}_{4} \mathrm{Co} \mathrm{Mo} \mathrm{N} \mathrm{O}_{0.25}$

or

$\left[\mathrm{CoCp}_{2}\right]\left[\mathrm{MoCl}_{4}(\right.$ phen $\left.)\right] \cdot 0.25\left(\mathrm{H}_{2} \mathrm{O}\right)$

Report prepared for:

Prof. W. Eckenhoff

October 17, 2018

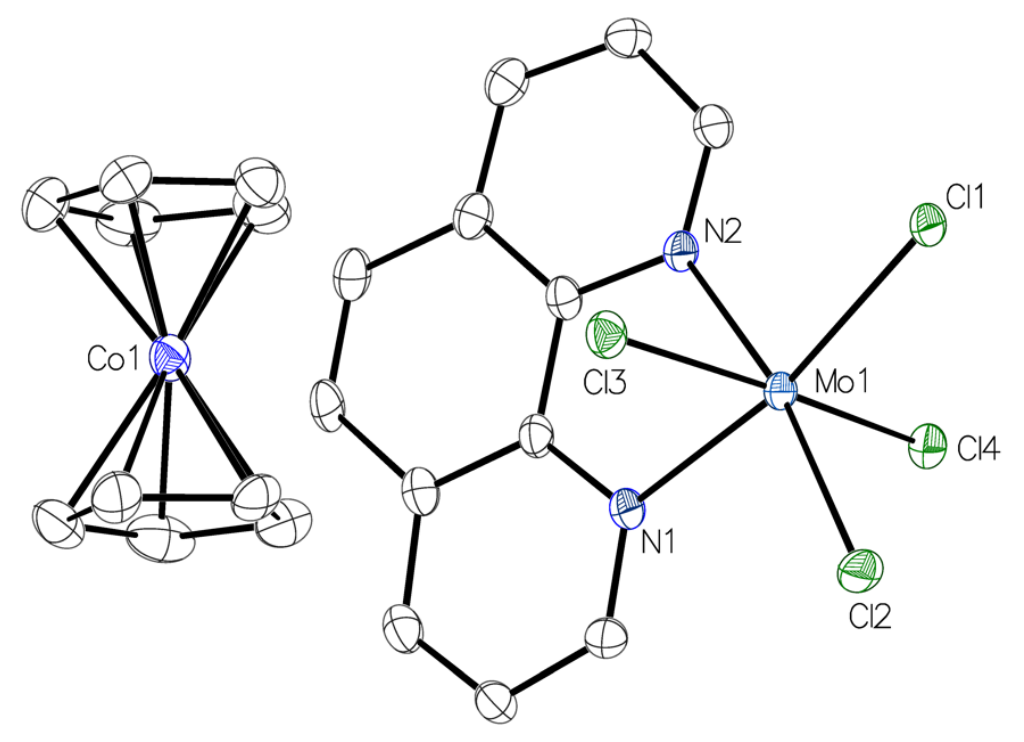

William W. Brennessel

X-ray Crystallographic Facility

Department of Chemistry, University of Rochester

120 Trustee Road

Rochester, NY 14627 


\section{Data collection}

A crystal $\left(0.159 \times 0.065 \times 0.038 \mathrm{~mm}^{3}\right)$ was placed onto a thin glass optical fiber or a nylon loop and mounted on a XtaLab Synergy-S Dualflex diffractometer equipped with a HyPix-6000HE HPC area detector for data collection at 99.99(10) K. A preliminary set of cell constants and an orientation matrix were calculated from a small sampling of reflections. ${ }^{1}$ A short pre-experiment was run, from which an optimal data collection strategy was determined. The full data collection was carried out using a PhotonJet $(\mathrm{Cu}) \mathrm{X}$-ray Source with frame times of 0.88 and 3.51 seconds and a detector distance of $31.2 \mathrm{~mm}$. Series of frames were collected in $0.50^{\circ}$ steps in $\omega$ at different $2 \theta$, $\kappa$, and $\phi$ settings. After the intensity data were corrected for absorption, the final cell constants were calculated from the xyz centroids of 14087 strong reflections from the actual data collection after integration. ${ }^{1}$ See Table 1 for additional crystal and refinement information.

Structure solution and refinement

The structure was solved using ShelXT ${ }^{2}$ and refined using ShelXL. ${ }^{3}$ The space group $C 2 / c$ was determined based on systematic absences and intensity statistics. Most or all non-hydrogen atoms were assigned from the solution. Full-matrix least squares / difference Fourier cycles were performed which located any remaining non-hydrogen atoms. All non-hydrogen atoms were refined with anisotropic displacement parameters. The hydrogen atoms on the cocrystallized water solvent molecule were placed in positions directed at $\mathrm{Cl}$ acceptors and then given a riding model. The H...A and D...A distances are greater than the sums of the individual van der Waals radii; however, it was deemed best to place them in directions of acceptors, which may have directed the packing, even if the distances in the crystal itself are large. The other option for acceptors were the pi systems of the phenanthroline ligands, but these are also beyond the sums of the van der Waals radii. All other hydrogen atoms were placed in ideal positions and refined as riding atoms with relative isotropic displacement parameters. The final full matrix least squares refinement converged to $R 1=0.0295\left(F^{2}, I>2 \sigma(I)\right)$ and $w R 2=0.0847$ ( $F^{2}$, all data).

\section{Structure description}

The structure is the one suggested. The asymmetric unit contains cobaltocenium cation and one monoanionic molybdenum complex in general positions and a cocrystallized water solvent molecule along a crystallographic twofold axis. The water molecule is modeled as partially occupied ( 0.51$)$.

Structure manipulation and figure generation were performed using Olex2. ${ }^{4}$ Unless noted otherwise all structural diagrams containing thermal displacement ellipsoids are drawn at the $50 \%$ probability level.

Data collection, structure solution, and structure refinement were conducted at the X-ray Crystallographic Facility, B04 Hutchison Hall, Department of Chemistry, University of Rochester. The instrument was purchased with funding from NSF MRI program grant CHE-1725028. All publications arising from this report MUST either 1) include William W. Brennessel as a coauthor or 2) acknowledge William W. Brennessel and the X-ray 
Crystallographic Facility of the Department of Chemistry at the University of Rochester.

1 CrysAlisPro, version 171.39.46; Rigaku Corporation: Oxford, UK, 2018.

2 Sheldrick, G. M. SHELXT, version 2018/2; Acta. Crystallogr. 2015, A71, 3-8.

3 Sheldrick, G. M. SHELXL, version 2018/3; Acta. Crystallogr. 2015, C71, 3-8.

4 Dolomanov, O. V.; Bourhis, L. J.; Gildea, R. J.; Howard, J. A. K.; Puschmann, H. Olex2, version 1.2-ac3; J. Appl. Cryst. 2009, 42, 339-341.

Some equations of interest:

$$
\begin{gathered}
R_{\mathrm{int}}=\Sigma\left|F_{\mathrm{o}}{ }^{2}-<F_{\mathrm{o}}{ }^{2}>\right| / \Sigma\left|F_{\mathrm{o}}{ }^{2}\right| \\
R 1=\Sigma|| F_{\mathrm{o}}|-| F_{\mathrm{c}} \| / \Sigma\left|F_{\mathrm{o}}\right| \\
w R 2=\left[\Sigma\left[w\left(F_{\mathrm{o}}{ }^{2}-F_{\mathrm{c}}{ }^{2}\right)^{2}\right] / \Sigma\left[w\left(F_{\mathrm{o}}{ }^{2}\right)^{2}\right]\right]^{1 / 2} \\
\text { where } w=1 /\left[\sigma^{2}\left(F_{\mathrm{o}}{ }^{2}\right)+(a P)^{2}+b P\right] \text { and } \\
P=1 / 3 \max \left(0, F_{\mathrm{o}}{ }^{2}\right)+2 / 3 F_{\mathrm{c}}{ }^{2} \\
\text { GOF }=S=\left[\Sigma\left[w\left(F_{\mathrm{o}}{ }^{2}-F_{\mathrm{c}}{ }^{2}\right)^{2}\right] /(m-n)\right]^{1 / 2}
\end{gathered}
$$

where $m=$ number of reflections and $n=$ number of parameters
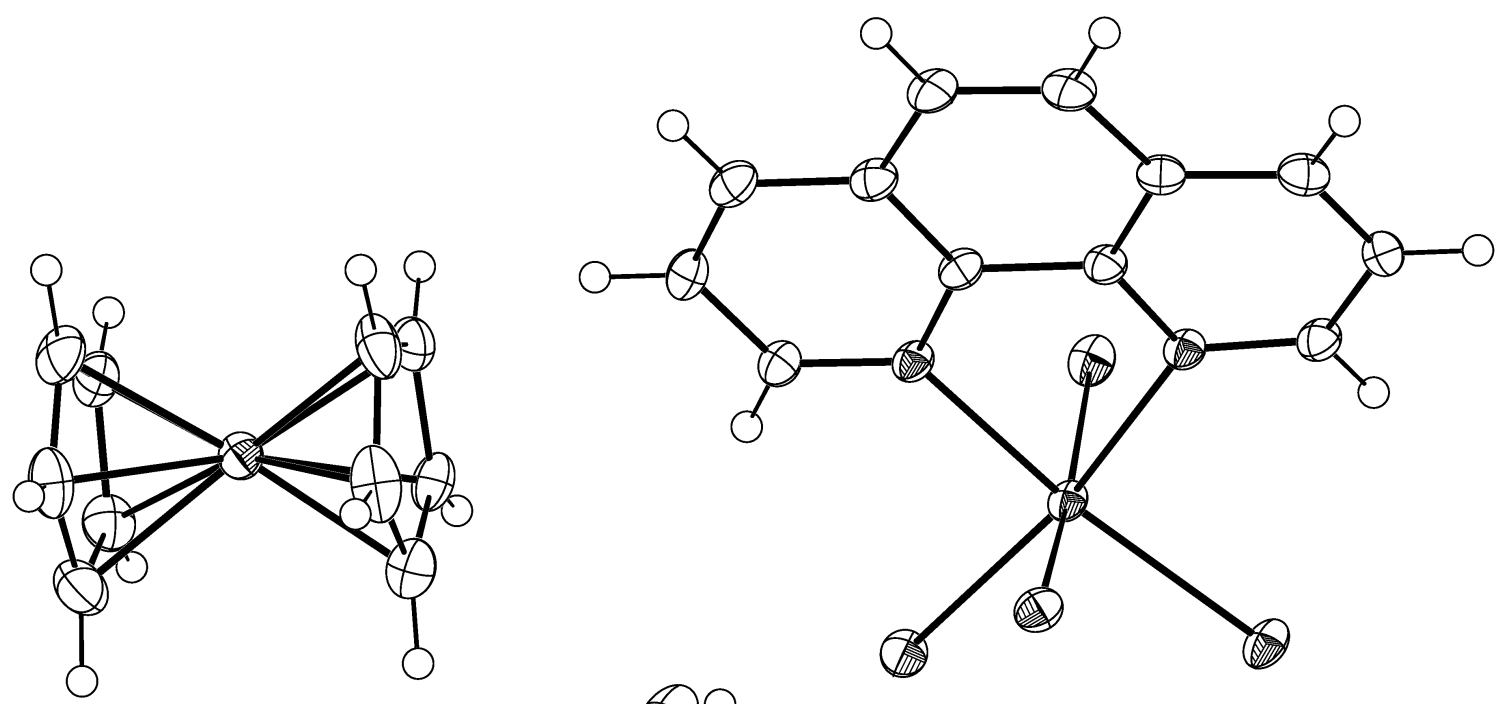


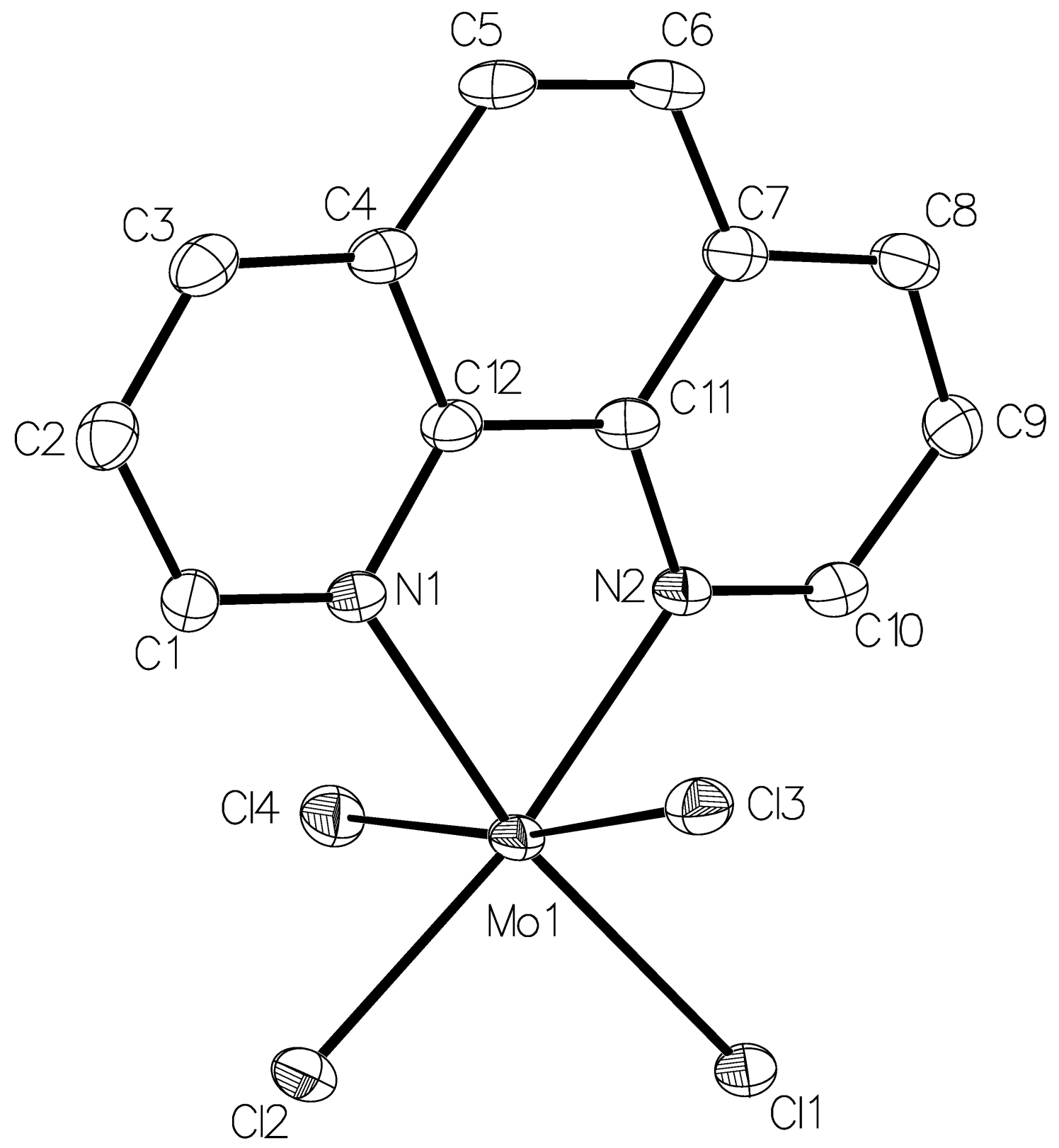




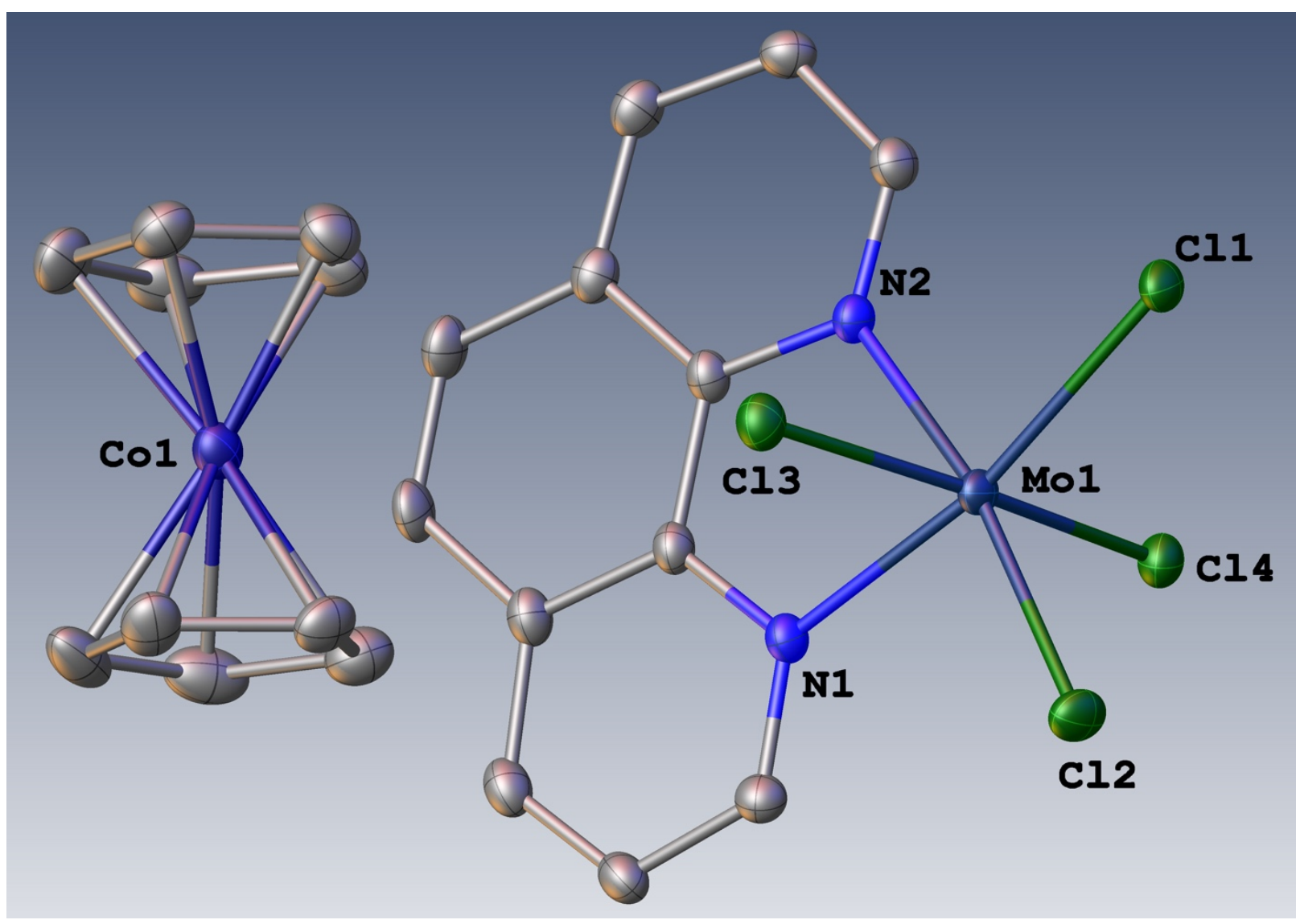


Table 1. Crystal data and structure refinement for rhowe28.

\begin{tabular}{|c|c|c|}
\hline Identification code & \multicolumn{2}{|l|}{ rhowe28 } \\
\hline Empirical formula & \multicolumn{2}{|c|}{ C22 H18.51 Cl4 Co Mo N2 O0.25 } \\
\hline Formula weight & \multicolumn{2}{|l|}{611.62} \\
\hline Temperature & \multicolumn{2}{|l|}{$99.99(10) \mathrm{K}$} \\
\hline Wavelength & \multicolumn{2}{|l|}{$1.54184 \AA$} \\
\hline Crystal system & \multicolumn{2}{|l|}{ monoclinic } \\
\hline Space group & \multicolumn{2}{|l|}{$C 2 / c$} \\
\hline \multirow[t]{3}{*}{ Unit cell dimensions } & $a=16.6327(3) \AA$ & $\alpha=90^{\circ}$ \\
\hline & $b=19.7226(2) \AA$ & $\beta=115.682(2)^{\circ}$ \\
\hline & $c=14.9988(3) \AA$ & $\gamma=90^{\circ}$ \\
\hline Volume & \multicolumn{2}{|l|}{$4434.16(14) \AA^{3}$} \\
\hline$Z$ & \multicolumn{2}{|l|}{8} \\
\hline Density (calculated) & \multicolumn{2}{|l|}{$1.832 \mathrm{Mg} / \mathrm{m}^{3}$} \\
\hline Absorption coefficient & \multicolumn{2}{|l|}{$15.042 \mathrm{~mm}^{-1}$} \\
\hline$F(000)$ & \multicolumn{2}{|l|}{2428} \\
\hline Crystal color, morphology & \multicolumn{2}{|l|}{ red, needle } \\
\hline Crystal size & \multicolumn{2}{|c|}{$0.159 \times 0.065 \times 0.038 \mathrm{~mm}^{3}$} \\
\hline Theta range for data collection & \multicolumn{2}{|l|}{3.704 to $77.793^{\circ}$} \\
\hline Index ranges & \multicolumn{2}{|c|}{$-21 \leq h \leq 20,-20 \leq k \leq 24,-16 \leq l \leq 18$} \\
\hline Reflections collected & \multicolumn{2}{|l|}{19476} \\
\hline Independent reflections & \multicolumn{2}{|c|}{$4624[R($ int $)=0.0339]$} \\
\hline Observed reflections & \multicolumn{2}{|l|}{4422} \\
\hline Completeness to theta $=74.504^{\circ}$ & \multicolumn{2}{|l|}{$99.3 \%$} \\
\hline Absorption correction & \multicolumn{2}{|l|}{ Multi-scan } \\
\hline Max. and min. transmission & \multicolumn{2}{|c|}{1.00000 and 0.14880} \\
\hline Refinement method & \multicolumn{2}{|c|}{ Full-matrix least-squares on $F^{2}$} \\
\hline Data / restraints / parameters & \multicolumn{2}{|l|}{4624 / 0 / 277} \\
\hline Goodness-of-fit on $F^{2}$ & \multicolumn{2}{|l|}{1.082} \\
\hline Final $R$ indices $[I>2 \operatorname{sigma}(I)]$ & \multicolumn{2}{|c|}{$R 1=0.0295, w R 2=0.0841$} \\
\hline$R$ indices (all data) & \multicolumn{2}{|c|}{$R 1=0.0305, w R 2=0.0847$} \\
\hline Largest diff. peak and hole & \multicolumn{2}{|c|}{0.881 and -0.674 e. $\AA^{-3}$} \\
\hline
\end{tabular}


Table 2. Atomic coordinates (x $\left.10^{4}\right)$ and equivalent isotropic displacement parameters $\left(\AA^{2} \times 10^{3}\right)$ for rhowe28. $U_{\text {eq }}$ is defined as one third of the trace of the orthogonalized $U_{i j}$ tensor.

\begin{tabular}{|c|c|c|c|c|}
\hline & $\mathrm{x}$ & $\mathrm{y}$ & $\mathrm{z}$ & $\mathrm{U}_{\mathrm{eq}}$ \\
\hline Mo1 & $7875(1)$ & $5876(1)$ & $2582(1)$ & $16(1)$ \\
\hline $\mathrm{Cl1}$ & $7925(1)$ & $6792(1)$ & $1514(1)$ & $22(1)$ \\
\hline $\mathrm{Cl} 2$ & 7001(1) & $6500(1)$ & $3246(1)$ & $24(1)$ \\
\hline $\mathrm{Cl} 3$ & $6542(1)$ & $5398(1)$ & $1244(1)$ & $21(1)$ \\
\hline $\mathrm{Cl} 4$ & $9284(1)$ & $6176(1)$ & $3957(1)$ & $22(1)$ \\
\hline N1 & 7861(1) & $4946(1)$ & $3341(2)$ & $17(1)$ \\
\hline N2 & 8591(1) & $5167(1)$ & 2083(2) & $16(1)$ \\
\hline $\mathrm{C} 1$ & $7543(2)$ & $4852(1)$ & $4006(2)$ & $19(1)$ \\
\hline $\mathrm{C} 2$ & $7496(2)$ & $4210(1)$ & $4384(2)$ & $21(1)$ \\
\hline $\mathrm{C} 3$ & $7761(2)$ & $3650(1)$ & $4040(2)$ & $22(1)$ \\
\hline $\mathrm{C} 4$ & $8089(2)$ & $3727(1)$ & $3324(2)$ & $20(1)$ \\
\hline $\mathrm{C} 5$ & $8362(2)$ & $3170(1)$ & $2895(2)$ & $22(1)$ \\
\hline C6 & $8692(2)$ & $3283(1)$ & $2226(2)$ & $22(1)$ \\
\hline $\mathrm{C} 7$ & $8800(2)$ & $3959(1)$ & 1931(2) & 19(1) \\
\hline $\mathrm{C} 8$ & $9169(2)$ & $4111(1)$ & $1270(2)$ & $22(1)$ \\
\hline C9 & $9291(2)$ & $4774(1)$ & $1082(2)$ & $20(1)$ \\
\hline $\mathrm{C} 10$ & $8995(2)$ & $5292(1)$ & $1509(2)$ & $19(1)$ \\
\hline $\mathrm{C} 11$ & $8518(2)$ & $4508(1)$ & $2323(2)$ & $17(1)$ \\
\hline $\mathrm{C} 12$ & $8144(2)$ & 4391(1) & $3009(2)$ & $17(1)$ \\
\hline Co1 & $4553(1)$ & $3322(1)$ & $4456(1)$ & $21(1)$ \\
\hline C13 & $4312(2)$ & $3856(2)$ & $5470(2)$ & $29(1)$ \\
\hline $\mathrm{C} 14$ & $4915(2)$ & $3317(1)$ & $5935(2)$ & $27(1)$ \\
\hline $\mathrm{C} 15$ & $4478(2)$ & $2704(2)$ & $5490(2)$ & $32(1)$ \\
\hline $\mathrm{C} 16$ & $3611(2)$ & $2858(2)$ & $4760(2)$ & $33(1)$ \\
\hline $\mathrm{C} 17$ & $3508(2)$ & $3577(2)$ & $4748(2)$ & $34(1)$ \\
\hline $\mathrm{C} 18$ & $5225(2)$ & $3939(1)$ & $3929(2)$ & $25(1)$ \\
\hline C19 & $5700(2)$ & $3323(1)$ & $4289(2)$ & $29(1)$ \\
\hline $\mathrm{C} 20$ & $5134(2)$ & $2787(2)$ & $3738(3)$ & $34(1)$ \\
\hline $\mathrm{C} 21$ & $4314(2)$ & $3067(2)$ & $3054(2)$ & $35(1)$ \\
\hline $\mathrm{C} 22$ & $4372(2)$ & $3783(2)$ & $3167(2)$ & $30(1)$ \\
\hline $\mathrm{O} 1$ & 5000 & $5292(3)$ & 2500 & $40(2)$ \\
\hline
\end{tabular}


Table 3. Bond lengths $[\AA]$ and angles $\left[{ }^{\circ}\right]$ for rhowe 28 .

\begin{tabular}{|c|c|c|c|}
\hline $\operatorname{Mo}(1)-\mathrm{Cl}(1)$ & $2.4396(6)$ & $\mathrm{Co}(1)-\mathrm{C}(17)$ & $2.031(3)$ \\
\hline $\mathrm{Mo}(1)-\mathrm{Cl}(2)$ & $2.4234(6)$ & $\mathrm{Co}(1)-\mathrm{C}(18)$ & $2.029(3)$ \\
\hline $\mathrm{Mo}(1)-\mathrm{Cl}(3)$ & $2.4453(6)$ & $\mathrm{Co}(1)-\mathrm{C}(19)$ & $2.028(3)$ \\
\hline $\mathrm{Mo}(1)-\mathrm{Cl}(4)$ & $2.4300(6)$ & $\mathrm{Co}(1)-\mathrm{C}(20)$ & $2.026(3)$ \\
\hline $\mathrm{Mo}(1)-\mathrm{N}(1)$ & $2.164(2)$ & $\mathrm{Co}(1)-\mathrm{C}(21)$ & $2.027(3)$ \\
\hline $\mathrm{Mo}(1)-\mathrm{N}(2)$ & $2.170(2)$ & $\mathrm{Co}(1)-\mathrm{C}(22)$ & $2.037(3)$ \\
\hline $\mathrm{N}(1)-\mathrm{C}(1)$ & $1.329(3)$ & $\mathrm{C}(13)-\mathrm{H}(13)$ & 0.9300 \\
\hline $\mathrm{N}(1)-\mathrm{C}(12)$ & $1.368(3)$ & $C(13)-C(14)$ & $1.419(4)$ \\
\hline $\mathrm{N}(2)-\mathrm{C}(10)$ & $1.325(3)$ & $\mathrm{C}(13)-\mathrm{C}(17)$ & $1.417(4)$ \\
\hline $\mathrm{N}(2)-\mathrm{C}(11)$ & $1.366(3)$ & $\mathrm{C}(14)-\mathrm{H}(14)$ & 0.9300 \\
\hline $\mathrm{C}(1)-\mathrm{H}(1)$ & 0.9300 & $C(14)-C(15)$ & $1.421(4)$ \\
\hline $\mathrm{C}(1)-\mathrm{C}(2)$ & $1.404(3)$ & $\mathrm{C}(15)-\mathrm{H}(15)$ & 0.9300 \\
\hline $\mathrm{C}(2)-\mathrm{H}(2)$ & 0.9300 & $C(15)-C(16)$ & $1.416(5)$ \\
\hline $\mathrm{C}(2)-\mathrm{C}(3)$ & $1.370(4)$ & $\mathrm{C}(16)-\mathrm{H}(16)$ & 0.9300 \\
\hline $\mathrm{C}(3)-\mathrm{H}(3)$ & 0.9300 & $C(16)-C(17)$ & $1.427(5)$ \\
\hline $\mathrm{C}(3)-\mathrm{C}(4)$ & $1.409(4)$ & $\mathrm{C}(17)-\mathrm{H}(17)$ & 0.9300 \\
\hline$C(4)-C(5)$ & $1.442(4)$ & $\mathrm{C}(18)-\mathrm{H}(18)$ & 0.9300 \\
\hline$C(4)-C(12)$ & $1.408(3)$ & $\mathrm{C}(18)-\mathrm{C}(19)$ & $1.422(4)$ \\
\hline $\mathrm{C}(5)-\mathrm{H}(5)$ & 0.9300 & $\mathrm{C}(18)-\mathrm{C}(22)$ & $1.416(4)$ \\
\hline$C(5)-C(6)$ & $1.355(4)$ & $\mathrm{C}(19)-\mathrm{H}(19)$ & 0.9300 \\
\hline $\mathrm{C}(6)-\mathrm{H}(6)$ & 0.9300 & $C(19)-C(20)$ & $1.419(4)$ \\
\hline$C(6)-C(7)$ & $1.440(4)$ & $\mathrm{C}(20)-\mathrm{H}(20)$ & 0.9300 \\
\hline$C(7)-C(8)$ & $1.405(4)$ & $C(20)-C(21)$ & $1.416(5)$ \\
\hline$C(7)-C(11)$ & $1.407(3)$ & $\mathrm{C}(21)-\mathrm{H}(21)$ & 0.9300 \\
\hline $\mathrm{C}(8)-\mathrm{H}(8)$ & 0.9300 & $C(21)-C(22)$ & $1.422(4)$ \\
\hline $\mathrm{C}(8)-\mathrm{C}(9)$ & $1.372(4)$ & $\mathrm{C}(22)-\mathrm{H}(22)$ & 0.9300 \\
\hline $\mathrm{C}(9)-\mathrm{H}(9)$ & 0.9300 & $\mathrm{O}(1)-\mathrm{H}(1 \mathrm{~A}) \# 1$ & 0.8499 \\
\hline$C(9)-C(10)$ & $1.404(4)$ & $\mathrm{O}(1)-\mathrm{H}(1 \mathrm{~A})$ & 0.8500 \\
\hline $\mathrm{C}(10)-\mathrm{H}(10)$ & 0.9300 & $\mathrm{O}(1)-\mathrm{H}(1 \mathrm{~B}) \# 1$ & 0.8500 \\
\hline$C(11)-C(12)$ & $1.434(3)$ & $\mathrm{O}(1)-\mathrm{H}(1 \mathrm{~B})$ & 0.8499 \\
\hline $\mathrm{Co}(1)-\mathrm{C}(13)$ & $2.028(3)$ & $\mathrm{Cl}(1)-\mathrm{Mo}(1)-\mathrm{Cl}(3)$ & $91.33(2)$ \\
\hline $\mathrm{Co}(1)-\mathrm{C}(14)$ & $2.032(3)$ & $\mathrm{Cl}(2)-\mathrm{Mo}(1)-\mathrm{Cl}(1)$ & $95.19(2)$ \\
\hline $\mathrm{Co}(1)-\mathrm{C}(15)$ & $2.018(3)$ & $\mathrm{Cl}(2)-\mathrm{Mo}(1)-\mathrm{Cl}(3)$ & $92.36(2)$ \\
\hline $\mathrm{Co}(1)-\mathrm{C}(16)$ & $2.029(3)$ & $\mathrm{Cl}(2)-\mathrm{Mo}(1)-\mathrm{Cl}(4)$ & $93.29(2)$ \\
\hline
\end{tabular}




\begin{tabular}{|c|c|c|c|}
\hline $\mathrm{Cl}(4)-\mathrm{Mo}(1)-\mathrm{Cl}(1)$ & $94.72(2)$ & $C(8)-C(7)-C(11)$ & $117.2(2)$ \\
\hline $\mathrm{Cl}(4)-\mathrm{Mo}(1)-\mathrm{Cl}(3)$ & $171.32(2)$ & $C(11)-C(7)-C(6)$ & $118.5(2)$ \\
\hline $\mathrm{N}(1)-\mathrm{Mo}(1)-\mathrm{Cl}(1)$ & $169.79(6)$ & $\mathrm{C}(7)-\mathrm{C}(8)-\mathrm{H}(8)$ & 120.1 \\
\hline $\mathrm{N}(1)-\mathrm{Mo}(1)-\mathrm{Cl}(2)$ & $93.97(6)$ & $\mathrm{C}(9)-\mathrm{C}(8)-\mathrm{C}(7)$ & $119.8(2)$ \\
\hline $\mathrm{N}(1)-\mathrm{Mo}(1)-\mathrm{Cl}(3)$ & $83.80(6)$ & $\mathrm{C}(9)-\mathrm{C}(8)-\mathrm{H}(8)$ & 120.1 \\
\hline $\mathrm{N}(1)-\mathrm{Mo}(1)-\mathrm{Cl}(4)$ & $89.24(6)$ & $\mathrm{C}(8)-\mathrm{C}(9)-\mathrm{H}(9)$ & 120.4 \\
\hline $\mathrm{N}(1)-\mathrm{Mo}(1)-\mathrm{N}(2)$ & $76.58(8)$ & $C(8)-C(9)-C(10)$ & $119.2(2)$ \\
\hline $\mathrm{N}(2)-\mathrm{Mo}(1)-\mathrm{Cl}(1)$ & $94.08(6)$ & $\mathrm{C}(10)-\mathrm{C}(9)-\mathrm{H}(9)$ & 120.4 \\
\hline $\mathrm{N}(2)-\mathrm{Mo}(1)-\mathrm{Cl}(2)$ & $170.32(6)$ & $\mathrm{N}(2)-\mathrm{C}(10)-\mathrm{C}(9)$ & $122.5(2)$ \\
\hline $\mathrm{N}(2)-\mathrm{Mo}(1)-\mathrm{Cl}(3)$ & $84.62(6)$ & $\mathrm{N}(2)-\mathrm{C}(10)-\mathrm{H}(10)$ & 118.7 \\
\hline $\mathrm{N}(2)-\mathrm{Mo}(1)-\mathrm{Cl}(4)$ & $88.74(6)$ & $\mathrm{C}(9)-\mathrm{C}(10)-\mathrm{H}(10)$ & 118.7 \\
\hline $\mathrm{C}(1)-\mathrm{N}(1)-\mathrm{Mo}(1)$ & $127.71(17)$ & $\mathrm{N}(2)-\mathrm{C}(11)-\mathrm{C}(7)$ & $122.6(2)$ \\
\hline $\mathrm{C}(1)-\mathrm{N}(1)-\mathrm{C}(12)$ & $118.2(2)$ & $\mathrm{N}(2)-\mathrm{C}(11)-\mathrm{C}(12)$ & $117.3(2)$ \\
\hline $\mathrm{C}(12)-\mathrm{N}(1)-\mathrm{Mo}(1)$ & $113.78(16)$ & $C(7)-C(11)-C(12)$ & $120.2(2)$ \\
\hline $\mathrm{C}(10)-\mathrm{N}(2)-\mathrm{Mo}(1)$ & $127.76(16)$ & $\mathrm{N}(1)-\mathrm{C}(12)-\mathrm{C}(4)$ & $122.7(2)$ \\
\hline $\mathrm{C}(10)-\mathrm{N}(2)-\mathrm{C}(11)$ & $118.4(2)$ & $\mathrm{N}(1)-\mathrm{C}(12)-\mathrm{C}(11)$ & $117.1(2)$ \\
\hline $\mathrm{C}(11)-\mathrm{N}(2)-\mathrm{Mo}(1)$ & $113.41(16)$ & $\mathrm{C}(4)-\mathrm{C}(12)-\mathrm{C}(11)$ & $120.1(2)$ \\
\hline $\mathrm{N}(1)-\mathrm{C}(1)-\mathrm{H}(1)$ & 118.7 & $\mathrm{C}(13)-\mathrm{Co}(1)-\mathrm{C}(14)$ & $40.92(12)$ \\
\hline $\mathrm{N}(1)-\mathrm{C}(1)-\mathrm{C}(2)$ & $122.6(2)$ & $\mathrm{C}(13)-\mathrm{Co}(1)-\mathrm{C}(16)$ & $69.05(12)$ \\
\hline $\mathrm{C}(2)-\mathrm{C}(1)-\mathrm{H}(1)$ & 118.7 & $\mathrm{C}(13)-\mathrm{Co}(1)-\mathrm{C}(17)$ & $40.87(12)$ \\
\hline $\mathrm{C}(1)-\mathrm{C}(2)-\mathrm{H}(2)$ & 120.3 & $\mathrm{C}(13)-\mathrm{Co}(1)-\mathrm{C}(18)$ & $107.59(12)$ \\
\hline$C(3)-C(2)-C(1)$ & $119.5(2)$ & $\mathrm{C}(13)-\mathrm{Co}(1)-\mathrm{C}(19)$ & $126.43(13)$ \\
\hline $\mathrm{C}(3)-\mathrm{C}(2)-\mathrm{H}(2)$ & 120.3 & $\mathrm{C}(13)-\mathrm{Co}(1)-\mathrm{C}(22)$ & $118.94(12)$ \\
\hline $\mathrm{C}(2)-\mathrm{C}(3)-\mathrm{H}(3)$ & 120.2 & $\mathrm{C}(14)-\mathrm{Co}(1)-\mathrm{C}(22)$ & $152.88(12)$ \\
\hline$C(2)-C(3)-C(4)$ & $119.6(2)$ & $\mathrm{C}(15)-\mathrm{Co}(1)-\mathrm{C}(13)$ & $68.85(12)$ \\
\hline $\mathrm{C}(4)-\mathrm{C}(3)-\mathrm{H}(3)$ & 120.2 & $\mathrm{C}(15)-\mathrm{Co}(1)-\mathrm{C}(14)$ & $41.07(12)$ \\
\hline$C(3)-C(4)-C(5)$ & $124.0(2)$ & $C(15)-C o(1)-C(16)$ & $40.95(13)$ \\
\hline $\mathrm{C}(12)-\mathrm{C}(4)-\mathrm{C}(3)$ & $117.3(2)$ & $\mathrm{C}(15)-\mathrm{Co}(1)-\mathrm{C}(17)$ & $68.94(13)$ \\
\hline$C(12)-C(4)-C(5)$ & $118.8(2)$ & $\mathrm{C}(15)-\mathrm{Co}(1)-\mathrm{C}(18)$ & $152.75(13)$ \\
\hline $\mathrm{C}(4)-\mathrm{C}(5)-\mathrm{H}(5)$ & 119.6 & $\mathrm{C}(15)-\mathrm{Co}(1)-\mathrm{C}(19)$ & $118.00(13)$ \\
\hline$C(6)-C(5)-C(4)$ & $120.8(2)$ & $\mathrm{C}(15)-\mathrm{Co}(1)-\mathrm{C}(20)$ & $107.23(13)$ \\
\hline $\mathrm{C}(6)-\mathrm{C}(5)-\mathrm{H}(5)$ & 119.6 & $\mathrm{C}(15)-\mathrm{Co}(1)-\mathrm{C}(21)$ & $126.68(13)$ \\
\hline$C(5)-C(6)-H(6)$ & 119.2 & $\mathrm{C}(15)-\mathrm{Co}(1)-\mathrm{C}(22)$ & $164.99(13)$ \\
\hline$C(5)-C(6)-C(7)$ & $121.6(2)$ & $\mathrm{C}(16)-\mathrm{Co}(1)-\mathrm{C}(14)$ & $69.22(12)$ \\
\hline$C(7)-C(6)-H(6)$ & 119.2 & $\mathrm{C}(16)-\mathrm{Co}(1)-\mathrm{C}(17)$ & $41.15(13)$ \\
\hline$C(8)-C(7)-C(6)$ & $124.4(2)$ & $\mathrm{C}(16)-\mathrm{Co}(1)-\mathrm{C}(18)$ & $165.14(13)$ \\
\hline
\end{tabular}




\begin{tabular}{|c|c|}
\hline $\mathrm{C}(16)-\mathrm{Co}(1)-\mathrm{C}(22)$ & $127.12(13)$ \\
\hline $\mathrm{C}(17)-\mathrm{Co}(1)-\mathrm{C}(14)$ & $69.03(12)$ \\
\hline $\mathrm{C}(17)-\mathrm{Co}(1)-\mathrm{C}(22)$ & $107.80(13)$ \\
\hline $\mathrm{C}(18)-\mathrm{Co}(1)-\mathrm{C}(14)$ & $118.38(11)$ \\
\hline $\mathrm{C}(18)-\mathrm{Co}(1)-\mathrm{C}(17)$ & $127.04(13)$ \\
\hline $\mathrm{C}(18)-\mathrm{Co}(1)-\mathrm{C}(22)$ & $40.74(11)$ \\
\hline $\mathrm{C}(19)-\mathrm{Co}(1)-\mathrm{C}(14)$ & $106.54(13)$ \\
\hline $\mathrm{C}(19)-\mathrm{Co}(1)-\mathrm{C}(16)$ & $152.52(13)$ \\
\hline C(19)-Co(1)-C(17) & $164.69(13)$ \\
\hline C(19)-Co(1)-C(18) & $41.03(11)$ \\
\hline $\mathrm{C}(19)-\mathrm{Co}(1)-\mathrm{C}(22)$ & $69.07(12)$ \\
\hline $\mathrm{C}(20)-\mathrm{Co}(1)-\mathrm{C}(13)$ & $164.48(14)$ \\
\hline $\mathrm{C}(20)-\mathrm{Co}(1)-\mathrm{C}(14)$ & $126.43(13)$ \\
\hline $\mathrm{C}(20)-\mathrm{Co}(1)-\mathrm{C}(16)$ & $118.51(12)$ \\
\hline $\mathrm{C}(20)-\mathrm{Co}(1)-\mathrm{C}(17)$ & $153.26(13)$ \\
\hline $\mathrm{C}(20)-\mathrm{Co}(1)-\mathrm{C}(18)$ & $68.61(12)$ \\
\hline $\mathrm{C}(20)-\mathrm{Co}(1)-\mathrm{C}(19)$ & $40.96(12)$ \\
\hline $\mathrm{C}(20)-\mathrm{Co}(1)-\mathrm{C}(21)$ & $40.89(14)$ \\
\hline $\mathrm{C}(20)-\mathrm{Co}(1)-\mathrm{C}(22)$ & $68.70(13)$ \\
\hline $\mathrm{C}(21)-\mathrm{Co}(1)-\mathrm{C}(13)$ & $153.32(14)$ \\
\hline $\mathrm{C}(21)-\mathrm{Co}(1)-\mathrm{C}(14)$ & $164.56(13)$ \\
\hline $\mathrm{C}(21)-\mathrm{Co}(1)-\mathrm{C}(16)$ & $107.32(12)$ \\
\hline $\mathrm{C}(21)-\mathrm{Co}(1)-\mathrm{C}(17)$ & $118.92(14)$ \\
\hline $\mathrm{C}(21)-\mathrm{Co}(1)-\mathrm{C}(18)$ & $68.80(12)$ \\
\hline $\mathrm{C}(21)-\mathrm{Co}(1)-\mathrm{C}(19)$ & $69.20(13)$ \\
\hline $\mathrm{C}(21)-\mathrm{Co}(1)-\mathrm{C}(22)$ & $40.96(13)$ \\
\hline $\mathrm{Co}(1)-\mathrm{C}(13)-\mathrm{H}(13)$ & 126.4 \\
\hline $\mathrm{C}(14)-\mathrm{C}(13)-\mathrm{Co}(1)$ & $69.69(17)$ \\
\hline $\mathrm{C}(14)-\mathrm{C}(13)-\mathrm{H}(13)$ & 125.7 \\
\hline$C(17)-C(13)-C o(1)$ & $69.71(17)$ \\
\hline $\mathrm{C}(17)-\mathrm{C}(13)-\mathrm{H}(13)$ & 125.7 \\
\hline$C(17)-C(13)-C(14)$ & $108.5(3)$ \\
\hline $\mathrm{Co}(1)-\mathrm{C}(14)-\mathrm{H}(14)$ & 126.9 \\
\hline $\mathrm{C}(13)-\mathrm{C}(14)-\mathrm{Co}(1)$ & $69.39(17)$ \\
\hline $\mathrm{C}(13)-\mathrm{C}(14)-\mathrm{H}(14)$ & 126.3 \\
\hline $\mathrm{C}(13)-\mathrm{C}(14)-\mathrm{C}(15)$ & $107.3(3)$ \\
\hline
\end{tabular}

\begin{tabular}{|c|c|}
\hline $\mathrm{C}(15)-\mathrm{C}(14)-\mathrm{Co}(1)$ & $68.96(17)$ \\
\hline $\mathrm{C}(15)-\mathrm{C}(14)-\mathrm{H}(14)$ & 126.3 \\
\hline $\mathrm{Co}(1)-\mathrm{C}(15)-\mathrm{H}(15)$ & 126.1 \\
\hline $\mathrm{C}(14)-\mathrm{C}(15)-\mathrm{Co}(1)$ & $69.97(16)$ \\
\hline $\mathrm{C}(14)-\mathrm{C}(15)-\mathrm{H}(15)$ & 125.6 \\
\hline $\mathrm{C}(16)-\mathrm{C}(15)-\mathrm{Co}(1)$ & $69.93(17)$ \\
\hline $\mathrm{C}(16)-\mathrm{C}(15)-\mathrm{C}(14)$ & $108.8(3)$ \\
\hline $\mathrm{C}(16)-\mathrm{C}(15)-\mathrm{H}(15)$ & 125.6 \\
\hline $\mathrm{Co}(1)-\mathrm{C}(16)-\mathrm{H}(16)$ & 126.7 \\
\hline $\mathrm{C}(15)-\mathrm{C}(16)-\mathrm{Co}(1)$ & $69.12(16)$ \\
\hline $\mathrm{C}(15)-\mathrm{C}(16)-\mathrm{H}(16)$ & 126.3 \\
\hline$C(15)-C(16)-C(17)$ & $107.5(3)$ \\
\hline $\mathrm{C}(17)-\mathrm{C}(16)-\mathrm{Co}(1)$ & $69.52(17)$ \\
\hline $\mathrm{C}(17)-\mathrm{C}(16)-\mathrm{H}(16)$ & 126.3 \\
\hline $\mathrm{Co}(1)-\mathrm{C}(17)-\mathrm{H}(17)$ & 126.8 \\
\hline $\mathrm{C}(13)-\mathrm{C}(17)-\mathrm{Co}(1)$ & $69.42(17)$ \\
\hline C(13)-C(17)-C(16) & $107.9(3)$ \\
\hline C(13)-C(17)-H(17) & 126.1 \\
\hline $\mathrm{C}(16)-\mathrm{C}(17)-\mathrm{Co}(1)$ & $69.33(17)$ \\
\hline $\mathrm{C}(16)-\mathrm{C}(17)-\mathrm{H}(17)$ & 126.1 \\
\hline $\mathrm{Co}(1)-\mathrm{C}(18)-\mathrm{H}(18)$ & 126.5 \\
\hline C(19)-C(18)-Co(1) & $69.44(15)$ \\
\hline $\mathrm{C}(19)-\mathrm{C}(18)-\mathrm{H}(18)$ & 125.7 \\
\hline $\mathrm{C}(22)-\mathrm{C}(18)-\mathrm{Co}(1)$ & $69.93(16)$ \\
\hline $\mathrm{C}(22)-\mathrm{C}(18)-\mathrm{H}(18)$ & 125.7 \\
\hline C(22)-C(18)-C(19) & $108.6(3)$ \\
\hline $\mathrm{Co}(1)-\mathrm{C}(19)-\mathrm{H}(19)$ & 126.2 \\
\hline C(18)-C(19)-Co(1) & $69.53(16)$ \\
\hline C(18)-C(19)-H(19) & 126.4 \\
\hline $\mathrm{C}(20)-\mathrm{C}(19)-\mathrm{Co}(1)$ & $69.44(16)$ \\
\hline C(20)-C(19)-C(18) & $107.2(3)$ \\
\hline $\mathrm{C}(20)-\mathrm{C}(19)-\mathrm{H}(19)$ & 126.4 \\
\hline $\mathrm{Co}(1)-\mathrm{C}(20)-\mathrm{H}(20)$ & 126.7 \\
\hline $\mathrm{C}(19)-\mathrm{C}(20)-\mathrm{Co}(1)$ & $69.59(17)$ \\
\hline $\mathrm{C}(19)-\mathrm{C}(20)-\mathrm{H}(20)$ & 125.7 \\
\hline $\mathrm{C}(21)-\mathrm{C}(20)-\mathrm{Co}(1)$ & $69.58(17)$ \\
\hline
\end{tabular}




$\begin{array}{lllc}\mathrm{C}(21)-\mathrm{C}(20)-\mathrm{C}(19) & 108.7(3) & \mathrm{C}(18)-\mathrm{C}(22)-\mathrm{C}(21) & 107.7(3) \\ \mathrm{C}(21)-\mathrm{C}(20)-\mathrm{H}(20) & 125.7 & \mathrm{C}(18)-\mathrm{C}(22)-\mathrm{H}(22) & 126.1 \\ \mathrm{Co}(1)-\mathrm{C}(21)-\mathrm{H}(21) & 126.0 & \mathrm{C}(21)-\mathrm{C}(22)-\mathrm{Co}(1) & 69.12(18) \\ \mathrm{C}(20)-\mathrm{C}(21)-\mathrm{Co}(1) & 69.53(18) & \mathrm{C}(21)-\mathrm{C}(22)-\mathrm{H}(22) & 126.1 \\ \mathrm{C}(20)-\mathrm{C}(21)-\mathrm{H}(21) & 126.1 & \mathrm{H}(1 \mathrm{~A})-\mathrm{O}(1)-\mathrm{H}(1 \mathrm{~A}) \# 1 & 173.8 \\ \mathrm{C}(20)-\mathrm{C}(21)-\mathrm{C}(22) & 107.8(3) & \mathrm{H}(1 \mathrm{~A})-\mathrm{O}(1)-\mathrm{H}(1 \mathrm{~B}) \# 1 & 54.8 \\ \mathrm{C}(22)-\mathrm{C}(21)-\mathrm{Co}(1) & 69.92(17) & \mathrm{H}(1 \mathrm{~A})-\mathrm{O}(1)-\mathrm{H}(1 \mathrm{~B}) & 120.6 \\ \mathrm{C}(22)-\mathrm{C}(21)-\mathrm{H}(21) & 126.1 & \mathrm{H}(1 \mathrm{~A}) \# 1-\mathrm{O}(1)-\mathrm{H}(1 \mathrm{~B}) \# 1 & 120.6 \\ \mathrm{Co}(1)-\mathrm{C}(22)-\mathrm{H}(22) & 127.0 & \mathrm{H}(1 \mathrm{~B})-\mathrm{O}(1)-\mathrm{H}(1 \mathrm{~A}) \# 1 & 54.8 \\ \mathrm{C}(18)-\mathrm{C}(22)-\mathrm{Co}(1) & 69.33(16) & \mathrm{H}(1 \mathrm{~B})-\mathrm{O}(1)-\mathrm{H}(1 \mathrm{~B}) \# 1 & 103.5\end{array}$

Symmetry transformations used to generate equivalent atoms:

$\# 1-\mathrm{x}+1, \mathrm{y},-\mathrm{z}+1 / 2$ 
Table 4. Anisotropic displacement parameters $\left(\AA^{2} \times 10^{3}\right)$ for rhowe28. The anisotropic displacement factor exponent takes the form: $-2 \pi^{2}\left[h^{2} a^{* 2} U_{11}+\ldots+2 h k a^{*} b^{*} U_{12}\right]$

\begin{tabular}{|c|c|c|c|c|c|c|}
\hline & $\mathrm{U}_{11}$ & $\mathrm{U}_{22}$ & $\mathrm{U}_{33}$ & $\mathrm{U}_{23}$ & $\mathrm{U}_{13}$ & $\mathrm{U}_{12}$ \\
\hline Mo1 & $24(1)$ & $9(1)$ & $18(1)$ & $0(1)$ & $12(1)$ & $1(1)$ \\
\hline $\mathrm{Cl1}$ & $34(1)$ & $12(1)$ & $22(1)$ & $0(1)$ & $15(1)$ & $-1(1)$ \\
\hline $\mathrm{Cl} 2$ & $36(1)$ & $16(1)$ & $27(1)$ & $0(1)$ & $20(1)$ & $6(1)$ \\
\hline $\mathrm{Cl} 3$ & $24(1)$ & $16(1)$ & $23(1)$ & $1(1)$ & $10(1)$ & $1(1)$ \\
\hline $\mathrm{Cl} 4$ & $29(1)$ & $16(1)$ & $22(1)$ & $-2(1)$ & $12(1)$ & $-3(1)$ \\
\hline N1 & $21(1)$ & $12(1)$ & $18(1)$ & $1(1)$ & $9(1)$ & $0(1)$ \\
\hline N2 & $21(1)$ & $11(1)$ & $18(1)$ & $0(1)$ & $9(1)$ & $0(1)$ \\
\hline $\mathrm{C} 1$ & $22(1)$ & $19(1)$ & $17(1)$ & $0(1)$ & $9(1)$ & $-1(1)$ \\
\hline $\mathrm{C} 2$ & $24(1)$ & $20(1)$ & $20(1)$ & $2(1)$ & $10(1)$ & $-4(1)$ \\
\hline $\mathrm{C} 3$ & $24(1)$ & $17(1)$ & $22(1)$ & $3(1)$ & $8(1)$ & $-3(1)$ \\
\hline $\mathrm{C} 4$ & $21(1)$ & $14(1)$ & $23(1)$ & $2(1)$ & $8(1)$ & $-1(1)$ \\
\hline $\mathrm{C} 5$ & $27(1)$ & $10(1)$ & $30(1)$ & $2(1)$ & $12(1)$ & $0(1)$ \\
\hline C6 & $24(1)$ & $13(1)$ & $29(1)$ & $-2(1)$ & $11(1)$ & $2(1)$ \\
\hline C7 & $18(1)$ & $16(1)$ & $20(1)$ & $-1(1)$ & $6(1)$ & $2(1)$ \\
\hline $\mathrm{C} 8$ & $23(1)$ & $18(1)$ & $24(1)$ & $-2(1)$ & $9(1)$ & $4(1)$ \\
\hline C9 & $21(1)$ & $22(1)$ & $20(1)$ & $1(1)$ & $11(1)$ & $2(1)$ \\
\hline $\mathrm{C} 10$ & $23(1)$ & $15(1)$ & $21(1)$ & $2(1)$ & $10(1)$ & $1(1)$ \\
\hline C11 & $18(1)$ & $13(1)$ & $21(1)$ & $1(1)$ & $8(1)$ & $0(1)$ \\
\hline $\mathrm{C} 12$ & $20(1)$ & $12(1)$ & $18(1)$ & $0(1)$ & $7(1)$ & $-1(1)$ \\
\hline Co1 & $23(1)$ & $15(1)$ & $28(1)$ & $-1(1)$ & $14(1)$ & $-2(1)$ \\
\hline $\mathrm{C} 13$ & $34(2)$ & $24(1)$ & $31(1)$ & $-4(1)$ & $18(1)$ & $-1(1)$ \\
\hline C14 & $30(1)$ & $27(1)$ & $27(1)$ & $4(1)$ & $15(1)$ & $-2(1)$ \\
\hline C15 & $42(2)$ & $20(1)$ & $41(2)$ & $5(1)$ & $24(1)$ & $-3(1)$ \\
\hline $\mathrm{C} 16$ & $33(2)$ & $36(2)$ & $36(2)$ & $-6(1)$ & $22(1)$ & $-14(1)$ \\
\hline $\mathrm{C} 17$ & $29(1)$ & $41(2)$ & $35(2)$ & $-1(1)$ & $18(1)$ & $6(1)$ \\
\hline $\mathrm{C} 18$ & $32(1)$ & $20(1)$ & $27(1)$ & $1(1)$ & $16(1)$ & $-8(1)$ \\
\hline C19 & $27(1)$ & $26(1)$ & $40(2)$ & $5(1)$ & $21(1)$ & $-2(1)$ \\
\hline $\mathrm{C} 20$ & $45(2)$ & $21(1)$ & $54(2)$ & $-6(1)$ & $39(2)$ & $-4(1)$ \\
\hline $\mathrm{C} 21$ & $40(2)$ & $37(2)$ & $35(2)$ & $-16(1)$ & $24(1)$ & $-17(1)$ \\
\hline $\mathrm{C} 22$ & $34(2)$ & $32(2)$ & $26(1)$ & $0(1)$ & $14(1)$ & $-4(1)$ \\
\hline O1 & $68(5)$ & $24(3)$ & $35(4)$ & 0 & $28(3)$ & 0 \\
\hline
\end{tabular}


Table 5. Hydrogen coordinates $\left(\times 10^{4}\right)$ and isotropic displacement parameters $\left(\AA^{2} \times 10^{3}\right)$ for rhowe28.

\begin{tabular}{|c|c|c|c|c|}
\hline & $\mathrm{x}$ & $\mathrm{y}$ & $\mathrm{z}$ & $\mathrm{U}(\mathrm{eq})$ \\
\hline H1 & 7343 & 5227 & 4229 & 23 \\
\hline $\mathrm{H} 2$ & 7287 & 4165 & 4864 & 26 \\
\hline $\mathrm{H} 3$ & 7724 & 3221 & 4278 & 26 \\
\hline H5 & 8311 & 2728 & 3080 & 27 \\
\hline H6 & 8854 & 2915 & 1951 & 27 \\
\hline $\mathrm{H} 8$ & 9330 & 3762 & 962 & 26 \\
\hline H9 & 9567 & 4879 & 676 & 24 \\
\hline $\mathrm{H} 10$ & 9085 & 5740 & 1383 & 23 \\
\hline H13 & 4426 & 4314 & 5615 & 34 \\
\hline H14 & 5490 & 3358 & 6438 & 33 \\
\hline H15 & 4722 & 2271 & 5651 & 38 \\
\hline H16 & 3186 & 2548 & 4361 & 39 \\
\hline H17 & 3003 & 3820 & 4339 & 40 \\
\hline H18 & 5438 & 4371 & 4155 & 30 \\
\hline H19 & 6275 & 3280 & 4793 & 34 \\
\hline $\mathrm{H} 20$ & 5277 & 2328 & 3813 & 41 \\
\hline $\mathrm{H} 21$ & 3824 & 2825 & 2609 & 42 \\
\hline $\mathrm{H} 22$ & 3930 & 4094 & 2806 & 36 \\
\hline $\mathrm{H} 1 \mathrm{~A}$ & 4654 & 5316 & 2782 & 60 \\
\hline H1B & 5443 & 5559 & 2665 & 60 \\
\hline
\end{tabular}


Table 6. Torsion angles $\left[{ }^{\circ}\right]$ for rhowe28.

\begin{tabular}{|c|c|c|c|}
\hline Mo1-N1-C1-C2 & $174.05(18)$ & $\mathrm{C} 11-\mathrm{C} 7-\mathrm{C} 8-\mathrm{C} 9$ & $4.0(4)$ \\
\hline Mo1-N1-C12-C4 & $-172.55(18)$ & C12-N1-C1-C2 & $0.7(4)$ \\
\hline Mo1-N1-C12-C11 & $8.2(3)$ & $\mathrm{C} 12-\mathrm{C} 4-\mathrm{C} 5-\mathrm{C} 6$ & $2.1(4)$ \\
\hline Mo1-N2-C10-C9 & $-167.62(18)$ & Co1-C13-C14-C15 & $-58.7(2)$ \\
\hline Mo1-N2-C11-C7 & $168.97(18)$ & Co1-C13-C17-C16 & $58.9(2)$ \\
\hline Mo1-N2-C11-C12 & $-11.5(3)$ & Co1-C14-C15-C16 & $-59.3(2)$ \\
\hline $\mathrm{N} 1-\mathrm{C} 1-\mathrm{C} 2-\mathrm{C} 3$ & $-2.0(4)$ & Co1-C15-C16-C17 & $-59.2(2)$ \\
\hline N2-C11-C12-N1 & $2.3(3)$ & Co1-C16-C17-C13 & $-58.9(2)$ \\
\hline $\mathrm{N} 2-\mathrm{C} 11-\mathrm{C} 12-\mathrm{C} 4$ & $-177.0(2)$ & Co1-C18-C19-C20 & $59.5(2)$ \\
\hline C1-N1-C12-C4 & $1.7(4)$ & Co1-C18-C22-C21 & $-58.6(2)$ \\
\hline C1-N1-C12-C11 & $-177.5(2)$ & Co1-C19-C20-C21 & $58.8(2)$ \\
\hline $\mathrm{C} 1-\mathrm{C} 2-\mathrm{C} 3-\mathrm{C} 4$ & $1.0(4)$ & $\mathrm{Co} 1-\mathrm{C} 20-\mathrm{C} 21-\mathrm{C} 22$ & $59.7(2)$ \\
\hline $\mathrm{C} 2-\mathrm{C} 3-\mathrm{C} 4-\mathrm{C} 5$ & $-178.3(3)$ & Co1-C21-C22-C18 & $58.8(2)$ \\
\hline $\mathrm{C} 2-\mathrm{C} 3-\mathrm{C} 4-\mathrm{C} 12$ & $1.2(4)$ & $\mathrm{C} 13-\mathrm{C} 14-\mathrm{C} 15-\mathrm{Co} 1$ & $59.0(2)$ \\
\hline $\mathrm{C} 3-\mathrm{C} 4-\mathrm{C} 5-\mathrm{C} 6$ & $-178.4(3)$ & $\mathrm{C} 13-\mathrm{C} 14-\mathrm{C} 15-\mathrm{C} 16$ & $-0.3(3)$ \\
\hline $\mathrm{C} 3-\mathrm{C} 4-\mathrm{C} 12-\mathrm{N} 1$ & $-2.7(4)$ & $\mathrm{C} 14-\mathrm{C} 13-\mathrm{C} 17-\mathrm{Co} 1$ & $-59.1(2)$ \\
\hline C3-C4-C12-C11 & $176.5(2)$ & $\mathrm{C} 14-\mathrm{C} 13-\mathrm{C} 17-\mathrm{C} 16$ & $-0.2(3)$ \\
\hline $\mathrm{C} 4-\mathrm{C} 5-\mathrm{C} 6-\mathrm{C} 7$ & $1.2(4)$ & $\mathrm{C} 14-\mathrm{C} 15-\mathrm{C} 16-\mathrm{Co} 1$ & $59.4(2)$ \\
\hline $\mathrm{C} 5-\mathrm{C} 4-\mathrm{C} 12-\mathrm{N} 1$ & $176.9(2)$ & $\mathrm{C} 14-\mathrm{C} 15-\mathrm{C} 16-\mathrm{C} 17$ & $0.2(3)$ \\
\hline C5-C4-C12-C11 & $-3.9(4)$ & $\mathrm{C} 15-\mathrm{C} 16-\mathrm{C} 17-\mathrm{Co} 1$ & $58.9(2)$ \\
\hline C5-C6-C7-C8 & $177.5(3)$ & $\mathrm{C} 15-\mathrm{C} 16-\mathrm{C} 17-\mathrm{C} 13$ & $0.0(3)$ \\
\hline C5-C6-C7-C11 & $-2.6(4)$ & $\mathrm{C} 17-\mathrm{C} 13-\mathrm{C} 14-\mathrm{Co} 1$ & $59.1(2)$ \\
\hline C6-C7-C8-C9 & $-176.1(2)$ & C17-C13-C14-C15 & $0.3(3)$ \\
\hline C6-C7-C11-N2 & $-179.8(2)$ & $\mathrm{C} 18-\mathrm{C} 19-\mathrm{C} 20-\mathrm{Co} 1$ & $-59.6(2)$ \\
\hline C6-C7-C11-C12 & $0.7(4)$ & C18-C19-C20-C21 & $-0.8(3)$ \\
\hline C7-C8-C9-C10 & $-3.8(4)$ & C19-C18-C22-Co1 & $58.8(2)$ \\
\hline C7-C11-C12-N1 & $-178.2(2)$ & $\mathrm{C} 19-\mathrm{C} 18-\mathrm{C} 22-\mathrm{C} 21$ & $0.2(3)$ \\
\hline $\mathrm{C} 7-\mathrm{C} 11-\mathrm{C} 12-\mathrm{C} 4$ & $2.6(4)$ & $\mathrm{C} 19-\mathrm{C} 20-\mathrm{C} 21-\mathrm{Co} 1$ & $-58.8(2)$ \\
\hline $\mathrm{C} 8-\mathrm{C} 7-\mathrm{C} 11-\mathrm{N} 2$ & $0.1(4)$ & C19-C20-C21-C22 & $0.9(3)$ \\
\hline C8-C7-C11-C12 & $-179.4(2)$ & $\mathrm{C} 20-\mathrm{C} 21-\mathrm{C} 22-\mathrm{Co} 1$ & $-59.4(2)$ \\
\hline $\mathrm{C} 8-\mathrm{C} 9-\mathrm{C} 10-\mathrm{N} 2$ & $-0.7(4)$ & $\mathrm{C} 20-\mathrm{C} 21-\mathrm{C} 22-\mathrm{C} 18$ & $-0.7(3)$ \\
\hline C10-N2-C11-C7 & $-4.4(3)$ & C22-C18-C19-Co1 & $-59.1(2)$ \\
\hline C10-N2-C11-C12 & $175.1(2)$ & C22-C18-C19-C20 & $0.4(3)$ \\
\hline C11-N2-C10-C9 & $4.7(4)$ & & \\
\hline
\end{tabular}


Symmetry transformations used to generate equivalent atoms:

$\# 1-\mathrm{x}+1, \mathrm{y},-\mathrm{z}+1 / 2$

Table 7. Hydrogen bonds and close contacts for rhowe28 [ $\AA$ and $\left.{ }^{\circ}\right]$.

\begin{tabular}{lcccc}
\hline D-H...A & d(D-H) & d(H...A & $d($ D...A $)$ & $<$ (DHA) \\
\hline O1-H1A...Cl3\#1 & 0.85 & 2.94 & $3.7892(7)$ & 179.9 \\
O1-H1B...Cl2 & 0.85 & 2.99 & $3.845(4)$ & 180.0 \\
\hline
\end{tabular}

Symmetry transformations used to generate equivalent atoms:

$\# 1-\mathrm{x}+1, \mathrm{y},-\mathrm{z}+1 / 2$ 
REFERENCE NUMBER: rhowe36

CRYSTAL STRUCTURE REPORT

$\mathrm{C}_{24} \mathrm{H}_{25} \mathrm{Cl}_{4}$ Co Mo N 3

or

$\left[\mathrm{CoCp}_{2}\right]\left[\mathrm{Mo}\left(\mathrm{dmbpy} \mathrm{Cl}_{4}\right] \cdot \mathrm{CH}_{3} \mathrm{CN}\right.$

Report prepared for:

Prof. W. Eckenhoff
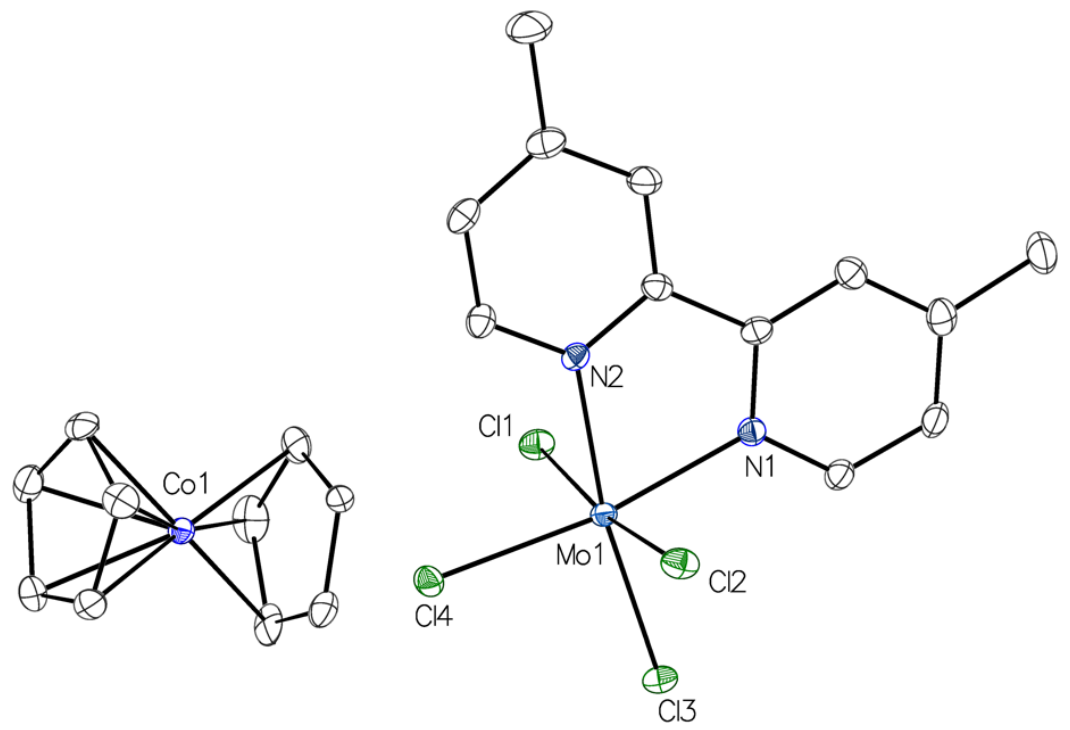

William W. Brennessel

X-ray Crystallographic Facility

Department of Chemistry, University of Rochester

120 Trustee Road

Rochester, NY 14627 


\section{Data collection}

A crystal $\left(0.155 \times 0.134 \times 0.066 \mathrm{~mm}^{3}\right)$ was placed onto a thin glass optical fiber or a nylon loop and mounted on a Rigaku XtaLab Synergy-S Dualflex diffractometer equipped with a HyPix-6000HE HPC area detector for data collection at 99.99(10) K. A preliminary set of cell constants and an orientation matrix were calculated from a small sampling of reflections. ${ }^{1}$ A short pre-experiment was run, from which an optimal data collection strategy was determined. The full data collection was carried out using a PhotonJet $(\mathrm{Cu}) \mathrm{X}$-ray Source with frame times of 0.06 and 0.23 seconds and a detector distance of $31.2 \mathrm{~mm}$. Series of frames were collected in $0.50^{\circ}$ steps in $\omega$ at different $2 \theta, \kappa$, and $\phi$ settings. After the intensity data were corrected for absorption, the final cell constants were calculated from the xyz centroids of 15290 strong reflections from the actual data collection after integration. ${ }^{1}$ See Table 1 for additional crystal and refinement information.

\section{Structure solution and refinement}

The structure was solved using ShelXT ${ }^{2}$ and refined using ShelXL. ${ }^{3}$ The space group $P 2{ }_{1} / c$ was determined based on systematic absences. Most or all non-hydrogen atoms were assigned from the solution. Full-matrix least squares / difference Fourier cycles were performed which located any remaining non-hydrogen atoms. All non-hydrogen atoms were refined with anisotropic displacement parameters. All hydrogen atoms were placed in ideal positions and refined as riding atoms with relative isotropic displacement parameters. The final full matrix least squares refinement converged to $R 1=0.0269\left(F^{2}, I>2 \sigma(I)\right)$ and $w R 2=0.0676\left(F^{2}\right.$, all data).

\section{Structure description}

The structure is the one suggested. The asymmetric unit contains one cation, one anion, and one cocrystallized acetonitrile solvent molecule, all in general positions. Cyclopentadienyl ring ligand C18-C22 is modeled as disordered over two positions (0.60:0.40).

Structure manipulation and figure generation were performed using Olex2. ${ }^{4}$ Unless noted otherwise all structural diagrams containing thermal displacement ellipsoids are drawn at the $50 \%$ probability level.

Data collection, structure solution, and structure refinement were conducted at the X-ray Crystallographic Facility, B04 Hutchison Hall, Department of Chemistry, University of Rochester. The instrument was purchased with funding from NSF MRI program grant CHE-1725028. All publications arising from this report MUST either 1) include William W. Brennessel as a coauthor or 2) acknowledge William W. Brennessel and the X-ray Crystallographic Facility of the Department of Chemistry at the University of Rochester. 
1 CrysAlisPro, version 171.39.46; Rigaku Corporation: Oxford, UK, 2018.

2 Sheldrick, G. M. SHELXT, version 2018/2; Acta. Crystallogr. 2015, A71, 3-8.

3 Sheldrick, G. M. SHELXL, version 2018/3; Acta. Crystallogr. 2015, C71, 3-8.

4 Dolomanov, O. V.; Bourhis, L. J.; Gildea, R. J.; Howard, J. A. K.; Puschmann, H. Olex2, version 1.2-ac3; J. Appl. Cryst. 2009, 42, 339-341.

Some equations of interest:

$$
\begin{gathered}
R_{\mathrm{int}}=\Sigma\left|F_{\mathrm{o}}^{2}-<F_{\mathrm{o}}^{2}>\right| / \Sigma\left|F_{\mathrm{o}}{ }^{2}\right| \\
R 1=\Sigma|| F_{\mathrm{o}}|-| F_{\mathrm{c}}|/ \Sigma| F_{\mathrm{o}} \mid \\
w R 2=\left[\Sigma\left[w\left(F_{\mathrm{o}}{ }^{2} F_{\mathrm{c}}{ }^{2}\right)^{2}\right] / \Sigma\left[w\left(F_{\mathrm{o}}{ }^{2}\right)^{2}\right]\right]^{1 / 2} \\
\text { where } w=1 /\left[\sigma^{2}\left(F_{\mathrm{o}}{ }^{2}\right)+(a P)^{2}+b P\right] \text { and } \\
P=1 / 3 \max \left(0, F_{\mathrm{o}}{ }^{2}\right)+2 / 3 F_{\mathrm{c}}{ }^{2} \\
\mathrm{GOF}=S=\left[\Sigma\left[w\left(F_{\mathrm{o}}{ }^{2}-F_{\mathrm{c}}{ }^{2}\right)^{2}\right] /(m-n)\right]^{1 / 2}
\end{gathered}
$$

where $m=$ number of reflections and $n=$ number of parameters
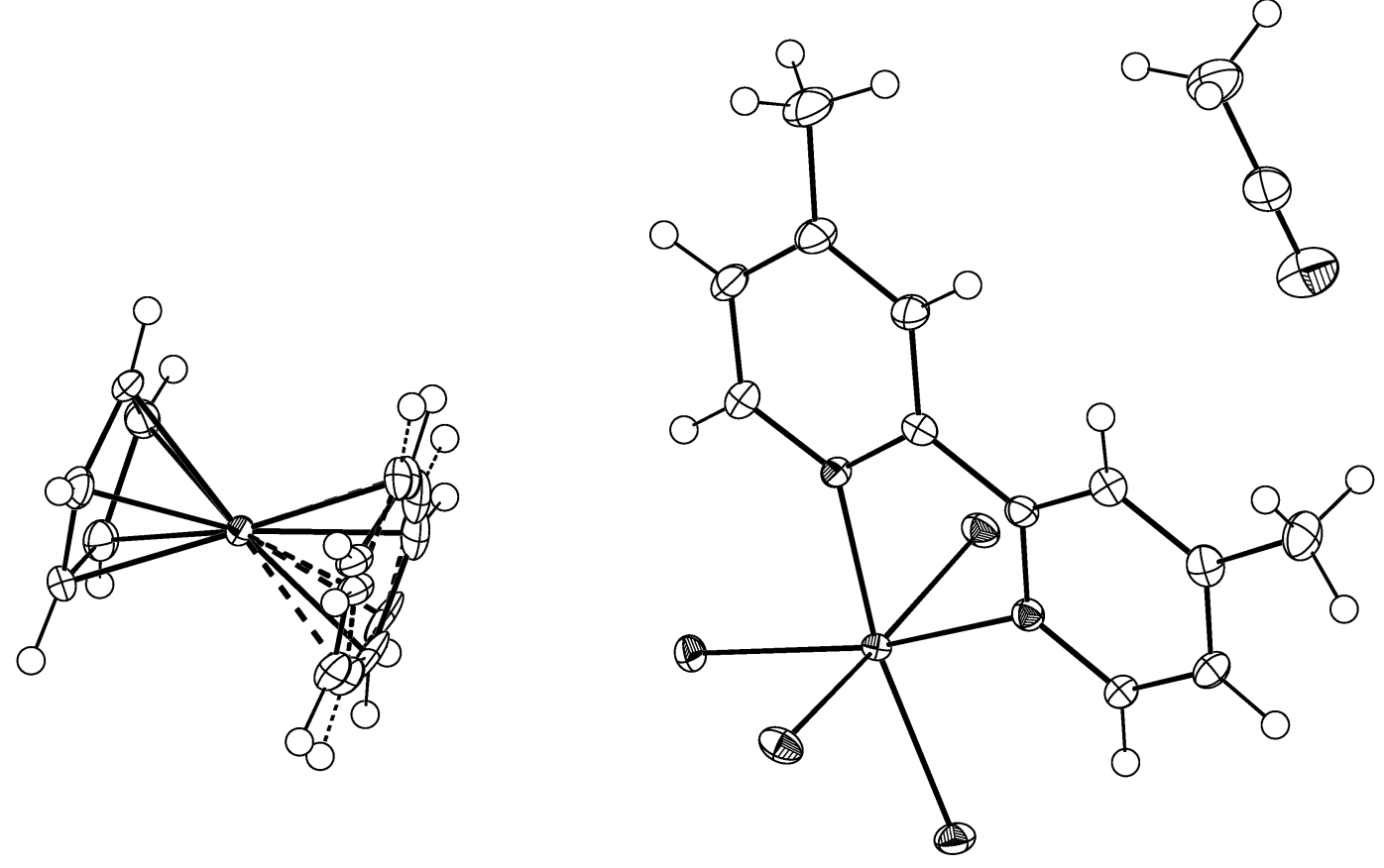


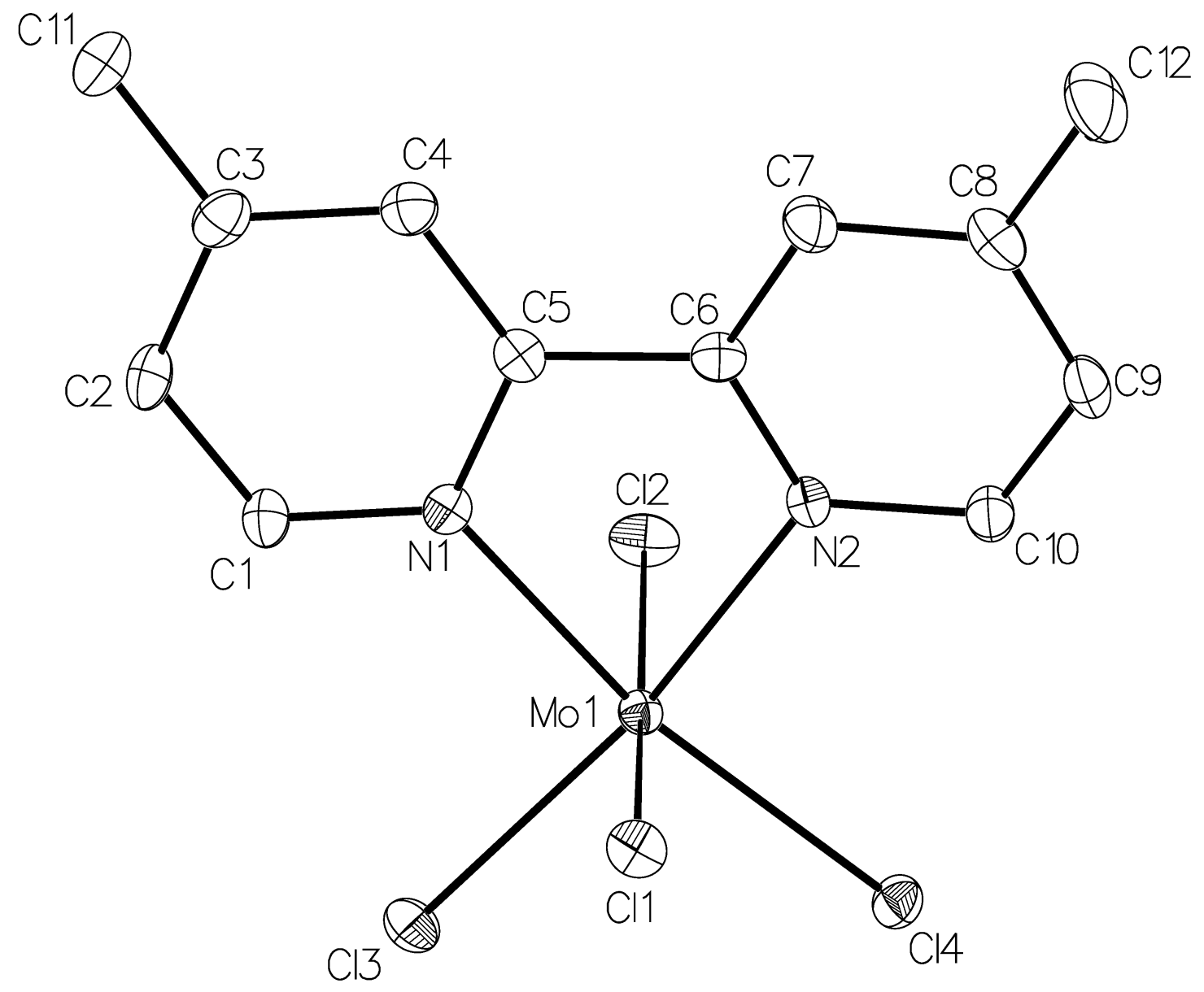



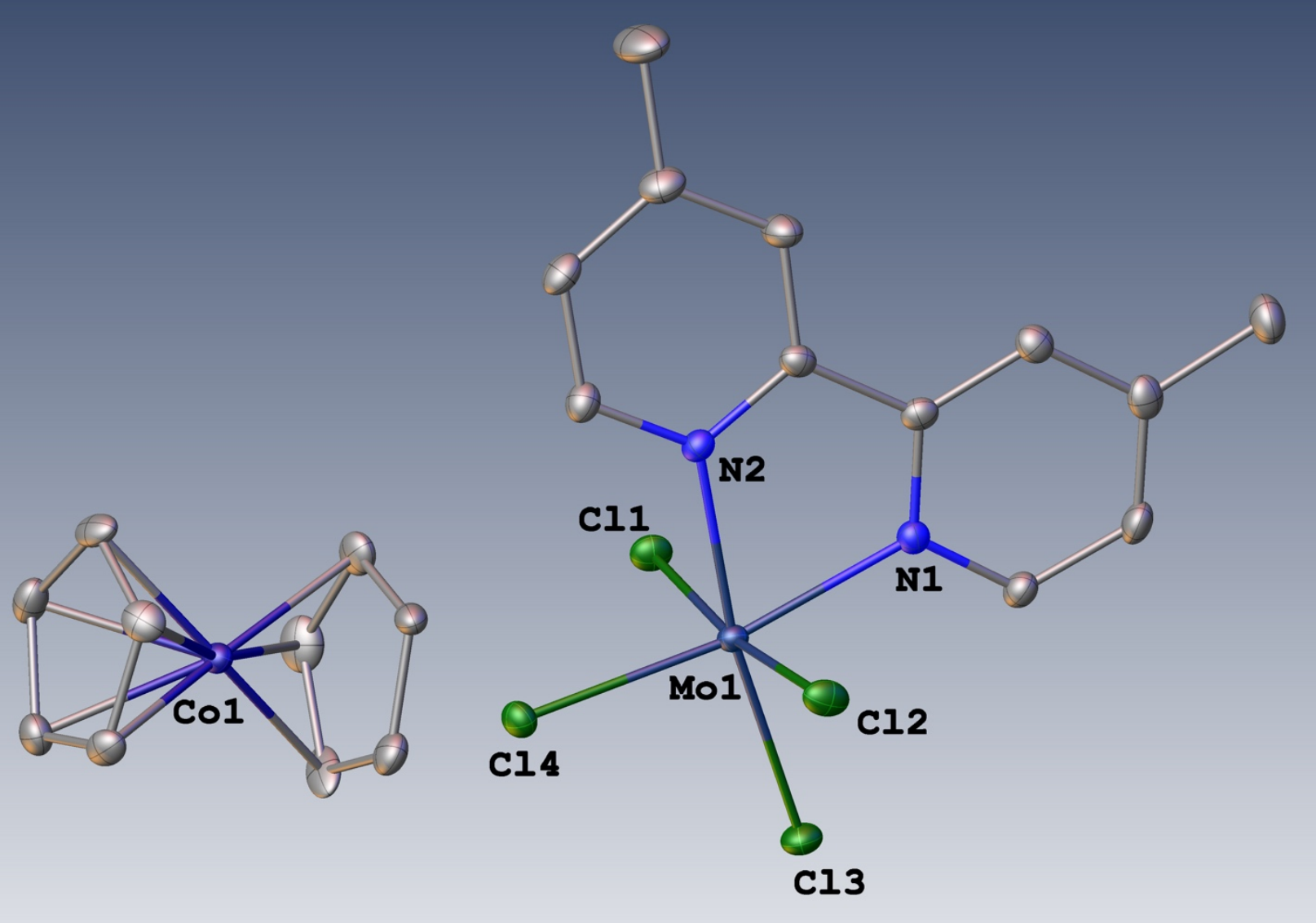
Table 1. Crystal data and structure refinement for rhowe36.

\begin{tabular}{|c|c|c|}
\hline Identification code & \multicolumn{2}{|l|}{ rhowe36 } \\
\hline Empirical formula & \multicolumn{2}{|c|}{ C24 H25 Cl4 Co Mo N3 } \\
\hline Formula weight & \multicolumn{2}{|l|}{652.14} \\
\hline Temperature & \multicolumn{2}{|l|}{$99.99(10) \mathrm{K}$} \\
\hline Wavelength & \multicolumn{2}{|l|}{$1.54184 \AA$} \\
\hline Crystal system & \multicolumn{2}{|l|}{ monoclinic } \\
\hline Space group & \multicolumn{2}{|l|}{$P 2{ }_{1} / c$} \\
\hline \multirow[t]{3}{*}{ Unit cell dimensions } & $a=12.35370(10) \AA$ & $\alpha=90^{\circ}$ \\
\hline & $b=9.96440(10) \AA$ & $\beta=93.6200(10)^{\circ}$ \\
\hline & $c=21.2064(2) \AA$ & $\gamma=90^{\circ}$ \\
\hline Volume & \multicolumn{2}{|l|}{$2605.24(4) \AA^{3}$} \\
\hline$Z$ & \multicolumn{2}{|l|}{4} \\
\hline Density (calculated) & \multicolumn{2}{|l|}{$1.663 \mathrm{Mg} / \mathrm{m}^{3}$} \\
\hline Absorption coefficient & \multicolumn{2}{|l|}{$12.844 \mathrm{~mm}^{-1}$} \\
\hline$F(000)$ & \multicolumn{2}{|l|}{1308} \\
\hline Crystal color, morphology & \multicolumn{2}{|l|}{ dark red, needle } \\
\hline Crystal size & \multicolumn{2}{|c|}{$0.155 \times 0.134 \times 0.066 \mathrm{~mm}^{3}$} \\
\hline Theta range for data collection & \multicolumn{2}{|l|}{3.585 to $78.064^{\circ}$} \\
\hline Index ranges & \multicolumn{2}{|c|}{$-15 \leq h \leq 15,-12 \leq k \leq 12,-19 \leq l \leq 26$} \\
\hline Reflections collected & \multicolumn{2}{|l|}{22947} \\
\hline Independent reflections & \multicolumn{2}{|l|}{$5476[R($ int $)=0.0386]$} \\
\hline Observed reflections & \multicolumn{2}{|l|}{5200} \\
\hline Completeness to theta $=74.504^{\circ}$ & \multicolumn{2}{|l|}{$99.9 \%$} \\
\hline Absorption correction & \multicolumn{2}{|l|}{ Multi-scan } \\
\hline Max. and min. transmission & \multicolumn{2}{|l|}{1.00000 and 0.75938} \\
\hline Refinement method & \multicolumn{2}{|c|}{ Full-matrix least-squares on $F^{2}$} \\
\hline Data / restraints / parameters & \multicolumn{2}{|l|}{$5476 / 56 / 317$} \\
\hline Goodness-of-fit on $F^{2}$ & \multicolumn{2}{|l|}{1.041} \\
\hline Final $R$ indices $[I>2 \operatorname{sigma}(I)]$ & \multicolumn{2}{|c|}{$R 1=0.0269, w R 2=0.0669$} \\
\hline$R$ indices (all data) & \multicolumn{2}{|c|}{$R 1=0.0285, w R 2=0.0676$} \\
\hline Largest diff. peak and hole & \multicolumn{2}{|l|}{0.397 and -0.777 e. $\AA^{-3}$} \\
\hline
\end{tabular}


Table 2. Atomic coordinates $\left(\times 10^{4}\right)$ and equivalent isotropic displacement parameters $\left(\AA^{2} \times 10^{3}\right)$ for rhowe $36 . \mathrm{U}_{\mathrm{eq}}$ is defined as one third of the trace of the orthogonalized $\mathrm{U}_{\mathrm{ij}}$ tensor.

\begin{tabular}{|c|c|c|c|c|}
\hline & $\mathrm{x}$ & $\mathrm{y}$ & $\mathrm{z}$ & $\mathrm{U}_{\mathrm{eq}}$ \\
\hline Mo1 & $2650(1)$ & $6888(1)$ & $6016(1)$ & 11(1) \\
\hline $\mathrm{Cl1}$ & $3642(1)$ & $8441(1)$ & $5377(1)$ & $17(1)$ \\
\hline $\mathrm{Cl} 2$ & $1524(1)$ & 5271(1) & $6540(1)$ & $19(1)$ \\
\hline $\mathrm{Cl} 3$ & 2581(1) & $8514(1)$ & $6869(1)$ & $18(1)$ \\
\hline $\mathrm{Cl} 4$ & 4331(1) & $5869(1)$ & $6435(1)$ & $16(1)$ \\
\hline N1 & $1149(2)$ & $7517(2)$ & $5525(1)$ & $13(1)$ \\
\hline N2 & $2514(2)$ & $5638(2)$ & $5178(1)$ & $12(1)$ \\
\hline $\mathrm{C} 1$ & $475(2)$ & $8451(2)$ & $5733(1)$ & $16(1)$ \\
\hline $\mathrm{C} 2$ & $-521(2)$ & $8744(3)$ & $5421(1)$ & $19(1)$ \\
\hline $\mathrm{C} 3$ & $-850(2)$ & $8038(2)$ & $4876(1)$ & $18(1)$ \\
\hline $\mathrm{C} 4$ & $-156(2)$ & $7063(2)$ & $4668(1)$ & $17(1)$ \\
\hline $\mathrm{C} 5$ & $842(2)$ & 6829(2) & $4990(1)$ & $14(1)$ \\
\hline C6 & $1633(2)$ & $5849(2)$ & $4777(1)$ & $14(1)$ \\
\hline $\mathrm{C} 7$ & $1523(2)$ & $5217(3)$ & $4192(1)$ & $20(1)$ \\
\hline $\mathrm{C} 8$ & $2332(2)$ & 4368(3) & 4001(1) & $21(1)$ \\
\hline C9 & $3221(2)$ & 4141(2) & $4424(1)$ & $18(1)$ \\
\hline $\mathrm{C} 10$ & $3284(2)$ & 4784(2) & $5003(1)$ & $17(1)$ \\
\hline C11 & $-1901(2)$ & $8340(3)$ & $4510(1)$ & $24(1)$ \\
\hline $\mathrm{C} 12$ & 2257(3) & $3735(3)$ & $3358(1)$ & $36(1)$ \\
\hline Co1 & $5789(1)$ & $975(1)$ & $6536(1)$ & $12(1)$ \\
\hline $\mathrm{C} 13$ & $6404(2)$ & $-485(3)$ & $6005(1)$ & $20(1)$ \\
\hline C14 & $7233(2)$ & $418(3)$ & $6223(1)$ & $21(1)$ \\
\hline C15 & 7292(2) & $417(3)$ & $6895(1)$ & $19(1)$ \\
\hline $\mathrm{C} 16$ & $6490(2)$ & $-483(2)$ & 7094(1) & $17(1)$ \\
\hline $\mathrm{C} 17$ & $5938(2)$ & $-1049(2)$ & $6545(1)$ & $19(1)$ \\
\hline $\mathrm{C} 18$ & $4736(14)$ & 2049(18) & $5959(4)$ & $20(1)$ \\
\hline C19 & $5479(9)$ & $2898(10)$ & $6290(4)$ & $25(2)$ \\
\hline $\mathrm{C} 20$ & $5352(6)$ & 2713(8) & $6959(4)$ & $25(1)$ \\
\hline $\mathrm{C} 21$ & $4532(6)$ & $1720(8)$ & $7012(3)$ & $20(1)$ \\
\hline $\mathrm{C} 22$ & 4153(9) & $1294(11)$ & $6395(4)$ & $16(1)$ \\
\hline C18' & $4630(20)$ & $1870(30)$ & $5967(7)$ & $20(1)$ \\
\hline
\end{tabular}




\begin{tabular}{lclll}
$\mathrm{C} 19^{\prime}$ & $5480(15)$ & $2776(17)$ & $6104(6)$ & $25(2)$ \\
$\mathrm{C}^{\prime} 0^{\prime}$ & $5600(9)$ & $2924(12)$ & $6763(6)$ & $25(1)$ \\
$\mathrm{C} 21^{\prime}$ & $4806(10)$ & $2100(12)$ & $7043(5)$ & $20(1)$ \\
$\mathrm{C}^{\prime} 2^{\prime}$ & $4210(14)$ & $1452(18)$ & $6543(6)$ & $16(1)$ \\
$\mathrm{N} 3$ & $61(2)$ & $8845(3)$ & $3346(1)$ & $38(1)$ \\
$\mathrm{C} 23$ & $28(2)$ & $8010(3)$ & $2978(1)$ & $26(1)$ \\
$\mathrm{C} 24$ & $-1(3)$ & $6954(3)$ & $2505(2)$ & $33(1)$ \\
\hline
\end{tabular}


Table 3. Bond lengths $[\AA]$ and angles $\left[{ }^{\circ}\right]$ for rhowe 36 .

\begin{tabular}{|c|c|c|c|}
\hline $\mathrm{Mo}(1)-\mathrm{Cl}(1)$ & $2.4381(5)$ & $\operatorname{Co}(1)-C(14)$ & $2.020(2)$ \\
\hline $\mathrm{Mo}(1)-\mathrm{Cl}(2)$ & $2.4403(6)$ & $\mathrm{Co}(1)-\mathrm{C}(15)$ & $2.039(2)$ \\
\hline $\mathrm{Mo}(1)-\mathrm{Cl}(3)$ & $2.4333(5)$ & $\operatorname{Co}(1)-C(16)$ & $2.033(2)$ \\
\hline $\mathrm{Mo}(1)-\mathrm{Cl}(4)$ & $2.4294(5)$ & $\mathrm{Co}(1)-\mathrm{C}(17)$ & $2.025(2)$ \\
\hline $\mathrm{Mo}(1)-\mathrm{N}(1)$ & $2.1606(19)$ & $\mathrm{Co}(1)-\mathrm{C}(18)$ & $2.03(2)$ \\
\hline $\mathrm{Mo}(1)-\mathrm{N}(2)$ & $2.1681(18)$ & $\mathrm{Co}(1)-\mathrm{C}(19)$ & $2.016(11)$ \\
\hline $\mathrm{N}(1)-\mathrm{C}(1)$ & $1.342(3)$ & $\mathrm{Co}(1)-\mathrm{C}(20)$ & $2.039(6)$ \\
\hline $\mathrm{N}(1)-\mathrm{C}(5)$ & $1.360(3)$ & $\mathrm{Co}(1)-\mathrm{C}\left(18^{\prime}\right)$ & $2.02(3)$ \\
\hline $\mathrm{N}(2)-\mathrm{C}(6)$ & $1.354(3)$ & $\mathrm{Co}(1)-\mathrm{C}\left(20^{\prime}\right)$ & $2.018(11)$ \\
\hline $\mathrm{N}(2)-\mathrm{C}(10)$ & $1.346(3)$ & $\mathrm{Co}(1)-\mathrm{C}\left(21^{\prime}\right)$ & $2.013(11)$ \\
\hline $\mathrm{C}(1)-\mathrm{H}(1)$ & 0.9300 & $\mathrm{Co}(1)-\mathrm{C}\left(22^{\prime}\right)$ & $2.008(19)$ \\
\hline $\mathrm{C}(1)-\mathrm{C}(2)$ & $1.390(3)$ & $\mathrm{C}(13)-\mathrm{H}(13)$ & 0.9300 \\
\hline $\mathrm{C}(2)-\mathrm{H}(2)$ & 0.9300 & $C(13)-C(14)$ & $1.419(4)$ \\
\hline $\mathrm{C}(2)-\mathrm{C}(3)$ & $1.392(4)$ & $\mathrm{C}(13)-\mathrm{C}(17)$ & $1.427(4)$ \\
\hline$C(3)-C(4)$ & $1.387(3)$ & $\mathrm{C}(14)-\mathrm{H}(14)$ & 0.9300 \\
\hline$C(3)-C(11)$ & $1.500(3)$ & $C(14)-C(15)$ & $1.421(3)$ \\
\hline $\mathrm{C}(4)-\mathrm{H}(4)$ & 0.9300 & $\mathrm{C}(15)-\mathrm{H}(15)$ & 0.9300 \\
\hline$C(4)-C(5)$ & $1.391(3)$ & $C(15)-C(16)$ & $1.420(3)$ \\
\hline$C(5)-C(6)$ & $1.472(3)$ & $\mathrm{C}(16)-\mathrm{H}(16)$ & 0.9300 \\
\hline$C(6)-C(7)$ & $1.391(3)$ & $\mathrm{C}(16)-\mathrm{C}(17)$ & $1.429(3)$ \\
\hline $\mathrm{C}(7)-\mathrm{H}(7)$ & 0.9300 & $\mathrm{C}(17)-\mathrm{H}(17)$ & 0.9300 \\
\hline$C(7)-C(8)$ & $1.389(3)$ & $\mathrm{C}(18)-\mathrm{H}(18)$ & 0.9300 \\
\hline $\mathrm{C}(8)-\mathrm{C}(9)$ & $1.391(4)$ & $\mathrm{C}(18)-\mathrm{C}(19)$ & $1.404(8)$ \\
\hline$C(8)-C(12)$ & $1.499(3)$ & $\mathrm{C}(18)-\mathrm{C}(22)$ & $1.423(8)$ \\
\hline $\mathrm{C}(9)-\mathrm{H}(9)$ & 0.9300 & $\mathrm{C}(19)-\mathrm{H}(19)$ & 0.9300 \\
\hline$C(9)-C(10)$ & $1.382(3)$ & $C(19)-C(20)$ & $1.449(8)$ \\
\hline $\mathrm{C}(10)-\mathrm{H}(10)$ & 0.9300 & $\mathrm{C}(20)-\mathrm{H}(20)$ & 0.9300 \\
\hline $\mathrm{C}(11)-\mathrm{H}(11 \mathrm{~A})$ & 0.9600 & $\mathrm{C}(20)-\mathrm{C}(21)$ & $1.426(7)$ \\
\hline $\mathrm{C}(11)-\mathrm{H}(11 \mathrm{~B})$ & 0.9600 & $\mathrm{C}(21)-\mathrm{H}(21)$ & 0.9300 \\
\hline $\mathrm{C}(11)-\mathrm{H}(11 \mathrm{C})$ & 0.9600 & $\mathrm{C}(21)-\mathrm{C}(22)$ & $1.427(7)$ \\
\hline $\mathrm{C}(12)-\mathrm{H}(12 \mathrm{~A})$ & 0.9600 & $\mathrm{C}(22)-\mathrm{H}(22)$ & 0.9300 \\
\hline $\mathrm{C}(12)-\mathrm{H}(12 \mathrm{~B})$ & 0.9600 & $\mathrm{C}\left(18^{\prime}\right)-\mathrm{H}\left(18^{\prime}\right)$ & 0.9300 \\
\hline $\mathrm{C}(12)-\mathrm{H}(12 \mathrm{C})$ & 0.9600 & $\mathrm{C}\left(18^{\prime}\right)-\mathrm{C}\left(19^{\prime}\right)$ & $1.405(12)$ \\
\hline $\mathrm{Co}(1)-\mathrm{C}(13)$ & $2.017(2)$ & $\mathrm{C}\left(18^{\prime}\right)-\mathrm{C}\left(22^{\prime}\right)$ & $1.418(12)$ \\
\hline
\end{tabular}




\begin{tabular}{|c|c|}
\hline $\mathrm{C}\left(19^{\prime}\right)-\mathrm{H}\left(19^{\prime}\right)$ & 0.9300 \\
\hline $\mathrm{C}\left(19^{\prime}\right)-\mathrm{C}\left(20^{\prime}\right)$ & $1.405(10)$ \\
\hline $\mathrm{C}\left(20^{\prime}\right)-\mathrm{H}\left(20^{\prime}\right)$ & 0.9300 \\
\hline $\mathrm{C}\left(20^{\prime}\right)-\mathrm{C}\left(21^{\prime}\right)$ & $1.435(11)$ \\
\hline $\mathrm{C}\left(21^{\prime}\right)-\mathrm{H}\left(21^{\prime}\right)$ & 0.9300 \\
\hline$C\left(21^{\prime}\right)-C\left(22^{\prime}\right)$ & $1.408(11)$ \\
\hline $\mathrm{C}\left(22^{\prime}\right)-\mathrm{H}\left(22^{\prime}\right)$ & 0.9800 \\
\hline $\mathrm{N}(3)-\mathrm{C}(23)$ & $1.140(4)$ \\
\hline $\mathrm{C}(23)-\mathrm{C}(24)$ & $1.453(4)$ \\
\hline $\mathrm{C}(24)-\mathrm{H}(24 \mathrm{~A})$ & 0.9600 \\
\hline $\mathrm{C}(24)-\mathrm{H}(24 \mathrm{~B})$ & 0.9600 \\
\hline $\mathrm{C}(24)-\mathrm{H}(24 \mathrm{C})$ & 0.9600 \\
\hline $\mathrm{Cl}(1)-\mathrm{Mo}(1)-\mathrm{Cl}(2)$ & $173.116(19)$ \\
\hline $\mathrm{Cl}(3)-\mathrm{Mo}(1)-\mathrm{Cl}(1)$ & $91.873(19)$ \\
\hline $\mathrm{Cl}(3)-\mathrm{Mo}(1)-\mathrm{Cl}(2)$ & $93.080(19)$ \\
\hline $\mathrm{Cl}(4)-\mathrm{Mo}(1)-\mathrm{Cl}(1)$ & $91.160(19)$ \\
\hline $\mathrm{Cl}(4)-\mathrm{Mo}(1)-\mathrm{Cl}(2)$ & $93.229(19)$ \\
\hline $\mathrm{Cl}(4)-\mathrm{Mo}(1)-\mathrm{Cl}(3)$ & 94.322(19) \\
\hline $\mathrm{N}(1)-\mathrm{Mo}(1)-\mathrm{Cl}(1)$ & $89.68(5)$ \\
\hline $\mathrm{N}(1)-\mathrm{Mo}(1)-\mathrm{Cl}(2)$ & $85.10(5)$ \\
\hline $\mathrm{N}(1)-\mathrm{Mo}(1)-\mathrm{Cl}(3)$ & $95.50(5)$ \\
\hline $\mathrm{N}(1)-\mathrm{Mo}(1)-\mathrm{Cl}(4)$ & $170.11(5)$ \\
\hline $\mathrm{N}(1)-\mathrm{Mo}(1)-\mathrm{N}(2)$ & $75.64(7)$ \\
\hline $\mathrm{N}(2)-\mathrm{Mo}(1)-\mathrm{Cl}(1)$ & $85.66(5)$ \\
\hline $\mathrm{N}(2)-\mathrm{Mo}(1)-\mathrm{Cl}(2)$ & $88.70(5)$ \\
\hline $\mathrm{N}(2)-\mathrm{Mo}(1)-\mathrm{Cl}(3)$ & $170.79(5)$ \\
\hline $\mathrm{N}(2)-\mathrm{Mo}(1)-\mathrm{Cl}(4)$ & $94.60(5)$ \\
\hline $\mathrm{C}(1)-\mathrm{N}(1)-\mathrm{Mo}(1)$ & $125.04(15)$ \\
\hline $\mathrm{C}(1)-\mathrm{N}(1)-\mathrm{C}(5)$ & 118.54(19) \\
\hline $\mathrm{C}(5)-\mathrm{N}(1)-\mathrm{Mo}(1)$ & $116.23(15)$ \\
\hline $\mathrm{C}(6)-\mathrm{N}(2)-\mathrm{Mo}(1)$ & $116.28(15)$ \\
\hline C(10)-N(2)-Mo(1) & $124.75(15)$ \\
\hline$C(10)-N(2)-C(6)$ & $118.6(2)$ \\
\hline $\mathrm{N}(1)-\mathrm{C}(1)-\mathrm{H}(1)$ & 118.7 \\
\hline $\mathrm{N}(1)-\mathrm{C}(1)-\mathrm{C}(2)$ & $122.5(2)$ \\
\hline $\mathrm{C}(2)-\mathrm{C}(1)-\mathrm{H}(1)$ & 118.7 \\
\hline
\end{tabular}

\begin{tabular}{|c|c|}
\hline $\mathrm{C}(1)-\mathrm{C}(2)-\mathrm{H}(2)$ & 120.2 \\
\hline$C(1)-C(2)-C(3)$ & $119.5(2)$ \\
\hline $\mathrm{C}(3)-\mathrm{C}(2)-\mathrm{H}(2)$ & 120.2 \\
\hline$C(2)-C(3)-C(11)$ & $121.8(2)$ \\
\hline $\mathrm{C}(4)-\mathrm{C}(3)-\mathrm{C}(2)$ & $117.7(2)$ \\
\hline $\mathrm{C}(4)-\mathrm{C}(3)-\mathrm{C}(11)$ & $120.5(2)$ \\
\hline $\mathrm{C}(3)-\mathrm{C}(4)-\mathrm{H}(4)$ & 119.7 \\
\hline$C(3)-C(4)-C(5)$ & $120.5(2)$ \\
\hline $\mathrm{C}(5)-\mathrm{C}(4)-\mathrm{H}(4)$ & 119.7 \\
\hline $\mathrm{N}(1)-\mathrm{C}(5)-\mathrm{C}(4)$ & $121.2(2)$ \\
\hline $\mathrm{N}(1)-\mathrm{C}(5)-\mathrm{C}(6)$ & $115.7(2)$ \\
\hline$C(4)-C(5)-C(6)$ & $123.1(2)$ \\
\hline $\mathrm{N}(2)-\mathrm{C}(6)-\mathrm{C}(5)$ & $115.7(2)$ \\
\hline $\mathrm{N}(2)-\mathrm{C}(6)-\mathrm{C}(7)$ & $121.3(2)$ \\
\hline$C(7)-C(6)-C(5)$ & $123.0(2)$ \\
\hline $\mathrm{C}(6)-\mathrm{C}(7)-\mathrm{H}(7)$ & 119.8 \\
\hline$C(8)-C(7)-C(6)$ & $120.3(2)$ \\
\hline $\mathrm{C}(8)-\mathrm{C}(7)-\mathrm{H}(7)$ & 119.8 \\
\hline $\mathrm{C}(7)-\mathrm{C}(8)-\mathrm{C}(9)$ & $117.6(2)$ \\
\hline $\mathrm{C}(7)-\mathrm{C}(8)-\mathrm{C}(12)$ & $121.1(2)$ \\
\hline $\mathrm{C}(9)-\mathrm{C}(8)-\mathrm{C}(12)$ & $121.3(2)$ \\
\hline $\mathrm{C}(8)-\mathrm{C}(9)-\mathrm{H}(9)$ & 120.2 \\
\hline$C(10)-C(9)-C(8)$ & $119.7(2)$ \\
\hline $\mathrm{C}(10)-\mathrm{C}(9)-\mathrm{H}(9)$ & 120.2 \\
\hline $\mathrm{N}(2)-\mathrm{C}(10)-\mathrm{C}(9)$ & $122.5(2)$ \\
\hline $\mathrm{N}(2)-\mathrm{C}(10)-\mathrm{H}(10)$ & 118.8 \\
\hline $\mathrm{C}(9)-\mathrm{C}(10)-\mathrm{H}(10)$ & 118.8 \\
\hline $\mathrm{C}(3)-\mathrm{C}(11)-\mathrm{H}(11 \mathrm{~A})$ & 109.5 \\
\hline $\mathrm{C}(3)-\mathrm{C}(11)-\mathrm{H}(11 \mathrm{~B})$ & 109.5 \\
\hline $\mathrm{C}(3)-\mathrm{C}(11)-\mathrm{H}(11 \mathrm{C})$ & 109.5 \\
\hline $\mathrm{H}(11 \mathrm{~A})-\mathrm{C}(11)-\mathrm{H}(11 \mathrm{~B})$ & 109.5 \\
\hline $\mathrm{H}(11 \mathrm{~A})-\mathrm{C}(11)-\mathrm{H}(11 \mathrm{C})$ & 109.5 \\
\hline $\mathrm{H}(11 \mathrm{~B})-\mathrm{C}(11)-\mathrm{H}(11 \mathrm{C})$ & 109.5 \\
\hline $\mathrm{C}(8)-\mathrm{C}(12)-\mathrm{H}(12 \mathrm{~A})$ & 109.5 \\
\hline $\mathrm{C}(8)-\mathrm{C}(12)-\mathrm{H}(12 \mathrm{~B})$ & 109.5 \\
\hline $\mathrm{C}(8)-\mathrm{C}(12)-\mathrm{H}(12 \mathrm{C})$ & 109.5 \\
\hline
\end{tabular}




\begin{tabular}{|c|c|}
\hline $\mathrm{H}(12 \mathrm{~A})-\mathrm{C}(12)-\mathrm{H}(12 \mathrm{~B})$ & 109.5 \\
\hline $\mathrm{H}(12 \mathrm{~A})-\mathrm{C}(12)-\mathrm{H}(12 \mathrm{C})$ & 109.5 \\
\hline $\mathrm{H}(12 \mathrm{~B})-\mathrm{C}(12)-\mathrm{H}(12 \mathrm{C})$ & 109.5 \\
\hline $\mathrm{C}(13)-\mathrm{Co}(1)-\mathrm{C}(14)$ & $41.16(11)$ \\
\hline $\mathrm{C}(13)-\mathrm{Co}(1)-\mathrm{C}(15)$ & $69.22(10)$ \\
\hline $\mathrm{C}(13)-\mathrm{Co}(1)-\mathrm{C}(16)$ & $69.37(9)$ \\
\hline $\mathrm{C}(13)-\mathrm{Co}(1)-\mathrm{C}(17)$ & $41.36(10)$ \\
\hline $\mathrm{C}(13)-\mathrm{Co}(1)-\mathrm{C}(18)$ & $107.1(4)$ \\
\hline $\mathrm{C}(13)-\mathrm{Co}(1)-\mathrm{C}(20)$ & $167.9(3)$ \\
\hline $\mathrm{C}(13)-\mathrm{Co}(1)-\mathrm{C}\left(18^{\prime}\right)$ & $105.2(7)$ \\
\hline $\mathrm{C}(13)-\mathrm{Co}(1)-\mathrm{C}\left(20^{\prime}\right)$ & $151.7(4)$ \\
\hline $\mathrm{C}(14)-\mathrm{Co}(1)-\mathrm{C}(15)$ & $40.99(10)$ \\
\hline $\mathrm{C}(14)-\mathrm{Co}(1)-\mathrm{C}(16)$ & $69.03(10)$ \\
\hline $\mathrm{C}(14)-\mathrm{Co}(1)-\mathrm{C}(17)$ & $69.38(10)$ \\
\hline $\mathrm{C}(14)-\mathrm{Co}(1)-\mathrm{C}(18)$ & $119.5(4)$ \\
\hline $\mathrm{C}(14)-\mathrm{Co}(1)-\mathrm{C}(20)$ & $129.9(3)$ \\
\hline $\mathrm{C}(14)-\mathrm{Co}(1)-\mathrm{C}\left(18^{\prime}\right)$ & $122.2(6)$ \\
\hline $\mathrm{C}(16)-\mathrm{Co}(1)-\mathrm{C}(15)$ & $40.83(10)$ \\
\hline $\mathrm{C}(16)-\mathrm{Co}(1)-\mathrm{C}(20)$ & $117.7(2)$ \\
\hline $\mathrm{C}(17)-\mathrm{Co}(1)-\mathrm{C}(15)$ & $69.20(10)$ \\
\hline $\mathrm{C}(17)-\mathrm{Co}(1)-\mathrm{C}(16)$ & $41.23(10)$ \\
\hline $\mathrm{C}(17)-\mathrm{Co}(1)-\mathrm{C}(18)$ & $125.8(4)$ \\
\hline $\mathrm{C}(17)-\mathrm{Co}(1)-\mathrm{C}(20)$ & $150.3(3)$ \\
\hline $\mathrm{C}(18)-\mathrm{Co}(1)-\mathrm{C}(15)$ & $154.3(3)$ \\
\hline $\mathrm{C}(18)-\mathrm{Co}(1)-\mathrm{C}(16)$ & $163.6(3)$ \\
\hline $\mathrm{C}(18)-\mathrm{Co}(1)-\mathrm{C}(20)$ & $68.9(4)$ \\
\hline $\mathrm{C}(19)-\mathrm{Co}(1)-\mathrm{C}(13)$ & $127.8(3)$ \\
\hline $\mathrm{C}(19)-\mathrm{Co}(1)-\mathrm{C}(14)$ & $109.5(3)$ \\
\hline $\mathrm{C}(19)-\mathrm{Co}(1)-\mathrm{C}(15)$ & $120.6(3)$ \\
\hline $\mathrm{C}(19)-\mathrm{Co}(1)-\mathrm{C}(16)$ & $153.7(3)$ \\
\hline $\mathrm{C}(19)-\mathrm{Co}(1)-\mathrm{C}(17)$ & $164.7(3)$ \\
\hline $\mathrm{C}(19)-\mathrm{Co}(1)-\mathrm{C}(18)$ & $40.6(3)$ \\
\hline $\mathrm{C}(19)-\mathrm{Co}(1)-\mathrm{C}(20)$ & $41.9(2)$ \\
\hline $\mathrm{C}(20)-\mathrm{Co}(1)-\mathrm{C}(15)$ & $109.1(2)$ \\
\hline $\mathrm{C}\left(18^{\prime}\right)-\mathrm{Co}(1)-\mathrm{C}(15)$ & $159.7(5)$ \\
\hline $\mathrm{C}\left(18^{\prime}\right)-\mathrm{Co}(1)-\mathrm{C}(16)$ & $157.3(5)$ \\
\hline
\end{tabular}

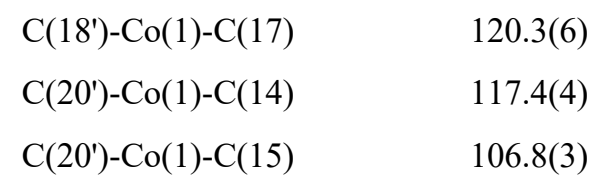




\begin{tabular}{|c|c|c|c|}
\hline $\mathrm{C}(16)-\mathrm{C}(15)-\mathrm{Co}(1)$ & $69.36(13)$ & $\mathrm{C}(20)-\mathrm{C}(21)-\mathrm{C}(22)$ & $109.1(6)$ \\
\hline$C(16)-C(15)-C(14)$ & $107.8(2)$ & $\mathrm{C}(22)-\mathrm{C}(21)-\mathrm{Co}(1)$ & $69.8(5)$ \\
\hline $\mathrm{C}(16)-\mathrm{C}(15)-\mathrm{H}(15)$ & 126.1 & $\mathrm{C}(22)-\mathrm{C}(21)-\mathrm{H}(21)$ & 125.4 \\
\hline $\mathrm{Co}(1)-\mathrm{C}(16)-\mathrm{H}(16)$ & 126.8 & $\mathrm{Co}(1)-\mathrm{C}(22)-\mathrm{H}(22)$ & 126.5 \\
\hline$C(15)-C(16)-C o(1)$ & $69.81(13)$ & $\mathrm{C}(18)-\mathrm{C}(22)-\mathrm{Co}(1)$ & $69.0(9)$ \\
\hline $\mathrm{C}(15)-\mathrm{C}(16)-\mathrm{H}(16)$ & 125.9 & $C(18)-C(22)-C(21)$ & $106.7(6)$ \\
\hline$C(15)-C(16)-C(17)$ & $108.2(2)$ & $\mathrm{C}(18)-\mathrm{C}(22)-\mathrm{H}(22)$ & 126.6 \\
\hline$C(17)-C(16)-C o(1)$ & $69.09(13)$ & $\mathrm{C}(21)-\mathrm{C}(22)-\mathrm{Co}(1)$ & $69.4(5)$ \\
\hline $\mathrm{C}(17)-\mathrm{C}(16)-\mathrm{H}(16)$ & 125.9 & $\mathrm{C}(21)-\mathrm{C}(22)-\mathrm{H}(22)$ & 126.6 \\
\hline $\mathrm{Co}(1)-\mathrm{C}(17)-\mathrm{H}(17)$ & 126.7 & $\mathrm{Co}(1)-\mathrm{C}\left(18^{\prime}\right)-\mathrm{H}\left(18^{\prime}\right)$ & 126.4 \\
\hline $\mathrm{C}(13)-\mathrm{C}(17)-\mathrm{Co}(1)$ & $69.00(14)$ & $\mathrm{C}\left(19^{\prime}\right)-\mathrm{C}\left(18^{\prime}\right)-\mathrm{Co}(1)$ & $70.5(13)$ \\
\hline$C(13)-C(17)-C(16)$ & $107.6(2)$ & $\mathrm{C}\left(19^{\prime}\right)-\mathrm{C}\left(18^{\prime}\right)-\mathrm{H}\left(18^{\prime}\right)$ & 125.7 \\
\hline $\mathrm{C}(13)-\mathrm{C}(17)-\mathrm{H}(17)$ & 126.2 & $\mathrm{C}\left(19^{\prime}\right)-\mathrm{C}\left(18^{\prime}\right)-\mathrm{C}\left(22^{\prime}\right)$ & $108.6(11)$ \\
\hline$C(16)-C(17)-C o(1)$ & $69.68(13)$ & $\mathrm{C}\left(22^{\prime}\right)-\mathrm{C}\left(18^{\prime}\right)-\mathrm{Co}(1)$ & $68.9(13)$ \\
\hline $\mathrm{C}(16)-\mathrm{C}(17)-\mathrm{H}(17)$ & 126.2 & $\mathrm{C}\left(22^{\prime}\right)-\mathrm{C}\left(18^{\prime}\right)-\mathrm{H}\left(18^{\prime}\right)$ & 125.7 \\
\hline $\mathrm{Co}(1)-\mathrm{C}(18)-\mathrm{H}(18)$ & 127.1 & $\mathrm{Co}(1)-\mathrm{C}\left(19^{\prime}\right)-\mathrm{H}\left(19^{\prime}\right)$ & 127.5 \\
\hline C(19)-C(18)-Co(1) & $69.1(8)$ & $\mathrm{C}\left(18^{\prime}\right)-\mathrm{C}\left(19^{\prime}\right)-\mathrm{Co}(1)$ & $69.0(14)$ \\
\hline $\mathrm{C}(19)-\mathrm{C}(18)-\mathrm{H}(18)$ & 125.2 & $\mathrm{C}\left(18^{\prime}\right)-\mathrm{C}\left(19^{\prime}\right)-\mathrm{H}\left(19^{\prime}\right)$ & 126.2 \\
\hline$C(19)-C(18)-C(22)$ & $109.7(7)$ & $\mathrm{C}\left(20^{\prime}\right)-\mathrm{C}\left(19^{\prime}\right)-\mathrm{Co}(1)$ & $68.9(8)$ \\
\hline $\mathrm{C}(22)-\mathrm{C}(18)-\mathrm{Co}(1)$ & $70.2(9)$ & $\mathrm{C}\left(20^{\prime}\right)-\mathrm{C}\left(19^{\prime}\right)-\mathrm{C}\left(18^{\prime}\right)$ & $107.7(11)$ \\
\hline $\mathrm{C}(22)-\mathrm{C}(18)-\mathrm{H}(18)$ & 125.2 & $\mathrm{C}\left(20^{\prime}\right)-\mathrm{C}\left(19^{\prime}\right)-\mathrm{H}\left(19^{\prime}\right)$ & 126.2 \\
\hline $\mathrm{Co}(1)-\mathrm{C}(19)-\mathrm{H}(19)$ & 125.2 & $\mathrm{Co}(1)-\mathrm{C}\left(20^{\prime}\right)-\mathrm{H}\left(20^{\prime}\right)$ & 126.3 \\
\hline C(18)-C(19)-Co(1) & 70.3(9) & $C\left(19^{\prime}\right)-C\left(20^{\prime}\right)-\operatorname{Co}(1)$ & $70.6(9)$ \\
\hline $\mathrm{C}(18)-\mathrm{C}(19)-\mathrm{H}(19)$ & 126.1 & $\mathrm{C}\left(19^{\prime}\right)-\mathrm{C}\left(20^{\prime}\right)-\mathrm{H}\left(20^{\prime}\right)$ & 125.7 \\
\hline$C(18)-C(19)-C(20)$ & $107.7(7)$ & $\mathrm{C}\left(19^{\prime}\right)-\mathrm{C}\left(20^{\prime}\right)-\mathrm{C}\left(21^{\prime}\right)$ & $108.6(9)$ \\
\hline$C(20)-C(19)-\mathrm{Co}(1)$ & $69.9(5)$ & $\mathrm{C}\left(21^{\prime}\right)-\mathrm{C}\left(20^{\prime}\right)-\mathrm{Co}(1)$ & $69.0(6)$ \\
\hline $\mathrm{C}(20)-\mathrm{C}(19)-\mathrm{H}(19)$ & 126.1 & $\mathrm{C}\left(21^{\prime}\right)-\mathrm{C}\left(20^{\prime}\right)-\mathrm{H}\left(20^{\prime}\right)$ & 125.7 \\
\hline $\mathrm{Co}(1)-\mathrm{C}(20)-\mathrm{H}(20)$ & 126.9 & $\mathrm{Co}(1)-\mathrm{C}\left(21^{\prime}\right)-\mathrm{H}\left(21^{\prime}\right)$ & 126.4 \\
\hline$C(19)-C(20)-\mathrm{Co}(1)$ & $68.3(5)$ & $C\left(20^{\prime}\right)-C\left(21^{\prime}\right)-C o(1)$ & $69.3(6)$ \\
\hline $\mathrm{C}(19)-\mathrm{C}(20)-\mathrm{H}(20)$ & 126.6 & $\mathrm{C}\left(20^{\prime}\right)-\mathrm{C}\left(21^{\prime}\right)-\mathrm{H}\left(21^{\prime}\right)$ & 126.6 \\
\hline $\mathrm{C}(21)-\mathrm{C}(20)-\mathrm{Co}(1)$ & $69.8(4)$ & $\mathrm{C}\left(22^{\prime}\right)-\mathrm{C}\left(21^{\prime}\right)-\mathrm{Co}(1)$ & 69.3(9) \\
\hline$C(21)-C(20)-C(19)$ & $106.7(6)$ & $\mathrm{C}\left(22^{\prime}\right)-\mathrm{C}\left(21^{\prime}\right)-\mathrm{C}\left(20^{\prime}\right)$ & $106.9(9)$ \\
\hline $\mathrm{C}(21)-\mathrm{C}(20)-\mathrm{H}(20)$ & 126.6 & $\mathrm{C}\left(22^{\prime}\right)-\mathrm{C}\left(21^{\prime}\right)-\mathrm{H}\left(21^{\prime}\right)$ & 126.6 \\
\hline $\mathrm{Co}(1)-\mathrm{C}(21)-\mathrm{H}(21)$ & 127.1 & $\mathrm{Co}(1)-\mathrm{C}\left(22^{\prime}\right)-\mathrm{H}\left(22^{\prime}\right)$ & 125.9 \\
\hline $\mathrm{C}(20)-\mathrm{C}(21)-\mathrm{Co}(1)$ & $69.3(3)$ & $\mathrm{C}\left(18^{\prime}\right)-\mathrm{C}\left(22^{\prime}\right)-\mathrm{Co}(1)$ & $69.8(15)$ \\
\hline $\mathrm{C}(20)-\mathrm{C}(21)-\mathrm{H}(21)$ & 125.4 & $\mathrm{C}\left(18^{\prime}\right)-\mathrm{C}\left(22^{\prime}\right)-\mathrm{H}\left(22^{\prime}\right)$ & 125.9 \\
\hline
\end{tabular}




$\begin{array}{llll}\mathrm{C}\left(21^{\prime}\right)-\mathrm{C}\left(22^{\prime}\right)-\mathrm{Co}(1) & 69.7(8) & \mathrm{C}(23)-\mathrm{C}(24)-\mathrm{H}(24 \mathrm{~B}) & 109.5 \\ \mathrm{C}\left(21^{\prime}\right)-\mathrm{C}\left(22^{\prime}\right)-\mathrm{C}\left(18^{\prime}\right) & 108.2(11) & \mathrm{C}(23)-\mathrm{C}(24)-\mathrm{H}(24 \mathrm{C}) & 109.5 \\ \mathrm{C}\left(21^{\prime}\right)-\mathrm{C}\left(22^{\prime}\right)-\mathrm{H}\left(22^{\prime}\right) & 125.9 & \mathrm{H}(24 \mathrm{~A})-\mathrm{C}(24)-\mathrm{H}(24 \mathrm{~B}) & 109.5 \\ \mathrm{~N}(3)-\mathrm{C}(23)-\mathrm{C}(24) & 179.2(3) & \mathrm{H}(24 \mathrm{~A})-\mathrm{C}(24)-\mathrm{H}(24 \mathrm{C}) & 109.5 \\ \mathrm{C}(23)-\mathrm{C}(24)-\mathrm{H}(24 \mathrm{~A}) & 109.5 & \mathrm{H}(24 \mathrm{~B})-\mathrm{C}(24)-\mathrm{H}(24 \mathrm{C}) & 109.5\end{array}$


Table 4. Anisotropic displacement parameters $\left(\AA^{2} \times 10^{3}\right)$ for rhowe 36 . The anisotropic displacement factor exponent takes the form: $-2 \pi^{2}\left[h^{2} a^{* 2} U_{11}+\ldots+2 h k a^{*} b^{*} U_{12}\right]$

\begin{tabular}{|c|c|c|c|c|c|c|}
\hline & $\mathrm{U}_{11}$ & $\mathrm{U}_{22}$ & $\mathrm{U}_{33}$ & $\mathrm{U}_{23}$ & $\mathrm{U}_{13}$ & $\mathrm{U}_{12}$ \\
\hline Mo1 & $12(1)$ & $11(1)$ & $9(1)$ & $0(1)$ & $1(1)$ & $0(1)$ \\
\hline $\mathrm{Cl1}$ & $20(1)$ & $17(1)$ & $14(1)$ & $3(1)$ & $3(1)$ & $-2(1)$ \\
\hline $\mathrm{Cl} 2$ & $21(1)$ & $21(1)$ & $14(1)$ & $2(1)$ & $1(1)$ & $-7(1)$ \\
\hline $\mathrm{Cl} 3$ & $21(1)$ & $19(1)$ & $14(1)$ & $-5(1)$ & $1(1)$ & $0(1)$ \\
\hline $\mathrm{Cl} 4$ & $16(1)$ & $16(1)$ & $15(1)$ & $1(1)$ & $-1(1)$ & $3(1)$ \\
\hline N1 & $13(1)$ & $14(1)$ & $13(1)$ & $1(1)$ & $1(1)$ & $0(1)$ \\
\hline N2 & $14(1)$ & $11(1)$ & $12(1)$ & $0(1)$ & $2(1)$ & $1(1)$ \\
\hline $\mathrm{C} 1$ & $17(1)$ & $16(1)$ & $15(1)$ & $0(1)$ & $3(1)$ & $4(1)$ \\
\hline $\mathrm{C} 2$ & $15(1)$ & $21(1)$ & $20(1)$ & $-1(1)$ & $5(1)$ & $6(1)$ \\
\hline $\mathrm{C} 3$ & $14(1)$ & $19(1)$ & 21(1) & $4(1)$ & $3(1)$ & $-1(1)$ \\
\hline $\mathrm{C} 4$ & $16(1)$ & $17(1)$ & $17(1)$ & $1(1)$ & $0(1)$ & $-1(1)$ \\
\hline $\mathrm{C} 5$ & $17(1)$ & $13(1)$ & $12(1)$ & $1(1)$ & $2(1)$ & $-1(1)$ \\
\hline C6 & $15(1)$ & $16(1)$ & $12(1)$ & $1(1)$ & $1(1)$ & $-1(1)$ \\
\hline C7 & $23(1)$ & $20(1)$ & $15(1)$ & $-4(1)$ & $-3(1)$ & $4(1)$ \\
\hline $\mathrm{C} 8$ & $27(1)$ & $17(1)$ & $18(1)$ & $-5(1)$ & $3(1)$ & $0(1)$ \\
\hline C9 & $21(1)$ & $14(1)$ & 21(1) & $-3(1)$ & $6(1)$ & $2(1)$ \\
\hline $\mathrm{C} 10$ & $16(1)$ & $15(1)$ & $20(1)$ & $-2(1)$ & $3(1)$ & $2(1)$ \\
\hline C11 & $16(1)$ & $26(1)$ & $30(1)$ & $0(1)$ & $-2(1)$ & $5(1)$ \\
\hline $\mathrm{C} 12$ & $46(2)$ & $38(2)$ & $23(1)$ & $-14(1)$ & $-5(1)$ & $16(1)$ \\
\hline Co1 & $13(1)$ & $11(1)$ & 11(1) & $-1(1)$ & $0(1)$ & $2(1)$ \\
\hline $\mathrm{C} 13$ & $29(1)$ & $18(1)$ & $14(1)$ & $-4(1)$ & $0(1)$ & $12(1)$ \\
\hline C14 & $19(1)$ & $24(1)$ & $20(1)$ & $7(1)$ & $7(1)$ & $7(1)$ \\
\hline C15 & $15(1)$ & $21(1)$ & $21(1)$ & $0(1)$ & $-1(1)$ & $4(1)$ \\
\hline $\mathrm{C} 16$ & $22(1)$ & $15(1)$ & $14(1)$ & $4(1)$ & $1(1)$ & $6(1)$ \\
\hline C17 & $24(1)$ & $10(1)$ & $22(1)$ & $-1(1)$ & $-2(1)$ & $1(1)$ \\
\hline C18 & $20(3)$ & $19(4)$ & $21(1)$ & $5(2)$ & $-1(1)$ & $9(2)$ \\
\hline C19 & $26(1)$ & $12(2)$ & $36(4)$ & $7(3)$ & $2(3)$ & $5(1)$ \\
\hline $\mathrm{C} 20$ & $19(3)$ & $16(2)$ & $40(4)$ & $-17(2)$ & $-4(2)$ & $9(2)$ \\
\hline $\mathrm{C} 21$ & $19(3)$ & $20(3)$ & $22(2)$ & $-3(2)$ & $4(2)$ & $6(2)$ \\
\hline $\mathrm{C} 22$ & $14(2)$ & $20(3)$ & $14(3)$ & $-6(3)$ & $-2(3)$ & $4(2)$ \\
\hline C18' & $20(3)$ & $19(4)$ & $21(1)$ & $5(2)$ & $-1(1)$ & $9(2)$ \\
\hline
\end{tabular}




\begin{tabular}{lllllll}
$\mathrm{C} 19^{\prime}$ & $26(1)$ & $12(2)$ & $36(4)$ & $7(3)$ & $2(3)$ & $5(1)$ \\
$\mathrm{C} 20^{\prime}$ & $19(3)$ & $16(2)$ & $40(4)$ & $-17(2)$ & $-4(2)$ & $9(2)$ \\
$\mathrm{C} 21^{\prime}$ & $19(3)$ & $20(3)$ & $22(2)$ & $-3(2)$ & $4(2)$ & $6(2)$ \\
$\mathrm{C} 22^{\prime}$ & $14(2)$ & $20(3)$ & $14(3)$ & $-6(3)$ & $-2(3)$ & $4(2)$ \\
$\mathrm{N} 3$ & $38(1)$ & $38(2)$ & $38(2)$ & $-8(1)$ & $11(1)$ & $-4(1)$ \\
$\mathrm{C} 23$ & $22(1)$ & $32(2)$ & $27(1)$ & $5(1)$ & $9(1)$ & $0(1)$ \\
$\mathrm{C} 24$ & $35(2)$ & $32(2)$ & $33(2)$ & $-4(1)$ & $13(1)$ & $-2(1)$ \\
\hline
\end{tabular}


Table 5. Hydrogen coordinates $\left(\times 10^{4}\right)$ and isotropic displacement parameters $\left(\AA^{2} \times 10^{3}\right)$ for rhowe36.

\begin{tabular}{|c|c|c|c|c|}
\hline & $\mathrm{x}$ & $\mathrm{y}$ & $\mathrm{z}$ & $\mathrm{U}(\mathrm{eq})$ \\
\hline H1 & 682 & 8921 & 6100 & 19 \\
\hline $\mathrm{H} 2$ & -963 & 9406 & 5576 & 23 \\
\hline $\mathrm{H} 4$ & -359 & 6562 & 4309 & 20 \\
\hline H7 & 906 & 5364 & 3927 & 23 \\
\hline H9 & 3770 & 3560 & 4318 & 22 \\
\hline H10 & 3881 & 4621 & 5282 & 20 \\
\hline H11A & -2388 & 8770 & 4782 & 37 \\
\hline H11B & -2219 & 7520 & 4350 & 37 \\
\hline $\mathrm{H} 11 \mathrm{C}$ & -1767 & 8926 & 4164 & 37 \\
\hline $\mathrm{H} 12 \mathrm{~A}$ & 2072 & 2804 & 3395 & 54 \\
\hline H12B & 2942 & 3815 & 3172 & 54 \\
\hline $\mathrm{H} 12 \mathrm{C}$ & 1707 & 4182 & 3096 & 54 \\
\hline H13 & 6200 & -677 & 5585 & 24 \\
\hline H14 & 7665 & 925 & 5970 & 25 \\
\hline H15 & 7770 & 917 & 7157 & 23 \\
\hline H16 & 6349 & -671 & 7511 & 21 \\
\hline H17 & 5377 & -1672 & 6539 & 23 \\
\hline H18 & 4639 & 1989 & 5522 & 24 \\
\hline H19 & 5966 & 3476 & 6111 & 30 \\
\hline $\mathrm{H} 20$ & 5733 & 3156 & 7289 & 30 \\
\hline $\mathrm{H} 21$ & 4284 & 1402 & 7389 & 24 \\
\hline $\mathrm{H} 22$ & 3626 & 647 & 6297 & 20 \\
\hline H18' & 4381 & 1583 & 5565 & 24 \\
\hline H19' & 5891 & 3203 & 5810 & 30 \\
\hline H20' & 6109 & 3464 & 6982 & 30 \\
\hline $\mathrm{H} 21^{\prime}$ & 4704 & 2012 & 7472 & 24 \\
\hline H22' & 3613 & 819 & 6586 & 20 \\
\hline $\mathrm{H} 24 \mathrm{~A}$ & 25 & 7345 & 2092 & 49 \\
\hline $\mathrm{H} 24 \mathrm{~B}$ & -658 & 6447 & 2526 & 49 \\
\hline $\mathrm{H} 24 \mathrm{C}$ & 612 & 6371 & 2584 & 49 \\
\hline
\end{tabular}


Table 6. Torsion angles $\left[{ }^{\circ}\right]$ for rhowe 36 .

\begin{tabular}{|c|c|c|c|}
\hline Mo1-N1-C1-C2 & $-174.97(18)$ & Co1-C16-C17-C13 & $58.79(16)$ \\
\hline Mo1-N1-C5-C4 & $174.04(17)$ & Co1-C18-C19-C20 & $-60.2(8)$ \\
\hline Mo1-N1-C5-C6 & $-6.6(2)$ & Co1-C18-C22-C21 & $59.4(9)$ \\
\hline Mo1-N2-C6-C5 & $-4.5(3)$ & Co1-C19-C20-C21 & $-59.5(5)$ \\
\hline Mo1-N2-C6-C7 & $172.62(18)$ & Co1-C20-C21-C22 & $-58.5(6)$ \\
\hline Mo1-N2-C10-C9 & $-171.32(18)$ & $\mathrm{Co} 1-\mathrm{C} 21-\mathrm{C} 22-\mathrm{C} 18$ & $-59.2(12)$ \\
\hline $\mathrm{N} 1-\mathrm{C} 1-\mathrm{C} 2-\mathrm{C} 3$ & $0.9(4)$ & Co1-C18'-C19'-C20' & $-58.2(13)$ \\
\hline N1-C5-C6-N2 & $7.4(3)$ & Co1-C18'-C22'-C21' & $59.3(14)$ \\
\hline N1-C5-C6-C7 & $-169.7(2)$ & Co1-C19'-C20'-C21' & $-58.6(8)$ \\
\hline N2-C6-C7-C8 & $-1.1(4)$ & Co1-C20'-C21'-C22' & $-59.4(10)$ \\
\hline C1-N1-C5-C4 & $-1.2(3)$ & Co1-C21'-C22'-C18' & $-59(2)$ \\
\hline C1-N1-C5-C6 & $178.2(2)$ & $\mathrm{C} 13-\mathrm{C} 14-\mathrm{C} 15-\mathrm{Co} 1$ & $-58.98(17)$ \\
\hline $\mathrm{C} 1-\mathrm{C} 2-\mathrm{C} 3-\mathrm{C} 4$ & $-0.1(4)$ & $\mathrm{C} 13-\mathrm{C} 14-\mathrm{C} 15-\mathrm{C} 16$ & $-0.5(3)$ \\
\hline $\mathrm{C} 1-\mathrm{C} 2-\mathrm{C} 3-\mathrm{C} 11$ & $-178.1(2)$ & C14-C13-C17-Co1 & $59.18(17)$ \\
\hline $\mathrm{C} 2-\mathrm{C} 3-\mathrm{C} 4-\mathrm{C} 5$ & $-1.2(4)$ & C14-C13-C17-C16 & $0.0(3)$ \\
\hline $\mathrm{C} 3-\mathrm{C} 4-\mathrm{C} 5-\mathrm{N} 1$ & $1.9(4)$ & $\mathrm{C} 14-\mathrm{C} 15-\mathrm{C} 16-\mathrm{Co} 1$ & $-58.15(16)$ \\
\hline C3-C4-C5-C6 & $-177.4(2)$ & $\mathrm{C} 14-\mathrm{C} 15-\mathrm{C} 16-\mathrm{C} 17$ & $0.4(3)$ \\
\hline $\mathrm{C} 4-\mathrm{C} 5-\mathrm{C} 6-\mathrm{N} 2$ & $-173.3(2)$ & C15-C16-C17-Co1 & $-59.03(16)$ \\
\hline C4-C5-C6-C7 & $9.6(4)$ & $\mathrm{C} 15-\mathrm{C} 16-\mathrm{C} 17-\mathrm{C} 13$ & $-0.2(3)$ \\
\hline C5-N1-C1-C2 & $-0.2(3)$ & C17-C13-C14-Co1 & $-59.25(16)$ \\
\hline C5-C6-C7-C8 & $175.8(2)$ & C17-C13-C14-C15 & $0.3(3)$ \\
\hline C6-N2-C10-C9 & $1.6(3)$ & C18-C19-C20-Co1 & $60.5(11)$ \\
\hline C6-C7-C8-C9 & $2.4(4)$ & C18-C19-C20-C21 & $1.0(13)$ \\
\hline C6-C7-C8-C12 & $-176.7(3)$ & $\mathrm{C} 19-\mathrm{C} 18-\mathrm{C} 22-\mathrm{Co} 1$ & $-57.9(13)$ \\
\hline C7-C8-C9-C10 & $-1.7(4)$ & C19-C18-C22-C21 & $2(2)$ \\
\hline C8-C9-C10-N2 & $-0.3(4)$ & C19-C20-C21-Co1 & $58.5(6)$ \\
\hline C10-N2-C6-C5 & $-178.0(2)$ & C19-C20-C21-C22 & $-0.1(9)$ \\
\hline C10-N2-C6-C7 & $-0.9(3)$ & $\mathrm{C} 20-\mathrm{C} 21-\mathrm{C} 22-\mathrm{Co} 1$ & $58.3(5)$ \\
\hline C11-C3-C4-C5 & $176.8(2)$ & $\mathrm{C} 20-\mathrm{C} 21-\mathrm{C} 22-\mathrm{C} 18$ & $-0.9(14)$ \\
\hline C12-C8-C9-C10 & $177.4(3)$ & C22-C18-C19-Co1 & $58.6(13)$ \\
\hline Co1-C13-C14-C15 & $59.55(17)$ & C22-C18-C19-C20 & $-1.6(19)$ \\
\hline Co1-C13-C17-C16 & $-59.22(16)$ & C18'-C19'-C20'-Co1 & $58.2(18)$ \\
\hline Co1-C14-C15-C16 & $58.52(16)$ & C18'-C19'-C20'-C21' & $0(2)$ \\
\hline Co1-C15-C16-C17 & $58.59(16)$ & C19'-C18'-C22'-Co1 & $-60(2)$ \\
\hline
\end{tabular}




$\begin{array}{lclc}\mathrm{C} 19^{\prime}-\mathrm{C} 18^{\prime}-\mathrm{C} 22^{\prime}-\mathrm{C} 21^{\prime} & 0(3) & \mathrm{C} 20^{\prime}-\mathrm{C} 21^{\prime}-\mathrm{C} 22^{\prime}-\mathrm{C} 18^{\prime} & 0(2) \\ \mathrm{C} 19^{\prime}-\mathrm{C} 20^{\prime}-\mathrm{C} 21^{\prime}-\mathrm{Co} 1 & 59.6(10) & \mathrm{C} 22^{\prime}-\mathrm{C} 18^{\prime}-\mathrm{C} 19^{\prime}-\mathrm{Co} 1 & 59(2) \\ \mathrm{C} 19^{\prime}-\mathrm{C} 20^{\prime}-\mathrm{C} 21^{\prime}-\mathrm{C} 22^{\prime} & 0.2(16) & \mathrm{C} 22^{\prime}-\mathrm{C} 18^{\prime}-\mathrm{C} 19^{\prime}-\mathrm{C} 20^{\prime} & 0(3) \\ \mathrm{C} 20^{\prime}-\mathrm{C} 21^{\prime}-\mathrm{C} 22^{\prime}-\mathrm{Co} 1 & 59.4(8) & & \end{array}$


REFERENCE NUMBER: rhowe38

CRYSTAL STRUCTURE REPORT

$\mathrm{C}_{24} \mathrm{H}_{21} \mathrm{Cl}_{4} \mathrm{Co} \mathrm{Mo} \mathrm{N} \mathrm{O}_{4}$

or

$\left[\mathrm{CoCp}_{2}\right]\left[\mathrm{Mo}(\mathrm{dcbpy}) \mathrm{Cl}_{4}\right] \cdot \mathrm{CH}_{3} \mathrm{CN}$

Report prepared for:

Prof. W. Eckenhoff

June 29, 2019
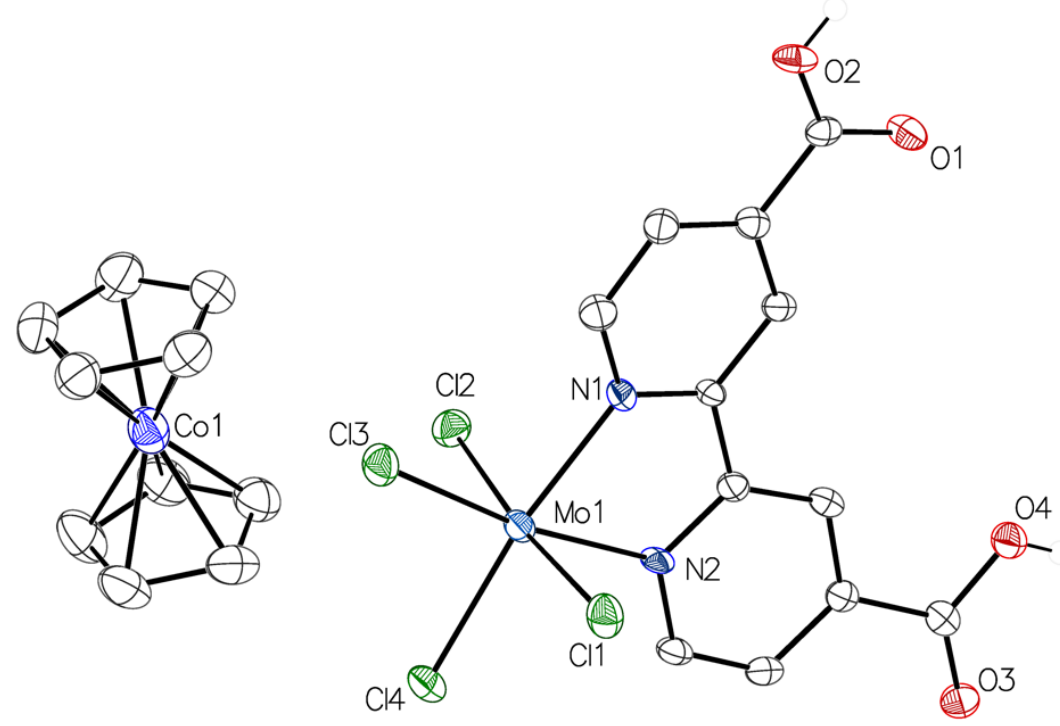

William W. Brennessel

X-ray Crystallographic Facility

Department of Chemistry, University of Rochester

120 Trustee Road 
Rochester, NY 14627 


\section{Data collection}

A crystal $\left(0.142 \times 0.06 \times 0.013 \mathrm{~mm}^{3}\right)$ was placed onto a thin glass optical fiber or a nylon loop and mounted on a Rigaku XtaLab Synergy-S Dualflex diffractometer equipped with a HyPix-6000HE HPC area detector for data collection at $100.00(10) \mathrm{K}$. A preliminary set of cell constants and an orientation matrix were calculated from a small sampling of reflections. ${ }^{1}$ A short pre-experiment was run, from which an optimal data collection strategy was determined. The full data collection was carried out using a PhotonJet $(\mathrm{Cu}) \mathrm{X}$-ray Source with a frame time of 1.34 seconds and a detector distance of $31.2 \mathrm{~mm}$. Series of frames were collected in $0.50^{\circ}$ steps in $\omega$ at different $2 \theta, \kappa$, and $\phi$ settings. After the intensity data were corrected for absorption, the final cell constants were calculated from the xyz centroids of 11825 strong reflections from the actual data collection after integration. ${ }^{1}$ See Table 1 for additional crystal and refinement information.

\section{Structure solution and refinement}

The structure was solved using ShelXT ${ }^{2}$ and refined using ShelXL. ${ }^{3}$ The space group P-1 was determined based on intensity statistics. Most or all non-hydrogen atoms were assigned from the solution. Full-matrix least squares / difference Fourier cycles were performed which located any remaining non-hydrogen atoms. All non-hydrogen atoms were refined with anisotropic displacement parameters. All hydrogen atoms were placed in ideal positions and refined as riding atoms with relative isotropic displacement parameters. The final full matrix least squares refinement converged to $R 1=0.0507\left(F^{2}, I>2 \sigma(I)\right)$ and $w R 2=0.1390\left(F^{2}\right.$, all data).

\section{Structure description}

The structure is the one suggested. The asymmetric unit contains one cation, one anion, and one cocrystallized solvent molecule, all in general positions. The $\mathrm{COOH}$ group containing atoms $\mathrm{O} 1$ and $\mathrm{O} 2$ is hydrogen bonded to its symmetry equivalent (see figure and Table 7). Because the $\mathrm{C} 11-\mathrm{O} 1$ and $\mathrm{C} 11-\mathrm{O} 2$ bond lengths are the same within $3 \sigma$, the hydrogen occupancies were fixed at 0.50:0.50.

Structure manipulation and figure generation were performed using Olex2. ${ }^{4}$ Unless noted otherwise all structural diagrams containing thermal displacement ellipsoids are drawn at the $50 \%$ probability level.

Data collection, structure solution, and structure refinement were conducted at the X-ray Crystallographic Facility, B04 Hutchison Hall, Department of Chemistry, University of Rochester. The instrument was purchased with funding from NSF MRI program grant CHE-1725028. All publications arising from this report MUST either 1) include William W. Brennessel as a coauthor or 2) acknowledge William W. Brennessel and the X-ray Crystallographic Facility of the Department of Chemistry at the University of Rochester. 
1 CrysAlisPro, version 171.40.57a; Rigaku Corporation: Oxford, UK, 2019.

2 Sheldrick, G. M. SHELXT, version 2018/2; Acta. Crystallogr. 2015, A71, 3-8.

3 Sheldrick, G. M. SHELXL, version 2018/3; Acta. Crystallogr. 2015, C71, 3-8.

4 Dolomanov, O. V.; Bourhis, L. J.; Gildea, R. J.; Howard, J. A. K.; Puschmann, H. Olex2, version 1.2-ac3; J. Appl. Cryst. 2009, 42, 339-341.

Some equations of interest:

$$
\begin{gathered}
R_{\mathrm{int}}=\Sigma\left|F_{\mathrm{o}}^{2}-<F_{\mathrm{o}}^{2}>\right| / \Sigma\left|F_{\mathrm{o}}{ }^{2}\right| \\
R 1=\Sigma|| F_{\mathrm{o}}|-| F_{\mathrm{c}}|/ \Sigma| F_{\mathrm{o}} \mid \\
w R 2=\left[\Sigma\left[w\left(F_{\mathrm{o}}{ }^{2} F_{\mathrm{c}}{ }^{2}\right)^{2}\right] / \Sigma\left[w\left(F_{\mathrm{o}}{ }^{2}\right)^{2}\right]\right]^{1 / 2} \\
\text { where } w=1 /\left[\sigma^{2}\left(F_{\mathrm{o}}{ }^{2}\right)+(a P)^{2}+b P\right] \text { and } \\
P=1 / 3 \max \left(0, F_{\mathrm{o}}{ }^{2}\right)+2 / 3 F_{\mathrm{c}}{ }^{2} \\
\mathrm{GOF}=S=\left[\Sigma\left[w\left(F_{\mathrm{o}}{ }^{2}-F_{\mathrm{c}}{ }^{2}\right)^{2}\right] /(m-n)\right]^{1 / 2}
\end{gathered}
$$

where $m=$ number of reflections and $n=$ number of parameters
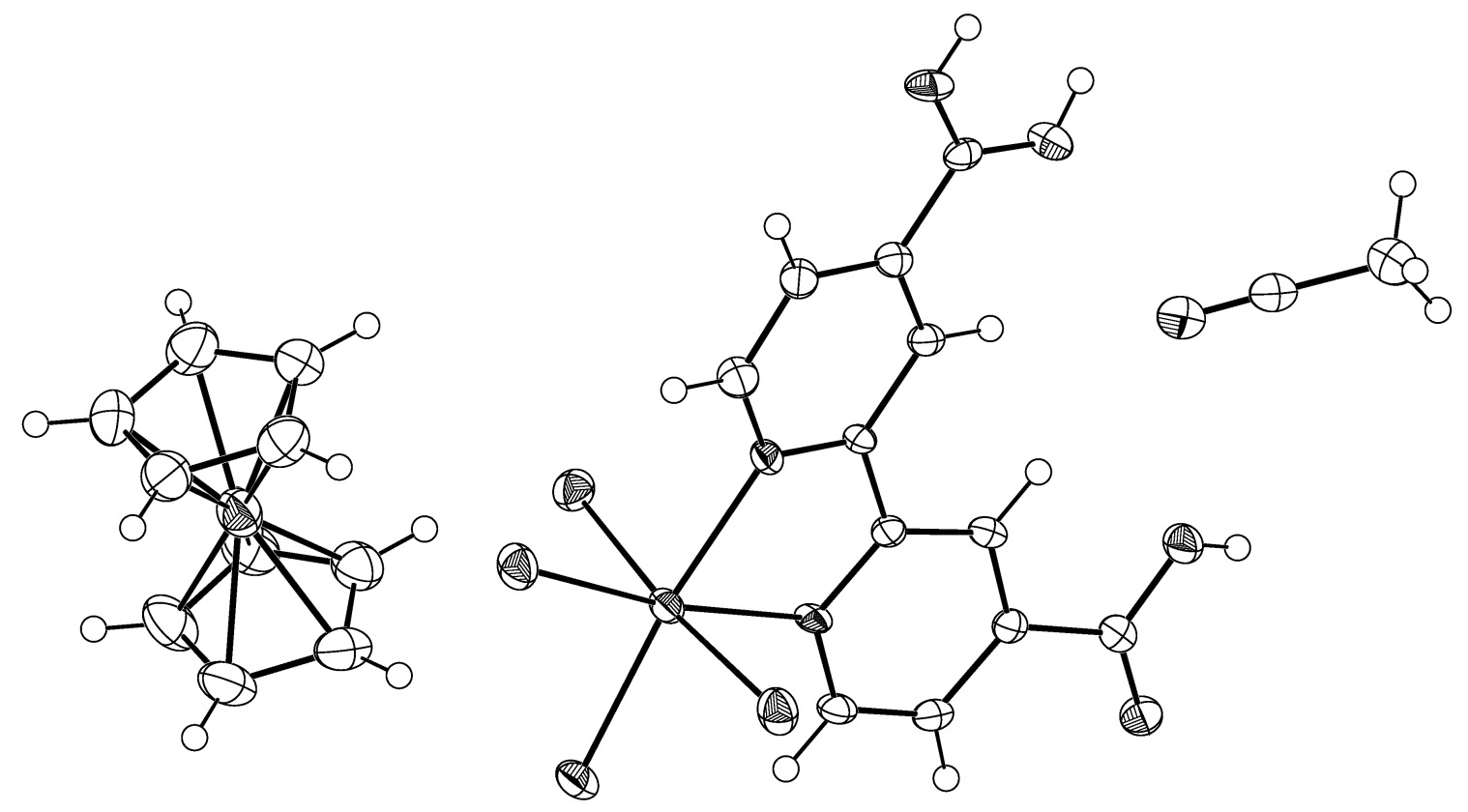

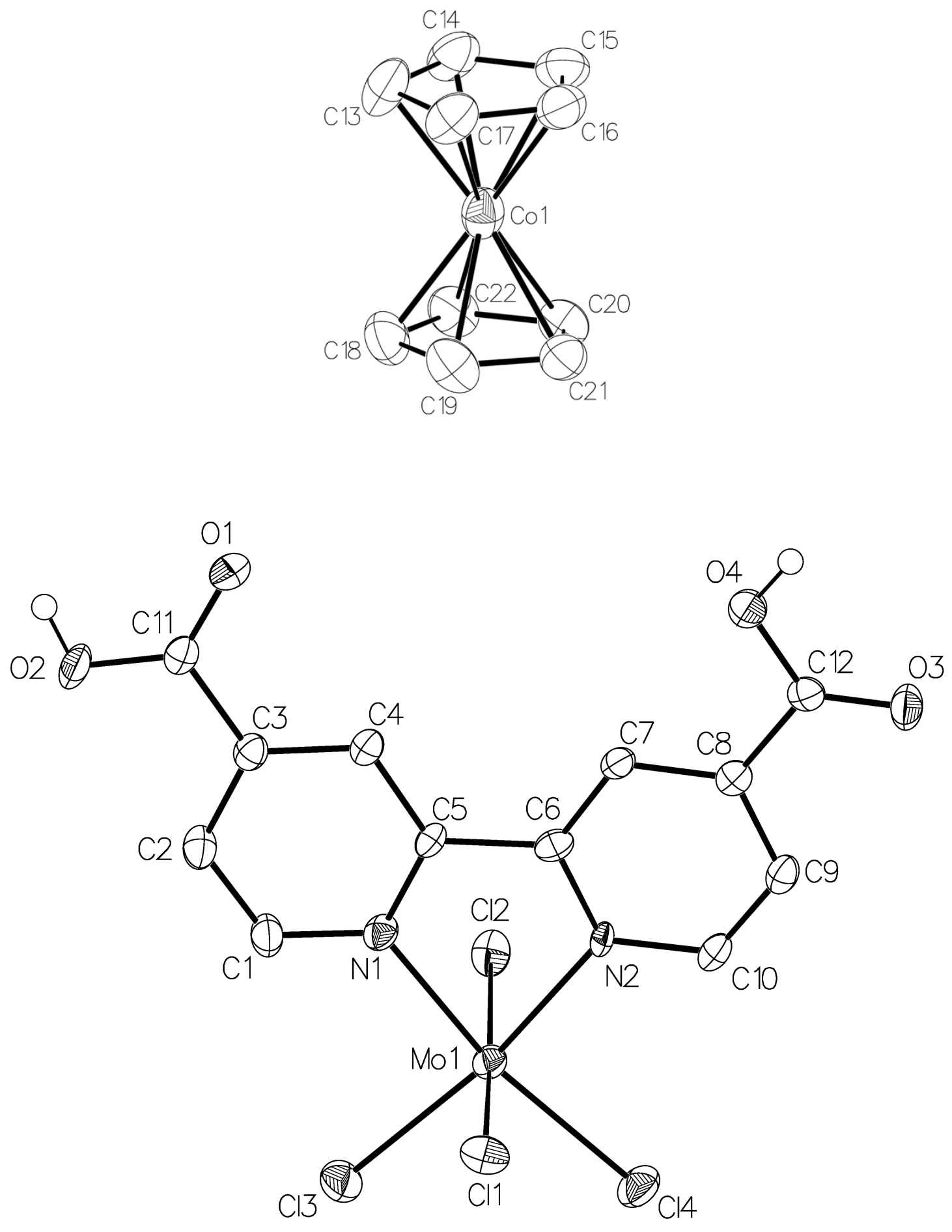


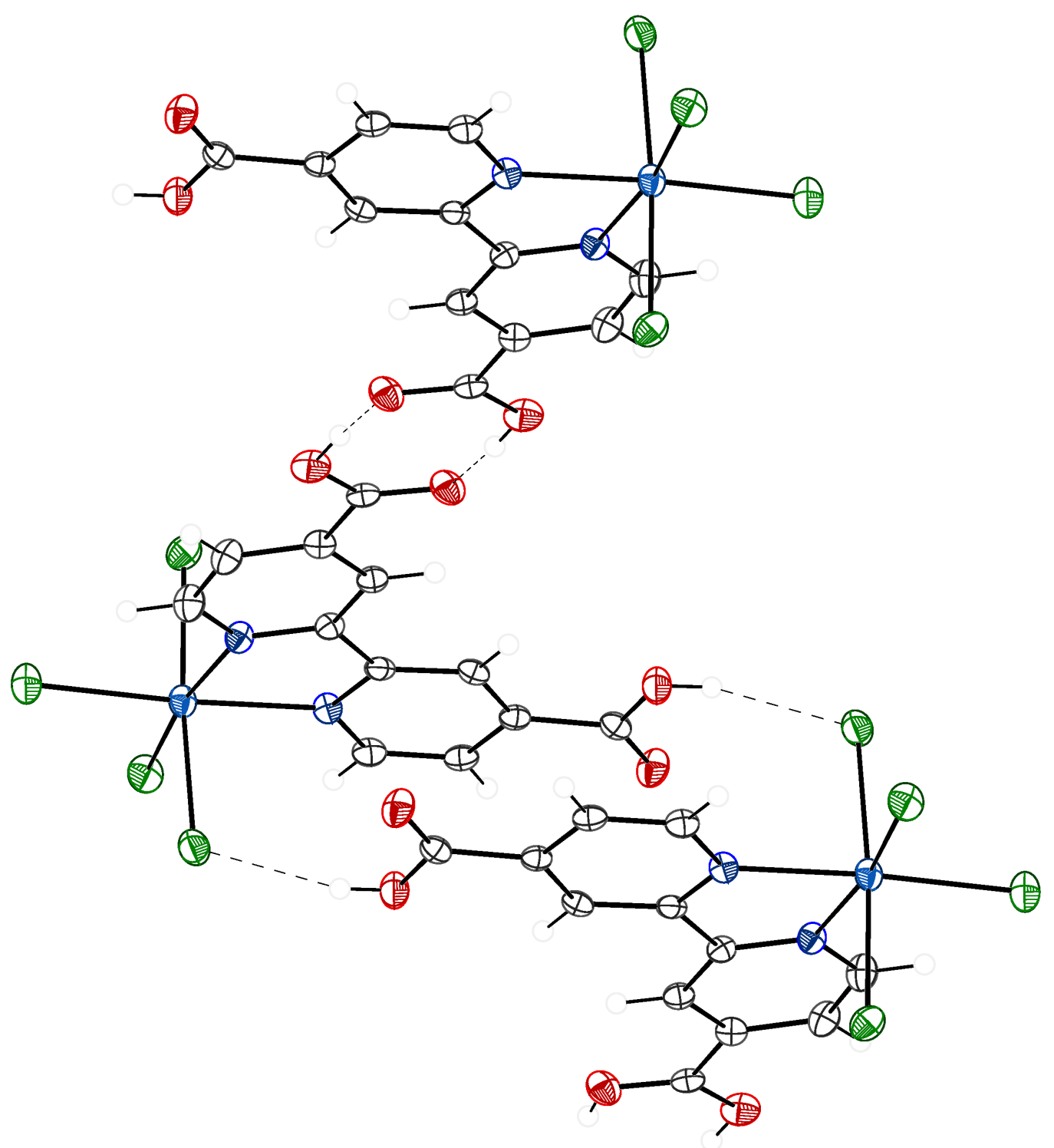



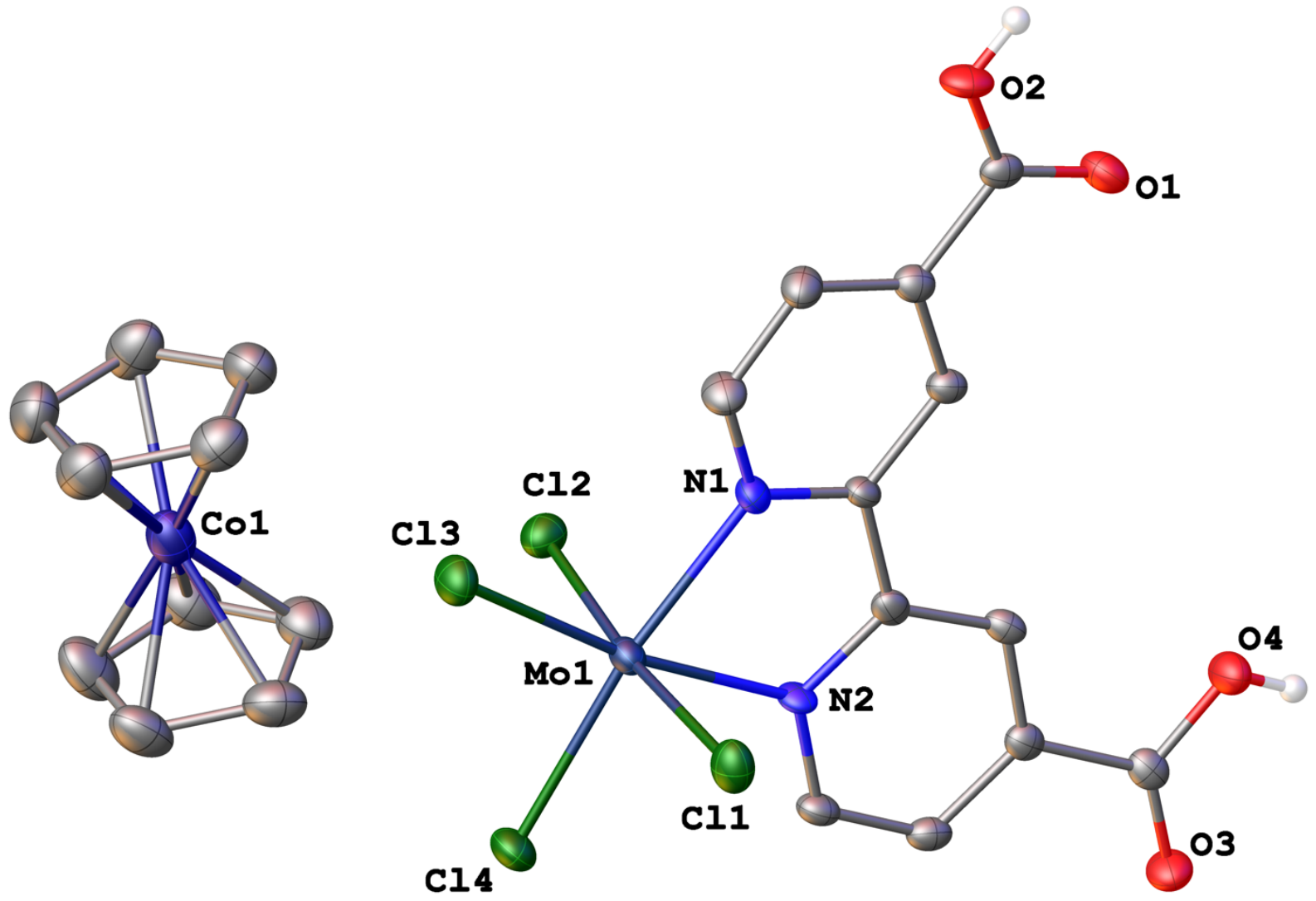
Table 1. Crystal data and structure refinement for rhowe38.

\begin{tabular}{|c|c|c|}
\hline Identification code & \multicolumn{2}{|l|}{ rhowe38 } \\
\hline Empirical formula & \multicolumn{2}{|c|}{ C24 H21 Cl4 Co Mo N3 O4 } \\
\hline Formula weight & \multicolumn{2}{|l|}{712.11} \\
\hline Temperature & \multicolumn{2}{|l|}{$100.00(10) \mathrm{K}$} \\
\hline Wavelength & \multicolumn{2}{|l|}{$1.54184 \AA$} \\
\hline Crystal system & \multicolumn{2}{|l|}{ triclinic } \\
\hline Space group & \multicolumn{2}{|l|}{$P-1$} \\
\hline \multirow[t]{3}{*}{ Unit cell dimensions } & $a=7.8058(2) \AA$ & $\alpha=94.456(2)^{\circ}$ \\
\hline & $b=9.6191(3) \AA$ & $\beta=94.772(2)^{\circ}$ \\
\hline & $c=18.6869(5) \AA$ & $\gamma=109.028(3)^{\circ}$ \\
\hline Volume & \multicolumn{2}{|l|}{$1313.62(7) \AA^{3}$} \\
\hline$Z$ & \multicolumn{2}{|l|}{2} \\
\hline Density (calculated) & \multicolumn{2}{|l|}{$1.800 \mathrm{Mg} / \mathrm{m}^{3}$} \\
\hline Absorption coefficient & \multicolumn{2}{|l|}{$12.921 \mathrm{~mm}^{-1}$} \\
\hline$F(000)$ & \multicolumn{2}{|l|}{710} \\
\hline Crystal color, morphology & \multicolumn{2}{|l|}{ dark green, needle } \\
\hline Crystal size & \multicolumn{2}{|c|}{$0.142 \times 0.06 \times 0.013 \mathrm{~mm}^{3}$} \\
\hline Theta range for data collection & \multicolumn{2}{|l|}{2.387 to $70.064^{\circ}$} \\
\hline Index ranges & \multicolumn{2}{|c|}{$-9 \leq h \leq 9,-11 \leq k \leq 10,-22 \leq l \leq 22$} \\
\hline Reflections collected & \multicolumn{2}{|l|}{25745} \\
\hline Independent reflections & \multicolumn{2}{|c|}{$4910[R($ int $)=0.1050]$} \\
\hline Observed reflections & \multicolumn{2}{|l|}{4405} \\
\hline Completeness to theta $=70.064^{\circ}$ & \multicolumn{2}{|l|}{$98.0 \%$} \\
\hline Absorption correction & \multicolumn{2}{|l|}{ Multi-scan } \\
\hline Max. and min. transmission & \multicolumn{2}{|c|}{1.00000 and 0.50234} \\
\hline Refinement method & \multicolumn{2}{|c|}{ Full-matrix least-squares on $F^{2}$} \\
\hline Data / restraints / parameters & \multicolumn{2}{|l|}{4910 / 0 / 338} \\
\hline Goodness-of-fit on $F^{2}$ & \multicolumn{2}{|l|}{1.091} \\
\hline Final $R$ indices $[I>2 \operatorname{sigma}(I)]$ & \multicolumn{2}{|c|}{$R 1=0.0507, w R 2=0.1339$} \\
\hline$R$ indices (all data) & \multicolumn{2}{|c|}{$R 1=0.0556, w R 2=0.1390$} \\
\hline Largest diff. peak and hole & \multicolumn{2}{|c|}{1.239 and -1.626 e. $\AA^{-3}$} \\
\hline
\end{tabular}


Table 2. Atomic coordinates $\left(\times 10^{4}\right)$ and equivalent isotropic displacement parameters $\left(\AA^{2} \times 10^{3}\right)$ for rhowe 38. $U_{\text {eq }}$ is defined as one third of the trace of the orthogonalized $U_{i j}$ tensor.

\begin{tabular}{|c|c|c|c|c|}
\hline & $\mathrm{x}$ & $\mathrm{y}$ & $\mathrm{z}$ & $\mathrm{U}_{\mathrm{eq}}$ \\
\hline Mo1 & $1807(1)$ & 2621(1) & 7704(1) & $19(1)$ \\
\hline $\mathrm{Cl1}$ & $1871(2)$ & $76(1)$ & $7626(1)$ & $28(1)$ \\
\hline $\mathrm{Cl} 2$ & $1866(1)$ & $5139(1)$ & $7665(1)$ & $26(1)$ \\
\hline $\mathrm{Cl} 3$ & $3730(2)$ & $3292(1)$ & $8846(1)$ & $29(1)$ \\
\hline $\mathrm{Cl} 4$ & $-1007(2)$ & 1912(1) & $8267(1)$ & $29(1)$ \\
\hline $\mathrm{O} 1$ & $7647(4)$ & $4396(4)$ & 4952(2) & $26(1)$ \\
\hline $\mathrm{O} 2$ & 9766(4) & $5028(4)$ & $5915(2)$ & $26(1)$ \\
\hline $\mathrm{O} 3$ & $-3461(4)$ & 291(4) & $4225(2)$ & $27(1)$ \\
\hline $\mathrm{O} 4$ & $-611(4)$ & 1292(4) & $3937(2)$ & $26(1)$ \\
\hline N1 & $4001(5)$ & $3181(4)$ & $7032(2)$ & $19(1)$ \\
\hline N2 & $474(4)$ & 2093(4) & $6604(2)$ & $16(1)$ \\
\hline $\mathrm{C} 1$ & $5796(6)$ & $3726(5)$ & 7293(2) & $24(1)$ \\
\hline $\mathrm{C} 2$ & $7162(6)$ & $4178(5)$ & $6853(2)$ & $22(1)$ \\
\hline $\mathrm{C} 3$ & $6683(6)$ & $4079(5)$ & $6115(2)$ & $18(1)$ \\
\hline $\mathrm{C} 4$ & $4863(6)$ & $3511(4)$ & $5834(2)$ & $18(1)$ \\
\hline $\mathrm{C} 5$ & $3530(5)$ & $3066(4)$ & $6314(2)$ & $16(1)$ \\
\hline C6 & $1566(6)$ & $2407(4)$ & $6068(2)$ & $18(1)$ \\
\hline $\mathrm{C} 7$ & $856(6)$ & $2064(4)$ & $5343(2)$ & $17(1)$ \\
\hline $\mathrm{C} 8$ & $-1016(6)$ & $1380(4)$ & $5166(2)$ & $17(1)$ \\
\hline C9 & $-2126(6)$ & $1058(4)$ & $5716(2)$ & 19(1) \\
\hline C10 & $-1343(6)$ & $1423(5)$ & $6419(2)$ & $20(1)$ \\
\hline $\mathrm{C} 11$ & $8120(6)$ & $4547(4)$ & $5619(2)$ & $18(1)$ \\
\hline $\mathrm{C} 12$ & $-1843(6)$ & $937(5)$ & 4394(2) & $18(1)$ \\
\hline Co1 & $953(1)$ & $7622(1)$ & $9346(1)$ & $35(1)$ \\
\hline $\mathrm{C} 13$ & $-1539(9)$ & $7638(7)$ & 9579(4) & $47(2)$ \\
\hline $\mathrm{C} 14$ & $-1206(8)$ & $8098(7)$ & $8876(3)$ & $42(1)$ \\
\hline $\mathrm{C} 15$ & $-916(7)$ & $6919(6)$ & $8456(3)$ & $38(1)$ \\
\hline $\mathrm{C} 16$ & $-1068(8)$ & $5739(6)$ & 8893(3) & $40(1)$ \\
\hline $\mathrm{C} 17$ & $-1466(8)$ & $6203(6)$ & 9583(3) & $42(1)$ \\
\hline $\mathrm{C} 18$ & $2829(9)$ & $9068(7)$ & $10089(3)$ & $43(1)$ \\
\hline C19 & 3099(9) & $9491(6)$ & 9391(3) & $42(1)$ \\
\hline
\end{tabular}




\begin{tabular}{lrrrr}
$\mathrm{C} 20$ & $3450(8)$ & $8326(6)$ & $8970(3)$ & $38(1)$ \\
$\mathrm{C} 21$ & $3358(8)$ & $7188(6)$ & $9415(3)$ & $38(1)$ \\
$\mathrm{C} 22$ & $2972(8)$ & $7654(6)$ & $10114(3)$ & $40(1)$ \\
$\mathrm{N} 3$ & $3640(6)$ & $2727(5)$ & $4008(2)$ & $34(1)$ \\
$\mathrm{C} 23$ & $4016(7)$ & $2288(5)$ & $3486(3)$ & $28(1)$ \\
$\mathrm{C} 24$ & $1708(6)$ & $2813(3)$ & $44(1)$ \\
\hline
\end{tabular}


Table 3. Bond lengths $[\AA]$ and angles $\left[{ }^{\circ}\right]$ for rhowe 38 .

\begin{tabular}{|c|c|c|c|}
\hline $\mathrm{Mo}(1)-\mathrm{Cl}(1)$ & $2.4588(12)$ & $\mathrm{Co}(1)-\mathrm{C}(13)$ & $2.032(6)$ \\
\hline $\mathrm{Mo}(1)-\mathrm{Cl}(2)$ & $2.4142(11)$ & $\mathrm{Co}(1)-\mathrm{C}(14)$ & $2.035(6)$ \\
\hline $\mathrm{Mo}(1)-\mathrm{Cl}(3)$ & $2.4180(11)$ & $\mathrm{Co}(1)-\mathrm{C}(15)$ & $2.033(6)$ \\
\hline $\mathrm{Mo}(1)-\mathrm{Cl}(4)$ & $2.4321(11)$ & $\mathrm{Co}(1)-\mathrm{C}(16)$ & $2.040(6)$ \\
\hline $\mathrm{Mo}(1)-\mathrm{N}(1)$ & $2.159(3)$ & $\mathrm{Co}(1)-\mathrm{C}(17)$ & $2.043(6)$ \\
\hline $\mathrm{Mo}(1)-\mathrm{N}(2)$ & $2.167(3)$ & $\mathrm{Co}(1)-\mathrm{C}(18)$ & $2.010(6)$ \\
\hline $\mathrm{O}(1)-\mathrm{H}(1)$ & 0.8200 & $\mathrm{Co}(1)-\mathrm{C}(19)$ & $2.009(6)$ \\
\hline $\mathrm{O}(1)-\mathrm{C}(11)$ & $1.255(5)$ & $\mathrm{Co}(1)-\mathrm{C}(20)$ & $2.048(6)$ \\
\hline $\mathrm{O}(2)-\mathrm{H}(2)$ & 0.8200 & $\mathrm{Co}(1)-\mathrm{C}(21)$ & $2.048(6)$ \\
\hline $\mathrm{O}(2)-\mathrm{C}(11)$ & $1.274(5)$ & $\mathrm{Co}(1)-\mathrm{C}(22)$ & $2.032(6)$ \\
\hline $\mathrm{O}(3)-\mathrm{C}(12)$ & $1.213(5)$ & $\mathrm{C}(13)-\mathrm{H}(13)$ & 0.9300 \\
\hline $\mathrm{O}(4)-\mathrm{H}(4)$ & 0.8200 & $\mathrm{C}(13)-\mathrm{C}(14)$ & $1.435(9)$ \\
\hline $\mathrm{O}(4)-\mathrm{C}(12)$ & $1.318(5)$ & $\mathrm{C}(13)-\mathrm{C}(17)$ & $1.400(9)$ \\
\hline $\mathrm{N}(1)-\mathrm{C}(1)$ & $1.357(6)$ & $\mathrm{C}(14)-\mathrm{H}(14)$ & 0.9300 \\
\hline $\mathrm{N}(1)-\mathrm{C}(5)$ & $1.348(5)$ & $\mathrm{C}(14)-\mathrm{C}(15)$ & $1.419(8)$ \\
\hline $\mathrm{N}(2)-\mathrm{C}(6)$ & $1.357(5)$ & $\mathrm{C}(15)-\mathrm{H}(15)$ & 0.9300 \\
\hline $\mathrm{N}(2)-\mathrm{C}(10)$ & $1.355(5)$ & $\mathrm{C}(15)-\mathrm{C}(16)$ & $1.429(8)$ \\
\hline $\mathrm{C}(1)-\mathrm{H}(1 \mathrm{~A})$ & 0.9300 & $\mathrm{C}(16)-\mathrm{H}(16)$ & 0.9300 \\
\hline $\mathrm{C}(1)-\mathrm{C}(2)$ & $1.379(6)$ & $\mathrm{C}(16)-\mathrm{C}(17)$ & $1.423(9)$ \\
\hline $\mathrm{C}(2)-\mathrm{H}(2 \mathrm{~A})$ & 0.9300 & $\mathrm{C}(17)-\mathrm{H}(17)$ & 0.9300 \\
\hline$C(2)-C(3)$ & $1.387(6)$ & $\mathrm{C}(18)-\mathrm{H}(18)$ & 0.9300 \\
\hline$C(3)-C(4)$ & $1.385(6)$ & $\mathrm{C}(18)-\mathrm{C}(19)$ & $1.410(8)$ \\
\hline$C(3)-C(11)$ & $1.492(6)$ & $\mathrm{C}(18)-\mathrm{C}(22)$ & $1.404(9)$ \\
\hline $\mathrm{C}(4)-\mathrm{H}(4 \mathrm{~A})$ & 0.9300 & $\mathrm{C}(19)-\mathrm{H}(19)$ & 0.9300 \\
\hline$C(4)-C(5)$ & $1.412(6)$ & $C(19)-C(20)$ & $1.431(8)$ \\
\hline$C(5)-C(6)$ & $1.472(6)$ & $\mathrm{C}(20)-\mathrm{H}(20)$ & 0.9300 \\
\hline$C(6)-C(7)$ & $1.391(6)$ & $\mathrm{C}(20)-\mathrm{C}(21)$ & $1.412(8)$ \\
\hline $\mathrm{C}(7)-\mathrm{H}(7)$ & 0.9300 & $\mathrm{C}(21)-\mathrm{H}(21)$ & 0.9300 \\
\hline$C(7)-C(8)$ & $1.392(6)$ & $C(21)-C(22)$ & $1.436(8)$ \\
\hline $\mathrm{C}(8)-\mathrm{C}(9)$ & $1.387(6)$ & $\mathrm{C}(22)-\mathrm{H}(22)$ & 0.9300 \\
\hline$C(8)-C(12)$ & $1.501(6)$ & $\mathrm{N}(3)-\mathrm{C}(23)$ & $1.130(7)$ \\
\hline $\mathrm{C}(9)-\mathrm{H}(9)$ & 0.9300 & $C(23)-C(24)$ & $1.455(7)$ \\
\hline$C(9)-C(10)$ & $1.370(6)$ & $\mathrm{C}(24)-\mathrm{H}(24 \mathrm{~A})$ & 0.9600 \\
\hline $\mathrm{C}(10)-\mathrm{H}(10)$ & 0.9300 & $\mathrm{C}(24)-\mathrm{H}(24 \mathrm{~B})$ & 0.9600 \\
\hline
\end{tabular}




\begin{tabular}{|c|c|}
\hline $\mathrm{C}(24)-\mathrm{H}(24 \mathrm{C})$ & 0.9600 \\
\hline $\mathrm{Cl}(2)-\mathrm{Mo}(1)-\mathrm{Cl}(1)$ & $174.25(4)$ \\
\hline $\mathrm{Cl}(2)-\mathrm{Mo}(1)-\mathrm{Cl}(3)$ & $91.14(4)$ \\
\hline $\mathrm{Cl}(2)-\mathrm{Mo}(1)-\mathrm{Cl}(4)$ & $92.98(4)$ \\
\hline $\mathrm{Cl}(3)-\mathrm{Mo}(1)-\mathrm{Cl}(1)$ & $91.96(4)$ \\
\hline $\mathrm{Cl}(3)-\mathrm{Mo}(1)-\mathrm{Cl}(4)$ & $93.66(4)$ \\
\hline $\mathrm{Cl}(4)-\mathrm{Mo}(1)-\mathrm{Cl}(1)$ & $91.65(4)$ \\
\hline N(1)-Mo(1)-Cl(1) & $88.91(10)$ \\
\hline N(1)-Mo(1)-Cl(2) & $85.95(10)$ \\
\hline $\mathrm{N}(1)-\mathrm{Mo}(1)-\mathrm{Cl}(3)$ & $96.15(10)$ \\
\hline $\mathrm{N}(1)-\mathrm{Mo}(1)-\mathrm{Cl}(4)$ & $170.15(10)$ \\
\hline $\mathrm{N}(1)-\mathrm{Mo}(1)-\mathrm{N}(2)$ & $75.01(13)$ \\
\hline $\mathrm{N}(2)-\mathrm{Mo}(1)-\mathrm{Cl}(1)$ & $87.12(10)$ \\
\hline $\mathrm{N}(2)-\mathrm{Mo}(1)-\mathrm{Cl}(2)$ & $89.08(10)$ \\
\hline N(2)-Mo(1)-Cl(3) & 171.12(9) \\
\hline $\mathrm{N}(2)-\mathrm{Mo}(1)-\mathrm{Cl}(4)$ & $95.19(9)$ \\
\hline $\mathrm{C}(11)-\mathrm{O}(1)-\mathrm{H}(1)$ & 109.5 \\
\hline $\mathrm{C}(11)-\mathrm{O}(2)-\mathrm{H}(2)$ & 109.5 \\
\hline $\mathrm{C}(12)-\mathrm{O}(4)-\mathrm{H}(4)$ & 109.5 \\
\hline $\mathrm{C}(1)-\mathrm{N}(1)-\mathrm{Mo}(1)$ & $124.0(3)$ \\
\hline $\mathrm{C}(5)-\mathrm{N}(1)-\mathrm{Mo}(1)$ & $117.0(3)$ \\
\hline $\mathrm{C}(5)-\mathrm{N}(1)-\mathrm{C}(1)$ & $118.9(4)$ \\
\hline $\mathrm{C}(6)-\mathrm{N}(2)-\mathrm{Mo}(1)$ & $116.9(3)$ \\
\hline $\mathrm{C}(10)-\mathrm{N}(2)-\mathrm{Mo}(1)$ & $124.7(3)$ \\
\hline$C(10)-N(2)-C(6)$ & $118.4(4)$ \\
\hline $\mathrm{N}(1)-\mathrm{C}(1)-\mathrm{H}(1 \mathrm{~A})$ & 118.7 \\
\hline $\mathrm{N}(1)-\mathrm{C}(1)-\mathrm{C}(2)$ & $122.6(4)$ \\
\hline $\mathrm{C}(2)-\mathrm{C}(1)-\mathrm{H}(1 \mathrm{~A})$ & 118.7 \\
\hline $\mathrm{C}(1)-\mathrm{C}(2)-\mathrm{H}(2 \mathrm{~A})$ & 120.7 \\
\hline $\mathrm{C}(1)-\mathrm{C}(2)-\mathrm{C}(3)$ & $118.7(4)$ \\
\hline $\mathrm{C}(3)-\mathrm{C}(2)-\mathrm{H}(2 \mathrm{~A})$ & 120.7 \\
\hline$C(2)-C(3)-C(11)$ & $120.4(4)$ \\
\hline $\mathrm{C}(4)-\mathrm{C}(3)-\mathrm{C}(2)$ & $119.8(4)$ \\
\hline$C(4)-C(3)-C(11)$ & $119.8(4)$ \\
\hline $\mathrm{C}(3)-\mathrm{C}(4)-\mathrm{H}(4 \mathrm{~A})$ & 120.7 \\
\hline$C(3)-C(4)-C(5)$ & $118.7(4)$ \\
\hline
\end{tabular}

\begin{tabular}{|c|c|}
\hline $\mathrm{C}(5)-\mathrm{C}(4)-\mathrm{H}(4 \mathrm{~A})$ & 120.7 \\
\hline $\mathrm{N}(1)-\mathrm{C}(5)-\mathrm{C}(4)$ & $121.3(4)$ \\
\hline$N(1)-C(5)-C(6)$ & $115.8(3)$ \\
\hline$C(4)-C(5)-C(6)$ & $122.9(4)$ \\
\hline $\mathrm{N}(2)-\mathrm{C}(6)-\mathrm{C}(5)$ & $115.0(3)$ \\
\hline $\mathrm{N}(2)-\mathrm{C}(6)-\mathrm{C}(7)$ & $121.5(4)$ \\
\hline $\mathrm{C}(7)-\mathrm{C}(6)-\mathrm{C}(5)$ & $123.4(4)$ \\
\hline $\mathrm{C}(6)-\mathrm{C}(7)-\mathrm{H}(7)$ & 120.5 \\
\hline $\mathrm{C}(6)-\mathrm{C}(7)-\mathrm{C}(8)$ & $119.1(4)$ \\
\hline $\mathrm{C}(8)-\mathrm{C}(7)-\mathrm{H}(7)$ & 120.5 \\
\hline$C(7)-C(8)-C(12)$ & $121.2(4)$ \\
\hline$C(9)-C(8)-C(7)$ & $119.2(4)$ \\
\hline$C(9)-C(8)-C(12)$ & $119.5(4)$ \\
\hline $\mathrm{C}(8)-\mathrm{C}(9)-\mathrm{H}(9)$ & 120.6 \\
\hline $\mathrm{C}(10)-\mathrm{C}(9)-\mathrm{C}(8)$ & $118.9(4)$ \\
\hline $\mathrm{C}(10)-\mathrm{C}(9)-\mathrm{H}(9)$ & 120.6 \\
\hline $\mathrm{N}(2)-\mathrm{C}(10)-\mathrm{C}(9)$ & $123.0(4)$ \\
\hline $\mathrm{N}(2)-\mathrm{C}(10)-\mathrm{H}(10)$ & 118.5 \\
\hline $\mathrm{C}(9)-\mathrm{C}(10)-\mathrm{H}(10)$ & 118.5 \\
\hline $\mathrm{O}(1)-\mathrm{C}(11)-\mathrm{O}(2)$ & $124.8(4)$ \\
\hline $\mathrm{O}(1)-\mathrm{C}(11)-\mathrm{C}(3)$ & $118.9(4)$ \\
\hline $\mathrm{O}(2)-\mathrm{C}(11)-\mathrm{C}(3)$ & $116.3(4)$ \\
\hline $\mathrm{O}(3)-\mathrm{C}(12)-\mathrm{O}(4)$ & $125.1(4)$ \\
\hline $\mathrm{O}(3)-\mathrm{C}(12)-\mathrm{C}(8)$ & $122.6(4)$ \\
\hline $\mathrm{O}(4)-\mathrm{C}(12)-\mathrm{C}(8)$ & $112.3(4)$ \\
\hline $\mathrm{C}(13)-\mathrm{Co}(1)-\mathrm{C}(14)$ & $41.3(2)$ \\
\hline $\mathrm{C}(13)-\mathrm{Co}(1)-\mathrm{C}(15)$ & $68.8(3)$ \\
\hline $\mathrm{C}(13)-\mathrm{Co}(1)-\mathrm{C}(16)$ & $68.6(3)$ \\
\hline $\mathrm{C}(13)-\mathrm{Co}(1)-\mathrm{C}(17)$ & $40.2(3)$ \\
\hline $\mathrm{C}(13)-\mathrm{Co}(1)-\mathrm{C}(20)$ & $158.5(2)$ \\
\hline $\mathrm{C}(13)-\mathrm{Co}(1)-\mathrm{C}(21)$ & $159.9(2)$ \\
\hline $\mathrm{C}(14)-\mathrm{Co}(1)-\mathrm{C}(16)$ & $69.0(2)$ \\
\hline $\mathrm{C}(14)-\mathrm{Co}(1)-\mathrm{C}(17)$ & $68.6(2)$ \\
\hline $\mathrm{C}(14)-\mathrm{Co}(1)-\mathrm{C}(20)$ & $121.2(2)$ \\
\hline $\mathrm{C}(14)-\mathrm{Co}(1)-\mathrm{C}(21)$ & $157.7(2)$ \\
\hline $\mathrm{C}(15)-\mathrm{Co}(1)-\mathrm{C}(14)$ & $40.8(2)$ \\
\hline
\end{tabular}




\begin{tabular}{|c|c|}
\hline $\mathrm{C}(15)-\mathrm{Co}(1)-\mathrm{C}(16)$ & $41.1(2)$ \\
\hline $\mathrm{C}(15)-\mathrm{Co}(1)-\mathrm{C}(17)$ & $68.6(2)$ \\
\hline $\mathrm{C}(15)-\mathrm{Co}(1)-\mathrm{C}(20)$ & $106.0(2)$ \\
\hline $\mathrm{C}(15)-\mathrm{Co}(1)-\mathrm{C}(21)$ & $122.3(2)$ \\
\hline $\mathrm{C}(16)-\mathrm{Co}(1)-\mathrm{C}(17)$ & $40.8(2)$ \\
\hline $\mathrm{C}(16)-\mathrm{Co}(1)-\mathrm{C}(20)$ & $122.0(2)$ \\
\hline $\mathrm{C}(16)-\mathrm{Co}(1)-\mathrm{C}(21)$ & $107.8(2)$ \\
\hline $\mathrm{C}(17)-\mathrm{Co}(1)-\mathrm{C}(20)$ & $159.2(2)$ \\
\hline $\mathrm{C}(17)-\mathrm{Co}(1)-\mathrm{C}(21)$ & $124.3(2)$ \\
\hline $\mathrm{C}(18)-\mathrm{Co}(1)-\mathrm{C}(13)$ & $107.5(3)$ \\
\hline $\mathrm{C}(18)-\mathrm{Co}(1)-\mathrm{C}(14)$ & $121.9(3)$ \\
\hline $\mathrm{C}(18)-\mathrm{Co}(1)-\mathrm{C}(15)$ & $157.7(2)$ \\
\hline $\mathrm{C}(18)-\mathrm{Co}(1)-\mathrm{C}(16)$ & $159.9(2)$ \\
\hline $\mathrm{C}(18)-\mathrm{Co}(1)-\mathrm{C}(17)$ & $123.7(3)$ \\
\hline $\mathrm{C}(18)-\mathrm{Co}(1)-\mathrm{C}(20)$ & $69.0(2)$ \\
\hline $\mathrm{C}(18)-\mathrm{Co}(1)-\mathrm{C}(21)$ & $68.7(2)$ \\
\hline $\mathrm{C}(18)-\mathrm{Co}(1)-\mathrm{C}(22)$ & $40.7(2)$ \\
\hline $\mathrm{C}(19)-\mathrm{Co}(1)-\mathrm{C}(13)$ & $122.1(3)$ \\
\hline $\mathrm{C}(19)-\mathrm{Co}(1)-\mathrm{C}(14)$ & $105.3(3)$ \\
\hline $\mathrm{C}(19)-\mathrm{Co}(1)-\mathrm{C}(15)$ & $120.9(2)$ \\
\hline $\mathrm{C}(19)-\mathrm{Co}(1)-\mathrm{C}(16)$ & $157.9(2)$ \\
\hline $\mathrm{C}(19)-\mathrm{Co}(1)-\mathrm{C}(17)$ & $158.9(3)$ \\
\hline $\mathrm{C}(19)-\mathrm{Co}(1)-\mathrm{C}(18)$ & $41.1(2)$ \\
\hline $\mathrm{C}(19)-\mathrm{Co}(1)-\mathrm{C}(20)$ & $41.3(2)$ \\
\hline $\mathrm{C}(19)-\mathrm{Co}(1)-\mathrm{C}(21)$ & $68.8(2)$ \\
\hline $\mathrm{C}(19)-\mathrm{Co}(1)-\mathrm{C}(22)$ & $69.0(2)$ \\
\hline $\mathrm{C}(20)-\mathrm{Co}(1)-\mathrm{C}(21)$ & $40.3(2)$ \\
\hline $\mathrm{C}(22)-\mathrm{Co}(1)-\mathrm{C}(13)$ & $123.2(3)$ \\
\hline $\mathrm{C}(22)-\mathrm{Co}(1)-\mathrm{C}(14)$ & $158.8(3)$ \\
\hline $\mathrm{C}(22)-\mathrm{Co}(1)-\mathrm{C}(15)$ & $159.7(2)$ \\
\hline $\mathrm{C}(22)-\mathrm{Co}(1)-\mathrm{C}(16)$ & $123.8(2)$ \\
\hline $\mathrm{C}(22)-\mathrm{Co}(1)-\mathrm{C}(17)$ & $108.9(2)$ \\
\hline $\mathrm{C}(22)-\mathrm{Co}(1)-\mathrm{C}(20)$ & $68.8(2)$ \\
\hline $\mathrm{C}(22)-\mathrm{Co}(1)-\mathrm{C}(21)$ & $41.2(2)$ \\
\hline $\mathrm{Co}(1)-\mathrm{C}(13)-\mathrm{H}(13)$ & 126.0 \\
\hline $\mathrm{C}(14)-\mathrm{C}(13)-\mathrm{Co}(1)$ & $69.5(3)$ \\
\hline
\end{tabular}

$\begin{array}{ll}\mathrm{C}(14)-\mathrm{C}(13)-\mathrm{H}(13) & 125.8 \\ \mathrm{C}(17)-\mathrm{C}(13)-\mathrm{Co}(1) & 70.3(3) \\ \mathrm{C}(17)-\mathrm{C}(13)-\mathrm{H}(13) & 125.8 \\ \mathrm{C}(17)-\mathrm{C}(13)-\mathrm{C}(14) & 108.4(5) \\ \mathrm{Co}(1)-\mathrm{C}(14)-\mathrm{H}(14) & 126.5\end{array}$

$\mathrm{C}(13)-\mathrm{C}(14)-\mathrm{Co}(1) \quad 69.2(4)$

$\mathrm{C}(13)-\mathrm{C}(14)-\mathrm{H}(14) \quad 126.4$

$\mathrm{C}(15)-\mathrm{C}(14)-\mathrm{Co}(1) \quad$ 69.5(3)

$\mathrm{C}(15)-\mathrm{C}(14)-\mathrm{C}(13) \quad 107.2(5)$

$\mathrm{C}(15)-\mathrm{C}(14)-\mathrm{H}(14) \quad 126.4$

$\mathrm{Co}(1)-\mathrm{C}(15)-\mathrm{H}(15) \quad 126.3$

$\mathrm{C}(14)-\mathrm{C}(15)-\mathrm{Co}(1) \quad 69.7(3)$

$\mathrm{C}(14)-\mathrm{C}(15)-\mathrm{H}(15) \quad 125.8$

C(14)-C(15)-C(16) 108.3(5)

$\mathrm{C}(16)-\mathrm{C}(15)-\mathrm{Co}(1) \quad 69.7(3)$

$\mathrm{C}(16)-\mathrm{C}(15)-\mathrm{H}(15) \quad 125.8$

$\mathrm{Co}(1)-\mathrm{C}(16)-\mathrm{H}(16) \quad 126.3$

$\mathrm{C}(15)-\mathrm{C}(16)-\mathrm{Co}(1) \quad$ 69.2(3)

$\mathrm{C}(15)-\mathrm{C}(16)-\mathrm{H}(16) \quad 126.3$

$\mathrm{C}(17)-\mathrm{C}(16)-\mathrm{Co}(1) \quad$ 69.7(3)

$\mathrm{C}(17)-\mathrm{C}(16)-\mathrm{C}(15) \quad 107.3(5)$

$\mathrm{C}(17)-\mathrm{C}(16)-\mathrm{H}(16) \quad 126.3$

$\mathrm{Co}(1)-\mathrm{C}(17)-\mathrm{H}(17) \quad 127.0$

$\mathrm{C}(13)-\mathrm{C}(17)-\mathrm{Co}(1) \quad$ 69.5(3)

$\mathrm{C}(13)-\mathrm{C}(17)-\mathrm{C}(16) \quad 108.7(5)$

$\mathrm{C}(13)-\mathrm{C}(17)-\mathrm{H}(17) \quad 125.7$

$\mathrm{C}(16)-\mathrm{C}(17)-\mathrm{Co}(1) \quad$ 69.5(3)

$\mathrm{C}(16)-\mathrm{C}(17)-\mathrm{H}(17) \quad 125.7$

$\mathrm{Co}(1)-\mathrm{C}(18)-\mathrm{H}(18) \quad 126.1$

C(19)-C(18)-Co(1) 69.4(3)

$\mathrm{C}(19)-\mathrm{C}(18)-\mathrm{H}(18) \quad 125.6$

$\mathrm{C}(22)-\mathrm{C}(18)-\mathrm{Co}(1) \quad 70.5(3)$

$\mathrm{C}(22)-\mathrm{C}(18)-\mathrm{H}(18) \quad 125.6$

$\mathrm{C}(22)-\mathrm{C}(18)-\mathrm{C}(19) \quad 108.9(5)$

$\mathrm{Co}(1)-\mathrm{C}(19)-\mathrm{H}(19) \quad 125.3$

C(18)-C(19)-Co(1) 69.5(3) 


$\begin{array}{lclc}\mathrm{C}(18)-\mathrm{C}(19)-\mathrm{H}(19) & 126.0 & \mathrm{C}(22)-\mathrm{C}(21)-\mathrm{H}(21) & 126.0 \\ \mathrm{C}(18)-\mathrm{C}(19)-\mathrm{C}(20) & 108.0(5) & \mathrm{Co}(1)-\mathrm{C}(22)-\mathrm{H}(22) & 126.5 \\ \mathrm{C}(20)-\mathrm{C}(19)-\mathrm{Co}(1) & 70.8(3) & \mathrm{C}(18)-\mathrm{C}(22)-\mathrm{Co}(1) & 68.8(3) \\ \mathrm{C}(20)-\mathrm{C}(19)-\mathrm{H}(19) & 126.0 & \mathrm{C}(18)-\mathrm{C}(22)-\mathrm{C}(21) & 107.5(5) \\ \mathrm{Co}(1)-\mathrm{C}(20)-\mathrm{H}(20) & 127.6 & \mathrm{C}(18)-\mathrm{C}(22)-\mathrm{H}(22) & 126.2 \\ \mathrm{C}(19)-\mathrm{C}(20)-\mathrm{Co}(1) & 67.9(3) & \mathrm{C}(21)-\mathrm{C}(22)-\mathrm{Co}(1) & 70.0(3) \\ \mathrm{C}(19)-\mathrm{C}(20)-\mathrm{H}(20) & 126.2 & \mathrm{C}(21)-\mathrm{C}(22)-\mathrm{H}(22) & 126.2 \\ \mathrm{C}(21)-\mathrm{C}(20)-\mathrm{Co}(1) & 69.9(3) & \mathrm{N}(3)-\mathrm{C}(23)-\mathrm{C}(24) & 179.2(6) \\ \mathrm{C}(21)-\mathrm{C}(20)-\mathrm{C}(19) & 107.5(5) & \mathrm{C}(23)-\mathrm{C}(24)-\mathrm{H}(24 \mathrm{~A}) & 109.5 \\ \mathrm{C}(21)-\mathrm{C}(20)-\mathrm{H}(20) & 126.2 & \mathrm{C}(23)-\mathrm{C}(24)-\mathrm{H}(24 \mathrm{~B}) & 109.5 \\ \mathrm{Co}(1)-\mathrm{C}(21)-\mathrm{H}(21) & 127.0 & \mathrm{C}(23)-\mathrm{C}(24)-\mathrm{H}(24 \mathrm{C}) & 109.5 \\ \mathrm{C}(20)-\mathrm{C}(21)-\mathrm{Co}(1) & 69.8(3) & \mathrm{H}(24 \mathrm{~A})-\mathrm{C}(24)-\mathrm{H}(24 \mathrm{~B}) & 109.5 \\ \mathrm{C}(20)-\mathrm{C}(21)-\mathrm{H}(21) & 126.0 & \mathrm{H}(24 \mathrm{~A})-\mathrm{C}(24)-\mathrm{H}(24 \mathrm{C}) & 109.5 \\ \mathrm{C}(20)-\mathrm{C}(21)-\mathrm{C}(22) & 108.1(5) & \mathrm{H}(24 \mathrm{~B})-\mathrm{C}(24)-\mathrm{H}(24 \mathrm{C}) & 109.5 \\ \mathrm{C}(22)-\mathrm{C}(21)-\mathrm{Co}(1) & 68.8(4) & & \end{array}$


Table 4. Anisotropic displacement parameters $\left(\AA^{2} \times 10^{3}\right)$ for rhowe 38. The anisotropic displacement factor exponent takes the form: $-2 \pi^{2}\left[h^{2} a^{* 2} U_{11}+\ldots+2 h k a^{*} b^{*} U_{12}\right]$

\begin{tabular}{|c|c|c|c|c|c|c|}
\hline & $\mathrm{U}_{11}$ & $\mathrm{U}_{22}$ & $\mathrm{U}_{33}$ & $\mathrm{U}_{23}$ & $\mathrm{U}_{13}$ & $\mathrm{U}_{12}$ \\
\hline Mo1 & $16(1)$ & $25(1)$ & $16(1)$ & $2(1)$ & $4(1)$ & $5(1)$ \\
\hline $\mathrm{Cl1}$ & $36(1)$ & $30(1)$ & $21(1)$ & $4(1)$ & $4(1)$ & $13(1)$ \\
\hline $\mathrm{Cl} 2$ & $22(1)$ & $26(1)$ & $29(1)$ & $-1(1)$ & $2(1)$ & $7(1)$ \\
\hline $\mathrm{Cl} 3$ & $27(1)$ & $37(1)$ & $20(1)$ & 1(1) & $1(1)$ & $10(1)$ \\
\hline $\mathrm{Cl} 4$ & $23(1)$ & $35(1)$ & $26(1)$ & $3(1)$ & $10(1)$ & $6(1)$ \\
\hline $\mathrm{O} 1$ & $23(2)$ & $30(2)$ & $25(2)$ & $8(1)$ & $8(1)$ & $7(1)$ \\
\hline $\mathrm{O} 2$ & $13(2)$ & $27(2)$ & $36(2)$ & $6(1)$ & $6(1)$ & $2(1)$ \\
\hline $\mathrm{O} 3$ & $15(2)$ & $34(2)$ & $26(2)$ & $-1(1)$ & $0(1)$ & $3(1)$ \\
\hline $\mathrm{O} 4$ & $22(2)$ & $34(2)$ & $18(2)$ & $2(1)$ & $2(1)$ & $3(1)$ \\
\hline N1 & $16(2)$ & $22(2)$ & $17(2)$ & $0(1)$ & $5(1)$ & $6(1)$ \\
\hline N2 & $7(2)$ & $23(2)$ & $19(2)$ & $4(1)$ & $3(1)$ & $4(1)$ \\
\hline $\mathrm{C} 1$ & $16(2)$ & $32(2)$ & $23(2)$ & $1(2)$ & $0(2)$ & $7(2)$ \\
\hline $\mathrm{C} 2$ & $16(2)$ & $27(2)$ & $23(2)$ & $-2(2)$ & $1(2)$ & $7(2)$ \\
\hline $\mathrm{C} 3$ & $17(2)$ & $18(2)$ & $22(2)$ & $3(2)$ & $4(2)$ & $7(2)$ \\
\hline $\mathrm{C} 4$ & $16(2)$ & $16(2)$ & $22(2)$ & $2(2)$ & $4(2)$ & $4(2)$ \\
\hline C5 & $13(2)$ & $16(2)$ & $18(2)$ & $1(2)$ & $6(2)$ & $3(2)$ \\
\hline C6 & $21(2)$ & $12(2)$ & $20(2)$ & $3(2)$ & $6(2)$ & $5(2)$ \\
\hline C7 & $16(2)$ & $17(2)$ & $21(2)$ & $7(2)$ & $6(2)$ & $7(2)$ \\
\hline $\mathrm{C} 8$ & $19(2)$ & $14(2)$ & $21(2)$ & $2(2)$ & $4(2)$ & $7(2)$ \\
\hline C9 & $15(2)$ & $15(2)$ & $27(2)$ & $3(2)$ & $4(2)$ & $4(2)$ \\
\hline C10 & $14(2)$ & $20(2)$ & $25(2)$ & $4(2)$ & $7(2)$ & $4(2)$ \\
\hline C11 & $17(2)$ & $12(2)$ & $26(2)$ & $2(2)$ & $4(2)$ & $5(2)$ \\
\hline $\mathrm{C} 12$ & $20(2)$ & $18(2)$ & $21(2)$ & $5(2)$ & $5(2)$ & $10(2)$ \\
\hline Co1 & $45(1)$ & $31(1)$ & $30(1)$ & $6(1)$ & 11(1) & $14(1)$ \\
\hline C13 & $51(4)$ & $50(3)$ & $51(4)$ & $12(3)$ & $26(3)$ & $26(3)$ \\
\hline C14 & $39(3)$ & $45(3)$ & $48(3)$ & $12(3)$ & $12(3)$ & 19(3) \\
\hline C15 & $30(3)$ & $47(3)$ & $37(3)$ & $5(2)$ & $6(2)$ & $10(2)$ \\
\hline C16 & $32(3)$ & $33(3)$ & $49(3)$ & $7(2)$ & $8(2)$ & $4(2)$ \\
\hline $\mathrm{C} 17$ & $43(3)$ & $38(3)$ & $50(3)$ & $16(3)$ & $22(3)$ & $12(3)$ \\
\hline $\mathrm{C} 18$ & $51(4)$ & $42(3)$ & $30(3)$ & $-4(2)$ & $3(2)$ & $10(3)$ \\
\hline \multirow[t]{2}{*}{ C19 } & $55(4)$ & $32(3)$ & $35(3)$ & $6(2)$ & $-1(2)$ & $10(3)$ \\
\hline & & & & S155 & & \\
\hline
\end{tabular}




\begin{tabular}{lllllll}
$\mathrm{C} 20$ & $37(3)$ & $39(3)$ & $33(3)$ & $4(2)$ & $8(2)$ & $6(2)$ \\
$\mathrm{C} 21$ & $38(3)$ & $35(3)$ & $38(3)$ & $3(2)$ & $-1(2)$ & $10(2)$ \\
$\mathrm{C} 22$ & $47(3)$ & $37(3)$ & $31(3)$ & $7(2)$ & $2(2)$ & $8(2)$ \\
$\mathrm{N} 3$ & $33(2)$ & $33(2)$ & $33(2)$ & $5(2)$ & $8(2)$ & $4(2)$ \\
$\mathrm{C} 23$ & $24(2)$ & $25(2)$ & $33(3)$ & $6(2)$ & $5(2)$ & $4(2)$ \\
$\mathrm{C} 24$ & $52(4)$ & $36(3)$ & $41(3)$ & $3(2)$ & $20(3)$ & $6(3)$ \\
\hline
\end{tabular}


Table 5. Hydrogen coordinates $\left(\times 10^{4}\right)$ and isotropic displacement parameters $\left(\AA^{2} \times 10^{3}\right)$ for rhowe38.

\begin{tabular}{|c|c|c|c|c|}
\hline & $\mathrm{x}$ & $\mathrm{y}$ & z & $\mathrm{U}(\mathrm{eq})$ \\
\hline $\mathrm{H} 1$ & 8554 & 4691 & 4740 & 39 \\
\hline $\mathrm{H} 2$ & 10449 & 5345 & 5611 & 39 \\
\hline $\mathrm{H} 4$ & -1122 & 1112 & 3522 & 40 \\
\hline H1A & 6116 & 3798 & 7788 & 29 \\
\hline $\mathrm{H} 2 \mathrm{~A}$ & 8381 & 4541 & 7048 & 27 \\
\hline $\mathrm{H} 4 \mathrm{~A}$ & 4525 & 3425 & 5339 & 22 \\
\hline H7 & 1622 & 2289 & 4982 & 20 \\
\hline H9 & -3381 & 602 & 5609 & 23 \\
\hline $\mathrm{H} 10$ & -2093 & 1200 & 6785 & 23 \\
\hline H13 & -1764 & 8198 & 9965 & 56 \\
\hline H14 & -1185 & 9001 & 8725 & 50 \\
\hline H15 & -669 & 6914 & 7978 & 46 \\
\hline H16 & -932 & 4836 & 8753 & 48 \\
\hline H17 & -1647 & 5646 & 9973 & 51 \\
\hline H18 & 2594 & 9632 & 10471 & 51 \\
\hline H19 & 3056 & 10373 & 9231 & 50 \\
\hline $\mathrm{H} 20$ & 3694 & 8320 & 8491 & 45 \\
\hline $\mathrm{H} 21$ & 3519 & 6292 & 9278 & 45 \\
\hline $\mathrm{H} 22$ & 2842 & 7117 & 10511 & 48 \\
\hline $\mathrm{H} 24 \mathrm{~A}$ & 3372 & 1183 & 2501 & 66 \\
\hline H24B & 5133 & 1047 & 2913 & 66 \\
\hline $\mathrm{H} 24 \mathrm{C}$ & 5213 & 2511 & 2581 & 66 \\
\hline
\end{tabular}


Table 6. Torsion angles $\left[{ }^{\circ}\right]$ for rhowe 38 .

\begin{tabular}{|c|c|c|c|}
\hline Mo1-N1-C1-C2 & $-175.0(3)$ & C10-N2-C6-C5 & $-177.1(4)$ \\
\hline Mo1-N1-C5-C4 & $175.2(3)$ & C10-N2-C6-C7 & $0.4(6)$ \\
\hline Mo1-N1-C5-C6 & $-6.8(5)$ & C11-C3-C4-C5 & $179.6(4)$ \\
\hline Mo1-N2-C6-C5 & $0.2(5)$ & C12-C8-C9-C10 & $177.7(4)$ \\
\hline Mo1-N2-C6-C7 & 177.7(3) & Co1-C13-C14-C15 & $59.3(4)$ \\
\hline Mo1-N2-C10-C9 & $-177.5(3)$ & Co1-C13-C17-C16 & $-58.5(4)$ \\
\hline N1-C1-C2-C3 & $0.4(7)$ & Co1-C14-C15-C16 & $59.2(4)$ \\
\hline N1-C5-C6-N2 & $4.3(5)$ & Co1-C15-C16-C17 & $59.5(4)$ \\
\hline N1-C5-C6-C7 & $-173.1(4)$ & Co1-C16-C17-C13 & $58.5(4)$ \\
\hline N2-C6-C7-C8 & $-0.2(6)$ & Co1-C18-C19-C20 & $-60.6(4)$ \\
\hline C1-N1-C5-C4 & $-0.5(6)$ & $\mathrm{Co1}-\mathrm{C} 18-\mathrm{C} 22-\mathrm{C} 21$ & $59.5(4)$ \\
\hline C1-N1-C5-C6 & $177.5(4)$ & Co1-C19-C20-C21 & $-58.8(4)$ \\
\hline $\mathrm{C} 1-\mathrm{C} 2-\mathrm{C} 3-\mathrm{C} 4$ & $-1.1(7)$ & Co1-C20-C21-C22 & $-58.3(4)$ \\
\hline $\mathrm{C} 1-\mathrm{C} 2-\mathrm{C} 3-\mathrm{C} 11$ & $-179.6(4)$ & Co1-C21-C22-C18 & $-58.8(4)$ \\
\hline $\mathrm{C} 2-\mathrm{C} 3-\mathrm{C} 4-\mathrm{C} 5$ & $1.0(6)$ & C13-C14-C15-Co1 & $-59.2(4)$ \\
\hline C2-C3-C11-O1 & $177.5(4)$ & C13-C14-C15-C16 & $0.0(7)$ \\
\hline $\mathrm{C} 2-\mathrm{C} 3-\mathrm{C} 11-\mathrm{O} 2$ & $0.0(6)$ & C14-C13-C17-Co1 & $59.2(4)$ \\
\hline C3-C4-C5-N1 & $-0.2(6)$ & C14-C13-C17-C16 & $0.7(7)$ \\
\hline C3-C4-C5-C6 & $-178.0(4)$ & $\mathrm{C} 14-\mathrm{C} 15-\mathrm{C} 16-\mathrm{Co} 1$ & $-59.2(4)$ \\
\hline C4-C3-C11-O1 & $-1.0(6)$ & C14-C15-C16-C17 & $0.4(7)$ \\
\hline C4-C3-C11-O2 & $-178.5(4)$ & C15-C16-C17-Co1 & $-59.2(4)$ \\
\hline C4-C5-C6-N2 & $-177.8(4)$ & C15-C16-C17-C13 & $-0.7(7)$ \\
\hline C4-C5-C6-C7 & $4.8(6)$ & C17-C13-C14-Co1 & $-59.8(5)$ \\
\hline C5-N1-C1-C2 & $0.4(7)$ & C17-C13-C14-C15 & $-0.4(7)$ \\
\hline C5-C6-C7-C8 & 177.1(4) & C18-C19-C20-Co1 & $59.8(4)$ \\
\hline C6-N2-C10-C9 & $-0.5(6)$ & C18-C19-C20-C21 & $1.0(7)$ \\
\hline C6-C7-C8-C9 & $0.0(6)$ & C19-C18-C22-Co1 & $-59.0(4)$ \\
\hline C6-C7-C8-C12 & $-177.8(4)$ & C19-C18-C22-C21 & $0.5(7)$ \\
\hline C7-C8-C9-C10 & $-0.1(6)$ & C19-C20-C21-Co1 & $57.6(4)$ \\
\hline C7-C8-C12-O3 & $177.2(4)$ & C19-C20-C21-C22 & $-0.7(7)$ \\
\hline C7-C8-C12-O4 & $-0.9(5)$ & $\mathrm{C} 20-\mathrm{C} 21-\mathrm{C} 22-\mathrm{Co} 1$ & $58.9(4)$ \\
\hline C8-C9-C10-N2 & $0.3(6)$ & C20-C21-C22-C18 & $0.1(7)$ \\
\hline C9-C8-C12-O3 & $-0.6(6)$ & C22-C18-C19-Co1 & $59.7(4)$ \\
\hline C9-C8-C12-O4 & $-178.7(4)$ & C22-C18-C19-C20 & $-1.0(7)$ \\
\hline
\end{tabular}


Table 7. Hydrogen bonds and close contacts for rhowe 38 [ $\AA$ and $\left.{ }^{\circ}\right]$.

\begin{tabular}{lcccc}
\hline D-H...A & d(D-H) & $d(H \ldots A)$ & $d(D \ldots A)$ & $<($ DHA $)$ \\
\hline O1-H1...O2\#1 & 0.82 & 1.84 & $2.639(4)$ & 165.2 \\
O2-H2...O1\#1 & 0.82 & 1.85 & $2.639(4)$ & 160.1 \\
O4-H4...Cl1\#2 & 0.82 & 2.29 & $3.052(3)$ & 155.6 \\
\hline
\end{tabular}

Symmetry transformations used to generate equivalent atoms:

$\# 1-\mathrm{x}+2,-\mathrm{y}+1,-\mathrm{z}+1 \quad \# 2-\mathrm{x},-\mathrm{y},-\mathrm{z}+1$ 
REFERENCE NUMBER: rhowe19

CRYSTAL STRUCTURE REPORT

$\mathrm{C}_{24} \mathrm{H}_{23} \mathrm{Cl}_{2} \mathrm{Mo}_{2} \mathrm{~N}_{6} \mathrm{O}_{4.5}$

or

$[\mathrm{Mo}(\text { bpy })(\mathrm{Cl})(\mathrm{O})(\mu-\mathrm{O})]_{2} \cdot 2 \mathrm{CH}_{3} \mathrm{CN} \cdot 1 / 2 \mathrm{H}_{2} \mathrm{O}$

Report prepared for:

Prof. W. Eckenhoff

June 02,2018

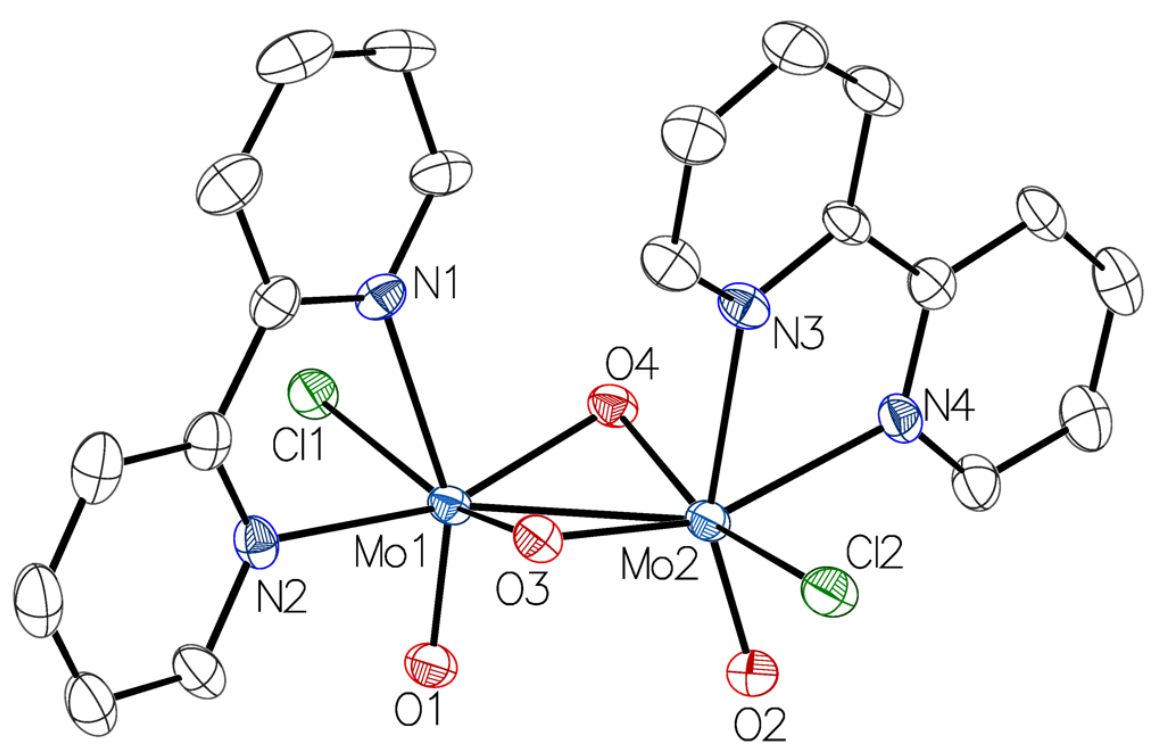

William W. Brennessel

X-ray Crystallographic Facility

Department of Chemistry, University of Rochester

120 Trustee Road 
Rochester, NY 14627 


\section{Data collection}

A crystal $\left(0.23 \times 0.153 \times 0.102 \mathrm{~mm}^{3}\right)$ was placed onto a thin glass optical fiber or a nylon loop and mounted on a XtaLab Synergy-S Dualflex diffractometer equipped with a HyPix-6000HE HPC area detector for data collection at 100.00(10) K. A preliminary set of cell constants and an orientation matrix were calculated from a small sampling of reflections. ${ }^{1}$ A short pre-experiment was run, from which an optimal data collection strategy was determined. The full data collection was carried out using a PhotonJet $(\mathrm{Cu})$ X-ray Source with frame times of 0.05 and 0.09 seconds and a detector distance of $31.2 \mathrm{~mm}$. Series of frames were collected in $0.50^{\circ}$ steps in $\omega$ at different $2 \theta, \kappa$, and $\phi$ settings. After the intensity data were corrected for absorption, the final cell constants were calculated from the xyz centroids of 27648 strong reflections from the actual data collection after integration. ${ }^{1}$ See Table 1 for additional crystal and refinement information.

\section{Structure solution and refinement}

The structure was solved using ShelXT ${ }^{2}$ and refined using ShelXL. ${ }^{3}$ The space group $P-1$ was determined based on intensity statistics. Most or all non-hydrogen atoms were assigned from the solution. Full-matrix least squares / difference Fourier cycles were performed which located any remaining non-hydrogen atoms. All non-hydrogen atoms were refined with anisotropic displacement parameters. The hydrogen atoms on the water molecule were placed in positions reasonable for hydrogen bonding and then given a riding model. All other hydrogen atoms were placed in ideal positions and refined as riding atoms with relative isotropic displacement parameters.

The intensity data were scaled and reduced ${ }^{1}$ according to non-merohedral twin law [ $10.012-0.159 / 0$ / 0 - 0 / 0 o -1 ], a 180 degree rotation around reciprocal lattice [100]. There were 8322 unique isolated reflections in the first component, 8309 unique isolated reflections in the second component, and 4435 unique overlapping reflections. Without twin modeling, $R 1$ (strong data) refined to 0.119 . The mass ratio of components refined to 0.5140(7):0.4860(7).

The final full matrix least squares refinement converged to $R 1=0.0474\left(F^{2}, I>2 \sigma(I)\right)$ and $w R 2=0.1396\left(F^{2}\right.$, all data).

\section{Structure description}

The structure is similar to the one suggested. The asymmetric unit contains two independent dinuclear Mo complexes, four independent cocrystallized acetonitrile solvent molecules, and one cocrystallized water solvent molecule, all in general positions.

Structure manipulation and figure generation were performed using Olex2. ${ }^{4}$ Unless noted otherwise all structural diagrams containing thermal displacement ellipsoids are drawn at the $50 \%$ probability level. 
Data collection, structure solution, and structure refinement were conducted at the X-ray Crystallographic Facility, B04 Hutchison Hall, Department of Chemistry, University of Rochester. The instrument was purchased with funding from NSF MRI program grant CHE-1725028. All publications arising from this report MUST either 1) include William W. Brennessel as a coauthor or 2) acknowledge William W. Brennessel and the X-ray Crystallographic Facility of the Department of Chemistry at the University of Rochester.

1 CrysAlisPro, version 171.39.46; Rigaku Corporation: Oxford, UK, 2018.

2 Sheldrick, G. M. SHELXT, version 2018/2; Acta. Crystallogr. 2015, A71, 3-8.

3 Sheldrick, G. M. SHELXL, version 2018/3; Acta. Crystallogr. 2015, C71, 3-8.

4 Dolomanov, O. V.; Bourhis, L. J.; Gildea, R. J.; Howard, J. A. K.; Puschmann, H. Olex2, version 1.2-ac3; J. Appl. Cryst. 2009, 42, 339-341.

Some equations of interest:

$$
\begin{gathered}
R_{\mathrm{int}}=\Sigma\left|F_{\mathrm{o}}{ }^{2}-<F_{\mathrm{o}}{ }^{2}>\right| / \Sigma\left|F_{\mathrm{o}}{ }^{2}\right| \\
R 1=\Sigma|| F_{\mathrm{o}}|-| F_{\mathrm{c}}|/ \Sigma| F_{\mathrm{o}} \mid \\
w R 2=\left[\Sigma\left[w\left(F_{\mathrm{o}}{ }^{2} F_{\mathrm{c}}{ }^{2}\right)^{2}\right] / \Sigma\left[w\left(F_{\mathrm{o}}{ }^{2}\right)^{2}\right]\right]^{1 / 2} \\
\text { where } w=1 /\left[\sigma^{2}\left(F_{\mathrm{o}}{ }^{2}\right)+(a P)^{2}+b P\right] \text { and } \\
P=1 / 3 \max \left(0, F_{\mathrm{o}}{ }^{2}\right)+2 / 3 F_{\mathrm{c}}{ }^{2} \\
\mathrm{GOF}=S=\left[\Sigma\left[w\left(F_{\mathrm{o}}{ }^{2}-F_{\mathrm{c}}{ }^{2}\right)^{2}\right] /(m-n)\right]^{1 / 2}
\end{gathered}
$$

where $m=$ number of reflections and $n=$ number of parameters 


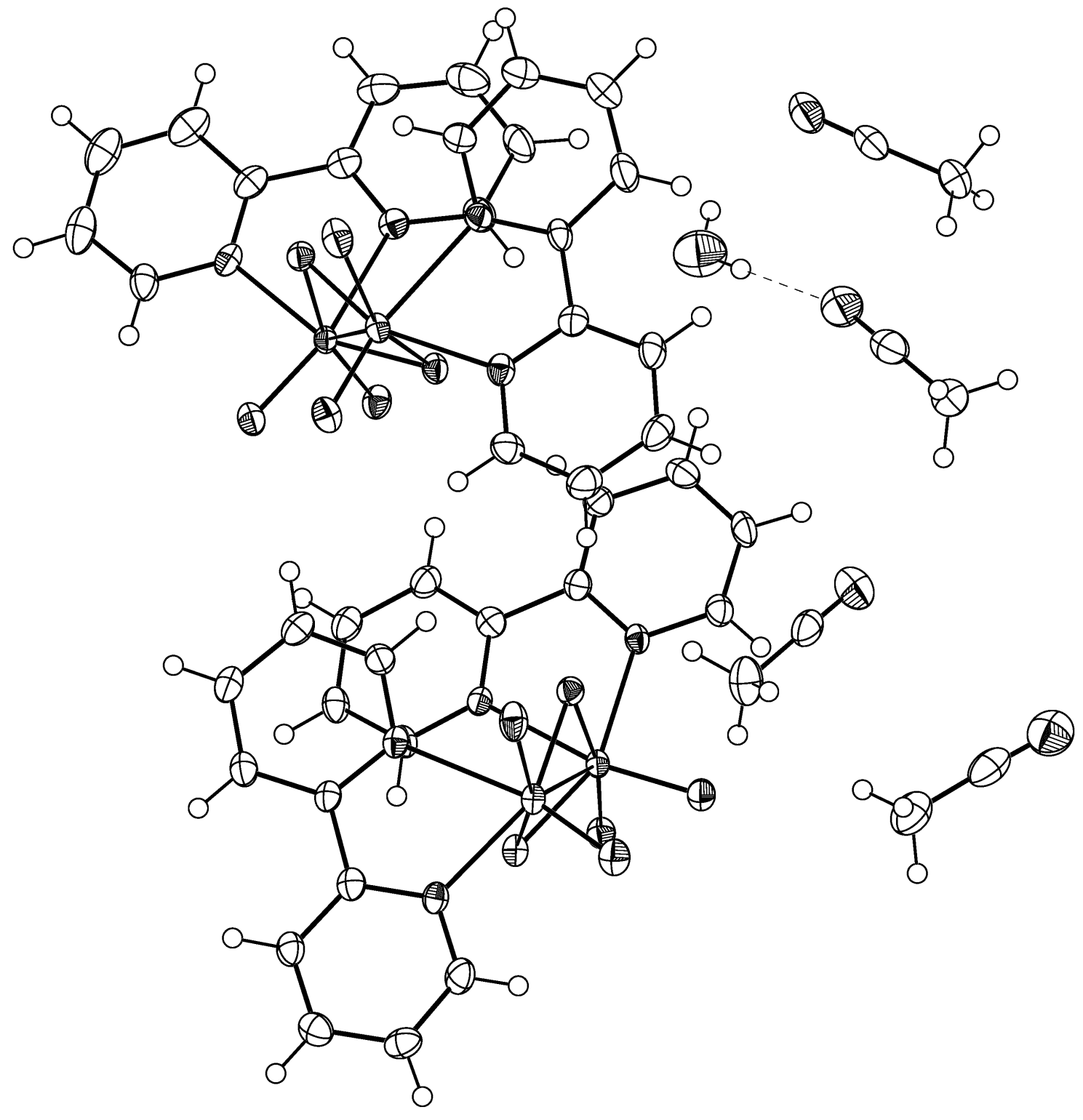



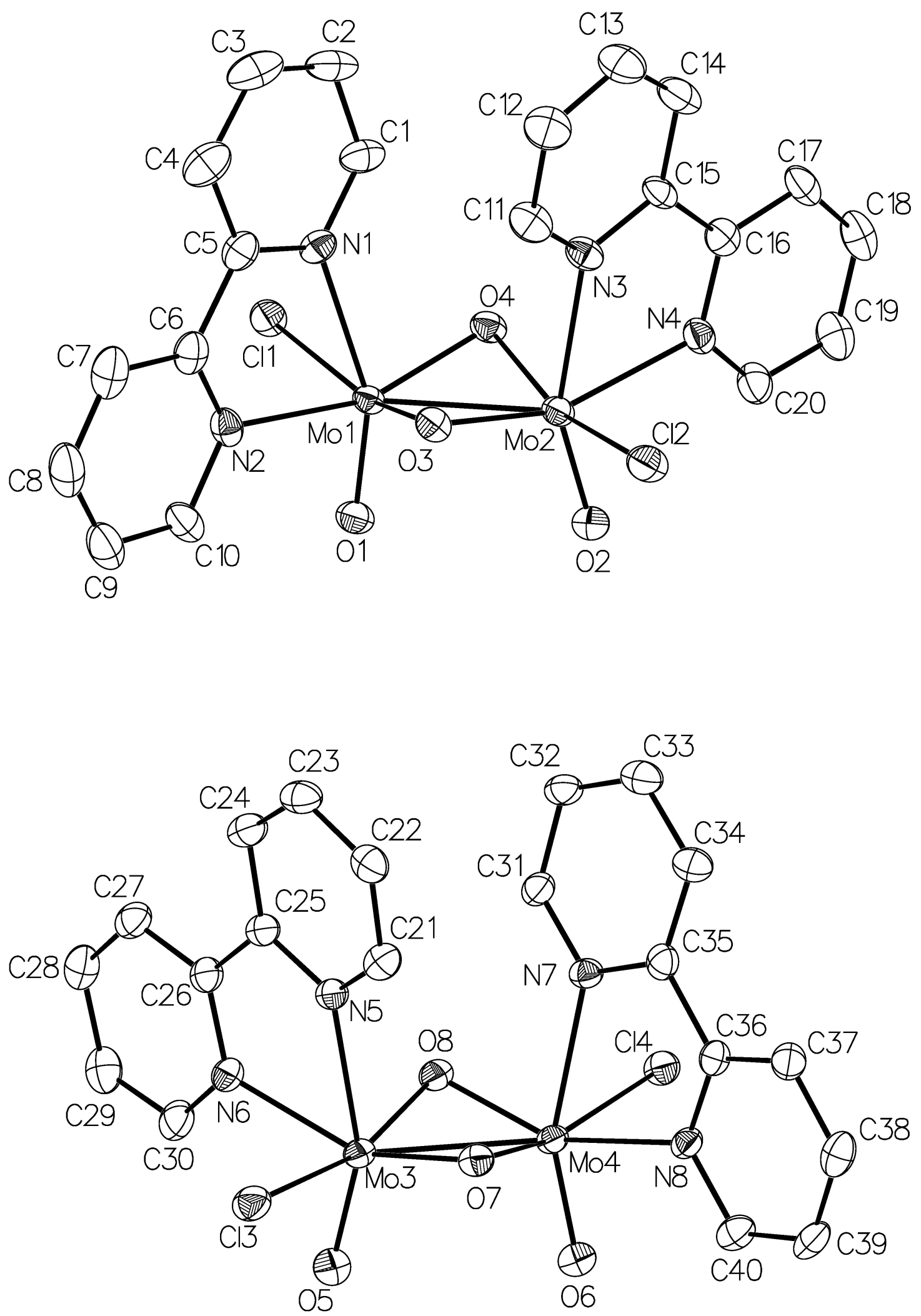

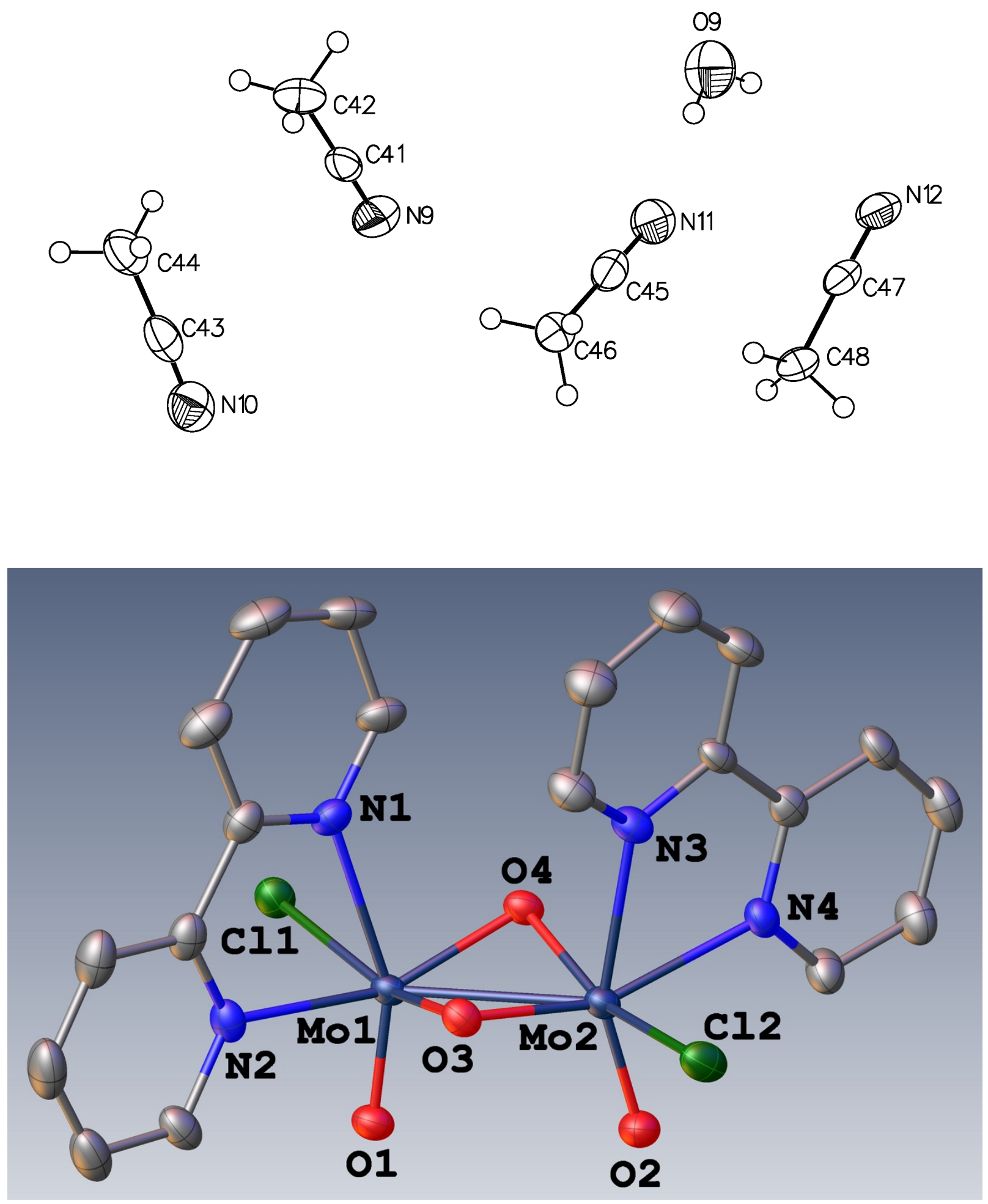
Table 1. Crystal data and structure refinement for rhowe19.

\begin{tabular}{|c|c|c|}
\hline Identification code & \multicolumn{2}{|l|}{ rhowe19 } \\
\hline Empirical formula & \multicolumn{2}{|c|}{$\mathrm{C} 24 \mathrm{H} 23 \mathrm{Cl} 2 \mathrm{Mo} 2 \mathrm{~N} 6 \mathrm{O} 4.50$} \\
\hline Formula weight & \multicolumn{2}{|l|}{730.26} \\
\hline Temperature & \multicolumn{2}{|l|}{$100.00(10) \mathrm{K}$} \\
\hline Wavelength & \multicolumn{2}{|l|}{$1.54184 \AA$} \\
\hline Crystal system & \multicolumn{2}{|l|}{ triclinic } \\
\hline Space group & \multicolumn{2}{|l|}{$P-1$} \\
\hline \multirow[t]{3}{*}{ Unit cell dimensions } & $a=13.1944(3) \AA$ & $\alpha=82.5899(8)^{\circ}$ \\
\hline & $b=13.48132(15) \AA$ & $\beta=84.6913(15)^{\circ}$ \\
\hline & $c=15.60517(11) \AA$ & $\gamma=89.6109(15)^{\circ}$ \\
\hline Volume & \multicolumn{2}{|l|}{$2740.79(8) \AA^{3}$} \\
\hline$Z$ & \multicolumn{2}{|l|}{4} \\
\hline Density (calculated) & \multicolumn{2}{|l|}{$1.770 \mathrm{Mg} / \mathrm{m}^{3}$} \\
\hline Absorption coefficient & \multicolumn{2}{|l|}{$9.670 \mathrm{~mm}^{-1}$} \\
\hline$F(000)$ & \multicolumn{2}{|l|}{1452} \\
\hline Crystal color, morphology & \multicolumn{2}{|l|}{ orange, needle } \\
\hline Crystal size & \multicolumn{2}{|c|}{$0.23 \times 0.153 \times 0.102 \mathrm{~mm}^{3}$} \\
\hline Theta range for data collection & \multicolumn{2}{|l|}{2.868 to $77.997^{\circ}$} \\
\hline Index ranges & \multicolumn{2}{|c|}{$-15 \leq h \leq 16,-17 \leq k \leq 17,-19 \leq l \leq 19$} \\
\hline Reflections collected & \multicolumn{2}{|l|}{21062} \\
\hline Independent reflections & \multicolumn{2}{|l|}{$21062[R(\mathrm{int})=?]$} \\
\hline Observed reflections & \multicolumn{2}{|l|}{19791} \\
\hline Completeness to theta $=74.504^{\circ}$ & \multicolumn{2}{|l|}{$99.7 \%$} \\
\hline Absorption correction & \multicolumn{2}{|l|}{ Multi-scan } \\
\hline Max. and min. transmission & \multicolumn{2}{|l|}{1.00000 and 0.23588} \\
\hline Refinement method & \multicolumn{2}{|c|}{ Full-matrix least-squares on $F^{2}$} \\
\hline Data / restraints / parameters & \multicolumn{2}{|l|}{21062 / 0 / 699} \\
\hline Goodness-of-fit on $F^{2}$ & \multicolumn{2}{|l|}{1.030} \\
\hline Final $R$ indices $[I>2 \operatorname{sigma}(I)]$ & \multicolumn{2}{|c|}{$R 1=0.0474, w R 2=0.1367$} \\
\hline$R$ indices (all data) & \multicolumn{2}{|c|}{$R 1=0.0495, w R 2=0.1396$} \\
\hline Largest diff. peak and hole & \multicolumn{2}{|l|}{1.063 and -1.434 e. $\AA^{-3}$} \\
\hline
\end{tabular}


Table 2. Atomic coordinates $\left(\times 10^{4}\right)$ and equivalent isotropic displacement parameters $\left(\AA^{2} \times 10^{3}\right)$ for rhowe19. $U_{e q}$ is defined as one third of the trace of the orthogonalized $U_{i j}$ tensor.

\begin{tabular}{|c|c|c|c|c|}
\hline & $\mathrm{x}$ & $\mathrm{y}$ & $\mathrm{z}$ & $\mathrm{U}_{\mathrm{eq}}$ \\
\hline Mo1 & $8448(1)$ & 6704(1) & 6293(1) & $17(1)$ \\
\hline Mo2 & $7659(1)$ & 8361(1) & $5708(1)$ & $18(1)$ \\
\hline Mo3 & $7307(1)$ & 8373(1) & 592(1) & $19(1)$ \\
\hline Mo4 & 6613(1) & 6716(1) & 1417(1) & $17(1)$ \\
\hline $\mathrm{Cl1}$ & $8210(1)$ & 4926(1) & $6196(1)$ & $24(1)$ \\
\hline $\mathrm{Cl} 2$ & 7693(1) & $10073(1)$ & $6090(1)$ & $29(1)$ \\
\hline $\mathrm{Cl} 3$ & 7133(1) & $10167(1)$ & 774(1) & $27(1)$ \\
\hline $\mathrm{Cl} 4$ & $6947(1)$ & 4906(1) & $1358(1)$ & $22(1)$ \\
\hline $\mathrm{O} 1$ & $9535(2)$ & $6748(2)$ & $5621(2)$ & $24(1)$ \\
\hline $\mathrm{O} 2$ & $8480(2)$ & $8680(2)$ & $4813(2)$ & $26(1)$ \\
\hline $\mathrm{O} 3$ & $8358(2)$ & 7996(2) & $6727(2)$ & $21(1)$ \\
\hline $\mathrm{O} 4$ & $7254(2)$ & 6972(2) & $5680(2)$ & $21(1)$ \\
\hline O5 & $6538(3)$ & $8536(3)$ & $-224(2)$ & $28(1)$ \\
\hline O6 & $5529(2)$ & 6599(2) & $934(2)$ & $24(1)$ \\
\hline $\mathrm{O} 7$ & $6579(2)$ & 8079(2) & $1723(2)$ & $20(1)$ \\
\hline $\mathrm{O} 8$ & $7795(2)$ & 6997(2) & 601(2) & $21(1)$ \\
\hline N1 & $7355(3)$ & $6200(3)$ & $7516(2)$ & $22(1)$ \\
\hline N2 & $9351(3)$ & 6351(3) & $7432(3)$ & $22(1)$ \\
\hline N3 & $6164(3)$ & 8399(3) & $6617(2)$ & $21(1)$ \\
\hline N4 & $6342(3)$ & 8689(3) & $4914(3)$ & $21(1)$ \\
\hline N5 & $8689(3)$ & 8599(3) & $1369(2)$ & $19(1)$ \\
\hline N6 & $8699(3)$ & 8670(3) & $-324(2)$ & $21(1)$ \\
\hline N7 & $7807(3)$ & 6369(3) & $2420(2)$ & $18(1)$ \\
\hline N8 & $5813(3)$ & $6367(3)$ & $2734(2)$ & $18(1)$ \\
\hline $\mathrm{C} 1$ & $6362(4)$ & 6043(4) & $7492(3)$ & $28(1)$ \\
\hline $\mathrm{C} 2$ & $5718(4)$ & 5762(4) & $8238(4)$ & $34(1)$ \\
\hline $\mathrm{C} 3$ & $6119(5)$ & $5659(4)$ & $9024(4)$ & $39(1)$ \\
\hline $\mathrm{C} 4$ & $7159(4)$ & 5801(4) & $9058(3)$ & $34(1)$ \\
\hline $\mathrm{C} 5$ & $7755(4)$ & $6066(3)$ & $8284(3)$ & $24(1)$ \\
\hline C6 & $8872(4)$ & $6176(3)$ & $8237(3)$ & $25(1)$ \\
\hline $\mathrm{C} 7$ & $9424(5)$ & $6074(4)$ & $8965(4)$ & $35(1)$ \\
\hline
\end{tabular}




\begin{tabular}{|c|c|c|c|c|}
\hline $\mathrm{C} 8$ & $10471(5)$ & 6164(4) & $8854(4)$ & $40(1)$ \\
\hline C9 & $10952(4)$ & $6363(4)$ & $8027(4)$ & $36(1)$ \\
\hline $\mathrm{C} 10$ & $10373(4)$ & $6452(4)$ & $7335(3)$ & $28(1)$ \\
\hline $\mathrm{C} 11$ & $6143(4)$ & $8370(4)$ & $7473(3)$ & $28(1)$ \\
\hline $\mathrm{C} 12$ & $5244(4)$ & $8391(4)$ & $8004(3)$ & $33(1)$ \\
\hline $\mathrm{C} 13$ & $4338(4)$ & $8443(4)$ & 7635(4) & $35(1)$ \\
\hline $\mathrm{C} 14$ & $4345(4)$ & $8481(4)$ & $6746(4)$ & $32(1)$ \\
\hline $\mathrm{C} 15$ & $5279(3)$ & $8476(3)$ & $6245(3)$ & $21(1)$ \\
\hline $\mathrm{C} 16$ & $5373(3)$ & $8611(3)$ & $5293(3)$ & $22(1)$ \\
\hline $\mathrm{C} 17$ & $4550(4)$ & $8696(3)$ & $4795(3)$ & $26(1)$ \\
\hline $\mathrm{C} 18$ & $4722(4)$ & $8843(4)$ & $3899(3)$ & $31(1)$ \\
\hline C19 & $5707(4)$ & 8923(4) & $3512(3)$ & $32(1)$ \\
\hline $\mathrm{C} 20$ & 6493(4) & $8842(4)$ & $4047(3)$ & $27(1)$ \\
\hline $\mathrm{C} 21$ & $8615(3)$ & $8641(3)$ & $2228(3)$ & $24(1)$ \\
\hline $\mathrm{C} 22$ & $9456(4)$ & $8707(3)$ & $2684(3)$ & $25(1)$ \\
\hline $\mathrm{C} 23$ & 10414(3) & $8749(3)$ & $2240(3)$ & $26(1)$ \\
\hline $\mathrm{C} 24$ & $10502(3)$ & $8727(3)$ & $1355(3)$ & $25(1)$ \\
\hline $\mathrm{C} 25$ & $9625(3)$ & $8666(3)$ & $930(3)$ & $19(1)$ \\
\hline $\mathrm{C} 26$ & $9624(3)$ & $8681(3)$ & $-16(3)$ & $21(1)$ \\
\hline $\mathrm{C} 27$ & $10513(3)$ & $8740(3)$ & $-573(3)$ & $24(1)$ \\
\hline $\mathrm{C} 28$ & 10461(4) & $8770(4)$ & $-1454(3)$ & $28(1)$ \\
\hline $\mathrm{C} 29$ & $9506(4)$ & $8754(4)$ & $-1767(3)$ & $28(1)$ \\
\hline $\mathrm{C} 30$ & $8653(4)$ & $8707(4)$ & $-1184(3)$ & $28(1)$ \\
\hline $\mathrm{C} 31$ & $8807(3)$ & $6276(3)$ & 2196(3) & $21(1)$ \\
\hline $\mathrm{C} 32$ & $9501(3)$ & $6010(3)$ & 2804(3) & $24(1)$ \\
\hline $\mathrm{C} 33$ & $9146(4)$ & $5827(4)$ & $3670(3)$ & $26(1)$ \\
\hline C34 & $8106(4)$ & $5914(4)$ & $3908(3)$ & $25(1)$ \\
\hline $\mathrm{C} 35$ & $7455(3)$ & $6189(3)$ & $3263(3)$ & $21(1)$ \\
\hline $\mathrm{C} 36$ & $6339(3)$ & $6254(3)$ & $3445(3)$ & $20(1)$ \\
\hline $\mathrm{C} 37$ & $5859(3)$ & $6178(3)$ & $4279(3)$ & $23(1)$ \\
\hline $\mathrm{C} 38$ & $4801(4)$ & $6187(4)$ & 4393(3) & $27(1)$ \\
\hline C39 & $4263(3)$ & $6274(3)$ & $3667(3)$ & $26(1)$ \\
\hline $\mathrm{C} 40$ & 4784(3) & $6372(3)$ & $2854(3)$ & $24(1)$ \\
\hline O9 & $3946(4)$ & $6104(4)$ & $6624(3)$ & $59(1)$ \\
\hline N9 & 2995(4) & $8272(5)$ & 2122(4) & $57(2)$ \\
\hline $\mathrm{C} 41$ & $3616(4)$ & $8680(4)$ & $1665(4)$ & $36(1)$ \\
\hline
\end{tabular}




$\begin{array}{lrrrr}\mathrm{C} 42 & 4433(4) & 9184(4) & 1069(4) & 41(1) \\ \mathrm{N} 10 & 1350(5) & 6458(4) & 902(4) & 51(1) \\ \mathrm{C} 43 & 2139(5) & 6687(4) & 598(4) & 40(1) \\ \mathrm{C} 44 & 3141(5) & 6989(5) & 177(4) & 45(1) \\ \mathrm{N} 11 & 2401(4) & 6956(4) & 5489(3) & 46(1) \\ \mathrm{C} 45 & 1956(4) & 7175(4) & 4903(4) & 35(1) \\ \mathrm{C} 46 & 1394(4) & 7465(4) & 4157(3) & 31(1) \\ \mathrm{N} 12 & 2017(4) & 9127(4) & 6749(3) & 44(1) \\ \mathrm{C} 47 & 1369(4) & 9114(4) & 6320(3) & 31(1) \\ \mathrm{C} 48 & 522(4) & 9109(4) & 5781(3) & 31(1) \\ \end{array}$


Table 3. Bond lengths $[\AA]$ and angles $\left[{ }^{\circ}\right]$ for rhowe 19 .

\begin{tabular}{|c|c|c|c|}
\hline $\operatorname{Mo}(1)-\operatorname{Mo}(2)$ & $2.5548(5)$ & $\mathrm{N}(5)-\mathrm{C}(21)$ & $1.343(6)$ \\
\hline $\operatorname{Mo}(1)-\mathrm{Cl}(1)$ & $2.4459(10)$ & $\mathrm{N}(5)-\mathrm{C}(25)$ & $1.352(6)$ \\
\hline $\operatorname{Mo}(1)-\mathrm{O}(1)$ & $1.694(3)$ & $\mathrm{N}(6)-\mathrm{C}(26)$ & $1.354(6)$ \\
\hline $\mathrm{Mo}(1)-\mathrm{O}(3)$ & $1.947(3)$ & $\mathrm{N}(6)-\mathrm{C}(30)$ & $1.344(6)$ \\
\hline $\mathrm{Mo}(1)-\mathrm{O}(4)$ & $1.926(3)$ & $\mathrm{N}(7)-\mathrm{C}(31)$ & $1.343(6)$ \\
\hline $\operatorname{Mo}(1)-\mathrm{N}(1)$ & $2.313(4)$ & $\mathrm{N}(7)-\mathrm{C}(35)$ & $1.347(6)$ \\
\hline $\operatorname{Mo}(1)-\mathrm{N}(2)$ & $2.230(4)$ & $\mathrm{N}(8)-\mathrm{C}(36)$ & $1.356(6)$ \\
\hline $\operatorname{Mo}(2)-\mathrm{Cl}(2)$ & $2.4587(11)$ & $\mathrm{N}(8)-\mathrm{C}(40)$ & $1.354(6)$ \\
\hline $\mathrm{Mo}(2)-\mathrm{O}(2)$ & $1.697(3)$ & $\mathrm{C}(1)-\mathrm{H}(1)$ & 0.9300 \\
\hline $\mathrm{Mo}(2)-\mathrm{O}(3)$ & $1.920(3)$ & $C(1)-C(2)$ & $1.389(7)$ \\
\hline $\operatorname{Mo}(2)-\mathrm{O}(4)$ & $1.958(3)$ & $\mathrm{C}(2)-\mathrm{H}(2)$ & 0.9300 \\
\hline $\mathrm{Mo}(2)-\mathrm{N}(3)$ & $2.326(4)$ & $C(2)-C(3)$ & $1.371(8)$ \\
\hline $\mathrm{Mo}(2)-\mathrm{N}(4)$ & $2.234(4)$ & $\mathrm{C}(3)-\mathrm{H}(3)$ & 0.9300 \\
\hline $\operatorname{Mo}(3)-\mathrm{Mo}(4)$ & $2.5598(4)$ & $C(3)-C(4)$ & $1.394(8)$ \\
\hline $\operatorname{Mo}(3)-\mathrm{Cl}(3)$ & $2.4788(11)$ & $\mathrm{C}(4)-\mathrm{H}(4)$ & 0.9300 \\
\hline $\mathrm{Mo}(3)-\mathrm{O}(5)$ & $1.692(3)$ & $C(4)-C(5)$ & $1.386(7)$ \\
\hline $\mathrm{Mo}(3)-\mathrm{O}(7)$ & $1.925(3)$ & $C(5)-C(6)$ & $1.476(7)$ \\
\hline $\mathrm{Mo}(3)-\mathrm{O}(8)$ & $1.957(3)$ & $C(6)-C(7)$ & $1.397(7)$ \\
\hline $\mathrm{Mo}(3)-\mathrm{N}(5)$ & $2.325(4)$ & $\mathrm{C}(7)-\mathrm{H}(7)$ & 0.9300 \\
\hline $\operatorname{Mo}(3)-\mathrm{N}(6)$ & $2.225(4)$ & $\mathrm{C}(7)-\mathrm{C}(8)$ & $1.381(8)$ \\
\hline $\mathrm{Mo}(4)-\mathrm{Cl}(4)$ & $2.4886(10)$ & $\mathrm{C}(8)-\mathrm{H}(8)$ & 0.9300 \\
\hline $\mathrm{Mo}(4)-\mathrm{O}(6)$ & 1.694(3) & $\mathrm{C}(8)-\mathrm{C}(9)$ & $1.380(9)$ \\
\hline $\mathrm{Mo}(4)-\mathrm{O}(7)$ & $1.956(3)$ & $\mathrm{C}(9)-\mathrm{H}(9)$ & 0.9300 \\
\hline $\mathrm{Mo}(4)-\mathrm{O}(8)$ & 1.927(3) & $\mathrm{C}(9)-\mathrm{C}(10)$ & $1.372(7)$ \\
\hline $\mathrm{Mo}(4)-\mathrm{N}(7)$ & $2.325(4)$ & $\mathrm{C}(10)-\mathrm{H}(10)$ & 0.9300 \\
\hline Mo(4)-N(8) & $2.217(4)$ & $\mathrm{C}(11)-\mathrm{H}(11)$ & 0.9300 \\
\hline $\mathrm{N}(1)-\mathrm{C}(1)$ & $1.333(6)$ & $\mathrm{C}(11)-\mathrm{C}(12)$ & $1.385(7)$ \\
\hline $\mathrm{N}(1)-\mathrm{C}(5)$ & $1.346(6)$ & $\mathrm{C}(12)-\mathrm{H}(12)$ & 0.9300 \\
\hline $\mathrm{N}(2)-\mathrm{C}(6)$ & $1.347(6)$ & $\mathrm{C}(12)-\mathrm{C}(13)$ & $1.372(8)$ \\
\hline $\mathrm{N}(2)-\mathrm{C}(10)$ & $1.349(6)$ & $\mathrm{C}(13)-\mathrm{H}(13)$ & 0.9300 \\
\hline $\mathrm{N}(3)-\mathrm{C}(11)$ & $1.329(6)$ & $\mathrm{C}(13)-\mathrm{C}(14)$ & $1.382(8)$ \\
\hline $\mathrm{N}(3)-\mathrm{C}(15)$ & $1.349(6)$ & $\mathrm{C}(14)-\mathrm{H}(14)$ & 0.9300 \\
\hline $\mathrm{N}(4)-\mathrm{C}(16)$ & $1.357(6)$ & $\mathrm{C}(14)-\mathrm{C}(15)$ & $1.396(6)$ \\
\hline $\mathrm{N}(4)-\mathrm{C}(20)$ & $1.339(6)$ & $C(15)-C(16)$ & $1.467(6)$ \\
\hline
\end{tabular}




\begin{tabular}{|c|c|c|c|}
\hline $\mathrm{C}(16)-\mathrm{C}(17)$ & $1.389(6)$ & $\mathrm{C}(37)-\mathrm{C}(38)$ & $1.391(7)$ \\
\hline C(17)-H(17) & 0.9300 & $\mathrm{C}(38)-\mathrm{H}(38)$ & 0.9300 \\
\hline C(17)-C(18) & $1.385(7)$ & $\mathrm{C}(38)-\mathrm{C}(39)$ & $1.383(7)$ \\
\hline C(18)-H(18) & 0.9300 & $\mathrm{C}(39)-\mathrm{H}(39)$ & 0.9300 \\
\hline C(18)-C(19) & $1.381(8)$ & $\mathrm{C}(39)-\mathrm{C}(40)$ & $1.377(7)$ \\
\hline C(19)-H(19) & 0.9300 & $\mathrm{C}(40)-\mathrm{H}(40)$ & 0.9300 \\
\hline C(19)-C(20) & $1.385(7)$ & $\mathrm{O}(9)-\mathrm{H}(9 \mathrm{~A})$ & 0.8500 \\
\hline C(20)-H(20) & 0.9300 & $\mathrm{O}(9)-\mathrm{H}(9 \mathrm{~B})$ & 0.8500 \\
\hline $\mathrm{C}(21)-\mathrm{H}(21)$ & 0.9300 & $\mathrm{~N}(9)-\mathrm{C}(41)$ & $1.132(8)$ \\
\hline$C(21)-C(22)$ & $1.382(6)$ & $\mathrm{C}(41)-\mathrm{C}(42)$ & $1.466(8)$ \\
\hline $\mathrm{C}(22)-\mathrm{H}(22)$ & 0.9300 & $\mathrm{C}(42)-\mathrm{H}(42 \mathrm{~A})$ & 0.9600 \\
\hline $\mathrm{C}(22)-\mathrm{C}(23)$ & $1.381(7)$ & $\mathrm{C}(42)-\mathrm{H}(42 \mathrm{~B})$ & 0.9600 \\
\hline $\mathrm{C}(23)-\mathrm{H}(23)$ & 0.9300 & $\mathrm{C}(42)-\mathrm{H}(42 \mathrm{C})$ & 0.9600 \\
\hline$C(23)-C(24)$ & $1.380(7)$ & $\mathrm{N}(10)-\mathrm{C}(43)$ & $1.131(9)$ \\
\hline $\mathrm{C}(24)-\mathrm{H}(24)$ & 0.9300 & $\mathrm{C}(43)-\mathrm{C}(44)$ & $1.457(9)$ \\
\hline$C(24)-C(25)$ & $1.393(6)$ & $\mathrm{C}(44)-\mathrm{H}(44 \mathrm{~A})$ & 0.9600 \\
\hline$C(25)-C(26)$ & $1.475(6)$ & $\mathrm{C}(44)-\mathrm{H}(44 \mathrm{~B})$ & 0.9600 \\
\hline$C(26)-C(27)$ & $1.389(6)$ & $\mathrm{C}(44)-\mathrm{H}(44 \mathrm{C})$ & 0.9600 \\
\hline C(27)-H(27) & 0.9300 & $\mathrm{~N}(11)-\mathrm{C}(45)$ & $1.138(8)$ \\
\hline $\mathrm{C}(27)-\mathrm{C}(28)$ & $1.378(7)$ & $\mathrm{C}(45)-\mathrm{C}(46)$ & $1.448(8)$ \\
\hline $\mathrm{C}(28)-\mathrm{H}(28)$ & 0.9300 & $\mathrm{C}(46)-\mathrm{H}(46 \mathrm{~A})$ & 0.9600 \\
\hline$C(28)-C(29)$ & $1.395(7)$ & $\mathrm{C}(46)-\mathrm{H}(46 \mathrm{~B})$ & 0.9600 \\
\hline C(29)-H(29) & 0.9300 & $\mathrm{C}(46)-\mathrm{H}(46 \mathrm{C})$ & 0.9600 \\
\hline$C(29)-C(30)$ & $1.376(7)$ & $\mathrm{N}(12)-\mathrm{C}(47)$ & $1.136(7)$ \\
\hline $\mathrm{C}(30)-\mathrm{H}(30)$ & 0.9300 & $\mathrm{C}(47)-\mathrm{C}(48)$ & $1.460(7)$ \\
\hline $\mathrm{C}(31)-\mathrm{H}(31)$ & 0.9300 & $\mathrm{C}(48)-\mathrm{H}(48 \mathrm{~A})$ & 0.9600 \\
\hline$C(31)-C(32)$ & $1.391(6)$ & $\mathrm{C}(48)-\mathrm{H}(48 \mathrm{~B})$ & 0.9600 \\
\hline $\mathrm{C}(32)-\mathrm{H}(32)$ & 0.9300 & $\mathrm{C}(48)-\mathrm{H}(48 \mathrm{C})$ & 0.9600 \\
\hline$C(32)-C(33)$ & $1.381(7)$ & $\mathrm{Cl}(1)-\mathrm{Mo}(1)-\mathrm{Mo}(2)$ & $137.39(3)$ \\
\hline $\mathrm{C}(33)-\mathrm{H}(33)$ & 0.9300 & $\mathrm{O}(1)-\mathrm{Mo}(1)-\mathrm{Mo}(2)$ & $99.49(11)$ \\
\hline$C(33)-C(34)$ & $1.397(7)$ & $\mathrm{O}(1)-\mathrm{Mo}(1)-\mathrm{Cl}(1)$ & $91.67(11)$ \\
\hline $\mathrm{C}(34)-\mathrm{H}(34)$ & 0.9300 & $\mathrm{O}(1)-\mathrm{Mo}(1)-\mathrm{O}(3)$ & $105.63(14)$ \\
\hline C(34)-C(35) & $1.395(6)$ & $\mathrm{O}(1)-\mathrm{Mo}(1)-\mathrm{O}(4)$ & $112.77(14)$ \\
\hline$C(35)-C(36)$ & $1.477(6)$ & $\mathrm{O}(1)-\mathrm{Mo}(1)-\mathrm{N}(1)$ & $157.38(15)$ \\
\hline$C(36)-C(37)$ & $1.386(6)$ & $\mathrm{O}(1)-\mathrm{Mo}(1)-\mathrm{N}(2)$ & $89.78(16)$ \\
\hline $\mathrm{C}(37)-\mathrm{H}(37)$ & 0.9300 & $\mathrm{O}(3)-\mathrm{Mo}(1)-\mathrm{Mo}(2)$ & $48.19(9)$ \\
\hline
\end{tabular}




\begin{tabular}{|c|c|}
\hline $\mathrm{O}(3)-\mathrm{Mo}(1)-\mathrm{Cl}(1)$ & $161.06(9)$ \\
\hline $\mathrm{O}(3)-\mathrm{Mo}(1)-\mathrm{N}(1)$ & $83.39(13)$ \\
\hline $\mathrm{O}(3)-\mathrm{Mo}(1)-\mathrm{N}(2)$ & $81.26(13)$ \\
\hline $\mathrm{O}(4)-\mathrm{Mo}(1)-\mathrm{Mo}(2)$ & $49.40(9)$ \\
\hline $\mathrm{O}(4)-\mathrm{Mo}(1)-\mathrm{Cl}(1)$ & $88.35(9)$ \\
\hline $\mathrm{O}(4)-\mathrm{Mo}(1)-\mathrm{O}(3)$ & $91.87(13)$ \\
\hline $\mathrm{O}(4)-\mathrm{Mo}(1)-\mathrm{N}(1)$ & $87.12(13)$ \\
\hline $\mathrm{O}(4)-\mathrm{Mo}(1)-\mathrm{N}(2)$ & $157.44(14)$ \\
\hline $\mathrm{N}(1)-\mathrm{Mo}(1)-\mathrm{Mo}(2)$ & $101.82(10)$ \\
\hline $\mathrm{N}(1)-\mathrm{Mo}(1)-\mathrm{Cl}(1)$ & $77.70(9)$ \\
\hline $\mathrm{N}(2)-\mathrm{Mo}(1)-\mathrm{Mo}(2)$ & $129.32(10)$ \\
\hline $\mathrm{N}(2)-\mathrm{Mo}(1)-\mathrm{Cl}(1)$ & $91.36(10)$ \\
\hline $\mathrm{N}(2)-\mathrm{Mo}(1)-\mathrm{N}(1)$ & $70.81(14)$ \\
\hline $\mathrm{Cl}(2)-\mathrm{Mo}(2)-\mathrm{Mo}(1)$ & $134.30(3)$ \\
\hline $\mathrm{O}(2)-\mathrm{Mo}(2)-\mathrm{Mo}(1)$ & $99.47(11)$ \\
\hline $\mathrm{O}(2)-\mathrm{Mo}(2)-\mathrm{Cl}(2)$ & $91.41(12)$ \\
\hline $\mathrm{O}(2)-\mathrm{Mo}(2)-\mathrm{O}(3)$ & $111.93(15)$ \\
\hline $\mathrm{O}(2)-\mathrm{Mo}(2)-\mathrm{O}(4)$ & $106.40(14)$ \\
\hline $\mathrm{O}(2)-\mathrm{Mo}(2)-\mathrm{N}(3)$ & $157.10(15)$ \\
\hline $\mathrm{O}(2)-\mathrm{Mo}(2)-\mathrm{N}(4)$ & $90.17(15)$ \\
\hline $\mathrm{O}(3)-\mathrm{Mo}(2)-\mathrm{Mo}(1)$ & 49.11(9) \\
\hline $\mathrm{O}(3)-\mathrm{Mo}(2)-\mathrm{Cl}(2)$ & $85.62(9)$ \\
\hline $\mathrm{O}(3)-\mathrm{Mo}(2)-\mathrm{O}(4)$ & $91.72(13)$ \\
\hline $\mathrm{O}(3)-\mathrm{Mo}(2)-\mathrm{N}(3)$ & $87.83(13)$ \\
\hline $\mathrm{O}(3)-\mathrm{Mo}(2)-\mathrm{N}(4)$ & $157.85(14)$ \\
\hline $\mathrm{O}(4)-\mathrm{Mo}(2)-\mathrm{Mo}(1)$ & 48.32(9) \\
\hline $\mathrm{O}(4)-\mathrm{Mo}(2)-\mathrm{Cl}(2)$ & 161.61(9) \\
\hline $\mathrm{O}(4)-\mathrm{Mo}(2)-\mathrm{N}(3)$ & $83.51(13)$ \\
\hline $\mathrm{O}(4)-\mathrm{Mo}(2)-\mathrm{N}(4)$ & $82.91(13)$ \\
\hline N(3)-Mo(2)-Mo(1) & $102.38(10)$ \\
\hline $\mathrm{N}(3)-\mathrm{Mo}(2)-\mathrm{Cl}(2)$ & $78.21(10)$ \\
\hline $\mathrm{N}(4)-\mathrm{Mo}(2)-\mathrm{Mo}(1)$ & $131.11(10)$ \\
\hline $\mathrm{N}(4)-\mathrm{Mo}(2)-\mathrm{Cl}(2)$ & $92.72(10)$ \\
\hline $\mathrm{N}(4)-\mathrm{Mo}(2)-\mathrm{N}(3)$ & $70.26(14)$ \\
\hline $\mathrm{Cl}(3)-\mathrm{Mo}(3)-\mathrm{Mo}(4)$ & $136.07(3)$ \\
\hline $\mathrm{O}(5)-\mathrm{Mo}(3)-\mathrm{Mo}(4)$ & $100.60(11)$ \\
\hline
\end{tabular}

\begin{tabular}{|c|c|}
\hline $\mathrm{O}(5)-\mathrm{Mo}(3)-\mathrm{Cl}(3)$ & $90.07(12)$ \\
\hline $\mathrm{O}(5)-\mathrm{Mo}(3)-\mathrm{O}(7)$ & $113.45(15)$ \\
\hline $\mathrm{O}(5)-\mathrm{Mo}(3)-\mathrm{O}(8)$ & $104.67(15)$ \\
\hline $\mathrm{O}(5)-\mathrm{Mo}(3)-\mathrm{N}(5)$ & $159.00(15)$ \\
\hline $\mathrm{O}(5)-\mathrm{Mo}(3)-\mathrm{N}(6)$ & $92.20(15)$ \\
\hline $\mathrm{O}(7)-\mathrm{Mo}(3)-\mathrm{Mo}(4)$ & $49.27(9)$ \\
\hline $\mathrm{O}(7)-\mathrm{Mo}(3)-\mathrm{Cl}(3)$ & $87.31(9)$ \\
\hline $\mathrm{O}(7)-\mathrm{Mo}(3)-\mathrm{O}(8)$ & $92.55(13)$ \\
\hline $\mathrm{O}(7)-\mathrm{Mo}(3)-\mathrm{N}(5)$ & $84.11(13)$ \\
\hline $\mathrm{O}(7)-\mathrm{Mo}(3)-\mathrm{N}(6)$ & $154.33(13)$ \\
\hline $\mathrm{O}(8)-\mathrm{Mo}(3)-\mathrm{Mo}(4)$ & $48.28(9)$ \\
\hline $\mathrm{O}(8)-\mathrm{Mo}(3)-\mathrm{Cl}(3)$ & 163.91(9) \\
\hline $\mathrm{O}(8)-\mathrm{Mo}(3)-\mathrm{N}(5)$ & $85.06(12)$ \\
\hline $\mathrm{O}(8)-\mathrm{Mo}(3)-\mathrm{N}(6)$ & $81.31(13)$ \\
\hline $\mathrm{N}(5)-\mathrm{Mo}(3)-\mathrm{Mo}(4)$ & $99.71(9)$ \\
\hline $\mathrm{N}(5)-\mathrm{Mo}(3)-\mathrm{Cl}(3)$ & 78.93(9) \\
\hline $\mathrm{N}(6)-\mathrm{Mo}(3)-\mathrm{Mo}(4)$ & $129.57(10)$ \\
\hline $\mathrm{N}(6)-\mathrm{Mo}(3)-\mathrm{Cl}(3)$ & $91.89(10)$ \\
\hline $\mathrm{N}(6)-\mathrm{Mo}(3)-\mathrm{N}(5)$ & $70.58(13)$ \\
\hline $\mathrm{Cl}(4)-\mathrm{Mo}(4)-\mathrm{Mo}(3)$ & $136.47(3)$ \\
\hline $\mathrm{O}(6)-\mathrm{Mo}(4)-\mathrm{Mo}(3)$ & $100.60(11)$ \\
\hline $\mathrm{O}(6)-\mathrm{Mo}(4)-\mathrm{Cl}(4)$ & $89.10(11)$ \\
\hline $\mathrm{O}(6)-\mathrm{Mo}(4)-\mathrm{O}(7)$ & $104.90(14)$ \\
\hline $\mathrm{O}(6)-\mathrm{Mo}(4)-\mathrm{O}(8)$ & $113.23(14)$ \\
\hline $\mathrm{O}(6)-\mathrm{Mo}(4)-\mathrm{N}(7)$ & $158.23(14)$ \\
\hline $\mathrm{O}(6)-\mathrm{Mo}(4)-\mathrm{N}(8)$ & $92.36(14)$ \\
\hline $\mathrm{O}(7)-\mathrm{Mo}(4)-\mathrm{Mo}(3)$ & $48.21(9)$ \\
\hline $\mathrm{O}(7)-\mathrm{Mo}(4)-\mathrm{Cl}(4)$ & $164.55(9)$ \\
\hline $\mathrm{O}(7)-\mathrm{Mo}(4)-\mathrm{N}(7)$ & $86.94(12)$ \\
\hline $\mathrm{O}(7)-\mathrm{Mo}(4)-\mathrm{N}(8)$ & $82.67(13)$ \\
\hline $\mathrm{O}(8)-\mathrm{Mo}(4)-\mathrm{Mo}(3)$ & $49.29(9)$ \\
\hline $\mathrm{O}(8)-\mathrm{Mo}(4)-\mathrm{Cl}(4)$ & $87.79(9)$ \\
\hline $\mathrm{O}(8)-\mathrm{Mo}(4)-\mathrm{O}(7)$ & $92.51(13)$ \\
\hline $\mathrm{O}(8)-\mathrm{Mo}(4)-\mathrm{N}(7)$ & $83.84(13)$ \\
\hline $\mathrm{O}(8)-\mathrm{Mo}(4)-\mathrm{N}(8)$ & $154.31(13)$ \\
\hline N(7)-Mo(4)-Mo(3) & $100.88(9)$ \\
\hline
\end{tabular}




\begin{tabular}{|c|c|}
\hline $\mathrm{N}(7)-\mathrm{Mo}(4)-\mathrm{Cl}(4)$ & $77.72(9)$ \\
\hline $\mathrm{N}(8)-\mathrm{Mo}(4)-\mathrm{Mo}(3)$ & $130.86(9)$ \\
\hline $\mathrm{N}(8)-\mathrm{Mo}(4)-\mathrm{Cl}(4)$ & $90.38(10)$ \\
\hline $\mathrm{N}(8)-\mathrm{Mo}(4)-\mathrm{N}(7)$ & $70.75(13)$ \\
\hline $\mathrm{Mo}(2)-\mathrm{O}(3)-\mathrm{Mo}(1)$ & $82.70(12)$ \\
\hline $\mathrm{Mo}(1)-\mathrm{O}(4)-\mathrm{Mo}(2)$ & $82.28(11)$ \\
\hline $\mathrm{Mo}(3)-\mathrm{O}(7)-\mathrm{Mo}(4)$ & $82.52(11)$ \\
\hline $\mathrm{Mo}(4)-\mathrm{O}(8)-\mathrm{Mo}(3)$ & $82.43(12)$ \\
\hline $\mathrm{C}(1)-\mathrm{N}(1)-\mathrm{Mo}(1)$ & $123.3(3)$ \\
\hline $\mathrm{C}(1)-\mathrm{N}(1)-\mathrm{C}(5)$ & $119.3(4)$ \\
\hline $\mathrm{C}(5)-\mathrm{N}(1)-\mathrm{Mo}(1)$ & $117.4(3)$ \\
\hline $\mathrm{C}(6)-\mathrm{N}(2)-\mathrm{Mo}(1)$ & $119.9(3)$ \\
\hline $\mathrm{C}(6)-\mathrm{N}(2)-\mathrm{C}(10)$ & 119.1(4) \\
\hline $\mathrm{C}(10)-\mathrm{N}(2)-\mathrm{Mo}(1)$ & $120.3(3)$ \\
\hline $\mathrm{C}(11)-\mathrm{N}(3)-\mathrm{Mo}(2)$ & $123.4(3)$ \\
\hline $\mathrm{C}(11)-\mathrm{N}(3)-\mathrm{C}(15)$ & $119.0(4)$ \\
\hline $\mathrm{C}(15)-\mathrm{N}(3)-\mathrm{Mo}(2)$ & $117.5(3)$ \\
\hline $\mathrm{C}(16)-\mathrm{N}(4)-\mathrm{Mo}(2)$ & $120.6(3)$ \\
\hline $\mathrm{C}(20)-\mathrm{N}(4)-\mathrm{Mo}(2)$ & $120.2(3)$ \\
\hline $\mathrm{C}(20)-\mathrm{N}(4)-\mathrm{C}(16)$ & $118.7(4)$ \\
\hline $\mathrm{C}(21)-\mathrm{N}(5)-\mathrm{Mo}(3)$ & $123.9(3)$ \\
\hline $\mathrm{C}(21)-\mathrm{N}(5)-\mathrm{C}(25)$ & $118.4(4)$ \\
\hline $\mathrm{C}(25)-\mathrm{N}(5)-\mathrm{Mo}(3)$ & $117.7(3)$ \\
\hline $\mathrm{C}(26)-\mathrm{N}(6)-\mathrm{Mo}(3)$ & $120.1(3)$ \\
\hline $\mathrm{C}(30)-\mathrm{N}(6)-\mathrm{Mo}(3)$ & $120.7(3)$ \\
\hline $\mathrm{C}(30)-\mathrm{N}(6)-\mathrm{C}(26)$ & $118.6(4)$ \\
\hline $\mathrm{C}(31)-\mathrm{N}(7)-\mathrm{Mo}(4)$ & $123.5(3)$ \\
\hline $\mathrm{C}(31)-\mathrm{N}(7)-\mathrm{C}(35)$ & $119.0(4)$ \\
\hline $\mathrm{C}(35)-\mathrm{N}(7)-\mathrm{Mo}(4)$ & $117.3(3)$ \\
\hline $\mathrm{C}(36)-\mathrm{N}(8)-\mathrm{Mo}(4)$ & $120.7(3)$ \\
\hline $\mathrm{C}(40)-\mathrm{N}(8)-\mathrm{Mo}(4)$ & $120.8(3)$ \\
\hline $\mathrm{C}(40)-\mathrm{N}(8)-\mathrm{C}(36)$ & $118.1(4)$ \\
\hline $\mathrm{N}(1)-\mathrm{C}(1)-\mathrm{H}(1)$ & 118.9 \\
\hline $\mathrm{N}(1)-\mathrm{C}(1)-\mathrm{C}(2)$ & $122.2(5)$ \\
\hline $\mathrm{C}(2)-\mathrm{C}(1)-\mathrm{H}(1)$ & 118.9 \\
\hline $\mathrm{C}(1)-\mathrm{C}(2)-\mathrm{H}(2)$ & 120.7 \\
\hline
\end{tabular}

\begin{tabular}{|c|c|}
\hline $\mathrm{C}(3)-\mathrm{C}(2)-\mathrm{C}(1)$ & $118.6(5)$ \\
\hline $\mathrm{C}(3)-\mathrm{C}(2)-\mathrm{H}(2)$ & 120.7 \\
\hline $\mathrm{C}(2)-\mathrm{C}(3)-\mathrm{H}(3)$ & 120.1 \\
\hline$C(2)-C(3)-C(4)$ & $119.8(5)$ \\
\hline $\mathrm{C}(4)-\mathrm{C}(3)-\mathrm{H}(3)$ & 120.1 \\
\hline $\mathrm{C}(3)-\mathrm{C}(4)-\mathrm{H}(4)$ & 120.9 \\
\hline$C(5)-C(4)-C(3)$ & $118.2(5)$ \\
\hline $\mathrm{C}(5)-\mathrm{C}(4)-\mathrm{H}(4)$ & 120.9 \\
\hline $\mathrm{N}(1)-\mathrm{C}(5)-\mathrm{C}(4)$ & $121.9(5)$ \\
\hline $\mathrm{N}(1)-\mathrm{C}(5)-\mathrm{C}(6)$ & $115.2(4)$ \\
\hline$C(4)-C(5)-C(6)$ & $122.8(5)$ \\
\hline $\mathrm{N}(2)-\mathrm{C}(6)-\mathrm{C}(5)$ & $115.6(4)$ \\
\hline $\mathrm{N}(2)-\mathrm{C}(6)-\mathrm{C}(7)$ & $120.7(5)$ \\
\hline$C(7)-C(6)-C(5)$ & $123.6(5)$ \\
\hline $\mathrm{C}(6)-\mathrm{C}(7)-\mathrm{H}(7)$ & 120.3 \\
\hline$C(8)-C(7)-C(6)$ & $119.3(5)$ \\
\hline $\mathrm{C}(8)-\mathrm{C}(7)-\mathrm{H}(7)$ & 120.3 \\
\hline $\mathrm{C}(7)-\mathrm{C}(8)-\mathrm{H}(8)$ & 120.3 \\
\hline $\mathrm{C}(9)-\mathrm{C}(8)-\mathrm{C}(7)$ & $119.4(5)$ \\
\hline $\mathrm{C}(9)-\mathrm{C}(8)-\mathrm{H}(8)$ & 120.3 \\
\hline $\mathrm{C}(8)-\mathrm{C}(9)-\mathrm{H}(9)$ & 120.6 \\
\hline$C(10)-C(9)-C(8)$ & $118.7(5)$ \\
\hline $\mathrm{C}(10)-\mathrm{C}(9)-\mathrm{H}(9)$ & 120.6 \\
\hline $\mathrm{N}(2)-\mathrm{C}(10)-\mathrm{C}(9)$ & $122.6(5)$ \\
\hline $\mathrm{N}(2)-\mathrm{C}(10)-\mathrm{H}(10)$ & 118.7 \\
\hline $\mathrm{C}(9)-\mathrm{C}(10)-\mathrm{H}(10)$ & 118.7 \\
\hline $\mathrm{N}(3)-\mathrm{C}(11)-\mathrm{H}(11)$ & 118.7 \\
\hline $\mathrm{N}(3)-\mathrm{C}(11)-\mathrm{C}(12)$ & $122.6(5)$ \\
\hline $\mathrm{C}(12)-\mathrm{C}(11)-\mathrm{H}(11)$ & 118.7 \\
\hline $\mathrm{C}(11)-\mathrm{C}(12)-\mathrm{H}(12)$ & 120.5 \\
\hline $\mathrm{C}(13)-\mathrm{C}(12)-\mathrm{C}(11)$ & $118.9(5)$ \\
\hline $\mathrm{C}(13)-\mathrm{C}(12)-\mathrm{H}(12)$ & 120.5 \\
\hline $\mathrm{C}(12)-\mathrm{C}(13)-\mathrm{H}(13)$ & 120.3 \\
\hline $\mathrm{C}(12)-\mathrm{C}(13)-\mathrm{C}(14)$ & $119.3(5)$ \\
\hline $\mathrm{C}(14)-\mathrm{C}(13)-\mathrm{H}(13)$ & 120.3 \\
\hline $\mathrm{C}(13)-\mathrm{C}(14)-\mathrm{H}(14)$ & 120.5 \\
\hline
\end{tabular}




\begin{tabular}{|c|c|c|c|}
\hline$C(13)-C(14)-C(15)$ & $118.9(5)$ & $\mathrm{N}(6)-\mathrm{C}(26)-\mathrm{C}(27)$ & 121.2(4) \\
\hline $\mathrm{C}(15)-\mathrm{C}(14)-\mathrm{H}(14)$ & 120.5 & $\mathrm{C}(27)-\mathrm{C}(26)-\mathrm{C}(25)$ & $122.7(4)$ \\
\hline N(3)-C(15)-C(14) & $121.2(4)$ & $\mathrm{C}(26)-\mathrm{C}(27)-\mathrm{H}(27)$ & 120.0 \\
\hline $\mathrm{N}(3)-\mathrm{C}(15)-\mathrm{C}(16)$ & $115.6(4)$ & $\mathrm{C}(28)-\mathrm{C}(27)-\mathrm{C}(26)$ & $120.0(4)$ \\
\hline$C(14)-C(15)-C(16)$ & $123.1(4)$ & $\mathrm{C}(28)-\mathrm{C}(27)-\mathrm{H}(27)$ & 120.0 \\
\hline N(4)-C(16)-C(15) & $115.0(4)$ & $\mathrm{C}(27)-\mathrm{C}(28)-\mathrm{H}(28)$ & 120.7 \\
\hline N(4)-C(16)-C(17) & $121.0(4)$ & $\mathrm{C}(27)-\mathrm{C}(28)-\mathrm{C}(29)$ & $118.6(4)$ \\
\hline$C(17)-C(16)-C(15)$ & $123.9(4)$ & $\mathrm{C}(29)-\mathrm{C}(28)-\mathrm{H}(28)$ & 120.7 \\
\hline $\mathrm{C}(16)-\mathrm{C}(17)-\mathrm{H}(17)$ & 120.3 & $\mathrm{C}(28)-\mathrm{C}(29)-\mathrm{H}(29)$ & 120.6 \\
\hline$C(18)-C(17)-C(16)$ & $119.4(4)$ & $\mathrm{C}(30)-\mathrm{C}(29)-\mathrm{C}(28)$ & $118.7(4)$ \\
\hline $\mathrm{C}(18)-\mathrm{C}(17)-\mathrm{H}(17)$ & 120.3 & $\mathrm{C}(30)-\mathrm{C}(29)-\mathrm{H}(29)$ & 120.6 \\
\hline $\mathrm{C}(17)-\mathrm{C}(18)-\mathrm{H}(18)$ & 120.2 & $\mathrm{~N}(6)-\mathrm{C}(30)-\mathrm{C}(29)$ & $122.9(4)$ \\
\hline$C(19)-C(18)-C(17)$ & $119.7(4)$ & $\mathrm{N}(6)-\mathrm{C}(30)-\mathrm{H}(30)$ & 118.5 \\
\hline $\mathrm{C}(19)-\mathrm{C}(18)-\mathrm{H}(18)$ & 120.2 & $\mathrm{C}(29)-\mathrm{C}(30)-\mathrm{H}(30)$ & 118.5 \\
\hline $\mathrm{C}(18)-\mathrm{C}(19)-\mathrm{H}(19)$ & 121.1 & $\mathrm{~N}(7)-\mathrm{C}(31)-\mathrm{H}(31)$ & 118.7 \\
\hline$C(18)-C(19)-C(20)$ & $117.9(5)$ & $\mathrm{N}(7)-\mathrm{C}(31)-\mathrm{C}(32)$ & $122.6(4)$ \\
\hline $\mathrm{C}(20)-\mathrm{C}(19)-\mathrm{H}(19)$ & 121.1 & $\mathrm{C}(32)-\mathrm{C}(31)-\mathrm{H}(31)$ & 118.7 \\
\hline N(4)-C(20)-C(19) & $123.3(4)$ & $\mathrm{C}(31)-\mathrm{C}(32)-\mathrm{H}(32)$ & 120.7 \\
\hline $\mathrm{N}(4)-\mathrm{C}(20)-\mathrm{H}(20)$ & 118.3 & $\mathrm{C}(33)-\mathrm{C}(32)-\mathrm{C}(31)$ & $118.7(4)$ \\
\hline $\mathrm{C}(19)-\mathrm{C}(20)-\mathrm{H}(20)$ & 118.3 & $\mathrm{C}(33)-\mathrm{C}(32)-\mathrm{H}(32)$ & 120.7 \\
\hline $\mathrm{N}(5)-\mathrm{C}(21)-\mathrm{H}(21)$ & 118.7 & $\mathrm{C}(32)-\mathrm{C}(33)-\mathrm{H}(33)$ & 120.4 \\
\hline N(5)-C(21)-C(22) & $122.6(4)$ & $\mathrm{C}(32)-\mathrm{C}(33)-\mathrm{C}(34)$ & 119.1(4) \\
\hline $\mathrm{C}(22)-\mathrm{C}(21)-\mathrm{H}(21)$ & 118.7 & $\mathrm{C}(34)-\mathrm{C}(33)-\mathrm{H}(33)$ & 120.4 \\
\hline $\mathrm{C}(21)-\mathrm{C}(22)-\mathrm{H}(22)$ & 120.5 & $\mathrm{C}(33)-\mathrm{C}(34)-\mathrm{H}(34)$ & 120.5 \\
\hline $\mathrm{C}(23)-\mathrm{C}(22)-\mathrm{C}(21)$ & $119.0(4)$ & $\mathrm{C}(35)-\mathrm{C}(34)-\mathrm{C}(33)$ & $119.0(4)$ \\
\hline $\mathrm{C}(23)-\mathrm{C}(22)-\mathrm{H}(22)$ & 120.5 & $\mathrm{C}(35)-\mathrm{C}(34)-\mathrm{H}(34)$ & 120.5 \\
\hline $\mathrm{C}(22)-\mathrm{C}(23)-\mathrm{H}(23)$ & 120.5 & $\mathrm{~N}(7)-\mathrm{C}(35)-\mathrm{C}(34)$ & $121.6(4)$ \\
\hline$C(24)-C(23)-C(22)$ & $119.0(4)$ & $\mathrm{N}(7)-\mathrm{C}(35)-\mathrm{C}(36)$ & $115.4(4)$ \\
\hline $\mathrm{C}(24)-\mathrm{C}(23)-\mathrm{H}(23)$ & 120.5 & $\mathrm{C}(34)-\mathrm{C}(35)-\mathrm{C}(36)$ & $123.0(4)$ \\
\hline $\mathrm{C}(23)-\mathrm{C}(24)-\mathrm{H}(24)$ & 120.3 & $\mathrm{~N}(8)-\mathrm{C}(36)-\mathrm{C}(35)$ & $114.9(4)$ \\
\hline$C(23)-C(24)-C(25)$ & $119.3(4)$ & $\mathrm{N}(8)-\mathrm{C}(36)-\mathrm{C}(37)$ & $122.2(4)$ \\
\hline $\mathrm{C}(25)-\mathrm{C}(24)-\mathrm{H}(24)$ & 120.3 & $\mathrm{C}(37)-\mathrm{C}(36)-\mathrm{C}(35)$ & $122.9(4)$ \\
\hline N(5)-C(25)-C(24) & $121.6(4)$ & $\mathrm{C}(36)-\mathrm{C}(37)-\mathrm{H}(37)$ & 120.5 \\
\hline $\mathrm{N}(5)-\mathrm{C}(25)-\mathrm{C}(26)$ & $114.4(4)$ & $\mathrm{C}(36)-\mathrm{C}(37)-\mathrm{C}(38)$ & 119.1(4) \\
\hline$C(24)-C(25)-C(26)$ & $124.0(4)$ & $\mathrm{C}(38)-\mathrm{C}(37)-\mathrm{H}(37)$ & 120.5 \\
\hline $\mathrm{N}(6)-\mathrm{C}(26)-\mathrm{C}(25)$ & 116.1(4) & $\mathrm{C}(37)-\mathrm{C}(38)-\mathrm{H}(38)$ & 120.6 \\
\hline
\end{tabular}




\begin{tabular}{|c|c|c|c|}
\hline $\mathrm{C}(39)-\mathrm{C}(38)-\mathrm{C}(37)$ & $118.8(4)$ & $\mathrm{C}(43)-\mathrm{C}(44)-\mathrm{H}(44 \mathrm{C})$ & 109.5 \\
\hline $\mathrm{C}(39)-\mathrm{C}(38)-\mathrm{H}(38)$ & 120.6 & $\mathrm{H}(44 \mathrm{~A})-\mathrm{C}(44)-\mathrm{H}(44 \mathrm{~B})$ & 109.5 \\
\hline $\mathrm{C}(38)-\mathrm{C}(39)-\mathrm{H}(39)$ & 120.3 & $\mathrm{H}(44 \mathrm{~A})-\mathrm{C}(44)-\mathrm{H}(44 \mathrm{C})$ & 109.5 \\
\hline $\mathrm{C}(40)-\mathrm{C}(39)-\mathrm{C}(38)$ & $119.5(4)$ & $\mathrm{H}(44 \mathrm{~B})-\mathrm{C}(44)-\mathrm{H}(44 \mathrm{C})$ & 109.5 \\
\hline C(40)-C(39)-H(39) & 120.3 & $\mathrm{~N}(11)-\mathrm{C}(45)-\mathrm{C}(46)$ & $179.4(6)$ \\
\hline $\mathrm{N}(8)-\mathrm{C}(40)-\mathrm{C}(39)$ & $122.4(4)$ & $\mathrm{C}(45)-\mathrm{C}(46)-\mathrm{H}(46 \mathrm{~A})$ & 109.5 \\
\hline $\mathrm{N}(8)-\mathrm{C}(40)-\mathrm{H}(40)$ & 118.8 & $\mathrm{C}(45)-\mathrm{C}(46)-\mathrm{H}(46 \mathrm{~B})$ & 109.5 \\
\hline $\mathrm{C}(39)-\mathrm{C}(40)-\mathrm{H}(40)$ & 118.8 & $\mathrm{C}(45)-\mathrm{C}(46)-\mathrm{H}(46 \mathrm{C})$ & 109.5 \\
\hline $\mathrm{H}(9 \mathrm{~A})-\mathrm{O}(9)-\mathrm{H}(9 \mathrm{~B})$ & 116.2 & $\mathrm{H}(46 \mathrm{~A})-\mathrm{C}(46)-\mathrm{H}(46 \mathrm{~B})$ & 109.5 \\
\hline $\mathrm{N}(9)-\mathrm{C}(41)-\mathrm{C}(42)$ & $178.6(7)$ & $\mathrm{H}(46 \mathrm{~A})-\mathrm{C}(46)-\mathrm{H}(46 \mathrm{C})$ & 109.5 \\
\hline $\mathrm{C}(41)-\mathrm{C}(42)-\mathrm{H}(42 \mathrm{~A})$ & 109.5 & $\mathrm{H}(46 \mathrm{~B})-\mathrm{C}(46)-\mathrm{H}(46 \mathrm{C})$ & 109.5 \\
\hline $\mathrm{C}(41)-\mathrm{C}(42)-\mathrm{H}(42 \mathrm{~B})$ & 109.5 & $\mathrm{~N}(12)-\mathrm{C}(47)-\mathrm{C}(48)$ & $178.7(6)$ \\
\hline $\mathrm{C}(41)-\mathrm{C}(42)-\mathrm{H}(42 \mathrm{C})$ & 109.5 & $\mathrm{C}(47)-\mathrm{C}(48)-\mathrm{H}(48 \mathrm{~A})$ & 109.5 \\
\hline $\mathrm{H}(42 \mathrm{~A})-\mathrm{C}(42)-\mathrm{H}(42 \mathrm{~B})$ & 109.5 & $\mathrm{C}(47)-\mathrm{C}(48)-\mathrm{H}(48 \mathrm{~B})$ & 109.5 \\
\hline $\mathrm{H}(42 \mathrm{~A})-\mathrm{C}(42)-\mathrm{H}(42 \mathrm{C})$ & 109.5 & $\mathrm{C}(47)-\mathrm{C}(48)-\mathrm{H}(48 \mathrm{C})$ & 109.5 \\
\hline $\mathrm{H}(42 \mathrm{~B})-\mathrm{C}(42)-\mathrm{H}(42 \mathrm{C})$ & 109.5 & $\mathrm{H}(48 \mathrm{~A})-\mathrm{C}(48)-\mathrm{H}(48 \mathrm{~B})$ & 109.5 \\
\hline $\mathrm{N}(10)-\mathrm{C}(43)-\mathrm{C}(44)$ & $178.0(7)$ & $\mathrm{H}(48 \mathrm{~A})-\mathrm{C}(48)-\mathrm{H}(48 \mathrm{C})$ & 109.5 \\
\hline $\mathrm{C}(43)-\mathrm{C}(44)-\mathrm{H}(44 \mathrm{~A})$ & 109.5 & H(48B)-C(48)-H(48C) & 109.5 \\
\hline $\mathrm{C}(43)-\mathrm{C}(44)-\mathrm{H}(44 \mathrm{~B})$ & 109.5 & & \\
\hline
\end{tabular}


Table 4. Anisotropic displacement parameters $\left(\AA^{2} \times 10^{3}\right)$ for rhowe19. The anisotropic displacement factor exponent takes the form: $-2 \pi^{2}\left[h^{2} a^{* 2} U_{11}+\ldots+2 h k a^{*} b^{*} U_{12}\right]$

\begin{tabular}{|c|c|c|c|c|c|c|}
\hline & $\mathrm{U}_{11}$ & $\mathrm{U}_{22}$ & $\mathrm{U}_{33}$ & $\mathrm{U}_{23}$ & $\mathrm{U}_{13}$ & $\mathrm{U}_{12}$ \\
\hline Mo1 & $13(1)$ & $17(1)$ & $20(1)$ & $-4(1)$ & $-2(1)$ & $-1(1)$ \\
\hline Mo2 & $14(1)$ & $18(1)$ & $23(1)$ & $-3(1)$ & $-2(1)$ & $0(1)$ \\
\hline Mo3 & $15(1)$ & $19(1)$ & $22(1)$ & $-1(1)$ & $-4(1)$ & $-1(1)$ \\
\hline Mo4 & $14(1)$ & $18(1)$ & $20(1)$ & $-3(1)$ & $-2(1)$ & $0(1)$ \\
\hline Cl1 & $22(1)$ & $19(1)$ & $31(1)$ & $-5(1)$ & $-5(1)$ & $-1(1)$ \\
\hline $\mathrm{Cl} 2$ & $22(1)$ & $19(1)$ & $46(1)$ & $-7(1)$ & $-1(1)$ & $-1(1)$ \\
\hline $\mathrm{Cl} 3$ & $21(1)$ & $20(1)$ & $41(1)$ & $-4(1)$ & $-6(1)$ & $-1(1)$ \\
\hline $\mathrm{C} 14$ & $20(1)$ & $20(1)$ & $26(1)$ & $-4(1)$ & $-3(1)$ & $0(1)$ \\
\hline $\mathrm{O} 1$ & $18(1)$ & $24(2)$ & $28(2)$ & $-4(1)$ & $-1(1)$ & $-1(1)$ \\
\hline $\mathrm{O} 2$ & $21(2)$ & $25(2)$ & $30(2)$ & $1(1)$ & $0(1)$ & $-1(1)$ \\
\hline $\mathrm{O} 3$ & $19(1)$ & $19(1)$ & $25(2)$ & $-7(1)$ & $-3(1)$ & $1(1)$ \\
\hline $\mathrm{O} 4$ & $16(1)$ & $19(1)$ & $26(2)$ & $-5(1)$ & $-2(1)$ & $-2(1)$ \\
\hline O5 & $25(2)$ & $27(2)$ & $31(2)$ & $0(1)$ & $-5(1)$ & $-1(1)$ \\
\hline O6 & $20(2)$ & $26(2)$ & $26(2)$ & $-4(1)$ & $-3(1)$ & $0(1)$ \\
\hline O7 & $18(1)$ & $20(1)$ & $22(1)$ & $-4(1)$ & $-3(1)$ & $1(1)$ \\
\hline O8 & $17(1)$ & $21(2)$ & $24(2)$ & $-2(1)$ & $-4(1)$ & $-1(1)$ \\
\hline N1 & $24(2)$ & $18(2)$ & $22(2)$ & $-1(1)$ & $-1(1)$ & $-4(1)$ \\
\hline N2 & $22(2)$ & $19(2)$ & $26(2)$ & $-5(1)$ & $-10(2)$ & $1(1)$ \\
\hline N3 & $17(2)$ & $22(2)$ & $25(2)$ & $-9(1)$ & $-1(1)$ & $1(1)$ \\
\hline N4 & $19(2)$ & $18(2)$ & $27(2)$ & $-4(1)$ & $-5(1)$ & $3(1)$ \\
\hline N5 & $17(2)$ & $14(2)$ & $26(2)$ & $-3(1)$ & $-5(1)$ & $0(1)$ \\
\hline N6 & $19(2)$ & $19(2)$ & $24(2)$ & $-2(1)$ & $-4(1)$ & $-4(1)$ \\
\hline N7 & $16(2)$ & $19(2)$ & $20(2)$ & $-4(1)$ & $-4(1)$ & $0(1)$ \\
\hline N8 & $15(2)$ & $17(2)$ & $24(2)$ & $-3(1)$ & $-2(1)$ & $-2(1)$ \\
\hline $\mathrm{C} 1$ & $27(2)$ & $24(2)$ & $33(2)$ & $-4(2)$ & $4(2)$ & $-8(2)$ \\
\hline $\mathrm{C} 2$ & $24(2)$ & $33(3)$ & $44(3)$ & $-9(2)$ & $9(2)$ & $-10(2)$ \\
\hline $\mathrm{C} 3$ & $46(3)$ & $36(3)$ & $32(3)$ & $-5(2)$ & $14(2)$ & $-12(2)$ \\
\hline $\mathrm{C} 4$ & $47(3)$ & $29(3)$ & $24(2)$ & $-4(2)$ & $2(2)$ & $-7(2)$ \\
\hline C5 & $31(2)$ & $19(2)$ & $23(2)$ & $-2(2)$ & $-3(2)$ & $-2(2)$ \\
\hline C6 & $32(2)$ & $17(2)$ & $26(2)$ & $-4(2)$ & $-9(2)$ & $0(2)$ \\
\hline $\mathrm{C} 7$ & $48(3)$ & $26(2)$ & $32(3)$ & $-2(2)$ & $-16(2)$ & $1(2)$ \\
\hline
\end{tabular}




\begin{tabular}{|c|c|c|c|c|c|c|}
\hline $\mathrm{C} 8$ & $44(3)$ & $30(3)$ & $48(3)$ & $-1(2)$ & $-25(3)$ & $4(2)$ \\
\hline C9 & 29(3) & $26(2)$ & $56(3)$ & $-5(2)$ & $-19(2)$ & $2(2)$ \\
\hline C10 & $20(2)$ & $24(2)$ & $40(3)$ & $-9(2)$ & $-7(2)$ & $4(2)$ \\
\hline $\mathrm{C} 11$ & $26(2)$ & $31(2)$ & $29(2)$ & $-12(2)$ & $-1(2)$ & $3(2)$ \\
\hline $\mathrm{C} 12$ & $33(3)$ & $36(3)$ & $31(2)$ & $-10(2)$ & $2(2)$ & $3(2)$ \\
\hline $\mathrm{C} 13$ & $25(2)$ & $43(3)$ & $37(3)$ & $-8(2)$ & $5(2)$ & $4(2)$ \\
\hline C14 & $17(2)$ & $38(3)$ & $42(3)$ & $-7(2)$ & $-2(2)$ & $2(2)$ \\
\hline $\mathrm{C} 15$ & $14(2)$ & $17(2)$ & $32(2)$ & $-7(2)$ & $-2(2)$ & $2(1)$ \\
\hline C16 & $23(2)$ & $16(2)$ & $29(2)$ & $-7(2)$ & $-4(2)$ & $2(2)$ \\
\hline $\mathrm{C} 17$ & $18(2)$ & $24(2)$ & $39(3)$ & $-8(2)$ & $-8(2)$ & $6(2)$ \\
\hline $\mathrm{C} 18$ & $30(2)$ & $30(2)$ & $36(3)$ & $-11(2)$ & $-15(2)$ & $9(2)$ \\
\hline C19 & $33(3)$ & $37(3)$ & $28(2)$ & $-7(2)$ & $-9(2)$ & $9(2)$ \\
\hline $\mathrm{C} 20$ & $26(2)$ & $30(2)$ & $25(2)$ & $-3(2)$ & $-2(2)$ & $8(2)$ \\
\hline $\mathrm{C} 21$ & $21(2)$ & $23(2)$ & $27(2)$ & $-6(2)$ & $-1(2)$ & $-1(2)$ \\
\hline $\mathrm{C} 22$ & $31(2)$ & $18(2)$ & $27(2)$ & $-4(2)$ & $-9(2)$ & $-1(2)$ \\
\hline $\mathrm{C} 23$ & $24(2)$ & $22(2)$ & $34(2)$ & $-7(2)$ & $-11(2)$ & $2(2)$ \\
\hline $\mathrm{C} 24$ & $18(2)$ & $24(2)$ & $33(2)$ & $-6(2)$ & $-4(2)$ & $1(2)$ \\
\hline $\mathrm{C} 25$ & $18(2)$ & $14(2)$ & $26(2)$ & $-5(2)$ & $-5(2)$ & $0(1)$ \\
\hline $\mathrm{C} 26$ & $21(2)$ & $16(2)$ & $28(2)$ & $-3(2)$ & $-3(2)$ & $-2(2)$ \\
\hline $\mathrm{C} 27$ & $20(2)$ & $22(2)$ & $31(2)$ & $-5(2)$ & $-3(2)$ & $-3(2)$ \\
\hline $\mathrm{C} 28$ & $29(2)$ & $24(2)$ & $30(2)$ & $-3(2)$ & $4(2)$ & $-4(2)$ \\
\hline C29 & $38(3)$ & $24(2)$ & $24(2)$ & $-5(2)$ & $-2(2)$ & $-6(2)$ \\
\hline $\mathrm{C} 30$ & $26(2)$ & $28(2)$ & $29(2)$ & $-1(2)$ & $-4(2)$ & $-9(2)$ \\
\hline $\mathrm{C} 31$ & $17(2)$ & $18(2)$ & $28(2)$ & $-3(2)$ & $-1(2)$ & $-1(2)$ \\
\hline C32 & $18(2)$ & $19(2)$ & $34(2)$ & $-4(2)$ & $-6(2)$ & $3(2)$ \\
\hline $\mathrm{C} 33$ & $26(2)$ & $24(2)$ & $30(2)$ & $-5(2)$ & $-10(2)$ & $3(2)$ \\
\hline C34 & $25(2)$ & $28(2)$ & $24(2)$ & $-3(2)$ & $-7(2)$ & $1(2)$ \\
\hline C35 & $22(2)$ & $17(2)$ & $24(2)$ & $-2(2)$ & $-1(2)$ & $-1(2)$ \\
\hline C36 & $20(2)$ & $17(2)$ & $23(2)$ & $-2(2)$ & $-2(2)$ & $-4(2)$ \\
\hline C37 & $24(2)$ & $23(2)$ & $22(2)$ & $-2(2)$ & $-2(2)$ & $-2(2)$ \\
\hline C38 & $27(2)$ & $24(2)$ & $28(2)$ & $-8(2)$ & $7(2)$ & $-5(2)$ \\
\hline C39 & $16(2)$ & $27(2)$ & $36(2)$ & $-6(2)$ & $2(2)$ & $-6(2)$ \\
\hline $\mathrm{C} 40$ & $19(2)$ & $22(2)$ & $30(2)$ & $-8(2)$ & $-2(2)$ & $-1(2)$ \\
\hline O9 & 71(3) & $54(3)$ & $49(3)$ & $0(2)$ & $-2(2)$ & $-1(2)$ \\
\hline N9 & $35(3)$ & $89(5)$ & $48(3)$ & $-14(3)$ & $5(2)$ & $-7(3)$ \\
\hline \multirow[t]{2}{*}{$\mathrm{C} 41$} & $28(3)$ & $42(3)$ & $44(3)$ & $-21(2)$ & $-15(2)$ & $12(2)$ \\
\hline & & & & S178 & & \\
\hline
\end{tabular}




\begin{tabular}{lllllll}
$\mathrm{C} 42$ & $29(3)$ & $39(3)$ & $53(3)$ & $4(3)$ & $-11(2)$ & $1(2)$ \\
$\mathrm{N} 10$ & $56(3)$ & $42(3)$ & $59(3)$ & $-15(2)$ & $-10(3)$ & $6(2)$ \\
$\mathrm{C} 43$ & $52(4)$ & $32(3)$ & $41(3)$ & $-15(2)$ & $-21(3)$ & $9(2)$ \\
$\mathrm{C} 44$ & $49(3)$ & $38(3)$ & $53(3)$ & $-17(3)$ & $-17(3)$ & $4(2)$ \\
$\mathrm{N} 11$ & $43(3)$ & $58(3)$ & $38(3)$ & $-4(2)$ & $-5(2)$ & $2(2)$ \\
$\mathrm{C} 45$ & $34(3)$ & $34(3)$ & $36(3)$ & $-9(2)$ & $6(2)$ & $-1(2)$ \\
$\mathrm{C} 46$ & $34(3)$ & $24(2)$ & $35(2)$ & $-3(2)$ & $-2(2)$ & $-1(2)$ \\
$\mathrm{N} 12$ & $26(2)$ & $65(3)$ & $41(3)$ & $-6(2)$ & $1(2)$ & $5(2)$ \\
$\mathrm{C} 47$ & $23(2)$ & $35(3)$ & $34(2)$ & $-5(2)$ & $7(2)$ & $1(2)$ \\
$\mathrm{C} 48$ & $23(2)$ & $30(2)$ & $37(3)$ & $0(2)$ & $3(2)$ & $-3(2)$ \\
& & & & & & \\
\hline
\end{tabular}


Table 5. Hydrogen coordinates $\left(\times 10^{4}\right)$ and isotropic displacement parameters $\left(\AA^{2} \times 10^{3}\right)$ for rhowe19.

\begin{tabular}{|c|c|c|c|c|}
\hline & $\mathrm{x}$ & $\mathrm{y}$ & $\mathrm{z}$ & $\mathrm{U}(\mathrm{eq})$ \\
\hline $\mathrm{H} 1$ & 6091 & 6125 & 6958 & 34 \\
\hline $\mathrm{H} 2$ & 5030 & 5645 & 8205 & 40 \\
\hline $\mathrm{H} 3$ & 5698 & 5494 & 9534 & 47 \\
\hline $\mathrm{H} 4$ & 7446 & 5720 & 9585 & 40 \\
\hline $\mathrm{H} 7$ & 9090 & 5947 & 9520 & 42 \\
\hline $\mathrm{H} 8$ & 10849 & 6092 & 9332 & 47 \\
\hline H9 & 11656 & 6436 & 7939 & 43 \\
\hline H10 & 10698 & 6586 & 6778 & 33 \\
\hline H11 & 6756 & 8333 & 7726 & 33 \\
\hline H12 & 5254 & 8371 & 8601 & 40 \\
\hline H13 & 3725 & 8453 & 7980 & 42 \\
\hline H14 & 3738 & 8508 & 6484 & 39 \\
\hline H17 & 3888 & 8654 & 5062 & 32 \\
\hline H18 & 4176 & 8887 & 3559 & 37 \\
\hline H19 & 5840 & 9029 & 2911 & 38 \\
\hline $\mathrm{H} 20$ & 7158 & 8897 & 3791 & 33 \\
\hline $\mathrm{H} 21$ & 7971 & 8625 & 2528 & 28 \\
\hline $\mathrm{H} 22$ & 9379 & 8724 & 3280 & 30 \\
\hline $\mathrm{H} 23$ & 10991 & 8792 & 2534 & 31 \\
\hline $\mathrm{H} 24$ & 11140 & 8751 & 1045 & 30 \\
\hline $\mathrm{H} 27$ & 11142 & 8759 & -351 & 29 \\
\hline $\mathrm{H} 28$ & 11051 & 8800 & -1832 & 34 \\
\hline $\mathrm{H} 29$ & 9446 & 8776 & -2358 & 34 \\
\hline H30 & 8017 & 8699 & -1395 & 33 \\
\hline H31 & 9045 & 6394 & 1612 & 25 \\
\hline H32 & 10191 & 5957 & 2630 & 28 \\
\hline H33 & 9594 & 5649 & 4090 & 31 \\
\hline H34 & 7850 & 5790 & 4488 & 30 \\
\hline H37 & 6239 & 6121 & 4757 & 28 \\
\hline H38 & 4461 & 6136 & 4946 & 32 \\
\hline
\end{tabular}




\begin{tabular}{lrrrr} 
H39 & 3555 & 6265 & 3728 & 32 \\
H40 & 4415 & 6445 & 2370 & 28 \\
H9A & 3503 & 6349 & 6296 & 88 \\
H9B & 3721 & 5848 & 7132 & 88 \\
H42A & 4939 & 9424 & 1396 & 61 \\
H42B & 4156 & 9739 & 716 & 61 \\
H42C & 4737 & 8722 & 703 & 61 \\
H44A & 3646 & 6820 & 576 & 67 \\
H44B & 3151 & 7698 & 0 & 67 \\
H44C & 3286 & 6647 & -323 & 67 \\
H46A & 694 & 7269 & 4296 & 47 \\
H46B & 1679 & 7140 & 3678 & 47 \\
H46C & 1437 & 8177 & 4002 & 47 \\
H48A & -41 & 9467 & 6026 & 46 \\
H48B & 319 & 8432 & 5757 & 46 \\
H48C & 731 & 9426 & 5205 & 46 \\
& & & & \\
\hline
\end{tabular}


Table 6. Torsion angles $\left[{ }^{\circ}\right]$ for rhowe 19.

\begin{tabular}{|c|c|c|c|}
\hline Mo1-N1-C1-C2 & $-178.5(4)$ & N5-C25-C26-C27 & $-179.5(4)$ \\
\hline Mo1-N1-C5-C4 & $177.6(4)$ & N6-C26-C27-C28 & $-1.2(7)$ \\
\hline Mo1-N1-C5-C6 & $-5.2(5)$ & N7-C31-C32-C33 & $0.5(6)$ \\
\hline Mo1-N2-C6-C5 & $9.9(5)$ & N7-C35-C36-N8 & $7.6(6)$ \\
\hline Mo1-N2-C6-C7 & $-172.2(3)$ & N7-C35-C36-C37 & $-174.2(4)$ \\
\hline Mo1-N2-C10-C9 & $171.9(4)$ & N8-C36-C37-C38 & $1.9(7)$ \\
\hline Mo2-N3-C11-C12 & $-179.9(4)$ & C1-N1-C5-C4 & $-2.1(7)$ \\
\hline Mo2-N3-C15-C14 & $178.5(4)$ & C1-N1-C5-C6 & $175.1(4)$ \\
\hline Mo2-N3-C15-C16 & $-4.9(5)$ & $\mathrm{C} 1-\mathrm{C} 2-\mathrm{C} 3-\mathrm{C} 4$ & $-2.3(8)$ \\
\hline Mo2-N4-C16-C15 & $10.1(5)$ & $\mathrm{C} 2-\mathrm{C} 3-\mathrm{C} 4-\mathrm{C} 5$ & $1.4(8)$ \\
\hline Mo2-N4-C16-C17 & $-172.2(3)$ & $\mathrm{C} 3-\mathrm{C} 4-\mathrm{C} 5-\mathrm{N} 1$ & $0.9(7)$ \\
\hline Mo2-N4-C20-C19 & $171.6(4)$ & $\mathrm{C} 3-\mathrm{C} 4-\mathrm{C} 5-\mathrm{C} 6$ & $-176.1(5)$ \\
\hline Mo3-N5-C21-C22 & $175.4(3)$ & $\mathrm{C} 4-\mathrm{C} 5-\mathrm{C} 6-\mathrm{N} 2$ & $174.4(4)$ \\
\hline Mo3-N5-C25-C24 & $-175.1(3)$ & C4-C5-C6-C7 & $-3.4(7)$ \\
\hline Mo3-N5-C25-C26 & $5.6(5)$ & $\mathrm{C} 5-\mathrm{N} 1-\mathrm{C} 1-\mathrm{C} 2$ & $1.1(7)$ \\
\hline Mo3-N6-C26-C25 & $-10.3(5)$ & $\mathrm{C} 5-\mathrm{C} 6-\mathrm{C} 7-\mathrm{C} 8$ & $178.3(5)$ \\
\hline Mo3-N6-C26-C27 & $171.9(3)$ & C6-N2-C10-C9 & $1.3(7)$ \\
\hline Mo3-N6-C30-C29 & $-171.1(4)$ & C6-C7-C8-C9 & $0.6(8)$ \\
\hline Mo4-N7-C31-C32 & $-176.1(3)$ & C7-C8-C9-C10 & $-0.9(8)$ \\
\hline Mo4-N7-C35-C34 & $176.0(3)$ & C8-C9-C10-N2 & $-0.1(8)$ \\
\hline Mo4-N7-C35-C36 & $-1.0(5)$ & C10-N2-C6-C5 & $-179.5(4)$ \\
\hline Mo4-N8-C36-C35 & $-11.1(5)$ & C10-N2-C6-C7 & $-1.5(7)$ \\
\hline Mo4-N8-C36-C37 & $170.6(3)$ & C11-N3-C15-C14 & $-2.9(7)$ \\
\hline Mo4-N8-C40-C39 & $-172.3(3)$ & C11-N3-C15-C16 & $173.8(4)$ \\
\hline $\mathrm{N} 1-\mathrm{C} 1-\mathrm{C} 2-\mathrm{C} 3$ & $1.1(8)$ & C11-C12-C13-C14 & $-0.5(8)$ \\
\hline N1-C5-C6-N2 & $-2.8(6)$ & $\mathrm{C} 12-\mathrm{C} 13-\mathrm{C} 14-\mathrm{C} 15$ & $-0.7(8)$ \\
\hline $\mathrm{N} 1-\mathrm{C} 5-\mathrm{C} 6-\mathrm{C} 7$ & $179.4(4)$ & $\mathrm{C} 13-\mathrm{C} 14-\mathrm{C} 15-\mathrm{N} 3$ & $2.5(7)$ \\
\hline N2-C6-C7-C8 & $0.6(7)$ & $\mathrm{C} 13-\mathrm{C} 14-\mathrm{C} 15-\mathrm{C} 16$ & $-173.9(5)$ \\
\hline N3-C11-C12-C13 & $0.2(8)$ & $\mathrm{C} 14-\mathrm{C} 15-\mathrm{C} 16-\mathrm{N} 4$ & $173.6(4)$ \\
\hline N3-C15-C16-N4 & $-3.0(6)$ & $\mathrm{C} 14-\mathrm{C} 15-\mathrm{C} 16-\mathrm{C} 17$ & $-4.1(7)$ \\
\hline N3-C15-C16-C17 & $179.4(4)$ & C15-N3-C11-C12 & $1.5(7)$ \\
\hline N4-C16-C17-C18 & $1.2(7)$ & C15-C16-C17-C18 & $178.7(4)$ \\
\hline N5-C21-C22-C23 & $1.1(7)$ & C16-N4-C20-C19 & $-0.3(7)$ \\
\hline N5-C25-C26-N6 & $2.7(5)$ & C16-C17-C18-C19 & $-1.3(7)$ \\
\hline
\end{tabular}




\begin{tabular}{|c|c|c|c|}
\hline C17-C18-C19-C20 & $0.6(8)$ & $\mathrm{C} 30-\mathrm{N} 6-\mathrm{C} 26-\mathrm{C} 25$ & $178.5(4)$ \\
\hline C18-C19-C20-N4 & $0.1(8)$ & C30-N6-C26-C27 & $0.7(6)$ \\
\hline C20-N4-C16-C15 & $-178.1(4)$ & C31-N7-C35-C34 & $0.3(6)$ \\
\hline C20-N4-C16-C17 & $-0.4(6)$ & C31-N7-C35-C36 & $-176.7(4)$ \\
\hline C21-N5-C25-C24 & $3.3(6)$ & C31-C32-C33-C34 & $0.0(7)$ \\
\hline $\mathrm{C} 21-\mathrm{N} 5-\mathrm{C} 25-\mathrm{C} 26$ & $-176.0(4)$ & C32-C33-C34-C35 & $-0.4(7)$ \\
\hline $\mathrm{C} 21-\mathrm{C} 22-\mathrm{C} 23-\mathrm{C} 24$ & $0.2(7)$ & C33-C34-C35-N7 & $0.2(7)$ \\
\hline $\mathrm{C} 22-\mathrm{C} 23-\mathrm{C} 24-\mathrm{C} 25$ & $0.3(7)$ & C33-C34-C35-C36 & $177.0(4)$ \\
\hline $\mathrm{C} 23-\mathrm{C} 24-\mathrm{C} 25-\mathrm{N} 5$ & $-2.0(7)$ & C34-C35-C36-N8 & $-169.4(4)$ \\
\hline $\mathrm{C} 23-\mathrm{C} 24-\mathrm{C} 25-\mathrm{C} 26$ & 177.2(4) & C34-C35-C36-C37 & $8.8(7)$ \\
\hline $\mathrm{C} 24-\mathrm{C} 25-\mathrm{C} 26-\mathrm{N} 6$ & $-176.6(4)$ & C35-N7-C31-C32 & $-0.7(6)$ \\
\hline $\mathrm{C} 24-\mathrm{C} 25-\mathrm{C} 26-\mathrm{C} 27$ & $1.2(7)$ & C35-C36-C37-C38 & $-176.2(4)$ \\
\hline C25-N5-C21-C22 & $-2.8(6)$ & C36-N8-C40-C39 & $0.4(6)$ \\
\hline $\mathrm{C} 25-\mathrm{C} 26-\mathrm{C} 27-\mathrm{C} 28$ & $-178.8(4)$ & C36-C37-C38-C39 & $-0.1(7)$ \\
\hline C26-N6-C30-C29 & $0.1(7)$ & C37-C38-C39-C40 & $-1.5(7)$ \\
\hline $\mathrm{C} 26-\mathrm{C} 27-\mathrm{C} 28-\mathrm{C} 29$ & $0.9(7)$ & C38-C39-C40-N8 & $1.4(7)$ \\
\hline C27-C28-C29-C30 & $-0.2(7)$ & C40-N8-C36-C35 & $176.2(4)$ \\
\hline C28-C29-C30-N6 & $-0.3(7)$ & C40-N8-C36-C37 & $-2.1(6)$ \\
\hline
\end{tabular}

Table 7. Hydrogen bonds and close contacts for rhowe $19\left[\AA\right.$ and $\left.^{\circ}\right]$.

\begin{tabular}{lcccc}
\hline D-H...A & d(D-H) & $d(H \ldots A)$ & $d($ D...A $)$ & $<($ DHA $)$ \\
\hline O9-H9A...N11 & 0.85 & 2.10 & $2.953(8)$ & 179.7 \\
O9-H9B...Cl4\#1 & 0.85 & 2.52 & $3.374(5)$ & 179.9 \\
\hline
\end{tabular}

Symmetry transformations used to generate equivalent atoms:

$\# 1-\mathrm{x}+1,-\mathrm{y}+1,-\mathrm{z}+1$ 
REFERENCE NUMBER: rhowe15

\section{CRYSTAL STRUCTURE REPORT}

$\mathrm{C}_{24} \mathrm{H}_{24} \mathrm{Cl}_{2} \mathrm{Mo}_{2} \mathrm{~N}_{4} \mathrm{O}_{5}$

Report prepared for:

Prof. W. Eckenhoff

April 29, 2018

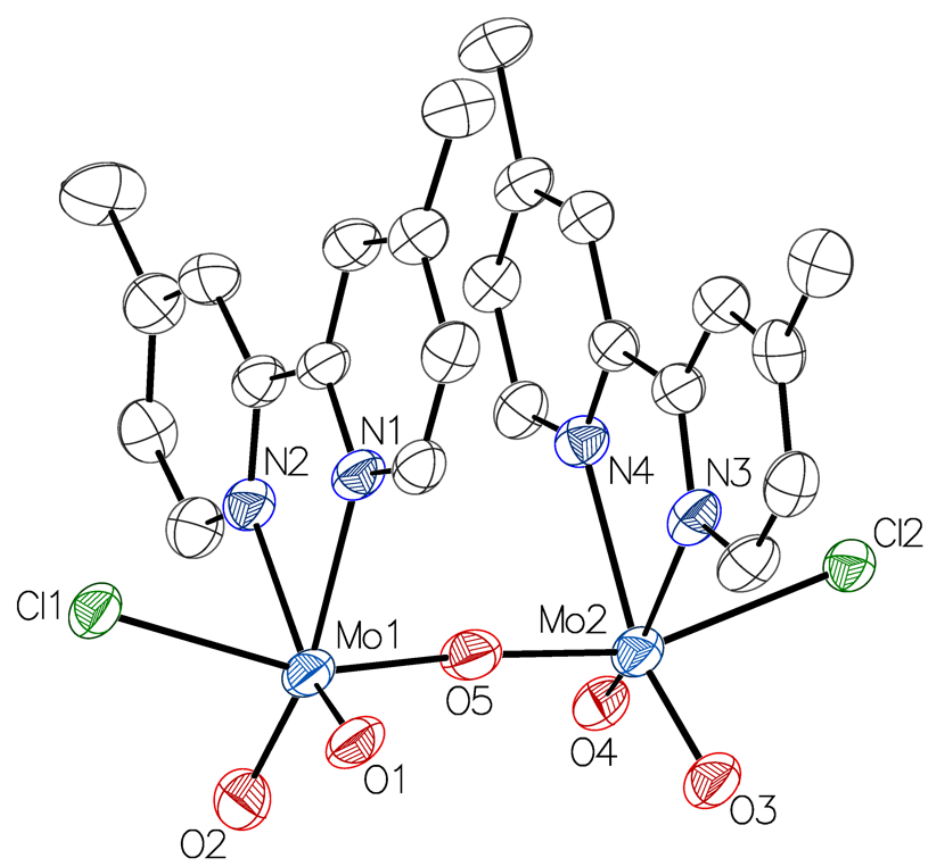

William W. Brennessel

X-ray Crystallographic Facility

Department of Chemistry, University of Rochester

120 Trustee Road 
Rochester, NY 14627 


\section{Data collection}

A crystal $\left(0.119 \times 0.078 \times 0.041 \mathrm{~mm}^{3}\right)$ was placed onto a thin glass optical fiber or a nylon loop and mounted on a XtaLab Synergy-S Dualflex diffractometer equipped with a HyPix-6000HE HPC area detector for data collection at 99.99(10) K. A preliminary set of cell constants and an orientation matrix were calculated from a small sampling of reflections. ${ }^{1}$ A short pre-experiment was run, from which an optimal data collection strategy was determined. The full data collection was carried out using a PhotonJet $(\mathrm{Cu}) \mathrm{X}$-ray Source with frame times of 0.08 and 0.32 seconds and a detector distance of $31.2 \mathrm{~mm}$. Series of frames were collected in $0.50^{\circ}$ steps in $\omega$ at different $2 \theta, \kappa$, and $\phi$ settings. After the intensity data were corrected for absorption, the final cell constants were calculated from the xyz centroids of 13967 strong reflections from the actual data collection after integration. ${ }^{1}$ See Table 1 for additional crystal and refinement information.

\section{Structure solution and refinement}

The structure was solved using ShelXT ${ }^{2}$ and refined using ShelXL. ${ }^{3}$ The space group $P 2{ }_{1} / n$ was determined based on systematic absences. Most or all non-hydrogen atoms were assigned from the solution. Full-matrix least squares / difference Fourier cycles were performed which located any remaining non-hydrogen atoms. All non-hydrogen atoms were refined with anisotropic displacement parameters. All hydrogen atoms were placed in ideal positions and refined as riding atoms with relative isotropic displacement parameters. The final full matrix least squares refinement converged to $R 1=0.0570\left(F^{2}, I>2 \sigma(I)\right)$ and $w R 2=0.1470\left(F^{2}\right.$, all data).

\section{Structure description}

The structure is the one suggested. The asymmetric unit contains one molecule in a general position.

Structure manipulation and figure generation were performed using Olex2. ${ }^{4}$ Unless noted otherwise all structural diagrams containing thermal displacement ellipsoids are drawn at the $50 \%$ probability level.

Data collection, structure solution, and structure refinement were conducted at the X-ray Crystallographic Facility, B04 Hutchison Hall, Department of Chemistry, University of Rochester. All publications arising from this report MUST either 1) include William W. Brennessel as a coauthor or 2) acknowledge William W. Brennessel and the Xray Crystallographic Facility of the Department of Chemistry at the University of Rochester. 
1 CrysAlisPro, version 171.39.46; Rigaku Corporation: Oxford, UK, 2018.

2 Sheldrick, G. M. SHELXT, version 2018/2; Acta. Crystallogr. 2015, A71, 3-8.

3 Sheldrick, G. M. SHELXL, version 2018/3; Acta. Crystallogr. 2015, C71, 3-8.

4 Dolomanov, O. V.; Bourhis, L. J.; Gildea, R. J.; Howard, J. A. K.; Puschmann, H. Olex2, version 1.2-ac3; J. Appl. Cryst. 2009, 42, 339-341.

Some equations of interest:

$$
\begin{gathered}
R_{\mathrm{int}}=\Sigma\left|F_{\mathrm{o}}^{2}-<F_{\mathrm{o}}^{2}>\right| / \Sigma\left|F_{\mathrm{o}}{ }^{2}\right| \\
R 1=\Sigma|| F_{\mathrm{o}}|-| F_{\mathrm{c}}|/ \Sigma| F_{\mathrm{o}} \mid \\
w R 2=\left[\Sigma\left[w\left(F_{\mathrm{o}}{ }^{2} F_{\mathrm{c}}{ }^{2}\right)^{2}\right] / \Sigma\left[w\left(F_{\mathrm{o}}{ }^{2}\right)^{2}\right]\right]^{1 / 2} \\
\text { where } w=1 /\left[\sigma^{2}\left(F_{\mathrm{o}}{ }^{2}\right)+(a P)^{2}+b P\right] \text { and } \\
P=1 / 3 \max \left(0, F_{\mathrm{o}}{ }^{2}\right)+2 / 3 F_{\mathrm{c}}{ }^{2} \\
\mathrm{GOF}=S=\left[\Sigma\left[w\left(F_{\mathrm{o}}{ }^{2}-F_{\mathrm{c}}{ }^{2}\right)^{2}\right] /(m-n)\right]^{1 / 2}
\end{gathered}
$$

where $m=$ number of reflections and $n=$ number of parameters

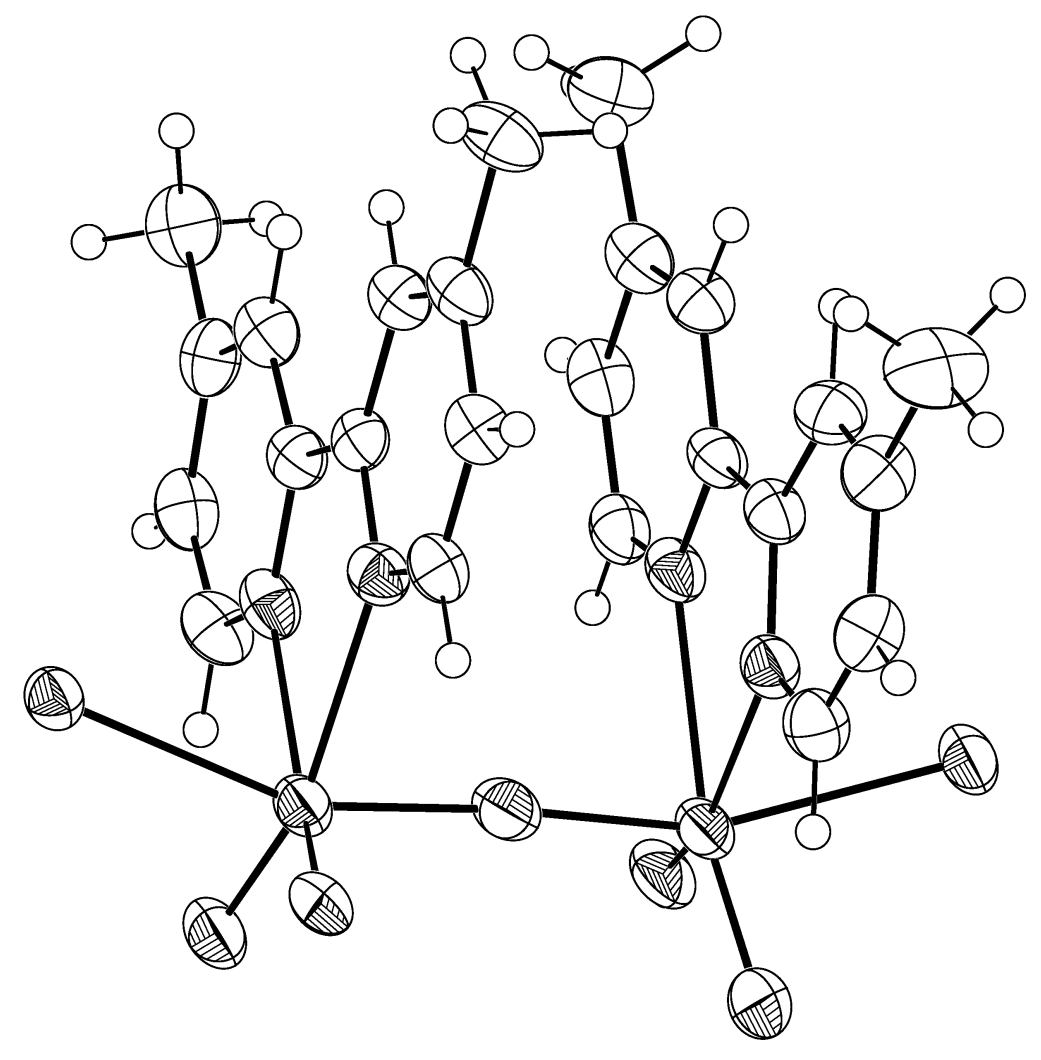



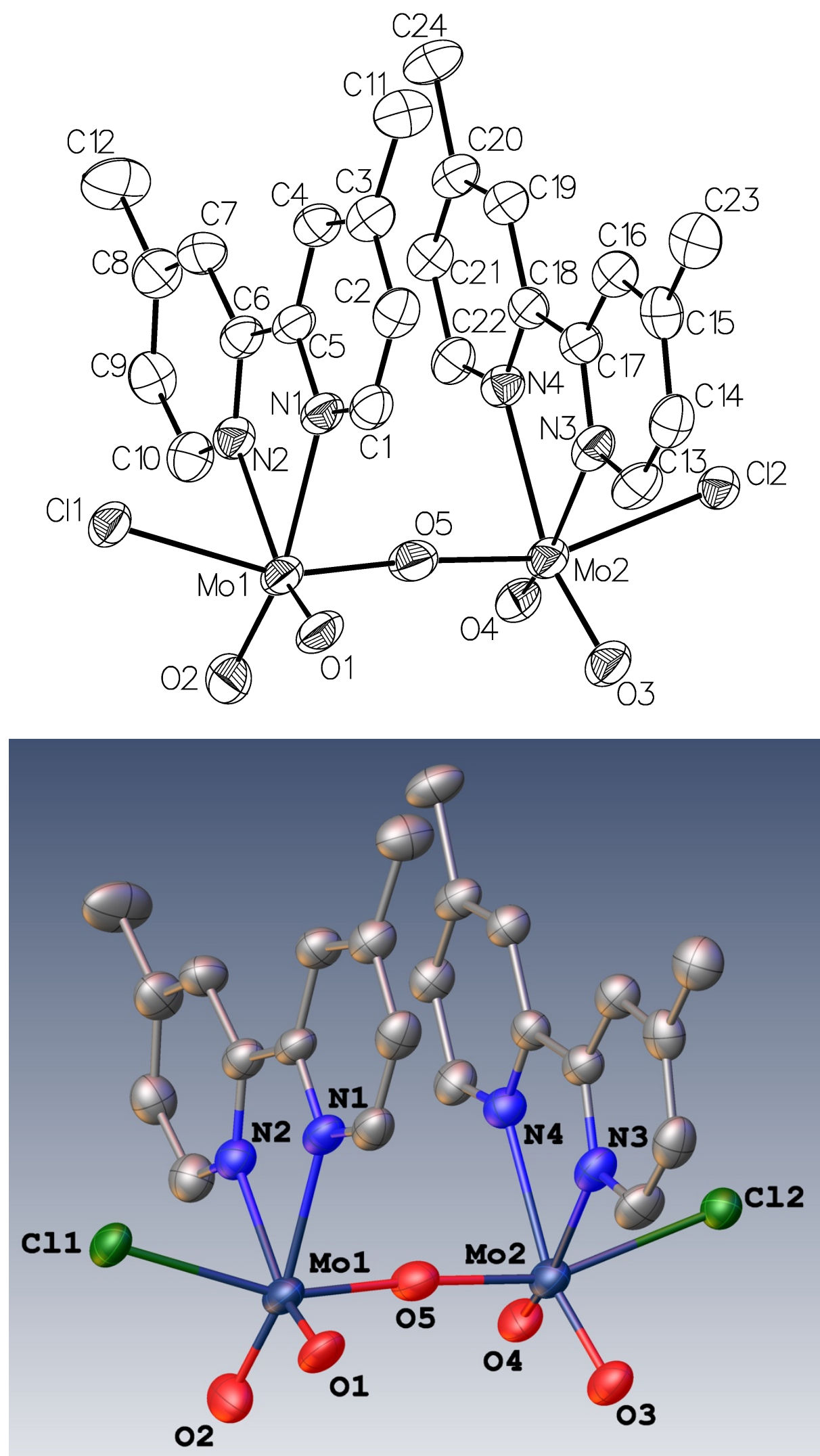
Table 1. Crystal data and structure refinement for rhowe15.

\begin{tabular}{|c|c|c|}
\hline Identification code & \multicolumn{2}{|l|}{ rhowe15 } \\
\hline Empirical formula & \multicolumn{2}{|c|}{$\mathrm{C} 24 \mathrm{H} 24 \mathrm{Cl} 2 \mathrm{Mo} 2 \mathrm{~N} 4 \mathrm{O} 5$} \\
\hline Formula weight & \multicolumn{2}{|l|}{711.25} \\
\hline Temperature & \multicolumn{2}{|l|}{$99.99(10) \mathrm{K}$} \\
\hline Wavelength & \multicolumn{2}{|l|}{$1.54184 \AA$} \\
\hline Crystal system & \multicolumn{2}{|l|}{ monoclinic } \\
\hline Space group & \multicolumn{2}{|l|}{$P 2{ }_{1} / n$} \\
\hline \multirow[t]{3}{*}{ Unit cell dimensions } & $a=13.5024(3) \AA$ & $\alpha=90^{\circ}$ \\
\hline & $b=13.7973(2) \AA$ & $\beta=108.984(3)^{\circ}$ \\
\hline & $c=14.8525(4) \AA$ & $\gamma=90^{\circ}$ \\
\hline Volume & \multicolumn{2}{|l|}{$2616.48(10) \AA^{3}$} \\
\hline$Z$ & \multicolumn{2}{|l|}{4} \\
\hline Density (calculated) & \multicolumn{2}{|l|}{$1.806 \mathrm{Mg} / \mathrm{m}^{3}$} \\
\hline Absorption coefficient & \multicolumn{2}{|l|}{$10.100 \mathrm{~mm}^{-1}$} \\
\hline$F(000)$ & \multicolumn{2}{|l|}{1416} \\
\hline Crystal color, morphology & \multicolumn{2}{|l|}{ orange, plate } \\
\hline Crystal size & \multicolumn{2}{|c|}{$0.119 \times 0.078 \times 0.041 \mathrm{~mm}^{3}$} \\
\hline Theta range for data collection & \multicolumn{2}{|l|}{3.847 to $78.048^{\circ}$} \\
\hline Index ranges & \multicolumn{2}{|c|}{$-17 \leq h \leq 17,-9 \leq k \leq 17,-18 \leq l \leq 18$} \\
\hline Reflections collected & \multicolumn{2}{|l|}{24511} \\
\hline Independent reflections & \multicolumn{2}{|c|}{$5496[R($ int $)=0.0607]$} \\
\hline Observed reflections & \multicolumn{2}{|l|}{5136} \\
\hline Completeness to theta $=74.504^{\circ}$ & \multicolumn{2}{|l|}{$99.7 \%$} \\
\hline Absorption correction & \multicolumn{2}{|l|}{ Multi-scan } \\
\hline Max. and min. transmission & \multicolumn{2}{|c|}{1.00000 and 0.44185} \\
\hline Refinement method & \multicolumn{2}{|c|}{ Full-matrix least-squares on $F^{2}$} \\
\hline Data / restraints / parameters & \multicolumn{2}{|l|}{$5496 / 0 / 338$} \\
\hline Goodness-of-fit on $F^{2}$ & \multicolumn{2}{|l|}{1.056} \\
\hline Final $R$ indices $[I>2 \operatorname{sigma}(I)]$ & \multicolumn{2}{|c|}{$R 1=0.0570, w R 2=0.1429$} \\
\hline$R$ indices (all data) & \multicolumn{2}{|c|}{$R 1=0.0608, w R 2=0.1470$} \\
\hline Largest diff. peak and hole & \multicolumn{2}{|c|}{3.143 and -1.774 e. $\AA^{-3}$} \\
\hline
\end{tabular}


Table 2. Atomic coordinates $\left(\times 10^{4}\right)$ and equivalent isotropic displacement parameters $\left(\AA^{2} \times 10^{3}\right)$ for rhowe 15. $U_{\text {eq }}$ is defined as one third of the trace of the orthogonalized $U_{i j}$ tensor.

\begin{tabular}{|c|c|c|c|c|}
\hline & $\mathrm{x}$ & $\mathrm{y}$ & $\mathrm{z}$ & $\mathrm{U}_{\mathrm{eq}}$ \\
\hline Mo1 & $4358(1)$ & $6787(1)$ & $5627(1)$ & $34(1)$ \\
\hline Mo2 & $3658(1)$ & $4623(1)$ & $6859(1)$ & $32(1)$ \\
\hline $\mathrm{Cl1}$ & $5338(1)$ & $8145(1)$ & $5307(1)$ & $36(1)$ \\
\hline $\mathrm{Cl} 2$ & $3508(1)$ & $3543(1)$ & 8131(1) & $32(1)$ \\
\hline $\mathrm{O} 1$ & $3235(3)$ & $7448(3)$ & $5409(3)$ & $42(1)$ \\
\hline $\mathrm{O} 2$ & $4237(4)$ & $6255(3)$ & $4543(3)$ & $44(1)$ \\
\hline $\mathrm{O} 3$ & 2321(3) & $4656(3)$ & $6327(3)$ & $41(1)$ \\
\hline $\mathrm{O} 4$ & $4077(3)$ & $3680(3)$ & $6333(3)$ & $39(1)$ \\
\hline O5 & $4071(3)$ & $5703(3)$ & $6282(3)$ & $39(1)$ \\
\hline N1 & 4938(3) & $7518(3)$ & $7116(3)$ & $32(1)$ \\
\hline $\mathrm{N} 2$ & $6073(4)$ & $6367(3)$ & $6440(3)$ & $32(1)$ \\
\hline N3 & $3564(3)$ & $5732(3)$ & $7996(4)$ & $32(1)$ \\
\hline N4 & $5276(4)$ & $4825(3)$ & 7993(3) & $31(1)$ \\
\hline $\mathrm{C} 1$ & $4305(4)$ & $8059(4)$ & $7432(4)$ & $36(1)$ \\
\hline $\mathrm{C} 2$ & $4617(4)$ & $8506(4)$ & $8307(5)$ & $36(1)$ \\
\hline $\mathrm{C} 3$ & $5646(5)$ & $8390(4)$ & $8912(4)$ & $36(1)$ \\
\hline $\mathrm{C} 4$ & $6310(4)$ & $7846(4)$ & $8572(4)$ & $33(1)$ \\
\hline $\mathrm{C} 5$ & $5937(4)$ & $7414(4)$ & $7670(4)$ & $30(1)$ \\
\hline C6 & $6589(4)$ & $6787(4)$ & $7284(4)$ & $32(1)$ \\
\hline $\mathrm{C} 7$ & $7659(5)$ & $6631(5)$ & $7736(4)$ & $37(1)$ \\
\hline $\mathrm{C} 8$ & $8198(5)$ & $6008(5)$ & $7330(5)$ & $41(1)$ \\
\hline C9 & $7645(5)$ & $5565(5)$ & $6471(5)$ & $40(1)$ \\
\hline $\mathrm{C} 10$ & $6594(5)$ & $5772(4)$ & $6049(5)$ & $38(1)$ \\
\hline $\mathrm{C} 11$ & $6010(5)$ & $8794(5)$ & $9897(5)$ & $45(2)$ \\
\hline $\mathrm{C} 12$ & $9354(5)$ & $5805(7)$ & $7816(6)$ & $63(2)$ \\
\hline $\mathrm{C} 13$ & $2665(4)$ & $6189(4)$ & $7942(5)$ & $37(1)$ \\
\hline $\mathrm{C} 14$ & $2578(5)$ & $6794(4)$ & $8644(5)$ & $38(1)$ \\
\hline $\mathrm{C} 15$ & $3428(5)$ & $6967(4)$ & $9445(4)$ & $35(1)$ \\
\hline $\mathrm{C} 16$ & $4366(4)$ & $6500(4)$ & $9498(4)$ & $34(1)$ \\
\hline $\mathrm{C} 17$ & $4411(4)$ & $5902(4)$ & $8761(4)$ & $30(1)$ \\
\hline C18 & $5383(4)$ & $5401(4)$ & $8758(4)$ & $28(1)$ \\
\hline
\end{tabular}




\begin{tabular}{lllll}
$\mathrm{C} 19$ & $6338(4)$ & $5516(4)$ & $9472(4)$ & $30(1)$ \\
$\mathrm{C} 20$ & $7220(4)$ & $5026(4)$ & $9413(4)$ & $34(1)$ \\
$\mathrm{C} 21$ & $7095(4)$ & $4443(4)$ & $8627(4)$ & $33(1)$ \\
$\mathrm{C} 22$ & $6126(4)$ & $4362(4)$ & $7929(4)$ & $34(1)$ \\
$\mathrm{C} 23$ & $3371(6)$ & $7644(5)$ & $10208(5)$ & $44(1)$ \\
$\mathrm{C} 24$ & $8253(5)$ & $5141(4)$ & $10195(5)$ & $42(1)$ \\
\hline
\end{tabular}


Table 3. Bond lengths $[\AA]$ and angles $\left[{ }^{\circ}\right]$ for rhowe 15 .

\begin{tabular}{|c|c|c|c|}
\hline $\mathrm{Mo}(1)-\mathrm{Cl}(1)$ & $2.4280(13)$ & $\mathrm{C}(9)-\mathrm{H}(9)$ & 0.9300 \\
\hline $\mathrm{Mo}(1)-\mathrm{O}(1)$ & $1.708(4)$ & $\mathrm{C}(9)-\mathrm{C}(10)$ & $1.382(9)$ \\
\hline $\mathrm{Mo}(1)-\mathrm{O}(2)$ & $1.728(4)$ & $\mathrm{C}(10)-\mathrm{H}(10)$ & 0.9300 \\
\hline $\mathrm{Mo}(1)-\mathrm{O}(5)$ & $1.891(4)$ & $\mathrm{C}(11)-\mathrm{H}(11 \mathrm{~A})$ & 0.9600 \\
\hline $\mathrm{Mo}(1)-\mathrm{N}(1)$ & $2.323(5)$ & $\mathrm{C}(11)-\mathrm{H}(11 \mathrm{~B})$ & 0.9600 \\
\hline $\mathrm{Mo}(1)-\mathrm{N}(2)$ & $2.310(5)$ & $\mathrm{C}(11)-\mathrm{H}(11 \mathrm{C})$ & 0.9600 \\
\hline $\mathrm{Mo}(2)-\mathrm{Cl}(2)$ & $2.4654(13)$ & $\mathrm{C}(12)-\mathrm{H}(12 \mathrm{~A})$ & 0.9600 \\
\hline $\mathrm{Mo}(2)-\mathrm{O}(3)$ & $1.720(4)$ & $\mathrm{C}(12)-\mathrm{H}(12 \mathrm{~B})$ & 0.9600 \\
\hline $\mathrm{Mo}(2)-\mathrm{O}(4)$ & $1.706(4)$ & $\mathrm{C}(12)-\mathrm{H}(12 \mathrm{C})$ & 0.9600 \\
\hline $\mathrm{Mo}(2)-\mathrm{O}(5)$ & $1.892(4)$ & $\mathrm{C}(13)-\mathrm{H}(13)$ & 0.9300 \\
\hline $\mathrm{Mo}(2)-\mathrm{N}(3)$ & $2.313(5)$ & $\mathrm{C}(13)-\mathrm{C}(14)$ & $1.370(9)$ \\
\hline $\mathrm{Mo}(2)-\mathrm{N}(4)$ & $2.301(5)$ & $\mathrm{C}(14)-\mathrm{H}(14)$ & 0.9300 \\
\hline $\mathrm{N}(1)-\mathrm{C}(1)$ & $1.330(8)$ & $\mathrm{C}(14)-\mathrm{C}(15)$ & $1.380(9)$ \\
\hline $\mathrm{N}(1)-\mathrm{C}(5)$ & $1.340(7)$ & $\mathrm{C}(15)-\mathrm{C}(16)$ & $1.400(8)$ \\
\hline $\mathrm{N}(2)-\mathrm{C}(6)$ & $1.351(7)$ & $\mathrm{C}(15)-\mathrm{C}(23)$ & $1.489(9)$ \\
\hline $\mathrm{N}(2)-\mathrm{C}(10)$ & $1.331(8)$ & $\mathrm{C}(16)-\mathrm{H}(16)$ & 0.9300 \\
\hline $\mathrm{N}(3)-\mathrm{C}(13)$ & $1.348(7)$ & $C(16)-C(17)$ & $1.387(8)$ \\
\hline $\mathrm{N}(3)-\mathrm{C}(17)$ & $1.345(7)$ & $\mathrm{C}(17)-\mathrm{C}(18)$ & $1.485(7)$ \\
\hline $\mathrm{N}(4)-\mathrm{C}(18)$ & $1.353(7)$ & $\mathrm{C}(18)-\mathrm{C}(19)$ & $1.387(7)$ \\
\hline $\mathrm{N}(4)-\mathrm{C}(22)$ & $1.344(7)$ & $\mathrm{C}(19)-\mathrm{H}(19)$ & 0.9300 \\
\hline $\mathrm{C}(1)-\mathrm{H}(1)$ & 0.9300 & $\mathrm{C}(19)-\mathrm{C}(20)$ & $1.396(8)$ \\
\hline$C(1)-C(2)$ & $1.375(9)$ & $\mathrm{C}(20)-\mathrm{C}(21)$ & $1.382(8)$ \\
\hline $\mathrm{C}(2)-\mathrm{H}(2)$ & 0.9300 & $C(20)-C(24)$ & $1.506(8)$ \\
\hline$C(2)-C(3)$ & $1.396(8)$ & $\mathrm{C}(21)-\mathrm{H}(21)$ & 0.9300 \\
\hline$C(3)-C(4)$ & $1.384(8)$ & $C(21)-C(22)$ & $1.384(8)$ \\
\hline$C(3)-C(11)$ & 1.493(9) & $\mathrm{C}(22)-\mathrm{H}(22)$ & 0.9300 \\
\hline $\mathrm{C}(4)-\mathrm{H}(4)$ & 0.9300 & $\mathrm{C}(23)-\mathrm{H}(23 \mathrm{~A})$ & 0.9600 \\
\hline$C(4)-C(5)$ & $1.403(8)$ & $\mathrm{C}(23)-\mathrm{H}(23 \mathrm{~B})$ & 0.9600 \\
\hline$C(5)-C(6)$ & $1.475(8)$ & $\mathrm{C}(23)-\mathrm{H}(23 \mathrm{C})$ & 0.9600 \\
\hline$C(6)-C(7)$ & $1.398(8)$ & $\mathrm{C}(24)-\mathrm{H}(24 \mathrm{~A})$ & 0.9600 \\
\hline $\mathrm{C}(7)-\mathrm{H}(7)$ & 0.9300 & $\mathrm{C}(24)-\mathrm{H}(24 \mathrm{~B})$ & 0.9600 \\
\hline $\mathrm{C}(7)-\mathrm{C}(8)$ & $1.385(9)$ & $\mathrm{C}(24)-\mathrm{H}(24 \mathrm{C})$ & 0.9600 \\
\hline$C(8)-C(9)$ & $1.393(9)$ & $\mathrm{O}(1)-\mathrm{Mo}(1)-\mathrm{Cl}(1)$ & $93.31(14)$ \\
\hline$C(8)-C(12)$ & $1.518(9)$ & $\mathrm{O}(1)-\mathrm{Mo}(1)-\mathrm{O}(2)$ & $104.0(2)$ \\
\hline
\end{tabular}




\begin{tabular}{|c|c|c|c|}
\hline $\mathrm{O}(1)-\mathrm{Mo}(1)-\mathrm{O}(5)$ & $101.6(2)$ & $\mathrm{C}(17)-\mathrm{N}(3)-\mathrm{Mo}(2)$ & $119.9(4)$ \\
\hline $\mathrm{O}(1)-\mathrm{Mo}(1)-\mathrm{N}(1)$ & $87.24(19)$ & $\mathrm{C}(17)-\mathrm{N}(3)-\mathrm{C}(13)$ & $118.1(5)$ \\
\hline $\mathrm{O}(1)-\mathrm{Mo}(1)-\mathrm{N}(2)$ & $155.5(2)$ & $\mathrm{C}(18)-\mathrm{N}(4)-\mathrm{Mo}(2)$ & $120.1(4)$ \\
\hline $\mathrm{O}(2)-\mathrm{Mo}(1)-\mathrm{Cl}(1)$ & $92.15(14)$ & $\mathrm{C}(22)-\mathrm{N}(4)-\mathrm{Mo}(2)$ & $121.3(4)$ \\
\hline $\mathrm{O}(2)-\mathrm{Mo}(1)-\mathrm{O}(5)$ & 99.81(19) & $\mathrm{C}(22)-\mathrm{N}(4)-\mathrm{C}(18)$ & $118.6(5)$ \\
\hline $\mathrm{O}(2)-\mathrm{Mo}(1)-\mathrm{N}(1)$ & $166.4(2)$ & $\mathrm{N}(1)-\mathrm{C}(1)-\mathrm{H}(1)$ & 118.3 \\
\hline $\mathrm{O}(2)-\mathrm{Mo}(1)-\mathrm{N}(2)$ & $98.9(2)$ & $\mathrm{N}(1)-\mathrm{C}(1)-\mathrm{C}(2)$ & $123.3(5)$ \\
\hline $\mathrm{O}(5)-\mathrm{Mo}(1)-\mathrm{Cl}(1)$ & $157.91(12)$ & $\mathrm{C}(2)-\mathrm{C}(1)-\mathrm{H}(1)$ & 118.3 \\
\hline $\mathrm{O}(5)-\mathrm{Mo}(1)-\mathrm{N}(1)$ & $85.18(17)$ & $\mathrm{C}(1)-\mathrm{C}(2)-\mathrm{H}(2)$ & 120.3 \\
\hline $\mathrm{O}(5)-\mathrm{Mo}(1)-\mathrm{N}(2)$ & $82.61(17)$ & $C(1)-C(2)-C(3)$ & $119.4(6)$ \\
\hline $\mathrm{N}(1)-\mathrm{Mo}(1)-\mathrm{Cl}(1)$ & $79.32(12)$ & $\mathrm{C}(3)-\mathrm{C}(2)-\mathrm{H}(2)$ & 120.3 \\
\hline $\mathrm{N}(2)-\mathrm{Mo}(1)-\mathrm{Cl}(1)$ & $77.20(12)$ & $C(2)-C(3)-C(11)$ & $122.0(6)$ \\
\hline $\mathrm{N}(2)-\mathrm{Mo}(1)-\mathrm{N}(1)$ & $68.98(17)$ & $C(4)-C(3)-C(2)$ & $117.1(6)$ \\
\hline $\mathrm{O}(3)-\mathrm{Mo}(2)-\mathrm{Cl}(2)$ & $91.86(14)$ & $C(4)-C(3)-C(11)$ & $120.9(5)$ \\
\hline $\mathrm{O}(3)-\mathrm{Mo}(2)-\mathrm{O}(5)$ & $101.00(18)$ & $\mathrm{C}(3)-\mathrm{C}(4)-\mathrm{H}(4)$ & 119.8 \\
\hline $\mathrm{O}(3)-\mathrm{Mo}(2)-\mathrm{N}(3)$ & $91.11(19)$ & $C(3)-C(4)-C(5)$ & $120.3(5)$ \\
\hline $\mathrm{O}(3)-\mathrm{Mo}(2)-\mathrm{N}(4)$ & $159.7(2)$ & $\mathrm{C}(5)-\mathrm{C}(4)-\mathrm{H}(4)$ & 119.8 \\
\hline $\mathrm{O}(4)-\mathrm{Mo}(2)-\mathrm{Cl}(2)$ & $90.71(15)$ & $\mathrm{N}(1)-\mathrm{C}(5)-\mathrm{C}(4)$ & $121.1(5)$ \\
\hline $\mathrm{O}(4)-\mathrm{Mo}(2)-\mathrm{O}(3)$ & $105.4(2)$ & $\mathrm{N}(1)-\mathrm{C}(5)-\mathrm{C}(6)$ & $115.7(5)$ \\
\hline $\mathrm{O}(4)-\mathrm{Mo}(2)-\mathrm{O}(5)$ & $101.7(2)$ & $C(4)-C(5)-C(6)$ & $123.1(5)$ \\
\hline $\mathrm{O}(4)-\mathrm{Mo}(2)-\mathrm{N}(3)$ & $160.75(19)$ & $\mathrm{N}(2)-\mathrm{C}(6)-\mathrm{C}(5)$ & $114.8(5)$ \\
\hline $\mathrm{O}(4)-\mathrm{Mo}(2)-\mathrm{N}(4)$ & $92.90(18)$ & $\mathrm{N}(2)-\mathrm{C}(6)-\mathrm{C}(7)$ & $121.3(5)$ \\
\hline $\mathrm{O}(5)-\mathrm{Mo}(2)-\mathrm{Cl}(2)$ & $158.93(13)$ & $C(7)-C(6)-C(5)$ & $123.9(5)$ \\
\hline $\mathrm{O}(5)-\mathrm{Mo}(2)-\mathrm{N}(3)$ & $84.45(18)$ & $\mathrm{C}(6)-\mathrm{C}(7)-\mathrm{H}(7)$ & 120.2 \\
\hline $\mathrm{O}(5)-\mathrm{Mo}(2)-\mathrm{N}(4)$ & $83.48(16)$ & $C(8)-C(7)-C(6)$ & $119.6(6)$ \\
\hline $\mathrm{N}(3)-\mathrm{Mo}(2)-\mathrm{Cl}(2)$ & $78.63(12)$ & $\mathrm{C}(8)-\mathrm{C}(7)-\mathrm{H}(7)$ & 120.2 \\
\hline $\mathrm{N}(4)-\mathrm{Mo}(2)-\mathrm{Cl}(2)$ & $78.91(12)$ & $C(7)-C(8)-C(9)$ & $118.1(6)$ \\
\hline $\mathrm{N}(4)-\mathrm{Mo}(2)-\mathrm{N}(3)$ & $69.48(16)$ & $C(7)-C(8)-C(12)$ & $120.8(6)$ \\
\hline $\mathrm{Mo}(1)-\mathrm{O}(5)-\mathrm{Mo}(2)$ & $174.8(2)$ & $C(9)-C(8)-C(12)$ & 121.1(6) \\
\hline $\mathrm{C}(1)-\mathrm{N}(1)-\mathrm{Mo}(1)$ & $121.5(4)$ & $\mathrm{C}(8)-\mathrm{C}(9)-\mathrm{H}(9)$ & 120.3 \\
\hline$C(1)-N(1)-C(5)$ & $118.7(5)$ & $\mathrm{C}(10)-\mathrm{C}(9)-\mathrm{C}(8)$ & $119.3(6)$ \\
\hline $\mathrm{C}(5)-\mathrm{N}(1)-\mathrm{Mo}(1)$ & $119.8(4)$ & $\mathrm{C}(10)-\mathrm{C}(9)-\mathrm{H}(9)$ & 120.3 \\
\hline $\mathrm{C}(6)-\mathrm{N}(2)-\mathrm{Mo}(1)$ & $120.2(4)$ & $\mathrm{N}(2)-\mathrm{C}(10)-\mathrm{C}(9)$ & $122.7(6)$ \\
\hline $\mathrm{C}(10)-\mathrm{N}(2)-\mathrm{Mo}(1)$ & $120.6(4)$ & $\mathrm{N}(2)-\mathrm{C}(10)-\mathrm{H}(10)$ & 118.6 \\
\hline $\mathrm{C}(10)-\mathrm{N}(2)-\mathrm{C}(6)$ & $118.9(5)$ & $\mathrm{C}(9)-\mathrm{C}(10)-\mathrm{H}(10)$ & 118.6 \\
\hline $\mathrm{C}(13)-\mathrm{N}(3)-\mathrm{Mo}(2)$ & $121.9(4)$ & $\mathrm{C}(3)-\mathrm{C}(11)-\mathrm{H}(11 \mathrm{~A})$ & 109.5 \\
\hline
\end{tabular}




\begin{tabular}{|c|c|c|c|}
\hline $\mathrm{C}(3)-\mathrm{C}(11)-\mathrm{H}(11 \mathrm{~B})$ & 109.5 & $\mathrm{~N}(4)-\mathrm{C}(18)-\mathrm{C}(19)$ & $121.9(5)$ \\
\hline $\mathrm{C}(3)-\mathrm{C}(11)-\mathrm{H}(11 \mathrm{C})$ & 109.5 & $C(19)-C(18)-C(17)$ & $123.1(5)$ \\
\hline $\mathrm{H}(11 \mathrm{~A})-\mathrm{C}(11)-\mathrm{H}(11 \mathrm{~B})$ & 109.5 & $\mathrm{C}(18)-\mathrm{C}(19)-\mathrm{H}(19)$ & 120.2 \\
\hline $\mathrm{H}(11 \mathrm{~A})-\mathrm{C}(11)-\mathrm{H}(11 \mathrm{C})$ & 109.5 & $\mathrm{C}(18)-\mathrm{C}(19)-\mathrm{C}(20)$ & $119.7(5)$ \\
\hline $\mathrm{H}(11 \mathrm{~B})-\mathrm{C}(11)-\mathrm{H}(11 \mathrm{C})$ & 109.5 & $\mathrm{C}(20)-\mathrm{C}(19)-\mathrm{H}(19)$ & 120.2 \\
\hline $\mathrm{C}(8)-\mathrm{C}(12)-\mathrm{H}(12 \mathrm{~A})$ & 109.5 & $\mathrm{C}(19)-\mathrm{C}(20)-\mathrm{C}(24)$ & $119.7(5)$ \\
\hline $\mathrm{C}(8)-\mathrm{C}(12)-\mathrm{H}(12 \mathrm{~B})$ & 109.5 & $C(21)-C(20)-C(19)$ & $117.6(5)$ \\
\hline $\mathrm{C}(8)-\mathrm{C}(12)-\mathrm{H}(12 \mathrm{C})$ & 109.5 & $C(21)-C(20)-C(24)$ & $122.7(5)$ \\
\hline $\mathrm{H}(12 \mathrm{~A})-\mathrm{C}(12)-\mathrm{H}(12 \mathrm{~B})$ & 109.5 & $\mathrm{C}(20)-\mathrm{C}(21)-\mathrm{H}(21)$ & 119.8 \\
\hline $\mathrm{H}(12 \mathrm{~A})-\mathrm{C}(12)-\mathrm{H}(12 \mathrm{C})$ & 109.5 & $C(20)-C(21)-C(22)$ & $120.3(5)$ \\
\hline $\mathrm{H}(12 \mathrm{~B})-\mathrm{C}(12)-\mathrm{H}(12 \mathrm{C})$ & 109.5 & $\mathrm{C}(22)-\mathrm{C}(21)-\mathrm{H}(21)$ & 119.8 \\
\hline $\mathrm{N}(3)-\mathrm{C}(13)-\mathrm{H}(13)$ & 118.7 & $\mathrm{~N}(4)-\mathrm{C}(22)-\mathrm{C}(21)$ & $122.0(5)$ \\
\hline $\mathrm{N}(3)-\mathrm{C}(13)-\mathrm{C}(14)$ & $122.6(6)$ & $\mathrm{N}(4)-\mathrm{C}(22)-\mathrm{H}(22)$ & 119.0 \\
\hline $\mathrm{C}(14)-\mathrm{C}(13)-\mathrm{H}(13)$ & 118.7 & $\mathrm{C}(21)-\mathrm{C}(22)-\mathrm{H}(22)$ & 119.0 \\
\hline $\mathrm{C}(13)-\mathrm{C}(14)-\mathrm{H}(14)$ & 119.7 & $\mathrm{C}(15)-\mathrm{C}(23)-\mathrm{H}(23 \mathrm{~A})$ & 109.5 \\
\hline$C(13)-C(14)-C(15)$ & $120.5(5)$ & $\mathrm{C}(15)-\mathrm{C}(23)-\mathrm{H}(23 \mathrm{~B})$ & 109.5 \\
\hline $\mathrm{C}(15)-\mathrm{C}(14)-\mathrm{H}(14)$ & 119.7 & $\mathrm{C}(15)-\mathrm{C}(23)-\mathrm{H}(23 \mathrm{C})$ & 109.5 \\
\hline$C(14)-C(15)-C(16)$ & $116.9(6)$ & $\mathrm{H}(23 \mathrm{~A})-\mathrm{C}(23)-\mathrm{H}(23 \mathrm{~B})$ & 109.5 \\
\hline$C(14)-C(15)-C(23)$ & $122.0(6)$ & $\mathrm{H}(23 \mathrm{~A})-\mathrm{C}(23)-\mathrm{H}(23 \mathrm{C})$ & 109.5 \\
\hline$C(16)-C(15)-C(23)$ & 121.1(6) & $\mathrm{H}(23 \mathrm{~B})-\mathrm{C}(23)-\mathrm{H}(23 \mathrm{C})$ & 109.5 \\
\hline $\mathrm{C}(15)-\mathrm{C}(16)-\mathrm{H}(16)$ & 120.0 & $\mathrm{C}(20)-\mathrm{C}(24)-\mathrm{H}(24 \mathrm{~A})$ & 109.5 \\
\hline$C(17)-C(16)-C(15)$ & $120.1(5)$ & $\mathrm{C}(20)-\mathrm{C}(24)-\mathrm{H}(24 \mathrm{~B})$ & 109.5 \\
\hline $\mathrm{C}(17)-\mathrm{C}(16)-\mathrm{H}(16)$ & 120.0 & $\mathrm{C}(20)-\mathrm{C}(24)-\mathrm{H}(24 \mathrm{C})$ & 109.5 \\
\hline $\mathrm{N}(3)-\mathrm{C}(17)-\mathrm{C}(16)$ & $121.8(5)$ & $\mathrm{H}(24 \mathrm{~A})-\mathrm{C}(24)-\mathrm{H}(24 \mathrm{~B})$ & 109.5 \\
\hline $\mathrm{N}(3)-\mathrm{C}(17)-\mathrm{C}(18)$ & $115.1(5)$ & $\mathrm{H}(24 \mathrm{~A})-\mathrm{C}(24)-\mathrm{H}(24 \mathrm{C})$ & 109.5 \\
\hline$C(16)-C(17)-C(18)$ & $123.1(5)$ & $\mathrm{H}(24 \mathrm{~B})-\mathrm{C}(24)-\mathrm{H}(24 \mathrm{C})$ & 109.5 \\
\hline $\mathrm{N}(4)-\mathrm{C}(18)-\mathrm{C}(17)$ & $115.0(5)$ & & \\
\hline
\end{tabular}


Table 4. Anisotropic displacement parameters $\left(\AA^{2} \times 10^{3}\right)$ for rhowe15. The anisotropic displacement factor exponent takes the form: $-2 \pi^{2}\left[h^{2} a^{* 2} U_{11}+\ldots+2 h \mathrm{k} \mathrm{a}^{*} \mathrm{~b}^{*} \mathrm{U}_{12}\right]$

\begin{tabular}{|c|c|c|c|c|c|c|}
\hline & $\mathrm{U}_{11}$ & $\mathrm{U}_{22}$ & $\mathrm{U}_{33}$ & $\mathrm{U}_{23}$ & $\mathrm{U}_{13}$ & $\mathrm{U}_{12}$ \\
\hline Mo1 & $28(1)$ & $34(1)$ & $34(1)$ & $5(1)$ & $2(1)$ & $-9(1)$ \\
\hline Mo2 & $30(1)$ & $30(1)$ & $33(1)$ & $2(1)$ & $6(1)$ & $-7(1)$ \\
\hline $\mathrm{Cl1}$ & $30(1)$ & $33(1)$ & $42(1)$ & $6(1)$ & $7(1)$ & $-8(1)$ \\
\hline $\mathrm{Cl} 2$ & $32(1)$ & $29(1)$ & $33(1)$ & $2(1)$ & $8(1)$ & $-5(1)$ \\
\hline $\mathrm{O} 1$ & $24(2)$ & $48(2)$ & $46(2)$ & $9(2)$ & $0(2)$ & $-10(2)$ \\
\hline $\mathrm{O} 2$ & $58(3)$ & $34(2)$ & $41(2)$ & $-2(2)$ & $16(2)$ & $-16(2)$ \\
\hline $\mathrm{O} 3$ & $34(2)$ & $36(2)$ & $45(2)$ & $9(2)$ & $4(2)$ & $-10(2)$ \\
\hline $\mathrm{O} 4$ & $42(2)$ & $35(2)$ & $39(2)$ & $-8(2)$ & $12(2)$ & $-17(2)$ \\
\hline O5 & $37(2)$ & $36(2)$ & $36(2)$ & $2(2)$ & $1(2)$ & $-9(2)$ \\
\hline N1 & $23(2)$ & $32(2)$ & $37(2)$ & $7(2)$ & $3(2)$ & $-2(2)$ \\
\hline N2 & $31(2)$ & $29(2)$ & $33(2)$ & $2(2)$ & $8(2)$ & $-5(2)$ \\
\hline N3 & $24(2)$ & $29(2)$ & $43(3)$ & $4(2)$ & $9(2)$ & $0(2)$ \\
\hline N4 & $30(2)$ & $26(2)$ & $33(2)$ & $0(2)$ & $8(2)$ & $-2(2)$ \\
\hline $\mathrm{C} 1$ & $27(3)$ & $36(3)$ & $40(3)$ & $10(2)$ & $6(2)$ & $-2(2)$ \\
\hline $\mathrm{C} 2$ & $27(3)$ & $32(3)$ & $48(3)$ & $8(2)$ & $11(2)$ & $6(2)$ \\
\hline $\mathrm{C} 3$ & $31(3)$ & $29(3)$ & $43(3)$ & $2(2)$ & $8(2)$ & $1(2)$ \\
\hline $\mathrm{C} 4$ & $26(2)$ & $32(3)$ & $36(3)$ & $2(2)$ & $6(2)$ & $3(2)$ \\
\hline $\mathrm{C} 5$ & $23(2)$ & $28(2)$ & $33(3)$ & $3(2)$ & $3(2)$ & $-3(2)$ \\
\hline C6 & $26(3)$ & $29(3)$ & $36(3)$ & $5(2)$ & $5(2)$ & $-1(2)$ \\
\hline C7 & $31(3)$ & $42(3)$ & $34(3)$ & $1(2)$ & $4(2)$ & $2(2)$ \\
\hline $\mathrm{C} 8$ & $30(3)$ & $51(4)$ & $43(3)$ & $4(3)$ & $12(2)$ & $8(3)$ \\
\hline C9 & $41(3)$ & $39(3)$ & $41(3)$ & $3(3)$ & $17(3)$ & $6(3)$ \\
\hline $\mathrm{C} 10$ & $40(3)$ & $33(3)$ & $43(3)$ & $0(2)$ & $15(3)$ & $-2(2)$ \\
\hline $\mathrm{C} 11$ & $38(3)$ & $46(3)$ & $47(4)$ & $-10(3)$ & $9(3)$ & $4(3)$ \\
\hline $\mathrm{C} 12$ & $31(3)$ & $96(6)$ & $58(4)$ & $-10(4)$ & $7(3)$ & 21(4) \\
\hline $\mathrm{C} 13$ & $23(2)$ & $36(3)$ & $49(3)$ & $3(3)$ & $6(2)$ & $5(2)$ \\
\hline C14 & $30(3)$ & $35(3)$ & $52(4)$ & $9(3)$ & $16(3)$ & $4(2)$ \\
\hline C15 & $41(3)$ & $25(2)$ & $41(3)$ & $9(2)$ & $17(3)$ & $5(2)$ \\
\hline $\mathrm{C} 16$ & $31(3)$ & $27(3)$ & $40(3)$ & $5(2)$ & $8(2)$ & $3(2)$ \\
\hline $\mathrm{C} 17$ & $30(3)$ & $25(2)$ & $32(3)$ & $5(2)$ & $6(2)$ & $0(2)$ \\
\hline $\mathrm{C} 18$ & $28(3)$ & $24(2)$ & $29(3)$ & $6(2)$ & $7(2)$ & $2(2)$ \\
\hline
\end{tabular}




\begin{tabular}{lllllll}
$\mathrm{C} 19$ & $29(3)$ & $24(2)$ & $35(3)$ & $1(2)$ & $8(2)$ & $1(2)$ \\
$\mathrm{C} 20$ & $29(3)$ & $24(2)$ & $43(3)$ & $5(2)$ & $3(2)$ & $-1(2)$ \\
$\mathrm{C} 21$ & $30(3)$ & $27(2)$ & $41(3)$ & $1(2)$ & $11(2)$ & $3(2)$ \\
$\mathrm{C} 22$ & $34(3)$ & $26(2)$ & $41(3)$ & $0(2)$ & $12(2)$ & $-4(2)$ \\
$\mathrm{C} 23$ & $48(4)$ & $33(3)$ & $53(4)$ & $3(3)$ & $17(3)$ & $4(3)$ \\
$\mathrm{C} 24$ & $28(3)$ & $34(3)$ & $53(4)$ & $-4(3)$ & $-1(3)$ & $2(2)$ \\
\hline
\end{tabular}


Table 5. Hydrogen coordinates $\left(\times 10^{4}\right)$ and isotropic displacement parameters $\left(\AA^{2} \times 10^{3}\right)$ for rhowe15.

\begin{tabular}{|c|c|c|c|c|}
\hline & $\mathrm{x}$ & $\mathrm{y}$ & z & $\mathrm{U}(\mathrm{eq})$ \\
\hline $\mathrm{H} 1$ & 3615 & 8140 & 7040 & 43 \\
\hline $\mathrm{H} 2$ & 4147 & 8882 & 8495 & 44 \\
\hline $\mathrm{H} 4$ & 7007 & 7767 & 8945 & 39 \\
\hline $\mathrm{H} 7$ & 8007 & 6944 & 8306 & 45 \\
\hline H9 & 7980 & 5134 & 6184 & 47 \\
\hline $\mathrm{H} 10$ & 6236 & 5483 & 5468 & 46 \\
\hline H11A & 5475 & 9204 & 9987 & 68 \\
\hline H11B & 6636 & 9166 & 9990 & 68 \\
\hline $\mathrm{H} 11 \mathrm{C}$ & 6153 & 8273 & 10350 & 68 \\
\hline $\mathrm{H} 12 \mathrm{~A}$ & 9718 & 6405 & 8022 & 95 \\
\hline H12B & 9636 & 5490 & 7376 & 95 \\
\hline $\mathrm{H} 12 \mathrm{C}$ & 9441 & 5393 & 8357 & 95 \\
\hline H13 & 2080 & 6090 & 7407 & 45 \\
\hline H14 & 1940 & 7091 & 8579 & 46 \\
\hline H16 & 4959 & 6591 & 10026 & 40 \\
\hline H19 & 6391 & 5917 & 9989 & 36 \\
\hline $\mathrm{H} 21$ & 7665 & 4104 & 8566 & 39 \\
\hline $\mathrm{H} 22$ & 6061 & 3976 & 7400 & 40 \\
\hline $\mathrm{H} 23 \mathrm{~A}$ & 3396 & 8300 & 10002 & 67 \\
\hline $\mathrm{H} 23 \mathrm{~B}$ & 3952 & 7528 & 10775 & 67 \\
\hline $\mathrm{H} 23 \mathrm{C}$ & 2727 & 7540 & 10337 & 67 \\
\hline $\mathrm{H} 24 \mathrm{~A}$ & 8737 & 5474 & 9952 & 63 \\
\hline H24B & 8528 & 4513 & 10424 & 63 \\
\hline $\mathrm{H} 24 \mathrm{C}$ & 8153 & 5509 & 10707 & 63 \\
\hline
\end{tabular}


Table 6. Torsion angles $\left[{ }^{\circ}\right]$ for rhowe 15 .

\begin{tabular}{|c|c|c|c|}
\hline Mo1-N1-C1-C2 & $-179.9(4)$ & $\mathrm{C} 5-\mathrm{C} 6-\mathrm{C} 7-\mathrm{C} 8$ & $178.2(6)$ \\
\hline Mo1-N1-C5-C4 & $-179.9(4)$ & C6-N2-C10-C9 & $0.0(9)$ \\
\hline Mo1-N1-C5-C6 & $-2.5(6)$ & C6-C7-C8-C9 & $0.7(9)$ \\
\hline Mo1-N2-C6-C5 & $7.1(6)$ & C6-C7-C8-C12 & $-178.5(7)$ \\
\hline Mo1-N2-C6-C7 & $-172.5(4)$ & C7-C8-C9-C10 & $1.1(10)$ \\
\hline Mo1-N2-C10-C9 & $174.3(5)$ & C8-C9-C10-N2 & $-1.5(10)$ \\
\hline Mo2-N3-C13-C14 & 175.1(4) & C10-N2-C6-C5 & $-178.5(5)$ \\
\hline Mo2-N3-C17-C16 & $-174.6(4)$ & C10-N2-C6-C7 & $1.9(8)$ \\
\hline Mo2-N3-C17-C18 & $5.1(6)$ & C11-C3-C4-C5 & $175.5(6)$ \\
\hline Mo2-N4-C18-C17 & $-3.0(6)$ & C12-C8-C9-C10 & $-179.7(7)$ \\
\hline Mo2-N4-C18-C19 & $177.8(4)$ & C13-N3-C17-C16 & $2.3(8)$ \\
\hline Mo2-N4-C22-C21 & $-177.1(4)$ & C13-N3-C17-C18 & $-178.0(5)$ \\
\hline N1-C1-C2-C3 & $-0.6(9)$ & C13-C14-C15-C16 & $0.2(8)$ \\
\hline N1-C5-C6-N2 & $-2.9(7)$ & $\mathrm{C} 13-\mathrm{C} 14-\mathrm{C} 15-\mathrm{C} 23$ & $178.1(6)$ \\
\hline N1-C5-C6-C7 & $176.7(5)$ & C14-C15-C16-C17 & $0.4(8)$ \\
\hline N2-C6-C7-C8 & $-2.2(9)$ & C15-C16-C17-N3 & $-1.7(8)$ \\
\hline N3-C13-C14-C15 & $0.5(9)$ & C15-C16-C17-C18 & $178.6(5)$ \\
\hline N3-C17-C18-N4 & $-1.4(7)$ & C16-C17-C18-N4 & $178.3(5)$ \\
\hline N3-C17-C18-C19 & $177.8(5)$ & C16-C17-C18-C19 & $-2.5(8)$ \\
\hline N4-C18-C19-C20 & $-0.3(8)$ & C17-N3-C13-C14 & $-1.7(9)$ \\
\hline C1-N1-C5-C4 & $1.3(8)$ & C17-C18-C19-C20 & $-179.4(5)$ \\
\hline C1-N1-C5-C6 & $178.7(5)$ & C18-N4-C22-C21 & $1.2(8)$ \\
\hline $\mathrm{C} 1-\mathrm{C} 2-\mathrm{C} 3-\mathrm{C} 4$ & $2.1(9)$ & C18-C19-C20-C21 & $0.3(8)$ \\
\hline C1-C2-C3-C11 & $-175.4(6)$ & C18-C19-C20-C24 & $-179.2(5)$ \\
\hline C2-C3-C4-C5 & $-1.9(8)$ & C19-C20-C21-C22 & $0.3(8)$ \\
\hline C3-C4-C5-N1 & $0.3(8)$ & $\mathrm{C} 20-\mathrm{C} 21-\mathrm{C} 22-\mathrm{N} 4$ & $-1.2(9)$ \\
\hline C3-C4-C5-C6 & $-177.0(5)$ & C22-N4-C18-C17 & $178.7(5)$ \\
\hline $\mathrm{C} 4-\mathrm{C} 5-\mathrm{C} 6-\mathrm{N} 2$ & $174.5(5)$ & C22-N4-C18-C19 & $-0.5(8)$ \\
\hline C4-C5-C6-C7 & $-5.9(9)$ & $\mathrm{C} 23-\mathrm{C} 15-\mathrm{C} 16-\mathrm{C} 17$ & $-177.5(5)$ \\
\hline C5-N1-C1-C2 & $-1.2(8)$ & С24-C20-C21-C22 & $179.8(6)$ \\
\hline
\end{tabular}


REFERENCE NUMBER: rhowe14

\section{CRYSTAL STRUCTURE REPORT}

$\mathrm{C}_{24} \mathrm{H}_{24} \mathrm{Cl}_{4} \mathrm{Mo}_{2} \mathrm{~N}_{4} \mathrm{O}_{3}$

Report prepared for:

Prof. W. Eckenhoff

April 29, 2018

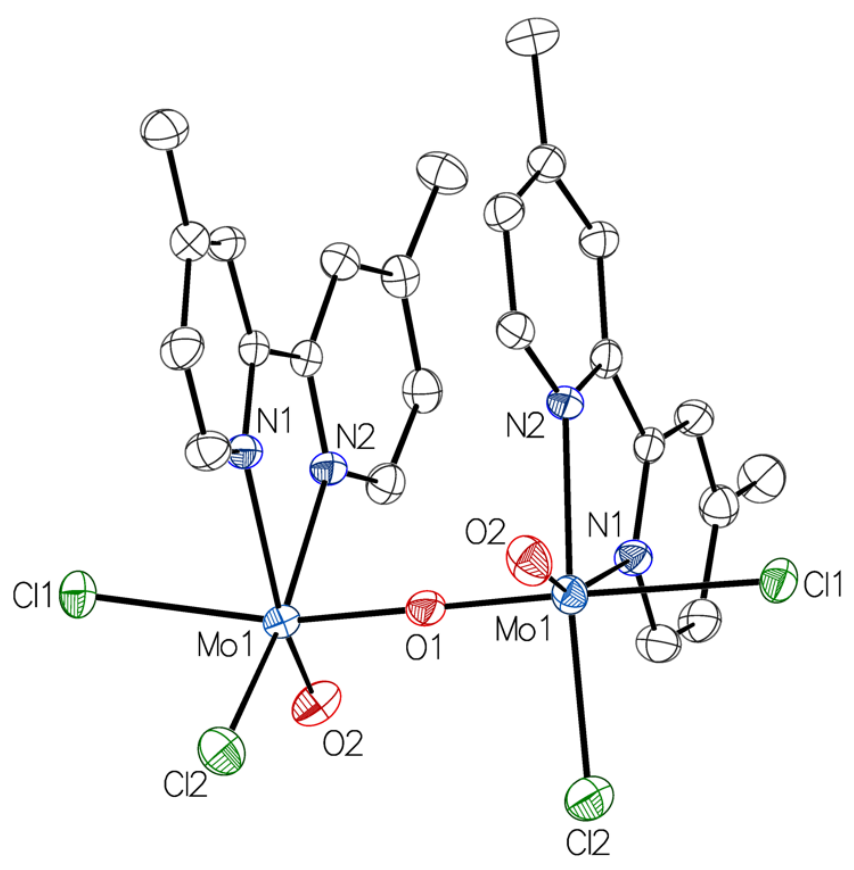

William W. Brennessel

X-ray Crystallographic Facility

Department of Chemistry, University of Rochester

120 Trustee Road 
Rochester, NY 14627 


\section{Data collection}

A crystal $\left(0.16 \times 0.068 \times 0.042 \mathrm{~mm}^{3}\right)$ was placed onto a thin glass optical fiber or a nylon loop and mounted on a XtaLab Synergy-S Dualflex diffractometer equipped with a HyPix-6000HE HPC area detector for data collection at 99.99(10) K. A preliminary set of cell constants and an orientation matrix were calculated from a small sampling of reflections. ${ }^{1}$ A short pre-experiment was run, from which an optimal data collection strategy was determined. The full data collection was carried out using a PhotonJet $(\mathrm{Cu}) \mathrm{X}$-ray Source with frame times of 0.23 and 0.91 seconds and a detector distance of $31.2 \mathrm{~mm}$. Series of frames were collected in $0.50^{\circ}$ steps in $\omega$ at different $2 \theta$, $\kappa$, and $\phi$ settings. After the intensity data were corrected for absorption, the final cell constants were calculated from the xyz centroids of 7018 strong reflections from the actual data collection after integration. ${ }^{1}$ See Table 1 for additional crystal and refinement information.

\section{Structure solution and refinement}

The structure was solved using ShelXT ${ }^{2}$ and refined using ShelXL. ${ }^{3}$ The space group $C 2 / c$ was determined based on systematic absences and intensity statistics. Most or all non-hydrogen atoms were assigned from the solution. Full-matrix least squares / difference Fourier cycles were performed which located any remaining non-hydrogen atoms. All non-hydrogen atoms were refined with anisotropic displacement parameters. All hydrogen atoms were placed in ideal positions and refined as riding atoms with relative isotropic displacement parameters. The final full matrix least squares refinement converged to $R 1=0.0420\left(F^{2}, I>2 \sigma(I)\right)$ and $w R 2=0.1020\left(F^{2}\right.$, all data).

\section{Structure description}

The structure is the one suggested. The asymmetric unit contains one half of the dinuclear complex located at a crystallographic two-fold axis that includes the bridging oxo ligand.

Structure manipulation and figure generation were performed using Olex2. ${ }^{4}$ Unless noted otherwise all structural diagrams containing thermal displacement ellipsoids are drawn at the $50 \%$ probability level.

Data collection, structure solution, and structure refinement were conducted at the X-ray Crystallographic Facility, B04 Hutchison Hall, Department of Chemistry, University of Rochester. All publications arising from this report MUST either 1) include William W. Brennessel as a coauthor or 2) acknowledge William W. Brennessel and the Xray Crystallographic Facility of the Department of Chemistry at the University of Rochester. 
1 CrysAlisPro, version 171.39.46; Rigaku Corporation: Oxford, UK, 2018.

2 Sheldrick, G. M. SHELXT, version 2018/2; Acta. Crystallogr. 2015, A71, 3-8.

3 Sheldrick, G. M. SHELXL, version 2018/3; Acta. Crystallogr. 2015, C71, 3-8.

4 Dolomanov, O. V.; Bourhis, L. J.; Gildea, R. J.; Howard, J. A. K.; Puschmann, H. Olex2, version 1.2-ac3; J. Appl. Cryst. 2009, 42, 339-341.

Some equations of interest:

$$
\begin{gathered}
R_{\text {int }}=\Sigma\left|F_{\mathrm{o}}^{2}-<F_{\mathrm{o}}{ }^{2}>\right| / \Sigma\left|F_{\mathrm{o}}{ }^{2}\right| \\
R 1=\Sigma|| F_{\mathrm{o}}|-| F_{\mathrm{c}}|| / \Sigma\left|F_{\mathrm{o}}\right| \\
w R 2=\left[\Sigma\left[w\left(F_{\mathrm{o}}{ }^{2} F_{\mathrm{c}}{ }^{2}\right)^{2}\right] / \Sigma\left[w\left(F_{\mathrm{o}}{ }^{2}\right)^{2}\right]\right]^{1 / 2} \\
\text { where } w=1 /\left[\sigma^{2}\left(F_{\mathrm{o}}{ }^{2}\right)+(a P)^{2}+b P\right] \text { and } \\
P=1 / 3 \max \left(0, F_{\mathrm{o}}{ }^{2}\right)+2 / 3 F_{\mathrm{c}}{ }^{2} \\
\mathrm{GOF}=S=\left[\Sigma\left[w\left(F_{\mathrm{o}}{ }^{2}-F_{\mathrm{c}}{ }^{2}\right)^{2}\right] /(m-n)\right]^{1 / 2}
\end{gathered}
$$

where $m=$ number of reflections and $n=$ number of parameters

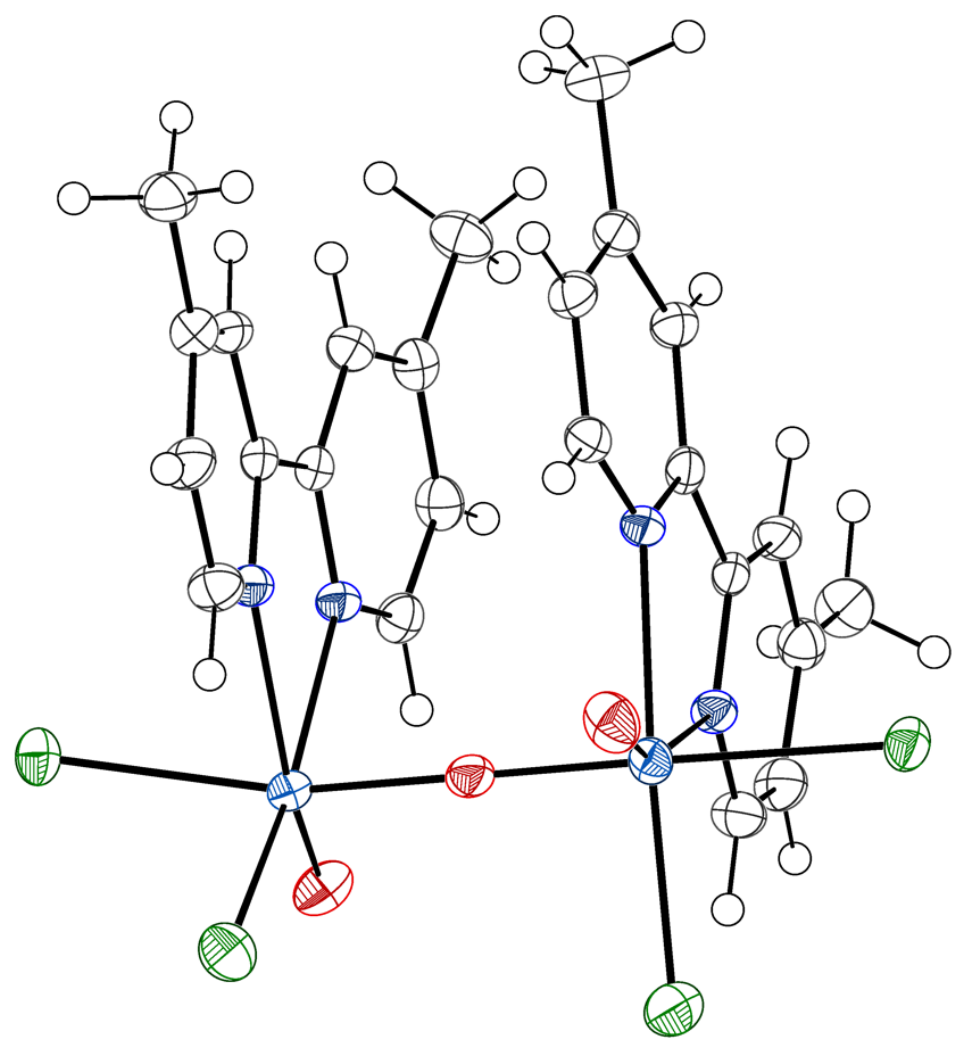



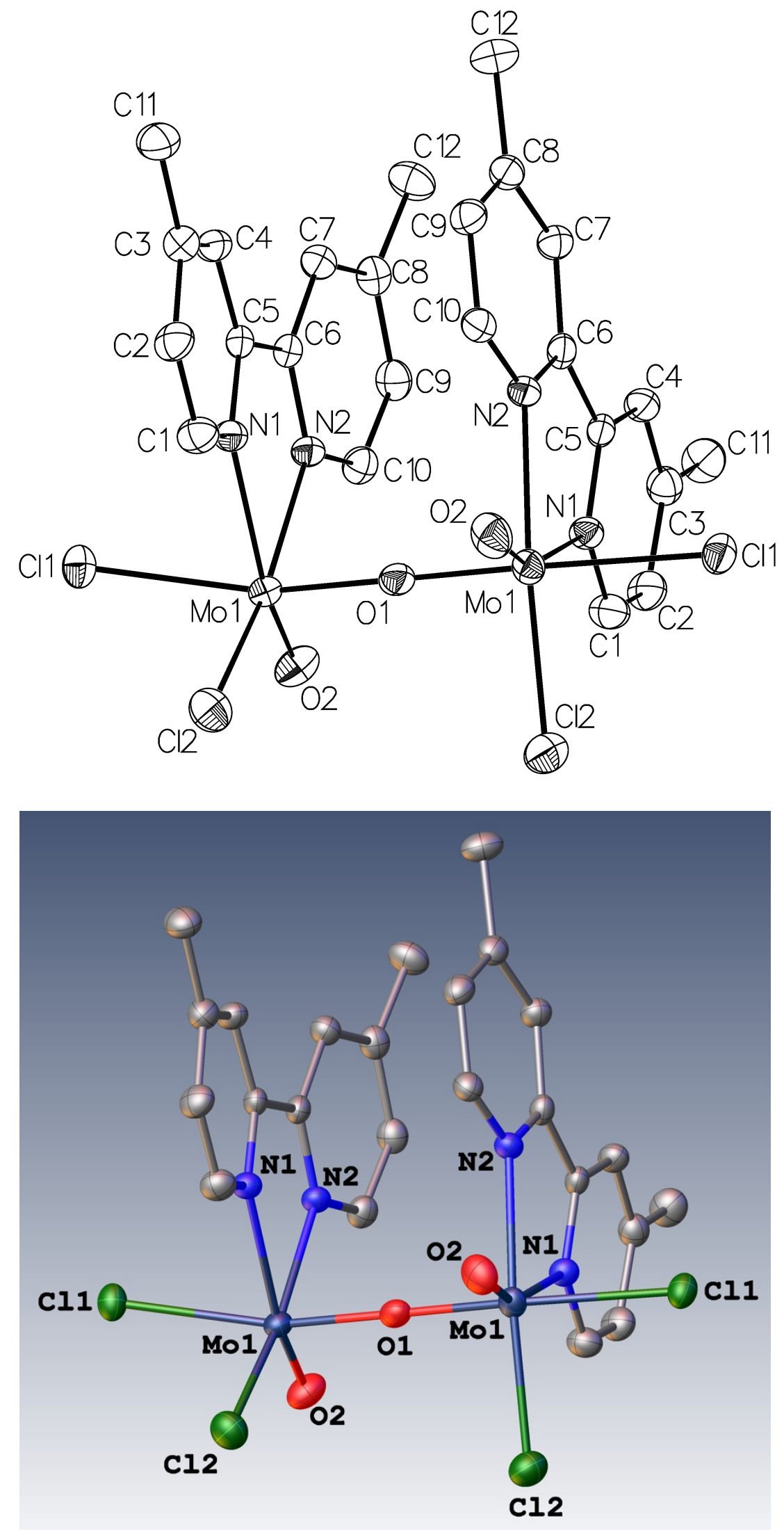
Table 1. Crystal data and structure refinement for rhowe14.

\begin{tabular}{|c|c|c|}
\hline Identification code & \multicolumn{2}{|l|}{ rhowe14 } \\
\hline Empirical formula & \multicolumn{2}{|c|}{$\mathrm{C} 24 \mathrm{H} 24 \mathrm{Cl} 4 \mathrm{Mo} 2 \mathrm{~N} 4 \mathrm{O} 3$} \\
\hline Formula weight & \multicolumn{2}{|l|}{750.15} \\
\hline Temperature & \multicolumn{2}{|l|}{$99.99(10) \mathrm{K}$} \\
\hline Wavelength & \multicolumn{2}{|l|}{$1.54184 \AA$} \\
\hline Crystal system & \multicolumn{2}{|l|}{ monoclinic } \\
\hline Space group & \multicolumn{2}{|l|}{$C 2 / c$} \\
\hline \multirow[t]{3}{*}{ Unit cell dimensions } & $a=13.4906(3) \AA$ & $\alpha=90^{\circ}$ \\
\hline & $b=15.4256(3) \AA$ & $\beta=106.847(2)^{\circ}$ \\
\hline & $c=13.6520(3) \AA$ & $\gamma=90^{\circ}$ \\
\hline Volume & \multicolumn{2}{|l|}{$2719.06(10) \AA^{3}$} \\
\hline$Z$ & \multicolumn{2}{|l|}{4} \\
\hline Density (calculated) & \multicolumn{2}{|l|}{$1.832 \mathrm{Mg} / \mathrm{m}^{3}$} \\
\hline Absorption coefficient & \multicolumn{2}{|l|}{$11.465 \mathrm{~mm}^{-1}$} \\
\hline$F(000)$ & \multicolumn{2}{|l|}{1488} \\
\hline Crystal color, morphology & \multicolumn{2}{|l|}{ orange, needle } \\
\hline Crystal size & \multicolumn{2}{|c|}{$0.16 \times 0.068 \times 0.042 \mathrm{~mm}^{3}$} \\
\hline Theta range for data collection & \multicolumn{2}{|l|}{4.466 to $77.832^{\circ}$} \\
\hline Index ranges & \multicolumn{2}{|c|}{$-16 \leq h \leq 17,-19 \leq k \leq 15,-12 \leq l \leq 17$} \\
\hline Reflections collected & \multicolumn{2}{|l|}{10786} \\
\hline Independent reflections & \multicolumn{2}{|c|}{$2847[R($ int $)=0.0327]$} \\
\hline Observed reflections & \multicolumn{2}{|l|}{2759} \\
\hline Completeness to theta $=74.504^{\circ}$ & \multicolumn{2}{|l|}{$99.7 \%$} \\
\hline Absorption correction & \multicolumn{2}{|l|}{ Multi-scan } \\
\hline Max. and min. transmission & \multicolumn{2}{|c|}{1.00000 and 0.55336} \\
\hline Refinement method & \multicolumn{2}{|c|}{ Full-matrix least-squares on $F^{2}$} \\
\hline Data / restraints / parameters & \multicolumn{2}{|l|}{$2847 / 0 / 170$} \\
\hline Goodness-of-fit on $F^{2}$ & \multicolumn{2}{|l|}{1.095} \\
\hline Final $R$ indices $[I>2 \operatorname{sigma}(I)]$ & \multicolumn{2}{|c|}{$R 1=0.0420, w R 2=0.1015$} \\
\hline$R$ indices (all data) & \multicolumn{2}{|c|}{$R 1=0.0429, w R 2=0.1020$} \\
\hline Largest diff. peak and hole & \multicolumn{2}{|c|}{1.421 and -1.003 e. $\AA^{-3}$} \\
\hline
\end{tabular}


Table 2. Atomic coordinates (x $\left.10^{4}\right)$ and equivalent isotropic displacement parameters $\left(\AA^{2} \times 10^{3}\right)$ for rhowe14. $U_{\text {eq }}$ is defined as one third of the trace of the orthogonalized $U_{i j}$ tensor.

\begin{tabular}{|c|c|c|c|c|}
\hline & $\mathrm{x}$ & $\mathrm{y}$ & $\mathrm{z}$ & $\mathrm{U}_{\mathrm{eq}}$ \\
\hline Mo1 & $3562(1)$ & $5552(1)$ & 2032(1) & $19(1)$ \\
\hline $\mathrm{Cl1}$ & $1747(1)$ & $5720(1)$ & 1939(1) & $24(1)$ \\
\hline $\mathrm{Cl} 2$ & 3553(1) & $4204(1)$ & $2817(1)$ & $32(1)$ \\
\hline $\mathrm{O} 1$ & 5000 & 5551(3) & 2500 & $23(1)$ \\
\hline $\mathrm{O} 2$ & $3353(3)$ & $5359(2)$ & $777(3)$ & $30(1)$ \\
\hline N1 & $3733(3)$ & $6389(2)$ & $3466(3)$ & $18(1)$ \\
\hline $\mathrm{N} 2$ & 3611(3) & $6947(2)$ & $1627(3)$ & $18(1)$ \\
\hline $\mathrm{C} 1$ & $3868(4)$ & $6045(3)$ & $4400(3)$ & $24(1)$ \\
\hline $\mathrm{C} 2$ & 3942(4) & $6551(3)$ & $5255(3)$ & $25(1)$ \\
\hline $\mathrm{C} 3$ & $3855(3)$ & $7449(3)$ & $5164(3)$ & $23(1)$ \\
\hline $\mathrm{C} 4$ & $3698(3)$ & $7796(3)$ & 4183(3) & $21(1)$ \\
\hline $\mathrm{C} 5$ & $3664(3)$ & $7260(3)$ & $3359(3)$ & $17(1)$ \\
\hline $\mathrm{C} 6$ & $3640(3)$ & 7571(3) & $2332(3)$ & $18(1)$ \\
\hline $\mathrm{C} 7$ & $3722(3)$ & $8443(3)$ & $2112(3)$ & $22(1)$ \\
\hline $\mathrm{C} 8$ & $3800(3)$ & 8693(3) & $1150(3)$ & $21(1)$ \\
\hline C9 & $3778(3)$ & $8034(3)$ & $449(3)$ & $23(1)$ \\
\hline $\mathrm{C} 10$ & $3681(3)$ & $7180(3)$ & $706(3)$ & $21(1)$ \\
\hline $\mathrm{C} 11$ & $3946(4)$ & 8013(3) & $6070(4)$ & $30(1)$ \\
\hline $\mathrm{C} 12$ & 3942(4) & $9624(3)$ & $922(4)$ & $28(1)$ \\
\hline
\end{tabular}


Table 3. Bond lengths $[\AA]$ and angles $\left[{ }^{\circ}\right]$ for rhowe 14 .

\begin{tabular}{|c|c|c|c|}
\hline $\operatorname{Mo}(1)-\mathrm{Cl}(1)$ & $2.4282(10)$ & $\mathrm{N}(1)-\mathrm{Mo}(1)-\mathrm{Cl}(1)$ & $80.54(9)$ \\
\hline $\operatorname{Mo}(1)-\mathrm{Cl}(2)$ & $2.3413(12)$ & $\mathrm{N}(1)-\mathrm{Mo}(1)-\mathrm{Cl}(2)$ & $97.00(9)$ \\
\hline $\mathrm{Mo}(1)-\mathrm{O}(1)$ & $1.8582(3)$ & $\mathrm{N}(2)-\mathrm{Mo}(1)-\mathrm{Cl}(1)$ & $89.22(9)$ \\
\hline $\operatorname{Mo}(1)-\mathrm{O}(2)$ & $1.682(3)$ & $\mathrm{N}(2)-\mathrm{Mo}(1)-\mathrm{Cl}(2)$ & $167.51(10)$ \\
\hline $\operatorname{Mo}(1)-\mathrm{N}(1)$ & $2.300(3)$ & $\mathrm{N}(2)-\mathrm{Mo}(1)-\mathrm{N}(1)$ & $70.51(13)$ \\
\hline $\operatorname{Mo}(1)-\mathrm{N}(2)$ & $2.227(3)$ & $\operatorname{Mo}(1) \# 1-\mathrm{O}(1)-\mathrm{Mo}(1)$ & $179.9(3)$ \\
\hline $\mathrm{N}(1)-\mathrm{C}(1)$ & $1.344(6)$ & $\mathrm{C}(1)-\mathrm{N}(1)-\mathrm{Mo}(1)$ & $122.6(3)$ \\
\hline $\mathrm{N}(1)-\mathrm{C}(5)$ & $1.351(5)$ & $\mathrm{C}(1)-\mathrm{N}(1)-\mathrm{C}(5)$ & $118.7(4)$ \\
\hline $\mathrm{N}(2)-\mathrm{C}(6)$ & $1.355(5)$ & $\mathrm{C}(5)-\mathrm{N}(1)-\mathrm{Mo}(1)$ & $118.7(3)$ \\
\hline $\mathrm{N}(2)-\mathrm{C}(10)$ & $1.336(5)$ & $\mathrm{C}(6)-\mathrm{N}(2)-\mathrm{Mo}(1)$ & $120.5(3)$ \\
\hline$C(1)-C(2)$ & $1.383(6)$ & $\mathrm{C}(10)-\mathrm{N}(2)-\mathrm{Mo}(1)$ & $120.6(3)$ \\
\hline $\mathrm{C}(2)-\mathrm{C}(3)$ & $1.392(6)$ & $\mathrm{C}(10)-\mathrm{N}(2)-\mathrm{C}(6)$ & $118.8(4)$ \\
\hline$C(3)-C(4)$ & $1.400(6)$ & $\mathrm{N}(1)-\mathrm{C}(1)-\mathrm{C}(2)$ & $122.3(4)$ \\
\hline$C(3)-C(11)$ & $1.489(6)$ & $\mathrm{C}(1)-\mathrm{C}(2)-\mathrm{C}(3)$ & $120.1(4)$ \\
\hline$C(4)-C(5)$ & $1.386(6)$ & $\mathrm{C}(2)-\mathrm{C}(3)-\mathrm{C}(4)$ & $116.8(4)$ \\
\hline$C(5)-C(6)$ & $1.474(6)$ & $\mathrm{C}(2)-\mathrm{C}(3)-\mathrm{C}(11)$ & $121.5(4)$ \\
\hline$C(6)-C(7)$ & $1.389(6)$ & $C(4)-C(3)-C(11)$ & $121.6(4)$ \\
\hline$C(7)-C(8)$ & $1.401(6)$ & $C(5)-C(4)-C(3)$ & $120.5(4)$ \\
\hline $\mathrm{C}(8)-\mathrm{C}(9)$ & $1.390(6)$ & $\mathrm{N}(1)-\mathrm{C}(5)-\mathrm{C}(4)$ & $121.4(4)$ \\
\hline$C(8)-C(12)$ & $1.493(6)$ & $\mathrm{N}(1)-\mathrm{C}(5)-\mathrm{C}(6)$ & 114.1(4) \\
\hline$C(9)-C(10)$ & $1.381(6)$ & $\mathrm{C}(4)-\mathrm{C}(5)-\mathrm{C}(6)$ & $124.3(4)$ \\
\hline $\mathrm{Cl}(2)-\mathrm{Mo}(1)-\mathrm{Cl}(1)$ & $88.86(4)$ & $\mathrm{N}(2)-\mathrm{C}(6)-\mathrm{C}(5)$ & $115.6(4)$ \\
\hline $\mathrm{O}(1)-\mathrm{Mo}(1)-\mathrm{Cl}(1)$ & $162.65(5)$ & $\mathrm{N}(2)-\mathrm{C}(6)-\mathrm{C}(7)$ & $121.4(4)$ \\
\hline $\mathrm{O}(1)-\mathrm{Mo}(1)-\mathrm{Cl}(2)$ & $89.13(12)$ & $C(7)-C(6)-C(5)$ & $122.7(4)$ \\
\hline $\mathrm{O}(1)-\mathrm{Mo}(1)-\mathrm{N}(1)$ & $82.60(11)$ & $C(6)-C(7)-C(8)$ & $120.2(4)$ \\
\hline $\mathrm{O}(1)-\mathrm{Mo}(1)-\mathrm{N}(2)$ & $89.04(15)$ & $C(7)-C(8)-C(12)$ & $120.7(4)$ \\
\hline $\mathrm{O}(2)-\mathrm{Mo}(1)-\mathrm{Cl}(1)$ & $95.57(12)$ & $\mathrm{C}(9)-\mathrm{C}(8)-\mathrm{C}(7)$ & $116.8(4)$ \\
\hline $\mathrm{O}(2)-\mathrm{Mo}(1)-\mathrm{Cl}(2)$ & $106.72(13)$ & $\mathrm{C}(9)-\mathrm{C}(8)-\mathrm{C}(12)$ & $122.5(4)$ \\
\hline $\mathrm{O}(2)-\mathrm{Mo}(1)-\mathrm{O}(1)$ & $101.51(12)$ & $\mathrm{C}(10)-\mathrm{C}(9)-\mathrm{C}(8)$ & $120.5(4)$ \\
\hline $\mathrm{O}(2)-\mathrm{Mo}(1)-\mathrm{N}(1)$ & $155.91(15)$ & $\mathrm{N}(2)-\mathrm{C}(10)-\mathrm{C}(9)$ & $122.2(4)$ \\
\hline $\mathrm{O}(2)-\mathrm{Mo}(1)-\mathrm{N}(2)$ & $85.74(15)$ & & \\
\hline
\end{tabular}

Symmetry transformations used to generate equivalent atoms:

$\# 1-\mathrm{x}+1, \mathrm{y},-\mathrm{z}+1 / 2$ 
Table 4. Anisotropic displacement parameters $\left(\AA^{2} \times 10^{3}\right)$ for rhowe14. The anisotropic displacement factor exponent takes the form: $-2 \pi^{2}\left[h^{2} a^{* 2} U_{11}+\ldots+2 h k a^{*} b^{*} U_{12}\right]$

\begin{tabular}{|c|c|c|c|c|c|c|}
\hline & $\mathrm{U}_{11}$ & $\mathrm{U}_{22}$ & $\mathrm{U}_{33}$ & $\mathrm{U}_{23}$ & $\mathrm{U}_{13}$ & $\mathrm{U}_{12}$ \\
\hline Mo1 & $20(1)$ & $17(1)$ & $22(1)$ & $-4(1)$ & $9(1)$ & $0(1)$ \\
\hline $\mathrm{Cl1}$ & $21(1)$ & $30(1)$ & $22(1)$ & $2(1)$ & $9(1)$ & $-1(1)$ \\
\hline $\mathrm{Cl} 2$ & $34(1)$ & $28(1)$ & $35(1)$ & $2(1)$ & $13(1)$ & $-3(1)$ \\
\hline $\mathrm{O} 1$ & $28(2)$ & $15(2)$ & $30(2)$ & 0 & $15(2)$ & 0 \\
\hline $\mathrm{O} 2$ & $34(2)$ & $32(2)$ & $27(2)$ & $-4(1)$ & $13(1)$ & $6(1)$ \\
\hline N1 & $21(2)$ & $16(2)$ & $19(2)$ & $-1(1)$ & $7(1)$ & $-1(1)$ \\
\hline $\mathrm{N} 2$ & $20(2)$ & $14(2)$ & $22(2)$ & $0(1)$ & $8(1)$ & $1(1)$ \\
\hline $\mathrm{C} 1$ & $30(2)$ & $20(2)$ & $24(2)$ & $2(2)$ & $9(2)$ & $2(2)$ \\
\hline $\mathrm{C} 2$ & $32(2)$ & $26(2)$ & $22(2)$ & $0(2)$ & $14(2)$ & $1(2)$ \\
\hline $\mathrm{C} 3$ & $22(2)$ & $23(2)$ & $25(2)$ & $-2(2)$ & $8(2)$ & $-1(2)$ \\
\hline $\mathrm{C} 4$ & $26(2)$ & $16(2)$ & $26(2)$ & $-1(2)$ & $12(2)$ & $0(2)$ \\
\hline $\mathrm{C} 5$ & $15(2)$ & $17(2)$ & $21(2)$ & $0(2)$ & $8(2)$ & $-1(2)$ \\
\hline C6 & $16(2)$ & $19(2)$ & $21(2)$ & $-2(2)$ & $8(2)$ & $-1(2)$ \\
\hline $\mathrm{C} 7$ & $23(2)$ & $18(2)$ & $25(2)$ & $0(2)$ & $9(2)$ & $-1(2)$ \\
\hline $\mathrm{C} 8$ & $19(2)$ & $23(2)$ & $20(2)$ & $3(2)$ & $5(2)$ & $1(2)$ \\
\hline C9 & $23(2)$ & $25(2)$ & $22(2)$ & $4(2)$ & $7(2)$ & $0(2)$ \\
\hline $\mathrm{C} 10$ & $21(2)$ & $21(2)$ & $22(2)$ & $3(2)$ & $7(2)$ & $3(2)$ \\
\hline $\mathrm{C} 11$ & $36(3)$ & $28(2)$ & $29(2)$ & $-7(2)$ & $13(2)$ & $-3(2)$ \\
\hline $\mathrm{C} 12$ & $37(3)$ & $21(2)$ & $26(2)$ & $3(2)$ & $8(2)$ & $-5(2)$ \\
\hline
\end{tabular}


Table 5. Hydrogen coordinates $\left(\times 10^{4}\right)$ and isotropic displacement parameters $\left(\AA^{2} \times 10^{3}\right)$ for rhowe14.

\begin{tabular}{lrrrl}
\hline & $x$ & $y$ & $z$ & $U(e q)$ \\
& & & & \\
H1 & 3913 & 5446 & 4474 & 29 \\
H2 & 4051 & 6291 & 5892 & 30 \\
H4 & 3615 & 8391 & 4083 & 26 \\
H7 & 3726 & 8861 & 2604 & 26 \\
H9 & 3829 & 8171 & -198 & 27 \\
H10 & 3662 & 6751 & 222 & 25 \\
H11A & 3853 & 7670 & 6624 & 45 \\
H11B & 3424 & 8456 & 5897 & 45 \\
H11C & 4619 & 8277 & 6275 & 45 \\
H12A & 3426 & 9968 & 1098 & 43 \\
H12B & 3873 & 9689 & 206 & 43 \\
H12C & 4618 & 9812 & 1315 & 43 \\
& & & & \\
\hline
\end{tabular}


Table 6. Torsion angles $\left[{ }^{\circ}\right]$ for rhowe 14 .

\begin{tabular}{lccc}
\hline Mo1-N1-C1-C2 & $-178.2(3)$ & C3-C4-C5-N1 & $3.1(6)$ \\
Mo1-N1-C5-C4 & $176.0(3)$ & C3-C4-C5-C6 & $-171.7(4)$ \\
Mo1-N1-C5-C6 & $-8.7(5)$ & C4-C5-C6-N2 & $179.8(4)$ \\
Mo1-N2-C6-C5 & $1.6(5)$ & C4-C5-C6-C7 & $4.7(6)$ \\
Mo1-N2-C6-C7 & $176.8(3)$ & C5-N1-C1-C2 & $-0.2(7)$ \\
Mo1-N2-C10-C9 & $-175.9(3)$ & C5-C6-C7-C8 & $173.5(4)$ \\
N1-C1-C2-C3 & $1.4(7)$ & C6-N2-C10-C9 & $-0.1(6)$ \\
N1-C5-C6-N2 & $4.7(5)$ & C6-C7-C8-C9 & $0.7(6)$ \\
N1-C5-C6-C7 & $-170.4(4)$ & C6-C7-C8-C12 & $-176.9(4)$ \\
N2-C6-C7-C8 & $-1.3(6)$ & C7-C8-C9-C10 & $0.2(6)$ \\
C1-N1-C5-C4 & $-2.0(6)$ & C8-C9-C10-N2 & $-0.5(7)$ \\
C1-N1-C5-C6 & $173.2(4)$ & C10-N2-C6-C5 & $-174.2(4)$ \\
C1-C2-C3-C4 & $-0.4(7)$ & C10-N2-C6-C7 & $1.0(6)$ \\
C1-C2-C3-C11 & $-179.1(4)$ & C11-C3-C4-C5 & $176.9(4)$ \\
C2-C3-C4-C5 & $-1.8(6)$ & C12-C8-C9-C10 & $177.7(4)$
\end{tabular}

Symmetry transformations used to generate equivalent atoms:

$\# 1-\mathrm{x}+1, \mathrm{y},-\mathrm{z}+1 / 2$ 